Prepared in cooperation with the North Dakota State Water Commission and the North Dakota Department of Health

\title{
Evaluation of Water-Quality Characteristics and Sampling Design for Streams in North Dakota, 1970-2008
}

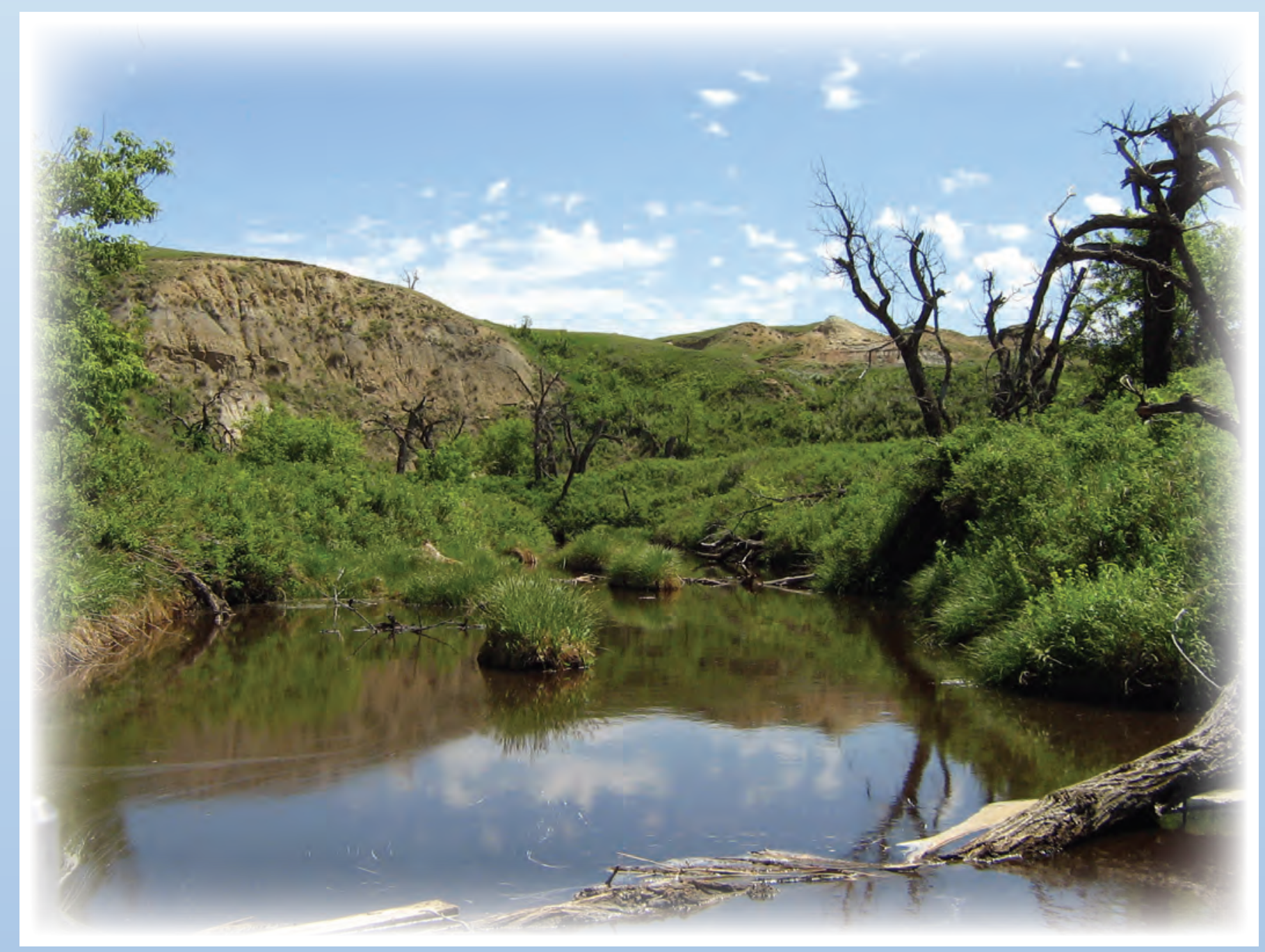

Scientific Investigations Report 2012-5216 
Cover photograph. Short Creek (a tributary to the Souris River) below the International Boundary near Roche Percee, Saskatchewan (photograph by Laurel Cottengaim, U.S. Geological Survey, 2008). 


\section{Evaluation of Water-Quality Characteristics and Sampling Design for Streams in North Dakota, 1970-2008}

By Joel M. Galloway, Aldo V. Vecchia, Kevin C. Vining, Brenda K. Densmore, and Robert F. Lundgren

Prepared in cooperation with the North Dakota State Water Commission and the North Dakota Department of Health

Scientific Investigations Report 2012-5216 


\title{
U.S. Department of the Interior \\ KEN SALAZAR, Secretary \\ U.S. Geological Survey \\ Marcia K. McNutt, Director
}

\author{
U.S. Geological Survey, Reston, Virginia: 2012
}

For more information on the USGS - the Federal source for science about the Earth, its natural and living resources, natural hazards, and the environment, visit http://www.usgs.gov or call 1-888-ASK-USGS.

For an overview of USGS information products, including maps, imagery, and publications, visit http://www.usgs.gov/pubprod

To order other USGS information products, visit http://store.usgs.gov

Any use of trade, firm, or product names is for descriptive purposes only and does not imply endorsement by the U.S. Government.

Although this information product, for the most part, is in the public domain, it also may contain copyrighted materials as noted in the text. Permission to reproduce copyrighted items must be secured from the copyright owner.

Suggested citation:

Galloway, J.M., Vecchia, A.V., Vining, K.C., Densmore, B.K., and Lundgren, R.F., 2012, Evaluation of water-quality characteristics and sampling design for streams in North Dakota, 1970-2008: U.S. Geological Survey Scientific Investigations Report 2012-5216, 304 p. 


\section{Contents}

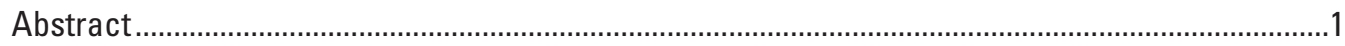

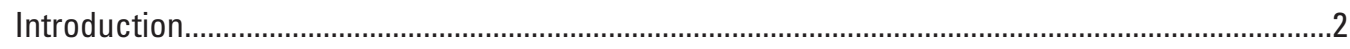

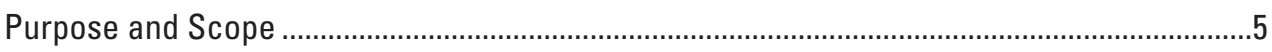

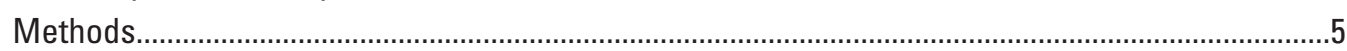

Site Selection and Water-Quality Data Screening ................................................................

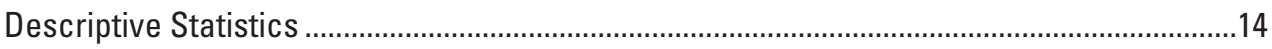

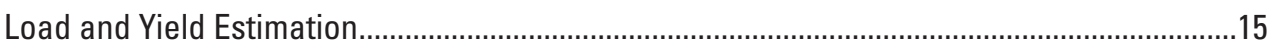

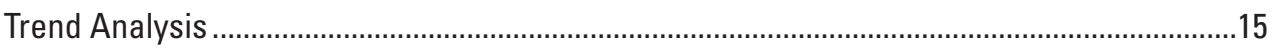

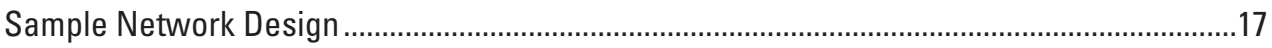

Median Concentrations of Selected Constituents ……................................................................17

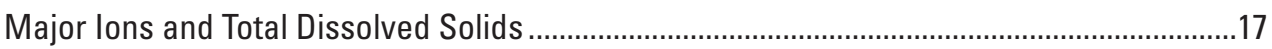

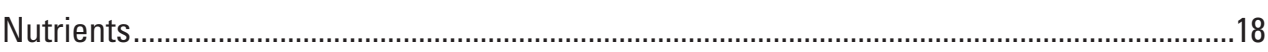

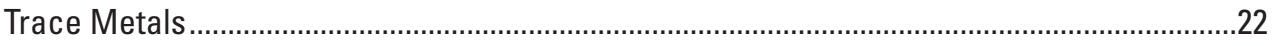

Suspended Sediment and Suspended Solids .......................................................................

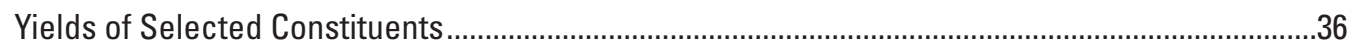

Sulfate and Total Dissolved Solids .......................................................................................36

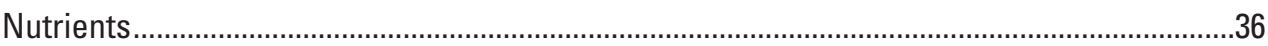

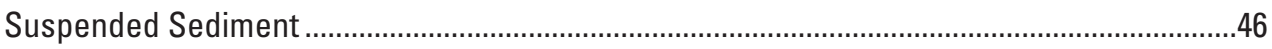

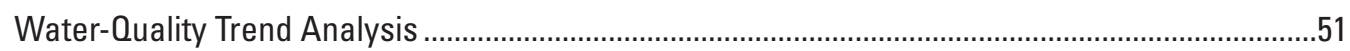

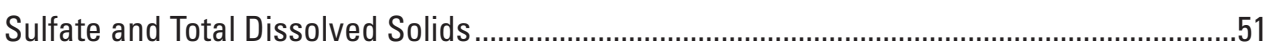

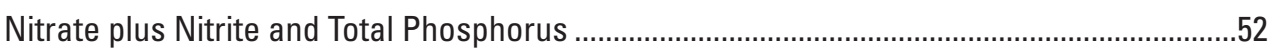

Sampling Designs to Monitor Water-Quality Trends and Loads ......................................................61

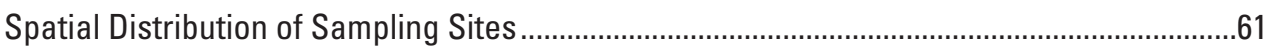

Sample Frequency for Detection of Seasonal Trends ..........................................................

Sample Frequency for Estimation of Constituent Loads .........................................................77

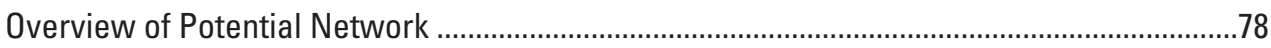

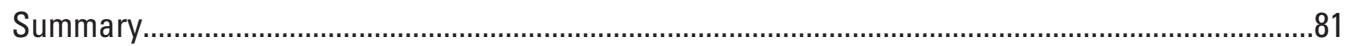

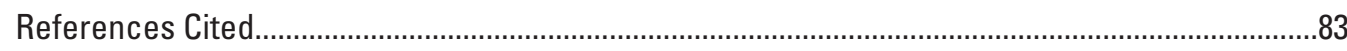

Appendix 1. Summary statistics for constituents and field measurements at selected sites in North Dakota ...................................................................................................

Appendix 2. Weighted regression methods for relating flow and concentration

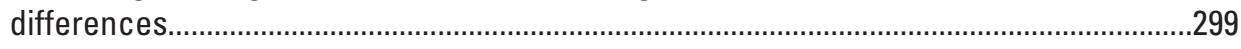

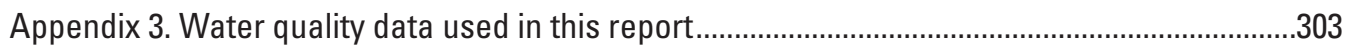




\section{Figures}

1. Map showing selected stream water-quality sample collection sites in North Dakota, 1970-2008

2. Map showing spatial distribution of median sulfate concentrations, in milligrams per liter, for selected stream sites in North Dakota for the period of record, spring (March through June), summer (July through October), and winter (November through February).

3. Map showing spatial distribution of median chloride concentrations, in milligrams per liter, for selected stream sites in North Dakota for the period of record, spring (March through June), summer (July through October), and winter (November through February)

4. Map showing spatial distribution of median total dissolved solids concentrations, in milligrams per liter, for selected stream sites in North Dakota for the period of record, spring (March through June), summer (July through October), and winter (November through February)

5. Map showing spatial distribution of median ammonia concentrations (total and dissolved), in milligrams per liter as nitrogen, for selected stream sites in North Dakota for the period of record (1970 through 2008), spring (March through June), summer (July through October), and winter (November through February).

6. Map showing spatial distribution of median nitrate plus nitrite concentrations (total and dissolved), in milligrams per liter as nitrogen, for selected stream sites in North Dakota for the period of record (1970 through 2008), spring (March through June), summer (July through October), and winter (November through February).

7. Map showing spatial distribution of median dissolved phosphorus concentrations, in milligrams per liter as phosphorus, for selected stream sites in North Dakota for the period of record (1970 through 2008), spring (March through June), summer (July through October), and winter (November through February)

8. Map showing spatial distribution of median total phosphorus concentrations, in milligrams per liter as phosphorus, for selected stream sites in North Dakota for the period of record (1970 through 2008), spring (March through June), summer (July through 0ctober), and winter (November through February)...

9. Map showing spatial distribution of median dissolved organic carbon concentrations, in milligrams per liter as carbon, for selected stream sites in North Dakota for the period of record (1970 through 2008), spring (March through June), summer (July through October), and winter (November through February).

10. Map showing spatial distribution of median total organic carbon concentrations, in milligrams per liter as carbon, for selected stream sites in North Dakota for the period of record (1970 through 2008), spring (March through June), summer (July through October), and winter (November through February).

11. Map showing spatial distribution of median total aluminum concentrations, in micrograms per liter, for selected stream sites in North Dakota for the period of record (1993 through 2008), spring (March through June), summer (July through October), and winter (November through February).

12. Map showing spatial distribution of median total arsenic concentrations, in micrograms per liter, for selected stream sites in North Dakota for the period of record (1993 through 2008), spring (March through June), summer (July through October), and winter (November through February) 
13. Map showing spatial distribution of median total chromium concentrations, in micrograms per liter, for selected stream sites in North Dakota for the period of record (1993 through 2008), spring (March through June), summer (July through October), and winter (November through February).....

14. Map showing spatial distribution of median total iron concentrations, in micrograms per liter, for selected stream sites in North Dakota for the period of record (1993 through 2008), spring (March through June), summer (July through October), and winter (November through February)

15. Map showing spatial distribution of median total lead concentrations, in micrograms per liter, for selected stream sites in North Dakota for the period of record (1993 through 2008), spring (March through June), summer (July through October), and winter (November through February)

16. Map showing spatial distribution of median total nickel concentrations, in micrograms per liter, for selected stream sites in North Dakota for the period of record (1993 through 2008), spring (March through June), summer (July through October), and winter (November through February)

17. Map showing spatial distribution of suspended-sediment concentrations, in milligrams per liter, for selected stream sites in North Dakota for the period of record (1970 through 2008), spring (March through June), summer (July through October), and winter (November through February)

18. Map showing spatial distribution of total suspended-solids concentrations, in milligrams per liter, for selected stream sites in North Dakota for the period of record (1970 through 2008), spring (March through June), summer (July through October), and winter (November through February).

19. Map showing spatial distribution of normalized annual sulfate and total dissolved solids yields for selected stream sites in North Dakota...

20. Map showing spatial distribution of normalized annual nitrogen and phosphorus yields for selected stream sites in North Dakota

21. Map showing spatial distribution of normalized annual total organic carbon yields for selected stream sites in North Dakota.

22. Map showing spatial distribution of normalized annual suspended-sediment yields for selected stream sites in North Dakota.

23. Graphs showing measured sulfate concentrations and fitted annual median concentrations from 1975 through 2008 for selected sites in North Dakota..

24. Graphs showing standardized sulfate concentrations and fitted trends for 1975 through 2008 for selected sites in North Dakota

25. Graphs showing measured total dissolved solids concentrations and fitted annual median concentrations for 1975 through 2008 for selected sites in North Dakota..........56

26. Graphs showing standardized total dissolved solids concentrations and fitted trends for 1975 through 2008 for selected sites in North Dakota.

27. Graphs showing measured nitrate plus nitrite concentrations and fitted annual median concentrations for 1975 through 2008 for selected sites in North Dakota. . .58

28. Graphs showing standardized nitrate plus nitrite concentrations and fitted trends for 1975 through 2008 for selected sites in North Dakota

29. Graphs showing measured total phosphorus concentrations and fitted annual median concentrations for 1975 through 2008 for selected sites in North Dakota..........60

30. Graphs showing standardized total phosphorus concentrations and fitted trends for 1975 through 2008 for selected sites in North Dakota 
31. Graphs showing relation between streamflow differences and sulfate

concentration difference $s$ for selected sites in North Dakota

32. Graphs showing relation between streamflow differences and total phosphorus concentration differences for selected sites in North Dakota

33. Map showing potential spatial water-quality network design for North Dakota .68

34. Graphs showing characteristic seasonal trends for selected sampling designs for sulfate at selected sites in North Dakota

35. Graphs showing characteristic seasonal trends for selected sampling designs for total dissolved solids at selected sites in North Dakota

36. Graphs showing characteristic seasonal trends for selected sampling designs for nitrate plus nitrite at selected sites in North Dakota.

37. Graphs showing characteristic seasonal trends for selected sampling designs for total phosphorus at selected sites in North Dakota

38. Graphs showing efficiency of sample designs for load estimation in terms of standardized difference between the average of log-transformed flows for the calibration dataset and log-transformed flows for the full dataset for 5-year moving windows.

39. Graphs showing efficiency of sample designs for load estimation in terms of ratio between the standard deviation of log-transformed flows for the calibration dataset and the standard deviation of log-transformed flows for the full dataset for 5 -year moving windows

\section{Appendix Figures}

2-1. Graphs showing weighted linear regression model for mean relative differences in streamflow and the logarithm of mean relative differences in sulfate concentration

2-2. Graphs showing partially weighted residuals from the weighted regression model for sulfate and sample size used to compute mean relative streamflow and concentration differences. SD is the standard deviation of the partially weighted residuals as a function of sample size

2-3. Graphs showing partially weighted residuals from the weighted regression model for sulfate and mean relative streamflow differences. SD is the standard deviation of the partially weighted residuals as a function of the mean absolute difference between downstream and upstream flow...

2-4. Graphs showing weighted linear regression model for mean relative differences in streamflow and the logarithm of mean relative differences in sulfate concentration, standardized to a sample size of $n=40$. 


\section{Tables}

1. Selected stream water-quality sites for North Dakota from 1970 through 2008 ...............6

2. Water-quality constituents and measurements selected for sites in North Dakota from 1970 through 2008 ...........................................................................................13

3. Regression model characteristics for S-LOADEST models for selected sites in North Dakota from 1970 through 2008 .........................................................................39

4. Estimated normalized annual loads and yields for selected sites and water-quality constituents in North Dakota .........................................................................................4

5. Fitted trends for selected standardized constituent concentrations at selected sites

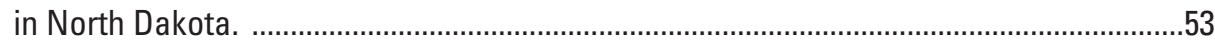

6. Current and potential state-wide water-quality sampling network for North Dakota ....63

7. Summary of potential state-wide water-quality sampling network for North Dakota ....81

\section{Appendix Tables}

1-1. Summary statistics for major ion constituents at selected sites in North Dakota from 1970 through 2008

1-2. Summary statistics for nutrient constituents at selected sites in North Dakota from 1970 through 2008

1-3. Summary statistics for trace-metal constituents at selected sites in North Dakota from 1993 through 2008

1-4. Summary statistics for field measurements, total suspended solids, and suspended sediment at selected sites in North Dakota from 1970 through 2008.

1-5. Median seasonal concentrations for selected constituents at selected sites in North Dakota from 1970 through 2008. 


\section{Conversion Factors and Datums}

\begin{tabular}{lcl}
\hline \multicolumn{1}{c}{ Multiply } & \multicolumn{1}{c}{ By } & \multicolumn{1}{c}{ To obtain } \\
\hline foot $(\mathrm{ft})$ & Length & \\
mile $(\mathrm{mi})$ & 0.3048 & meter $(\mathrm{m})$ \\
& 1.609 & kilometer $(\mathrm{km})$ \\
\hline acre & Area & \\
square foot $\left(\mathrm{ft}^{2}\right)$ & 4,047 & square meter $\left(\mathrm{m}^{2}\right)$ \\
square inch $\left(\mathrm{in}^{2}\right)$ & 0.09290 & square meter $\left(\mathrm{m}^{2}\right)$ \\
square mile $\left(\mathrm{mi}^{2}\right)$ & 6.452 & square centimeter $\left(\mathrm{cm}^{2}\right)$ \\
& 2.590 & square kilometer $\left(\mathrm{km}^{2}\right)$ \\
\hline cubic foot $\left(\mathrm{ft}^{3}\right)$ & Volume & \\
cubic foot per second $\left(\mathrm{ft}^{3} / \mathrm{s}\right)$ & 0.02832 & cubic meter $\left(\mathrm{m}^{2}\right)$ \\
\hline & 0.02832 & cubic meter per second $\left(\mathrm{m}^{3} / \mathrm{s}\right)$ \\
\hline pound, avoirdupois $(\mathrm{lb})$ & Mass & \\
pounds per year per square mile & 0.4536 & kilogram $(\mathrm{kg})$ \\
$\quad\left(\mathrm{lbs} / \mathrm{yr} / \mathrm{mi}^{2}\right)$ & & kilograms per year per square \\
ton, short $(2,000 \mathrm{lb})$ & & kilometer \\
\hline
\end{tabular}

Temperature in degrees Celsius $\left({ }^{\circ} \mathrm{C}\right)$ may be converted to degrees Fahrenheit $\left({ }^{\circ} \mathrm{F}\right)$ as follows:

$$
{ }^{\circ} \mathrm{F}=\left(1.8 \times{ }^{\circ} \mathrm{C}\right)+32
$$

Vertical coordinate information is referenced to North American Vertical Datum of 1988 (NAVD 88)

Horizontal coordinate information is referenced to North American Datum of 1983 (NAD 83)

Specific conductance is given in microsiemens per centimeter at 25 degrees Celsius $(\mu \mathrm{S} / \mathrm{cm}$ at $\left.25^{\circ} \mathrm{C}\right)$.

Concentrations of chemical constituents in water are given either in milligrams per liter (mg/L) or micrograms per liter $(\mu \mathrm{g} / \mathrm{L})$. 


\title{
Evaluation of Water-Quality Characteristics and Sampling Design for Streams in North Dakota, 1970-2008
}

\author{
By Joel M. Galloway, Aldo V. Vecchia, Kevin C. Vining, Brenda K. Densmore, and Robert F. Lundgren
}

\section{Abstract}

In response to the need to examine the large amount of historic water-quality data comprehensively across North Dakota and evaluate the efficiency of the State-wide sampling programs, a study was done by the U.S. Geological Survey in cooperation with the North Dakota State Water Commission and the North Dakota Department of Health to describe the water-quality data collected for the various programs and determine an efficient State-wide sampling design for monitoring future water-quality conditions. Although data collected for the North Dakota State Water Commission High-Low Sampling Program, the North Dakota Department of Health Ambient Water-Quality Network, and other projects and programs provide valuable information on the quality of water in streams in North Dakota, the objectives vary among the programs, some of the programs overlap spatially and temporally, and the various sampling designs may not be the most efficient or relevant to the objectives of the individual programs as they have changed through time.

Median concentrations of water-quality constituents at sampling locations in North Dakota were determined for the period of record. In general, median sulfate concentrations tended to be higher in the southwest part of the State compared to the northeast part of North Dakota in the Red River of the North Basin. Median chloride concentrations tended to be lower in the southwest and higher in the northeast parts of the State, nearly the opposite of the pattern seen in median sulfate concentrations. In general, median total dissolved solid concentrations tended to be lower for sites in the Red River of the North, Souris River, and James River Basins compared to sites in southwest North Dakota.

In general, overall median ammonia concentrations were spatially variable across the State. The highest overall median nitrate plus nitrite concentrations tended to occur in the Red River of the North Basin. Median dissolved and total phosphorus concentrations across North Dakota generally were higher for the Red, Souris, and James River Basins than river basins in the southwest part of the State. Median dissolved and total organic carbon concentrations tended to be the lowest at sites located in the eastern part of the State compared to sites throughout the remainder of the State.
Median total aluminum concentrations generally were higher in samples from sites in the southern and eastern parts of the State compared to other parts of the State. Median total arsenic concentrations in North Dakota were the lowest mostly in the southwestern parts of the State, and highest mostly in the eastern parts of the State. Median total chromium concentrations across North Dakota generally were higher in the far eastern and western areas of the State. Median total iron concentrations in North Dakota were lowest in the Souris, Sheyenne, and James River Basins compared to sites in other parts of North Dakota. Median total lead concentrations were highest at sites scattered across North Dakota. Median total nickel concentrations were higher at a few sites in the southwest and eastern parts of North Dakota compared to other sites. Median suspended-sediment concentrations and total suspended solids in water samples obtained across North Dakota were higher at sites located in parts of southwestern and eastern North Dakota compared to sites located in other areas of the State.

Normalized annual loads and yields were estimated for selected constituents at 34 sites across North Dakota. Normalized annual sulfate yields ranged from 9,250 to 56,200 pounds per year per square mile in the Missouri River Basin and from 4,680 to 72,500 pounds per year per square mile in the Red River of the North Basin. Total dissolved solids yields ranged from 30,400 to 119,000 pounds per year per square mile in the Missouri River Basin and from 13,000 to 169,000 pounds per year per square mile in the Red River of the North Basin. Nitrate plus nitrite yields ranged from 24 to 120 pounds per year per square mile as nitrogen in the Missouri River Basin and from 2 to 1,260 pounds per year per square mile as nitrogen in the Red River of the North Basin. Normalized annual total phosphorus yields ranged from less than 1 to 167 pounds per year per square mile as phosphorus in the Missouri River Basin and from 6 to 76 pounds per year per square mile as phosphorus in the Red River of the North Basin. Suspendedsediment yields ranged from 519 to 622,000 pounds per year per square mile in the Missouri River Basin and from 1,500 to 78,800 pounds per year per square mile in the Red River of the North Basin.

Ten sites were selected for trend analysis for sulfate, total dissolved solids, nitrate plus nitrite, and total phosphorus. The fitted trends for standardized sulfate concentrations indicated significant increases in concentration at five sites and 
a significant decrease in concentration at one site from 1975 through 2008. Median standardized sulfate concentrations increased from about 55 to 106 milligrams per liter for the Red River of the North at Grand Forks, North Dakota, 262 to 308 milligrams per liter for the Souris River near Sherwood, North Dakota, 455 to 506 milligrams per liter for Spring Creek at Zap, North Dakota, 440 to 520 milligrams per liter for the Knife River at Hazen, North Dakota, and 380 to 435 milligrams per liter for the Heart River near Mandan, North Dakota. The fitted trend for the Little Missouri River near Watford City, North Dakota indicated a decrease in median standardized concentration from about 492 to 417 milligrams per liter. The fitted trends for standardized total dissolved solid concentrations indicated significant increases in concentration at five sites from 1975 through 2008, including the Heart River near Mandan, North Dakota, Knife River at Hazen, North Dakota, Spring Creek at Zap, North Dakota, and the Red River of the North at Grand Forks, North Dakota. The greatest increase in median standardized total dissolved solid concentrations was detected for the Wild Rice River near Abercrombie, North Dakota, where median concentrations increased from about 690 to 1,120 milligrams per liter from 1975 through 2008.

The fitted trends for standardized nitrate plus nitrite concentrations indicated no significant increases or decreases in concentration for 7 of the 8 sites analyzed for trends; however, median standardized nitrate plus nitrite concentrations for the Red River of the North near Grand Forks, North Dakota increased from about 0.26 to 0.45 milligrams per liter as nitrogen during 1990 to 2008. The fitted trends for standardized total phosphorus concentrations indicated significant decreases in concentration at 4 sites and a significant increase at 1 out of the 10 sites analyzed for trends. All of the significant decreases were for sites in western North Dakota, including decreases in median standardized total phosphorus concentration for the Cannonball River at Breien, North Dakota, Heart River near Mandan, North Dakota, Knife River at Hazen, North Dakota, and Spring Creek at Zap, North Dakota. Conversely, median standardized total phosphorus concentrations for the Red River of the North at Grand Forks, North Dakota increased from about 0.10 to 0.16 milligrams per liter from 1990 through 2008.

One objective of a State-wide sampling program was to evaluate ways to describe the spatial variability of water-quality conditions across the State in the most efficient manner. Weighted least-squares regression analysis was used to relate the average absolute difference between paired downstream and upstream concentrations, expressed as a percent of the average downstream concentration, to the average absolute difference in daily flow between the downstream and upstream pairs, expressed as a percent of the average downstream flow. The analysis showed that a reasonable spatial network would consist of including the most downstream sites in large basins first, followed by the next upstream site(s) that roughly bisect the downstream flows at the first sites, followed by the next upstream site(s) that roughly bisect flows for the second sites. Sampling sites to be included in a potential State-wide network were prioritized into 3 design levels: level 1 (highest priority), level 2 (second priority), and level 3 (third priority). The level-1 sites included most of the 34 active Ambient Water-Quality Network sites plus two additional Canadian/ U.S. border sites in the Souris River Basin. The level-2 sites were selected from among the active High-Low Sampling Program sites to fill major gaps in coverage of the level-1 sites while minimizing redundancy of the network. The level-2 sites generally were well upstream of the level-1 sites or on large tributaries with no level-1 sites. The level-3 sites were selected from among active High-Low Sampling Program sites to fill gaps in coverage remaining after inclusion of the level-1 and level-2 sites. The level-3 sites generally were on smaller tributaries with no level-1 or level-2 sites or in larger basins well upstream of the level-1 or level-2 sites. In all, the potential network consists of 81 sites, with 34 level-1 sites, 21 level-2 sites, and 26 level- 3 sites.

Given the spatial distribution and priority designation (levels 1-3) of sites in the potential spatial network, the next consideration was to determine the appropriate temporal sampling frequency to use for monitoring future water-quality conditions. The time-series model used to detect concentration trends for this report also was used to evaluate sampling designs to monitor future water-quality trends. Sampling designs were evaluated with regard to their sensitivity to detect seasonal trends that occurred during three 4-month seasonsMarch through June, July through October, and November through February. Unlike sample designs for detecting trends that are targeting flow-adjusted concentration through time, sample designs for the estimation of constituent loads should focus on the main contributing factor that can affect the annual load, which are changes in concentration with streamflow.

For the 34 level-1 sites, samples would be collected for major ions, trace metals, nutrients, bacteria, and sediment eight times per year, with samples in January, April (2 samples), May, June, July, August, and October. For the 21 level-2 sites, samples would be collected for major ions, trace metals, and nutrients six times per year (January, April, May, June, August, and October), and for the 26 level-3 sites, samples would be collected for these constituents four times per year (April, June, August, and October).

\section{Introduction}

Various agencies have conducted water-quality sampling programs and projects for streams in North Dakota for numerous years for several purposes. Various programs and projects have different sampling designs, different selected waterquality constituents, and different laboratories that completed the sample analyses based on the program objectives. Some of the data from these programs have been used for regulation of water-quality standards, some have been described in numerous reports with different objectives, and some of the data 
have not been evaluated at all. Two of the major programs that have provided a large amount of data across North Dakota are the North Dakota State Water Commission (NDSWC) HighLow Flow Sampling Program (HLSP) and the North Dakota Department of Health (NDDH) Ambient Water-Quality Network (hereafter referred to as ambient network)(fig. 1).

The U.S. Geological Survey (USGS), in cooperation with the NDSWC, currently (2010) collects 162 water-quality samples per year at 81 HLSP sites throughout North Dakota (fig. 1). Some of these HLSP sites are colocated with sites that are sampled as part of other sampling programs described later. The program began in the late 1960s with collecting samples every time a streamflow measurement was made or approximately monthly at about $12-15$ stations throughout the state. These stations were rotated to obtain data from across North Dakota. The purpose was to begin collecting enough water-quality information to help understand the water quality of North Dakota streams. Monthly sampling ended about 1971. In 1972, a water-quality program was started in which water samples were collected at most of the streamflow-gaging stations that did not have another water-quality sampling program. This program, which started with only one sample at each gaging station in 1972, evolved to the current program (2010) that consists of collecting two samples each year-one during high-flow conditions, which is usually during snowmelt runoff (March through May), and one during low-flow conditions, which is usually during middle to late summer (August through October). The NDSWC laboratory in Bismarck, N. Dak. analyzed HLSP samples until June 2003 when the laboratory closed, and the NDDH laboratory in Bismarck, N. Dak. began analyzing the samples after that time. Samples collected for the HLSP are analyzed for major ions and selected trace metals.

NDSWC water-resource managers have indicated the HLSP data are used to make management decisions that were never anticipated when the HLSP began; however, after 35 years of sampling program operation, the NDSWC recognize that the goals, objectives, and uses of the data need to be more clearly identified and prioritized. For example, the temporal and spatial distribution of the 162 samples that are currently (2010) collected per year would probably include different sites and temporal allocation if the emphasis is on evaluating time trends or estimating loads rather than characterizing concentrations during high- or low-flow conditions.

Currently, in 2010, the NDDH ambient network consists of 34 monitoring stations located on 19 rivers (fig. 1). Samples are collected by the NDDH at 26 sites and by the USGS at eight sites. The primary purpose of this network was to provide data for trend analysis, general water-quality characterization, determination of compliance of water-quality standards, and pollutant loading calculations. Samples are analyzed for major ions, nutrients, trace metals, total suspended solids, total and dissolved organic carbon, and fecal indicator bacteria by the NDDH Laboratory in Bismarck, N. Dak. Early water-quality data collection by the NDDH began in the 1960 's with a statewide monitoring program consisting of a network of stations that were located immediately below point source discharges or near the confluences of major rivers (North Dakota Department of Health, 2005). The primary purpose of the historic monitoring program was to assess the general chemical character of the state's rivers and streams and, to the extent practical, assess point source discharge compliance with state water-quality standards. In 1972, Congress passed the Federal Water Pollution Control Act, commonly referred to as the Clean Water Act (U.S. Environmental Protection Agency, 1972) that directed States to submit biennial reports to the U.S. Environmental Protection Agency (USEPA) and Congress that includes a comprehensive water-quality assessment [305(b) report] and listing of water-quality impaired (degraded) streams or stream segments [303(d) report] within the States (Pope and others, 2004). In response to the provisions of the Clean Water Act, states established, modified, or enhanced their ambient stream-water-quality monitoring networks to provide the data necessary to assess stream water quality relative to state-established water-quality standards. By 1993, the NDDH had a monitoring network that included 61 ambient monitoring stations on 31 rivers and streams. Through time, as budgetary considerations and program needs changed, the program was reduced to the current (2010) network of 34 stations (fig. 1). Ambient network stations that are not currently active are designated as "other" in figure 1.

In 2010, the frequency of sampling for the NDDH ambient network was eight samples collected from each station per year (4 during April-June, 3 during July-October, and 1 during November-March). This design was selected for estimating average annual loads during a 5- to 10-year period (NDDH, 2005); however, the data have not been fully evaluated to determine if they provide an efficient design for estimating average annual loads or monitoring long-term trends.

The USGS has maintained other long-term stream sampling networks in North Dakota, particularly in the Souris and James River Basins. The USGS, in cooperation with U.S. Army Corps of Engineers and the City of Minot, have sampled as many as five sites on the Souris River (sites 82, 83, 85, 87 , and 98 on fig. 1), two of which have a complete period of record from 1972 through present (2010). Two of the sites (Souris River near Sherwood, N. Dak. and near Westhope, N. Dak.; sites 82 and 98 on fig. 1) are located near the U.S.Canada border and are maintained as part of the International Souris River Board mandate to monitor aquatic ecosystem health at the International border (International Souris River Board, 2009). Samples are currently collected seven times per year at three sites (Souris River near Sherwood, Minot, and Verendrye, N. Dak.; sites 82, 85, and 87 on fig. 1) and are analyzed for nutrient, major ion, and trace-metal constituents. Although USGS discontinued sampling the Souris River near Westhope, N. Dak. in 1995, Environment Canada has continued sampling that site eight times per year. Two sites have been sampled by the USGS in cooperation with the U.S. Bureau of Reclamation on the James River and the Arrowwood National Wildlife Refuge as part of its refuge-monitoring program and as part of an environmental impact statement 


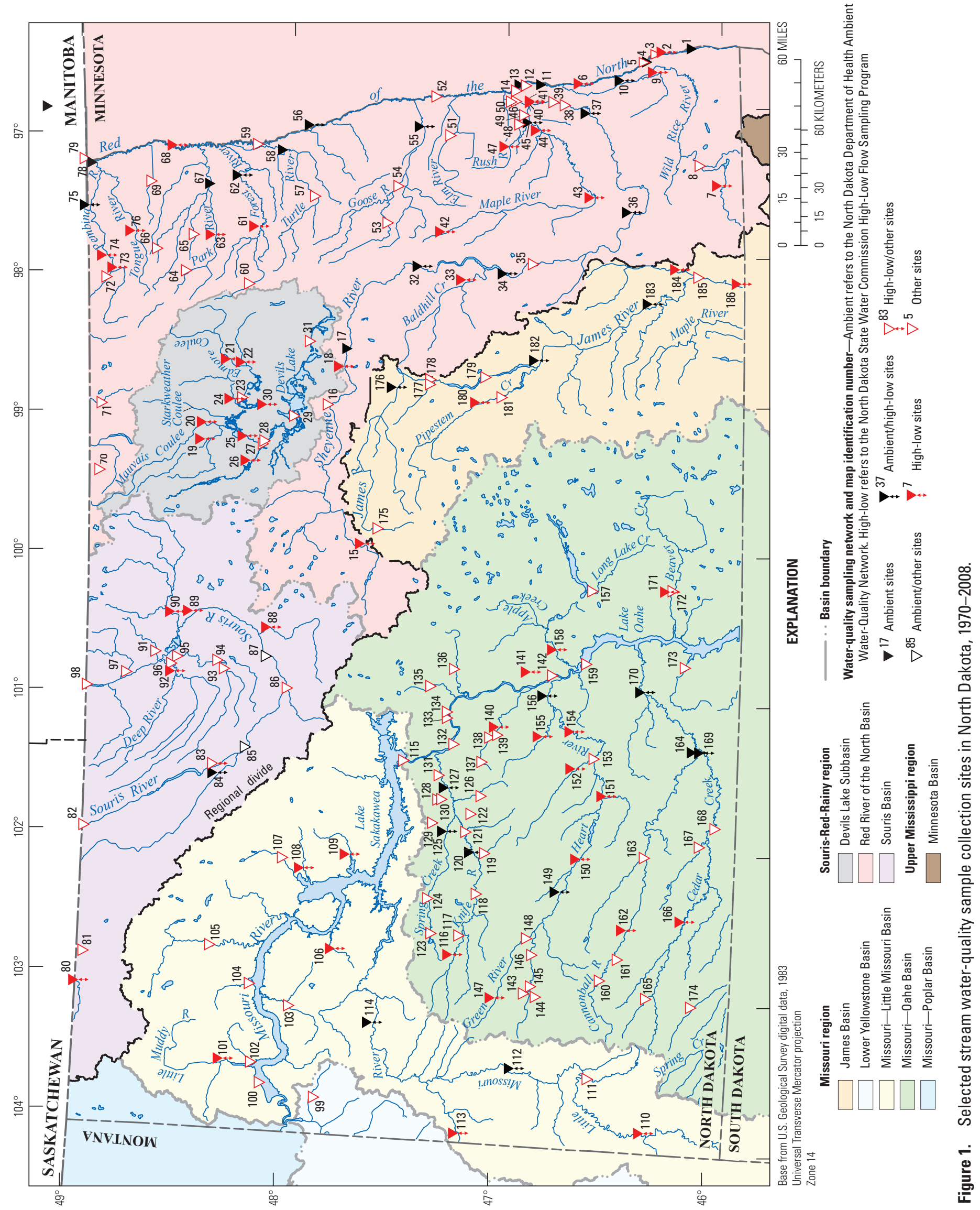


commitment for various periods between 1987 and present (2010) (Ryberg and Hiemenz, 2009) (sites 177 and 179 on fig. 1). Currently (2010), samples are collected at the two sites five times per year and are analyzed for nutrients, major ions, and suspended-sediment concentration.

Although data collected for the NDSWC HLSP, NDDH ambient network, and other projects and programs provide valuable information on the quality of water in streams of the State, the objectives vary among the programs; some of the programs overlap spatially and temporally, and the various sampling designs may not be the most efficient or relevant to the objectives of the individual programs as they have changed through time. In response to the need to comprehensively examine the large amount of historic water-quality data across North Dakota and evaluate the efficiency of the State-wide sampling program, a study was conducted by the USGS in cooperation with the NDSWC and the NDDH to describe the water-quality data collected for the various programs and determine an efficient State-wide sampling design for monitoring future water-quality conditions.

\section{Purpose and Scope}

The purpose of this report is to provide descriptive statistics and graphical summaries of water-quality data from sites throughout the State, determine annual loads and yields and trends in concentration for selected constituents and sites with sufficient concentration and streamflow data, and determine an efficient potential State-wide network sampling design for monitoring future water-quality conditions. All data from streams throughout North Dakota collected as part of various NDDH, NDSWC, and USGS water-quality programs and projects from 1970 to 2008 were evaluated. For most constituents, data from the entire historical period (1970-2008) were used in the evaluations, however, trace-metal data collected before 1993 were not included.

\section{Methods}

This section describes the methods used for site selection, water-quality data preparation, computation of descriptive statistics, load and yield estimation, trend analysis, and sampling design analysis. Water-quality and streamflow data for North Dakota were obtained from the USGS National Water Information System (NWIS) database (http://nwis.waterdata. usgs.gov/nd/nwis/), the USEPA STORET database (http:// www.epa.gov/storet/dw_home.html), and electronic files from the NDDH (Joe Gross, North Dakota Department of Health, written commun., 2010).

\section{Site Selection and Water-Quality Data Screening}

Water-quality data from the various databases were screened to develop a data set that could be used to describe water-quality conditions across North Dakota in terms of spatial and temporal variability. Data from 1970 through 2008 were initially considered for all monitored constituents except for trace metals. Only trace-metal data collected from 1993 through 2008 were considered for this report because there were substantial changes in sample collection and analysis methods for trace metals beginning in 1993 (U.S. Geological Survey, 1992 and 1993). The first level of site selection involved removing sites that had fewer than 10 samples for all of the constituents, which resulted in 186 sites across North Dakota available for evaluation (fig. 1 and table 1). Site selection criteria for determining loads and trends are described below.

Water-quality data were screened by eliminating certain constituents with large ranges of laboratory detection limits, highly variable field collection, and variable laboratory analysis methods that prevented comparability between data sets and the computation of descriptive statistics and other analyses. Some of these constituents included fecal indicator bacteria (Escherichia coli, fecal coliform, and fecal streptococci), phytoplankton, chlorophyll $a$ and $b$, and organic compounds such as pesticides. Also, constituents that had more than 90 percent of the values less than the censoring level (the level below which concentrations could not be accurately quantified, determined as described next) were not evaluated.

Because water-quality analysis methods have varied for the various water-quality programs from 1970 to 2008 and were conducted by three different laboratories (NDDH Water-Quality Laboratory, NDSWC Water-Quality Laboratory, and the USGS National Water-Quality Laboratory), several of the constituents have a wide range in censoring levels. The censoring level is the "less-than" value reported when concentrations are too low to be accurately quantified. The censoring level can change through time and among laboratories because of changes in sensitivity of laboratory equipment or analytical methods or changes in protocol for determining the censoring level. The censoring level is generally higher than the method detection limit, which is the lowest concentration for which a compound can be detected (but not necessarily accurately quantified) (Childress and others, 1999). For comparability between data and for estimates of trends and loads, constituents were recensored to a single level. Generally, the highest censoring level for each constituent was used with a few exceptions. In some cases, some higher censoring levels were present in the data for only a few values. If there were less than 5 values for the higher censoring level in some cases, they were excluded from the data set (table 2). Fiftynine constituents and physical measurements were included in the final list for analysis. The constituents included nutrients, major ions, trace metals, and suspended solids/sediment. Physical measurements included $\mathrm{pH}$, dissolved oxygen, water 
Table 1. Selected stream water-quality sites for North Dakota from 1970 through 2008.

[ID, identification; USEPA, Environment Protection Agency; STORET, STOrage and RETrieval; USGS, U.S. Geological Survey; HUC, hydrologic unit code; --, not available; Ambient, North Dakota Department of Health Ambient Water-Quality Network; High-Low, North Dakota State Water Commission High-Low Flow Sampling Program; other, sampled as part of various other programs]

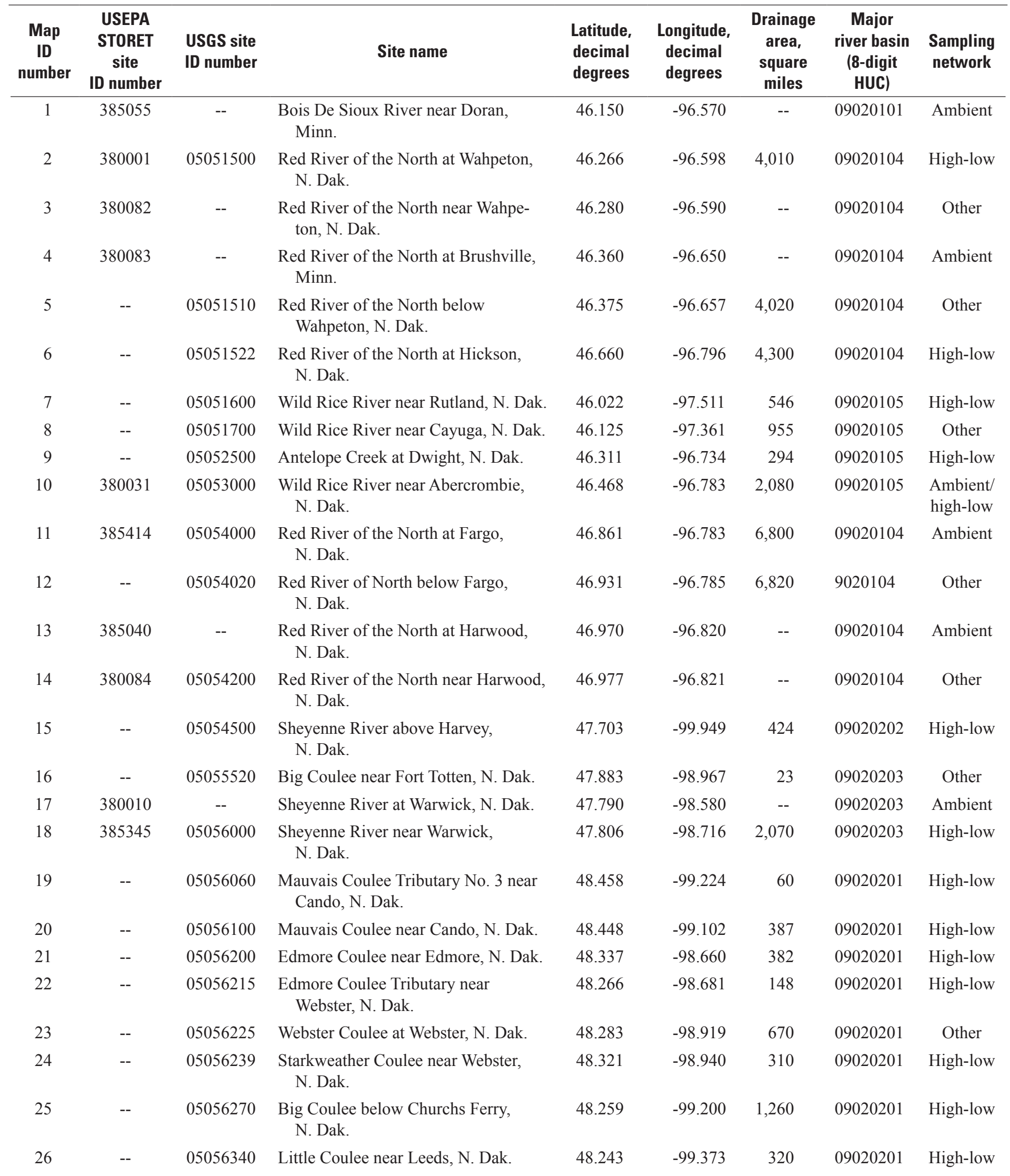


Table 1. Selected stream water-quality sites for North Dakota from 1970 through 2008.—Continued

[ID, identification; USEPA, Environment Protection Agency; STORET, STOrage and RETrieval; USGS, U.S. Geological Survey; HUC, hydrologic unit code; --, not available; Ambient, North Dakota Department of Health Ambient Water-Quality Network; High-Low, North Dakota State Water Commission High-Low Flow Sampling Program; other, sampled as part of various other programs]

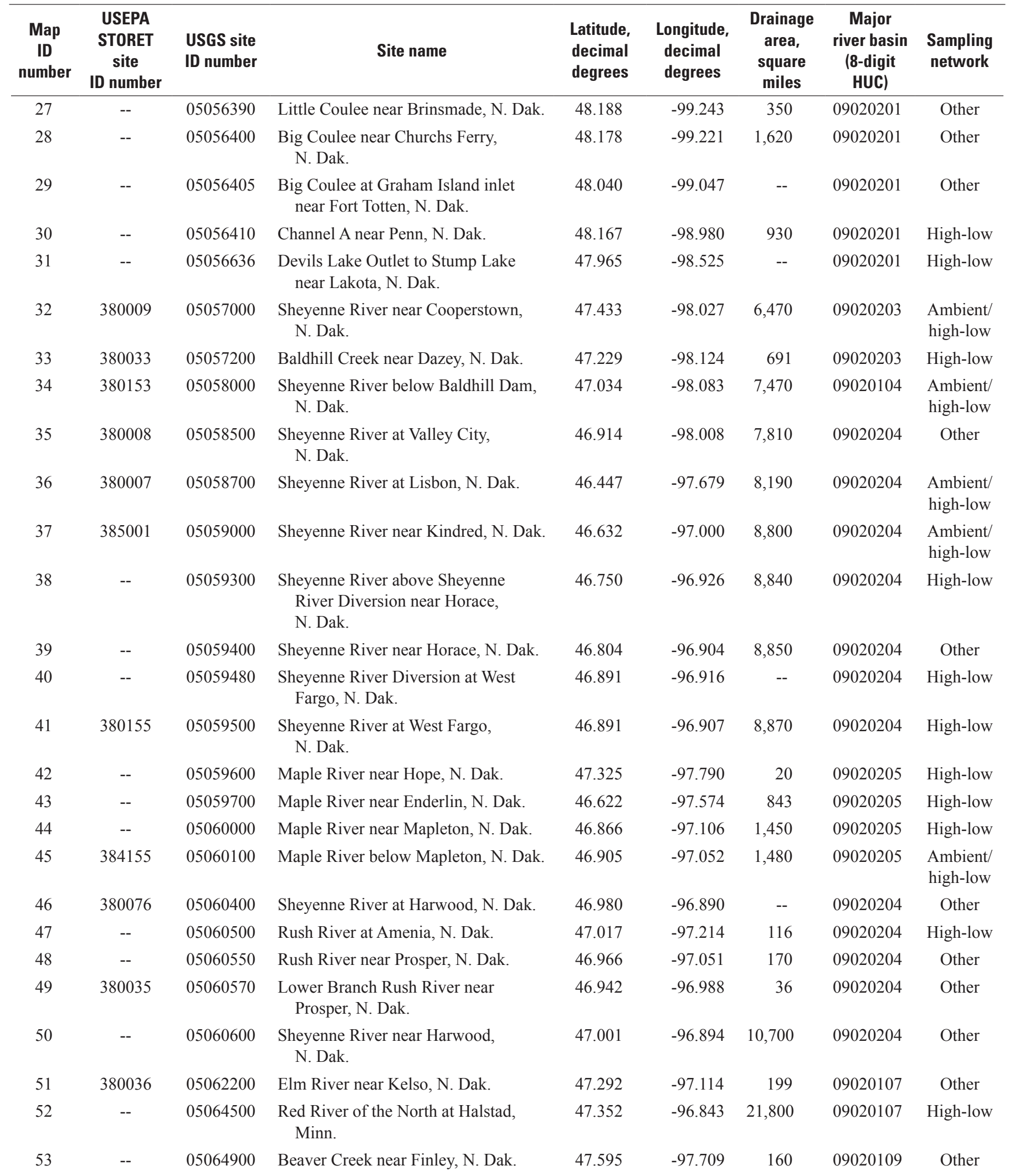


Table 1. Selected stream water-quality sites for North Dakota from 1970 through 2008. - Continued

[ID, identification; USEPA, Environment Protection Agency; STORET, STOrage and RETrieval; USGS, U.S. Geological Survey; HUC, hydrologic unit code; --, not available; Ambient, North Dakota Department of Health Ambient Water-Quality Network; High-Low, North Dakota State Water Commission High-Low Flow Sampling Program; other, sampled as part of various other programs]

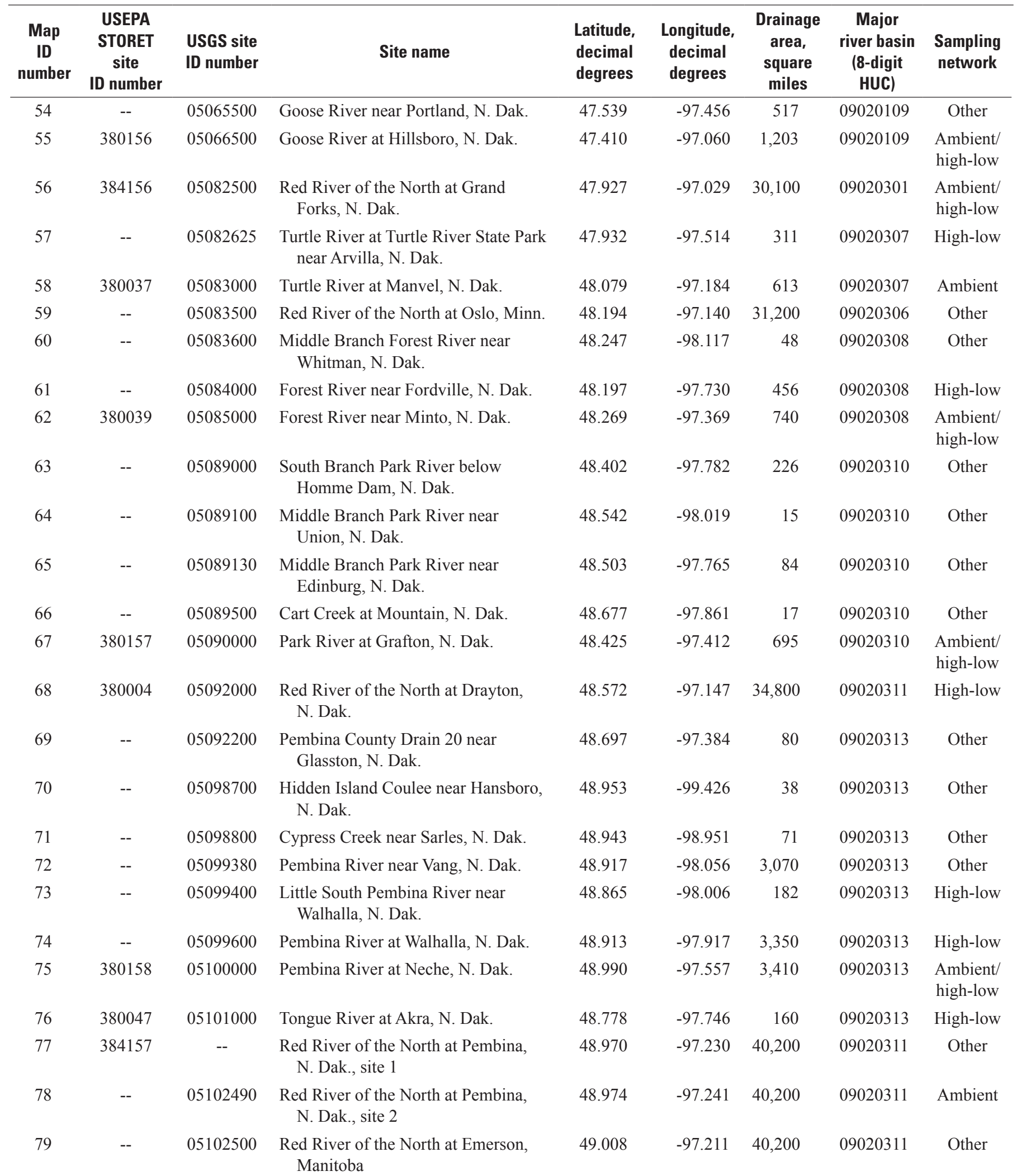


Table 1. Selected stream water-quality sites for North Dakota from 1970 through 2008._Continued

[ID, identification; USEPA, Environment Protection Agency; STORET, STOrage and RETrieval; USGS, U.S. Geological Survey; HUC, hydrologic unit code; --, not available; Ambient, North Dakota Department of Health Ambient Water-Quality Network; High-Low, North Dakota State Water Commission High-Low Flow Sampling Program; other, sampled as part of various other programs]

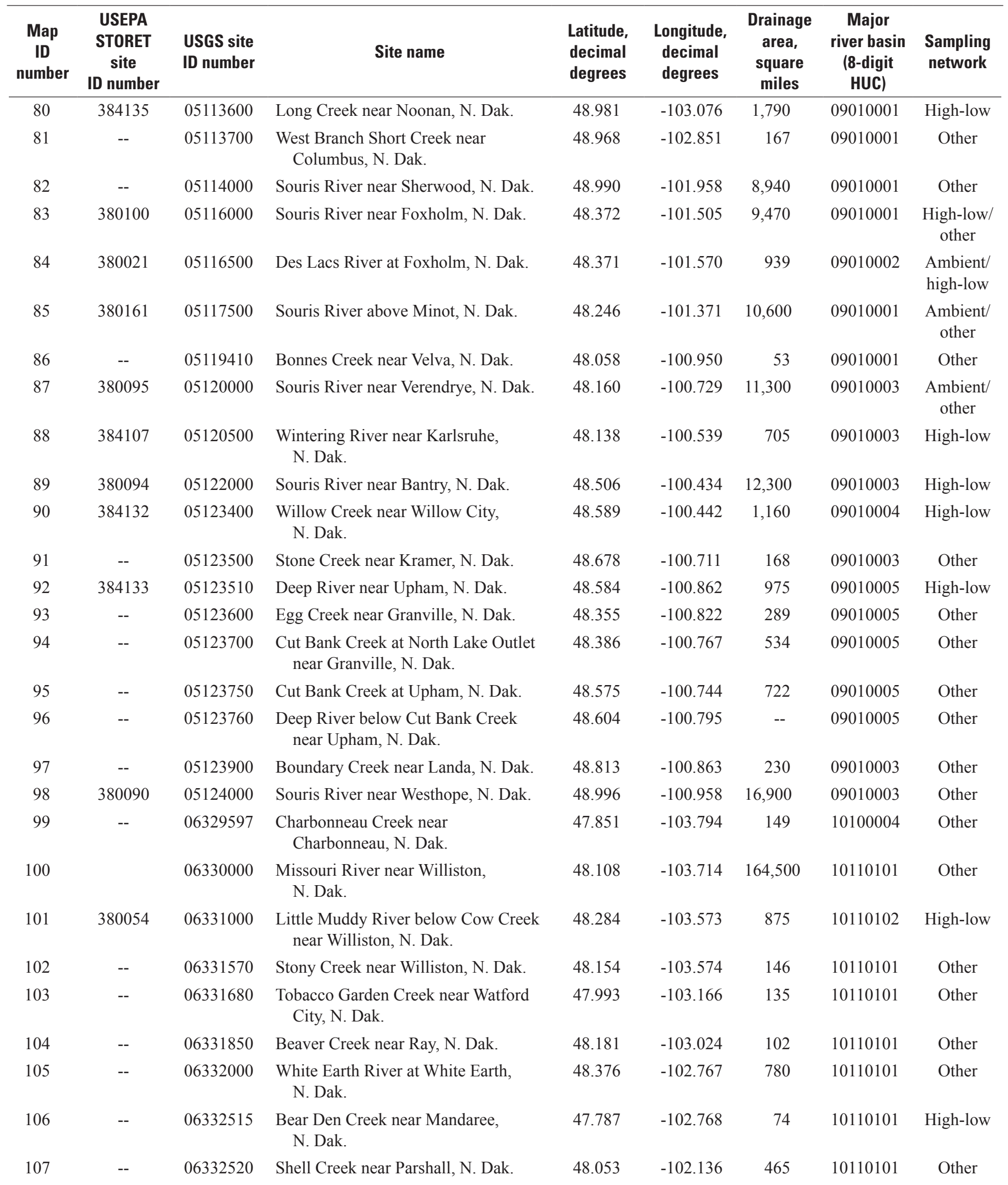


Table 1. Selected stream water-quality sites for North Dakota from 1970 through 2008.—Continued

[ID, identification; USEPA, Environment Protection Agency; STORET, STOrage and RETrieval; USGS, U.S. Geological Survey; HUC, hydrologic unit code; --, not available; Ambient, North Dakota Department of Health Ambient Water-Quality Network; High-Low, North Dakota State Water Commission High-Low Flow Sampling Program; other, sampled as part of various other programs]

\begin{tabular}{|c|c|c|c|c|c|c|c|c|}
\hline $\begin{array}{c}\text { Map } \\
\text { ID } \\
\text { number }\end{array}$ & $\begin{array}{c}\text { USEPA } \\
\text { STORET } \\
\text { site } \\
\text { ID number }\end{array}$ & $\begin{array}{l}\text { USGS site } \\
\text { ID number }\end{array}$ & Site name & $\begin{array}{l}\text { Latitude, } \\
\text { decimal } \\
\text { degrees }\end{array}$ & $\begin{array}{c}\text { Longitude, } \\
\text { decimal } \\
\text { degrees }\end{array}$ & $\begin{array}{c}\text { Drainage } \\
\text { area, } \\
\text { square } \\
\text { miles }\end{array}$ & $\begin{array}{c}\text { Major } \\
\text { river basin } \\
\text { (8-digit } \\
\text { HUC) }\end{array}$ & $\begin{array}{l}\text { Sampling } \\
\text { network }\end{array}$ \\
\hline 109 & -- & 06332770 & $\begin{array}{l}\text { Deepwater Creek near Mandaree, } \\
\text { N. Dak. }\end{array}$ & 47.738 & -102.107 & 220 & 10110101 & High-low \\
\hline 111 & -- & 06335750 & Deep Creek near Amidon, N. Dak. & 46.577 & -103.557 & 250 & 10110203 & Other \\
\hline 112 & 380022 & 06336000 & $\begin{array}{l}\text { Little Missouri River at Medora, } \\
\text { N. Dak. }\end{array}$ & 46.919 & -103.528 & 6,190 & 10110203 & $\begin{array}{l}\text { Ambient/ } \\
\text { high-low }\end{array}$ \\
\hline 113 & 385030 & 06336600 & Beaver Creek near Trotters, N. Dak. & 47.163 & -103.992 & 616 & 10110204 & High-low \\
\hline 116 & -- & 06339100 & Knife River at Manning, N. Dak. & 47.236 & -102.769 & 205 & 10130201 & High-low \\
\hline 117 & -- & 06339180 & Stray Creek near Manning, N. Dak. & 47.213 & -102.623 & 30 & 10130201 & Other \\
\hline 118 & -- & 06339300 & Knife River at Marshall, N. Dak. & 47.138 & -102.333 & 722 & 10130201 & Other \\
\hline 119 & -- & 06339490 & $\begin{array}{l}\text { Elm Creek near Golden Valley, } \\
\text { N. Dak. }\end{array}$ & 47.107 & -102.051 & 82 & 10130201 & Other \\
\hline 120 & 384131 & 06339500 & $\begin{array}{l}\text { Knife River near Golden Valley, } \\
\text { N. Dak. }\end{array}$ & 47.154 & -102.059 & 1,230 & 10130201 & $\begin{array}{l}\text { Ambient/ } \\
\text { high-low }\end{array}$ \\
\hline 121 & -- & 06339550 & Coyote Creek near Zap, N. Dak. & 47.199 & -101.912 & 65 & 10130201 & Other \\
\hline 126 & -- & 06340200 & $\begin{array}{l}\text { West Branch Otter Creek near Beulah, } \\
\text { N. Dak. }\end{array}$ & 47.135 & -101.660 & 27 & 10130201 & Other \\
\hline 127 & 380087 & 06340500 & Knife River at Hazen, N. Dak. & 47.285 & -101.622 & 2,240 & 10130201 & $\begin{array}{l}\text { Ambient/ } \\
\text { high-low }\end{array}$ \\
\hline 128 & -- & 06340520 & Antelope Creek above Hazen, N. Dak. & 47.335 & -101.695 & 47 & 10130201 & Other \\
\hline 129 & -- & 06340528 & $\begin{array}{l}\text { West Branch Antelope Creek No. } 4 \\
\text { near Zap, N. Dak. }\end{array}$ & 47.356 & -101.854 & 8 & 10130201 & Other \\
\hline 130 & -- & 06340540 & $\begin{array}{l}\text { West Branch Antelope Creek near } \\
\text { Hazen, N. Dak. }\end{array}$ & 47.317 & -101.691 & 38 & 10130201 & Other \\
\hline 131 & -- & 06340580 & Coal Creek near Stanton, N. Dak. & 47.334 & -101.527 & 16 & 10130201 & Other \\
\hline 132 & -- & 06340780 & $\begin{array}{l}\text { Alderin Creek near Fort Clark, } \\
\text { N. Dak. }\end{array}$ & 47.269 & -101.309 & 22 & 10130101 & Other \\
\hline 133 & -- & 06340905 & $\begin{array}{l}\text { Coal Lake Coulee near Hensler, } \\
\text { N. Dak. }\end{array}$ & 47.303 & -101.131 & 71 & 10130101 & Other \\
\hline 134 & -- & 06340930 & Buffalo Creek near Washburn, N. Dak. & 47.303 & -101.089 & 57 & 10130101 & Other \\
\hline
\end{tabular}


Table 1. Selected stream water-quality sites for North Dakota from 1970 through 2008._Continued

[ID, identification; USEPA, Environment Protection Agency; STORET, STOrage and RETrieval; USGS, U.S. Geological Survey; HUC, hydrologic unit code; --, not available; Ambient, North Dakota Department of Health Ambient Water-Quality Network; High-Low, North Dakota State Water Commission High-Low Flow Sampling Program; other, sampled as part of various other programs]

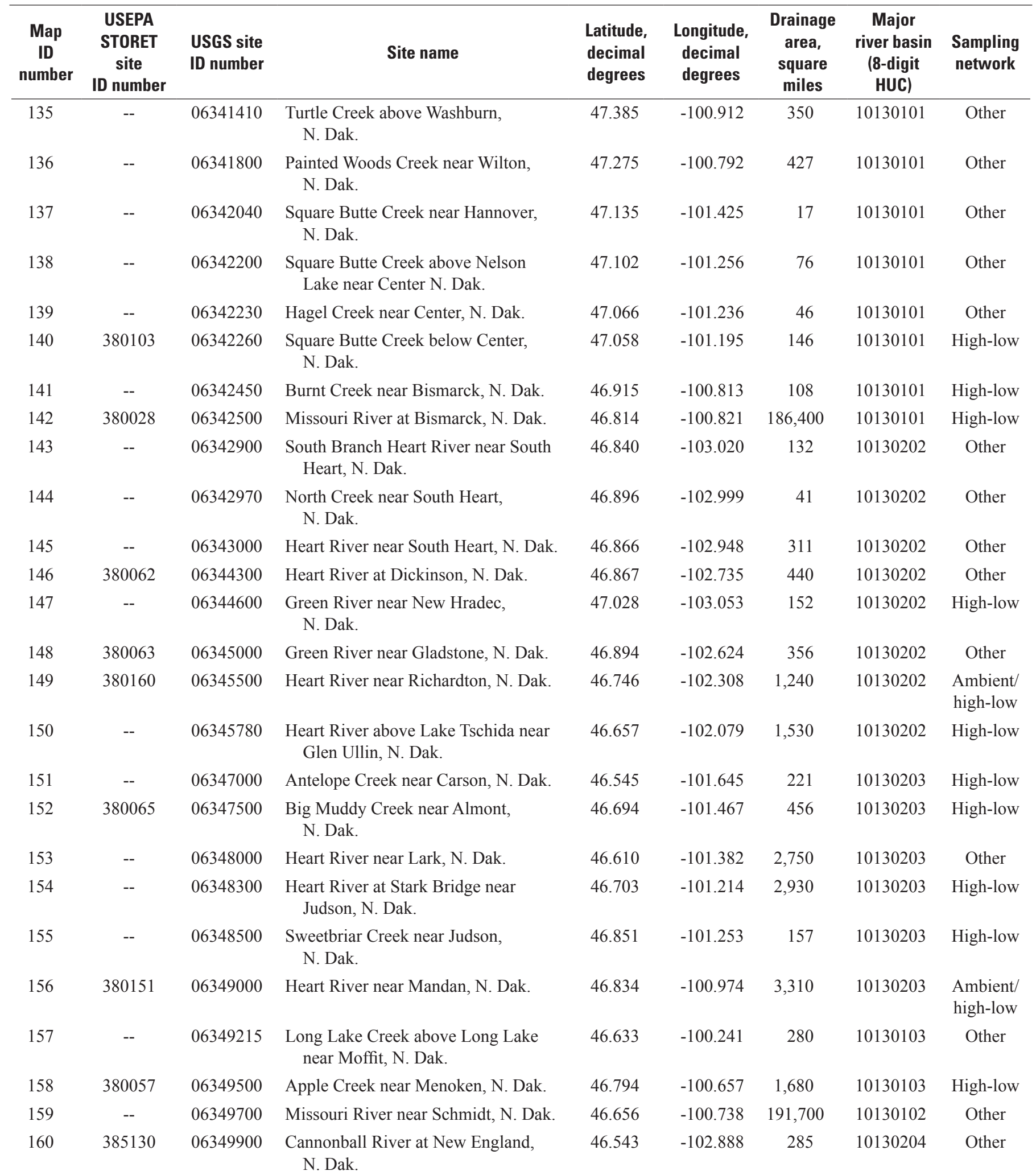


Table 1. Selected stream water-quality sites for North Dakota from 1970 through 2008.—Continued

[ID, identification; USEPA, Environment Protection Agency; STORET, STOrage and RETrieval; USGS, U.S. Geological Survey; HUC, hydrologic unit code; --, not available; Ambient, North Dakota Department of Health Ambient Water-Quality Network; High-Low, North Dakota State Water Commission High-Low Flow Sampling Program; other, sampled as part of various other programs]

\begin{tabular}{|c|c|c|c|c|c|c|c|c|}
\hline $\begin{array}{c}\text { Map } \\
\text { ID } \\
\text { number }\end{array}$ & $\begin{array}{c}\text { USEPA } \\
\text { STORET } \\
\text { site } \\
\text { ID number }\end{array}$ & $\begin{array}{l}\text { USGS site } \\
\text { ID number }\end{array}$ & Site name & $\begin{array}{l}\text { Latitude, } \\
\text { decimal } \\
\text { degrees }\end{array}$ & $\begin{array}{l}\text { Longitude, } \\
\text { decimal } \\
\text { degrees }\end{array}$ & $\begin{array}{c}\text { Drainage } \\
\text { area, } \\
\text { square } \\
\text { miles } \\
\end{array}$ & $\begin{array}{l}\text { Major } \\
\text { river basin } \\
\text { (8-digit } \\
\text { HUC) }\end{array}$ & $\begin{array}{l}\text { Sampling } \\
\text { network }\end{array}$ \\
\hline 161 & -- & 06349930 & $\begin{array}{l}\text { Coal Bank Creek near Havelock, } \\
\text { N. Dak. }\end{array}$ & 46.464 & -102.739 & 70 & 10130204 & Other \\
\hline 162 & -- & 06350000 & Cannonball River at Regent, N. Dak. & 46.427 & -102.551 & 580 & 10130204 & High-low \\
\hline 163 & -- & 06351000 & $\begin{array}{l}\text { Cannonball River below Bentley, } \\
\text { N. Dak. }\end{array}$ & 46.358 & -102.042 & 1,140 & 10130204 & Other \\
\hline 164 & 380105 & 06351200 & $\begin{array}{l}\text { Cannonball River near Raleigh, } \\
\text { N. Dak. }\end{array}$ & 46.127 & -101.333 & 1,640 & 10130204 & $\begin{array}{l}\text { Ambient/ } \\
\text { high-low }\end{array}$ \\
\hline 165 & -- & 06351680 & $\begin{array}{l}\text { White Butte Fork Cedar Creek near } \\
\text { Scranton, N. Dak. }\end{array}$ & 46.322 & -102.996 & 43 & 10130205 & Other \\
\hline 166 & -- & 06352000 & Cedar Creek near Haynes, N. Dak. & 46.155 & -102.475 & 553 & 10130205 & High-low \\
\hline 167 & -- & 06352400 & Timber Creek near Bentley, N. Dak. & 46.101 & -101.957 & 100 & 10130205 & Other \\
\hline 168 & -- & 06352500 & $\begin{array}{l}\text { Cedar Creek near Pretty Rock, } \\
\text { N. Dak. }\end{array}$ & 46.032 & -101.832 & 1,340 & 10130205 & Other \\
\hline 169 & 380077 & 06353000 & Cedar Creek near Raleigh, N. Dak. & 46.092 & -101.333 & 1,750 & 10130205 & $\begin{array}{l}\text { Ambient/ } \\
\text { high-low }\end{array}$ \\
\hline 170 & 380067 & 06354000 & Cannonball River at Breien, N. Dak. & 46.376 & -100.934 & 4,100 & 10130206 & $\begin{array}{l}\text { Ambient/ } \\
\text { high-low }\end{array}$ \\
\hline 171 & 380058 & 06354500 & Beaver Creek near Linton, N. Dak. & 46.258 & -100.233 & 717 & 10130104 & Other \\
\hline 172 & -- & 06354580 & Beaver Creek below Linton, N. Dak. & 46.269 & -100.251 & 765 & 10130104 & High-low \\
\hline 173 & -- & 06354815 & $\begin{array}{l}\text { Porcupine Creek near Fort Yates, } \\
\text { N. Dak. }\end{array}$ & 46.193 & -100.751 & 220 & 10130102 & Other \\
\hline 174 & -- & 06355310 & $\begin{array}{l}\text { Buffalo Creek Tributary near } \\
\text { Gascoyne, N. Dak. }\end{array}$ & 46.111 & -103.039 & 16 & 10130301 & Other \\
\hline 175 & 384210 & 06467600 & James River near Manfred, N. Dak. & 47.644 & -99.828 & 253 & 10160001 & Other \\
\hline 176 & 384130 & 06468170 & James River near Grace City, N. Dak. & 47.558 & -98.863 & 1,060 & 10160001 & $\begin{array}{l}\text { Ambient/ } \\
\text { high-low }\end{array}$ \\
\hline 177 & 384211 & 06468250 & $\begin{array}{l}\text { James River above Arrowwood Lake } \\
\text { near Kensal, N. Dak. }\end{array}$ & 47.400 & -98.797 & 1,200 & 10160001 & Other \\
\hline 178 & -- & 06468300 & $\begin{array}{l}\text { Kelly Creek below Niccum Reservoir } \\
\text { near Bordulac, N. Dak. }\end{array}$ & 47.401 & -98.829 & 188 & 10160001 & Other \\
\hline 179 & -- & 06468500 & James River near Pingree, N. Dak. & 47.142 & -98.783 & 1,670 & 10160001 & Other \\
\hline 180 & 380152 & 06469400 & Pipestem Creek near Pingree, N. Dak. & 47.168 & -98.969 & 700 & 10160002 & High-low \\
\hline 181 & 380015 & 06469500 & $\begin{array}{l}\text { Pipestem Creek near Buchanan, } \\
\text { N. Dak. }\end{array}$ & 47.066 & -98.919 & 758 & 10160002 & Other \\
\hline 182 & 380013 & 06470000 & James River at Jamestown, N. Dak. & 46.890 & -98.682 & 2,820 & 10160003 & $\begin{array}{l}\text { Ambient/ } \\
\text { high-low }\end{array}$ \\
\hline 183 & 380012 & 06470500 & James River at Lamoure, N. Dak. & 46.356 & -98.304 & 4,390 & 10160003 & $\begin{array}{l}\text { Ambient/ } \\
\text { high-low }\end{array}$ \\
\hline 184 & 384215 & 06470800 & Bear Creek near Oakes, N. Dak. & 46.225 & -98.071 & 357 & 10160003 & High-low \\
\hline 185 & 380074 & 06470830 & James River at Oakes, N. Dak. & 46.139 & -98.115 & 5,320 & 10160003 & Other \\
\hline 186 & 384217 & 06470878 & $\begin{array}{l}\text { James River at N. Dak.-S. Dak. } \\
\text { state line }\end{array}$ & 45.936 & -98.174 & 5,480 & 10160003 & High-low \\
\hline
\end{tabular}


Table 2. Water-quality constituents and measurements selected for sites in North Dakota from 1970 through 2008.

[USGS, U.S. Geological Survey; NDDH, North Dakota Department of Health; *, less than 5 values in data set, values were excluded; $\mu \mathrm{g} / \mathrm{L}$, micrograms per liter; mg/L, milligrams per liter; --, not available; $\mu \mathrm{S} / \mathrm{cm}$, microsiemens per centimeter; $\mathrm{C}$, Celsius]

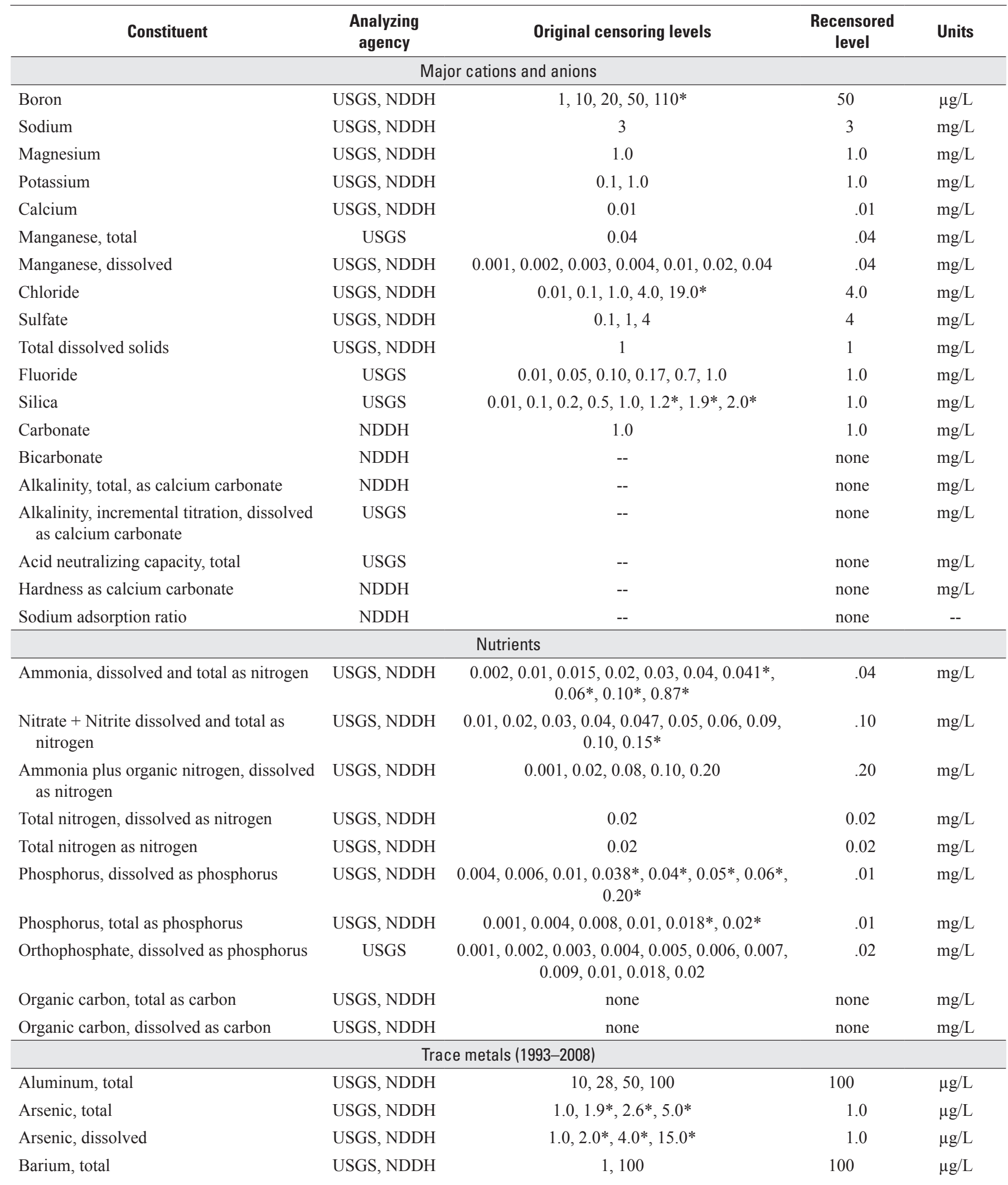


Table 2. Water-quality constituents and measurements selected for sites in North Dakota from 1970 through 2008._Continued

[USGS, U.S. Geological Survey; NDDH, North Dakota Department of Health; *, less than 5 values in data set, values were excluded; $\mu$ g/L, micrograms per liter; $\mathrm{mg} / \mathrm{L}$, milligrams per liter; --, not available; $\mu \mathrm{S} / \mathrm{cm}$, microsiemens per centimeter; $\mathrm{C}$, Celsius]

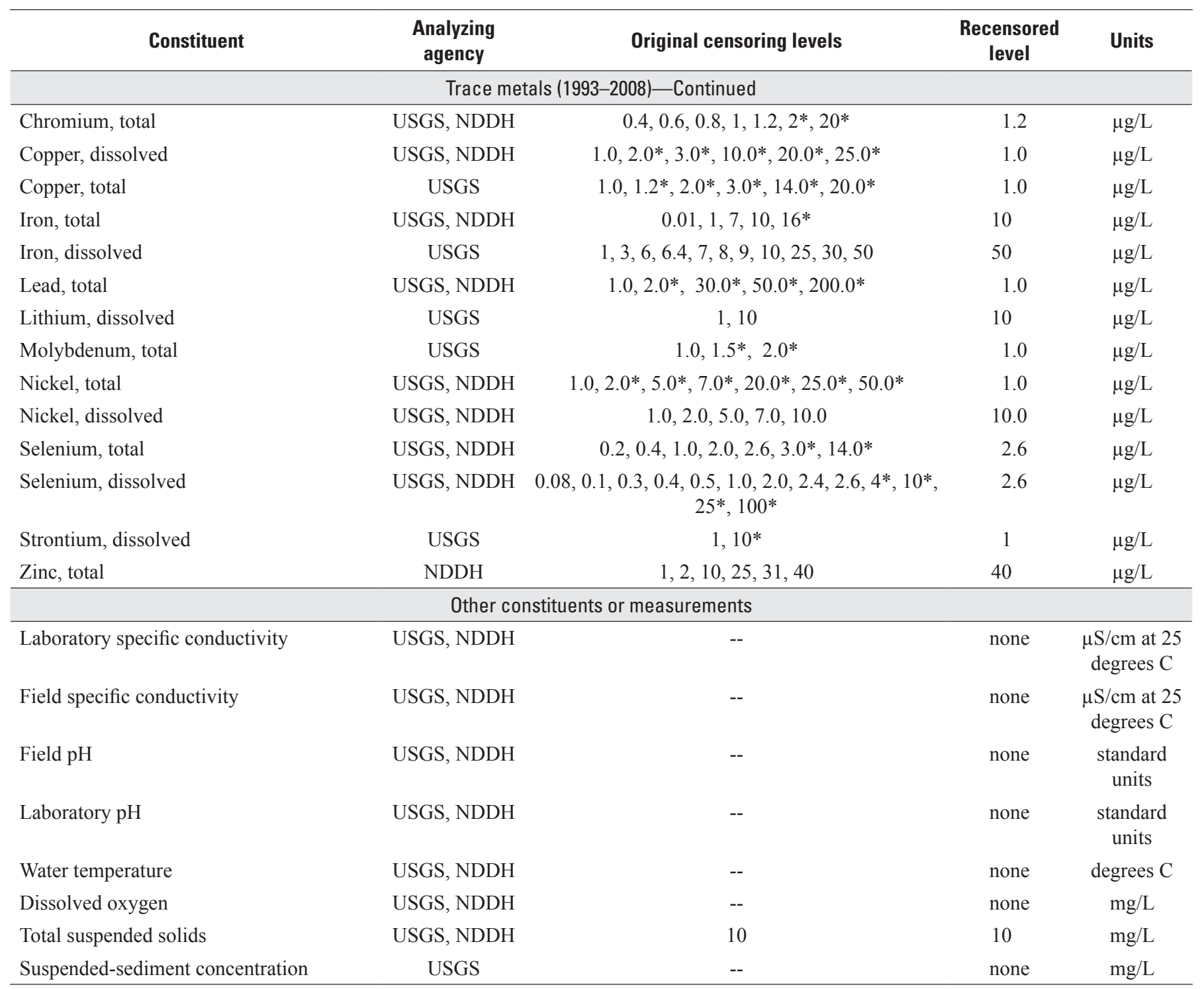

temperature, and specific conductivity (table 2). For two of the nutrients (ammonia and nitrate plus nitrite), dissolved and total concentrations were not substantially different and thus were combined to form a "dissolved and total" constituent concentration. The combined concentrations provided a more complete sampling record for analyzing loads and concentration trends than either the separate dissolved or total concentrations. On sampling dates for which both the dissolved and total concentrations were available, the dissolved concentration was used for the combined data.

\section{Descriptive Statistics}

Descriptive statistics (sample size, period of record, minimum, maximum, and median) were computed for each constituent and site included in the study (appendix tables 1-1 through 1-5). Median concentrations of selected water-quality constituents were mapped using Geographic Information Systems (GIS) to indicate the spatial distribution of constituent concentrations across the State. Median concentrations were determined for the period of record from 1970 through 2008 (1993 through 2008 for trace metals) and for the spring (March through June), summer (July through October), and winter (November through February) seasons at each sampling location. Median values were calculated with no consideration 
for period of record, sampling dates, sampling times, or number of samples taken at each sampling location.

\section{Load and Yield Estimation}

Load and yield estimates for water-quality constituents were calculated for selected sites from constituent concentrations and streamflows measured at each site. Constituent load (L) is a function of the volumetric rate of water passing a point in the stream $(\mathrm{Q})$ and the constituent concentration within the water (C). Constituent yield is a function of constituent load and the drainage area contributing to flow at the site. Sites and constituents used for the load and yield estimation were chosen on the basis of data availability and the importance of the constituent. Based on the availability of water-quality and streamflow data, 34 sites were determined to have adequate data (minimum of 20 samples) for the estimation of loads and yields for eight constituents.

Regression methods were used to estimate constituent loads. These methods use natural logarithm (ln)-transformed relations between $\mathrm{Q}$ and $\mathrm{C}$ to estimate daily $\mathrm{C}$ (or $\mathrm{L}$ ) for a particular constituent at a site (Cohn and others, 1989; Cohn and others, 1992; Cohn, 1995). The regression method can account for non-normal data distributions, seasonal and long-term cycles, censored data, biases associated with using logarithmic transformations, and serial correlations of the residuals (Cohn, 1995). Regression methods use discrete water-quality samples often collected over several years and a daily streamflow hydrograph. A regression model for estimating constituent load can be expressed as:

$$
\ln (L)=\beta_{0}+\beta_{1} \ln \left(Q_{d}\right)+\beta_{2} T+\beta_{3} \sin (2 \pi T)+\beta_{4} \cos (2 \pi T)+E
$$

where

$$
\begin{array}{cl}
\ln () & \begin{array}{c}
\text { represents the natural logarithm } \\
\text { function; }
\end{array} \\
\beta_{0}, \beta_{1}, \beta_{2}, \beta_{3}, \text { and } \beta_{4} & \begin{array}{l}
\text { are the coefficients of the model; } \\
Q_{d}
\end{array} \\
\text { is the daily mean discharge, in } \\
\text { cubic feet per second; } \\
E & \begin{array}{l}
\text { is decimal time, in years; and } \\
\text { is the model error. }
\end{array}
\end{array}
$$

In this model, relations between discharge and load are identified by the $\beta_{1}$ coefficient, temporal trends are identified by $\beta_{2}$, and seasonal effects are identified by $\beta_{3}$ and $\beta_{4}$. Transforming the results of the model from logarithmic space to linear space was accomplished using an adjusted maximum likelihood estimator (AMLE) (Cohn and others, 1992). The AMLE method can also handle censored values.

Best-fit load models for all constituents at each of the 34 sites were determined using the S-LOADEST computer program (Runkel and others, 2004). Best-fit models were selected based on residual plots, significance of included variables, results of normality tests, and coefficient of determination of the regression. For example, if the seasonality variables $\left(\beta_{3}\right.$ and $\beta_{4}$ ) were determined to be significant, they were included in the model, if not, they were not used in the model. Some models may have only included a significant relation between load and discharge $\left(\beta_{1}\right)$. Models were not determined if the calibration dataset was made up of more than 80 percent censored values. No minimum criteria for model performance were used; therefore, best-fit models of the group were selected even if the model was poor.

Normalized annual loads (tons per year) were calculated by creating a "reference" discharge period of 30 years of data around the median year of the model calibration dataset and using the average of the daily flows for the reference period to create a normalized set of daily flows (one for each day of the year). For each site, the calibration dataset, including all constituents, was reviewed to find the data range across time. The median of this range was used to select discharge data for that site, 15 years prior to the median date and 15 years after the median date was used. From this 30-year discharge record, the average of the mean daily discharge was calculated for each day of the year. These average daily values were grouped into a normalized discharge dataset spanning a full year, January 1 through December 31. This normalized discharge dataset for each site was used to calculate the normalized annual load from the best-fit model for each constituent. Normalized annual yields (pounds per year per square mile) for each constituent at each site were calculated by dividing the normalized annual loads by the drainage area (square miles) contributing to flow at the sampling site. Sites 45 and 112 (table 1) only had 15 and 16 years of streamflow data during the 30 -year period, respectively; however, the sites were still included and the average flow data was calculated from the data available. Site 5 (Red River of the North below Wahpeton, N. Dak.) had no discharge record; however, discharge data were available at site 2, just upstream from site 5 (fig. 1), and was used in the normalized load calculation for site 5 .

\section{Trend Analysis}

Sites and constituents used for the water-quality trend analysis were chosen on the basis of data availability and the importance of the constituent to aquatic health in the State. Ten sites were selected for trend analysis for sulfate, total dissolved solids (TDS), nitrate plus nitrite, and total phosphorus. The period of record chosen for the analysis was the 34-year period from 1975 through 2008. Concentration data for 1970 to 1974 were not used because the time-series model used in the trend analysis required 5 years of flow data prior to the first concentration sample.

A statistical time-series model for streamflow and constituent concentration developed by the USGS (Vecchia, 2000) was used in this report to detect water-quality trends. Other applications of the model include Jones and Armstrong (2001), Trench and Vecchia (2002), Vecchia (2003), and Vecchia (2005). Details on the theory and parameter estimation for the model are described in Vecchia (2005). 
In the time-series model, concentration data were partitioned into several components according to equation 2 :

$$
\log (C)=M+A N N+S E A S+T R E N D+H F V
$$

where

$$
\begin{aligned}
& \log \text { denotes the base-10 logarithm; } \\
& C \text { is the concentration, in milligrams per liter; } \\
& M \quad \text { is the long-term mean of the log-transformed } \\
& \text { concentration, as the base-10 logarithm of } \\
& \text { milligrams per liter; } \\
& A N N \quad \text { is the annual concentration anomaly } \\
& \text { (dimensionless); } \\
& \text { SEAS is the seasonal concentration anomaly } \\
& \text { (dimensionless); } \\
& \text { TREND is the concentration trend (dimensionless); } \\
& \text { and } \\
& H F V \text { is the high-frequency variability of the } \\
& \text { concentration (dimensionless). }
\end{aligned}
$$

The procedure used to partition the data is described in detail in Vecchia (2005). In equation 2, the annual concentration anomaly $(A N N)$, seasonal concentration anomaly $(S E A S)$, and high-frequency variability $(H F V)$ terms represent natural variability in concentration for different time scales. $A N N$ is an estimate of the inter-annual variability in concentration that can be attributed to long-term variability in streamflow. Extended droughts and wet periods can change the chemical composition of streamflow by changing the degree of contact between surface runoff and soil particles, and changing the relative composition of runoff among groundwater, overland flow, and subsurface flow (Vecchia, 2005).

SEAS is an estimate of the seasonal variability in concentration that can be attributed to seasonal variability in streamflow or to factors other than variability in streamflow. For example, the seasonal snow-accumulation and snowmelt cycle causes seasonal fluctuations in streamflow and water quality. Seasonal temperature differences also may affect the rate of chemical processes, such as reduction of ammonia, and thus cause seasonal differences in nutrient concentrations. Seasonality also may occur in the sources of chemical constituents (for example, fertilizer application or roadway deicing), independent of streamflow conditions (Vecchia, 2005).

TREND is an estimate of the long-term systematic changes in concentration that are unrelated to long-term variability in streamflow. A statistically significant trend might indicate changes in human activities (such as land use, fertilizer application, and sewage treatment) that change the amount of a particular chemical constituent available for solution or suspension in surface water, or that change the rate at which the constituent reaches the surface water. For this report, a trend was defined as a statistically significant increase or decrease in median concentration for a period of 10 years or more. The methods used to detect the trends and evaluate their statistical significance are described in Vecchia (2005).

$H F V$ is an estimate of the variability in concentration for time scales that are smaller than the seasonal time scale (time scales of several days to several weeks). Thus, high-frequency variability is the variability that remains after the removal of seasonal and annual anomalies and trends. Day-to-day changes in meteorological conditions may cause high-frequency variability in streamflow and concentration. The high-frequency variability depends on a time-series model, called a periodic autoregressive moving average model, that accounts for the presence of serial correlation among concentrations (for example, the tendency for high or low values to persist for several days to several weeks before returning to normal levels) (Vecchia, 2005).

Two types of time-series plots are used in this report to illustrate long-term changes in concentration. The first type shows the measured log-transformed concentrations $(\log (C)$ in eq. 2) as well as the fitted annual median concentrations obtained by adding together the mean, annual anomaly, and trend in equation 3 :

$$
F A M C=M+A N N+T R E N D
$$

where

FAMC is the fitted annual median concentration, as the base-10 logarithm of milligrams per liter.

Changes in FAMC through time indicate long-term (interannual) changes in median concentration resulting from both streamflow-related variability and trend.

The second type of time-series plot illustrates the longterm changes in median concentration that are due just to the trend. Log-transformed concentrations that have both the seasonal and annual anomalies removed are referred to in this report as standardized concentrations. Using equation 2, the standardized concentration is defined as:

$$
S C=\log (C)-A N N-S E A S=M+T R E N D+H F V
$$

where

$$
\begin{aligned}
& S C \quad \text { is the standardized concentration, as the base- } \\
& 10 \text { logarithm of milligrams per liter. }
\end{aligned}
$$

The standardized concentrations defined by equation 4 are analogous to the flow-adjusted concentrations defined in previous publications as the residuals from a regression model that relates concentration to concurrent daily streamflow (Hirsch and others, 1982); however, the approach used for this report generally is more effective than a regression-based approach for removing streamflow-related variability (Vecchia, 2005). Time series plots showing the standardized concentrations along with the fitted trend $(M+T R E N D)$ illustrate long-term changes in median concentration that are due to factors other than natural flow-related variability. 


\section{Sample Network Design}

Design of an effective State-wide water-quality sampling network requires both a spatial component (where to sample) and a temporal component (how often to sample). An efficient design is one that accomplishes the objectives of the sampling programs with a minimum of spatial and temporal redundancy. For this study, three sampling program objectives were considered: characterizing spatial water-quality variability, detecting temporal trends in water quality, and estimating constituent loads. To simplify the design analysis, these three objectives were considered separately. Also, to simplify the analysis, the network design was evaluated using four key water-quality constituents: dissolved sulfate, TDS, nitrate plus nitrite, and total phosphorus. Although sampling designs were not explicitly evaluated for other constituents, it was expected that an efficient network for these constituents would be relatively efficient for other constituents as well.

To evaluate the first objective (characterizing spatial water-quality variability), all 186 sites (table 1) were used, regardless of whether a site was an active or discontinued sampling site. Sites were nested in downstream to upstream order. For each downstream/upstream pair and each waterquality sample for the downstream site, a concurrent waterquality sample for the upstream site was defined as the closest upstream sample (in time) to the downstream sample such that the upstream sample was taken within 1 week before or after the downstream sample. If there was no upstream sample taken within 1 week of the downstream sample, the concurrent upstream sample was defined as missing. Only downstream/ upstream pairs with at least 10 concurrent samples defined in this manner were included. The average absolute difference between the paired downstream and upstream concentrations, expressed as a percent of the average downstream concentration, was related to the average absolute difference in daily flow between the downstream and upstream pairs, expressed as a percent of the average downstream flow. Relating the concentration differences to the flow differences provided information on redundancy of concentration values as the intervening flow between the downstream and upstream sites became small in relation to the downstream site. For example, if the upstream concentration had a 90 percent chance of being within 20 percent, on average, of the downstream concentration whenever the upstream flow was at least 80 percent, on average, of the downstream flow, water-quality samples at the upstream might be considered to be redundant given the downstream water-quality data.

To evaluate the second sampling objective (detecting temporal trends), the time-series model, described in the previous section for evaluating concentration trends, was used to evaluate the sensitivity of various temporal sampling designs for monitoring future concentration trends. The design methodology is described in Vecchia (2005). Temporal designs (specified by the number and timing of water-quality samples within each year) were evaluated with respect to their sensitivity to detect a trend within each of three seasons, a spring high-flow season (March-June), a summer low-flow season (July-October), and a winter low-flow season (NovemberFebruary). An efficient design is one that maximizes the sensitivity to detect trends in each season with the fewest number of samples. Sensitivity is measured by the characteristic trend, which is defined as the percent change in concentration that has an 80-percent chance of being detected after 5 years of sampling. An efficient design is one that has low characteristic trends (high sensitivity) for all of the constituents and all of the seasons subject to budgetary constraints.

To evaluate the third sampling objective (estimating constituent loads), the efficient designs described previously for detecting concentration trends were evaluated with respect to their ability to estimate loads. The accuracy of estimated loads is dependent on both flow and concentration, and adding extra samples during times of year when most of the load is expected to be transported can result in substantial improvements in the accuracy of estimated loads. Methods described by Gilroy and others (1990) were used to evaluate efficiency of the sampling designs for estimating loads during 5-year periods (the objective of the load component of the NDDH ambient program) and to determine if and when extra samples would be beneficial for estimating loads.

\section{Median Concentrations of Selected Constituents}

Median concentrations of water-quality constituents at sampling locations in North Dakota were determined for the period of record and for the spring (March through June), summer (July through October), and winter (November through February) seasons at each sampling location. Median values were calculated with no consideration for period of record, sampling dates, sampling times, or number of samples taken at each sampling location.

\section{Major Ions and Total Dissolved Solids}

Many water-quality samples were analyzed for several major ions included in this report; however, only the information on the concentrations of sulfate, chloride, and TDS are presented in this section. Period of record summary statistics for all of the constituents analyzed and seasonal medians for selected constituents in this report are presented in appendix 1 (tables 1-1 and 1-5).

Sulfur and chloride are naturally present in soils across North Dakota. Sulfur is readily oxidized to produce sulfate ions that are highly soluble (Hem, 1985). Sulfate in streams may be affected by land-use changes that can increase or decrease the exposure of naturally occurring sulfur to surface runoff. Human sources of sulfate, such as emissions from burning fossil fuels and wastewater discharge from mining and industrial operations, also may affect sulfate concentrations in 
streams. Chloride also is highly soluble, but generally occurs in much smaller amounts in soils compared to sulfur. In contrast to other ions, most of the chloride content in streams is in the form of ionized chloride (Hem, 1992). Human activities such as roadway and driveway de-icing and industrial and municipal wastewater discharge also may introduce chloride to streams.

Dissolved solids in streams are composed of major ions (such as calcium, magnesium, sodium, potassium, sulfate, and chloride) and many other constituents that are present in small quantities. TDS concentrations may be affected by different constituents in different locations in the State.

In general, median sulfate concentrations tended to be greater in the southwest part of the State compared to the northeast part of the State in the Red River of the North Basin (fig. 2). Seasonal variations indicated lower median concentrations in samples collected during spring and higher concentrations from samples collected during winter at many locations. This seasonal pattern is likely due to the dilution effect of higher streamflows that occur during the spring compared to the lower streamflows that occur during winter. Median sulfate concentrations exceeded the USEPA secondary drinking water regulation of 250 milligrams per liter (mg/L) (U.S. Environmental Protection Agency, 2009) and the North Dakota sulfate numerical standard for class 1 streams of $250 \mathrm{mg} / \mathrm{L}$ (30-day arithmetic average; North Dakota Department of Health, 2010 ) at 86 out of 183 sites in North Dakota (fig. 2). The lowest median sulfate concentrations for the period of record at a sampling site was $20 \mathrm{mg} / \mathrm{L}$ at Big Coulee near Fort Totten, N. Dak. (site 16), and the highest median sulfate concentration for the period of record at a sampling site was $2,400 \mathrm{mg} / \mathrm{L}$ at Buffalo Creek tributary near Gascoyne, N. Dak. (site 174) (appendix table 1-5).

Median chloride concentrations tended to be lower in the southwest and higher in the northeast parts of the State (fig. 3), nearly the opposite of the pattern seen for median sulfate concentrations (fig. 2). Substantial differences in median chloride concentrations were not evident among samples collected during different seasons. Median chloride concentrations exceeded the North Dakota numerical standard of $175 \mathrm{mg} / \mathrm{L}$ (30-day arithmetic average) (North Dakota Department of Health, 2010) at 3 of 184 sites in North Dakota. The lowest median chloride concentrations for the period of record at a sampling site were less than $4.0 \mathrm{mg} / \mathrm{L}$ at West Branch Antelope Creek No. 4 near Zap, N. Dak. and Coal Lake Coulee near Hensler, N. Dak. (sites 129 and 133, respectively), and the highest median chloride concentration for the period of record at a sampling site was $446.5 \mathrm{mg} / \mathrm{L}$ at the Turtle River at Manvel, N. Dak. (site 58) (appendix table 1-5).

In general, median TDS concentrations tended to be lower for sites in the Red River of the North, Souris, and James River Basins compared to sites in southwest North Dakota (figs. 1 and 4). Like sulfate, median TDS concentrations at many locations tended to be lower in samples collected during spring compared to samples collected during the summer and winter, likely because of the dilution effect of higher streamflows that occur during the spring compared to the lower streamflows that occur during other seasons. Median TDS concentrations exceeded the USEPA secondary drinking-water regulation for TDS of $500 \mathrm{mg} / \mathrm{L}$ (U.S. Environmental Protection Agency, 2009) at 136 out of 184 sites. The lowest median TDS concentration for the period of record at a sampling site was $172 \mathrm{mg} / \mathrm{L}$ at the Lower Branch Rush River near Prosper, N. Dak. (site 49), and the highest median TDS concentration for the period of record at a sampling site was 4,270 mg/L at Buffalo Creek tributary near Gascoyne, N. Dak. (site 174) (appendix table 1-5).

\section{Nutrients}

Nutrient dynamics are controlled by activities in the basin and processes that occur in the stream. Wastewater-treatment plant discharge can be a major point source of nitrogen (mainly nitrate), phosphorus, and organic material. Septic systems can act as point sources as nutrients migrate through the groundwater system into the stream. The influence of point sources usually is more evident during base-flow conditions in a stream because concentrations are less affected by dilution. Nonpoint sources of nitrogen, phosphorus, and organic carbon mainly are delivered during runoff events as rainfall washes material off the landscape into the stream, resulting in greater concentrations during high-flow conditions. Some nonpoint sources of nutrients include runoff from agricultural areas, where fertilizers are applied or livestock production occurs; runoff from urban areas, where fertilizers are applied to lawns, shrubs, and trees; and from atmospheric deposition of nitrogen. Natural sources of nitrogen and phosphorus include fixation of atmospheric nitrogen by plants and animals, dissolution of phosphorus-bearing rocks or minerals in the soil, and oxidation of organic matter, including soil organic matter and decaying plants and animals (Hem, 1985).

Instream processes also can affect nutrient concentrations (Allan, 1995). Aquatic vegetation, particularly algae, depends on nitrogen and phosphorus for its food supply. Nitrate is the most stable ion of nitrogen over a wide range of conditions and is readily assimilated by algae. Total phosphorus concentrations include inorganic phosphorus (in solution, complexed with iron or other trace elements, or adsorbed to sediment particles) and organic phosphorus. Sources of organic carbon in the water column can include those outside the aquatic system and within the aquatic system. Natural sources of organic carbon outside the aquatic system include soils and plants, and sources within the aquatic system include excretion from actively growing algae or the decomposition of dead algae and macrophytes. Anthropogenic (human influenced) sources of organic carbon include wastewater-treatment discharges, animal waste, and septic systems. Activities that cause land disturbance such as row-crop agriculture, animal grazing, timber harvesting, mining, road construction and maintenance, and urbanization also can result in increased stream concentrations of organic carbon (Allan, 1995). 

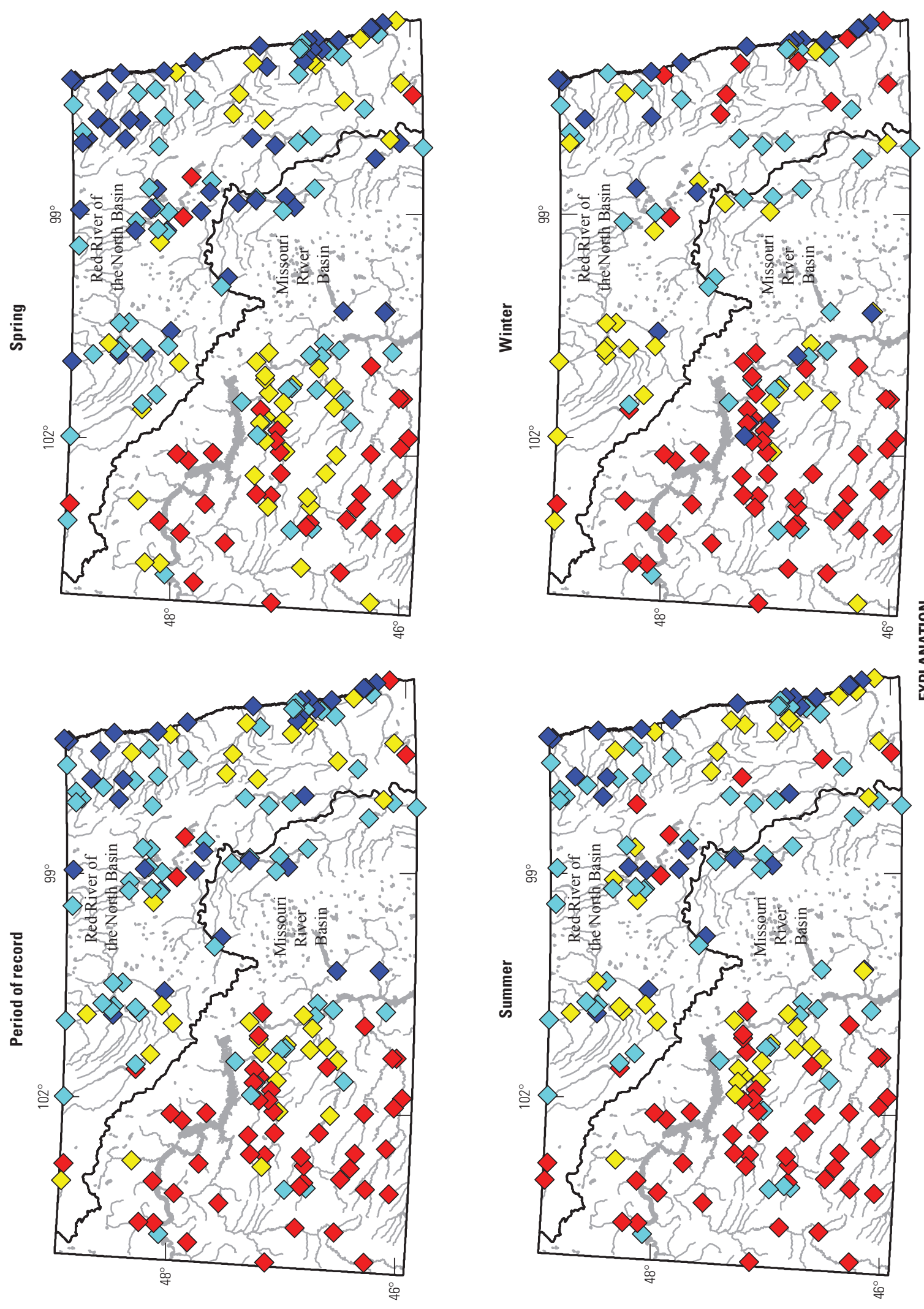

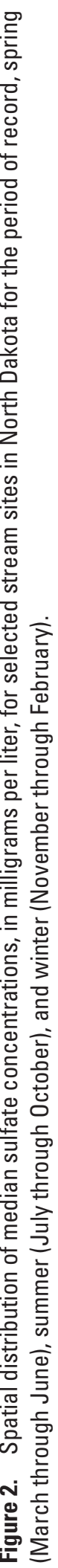



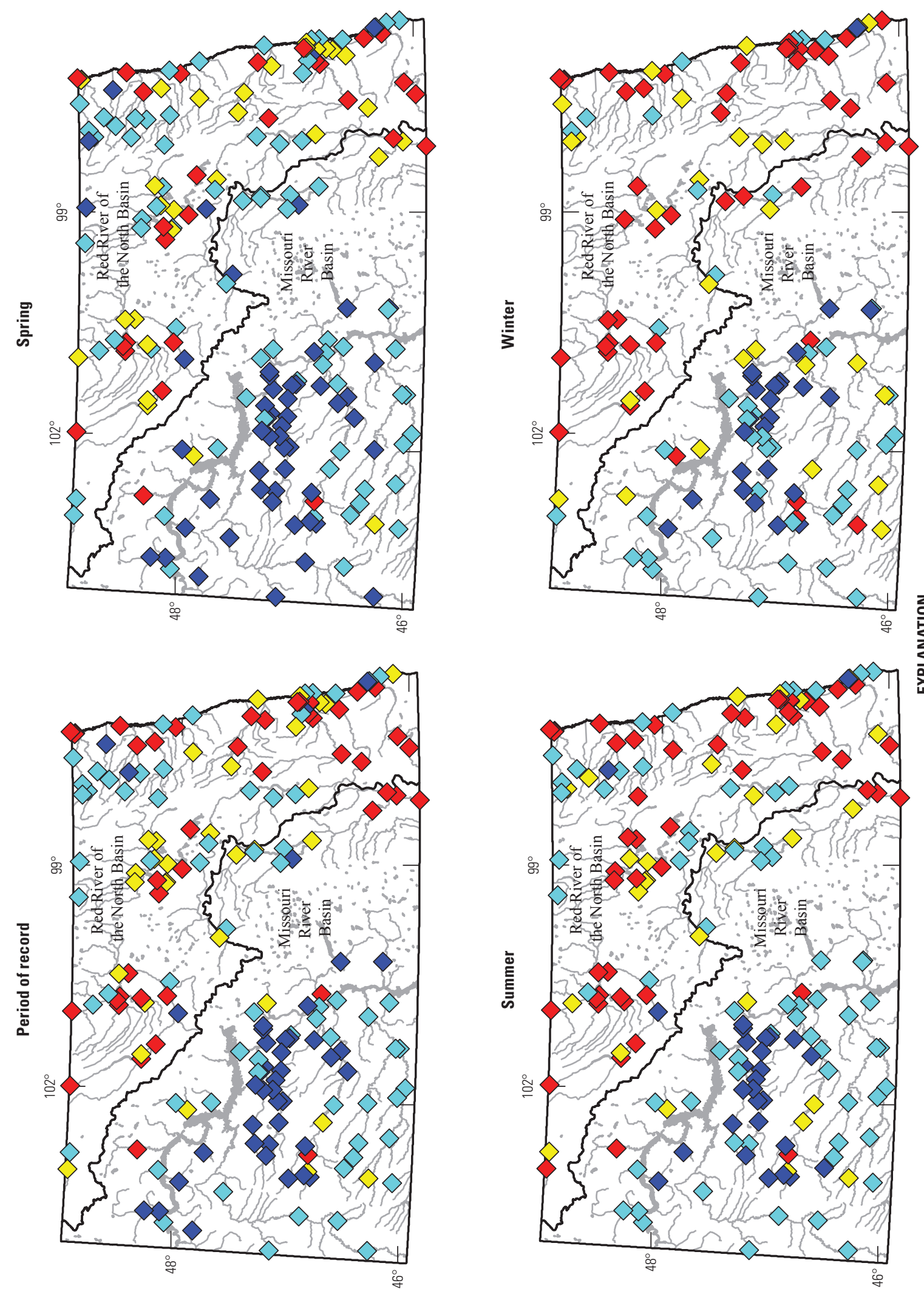

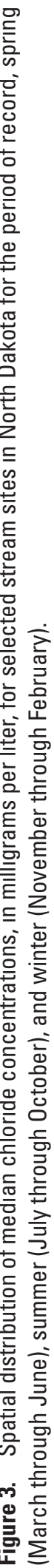



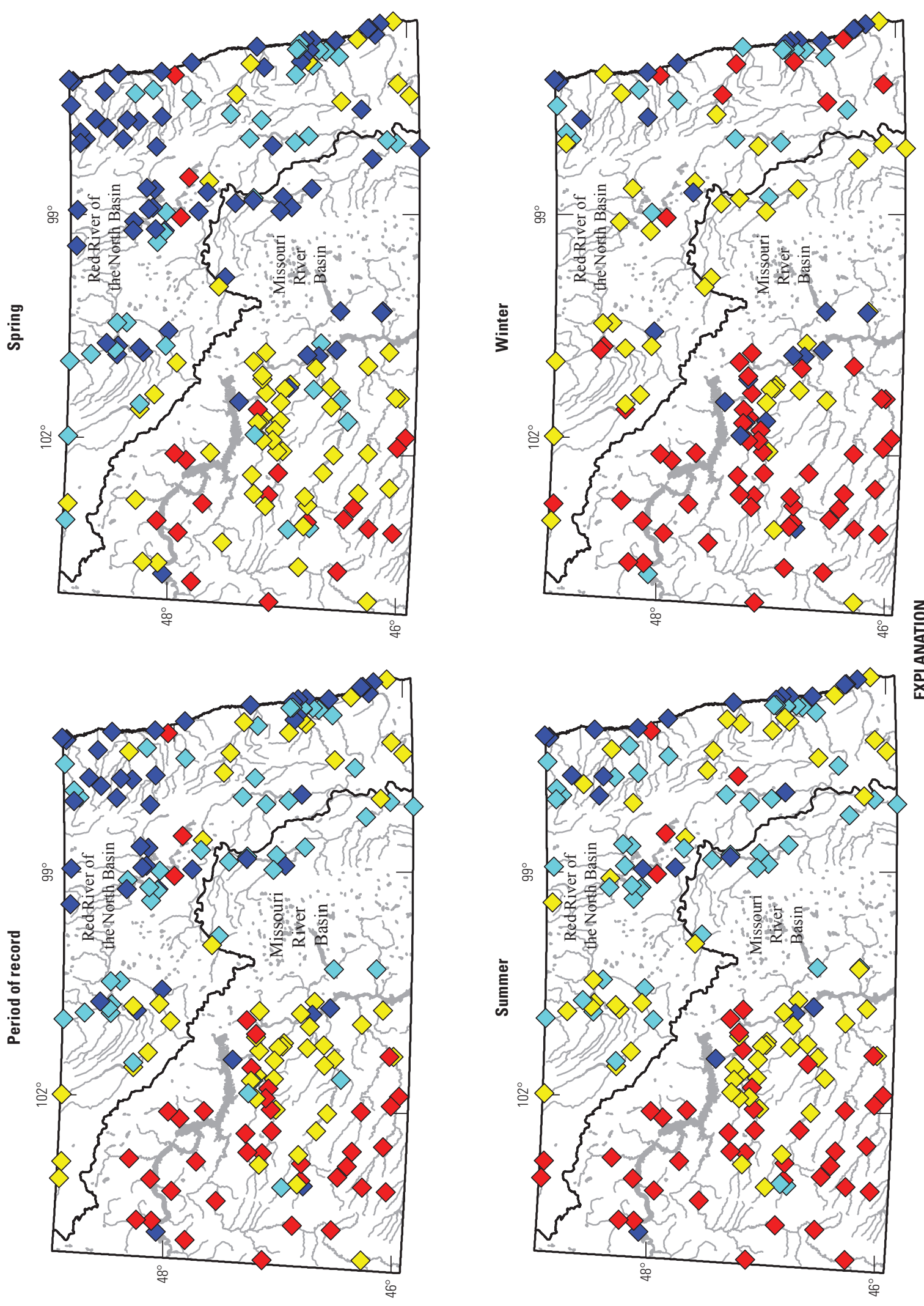

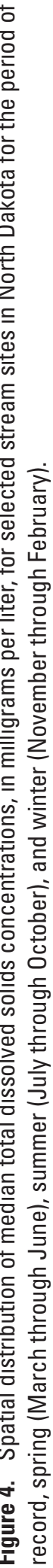


Water-quality samples at selected sites across the State were analyzed for various forms of nitrogen, phosphorus, and carbon. In this section, information describing concentrations of ammonia, nitrate plus nitrite, total nitrogen, dissolved phosphorus, total phosphorus, dissolved organic carbon, and total organic carbon are presented. Period of record summary statistics for all of the constituents analyzed and seasonal medians for selected constituents in this report are presented in appendix 1 (tables 1-2 and 1-5).

In general, overall median ammonia concentrations (total and dissolved) were spatially variable across the State (fig. 5). Seasonally, median ammonia concentrations generally were highest during winter and lowest in summer (fig. 5). No distinct national standards for ammonia exist and North Dakota State standards vary based on water temperature and pH (North Dakota Department of Health, 2010). Median ammonia concentrations for the period of record at all of the sites (139 sites) ranged from less than $0.04 \mathrm{mg} / \mathrm{L}$ as nitrogen at 64 sites to $0.59 \mathrm{mg} / \mathrm{L}$ as nitrogen at the Square Butte Creek below Center, N. Dak. (site 140) (appendix table 1-5).

The highest overall median nitrate plus nitrite concentrations (total and dissolved) tended to occur in the Red River of the North Basin (fig. 6). This pattern of high concentrations in the Red River of the North Basin particularly was evident during the spring season, and may reflect fertilizer application to row crops and other cropland in that area. This pattern was reversed during the winter season, with the lowest median concentrations occurring in the Red River of the North Basin. No median concentrations exceeded the North Dakota drinking-water standard. In North Dakota, nitrate plus nitrite concentrations cannot exceed $10 \mathrm{mg} / \mathrm{L}$ for any waters used as a municipal or domestic drinking-water supply (North Dakota Department of Health, 2010). Median nitrate plus nitrite concentrations for the period of records ranged from less than $0.10 \mathrm{mg} / \mathrm{L}$ as nitrogen at 98 out of 150 sites to $1.60 \mathrm{mg} / \mathrm{L}$ as nitrogen at Cart Creek at Mountain, N. Dak. (site 66) (appendix table 1-5).

Median dissolved and total phosphorus concentrations across North Dakota generally were greater for the Red, Souris, and James River Basins than river basins in the southwest part of the State (figs. 1, 7, and 8). Median dissolved phosphorus concentrations tended to be similar among the seasons (fig. 7), whereas median total phosphorus concentrations tended to be higher in the summer than other seasons (fig. 8), particularly in the Souris River, Devils Lake, and James River Basins. No water-quality standards have been defined by the USEPA or North Dakota for dissolved or total phosphorus concentrations. Median dissolved phosphorus concentrations for the period of record ranged from less than $0.01 \mathrm{mg} / \mathrm{L}$ as phosphorus at 11 out of 128 sites to $0.55 \mathrm{mg} / \mathrm{L}$ as phosphorus at Channel A near Penn, N. Dak. (site 30) (appendix table 1-5). Median total phosphorus concentrations for the period of record ranged from less than $0.01 \mathrm{mg} / \mathrm{L}$ as phosphorus at the Missouri River at Garrison Dam, N. Dak. (site 115 ) to $0.51 \mathrm{mg} / \mathrm{L}$ as phosphorus at the Elm River near Kelso, N. Dak. (site 51) (appendix table 1-5). The relatively low total phosphorus at site 115 may be because the site is below a dam. Total phosphorus includes inorganic and organic phosphorus. Inorganic phosphorus can be adsorbed to sediment particles that may be retained in the upstream reservoir by settling and organic phosphorus also may be retained in the upstream reservoir by biological processes (Hem, 1985).

Median dissolved and total organic carbon concentrations tended to be lowest in samples collected at sites located in the eastern part of the State compared with other median concentrations at sites throughout the remainder of the State (figs. 9 and 10). No water-quality standards have been defined by the USEPA or North Dakota for dissolved or total organic carbon concentrations. Median dissolved organic carbon concentrations for the period of record ranged from $3.3 \mathrm{mg} / \mathrm{L}$ as carbon at the Missouri River at Garrison Dam, N. Dak. (site 115) to $30.0 \mathrm{mg} / \mathrm{L}$ as carbon at Buffalo Creek Tributary near Gascoyne, N. Dak. (site 174) (table 1-5). Median total organic carbon concentrations for the period of record ranged from $4.0 \mathrm{mg} / \mathrm{L}$ as carbon at the Missouri River near Schmidt, N. Dak. (site 159) to $24.0 \mathrm{mg} / \mathrm{L}$ as carbon at the East Fork Shell Creek near Parshall, N. Dak. (site 108) (appendix table 1-5).

\section{Trace Metals}

Trace-metal concentrations generally are much lower than dissolved major ion and nutrient concentrations in streams in North Dakota. Trace metals in streams exist in both dissolved and particulate form. Constituents selected for presentation in this section were chosen dependent on the amount of data available, and included only the total concentration (includes dissolved and particulate concentrations). Analyses for many trace metals were performed on water samples collected from sites across North Dakota from 1993 through 2008, but only information concerning the concentrations of total aluminum, total arsenic, total chromium, total iron, total lead, and total nickel are presented in this section. Period of record summary statistics for all of the constituents analyzed and seasonal medians for selected constituents in this report are presented in appendix 1 (tables 1-3 and 1-5).

Median total aluminum concentrations generally were higher in samples from sites in the southern and eastern parts of the State compared to concentrations in other parts of the State (fig. 11). Median concentrations exceeded the USEPA secondary drinking-water regulation for aluminum of 200 micrograms per liter ( $\mu \mathrm{g} / \mathrm{L})$ (U.S. Environmental Protection Agency, 2009) at 45 out of 57 sites with available data. Seasonal variations indicated higher median total aluminum concentrations during spring and summer and lower concentrations during winter at many locations. Median total aluminum concentrations for the period of record ranged from less than $100 \mu \mathrm{g} / \mathrm{L}$ at 7 out 57 sites to $20,050 \mu \mathrm{g} / \mathrm{L}$ at the Little Missouri River near Marmarth, N. Dak. (site 110) (appendix table 1-5). 

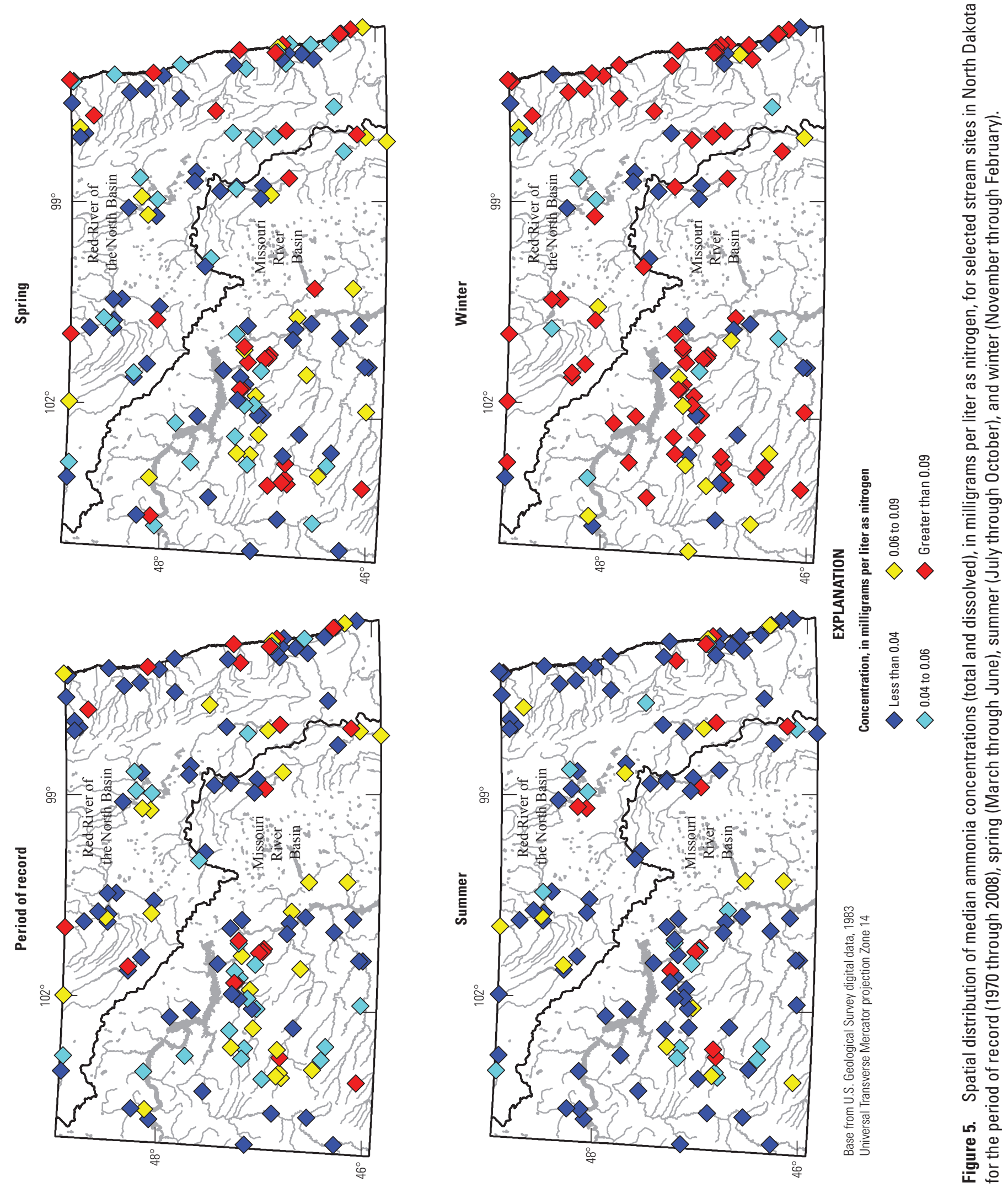

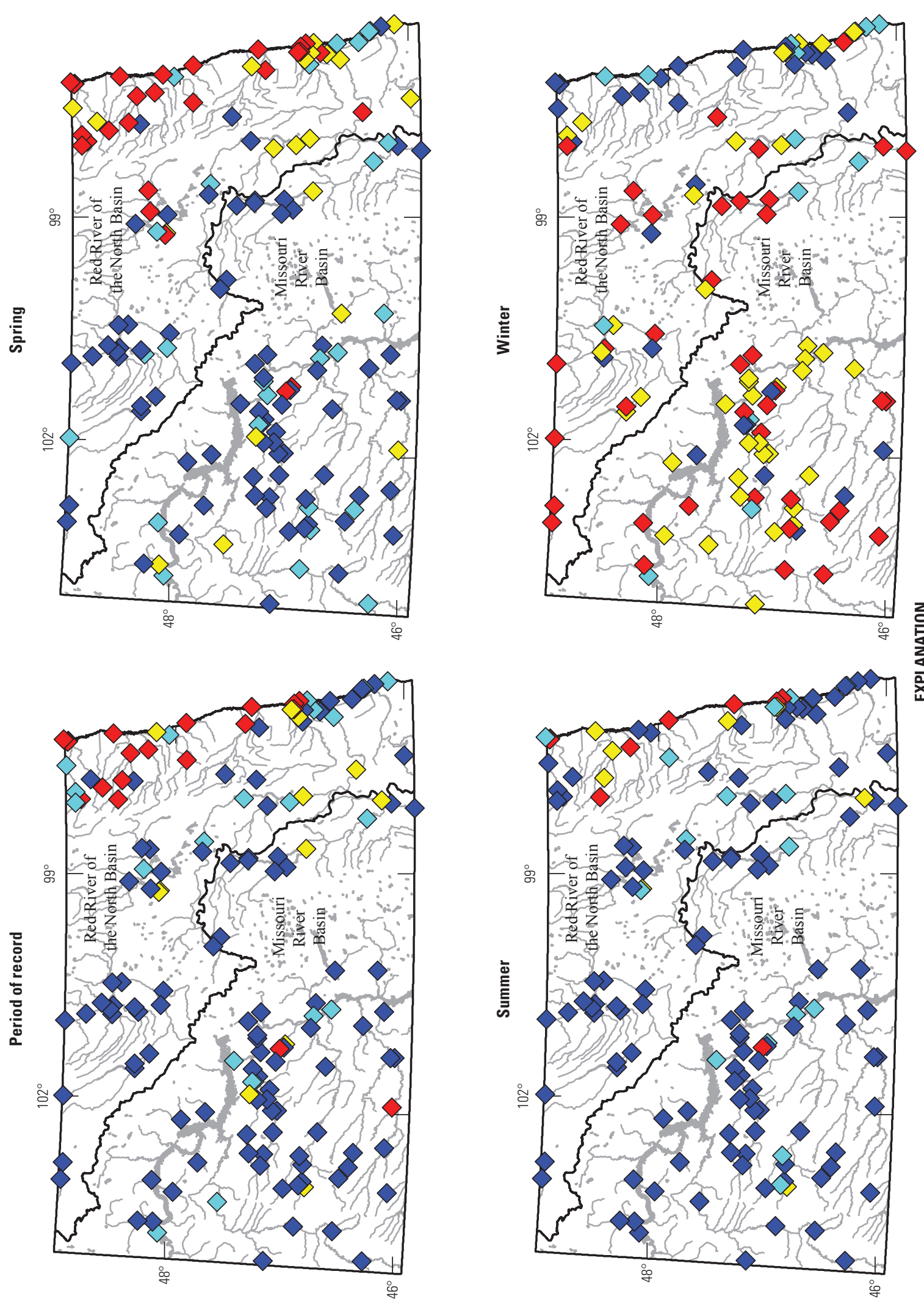

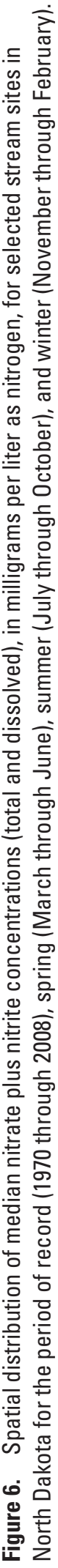



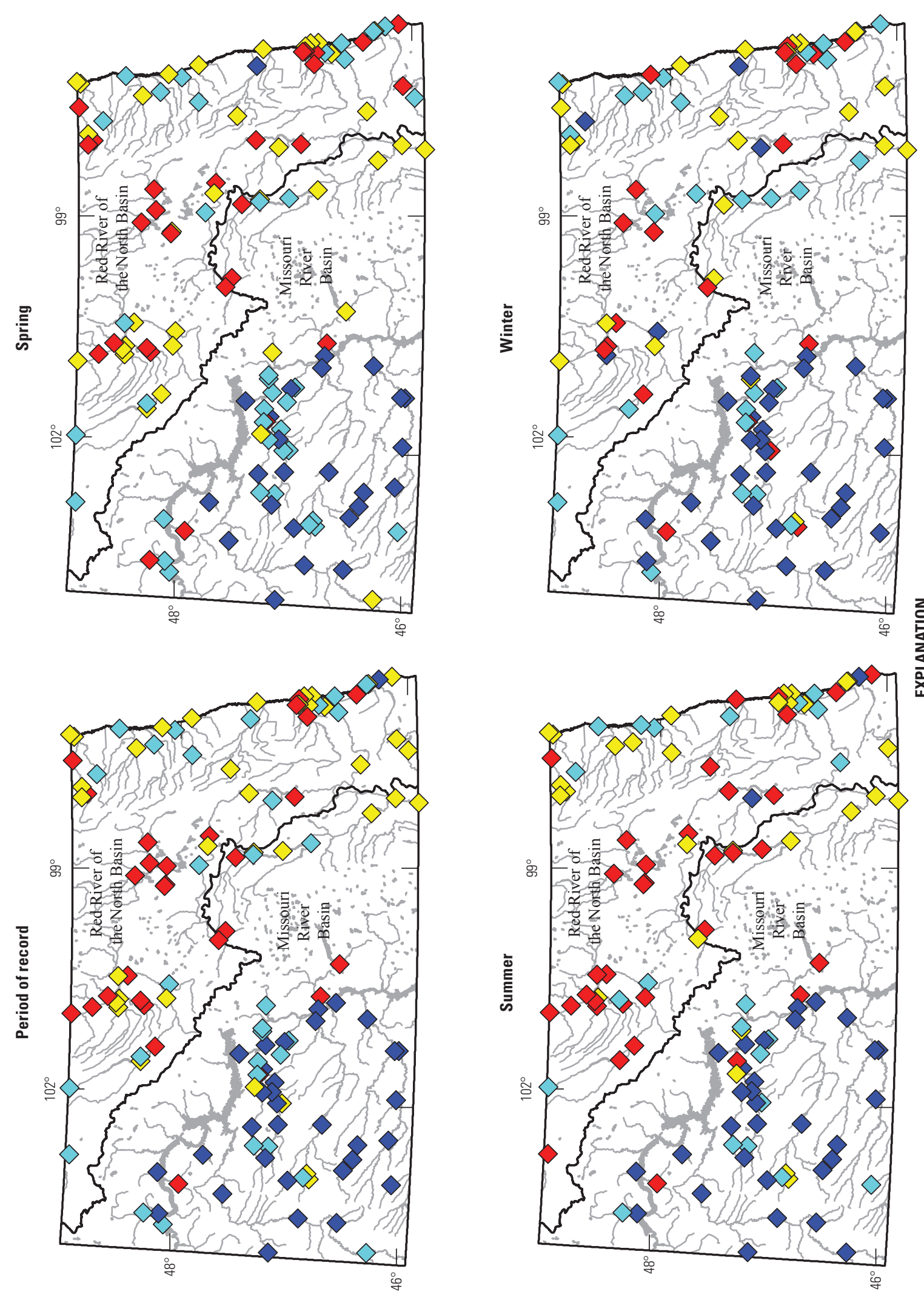

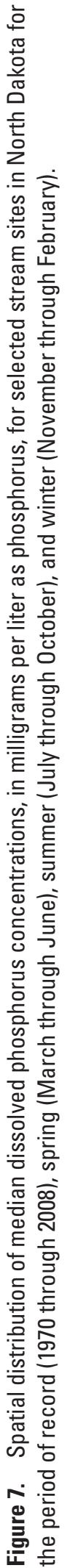



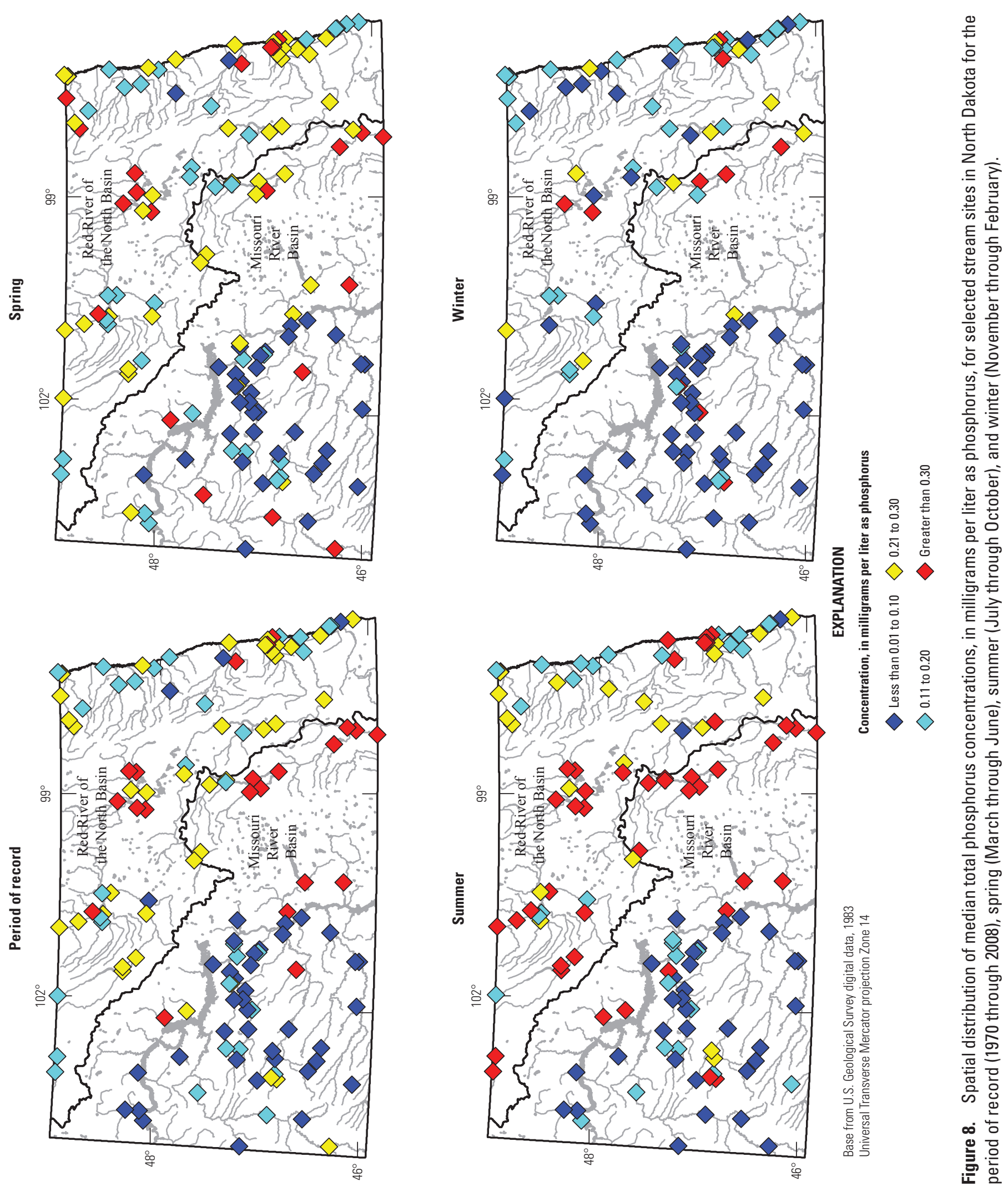

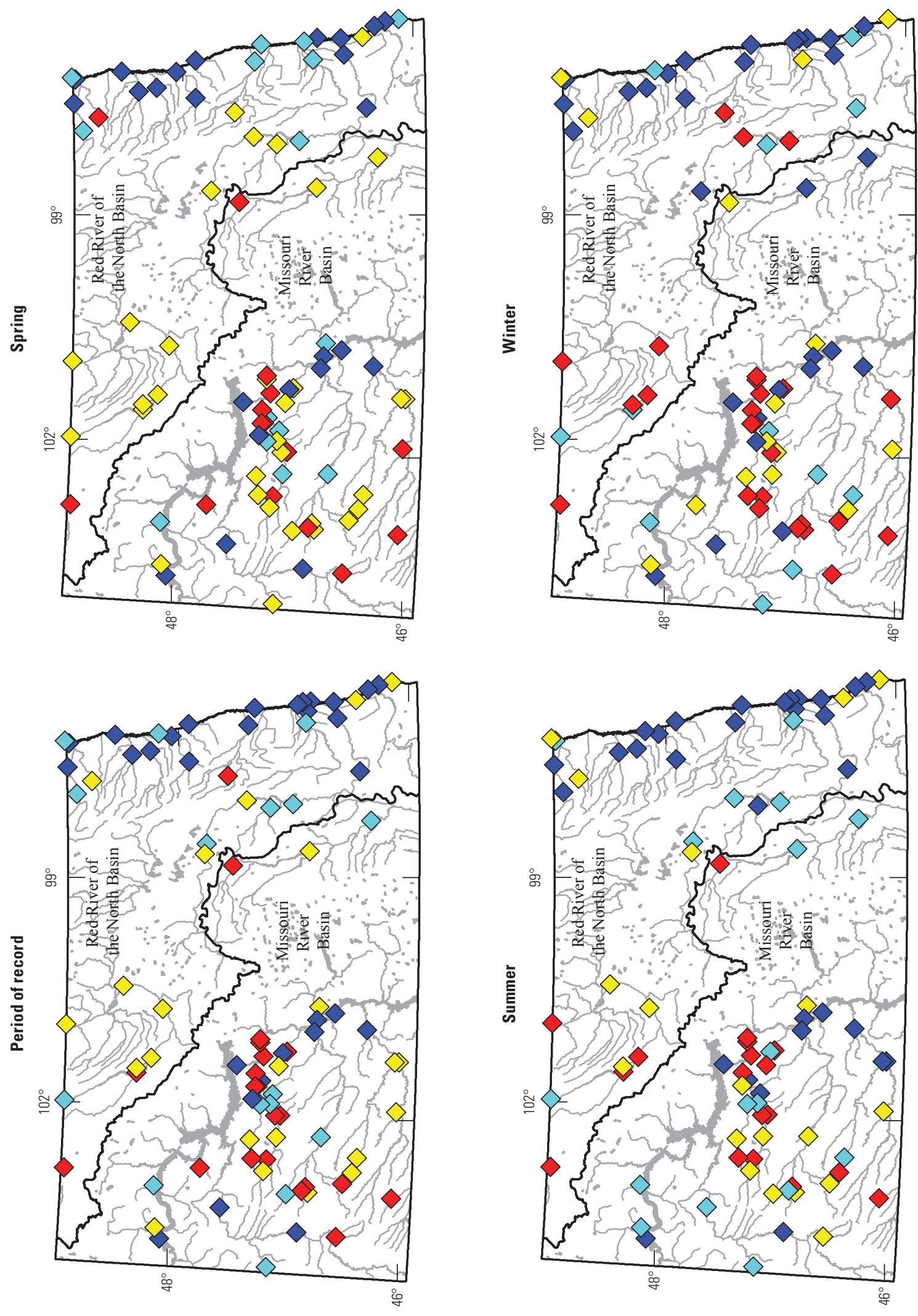

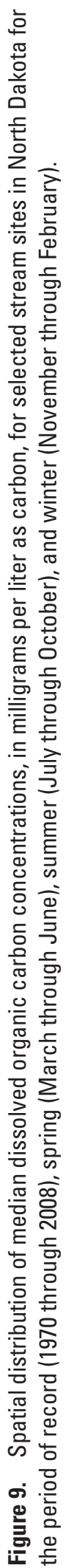



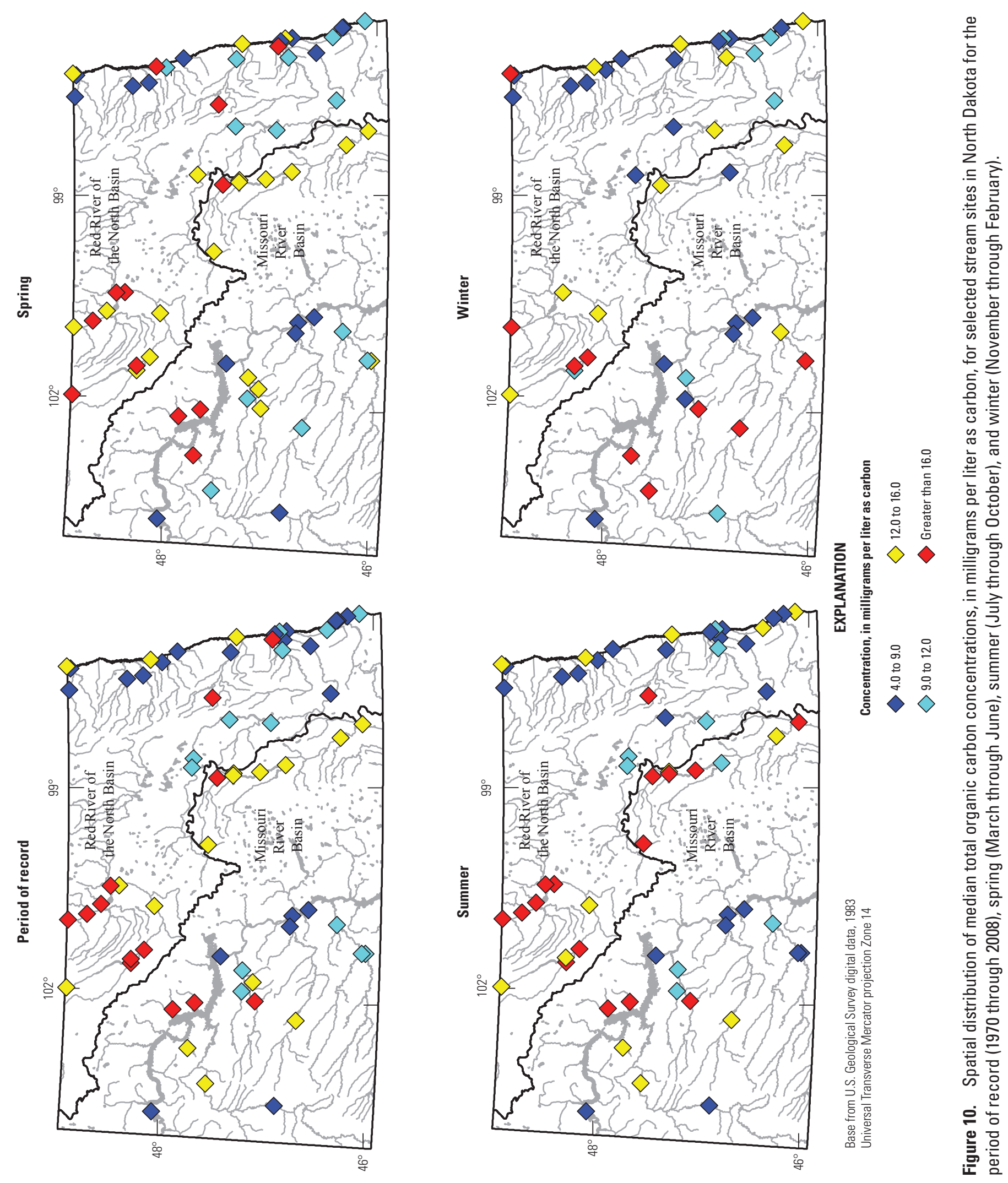

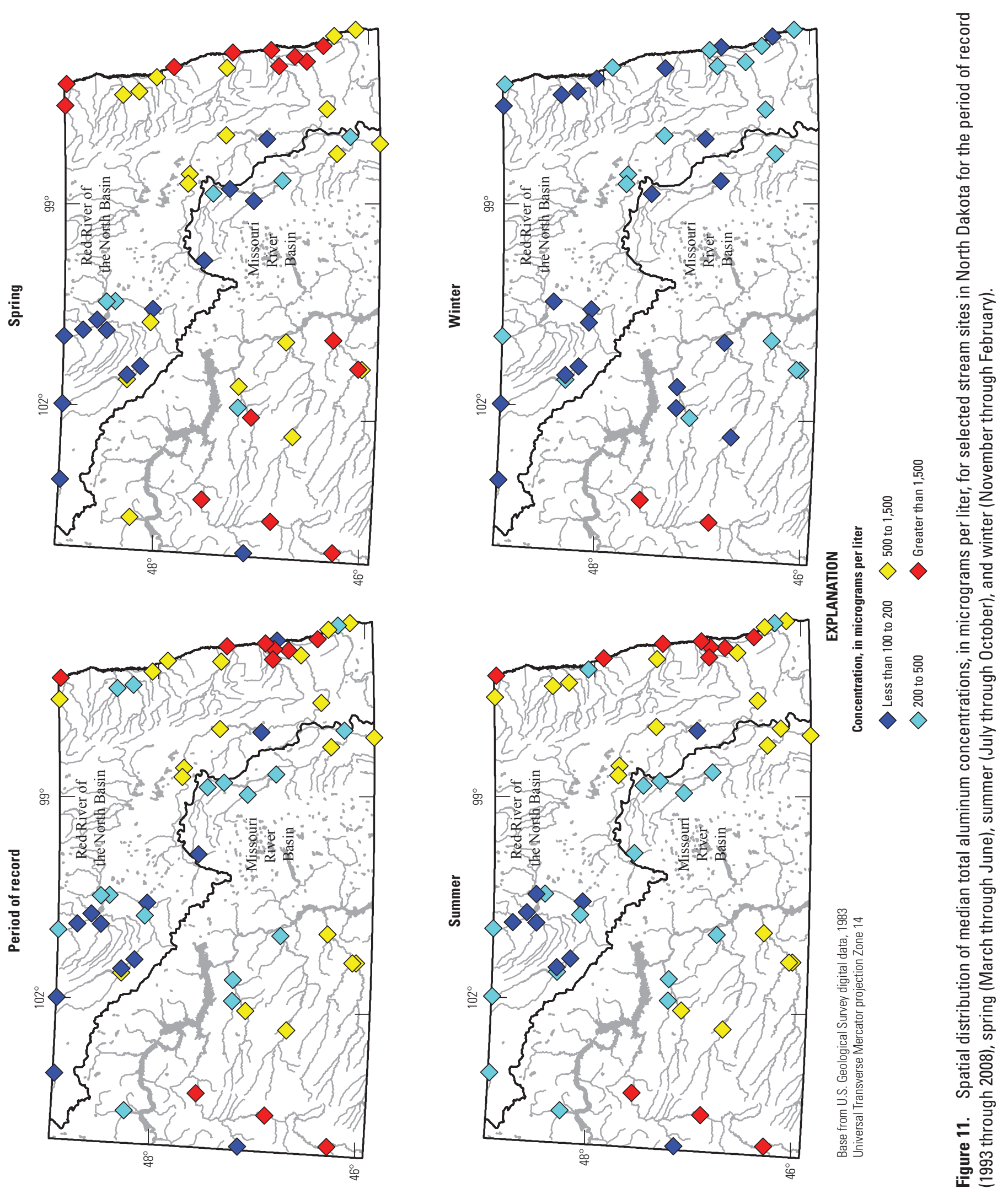
Median total arsenic concentrations in North Dakota indicate that the lowest values occurred mostly in the southwestern parts of the State, whereas the highest values occurred mostly in the eastern parts of the State (fig. 12). The median arsenic concentration exceeded the USEPA and North Dakota standards of $10.0 \mu \mathrm{g} / \mathrm{L}$ (U.S. Environmental Protection Agency, 2009 and North Dakota Department of Health, 2010) at only one site for the period of record (site 3; only one sample available). The median arsenic concentrations for the spring exceeded $10.0 \mu \mathrm{g} / \mathrm{L}$ at two sites, and the median concentrations for the summer exceeded $10.0 \mu \mathrm{g} / \mathrm{L}$ at three sites. Seasonal variations indicated higher median total arsenic concentrations in samples collected during summer and lower concentrations from samples collected during spring and winter at many locations. Median total arsenic concentrations for the period of record ranged from $1.3 \mu \mathrm{g} / \mathrm{L}$ at the Heart River near Mandan, N. Dak. (site 156) to $24.9 \mu \mathrm{g} / \mathrm{L}$ at the Red River of the North near Wahpeton, N. Dak. (site 3; only one sample available) (appendix table 1-5).

Median total chromium concentrations in water samples collected across North Dakota generally were greater in the far eastern and western areas of the State, and no median concentrations exceeded the USEPA and North Dakota standards for chromium of $100 \mu \mathrm{g} / \mathrm{L}$ (U.S. Environmental Protection Agency, 2009 and North Dakota Department of Health, 2010) (fig. 13). Seasonal variations indicated higher median total chromium concentrations in samples collected during spring and lower concentrations from samples collected during winter at many locations. Median total chromium concentrations for the period of record ranged from less than $1.2 \mu \mathrm{g} / \mathrm{L}$ at 34 out of 60 sites to $21.0 \mu \mathrm{g} / \mathrm{L}$ at the Little Missouri River at Marmarth, N. Dak. (site 110) (appendix table 1-5).

Median total iron concentrations in North Dakota were lowest in the Souris, Sheyenne, and James River Basins compared to sites in other parts of North Dakota (figs. 1 and 14). Median total iron concentrations exceeded the USEPA secondary drinking-water standard for iron of $300 \mu \mathrm{g} / \mathrm{L}$ (U.S. Environmental Protection Agency, 2009) at 66 out of 78 sites. Median total iron concentration for the period of record ranged from $11 \mu \mathrm{g} / \mathrm{L}$ at the Red River of the North at Emerson, Manitoba (site 79) to $20,150 \mu \mathrm{g} / \mathrm{L}$ at the Little Missouri River at Marmarth, N. Dak. (site 110) (appendix table 1-5).

The highest median total lead concentrations in water samples were scattered across North Dakota (fig. 15). Median total lead concentrations only exceeded the USEPA and North Dakota standards for lead concentration in drinking water of $15.0 \mu \mathrm{g} / \mathrm{L}$ (U.S. Environmental Protection Agency, 2009 and North Dakota Department of Health, 2010) at two sites from samples collected in the spring. Seasonal variations indicated higher median total lead concentrations during spring and lower concentrations during winter at many locations, particularly in the Red River of the North Basin. Median total lead concentrations for the period of record ranged from less than $1.0 \mu \mathrm{g} / \mathrm{L}$ (at 30 out of 60 sites) to $12.8 \mu \mathrm{g} / \mathrm{L}$ at the Little Missouri River at Marmarth, N. Dak. (site 110) (appendix table 1-5).
Median total nickel concentrations were higher at a few sites in the southwest and eastern parts of North Dakota compared to other sites (fig. 16). No median nickel concentrations exceeded the North Dakota regulation for nickel concentration in waters for human usage of $100.0 \mu \mathrm{g} / \mathrm{L}$ (North Dakota Department of Health, 2010). Seasonal variations indicated higher median total nickel concentrations in samples collected during spring and lower concentrations from samples collected during winter at many locations. Median total nickel concentrations for the period of record ranged from $2.0 \mu \mathrm{g} / \mathrm{L}$ at Boundary Creek near Landa, N. Dak. (site 97) to $26.0 \mu \mathrm{g} / \mathrm{L}$ at the Little Missouri River at Marmarth, N. Dak. (site 110) (appendix table 1-5).

\section{Suspended Sediment and Suspended Solids}

Suspended sediment in water is the particulate matter that consists of soil and rock particles eroded from the landscape. Sediment can be transported in the water column or can settle to the streambed. The movement of suspended sediment in streams is important in the fate and transport of chemicals in the environment because the particles can sorb nutrients, trace elements, and organic compounds. Large concentrations of suspended sediment often are associated with storm-runoff events that increase streamflow, erosion, and resuspension of bed material (Guy, 1970). Activities such as row-crop agriculture, animal grazing, timber harvesting, mining, road construction and maintenance, and urbanization can cause increased sediment concentrations in streams (Guy, 1970).

Suspended-sediment concentration (SSC) and total suspended solids (TSS) are commonly used to quantify concentrations of suspended solid-phase material in surface water. Often TSS is used as a surrogate for SSC, which includes the inorganic sand, silt, and clay matrix transported in streams, and SSC and TSS frequently have been used interchangeably; however, the analytical methods differ and the two may not be equivalent when solid-phase material, especially sand, becomes more concentrated (Gray and others, 2000). SSC analytical methods measure all the sediment and the mass of the entire water-sediment mixture of the original sample; TSS methods only use an aliquot of the original sample for subsequent analysis. SSC typically is used to analyze natural water, whereas TSS originally was designed for wastewater discharge analyses and later was extended operationally to include natural water (Gray and others, 2000). Gray and others (2000) show that TSS is fundamentally unreliable for the analysis of solid-phase material in natural-water samples with sand-size material, but concluded that the SSC method produces relatively reliable results regardless of the amount or percentage of sand-size material. Gray and others (2000) also concluded that SSC and TSS data collected from natural water are not comparable and should not be used interchangeably, and that the accuracy and comparability of suspended solid-phase concentrations of natural waters would be greatly enhanced if SSC data were used. 

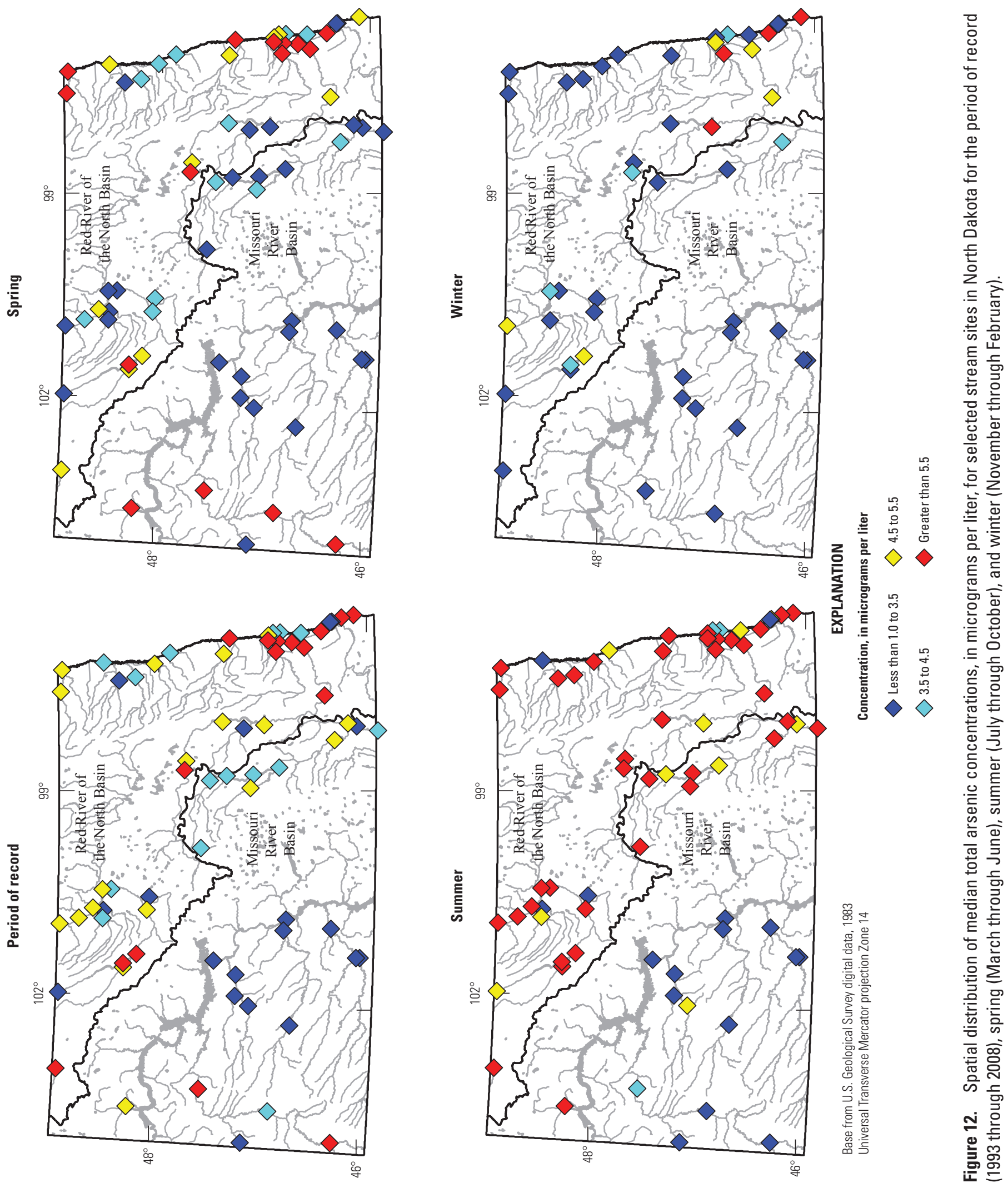

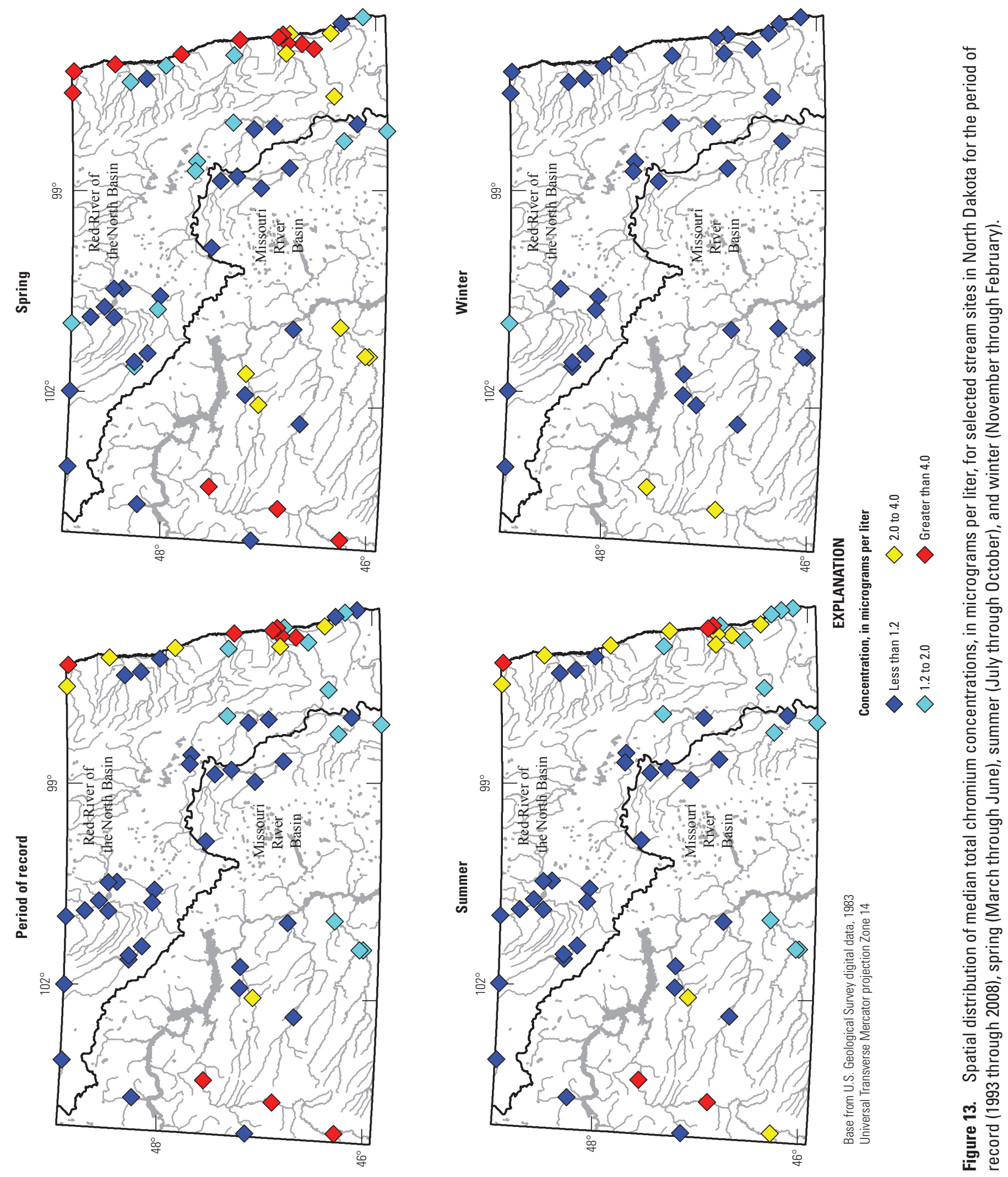

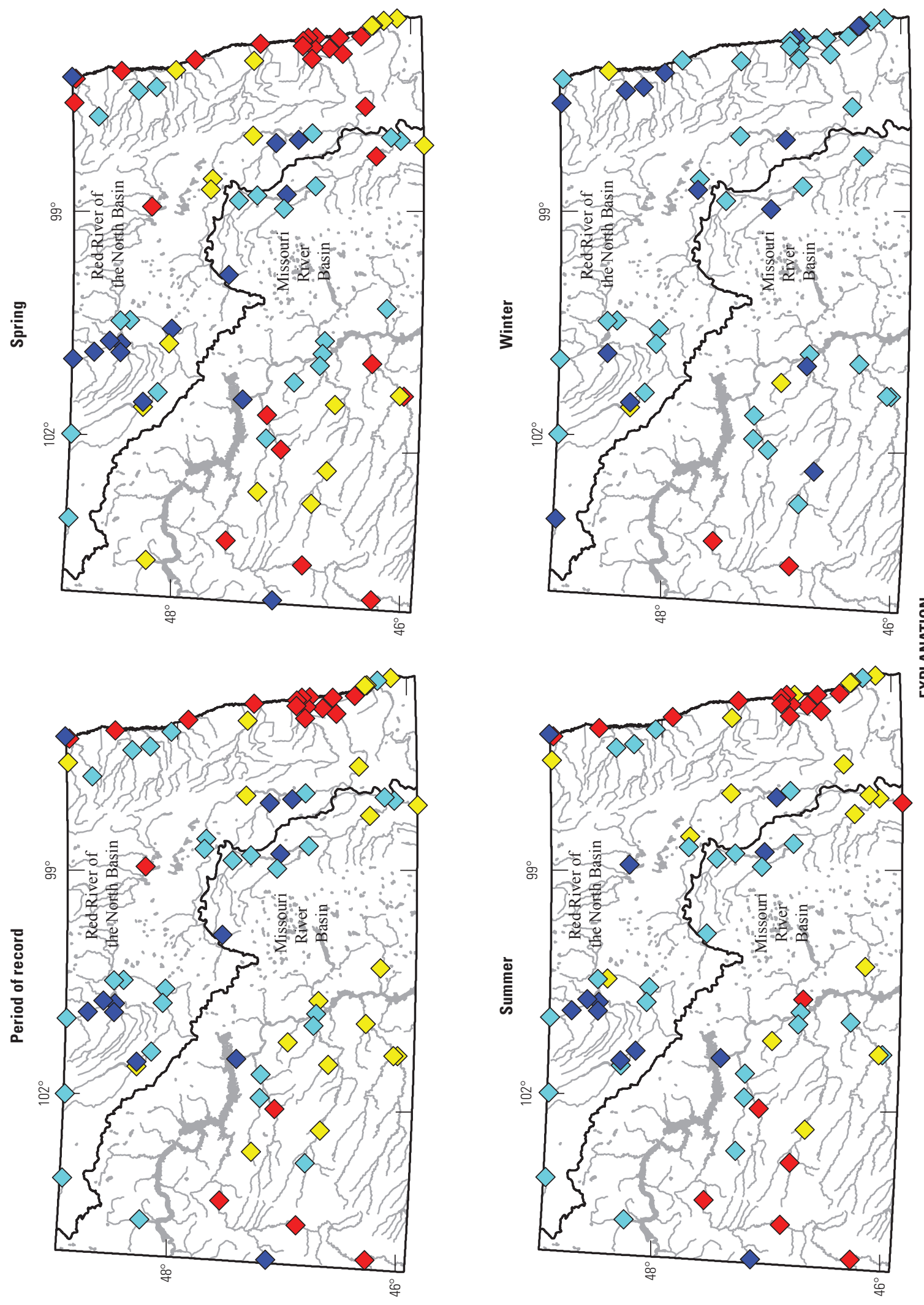

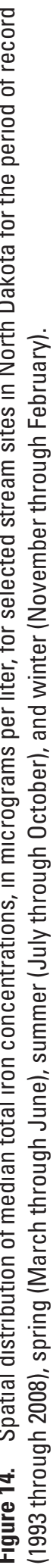



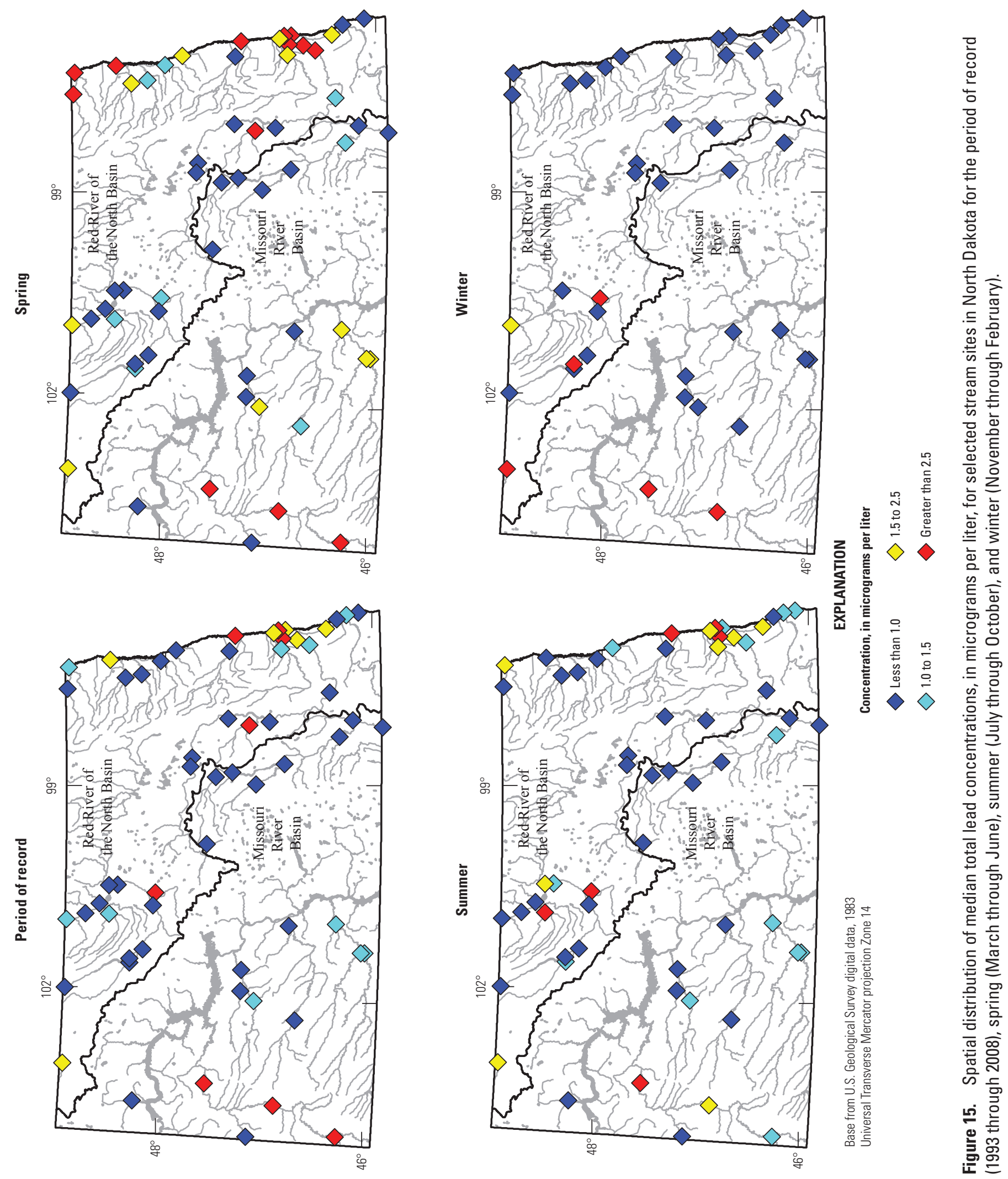

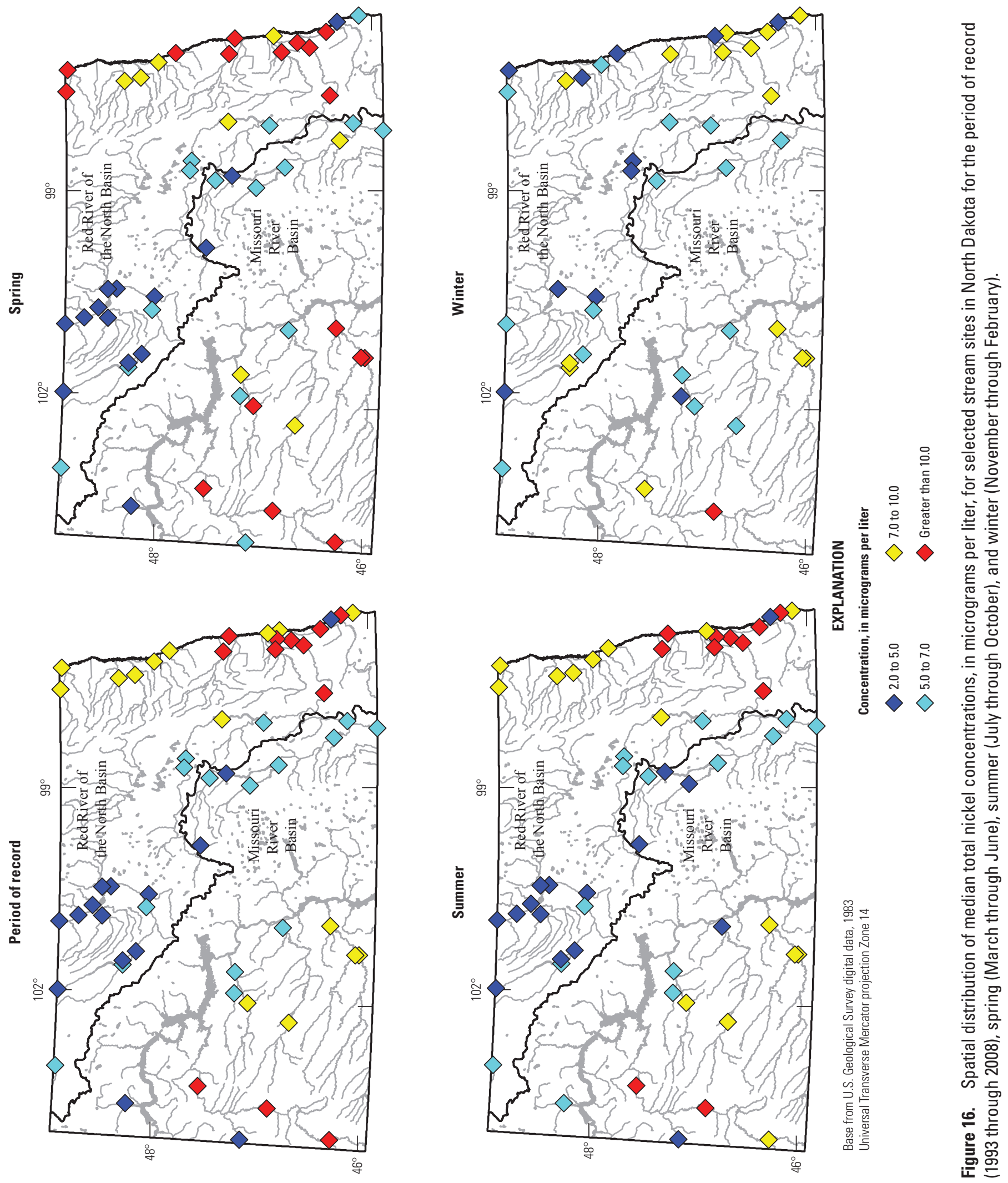
Median SSC and TSS in water samples obtained across North Dakota were higher at sites located in southwestern and eastern areas of North Dakota compared to sites located in other areas of the State (figs. 17 and 18). No USEPA or North Dakota standards have been established for TSS or SSC. Median SSC for the period of record at a sampling site ranged from $2 \mathrm{mg} / \mathrm{L}$ at the Missouri River at Garrison Dam, N. Dak. (site 115) to $1,028 \mathrm{mg} / \mathrm{L}$ at the Little Missouri River near Watford City, N. Dak. (site 114). Median TSS for the period of record at a sampling site ranged from less than $10 \mathrm{mg} / \mathrm{L}$ at 15 out of 63 sites to $314 \mathrm{mg} / \mathrm{L}$ at the Little Missouri River near Watford City, N. Dak. (site 114) (appendix table 1-5). The relatively low SSC and TSS at site 115 are likely because the site is located below a dam, where sediment particles are retained in the upstream reservoir through settling. The higher values in the Little Missouri River may be because of the occurrence of highly erodible soils in the area and the lack of any control structures or reservoirs on the Little Missouri River that would retain sediment.

\section{Yields of Selected Constituents}

Normalized annual loads and yields were estimated for sulfate, TDS, nitrogen (nitrate plus nitrite and ammonia), phosphorus (total and dissolved), total organic carbon, and suspended sediment at 34 sites across North Dakota. Information on the load model calibration periods and results are given in table 3 . To facilitate comparisons between sites, this section focuses on the estimated yields. Loads, which are highly dependent on streamflow, were computed primarily to obtain yields.

\section{Sulfate and Total Dissolved Solids}

Normalized annual sulfate yields ranged from 9,250 to $56,200 \mathrm{lbs} / \mathrm{yr} / \mathrm{mi}^{2}$ in the Missouri River Basin and from 4,680 to $72,500 \mathrm{lbs} / \mathrm{yr} / \mathrm{mi}^{2}$ in the Red River of the North Basin (fig. 19 and table 4). The highest yields (greater than $\left.45,200 \mathrm{lbs} / \mathrm{yr} / \mathrm{mi}^{2}\right)$ were at five sites in the Missouri River Basin (site 112 on the Little Missouri River; sites 149 and 156 on the Heart River; site 169 on Cedar Creek; and site 170 on the Cannonball River) and two sites in the Red River of the North Basin (site 45 on the Maple River and site 55 on the Goose River) (figs. 1 and 19; table 4). The lowest yields (less than $12,200 \mathrm{lbs} / \mathrm{yr} / \mathrm{mi}^{2}$ ) were at two sites in the Missouri River Basin (site 158 on Apple Creek and site 176 on the James River) and six sites in the Red River of the North Basin (sites 32 and 37 on the Sheyenne River; and sites 82, 85, 89, and 98 on the Souris River) (figs. 1 and 19; table 4)

Normalized annual TDS yields ranged from 30,400 to $119,000 \mathrm{lbs} / \mathrm{yr} / \mathrm{mi}^{2}$ in the Missouri River Basin and from 13,000 to $169,000 \mathrm{lbs} / \mathrm{yr} / \mathrm{mi}^{2}$ in the Red River of the North Basin (fig. 19 and table 4). The highest yields (greater than $107,000 \mathrm{lbs} / \mathrm{yr} / \mathrm{mi}^{2}$ ) were at three sites in the Missouri River
Basin (site 112 in the Little Missouri River and sites 149 and 156 on the Heart River) and at four sites in the Red River of the North Basin (site 45 on the Maple River; site 55 on the Goose River; and sites 11 and 79 on the Red River of the North) (figs. 1 and 19; table 4). The lowest yields (43,600 lbs/ $\mathrm{yr} / \mathrm{mi}^{2}$ or less) were at two sites in the Missouri River Basin (site 158 on Apple Creek and site 176 on the James River) and seven sites in the Red River of the North Basin (sites 32 and 37 on the Sheyenne River; site 33 on Baldhill Creek; and sites $82,85,89$, and 98 on the Souris River) (figs. 1 and 19; table 4).

\section{Nutrients}

Normalized annual nitrate plus nitrite yields ranged from 24 to $120 \mathrm{lbs} / \mathrm{yr} / \mathrm{mi}^{2}$ as nitrogen in the Missouri River Basin and from 2 to $1,260 \mathrm{lbs} / \mathrm{yr} / \mathrm{mi}^{2}$ as nitrogen in the Red River of the North Basin (fig. 20 and table 4). The highest yields (greater than $120 \mathrm{lbs} / \mathrm{yr} / \mathrm{mi}^{2}$ ) were at eight sites in the Red River of the North Basin (site 21 on Edmore Coulee; site 45 on the Maple River; site 55 on the Goose River; site 67 on the Park River; site 75 on the Pembina River; site 76 on the Tongue River; and sites 56 and 79 on the Red River of the North) (figs. 1 and 20; table 4). The lowest yields (less than $\left.33 \mathrm{lbs} / \mathrm{yr} / \mathrm{mi}^{2}\right)$ were at three sites in the Missouri River Basin (sites 115 and 142 on the Missouri River and site 158 on Apple Creek) and five sites in the Red River of the North Basin (site 32 on the Sheyenne River and sites 82, 85, 89, and 98 on the Souris River) (figs. 1 and 20; table 4).

Normalized annual ammonia yields ranged from 6 to $22 \mathrm{lbs} / \mathrm{yr} / \mathrm{mi}^{2}$ as nitrogen in the Missouri River Basin and from 4 to $62 \mathrm{lbs} / \mathrm{yr} / \mathrm{mi}^{2}$ as nitrogen in the Red River of the North Basin (fig. 20 and table 4). The highest yields (greater than $\left.22 \mathrm{lbs} / \mathrm{yr} / \mathrm{mi}^{2}\right)$ were at six sites in the Red River of the North Basin (site 10 on the Wild Rice River; site 45 on the Maple River; site 55 on the Goose River; site 76 on the Tongue River; and sites 5 and 79 on the Red River of the North) (figs. 1 and 20; table 4). The lowest yields (less than $8 \mathrm{lbs} / \mathrm{yr} / \mathrm{mi}^{2}$ ) were at one site in the Missouri River Basin (site 112 on the Little Missouri River) and five sites in the Red River of the North Basin (site 32 on the Sheyenne River and sites 82, 85, 89, and 98 on the Souris River) (figs. 1 and 20; table 4).

Normalized annual dissolved phosphorus yields ranged from less than 1 to $35 \mathrm{lbs} / \mathrm{yr} / \mathrm{mi}^{2}$ as phosphorus in the Missouri River Basin and from 5 to $47 \mathrm{lbs} / \mathrm{yr} / \mathrm{mi}^{2}$ as phosphorus in the Red River of the North Basin (fig. 20 and table 4). The highest yields (greater than $39 \mathrm{lbs} / \mathrm{yr} / \mathrm{mi}^{2}$ ) were at four sites in the Red River of the North Basin (site 55 on the Goose River and sites 11, 56, and 79 on the Red River of the North) (figs. 1 and 20 ; table 4). The lowest yields (less than $8 \mathrm{lbs} / \mathrm{yr} / \mathrm{mi}^{2}$ ) were at four sites in the Missouri River Basin (site 114 on the Little Missouri River; site 115 on the Missouri River; site 156 on the Heart River; and site 170 on the Cannonball River) and two sites in the Red River of the North Basin (sites 85 and 89 on the Souris River) (figs. 1 and 20; table 4). 

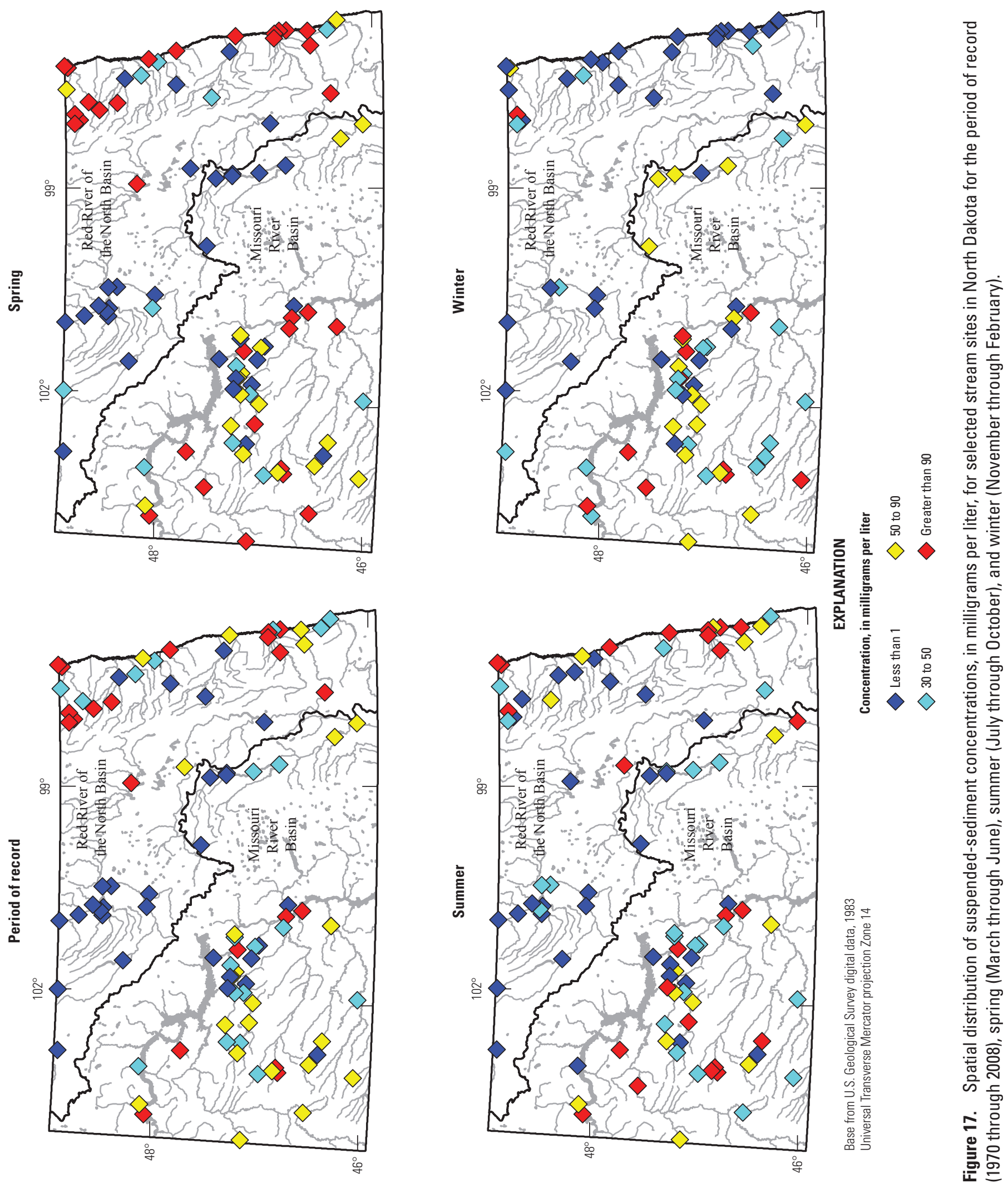

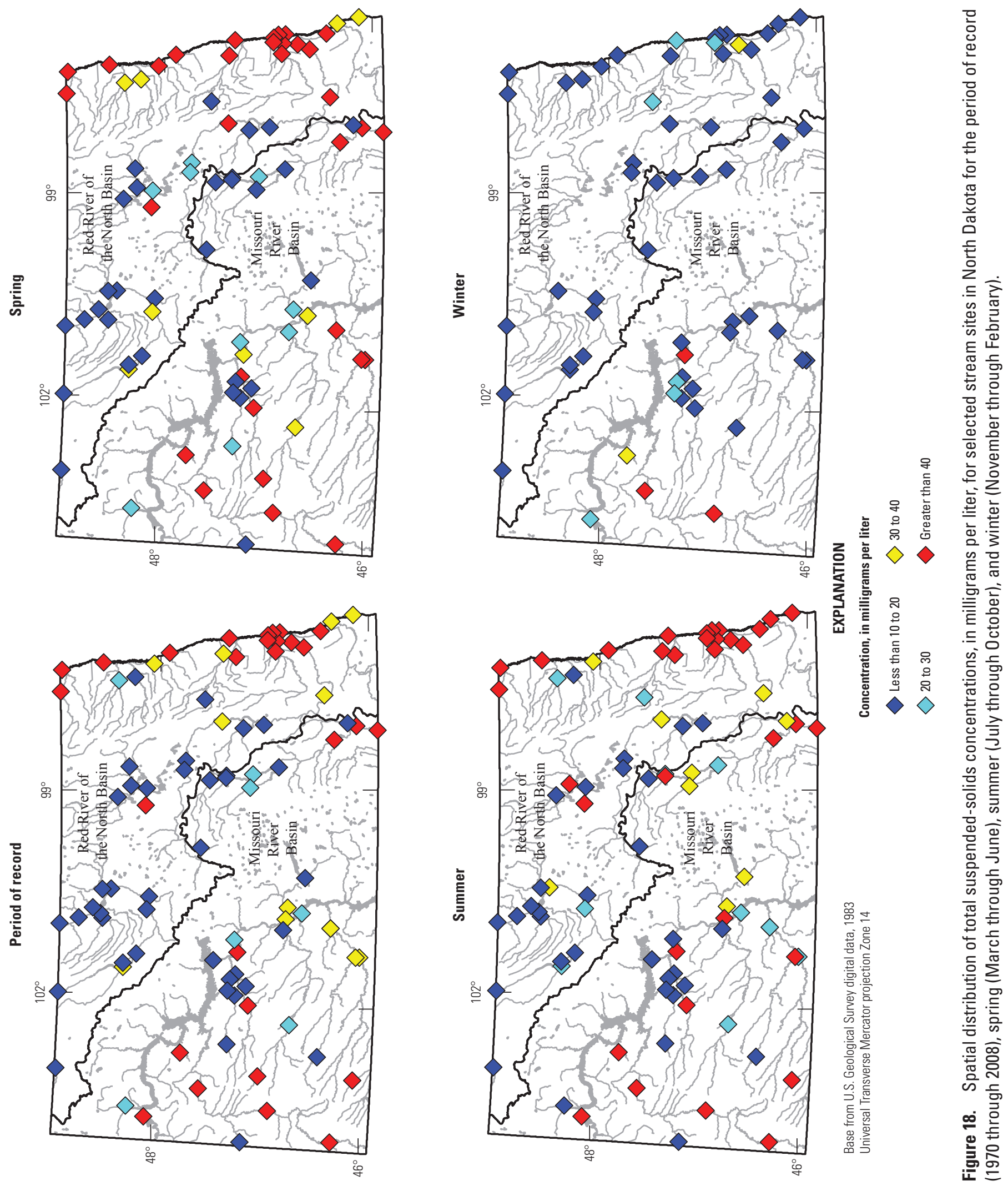
Table 3. Regression model characteristics for S-LOADEST models for selected sites in North Dakota from 1970 through 2008.

[Q, natural log discharge; BpQ, natural log of discharge with a break point; T, time; SS, season; CEN, data had more than 80 percent censored values; ISD, insufficient data; --, not used]

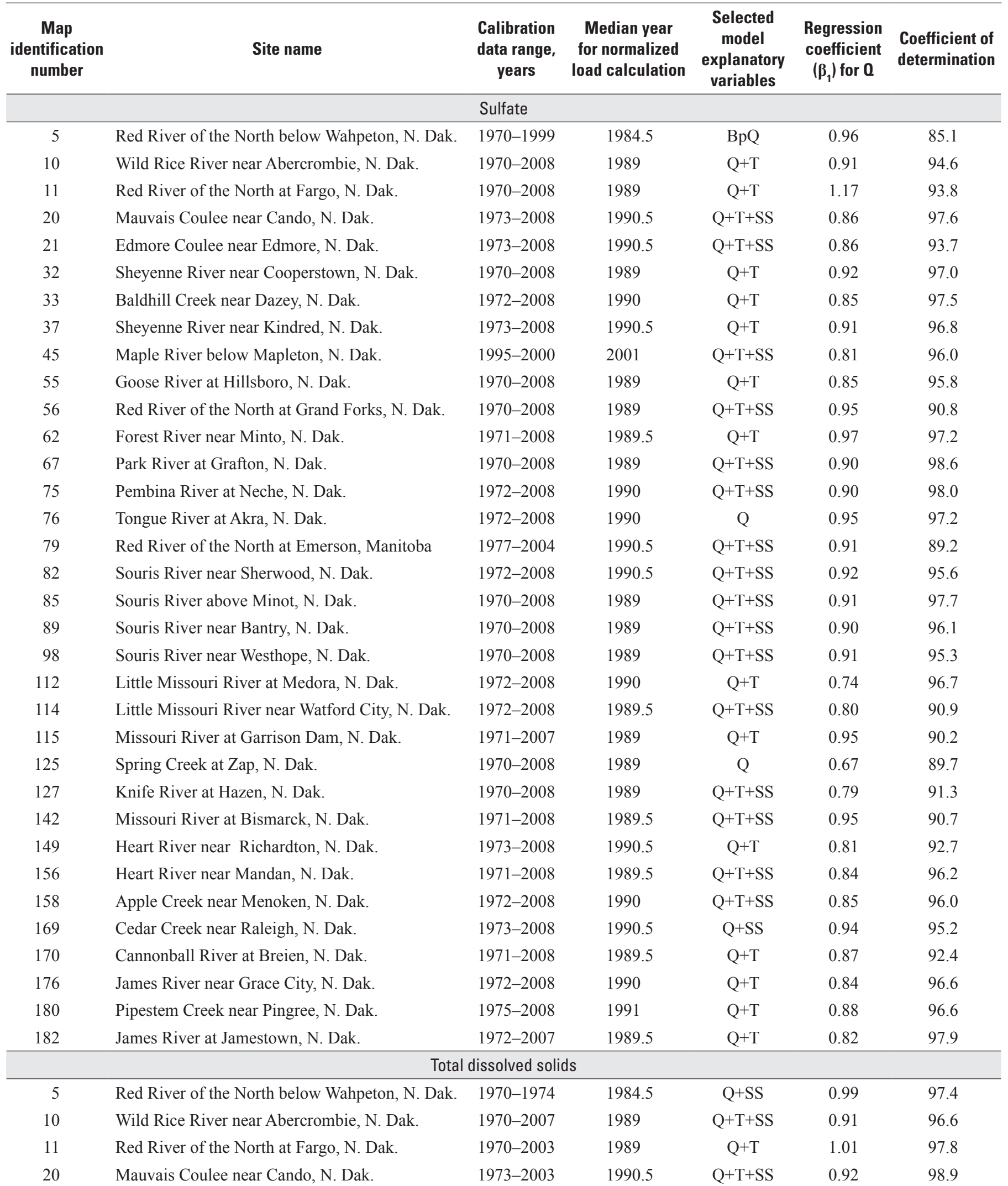


Table 3. Regression model characteristics for S-LOADEST models for selected sites in North Dakota from 1970 through 2008. Continued

[Q, natural log discharge; BpQ, natural log of discharge with a break point; T, time; SS, season; CEN, data had more than 80 percent censored values; ISD, insufficient data; --, not used]

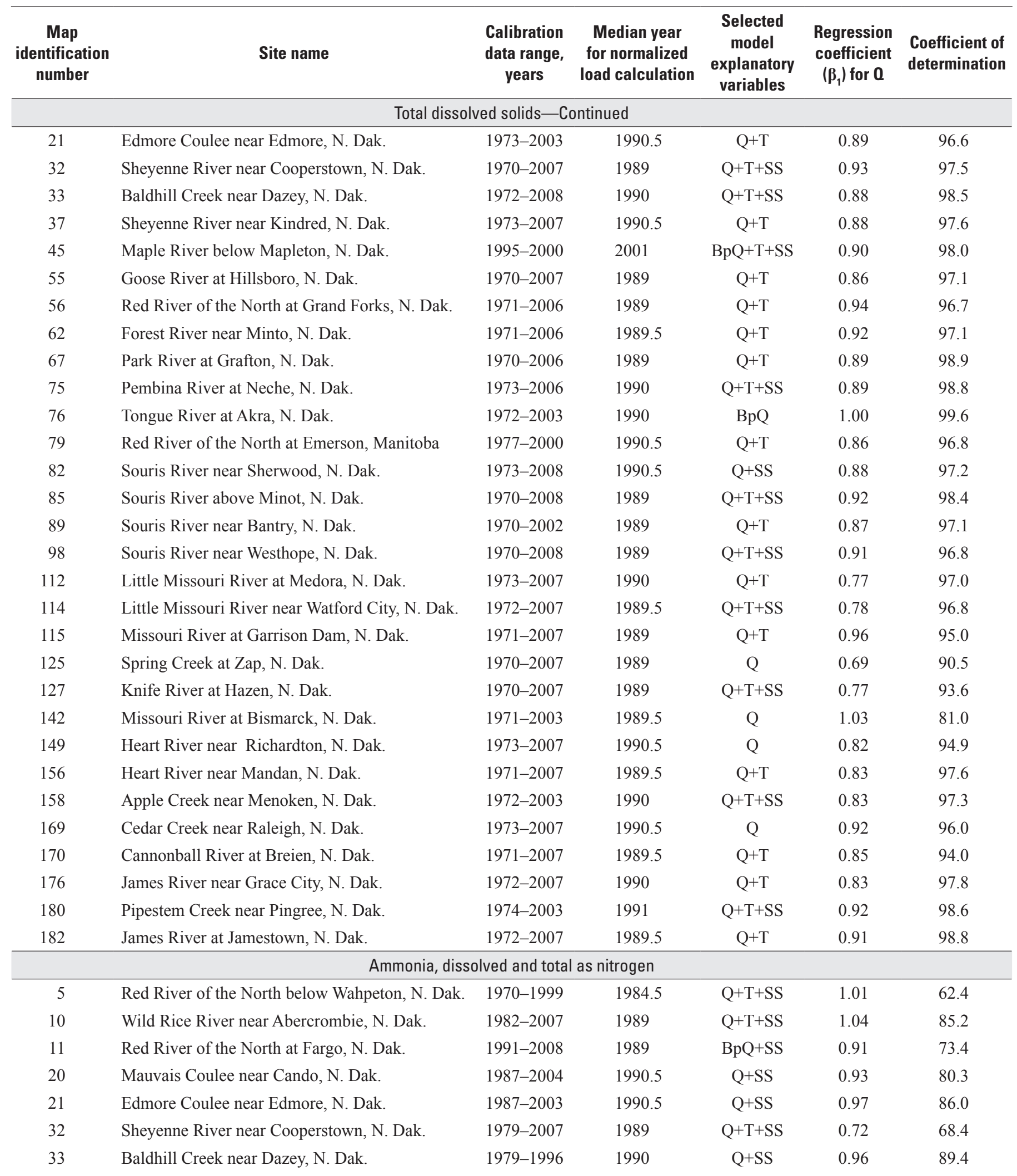


Table 3. Regression model characteristics for S-LOADEST models for selected sites in North Dakota from 1970 through 2008.Continued

[Q, natural log discharge; BpQ, natural log of discharge with a break point; T, time; SS, season; CEN, data had more than 80 percent censored values; ISD, insufficient data; --, not used]

\begin{tabular}{|c|c|c|c|c|c|c|}
\hline $\begin{array}{c}\text { Map } \\
\text { identification } \\
\text { number }\end{array}$ & Site name & $\begin{array}{c}\text { Calibration } \\
\text { data range, } \\
\text { years }\end{array}$ & $\begin{array}{l}\text { Median year } \\
\text { for normalized } \\
\text { load calculation }\end{array}$ & $\begin{array}{c}\text { Selected } \\
\text { model } \\
\text { explanatory } \\
\text { variables }\end{array}$ & $\begin{array}{c}\text { Regression } \\
\text { coefficient } \\
\left(\beta_{1}\right) \text { for } 0 \\
\end{array}$ & $\begin{array}{l}\text { Coefficient of } \\
\text { determination }\end{array}$ \\
\hline \multicolumn{7}{|c|}{ Ammonia, dissolved and total as nitrogen-Continued } \\
\hline 37 & Sheyenne River near Kindred, N. Dak. & $1977-2007$ & 1990.5 & $\mathrm{Q}+\mathrm{T}+\mathrm{SS}$ & 1.57 & 76.0 \\
\hline 45 & Maple River below Mapleton, N. Dak. & 1997-2007 & 2001 & $\mathrm{Q}+\mathrm{SS}$ & 1.08 & 82.7 \\
\hline 56 & Red River of the North at Grand Forks, N. Dak. & 1992-2008 & 1989 & $\mathrm{Q}+\mathrm{SS}$ & 1.28 & 79.5 \\
\hline 62 & Forest River near Minto, N. Dak. & 1994-2008 & 1989.5 & $\mathrm{Q}+\mathrm{SS}$ & 0.67 & 44.6 \\
\hline 67 & Park River at Grafton, N. Dak. & 1994-2007 & 1989 & $\mathrm{Q}+\mathrm{SS}$ & 0.84 & 80.2 \\
\hline 75 & Pembina River at Neche, N. Dak. & 1994-2008 & 1990 & CEN & CEN & CEN \\
\hline 85 & Souris River above Minot, N. Dak. & 1981-2008 & 1989 & $\mathrm{Q}+\mathrm{T}+\mathrm{SS}$ & 0.92 & 81.0 \\
\hline 89 & Souris River near Bantry, N. Dak. & $1981-2000$ & 1989 & $\mathrm{Q}+\mathrm{T}+\mathrm{SS}$ & 0.85 & 78.2 \\
\hline 98 & Souris River near Westhope, N. Dak. & 1970-2006 & 1989 & $\mathrm{Q}+\mathrm{T}+\mathrm{SS}$ & 0.77 & 60.3 \\
\hline 112 & Little Missouri River at Medora, N. Dak. & 1979-2007 & 1990 & Q & 0.98 & 52.0 \\
\hline 114 & Little Missouri River near Watford City, N. Dak. & 1977-2007 & 1989.5 & $\mathrm{Q}+\mathrm{T}+\mathrm{SS}$ & 0.96 & 79.0 \\
\hline 115 & Missouri River at Garrison Dam, N. Dak. & 1974-2007 & 1989 & $\mathrm{CEN}$ & CEN & $\mathrm{CEN}$ \\
\hline 125 & Spring Creek at Zap, N. Dak. & 1974-2007 & 1989 & $\mathrm{Q}+\mathrm{T}+\mathrm{SS}$ & 1.12 & 59.9 \\
\hline 127 & Knife River at Hazen, N. Dak. & 1974-2007 & 1989 & $\mathrm{Q}+\mathrm{T}+\mathrm{SS}$ & 1.19 & 76.0 \\
\hline 142 & Missouri River at Bismarck, N. Dak. & 1971-2001 & 1989.5 & CEN & CEN & CEN \\
\hline 180 & Pipestem Creek near Pingree, N. Dak. & ISD & ISD & ISD & ISD & ISD \\
\hline 182 & James River at Jamestown, N. Dak. & $1987-2007$ & 1989.5 & $\mathrm{Q}+\mathrm{SS}$ & 0.93 & 82.7 \\
\hline \multicolumn{7}{|c|}{ Nitrate + nitrite dissolved and total as nitrogen } \\
\hline 5 & Red River of the North below Wahpeton, N. Dak. & 1971-1999 & 1984.5 & $\mathrm{Q}+\mathrm{T}+\mathrm{SS}$ & 1.24 & 52.6 \\
\hline 10 & Wild Rice River near Abercrombie, N. Dak. & 1972-2007 & 1989 & $\mathrm{Q}+\mathrm{SS}$ & 1.34 & 86.3 \\
\hline 11 & Red River of the North at Fargo, N. Dak. & $1971-2008$ & 1989 & $\mathrm{BpQ}+\mathrm{SS}$ & -- & 86.5 \\
\hline 20 & Mauvais Coulee near Cando, N. Dak. & $1987-2004$ & 1990.5 & $\mathrm{Q}+\mathrm{SS}$ & 1.41 & 88.7 \\
\hline 21 & Edmore Coulee near Edmore, N. Dak. & 1983-2004 & 1990.5 & $\mathrm{Q}+\mathrm{SS}$ & 1.30 & 87.5 \\
\hline 32 & Sheyenne River near Cooperstown, N. Dak. & 1971-2007 & 1989 & $\mathrm{Q}+\mathrm{SS}$ & 1.26 & 76.6 \\
\hline 33 & Baldhill Creek near Dazey, N. Dak. & 1979-1996 & 1990 & $\mathrm{Q}+\mathrm{SS}$ & 1.45 & 95.6 \\
\hline 37 & Sheyenne River near Kindred, N. Dak. & 1977-2007 & 1990.5 & $\mathrm{Q}+\mathrm{SS}$ & 1.64 & 83.7 \\
\hline
\end{tabular}


Table 3. Regression model characteristics for S-LOADEST models for selected sites in North Dakota from 1970 through 2008. Continued

[Q, natural log discharge; BpQ, natural log of discharge with a break point; T, time; SS, season; CEN, data had more than 80 percent censored values; ISD, insufficient data; --, not used]

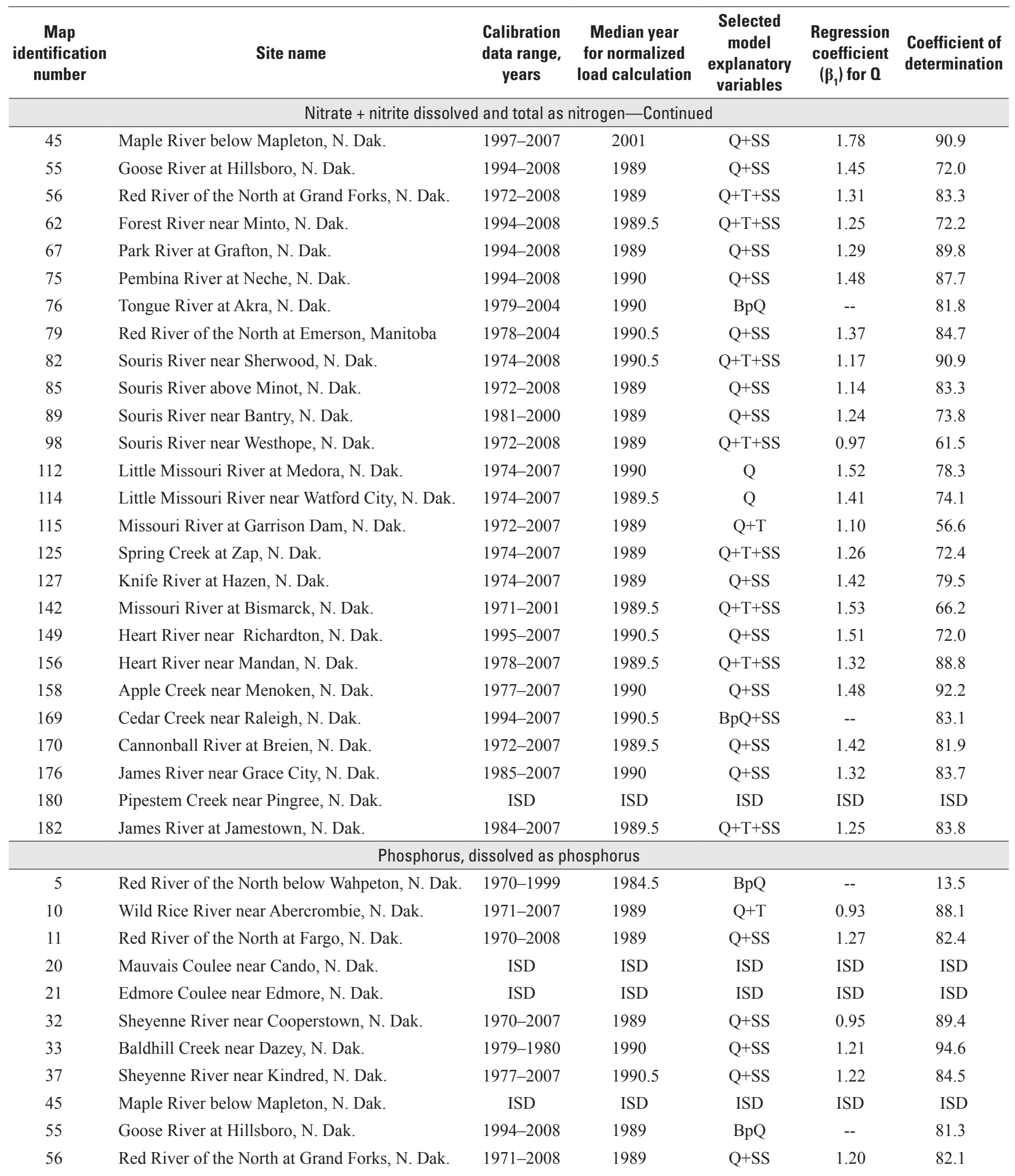


Table 3. Regression model characteristics for S-LOADEST models for selected sites in North Dakota from 1970 through 2008.Continued

[Q, natural log discharge; BpQ, natural log of discharge with a break point; T, time; SS, season; CEN, data had more than 80 percent censored values; ISD, insufficient data; --, not used]

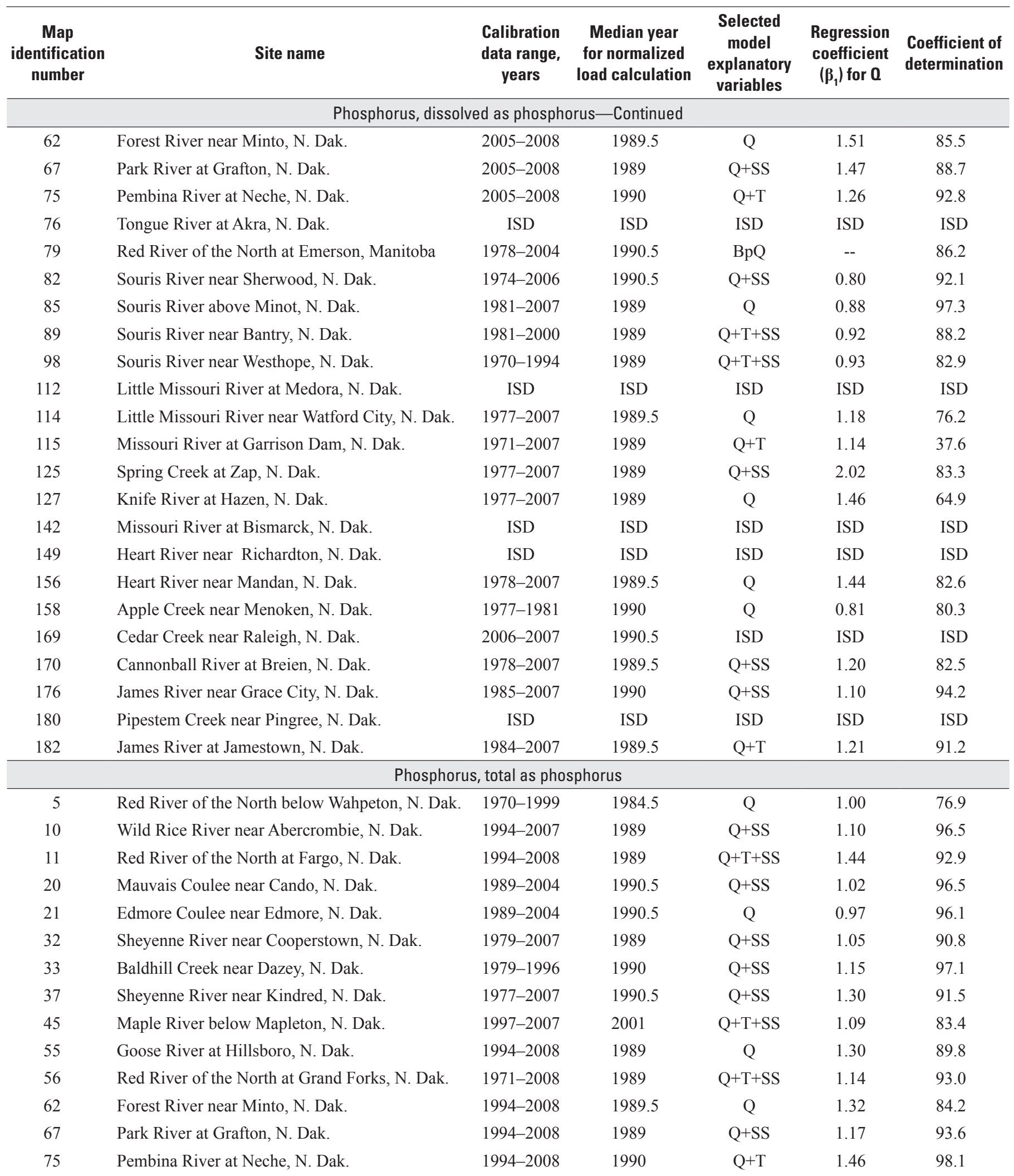


Table 3. Regression model characteristics for S-LOADEST models for selected sites in North Dakota from 1970 through 2008.— Continued

[Q, natural log discharge; BpQ, natural log of discharge with a break point; T, time; SS, season; CEN, data had more than 80 percent censored values; ISD, insufficient data; --, not used]

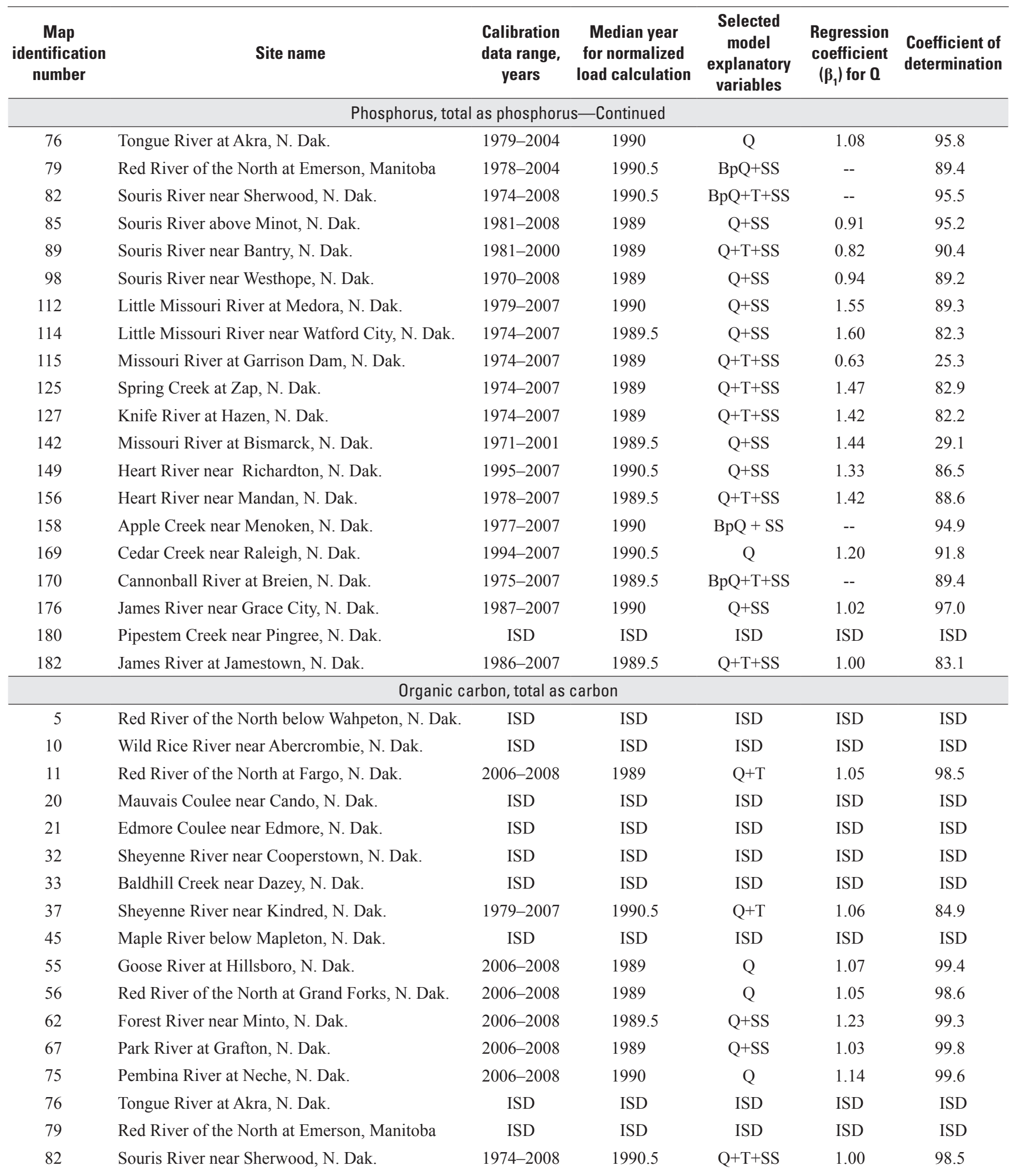


Table 3. Regression model characteristics for S-LOADEST models for selected sites in North Dakota from 1970 through 2008.Continued

[Q, natural log discharge; BpQ, natural log of discharge with a break point; T, time; SS, season; CEN, data had more than 80 percent censored values; ISD, insufficient data; --, not used]

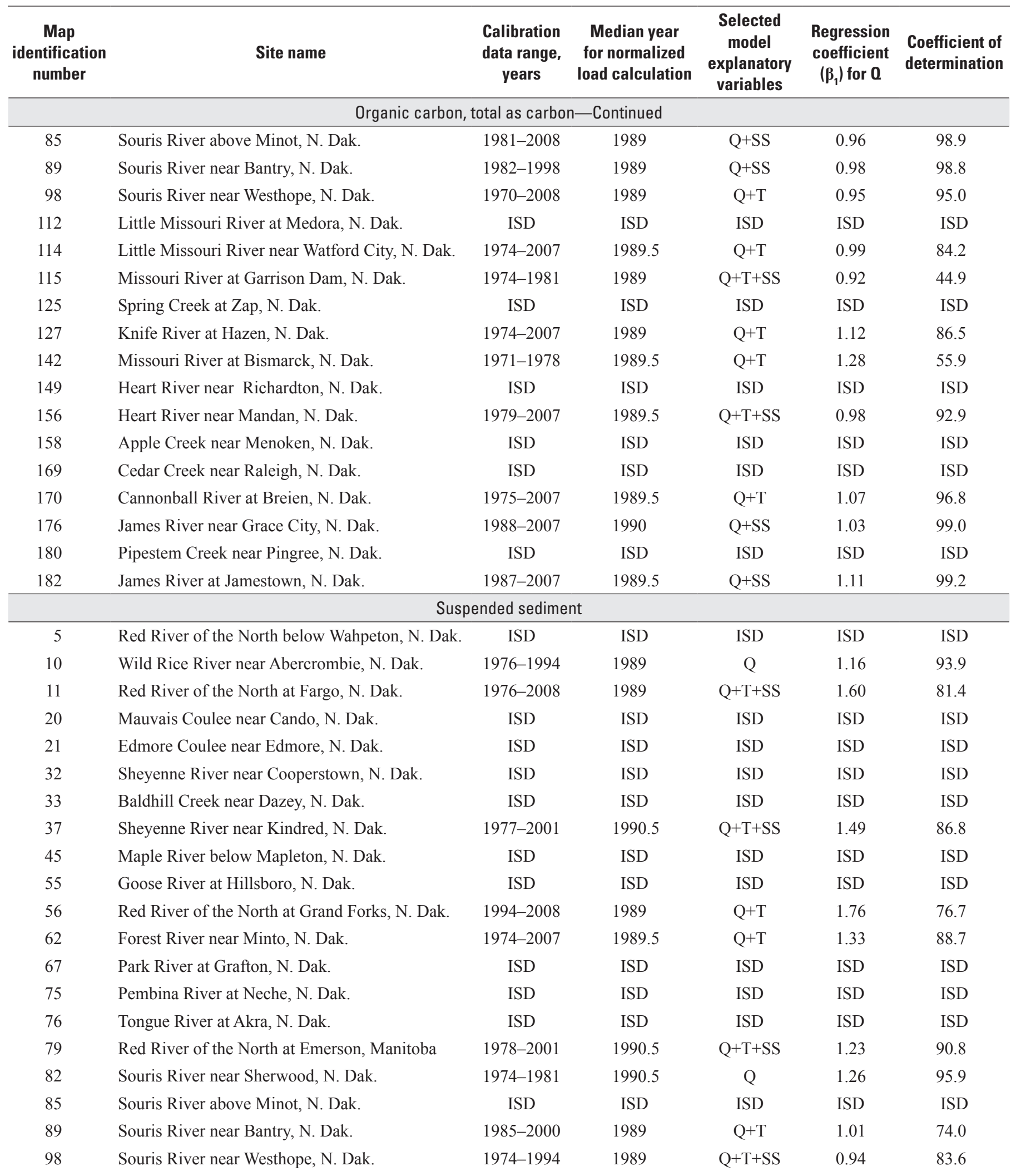


Table 3. Regression model characteristics for S-LOADEST models for selected sites in North Dakota from 1970 through 2008. Continued

[Q, natural log discharge; BpQ, natural log of discharge with a break point; T, time; SS, season; CEN, data had more than 80 percent censored values; ISD, insufficient data; --, not used]

\begin{tabular}{|c|c|c|c|c|c|c|}
\hline $\begin{array}{c}\text { Map } \\
\text { identification } \\
\text { number }\end{array}$ & Site name & $\begin{array}{c}\text { Calibration } \\
\text { data range, } \\
\text { years }\end{array}$ & $\begin{array}{l}\text { Median year } \\
\text { for normalized } \\
\text { load calculation }\end{array}$ & $\begin{array}{l}\text { Selected } \\
\text { model } \\
\text { explanatory } \\
\text { variables }\end{array}$ & $\begin{array}{c}\text { Regression } \\
\text { coefficient } \\
\left(\beta_{1}\right) \text { for } 0\end{array}$ & $\begin{array}{l}\text { Coefficient of } \\
\text { determination }\end{array}$ \\
\hline 112 & Little Missouri River at Medora, N. Dak. & ISD & ISD & ISD & ISD & ISD \\
\hline 114 & Little Missouri River near Watford City, N. Dak. & 1971-1994 & 1989.5 & $\mathrm{Q}+\mathrm{SS}$ & 1.67 & 86.2 \\
\hline 125 & Spring Creek at Zap, N. Dak. & 1975-2008 & 1989 & $\mathrm{Q}+\mathrm{T}+\mathrm{SS}$ & 1.49 & 89.5 \\
\hline 127 & Knife River at Hazen, N. Dak. & 1974-1993 & 1989 & $\mathrm{Q}+\mathrm{SS}$ & 1.53 & 88.3 \\
\hline 142 & Missouri River at Bismarck, N. Dak. & $1972-2001$ & 1989.5 & $\mathrm{Q}+\mathrm{T}$ & 2.01 & 46.5 \\
\hline 149 & Heart River near Richardton, N. Dak. & ISD & ISD & ISD & ISD & ISD \\
\hline 156 & Heart River near Mandan, N. Dak. & $1972-1993$ & 1989.5 & $\begin{array}{l}\mathrm{Q}+\mathrm{March}- \\
\text { June period }\end{array}$ & 0.95 & 93.9 \\
\hline 170 & Cannonball River at Breien, N. Dak. & 1973-1992 & 1989.5 & $\mathrm{Q}+\mathrm{T}+\mathrm{SS}$ & 1.57 & 90.7 \\
\hline 176 & James River near Grace City, N. Dak. & 1985-1995 & 1990 & $\mathrm{Q}+\mathrm{SS}$ & 0.94 & 90.6 \\
\hline 180 & Pipestem Creek near Pingree, N. Dak. & ISD & ISD & ISD & ISD & ISD \\
\hline 182 & James River at Jamestown, N. Dak. & 1985-1995 & 1989.5 & $\mathrm{Q}+\mathrm{SS}$ & 0.94 & 91.8 \\
\hline
\end{tabular}

Normalized annual total phosphorus yields ranged from less than 1 to $167 \mathrm{lbs} / \mathrm{yr} / \mathrm{mi}^{2}$ as phosphorus in the Missouri River Basin and from 6 to $76 \mathrm{lbs} / \mathrm{yr} / \mathrm{mi}^{2}$ as phosphorus in the Red River of the North Basin (fig. 20 and table 4). The highest yields (greater than $46 \mathrm{lbs} / \mathrm{yr} / \mathrm{mi}^{2}$ ) were at two sites in the Missouri River Basin (sites 112 and 114 on the Little Missouri River) and six sites in the Red River of the North Basin (site 20 on Mauvais Coulee; site 21 on Edmore Coulee; site 75 on the Pembina River; site 76 on the Tongue River; and sites 5 and 79 on the Red River of the North) (figs. 1 and 20; table 4). The lowest yields (less than $14 \mathrm{lbs} / \mathrm{yr} / \mathrm{mi}^{2}$ ) were at three sites in the Missouri River Basin (sites 115 and 142 on the Missouri River and site 169 on Cedar Creek) and five sites in the Red River of the North Basin (site 32 on the Sheyenne River; site 45 on the Maple River; and sites 82, 85 and 89 on the Souris River) (figs. 1 and 20; table 4).

Normalized annual total organic carbon yields ranged from 426 to 2,670 $\mathrm{lbs} / \mathrm{yr} / \mathrm{mi}^{2}$ as carbon in the Missouri River Basin and from 394 to $11,100 \mathrm{lbs} / \mathrm{yr} / \mathrm{mi}^{2}$ as carbon in the Red River of the North Basin (fig. 21 and table 4). The highest yields (greater than 2,100 lbs/yr/mi ${ }^{2}$ ) were at two sites in the Missouri River Basin (site 115 on the Missouri River and site 127 on the Knife River) and three sites in the Red River of the North Basin (site 11 and 56 on the Red River of the North and site 55 on the Goose River) (figs. 1 and 21; table 4). The lowest yields (less than $800 \mathrm{lbs} / \mathrm{yr} / \mathrm{mi}^{2}$ ) were at one site in the Missouri River Basin (site 142 on the Missouri River) and four sites in the Red River of the North Basin (site 37 on the Sheyenne River and sites 82,85 and 89 on the Souris River) (figs. 1 and 21; table 4).

\section{Suspended Sediment}

Normalized annual suspended-sediment yields ranged from 519 to $622,000 \mathrm{lbs} / \mathrm{yr} / \mathrm{mi}^{2}$ in the Missouri River Basin and from 1,500 to 78,800 lbs/yr/ $\mathrm{mi}^{2}$ in the Red River of the North Basin (fig. 22 and table 4). The highest yields (greater than $31,700 \mathrm{lbs} / \mathrm{yr} / \mathrm{mi}^{2}$ ) were at three sites in the Missouri River Basin (sites 114 on the Little Missouri River; site 156 

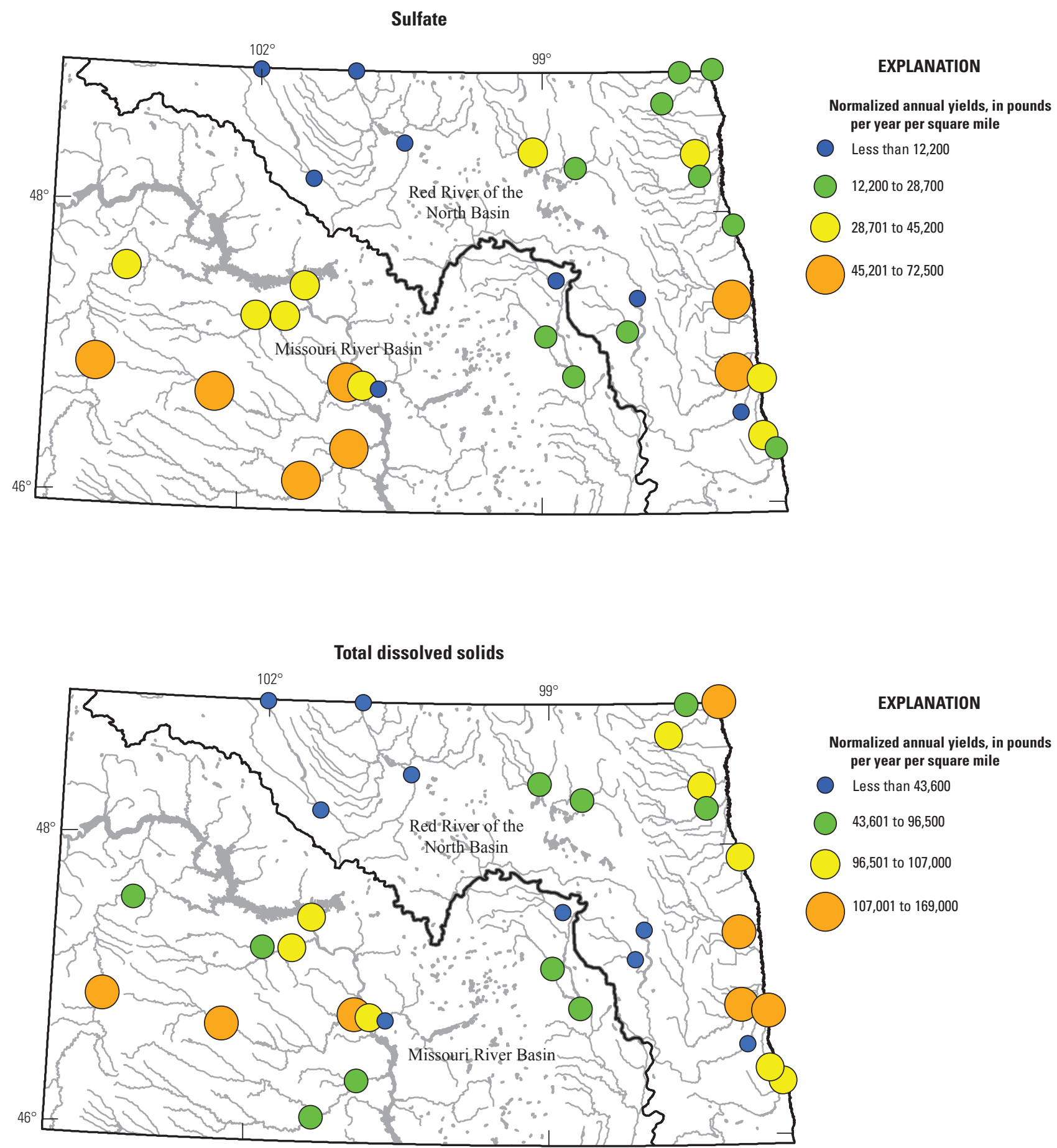

Figure 19. Spatial distribution of normalized annual sulfate and total dissolved solids yields for selected stream sites in North Dakota. 


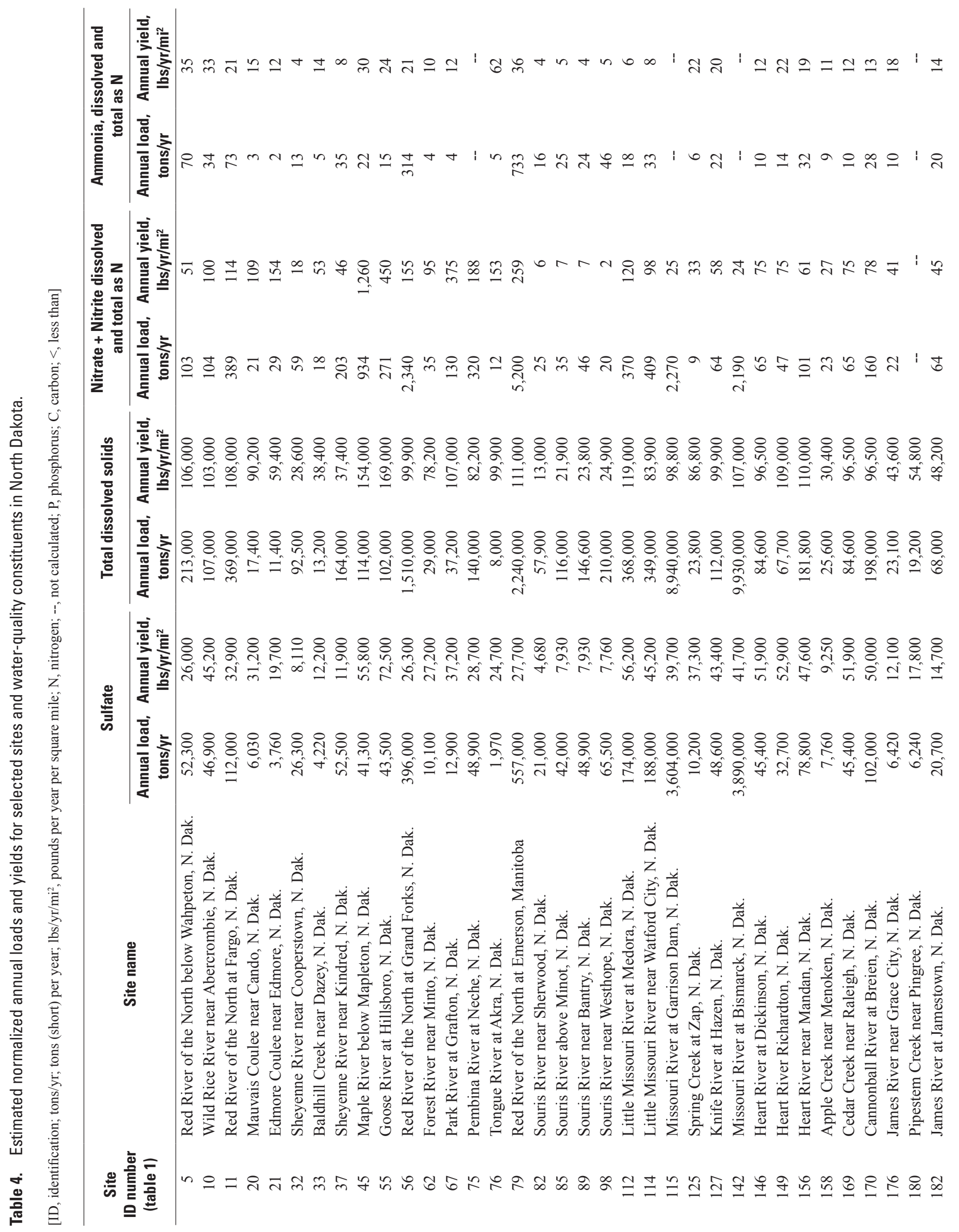




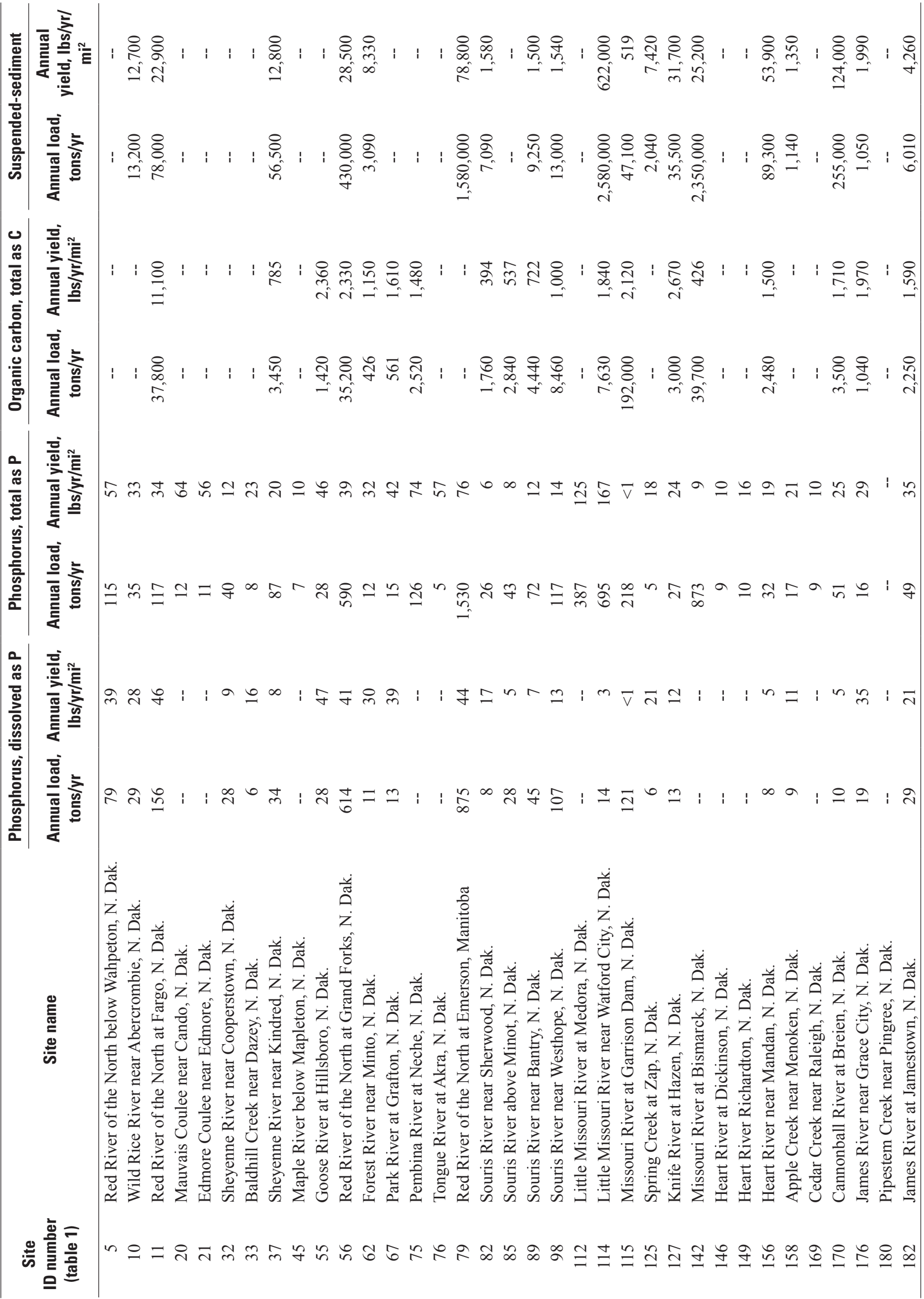



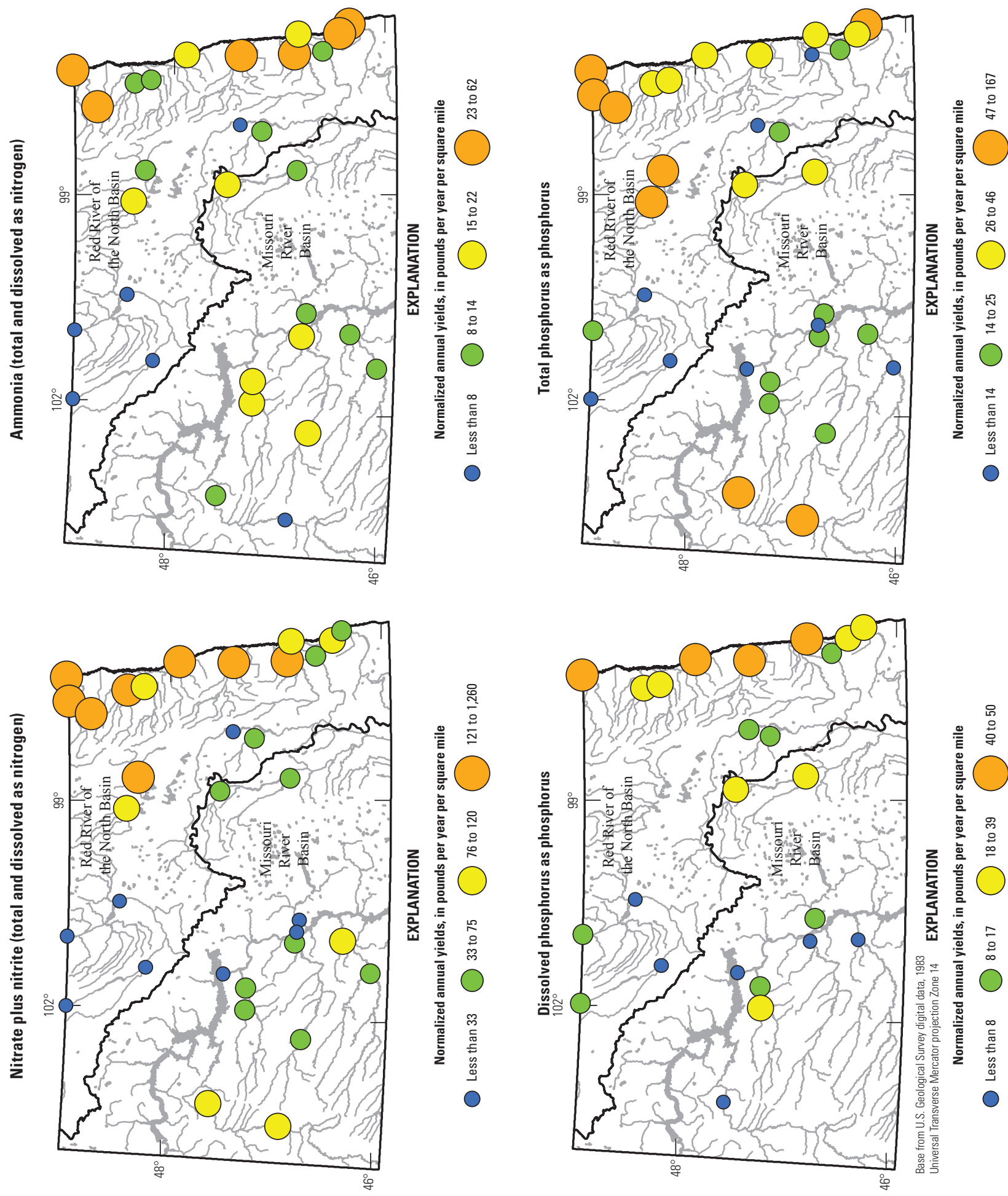

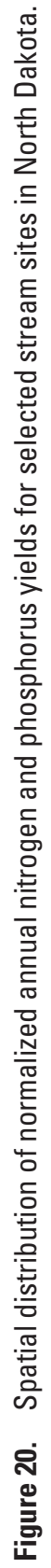




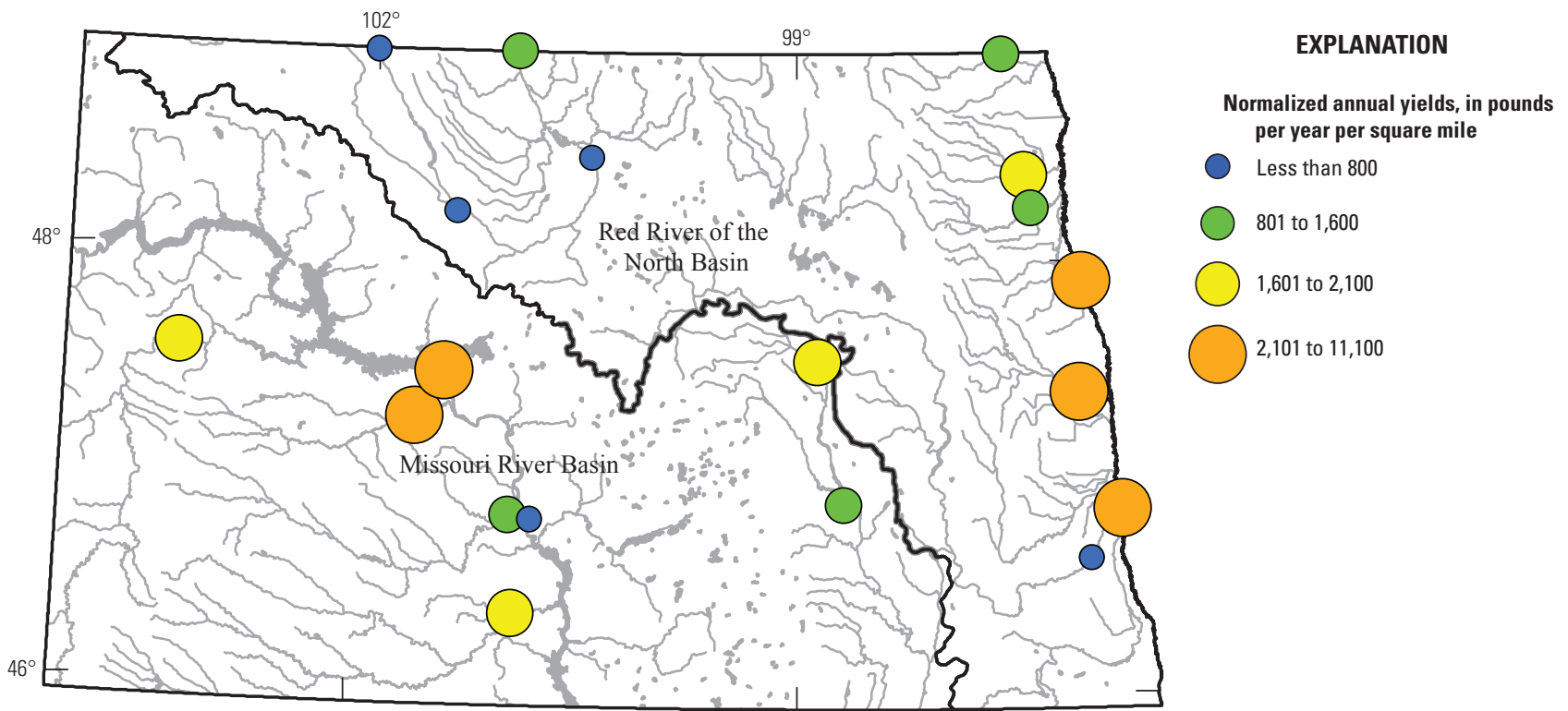

Figure 21. Spatial distribution of normalized annual total organic carbon yields for selected stream sites in North Dakota.

on the Heart River; and site 170 on the Cannonball River) and one site in the Red River of the North Basin (site 79 on the Red River of the North) (figs. 1 and 22; table 4). The lowest yields $\left(1,580 \mathrm{lbs} / \mathrm{yr} / \mathrm{mi}^{2}\right.$ or less $)$ were at two sites in the Missouri River Basin (site 115 on the Missouri River and site 158 on Apple Creek) and three sites in the Red River of the North Basin (sites 82, 89 and 98 on the Souris River) (figs. 1 and 22; table 4).

\section{Water-Quality Trend Analysis}

The results of the water-quality trend analysis for the stations and constituents used for this study are summarized in this section. Long-term variability in the fitted annual median of the measured concentrations (FAMC) (eq. 3) indicates the inter-annual variability in concentration that can be attributed to both the flow-related variability (ANN) and the trend (TREND). The trend indicates long-term changes in standardized concentrations (eq. 4) that are unrelated to streamflow. The fitted trends along with statistical significance levels (P-values) are given in table 5. Unless otherwise noted, the fitted trends discussed in this section were highly significant, as indicated by a generalized likelihood ratio test (Vecchia, 2005 ) with a p-value less than 0.01 . Thus, for any given station and constituent, the chance that the fitted trend could have occurred given the null hypothesis that the standardized concentrations were trend free was less than 1 percent (the data were trend free if their probability distribution remained the same for the entire trend-analysis period).

\section{Sulfate and Total Dissolved Solids}

The fitted annual median concentrations for sulfate at the 10 sites analyzed for trends from 1975 through 2008 indicated a pattern of generally higher median concentrations during the latter half of the period (1993-2008) compared to earlier years for most of the sites (fig. 23). This pattern was particularly evident for the sites in eastern North Dakota (sites 10, $32,55,56$, and 82) and for two sites in western North Dakota (sites 125 and 127). The latter period corresponds with an extended period of wet conditions and high streamflows, especially in eastern North Dakota. Conversely, for two sites in western North Dakota (sites 114 and 170), median concentrations during 1993-2008 were somewhat lower compared to earlier years. The greatest ranges in annual median sulfate concentration were from 341 to $867 \mathrm{mg} / \mathrm{L}$ for the Little Missouri River near Watford City, N. Dak. (site 114) and from 382 to $767 \mathrm{mg} / \mathrm{L}$ for the Cannonball River at Breien, N. Dak. (site 170).

The fitted trends for standardized sulfate concentrations indicated significant increases in concentration at five sites and a significant decrease in concentration at one site from 1975 through 2008 (fig. 24 and table 5). Median standardized sulfate concentrations increased from about 55 to $106 \mathrm{mg} / \mathrm{L}$ for the Red River of the North at Grand Forks, N. Dak. (site 56); 262 to $308 \mathrm{mg} / \mathrm{L}$ for the Souris River near Sherwood, N. Dak. (site 82); 455 to $506 \mathrm{mg} / \mathrm{L}$ for Spring Creek at Zap, N. Dak. (site 125); 440 to $520 \mathrm{mg} / \mathrm{L}$ for the Knife River at Hazen, N. Dak. (site 127); and 380 to $435 \mathrm{mg} / \mathrm{L}$ for the Heart River near Mandan, N. Dak. (site 156). The fitted trend for the Little Missouri River near Watford City, N. Dak. (site 114) showed a decrease in median standardized concentration from about 492 to $417 \mathrm{mg} / \mathrm{L}$. No significant increase or decrease in sulfate 


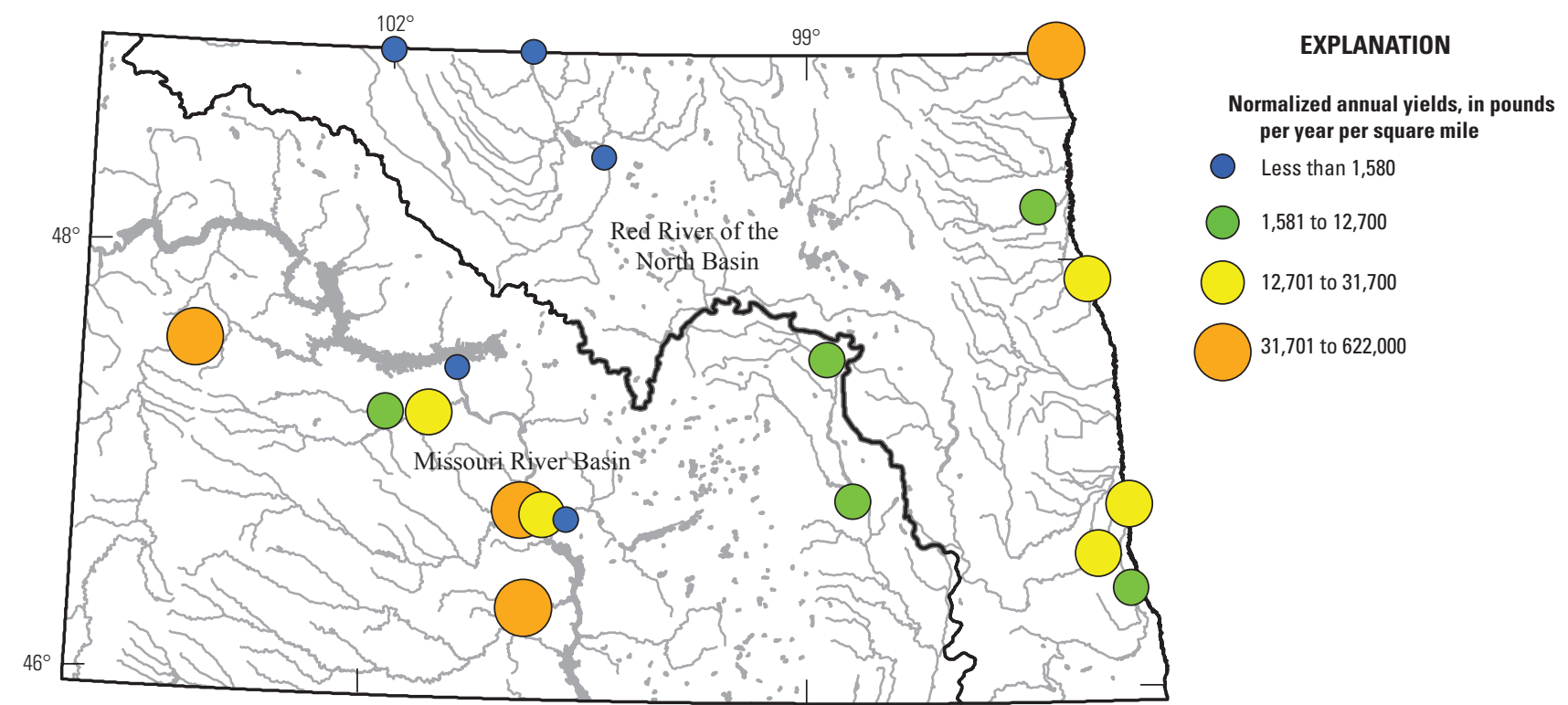

Figure 22. Spatial distribution of normalized annual suspended-sediment yields for selected stream sites in North Dakota.

concentrations was detected at the Wild Rice River near Abercrombie, N. Dak. (site 10), Sheyenne River near Cooperstown, N. Dak. (site 32), Goose River at Hillsboro, N. Dak. (site 55), or the Cannonball River at Breien, N. Dak. (site 170).

The fitted annual median TDS concentrations at the 10 sites analyzed for trends (fig. 25) were relatively variable compared to sulfate (fig. 23). The greatest ranges in annual median TDS concentration were from about 480 to $1,140 \mathrm{mg} / \mathrm{L}$ for the Wild Rice River near Abercrombie, N. Dak. (site 10), 630 to $1,170 \mathrm{mg} / \mathrm{L}$ for the Souris River near Sherwood, N. Dak. (site 82), and 720 to $1,950 \mathrm{mg} / \mathrm{L}$ for the Little Missouri River near Watford City, N. Dak. (site 114).

The fitted trends for standardized TDS concentrations indicated significant increases in concentration at five sites from 1975 through 2008 (fig. 26 and table 5). The greatest increase in median standardized TDS concentrations was detected for the Wild Rice River near Abercrombie, N. Dak. (site 10), where median concentrations increased from about 690 to $1,120 \mathrm{mg} / \mathrm{L}$ from 1975 through 2008. Median standardized TDS concentrations increased from about $880 \mathrm{mg} / \mathrm{L}$ to $1,000 \mathrm{mg} / \mathrm{L}$ for the Heart River near Mandan, N. Dak. (site 156); from about 1,090 to $1,210 \mathrm{mg} / \mathrm{L}$ for the Knife River at Hazen, N. Dak. (site 127); from about 1,000 to $1,110 \mathrm{mg} / \mathrm{L}$ for Spring Creek at Zap, N. Dak. (site 125); and from about 280 to $420 \mathrm{mg} / \mathrm{L}$ at the Red River of the North at Grand Forks, N. Dak. (site 56). No significant trend in TDS concentrations was detected at the Sheyenne River near Cooperstown, N. Dak. (site 32), Goose River at Hillsboro, N. Dak. (site 55), Souris River near Sherwood, N. Dak. (site 82), Little Missouri River near Watford City, N. Dak. (site 114), or Cannonball River at Breien, N. Dak. (site 170) (fig. 26).

\section{Nitrate plus Nitrite and Total Phosphorus}

The fitted annual median concentrations for nitrate plus nitrite (total and dissolved, as nitrogen) (fig. 27) were highly variable for sites in the Red River of the North Basin and the Little Missouri River near Watford City, N. Dak. (site 114). Concentrations varied from 0.12 to $0.50 \mathrm{mg} / \mathrm{L}$ for the Wild Rice River near Abercrombie, N. Dak. (site 10), from 0.11 to $0.41 \mathrm{mg} / \mathrm{L}$ for the Sheyenne River near Cooperstown, N. Dak. (site 32), from 0.23 to $0.66 \mathrm{mg} / \mathrm{L}$ for the Red River of the North near Grand Forks, N. Dak. (site 56), and from 0.19 to $0.59 \mathrm{mg} / \mathrm{L}$ for the Little Missouri River near Watford City, N. Dak. (site 114). Median concentrations for the other four sites did not vary more than $0.06 \mathrm{mg} / \mathrm{L}$.

The fitted trends for standardized nitrate plus nitrite concentrations indicated no significant increases or decreases in concentration for 7 of the 8 sites analyzed for trends from 1975 through 2008 (fig. 28 and table 5); however, median standardized nitrate plus nitrite concentration for the Red River of the North near Grand Forks, N. Dak. (site 56) increased from about 0.26 to $0.45 \mathrm{mg} / \mathrm{L}$ as nitrogen during 1990 to 2008 (data were not available for that site before 1990).

The fitted annual median concentrations for total phosphorus were not highly variable (fig. 29). The greatest variability in annual median total phosphorus concentration was observed for the Souris River near Sherwood, N. Dak. (site 82), where median concentrations ranged from about 0.11 to $0.40 \mathrm{mg} / \mathrm{L}$ as phosphorus, and the Little Missouri River near Watford city, N. Dak. (site 114), where median concentrations ranged from about 0.05 to $0.26 \mathrm{mg} / \mathrm{L}$ as phosphorus.

The fitted trends for standardized total phosphorus concentrations indicated significant decreases in concentration at 4 out of the 10 sites and a significant increase at 1 out 
Table 5. Fitted trends for selected standardized constituent concentrations at selected sites in North Dakota.

[ID, identification; mg/L, milligrams per liter; --, not calculated; <, less than; N, nitrogen; NA, insufficient data for analysis; P, phosphorus]

\begin{tabular}{|c|c|c|c|}
\hline $\begin{array}{c}\text { Site ID } \\
\text { number } \\
\text { (table 1) }\end{array}$ & Station Name & $\begin{array}{l}\text { Estimated change } \\
\text { in concentration from } \\
1975 \text { to } 2008 \text { (percent) }\end{array}$ & $\begin{array}{l}\text { P-value for } \\
\text { trend coefficient }\end{array}$ \\
\hline \multicolumn{4}{|c|}{ Sulfate, $\mathrm{mg} / \mathrm{L}$} \\
\hline 10 & Wild Rice River near Abercrombie, N. Dak. & No trend & -- \\
\hline 32 & Sheyenne River near Cooperstown, N. Dak. & No trend & -- \\
\hline 55 & Goose River at Hillsboro, N. Dak. & No trend & -- \\
\hline 56 & Red River of the North at Grand Forks, N. Dak. & 95 & 0.0009 \\
\hline 82 & Souris River near Sherwood, N. Dak. & 18 & $<0.0001$ \\
\hline 114 & Little Missouri River near Watford City, N. Dak. & -15 & 0.0047 \\
\hline 125 & Spring Creek at Zap, N. Dak. & 11 & 0.0016 \\
\hline 127 & Knife River at Hazen, N. Dak. & 19 & $<0.0001$ \\
\hline 156 & Heart River near Mandan, N. Dak. & 14 & 0.0005 \\
\hline 170 & Cannonball River at Breien, N. Dak. & No trend & -- \\
\hline \multicolumn{4}{|c|}{ Total dissolved solids, mg/L } \\
\hline 10 & Wild Rice River near Abercrombie, N. Dak. & 63 & $<0.0001$ \\
\hline 32 & Sheyenne River near Cooperstown, N. Dak. & No trend & -- \\
\hline 55 & Goose River at Hillsboro, N. Dak. & No trend & -- \\
\hline 56 & Red River of the North at Grand Forks, N. Dak. & 48 & $<0.0001$ \\
\hline 82 & Souris River near Sherwood, N. Dak. & No trend & -- \\
\hline 114 & Little Missouri River near Watford City, N. Dak. & No trend & -- \\
\hline 125 & Spring Creek at Zap, N. Dak. & 11 & 0.0005 \\
\hline 127 & Knife River at Hazen, N. Dak. & 11 & $<0.0001$ \\
\hline 156 & Heart River near Mandan, N. Dak. & 13 & 0.0001 \\
\hline 170 & Cannonball River at Breien, N. Dak. & No trend & -- \\
\hline \multicolumn{4}{|c|}{ Nitrate + Nitrite dissolved and total as $\mathrm{N}$, in $\mathrm{mg} / \mathrm{L}$} \\
\hline 10 & Wild Rice River near Abercrombie, N. Dak. & No trend & -- \\
\hline 32 & Sheyenne River near Cooperstown, N. Dak. & No trend & -- \\
\hline 55 & Goose River at Hillsboro, N. Dak. & NA & -- \\
\hline 56 & Red River of the North at Grand Forks, N. Dak. & ${ }^{1} 70$ & $<0.0001$ \\
\hline 82 & Souris River near Sherwood, N. Dak. & No trend & -- \\
\hline 114 & Little Missouri River near Watford City, N. Dak. & No trend & -- \\
\hline 125 & Spring Creek at Zap, N. Dak. & NA & -- \\
\hline 127 & Knife River at Hazen, N. Dak. & No trend & -- \\
\hline 156 & Heart River near Mandan, N. Dak. & No trend & -- \\
\hline 170 & Cannonball River at Breien, N. Dak. & No trend & -- \\
\hline \multicolumn{4}{|c|}{ Phosphorus, total as $\mathrm{P}$, in $\mathrm{mg} / \mathrm{L}$} \\
\hline 10 & Wild Rice River near Abercrombie, N. Dak. & No trend ${ }^{1}$ & -- \\
\hline 32 & Sheyenne River near Cooperstown, N. Dak. & No trend & -- \\
\hline 55 & Goose River at Hillsboro, N. Dak. & No trend ${ }^{1}$ & -- \\
\hline 56 & Red River of the North at Grand Forks, N. Dak. & ${ }^{1} 60$ & 0.0001 \\
\hline 82 & Souris River near Sherwood, N. Dak. & No trend & -- \\
\hline 114 & Little Missouri River near Watford City, N. Dak. & No trend & -- \\
\hline 125 & Spring Creek at Zap, N. Dak. & -51 & $<0.0001$ \\
\hline 127 & Knife River at Hazen, N. Dak. & -46 & $<0.0001$ \\
\hline 156 & Heart River near Mandan, N. Dak. & -47 & $<0.0001$ \\
\hline 170 & Cannonball River at Breien, N. Dak. & -51 & $<0.0001$ \\
\hline
\end{tabular}

${ }^{1}$ Change was analyzed only from 1990 to 2008. 


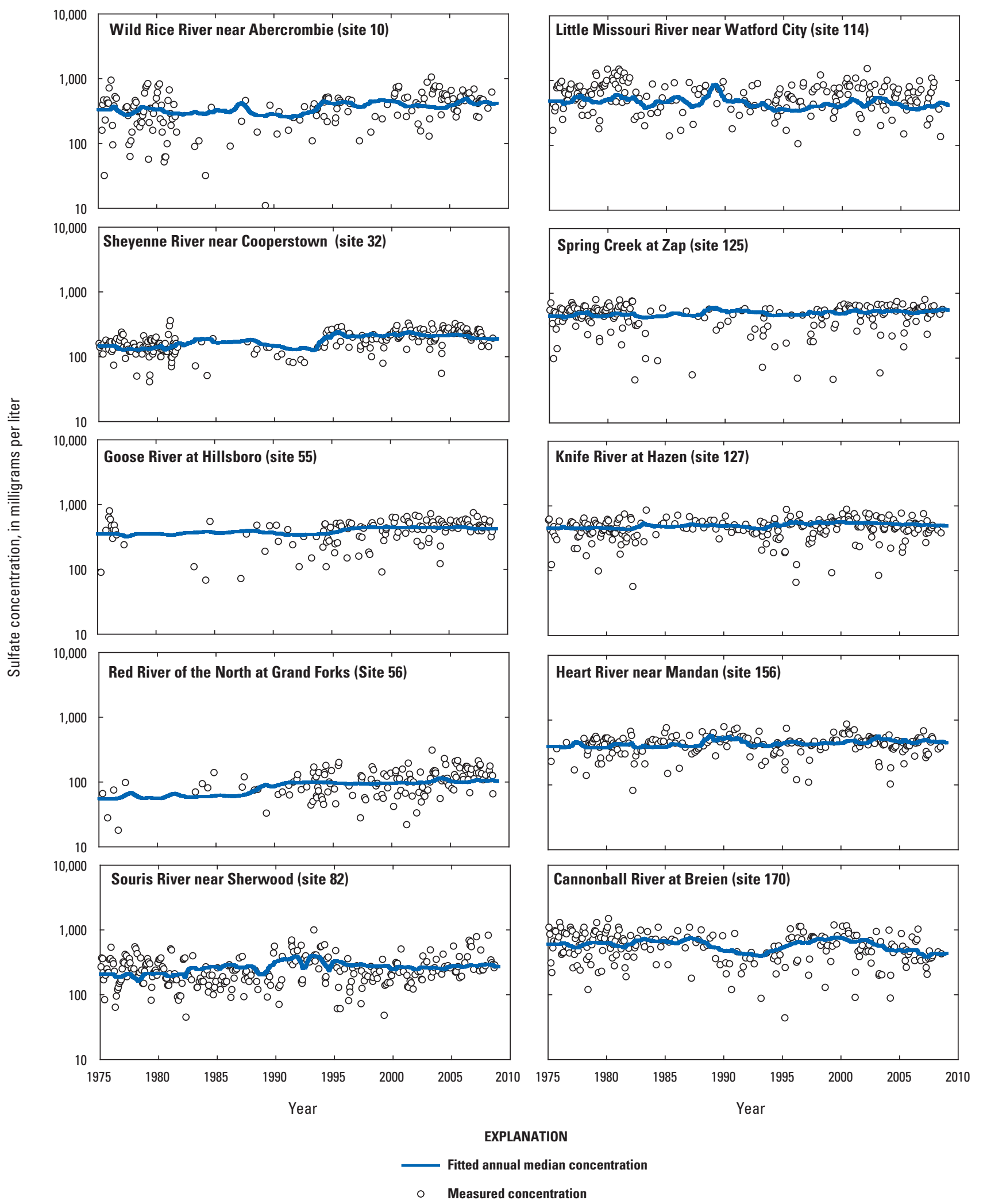

Figure 23. Measured sulfate concentrations and fitted annual median concentrations from 1975 through 2008 for selected sites in North Dakota. 


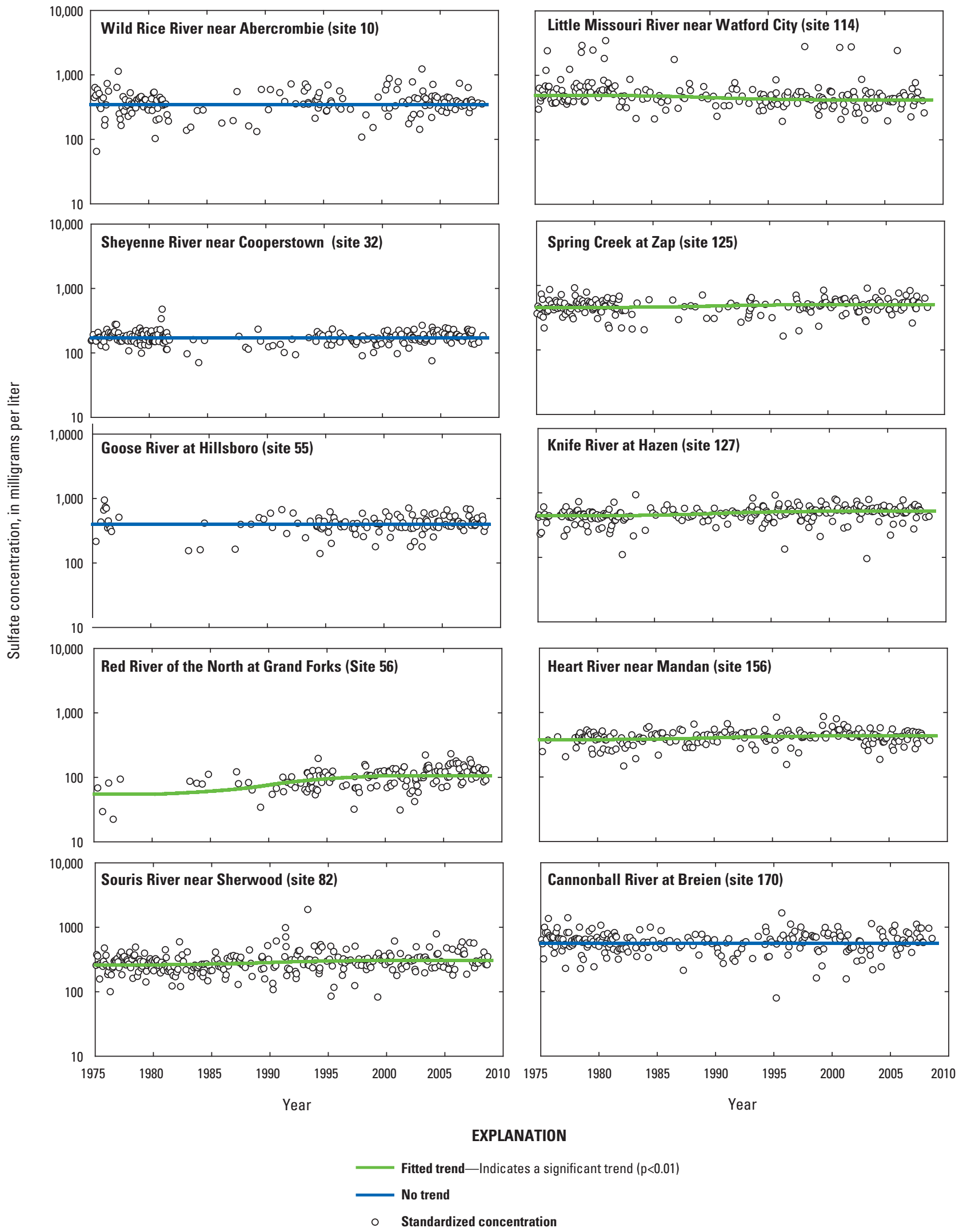

Figure 24. Standardized sulfate concentrations and fitted trends for 1975 through 2008 for selected sites in North Dakota. 


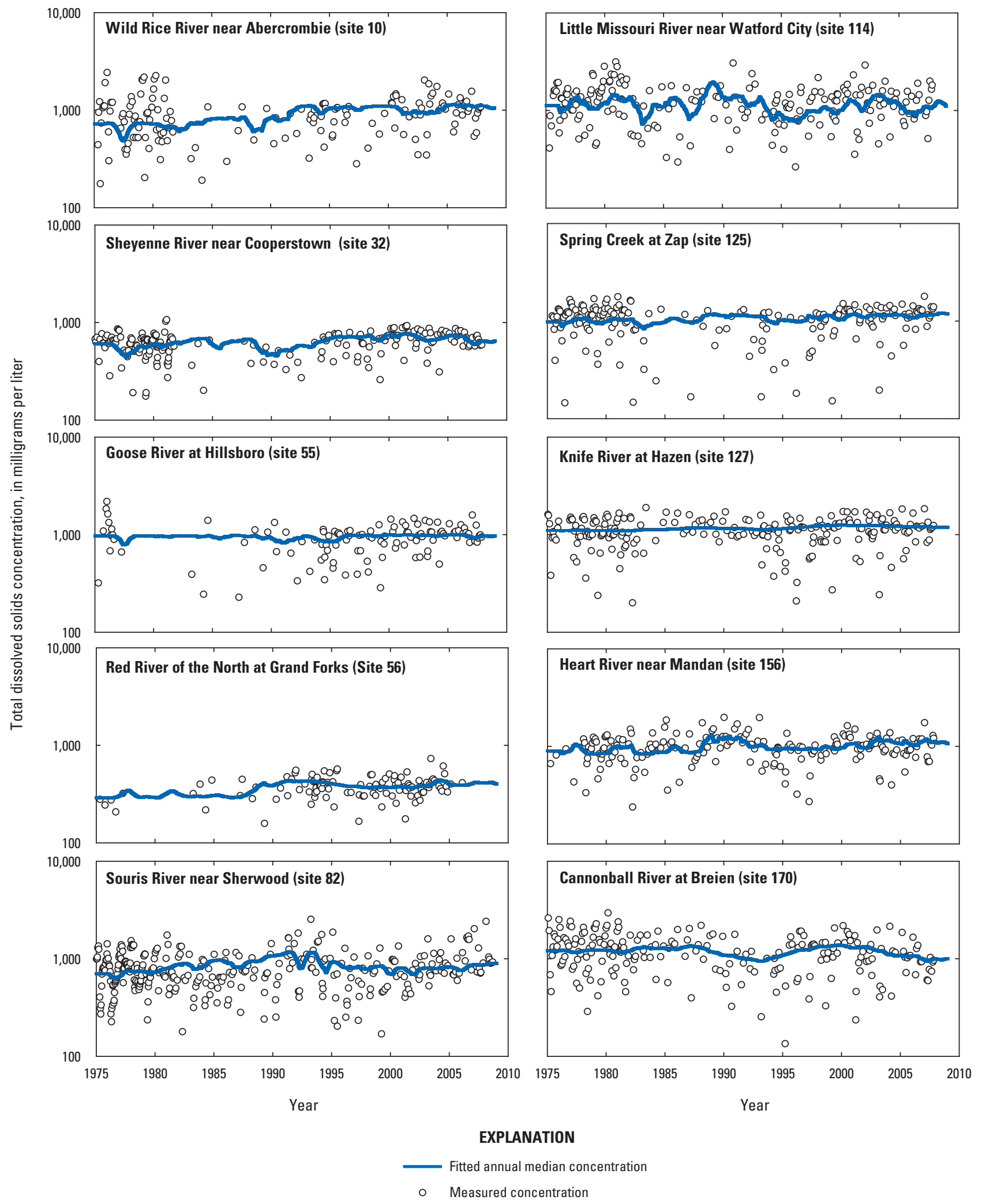

Figure 25. Measured total dissolved solids concentrations and fitted annual median concentrations for 1975 through 2008 for selected sites in North Dakota. 


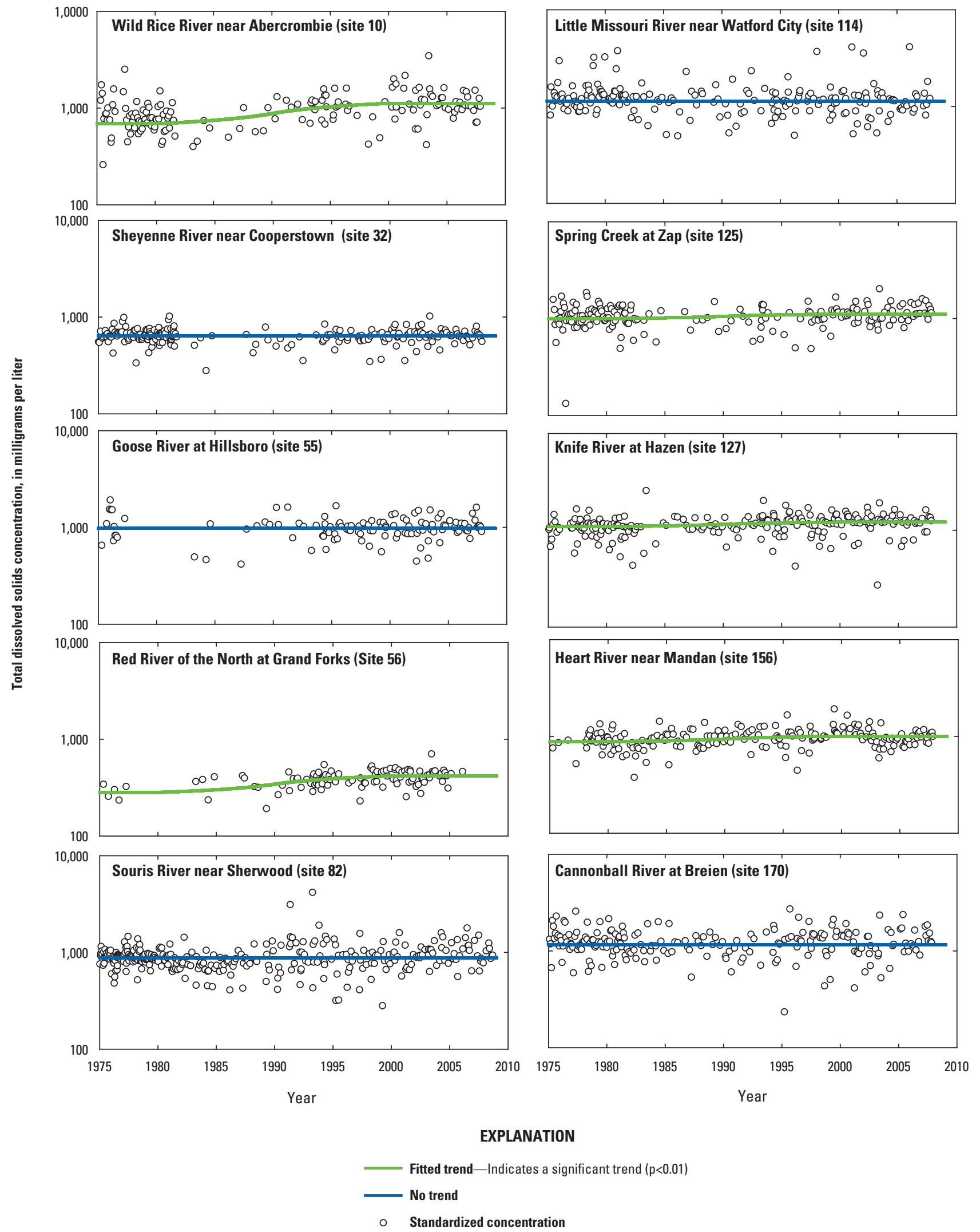

Figure 26. Standardized total dissolved solids concentrations and fitted trends for 1975 through 2008 for selected sites in North Dakota. 

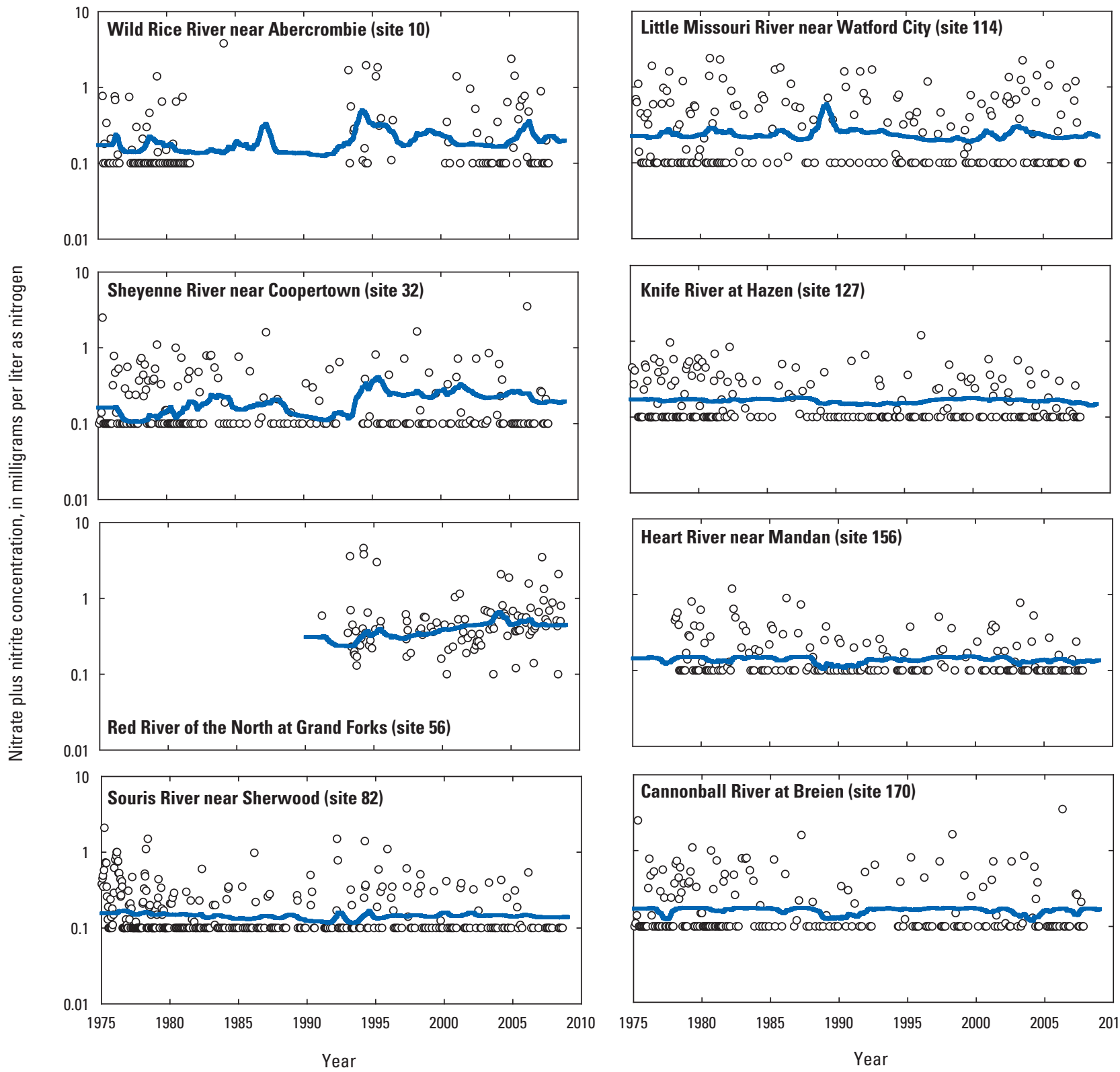

Heart River near Mandan (site 156)

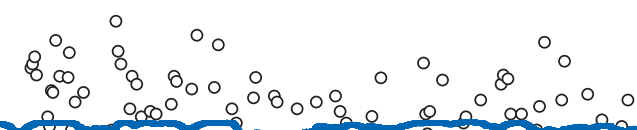

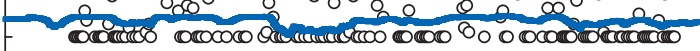

\section{EXPLANATION}

Cannonball River at Breien (site 170)

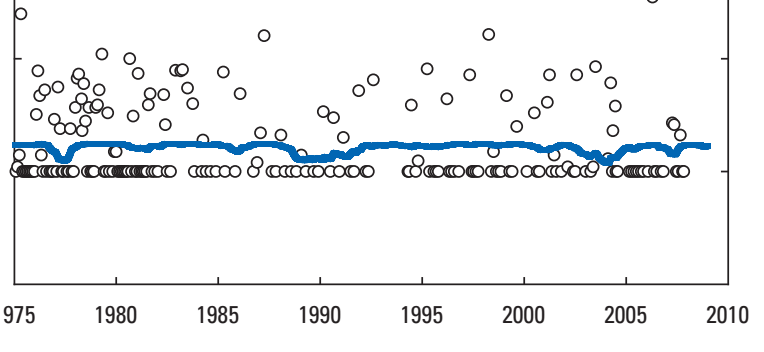

Fitted annual median concentration

- Measured concentration

Figure 27. Measured nitrate plus nitrite concentrations and fitted annual median concentrations for 1975 through 2008 for selected sites in North Dakota. 

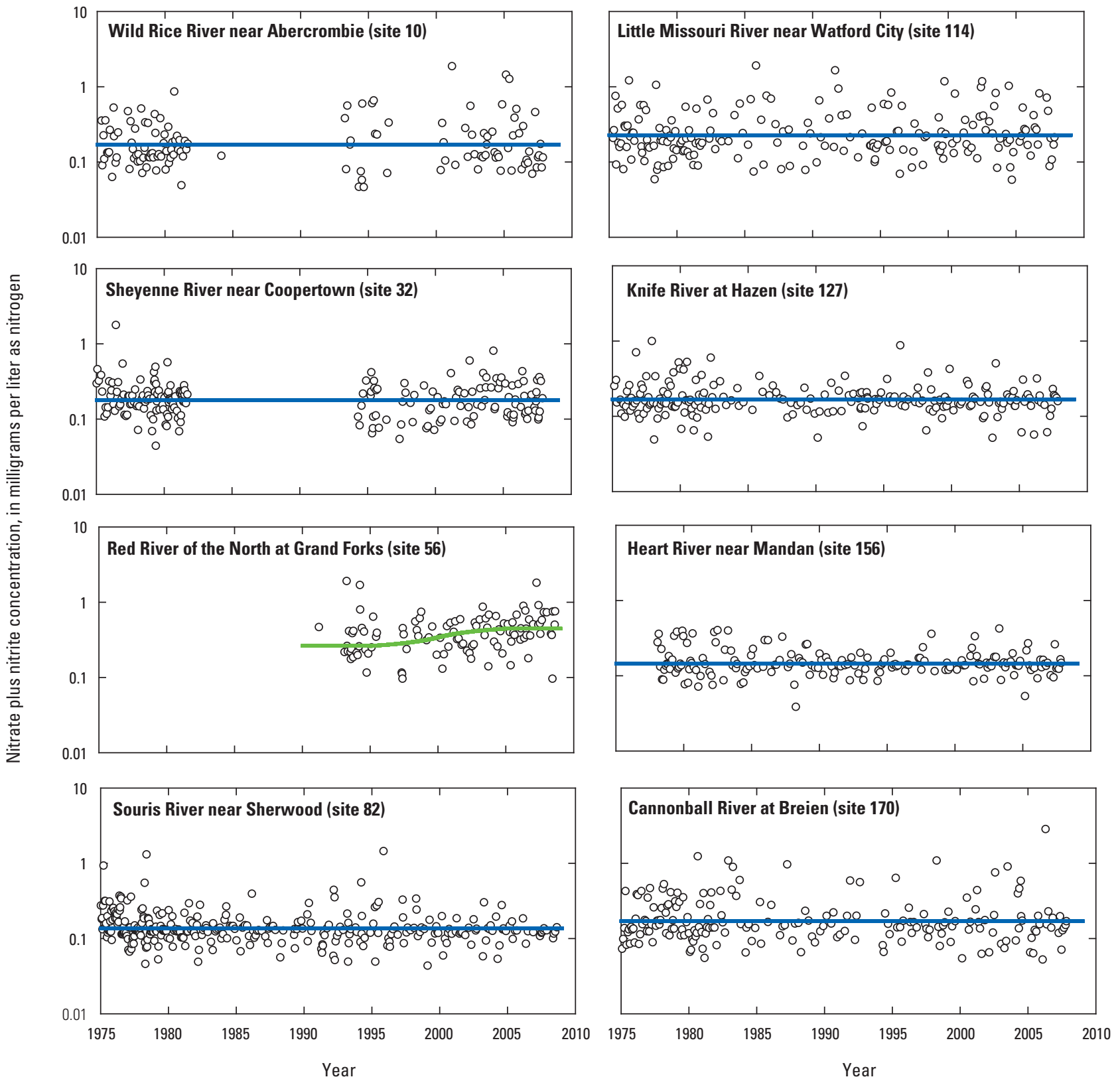

\section{EXPLANATION}

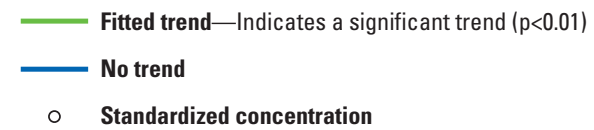

Figure 28. Standardized nitrate plus nitrite concentrations and fitted trends for 1975 through 2008 for selected sites in North Dakota. 

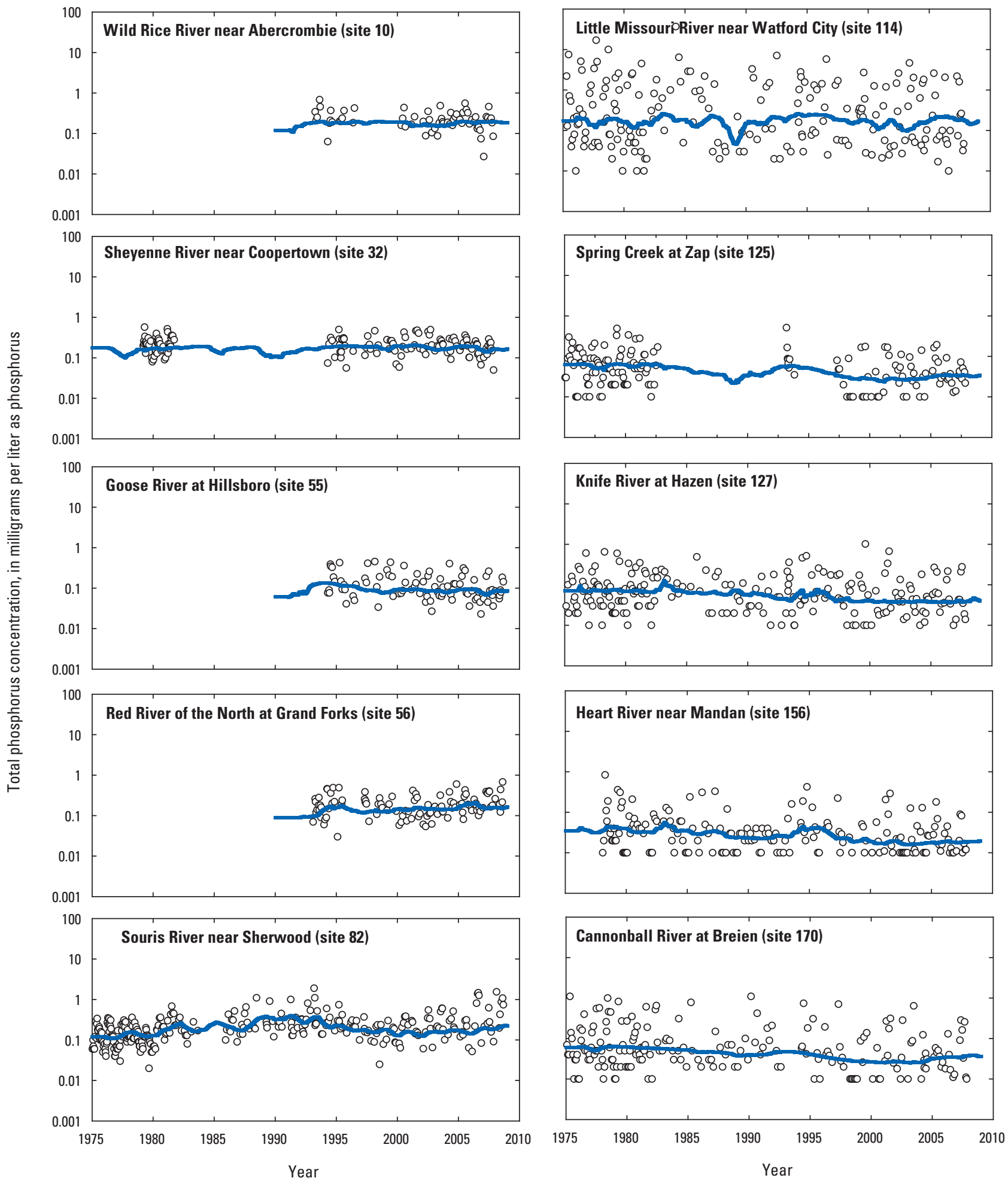

\section{EXPLANATION}

Fitted annual median concentration

- Measured concentration

Figure 29. Measured total phosphorus concentrations and fitted annual median concentrations for 1975 through 2008 for selected sites in North Dakota. 
of the 10 sites analyzed for trends (fig. 30 and table 5). All of the significant decreases were for sites in western North Dakota, including decreases in median standardized total phosphorus concentration from about 0.06 to $0.03 \mathrm{mg} / \mathrm{L}$ for the Cannonball River at Breien, N. Dak. (site 170); from about 0.04 to $0.02 \mathrm{mg} / \mathrm{L}$ for the Heart River near Mandan, N. Dak. (site 156); from about 0.07 to $0.04 \mathrm{mg} / \mathrm{L}$ for the Knife River at Hazen, N. Dak. (site 127); and from about 0.07 to 0.03 $\mathrm{mg} / \mathrm{L}$ for Spring Creek at Zap, N. Dak. (site 125). Conversely, median standardized total phosphorus concentration for the Red River of the North at Grand Forks, N. Dak. (site 56) increased from about 0.10 to $0.16 \mathrm{mg} / \mathrm{L}$ from 1990 through 2008 (data were not available for that site before 1990). No significant trend in total phosphorus concentrations was detected for the remaining sites.

\section{Sampling Designs to Monitor Water- Quality Trends and Loads}

Many different sampling programs with various objectives and sampling designs throughout North Dakota have contributed data to this report. This section describes the results of spatial and statistical analyses of the data described in previous sections of the report to provide guidance to waterresource agencies for efficient and effective sampling designs for the detection of trends and estimation of constituent loads when developing new water-quality programs or refining current programs. The single most important consideration for interpreting water-quality data is having complete streamflow records (Vecchia, 2005). This applies when interpreting spatial variability, temporal trends, or loads. Water-quality data from a location that does not have an active streamgage (or a nearby streamgage) is of limited use. The number and placement of streamflow gages is determined largely by considerations (cost, importance for flood warning or water supply, and so forth) unrelated to water quality. Therefore, rather than designing a sampling program that is optimal strictly with respect to water quality and thus might require relocation of streamgages, a much simpler and more practical approach of designing a water-quality sampling program that is optimal with respect to the existing placement of streamgage locations is the approach described here. Mean annual flows from 2000 (or the earliest available year of record if later than 2000) through 2011 are given in table 6 for streamgages that were active in 2011.

\section{Spatial Distribution of Sampling Sites}

One objective of a State-wide sampling program was to describe the spatial variability of water-quality conditions across the State in the most efficient manner. Given a fixed number of sampling sites that are colocated with streamgages, the locations of the sites should be selected to minimize redundancy in the network to accomplish this objective. Each active site should provide as much incremental information as possible for characterizing water-quality conditions in a representative area of the State (corresponding to a particular drainage basin) that is not adequately characterized by any of the other sites in the network. To provide a quantitative evaluation of spatial network efficiency, it was assumed that the goal of the spatial network design is to characterize spatial variability in the long-term mean concentrations of each of the chemical constituents. The long-term mean is assumed to be constant through time (for example, no temporal trends) for this analysis. Temporal variability and trends will be considered in the next section of this report.

Because there generally are streamgages near the mouth (or, in the case of basins that overlap with other states or provinces, near the downstream border) of major drainage basins in the state, high priority was given to collecting water-quality samples at these locations to describe aggregate water-quality conditions across large areas of the State. Sampling sites colocated with streamgages that are nested within the larger basins can then be selected to minimize redundancy in the overall network. To evaluate the spatial redundancy in nested samples, two constituents - sulfate and total phosphorus were selected. Sulfate generally is the major ion of greatest concern and phosphorus is the nutrient of greatest concern for evaluating water-quality conditions in the State (Mike Ell, North Dakota Department of Health, oral commun., 2011). As described previously in the Methods section, for each downstream/upstream pair of sites with at least 10 concurrent samples, the average absolute difference between the paired downstream and upstream concentrations, expressed as a percent of the average downstream concentration, was related to the average absolute difference in daily flow between the downstream and upstream pairs, expressed as a percent of the average downstream flow. The relation between the flow differences and concentration differences are shown in figure 31 for sulfate and figure 32 for total phosphorus. A nonlinear weighted regression procedure described in appendix 2 was used to fit the lines in these figures representing estimates of the 50th and 90th percentile of the concentration differences for given flow difference. Of the 186 sites considered for this analysis, which resulted in a total of 17,205 potential pairs of sites, only 178 pairs for sulfate and 73 pairs for phosphorus were upstream/downstream pairs with sufficient concurrent data to analyze. These site pairs are shown as the points in figure 31 (178 pairs) and fig. 32 (73 pairs). The sample sizes used to compute the mean percent differences varied widely among the station pairs, so the weighted regression procedure was used to adjust the pairs to represent a sample size of 40 and the sample-size adjusted data are shown in figures 31 and 32 (see appendix 2).

Several important observations regarding placement of sampling sites can be gleaned from figures 31 and 32. For pairs where the upstream site provided a large percentage of the downstream flows (50 percent or more), the concentration differences tend to be relatively small. For example, if the 

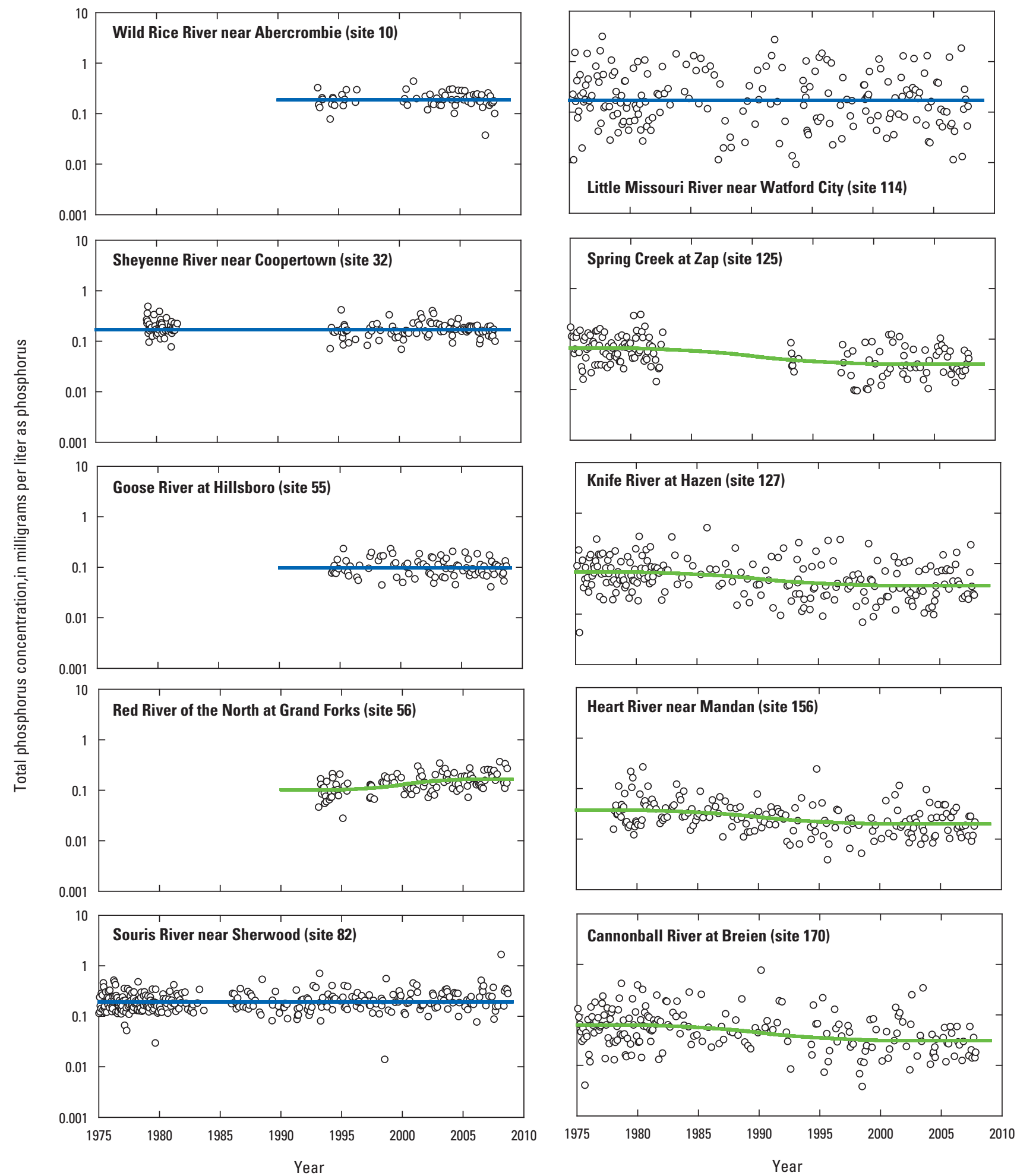

EXPLANATION

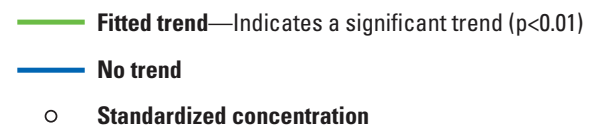

Figure 30. Standardized total phosphorus concentrations and fitted trends for 1975 through 2008 for selected sites in North Dakota. 
Table 6. Current and potential state-wide water-quality sampling network for North Dakota.

[All streamflow data obtained from http://nwis.waterdata.usgs.gov/nd/nwis/; USGS, U.S. Geological Survey; ft³, cubic foot per second; --, not included in potential state-wide network; NA, not available; *, streamflow obtained from nearby upstream gage; **, no nearby gage; ***, streamflow obtained from Environment Canada (http://www.wateroffice.ec.gc.ca/graph/graph_e.html?stn=05OC001)]

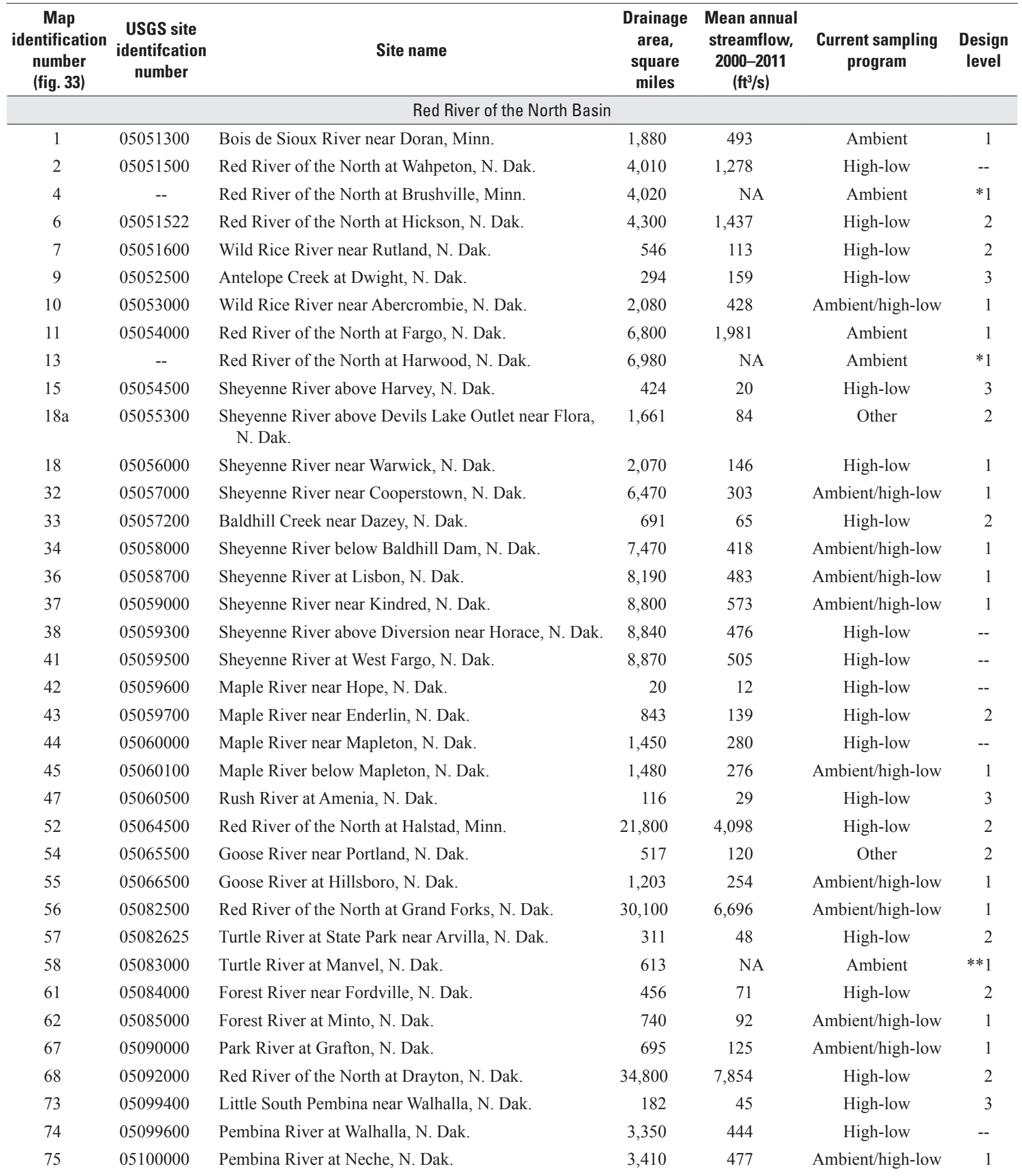


Table 6. Current and potential state-wide water-quality sampling network for North Dakota.—Continued

[All streamflow data obtained from http://nwis.waterdata.usgs.gov/nd/nwis/; USGS, U.S. Geological Survey; $\mathrm{ft}^{3} / \mathrm{s}$, cubic foot per second; --, not included in potential state-wide network; NA, not available; *, streamflow obtained from nearby upstream gage; **, no nearby gage; ***, streamflow obtained from Environment Canada (http://www.wateroffice.ec.gc.ca/graph/graph_e.html?stn=05OC001)]

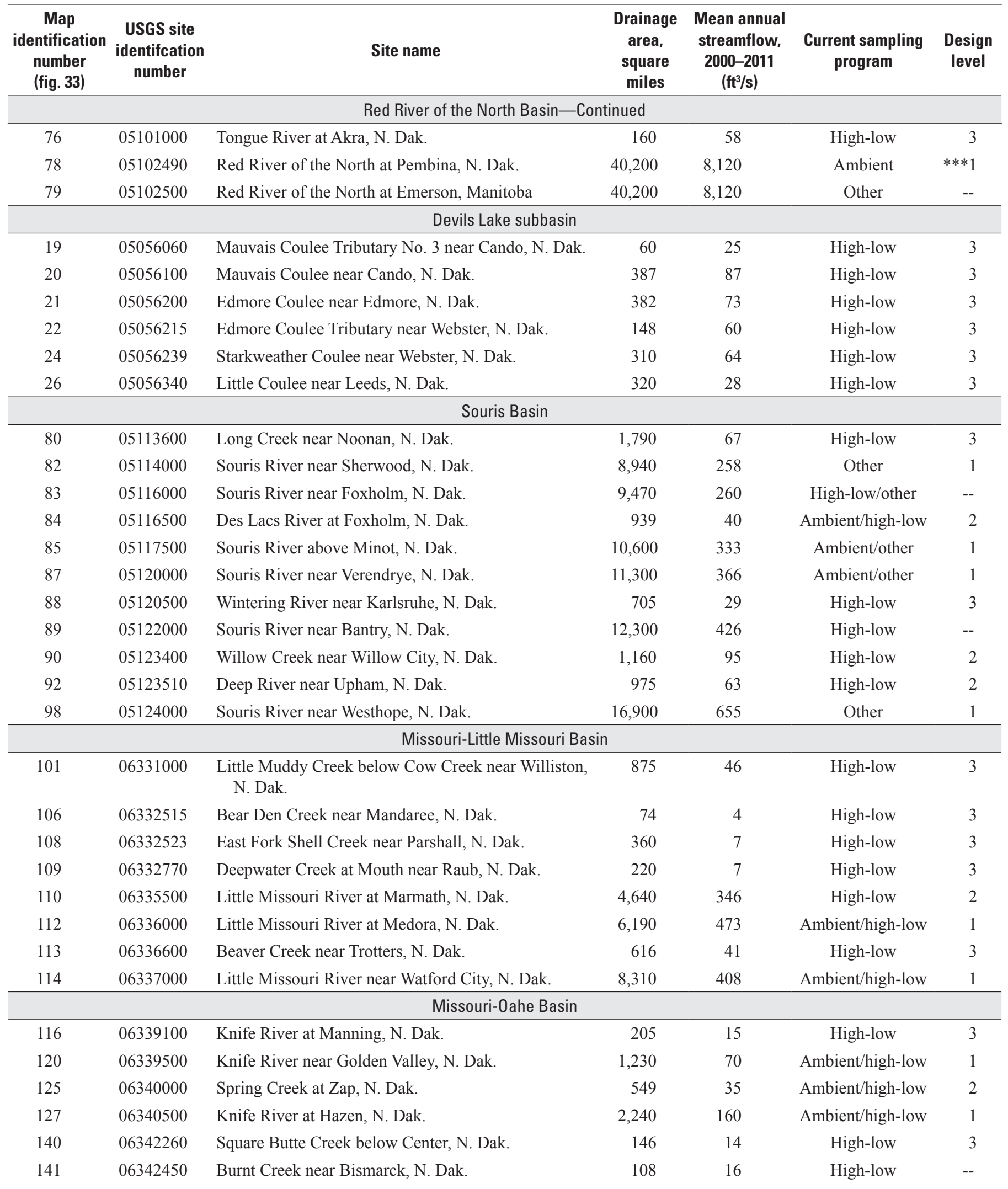


Table 6. Current and potential state-wide water-quality sampling network for North Dakota._Continued

[All streamflow data obtained from http://nwis.waterdata.usgs.gov/nd/nwis/; USGS, U.S. Geological Survey; ft³/s, cubic foot per second; --, not included in potential state-wide network; NA, not available; *, streamflow obtained from nearby upstream gage; **, no nearby gage; ***, streamflow obtained from Environment Canada (http://www.wateroffice.ec.gc.ca/graph/graph_e.html?.stn=050C001)]

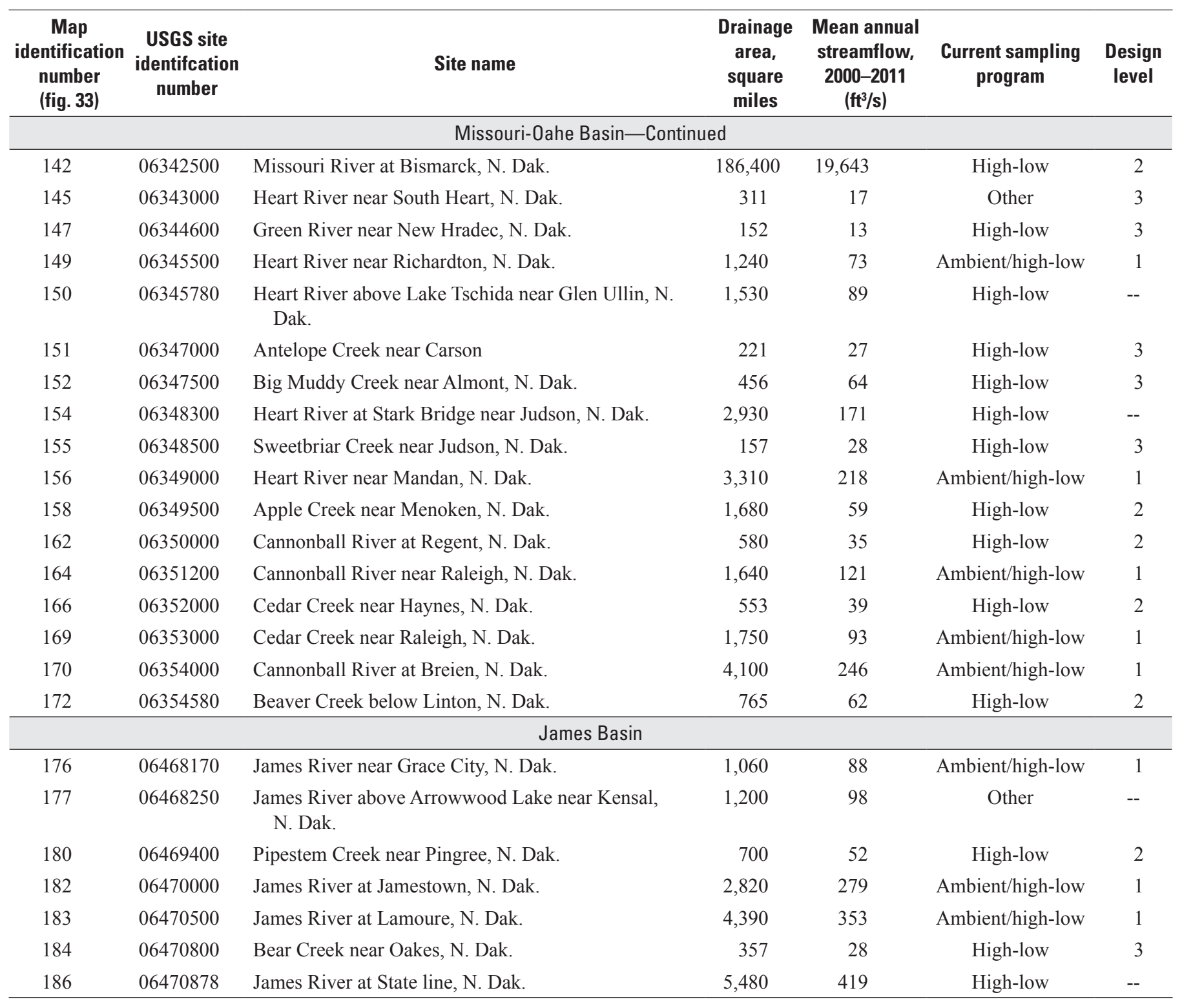




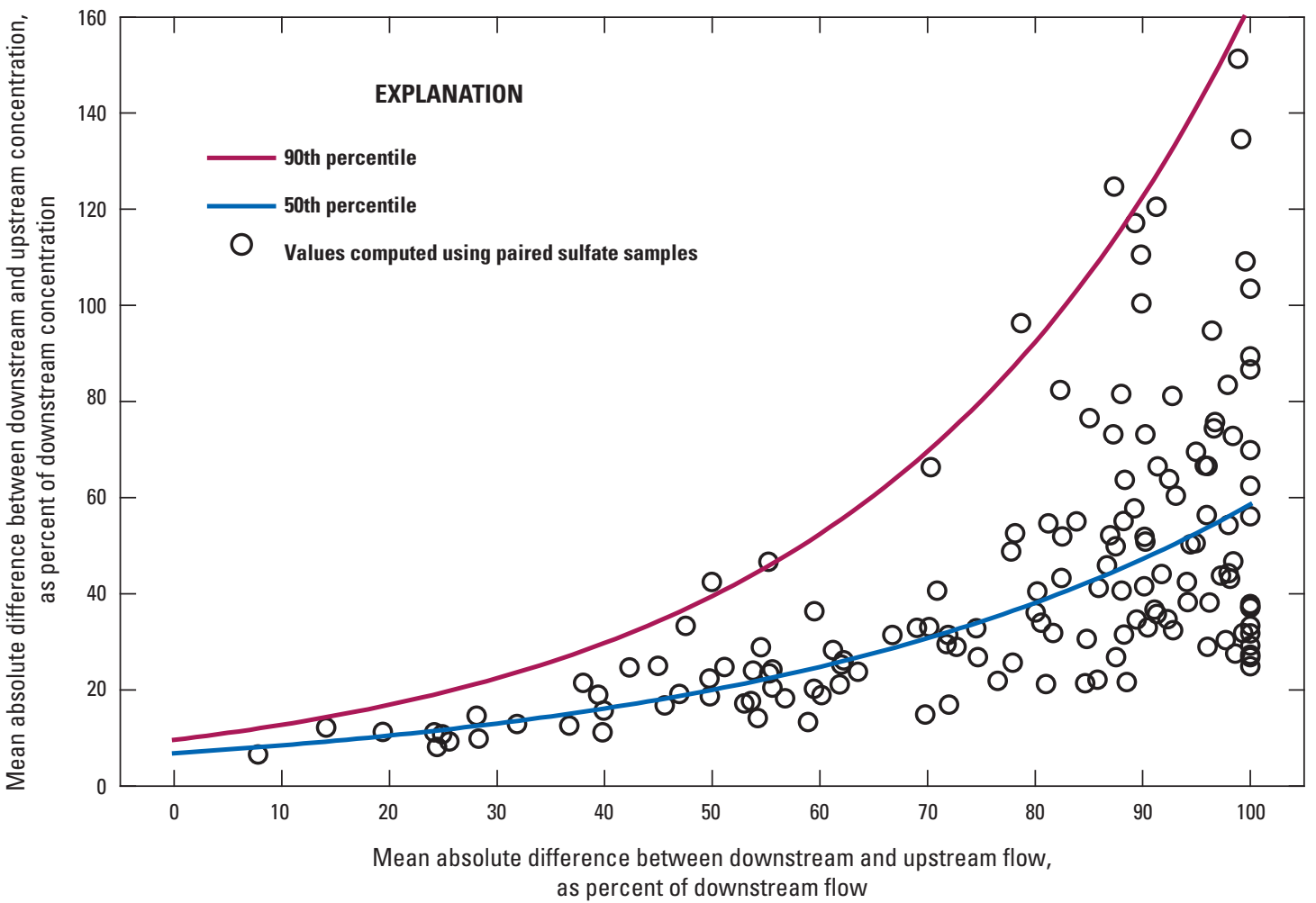

Figure 31. Relation between streamflow differences and sulfate concentration differences for selected sites in North Dakota.

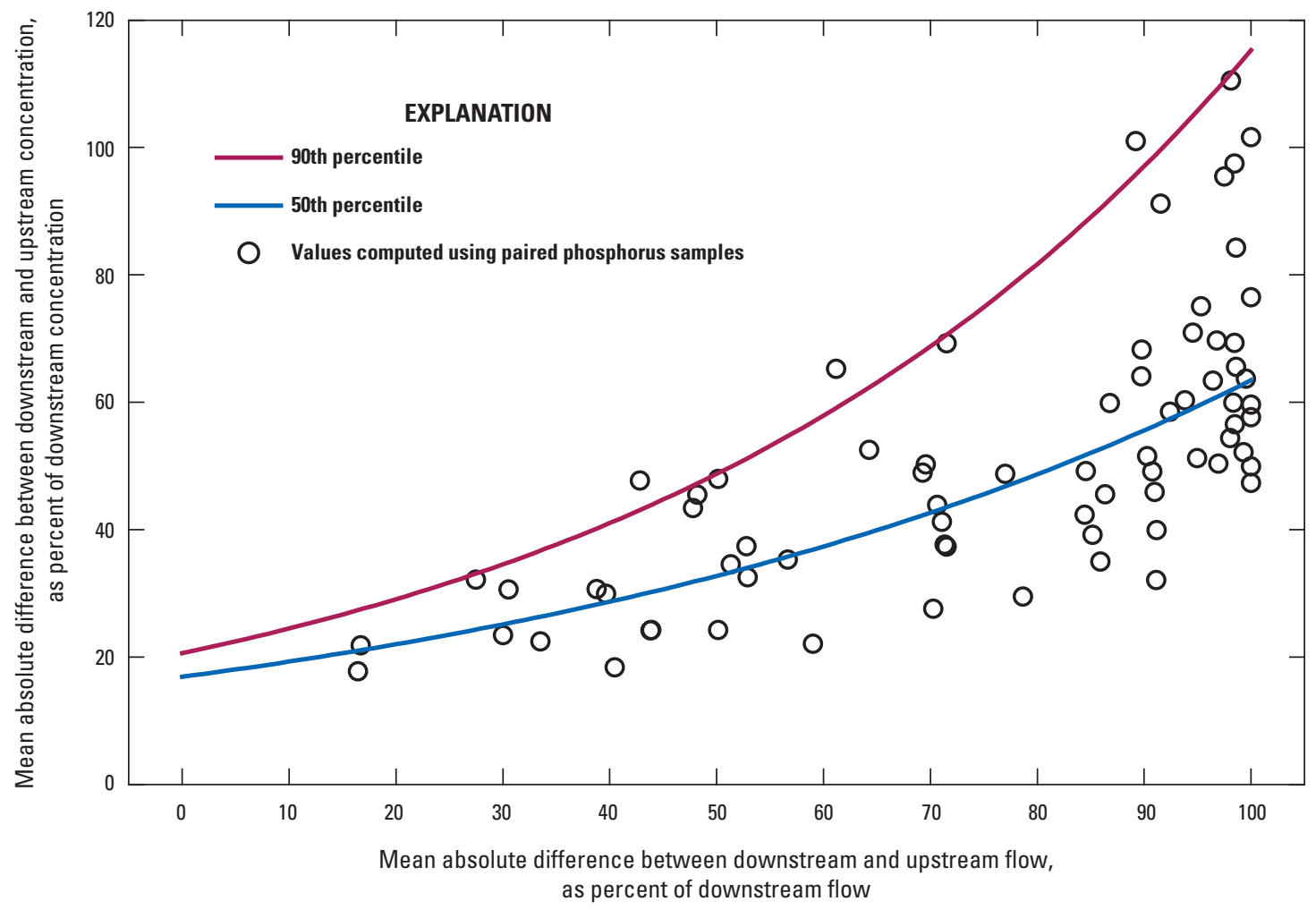

Figure 32. Relation between streamflow differences and total phosphorus concentration differences for selected sites in North Dakota. 
upstream flow is 80 percent, on average, of the downstream flow (corresponding to a percent difference of 20 percent), 90 percent of the station pairs would have upstream concentration within about 15 percent, on average, of downstream concentration for sulfate (fig. 31) and within about 25 percent, on average, for phosphorus (fig. 32). If the upstream flow is 50 percent, on average, of the downstream flow (a percent difference of 50 percent), 90 percent of the station pairs would have upstream concentration within about 40 percent (for sulfate) and 50 percent (for phosphorus), on average, of the downstream concentration. Conversely, if the upstream site provides a small percentage of the downstream flow, the concentration differences can become quite large. For example, if upstream flow is only 10 percent, on average, of downstream flow (90 percent difference), more than one-half of the pairs have upstream concentrations more than 50 percent different (for sulfate) and 55 percent different (for phosphorus), on average, from downstream concentrations, and more than 10 percent of the pairs have upstream concentrations more than 140 percent different (for sulfate) and 100 percent different (for phosphorus), on average, from downstream concentrations. Thus, a reasonable spatial network would consist of including the most downstream sites in large basins first, followed by the next upstream site(s) that roughly bisect the downstream flows at the first sites (given the constraints of existing streamgage locations), followed by the next upstream site(s) that roughly bisect flows for the second sites. Other special considerations, such as whether a site is located near a major city, water supply intake, or downstream of a major reservoir could be considered.

Considering the previous discussion, sampling sites to be included in the proposed State-wide network were prioritized into 3 design levels: level 1 (highest priority), level 2 (second priority), and level 3 (third priority) (table 6; fig. 33). Starting with the Red River of the North main stem sites, the downstream site on the North Dakota/Manitoba border (site 78, Red River of the North at Pembina, N. Dak.) was designated as a level-1 site and is currently (2010) a NDDH ambient network site. Site 52 (Red River of the North at Halstad, Minn.) has essentially one-half the flow of site 78 and would thus be an ideal location for the next level-1 site; however, site 56 (Red River of the North at Grand Forks, N. Dak.) currently (2010) is an established ambient network site. Therefore, site 56 was selected as a level- 1 site and site 52 was included as a level-2 site. Site 68 (Red River of the North at Drayton, N. Dak.), which is midway between sites 56 and 78, also was selected as a level-2 site because of the importance of Red River of the North main stem water quality and the relatively large intervening drainage area (about 10,000 square miles) between sites 56 and 78. Proceeding upstream from site 52, site 11 (Red River of the North at Fargo), which provides about one-half the flow for site 52 and is currently (2010) an ambient network site, was included as a level-1 site. Site 13 (Red River of the North at Harwood, N. Dak.) has only a slightly higher drainage area than site 11 and the nearest active streamgage is at site 11; however, this site is currently (2010) an ambient network site and was important for NDDH for estimating point sources from the Fargo-Moorhead area (Mike Ell, North Dakota Department of Health, oral commun., 2011). Therefore, site 13 was designated as a level-1 site although it is largely redundant with site 11. Site 4 (Red River of the North at Brushville, Minn.) and site 1 (Bois de Sioux River near Doran, Minn.) both are current (2010) ambient network sites and were designated as level-1 sites. Site 6 (Red River of the North at Hickson, N. Dak.), midway between sites 11 and 4 , was designated as a level-2 site. A currently (2010) active HLSP sampling site (site 2, table 6) could be discontinued because of redundancy with site 4 (fig. 1).

Among the Red River of the North tributaries (excluding the Sheyenne River, which is considered separately), sites 10 (Wild Rice River near Abercrombie, N. Dak.), 45 (Maple River below Mapleton, N. Dak.), 55 (Goose River at Hillsboro, N. Dak.), 58 (Turtle River at Manvel, N. Dak.), 62 (Forest River at Minto, N. Dak.), 67 (Park River at Grafton, N. Dak.), and 75 (Pembina River at Neche, N. Dak.) are all current (2010) ambient network sites and were all selected as level-1 sites (table 6, fig. 33). Although there is no active streamgage near site 58 , maintaining sampling at that site was important for estimating load contributions from the Turtle River (Mike Ell, North Dakota Department of Health, oral commun., 2011). Locating a streamgage near site 58 would be beneficial for interpreting water-quality data from that site. Level-2 sites among the Red River of the North tributaries were upstream of level-1 sites, including sites 7 (Wild Rice River near Rutland, N. Dak.), 43 (Maple River near Enderlin, N. Dak.), 54 (Goose River near Portland, N. Dak.), 57 (Turtle River near Arvilla, N. Dak.), and 61 (Forest River near Fordville, N. Dak.). Several level-3 sites were selected on smaller tributaries, including sites 9 (Antelope Creek near Dwight, N. Dak.), 47 (Rush River at Armenia, N. Dak.), 73 (Little South Pembina River near Walhalla, N. Dak.), and 76 (Tongue River at Akra, N. Dak.). Three currently (2010) active HLSP sampling sites in the Maple and Pembina Basins (sites 42,44 , and 74 ; table 6 ) could be discontinued in the proposed network because of redundancy with other sites or (in the case of site 42) small drainage area (fig. 1 and table 1).

Next, sites were selected within the Sheyenne River Basin, the largest North Dakota tributary to the Red River of the North (table 6 and fig. 33). Three level-1 sites that adhered closely to the principle of bisecting flows were sites 37 (Sheyenne River near Kindred, N. Dak.), 32 (Sheyenne River near Cooperstown, N. Dak.), and 18 (Sheyenne River near Warwick, N. Dak.). These sites are currently (2010) ambient network sites. Two other ambient network sites, sites 36 (Sheyenne River at Lisbon, N. Dak.) and 34 (Sheyenne River below Baldhill Dam, N. Dak.), were designated as level-1 sites although there may be considerable redundancy of these sites with the downstream site (site 37). These sites are important for monitoring the effects of the Devils Lake outlets (http:// www.swc.state.nd.us/4dlink9/4dcgi/GetSubCategoryRecord/ Devils\%20Lake\%20Flooding/Outlets) on water-quality in the Sheyenne River. Three additional sites were included in the 


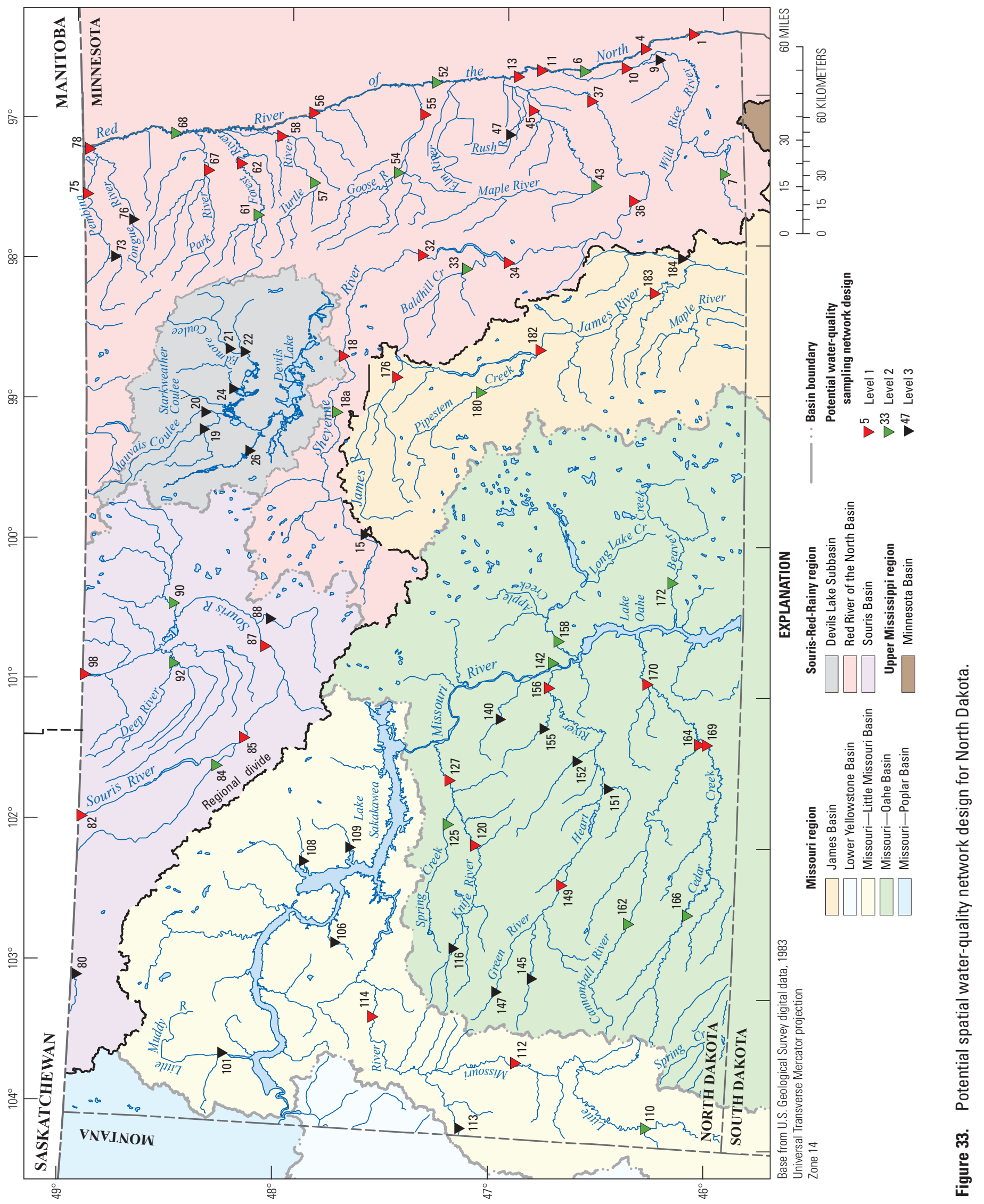


Sheyenne Basin: two level-2 sites (site 33, Baldhill Creek near Dazey, N. Dak. and site 18a, Sheyenne River above Devils Lake outlet near Flora, N. Dak.) and a level-3 site (site 15, Sheyenne River above Harvey, N. Dak.). Although waterquality data for the site near Flora, N. Dak. (site 18a) were not analyzed in this report, the site has been sampled recently as part of the Devils Lake outlet monitoring and would continue to be sampled in the proposed network. Two currently (2010) active HLSP sites in the Sheyenne Basin (sites 38 and 41 , table 6) could be discontinued in the proposed network because of redundancy with site 37 .

The Devils Lake Subbasin of the Sheyenne River has been experiencing extensive flooding since 1993 and there is concern of a potential spill from Devils Lake to the Sheyenne River, an event that has not happened for at least 800 years (Vecchia, 2008). The State has constructed outlets from the west end of Devils Lake in 2008 and from the east end of Devils Lake in 2012. Devils Lake water-quality issues are important for future outlet operation; therefore, six level-3 sites (sites 19, 20, 21, 22, 24, and 26) were designated on major tributaries to Devils Lake (fig. 33 and table 6).

Several sampling sites were designated in the Souris River Basin, which flows southeast from Saskatchewan into North Dakota before bending to the northwest and exiting North Dakota into Manitoba (table 6 and fig. 33). Site 98 (Souris River near Westhope, N. Dak.), which is the downstream site, and site 85 (Souris River above Minot, N. Dak.), which is about midway upstream from site 98 , were selected as level-1 sites. Site 98 currently (2010) is sampled as part of the International Souris River Board mandate to monitor water-quality at the International border and site 85 is an ambient network site. Additional level-1 sites were site 87 (Souris River near Verendrye, N. Dak.) and 82 (Souris River near Sherwood, N. Dak.). Although site 87 was highly redundant with site 85, it is an ambient network site considered important for estimating point sources from the Minot area (Mike Ell, North Dakota Department of Health, oral commun., 2011). Site 87 also is an ambient network site and site 82 is sampled as part of the International Souris River Board mandate. Three level-2 sites (site 84, Des Lacs River at Foxholm, N. Dak.; site 92, Deep River near Upham, N. Dak.; and site 90, Willow Creek near Willow City, N. Dak.) and two level-3 sites (site 88, Wintering River near Karlsruhe, N. Dak. and site 80, Long Creek near Noonan, N. Dak.) also are included in the proposed Souris River sampling sites. Two current (2010) HLSP sampling sites (sites 83 and 89 , table 6) could be discontinued because of redundancy with other sites.

The remaining sites in the proposed network are in the Missouri River Basin. Among the smaller tributaries, sites 172 (Beaver Creek below Linton, N. Dak.) and 158 (Apple Creek near Menoken, N. Dak.) were designated as level-2 sites and sites 140 (Square Butte Creek below Center, N. Dak.), 109 (Deepwater Creek near Raub, N. Dak.), 108 (East Fork Shell Creek near Parshall, N. Dak.), 106 (Bear Den Creek near Mandaree, N. Dak.), and site 101 (Little Muddy River below Cow Creek near Williston, N. Dak.) were designated as level-3 sites.
For the larger Missouri River tributaries, starting with the Cannonball River, sites 170 (Cannonball River at Breien, N. Dak.), 169 (Cedar Creek near Raleigh, N. Dak.), and 164 (Cannonball River near Raleigh, N. Dak.) were designated as level-1 sites. All of these sites are ambient network sites and adhere closely to the principle of bisecting flows. Upstream sites 166 (Cedar Creek near Haynes, N. Dak.) and 162 (Cannonball River at Regent, N. Dak.) were designated as level-2 sites. For the Heart River, ambient network sites 156 (Heart River near Mandan) and 149 (Heart River near Richardton, N. Dak.) were designated as level-1 sites, and sites 155 (Sweetbriar Creek near Judson, N. Dak.), 152 (Big Muddy Creek near Almont, N. Dak.), 151 (Antelope Creek near Carson, N. Dak.), 147 (Green River near New Hradec, N. Dak.), and 145 (Heart River near South Heart, N. Dak.) were designated as level-3 sites. Two HLSP sampling sites (sites 154 and 150, table 6) were not included in the proposed network because of redundancy with other sites. For the Knife River, sites 127 (Knife River at Hazen, N. Dak.) and 120 (Knife River near Golden Valley, N. Dak.), which are current (2010) ambient network sites, were designated as level-1 sites. Site 125 (Spring Creek at Zap, N. Dak.), a current (2010) ambient network site, was designated as a level-2 site and site 116 (Knife River at Manning, N. Dak.) was designated as a level-3 site. In addition to the previous sites, site 142 (Missouri River at Bismarck, N. Dak.) was designated as a level-2 site because it was the only site on the main stem Missouri River between Lake Sakakawea and Lake Oahe.

In the Little Missouri River Basin, site 114 (Little Missouri River near Watford City, N. Dak.) was designated as a level-1 site. Site 112 (Little Missouri River near Medora, N. Dak.), although similar in flow to site 114, is an ambient network site considered important for monitoring water quality for the undeveloped upper parts of the basin (Mike Ell, North Dakota Department of Health, oral commun., 2011) and thus was designated as a level-1 site. Remaining designated sites included a level-2 site (site 110, Little Missouri River near Marmarth, N. Dak.) and a level-3 site (site 113, Beaver Creek near Trotters, N. Dak.).

For the final Missouri River tributary, the James River, ambient network sites 183 (James River at Lamoure, N. Dak.), 182 (James River at Jamestown, N. Dak.), and 176 (James River near Grace City, N. Dak.) were designated at level-1 sites, site 180 (Pipestem Creek near Pingree, N. Dak.) a level-2 site, and site 184 (Bear Creek near Oakes, N. Dak.) a level-3 site. Two currently active (2010) sampling sites (sites 186 and 177, table 6) were not included in the potential network because of redundancy with other sites.

In all, the potential network consists of 81 sites, with 34 level-1 sites, 21 level-2 sites, and 26 level-3 sites (fig. 33). Given possible budget constraints of the sampling programs and considering the suggested temporal sampling frequencies described in the following section, this was considered to be the maximum potential number of sites for inclusion in the proposed network. 


\section{Sample Frequency for Detection of Seasonal Trends}

Given the spatial distribution and priority designation (levels $1-3$ ) of the sites in the potential network described in the previous section, the next consideration was to determine the appropriate temporal sampling frequency to use for monitoring changes in future water-quality conditions. The timeseries model used to detect concentration trends for this report also was used to evaluate sampling designs to monitor future water-quality trends. The interpretation of trends in terms of cause and effect can be difficult and, although the data indicated that trends have occurred, establishing a cause for the trends may require more extensive data gathering and model development; however, to evaluate the various sampling designs for this report, no interpretation of cause and effect was needed because the designs depended only on the statistical properties of the high-frequency variability in concentration (eq. 2), and the statistical properties of the high-frequency variability were independent of the trends.

To evaluate temporal sampling designs, the number of samples collected (on average) each year needed to be considered along with the sampling dates and whether the number of samples or sampling dates should be variable or fixed (the same year after year). Assuming that trends can occur at any time, in any direction, and for any duration, a reasonable approach for trend monitoring is to collect a fixed number of samples each year (Vecchia, 2005). The sampling dates also should remain relatively fixed; however, sampling on the exact same days each year is neither feasible nor necessary.

Given that the number of samples and the sampling dates remain fixed, two related concepts (sensitivity and efficiency) were used to evaluate sampling designs. Sensitivity measures the ability of a design to detect a trend - the smaller the trend that can be detected, the more sensitive the design. An efficient design maximizes the sensitivity to detect a trend for a fixed cost, which for this report was measured in terms of the number of samples per year. The only way to increase the sensitivity of an efficient design is to increase the cost - that is, to collect samples more frequently. For example, among all possible two-sample designs, the most efficient design might be to sample once during April and once during August, and among all possible three-sample designs, the most efficient design might be to sample once in April, once in August, and once in December. The best three-sample design is more sensitive than the best two-sample design but also is more costly. Therefore, to determine whether three samples are preferable to two samples, judgment is needed as to whether the increased sensitivity is worth the increased cost.

For the design analysis in this report, the assumptions were made that a streamgage would continue to be operated at each station (so that the future streamflow-related variability could be removed) and that concentration data would be collected no more than once each month. Concentration data potentially could be collected more often, but for trend analysis, sampling at most once in any given month generally is sufficient. For estimating loads (see the next section), more frequent sampling may be useful. The assumption also was made that the concentration data would be collected near the fifth day of the month; however, the actual sampling date could vary from 1 week before to 1 week after the fifth day without appreciably changing the results of the design analysis (Vecchia, 2005).

The sensitivity of a sampling design depends on the duration of the trend. If concentrations are increasing at a rate of 5 percent per year, the chance of detecting the trend after only 1 year may be extremely small; however, if the trend persists for 5 years, the chance of detecting the trend increases dramatically. In contrast to the sensitivity of a design, the efficiency of a design is not dependent on the duration of the trend. If a particular four-sample (per year) design is most efficient for detecting a trend that persists for 5 years, that design generally will be most efficient for detecting a trend that persists for 2 years or 10 years. Therefore, a 5-year duration was used as a benchmark to determine which designs in the design analysis were most efficient.

Even if a trend persists for 5 years or longer, sampling designs cannot detect trends with absolute certainty or prove that no trends exist with absolute certainty. Therefore, the size of the trend that can be detected depends on the acceptable level of tolerance for incorrectly identifying a trend. For this report, the acceptable level of tolerance was controlled by specifying two probabilities - the probability that a true trend was detected and the probability that a trend was detected when no true trend existed. Because the selection of an efficient design is not highly dependent on the values specified for the two probabilities, for this report, the first probability (also called the power for detecting a trend) was set equal to 0.8 and the second probability (also called the type-I error probability) was set equal to 0.1 . The designs then were compared in terms of their characteristic trends. The characteristic trend of a design, which was computed as described by Vecchia (2000), is interpreted as the increase or decrease in concentration during a 5-year period, in percent, that has an 80-percent chance of being detected using a type-I error probability of 0.1 .

Trends larger than the characteristic trend have more than an 80 -percent chance of being detected, and trends smaller than the characteristic trend have less than an 80-percent chance of being detected. The characteristic trend is used in this report as a benchmark to compare the sensitivity of the various sampling designs.

Sampling designs were evaluated with regard to their sensitivity to detect seasonal trends that occurred during three 4-month seasons - March through June (the high-flow period, referred to as the spring season in this report), July through October (the late summer/early fall low-flow period, referred to as the summer season in this report), and November through February (the late fall/winter low-flow period, referred to as the winter season in this report). Trends that can occur in certain constituents only during certain times of the year may not be detected in sampling programs designed on an annual basis. For example, many constituents can reach a stream from 
nonpoint sources when most of the snowmelt and rainfall runoff in North Dakota occurs, generally from March to June. Certain constituents may have substantial groundwater sources, therefore monitoring during low-flow conditions may be important for detecting trends. Many constituents may have a complex combination of point sources, groundwater sources, and nonpoint sources and may require monitoring during all flow conditions.

The design results for sulfate, TDS, nitrate plus nitrite, and total phosphorus at the sites examined in the Water Quality Trend Analysis section (table 5) were used to develop an efficient overall design. The most efficient design for one constituent may be an inefficient design for another constituent. Similarly, the most efficient design for one site may be an inefficient design for another site. Therefore, designs needed to be evaluated with regard to their overall ability to detect trends in various constituents and for various sites. The goal of the following discussion was to suggest designs that maintain reasonable efficiency and consistency for different sites and constituents. Implementing the single most efficient design for every individual constituent and site is neither logistically nor economically feasible.

The characteristic seasonal trends for sulfate and TDS for 10 selected sites are shown in figures 34 and 35 . The designs shown in this figure are a small subset of the many potential designs considered. Designs not included in the figures generally were less efficient than the designs shown. Designs 1 through 7 have samples selected during the spring season, designs 8 through 15 have samples selected in the summer season, and designs 16 through 22 have samples selected in the winter season. The simplest design considered was a single sample in each of the three periods. Based on discussions with NDDH and NDSWC personnel, a characteristic trend of 20 percent or less for sulfate and TDS was selected as a benchmark for having good sensitivity for detecting trends in those constituents.

For the spring season, the one-sample designs (designs 1 and 2) for most of the sites have characteristic trends greater than 20 percent and do not meet the sensitivity criteria (figs. 34 and 35); however, the two-sample designs (3 and 4) have characteristic trends less than 20 percent at most sites for both sulfate and TDS. Although samples collected in March and May (design 3) yield a slightly smaller characteristic trend at most sites, there is not much difference than if samples were collected in April and June (design 4). The only exception is the Wild Rice River near Abercrombie, N. Dak. (site 10), for which sampling in March and May provides substantially better sensitivity than sampling in April and June. Adding either one or two additional samples to the two-sample designs in the spring (designs 5-7) provides negligible increases in sensitivity of detecting trends for sulfate and TDS. Therefore, design 3 or 4 are efficient designs for detecting trends in the spring season.

For the summer season many of the sites had characteristic trends less than 20 percent with the one-sample designs for sulfate and TDS (designs 8 and 9; figs. 34 and 35); however, by increasing to two samples in the summer, the sensitivity of detecting a trend improved considerably. In particular, a twosample design with samples collected in August and October (design 11) is a particularly sensitive design for the detection of trends in sulfate and TDS in the summer season. For that design, characteristic trends were less than 20 percent for all of the sites and less than 10 percent for most of the sites. As was observed in the spring, additional samples in the summer (designs 12 through 15) provided a negligible increase in the sensitivity of detecting trends for sulfate and TDS over the best two-sample design.

During the winter season, a one-sample design would provide sufficient sensitivity to detect trends in sulfate and TDS (figs. 34 and 35); however, the month when the sample is collected seems to be important in meeting the 20 percent characteristic trend criterion. Overall, a sample collected in November (design 16) meets the criterion at all sites for TDS and at 9 out of 10 sites for sulfate. The characteristic trends increase substantially at many of the sites if the sample is collected in December (design 17), January (design 18), and February (design 19) particularly at the Little Missouri River near Watford City, N. Dak. (site 114); however, conditions in November generally consist of open-water and low-flow conditions that are similar to flows in late summer. Therefore, the November sample may not add much additional information if collected along with an October sample. If concentrations during winter conditions are of particular concern, a single sample in December (design 17) or January (design 18) may be preferable to the November sample.

Overall, the most efficient design for the detection of seasonal trends for sulfate and TDS appears to be one that includes five samples collected per year, including two in spring (either March and May or April and June), two in summer and early fall (August and October), and one in winter (December or January). For many of the sites, when two samples are collected in the spring and in the summer months, the sensitivity to detect seasonal trends is not strongly affected by which months are sampled; however, for the winter months, where a one-sample design was used, the month selected for sampling is important for sensitivity in detecting the seasonal trend in sulfate and TDS.

A greater variation in the characteristic seasonal trends was evident for nitrate plus nitrite and total phosphorus (figs. 36 and 37) compared to characteristic trends in sulfate and TDS (figs. 34 and 35). Because of the greater variation and the expense required to achieve the same level of sensitivity as major ions (20 percent), based on discussions with NDDH and NDSWC personnel, designs with characteristic trends less than 40 percent were considered to have acceptable sensitivity for detecting trends in nitrate plus nitrite and total phosphorus. The same 4-month periods that were considered for sulfate and TDS were used for the analysis of nitrate plus nitrite and total phosphorus designs.

For the spring season, the one-sample designs (designs 1 and 2) had poor sensitivity for detecting trends in nitrate plus nitrite and total phosphorus for most sites (figs. 36 and 37). 


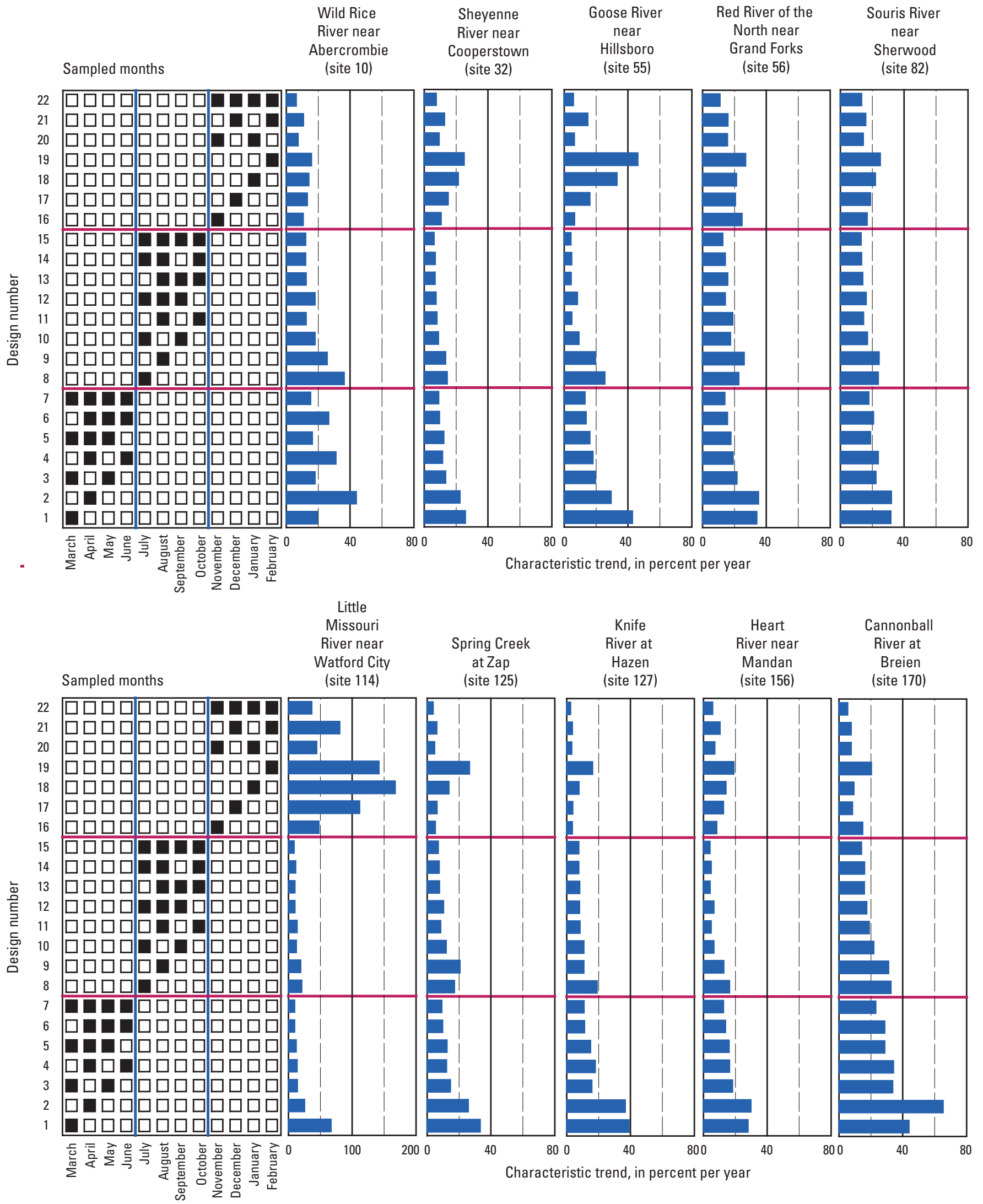

Figure 34. Characteristic seasonal trends for selected sampling designs for sulfate at selected sites in North Dakota. 


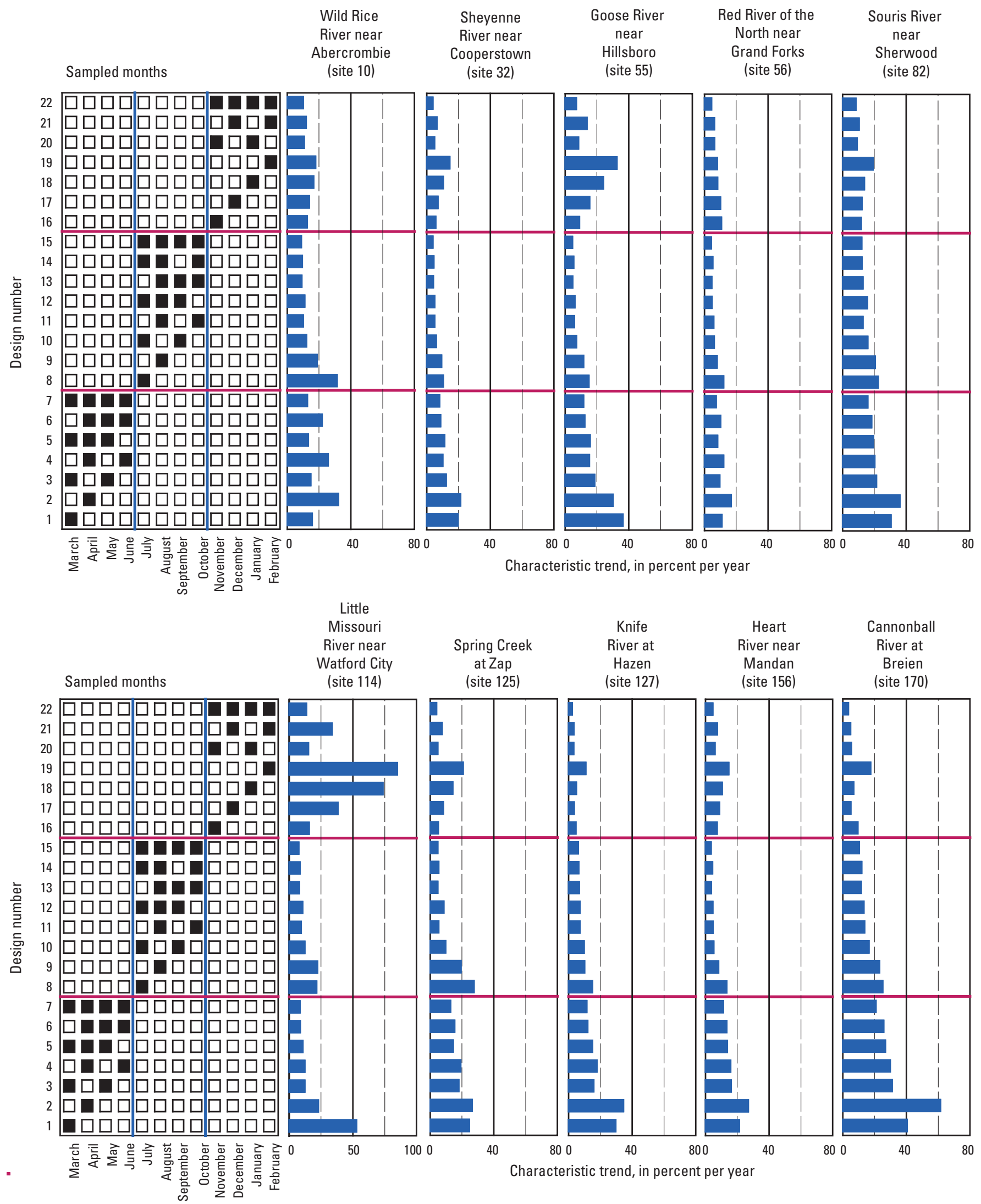

Figure 35. Characteristic seasonal trends for selected sampling designs for total dissolved solids at selected sites in North Dakota. 


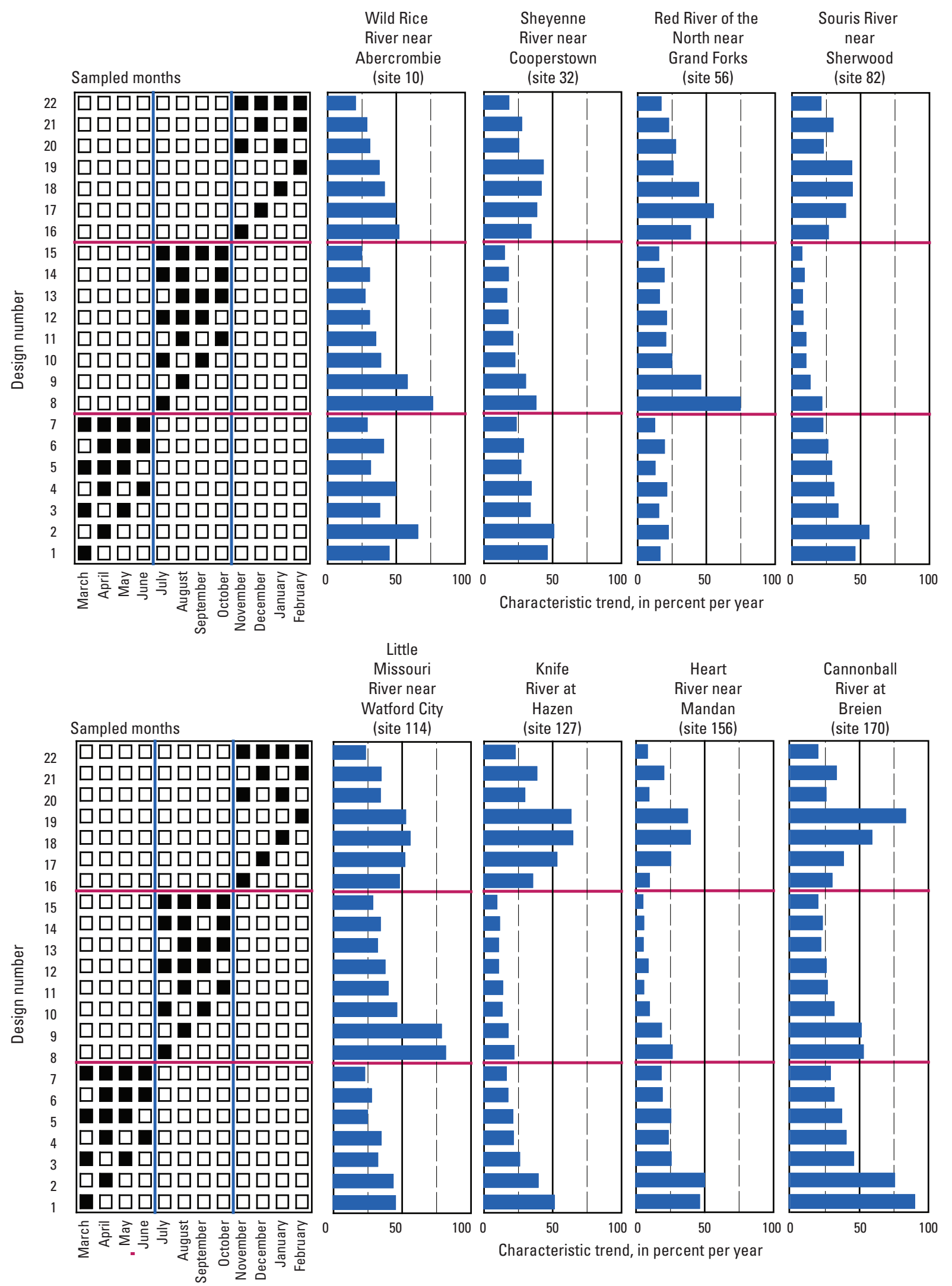

Figure 36. Characteristic seasonal trends for selected sampling designs for nitrate plus nitrite at selected sites in North Dakota. 
Sampled months

River near

Sheyenne

River near

Abercrombie

Cooperstown

(site 32)
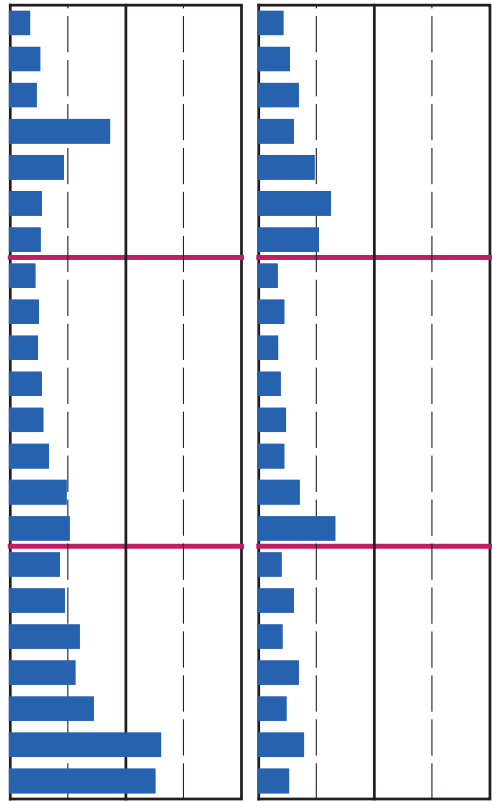

near
Hillsboro

North near

Grand Forks

near

Hillsboro

(site 56)

Sherwood

(site 82)
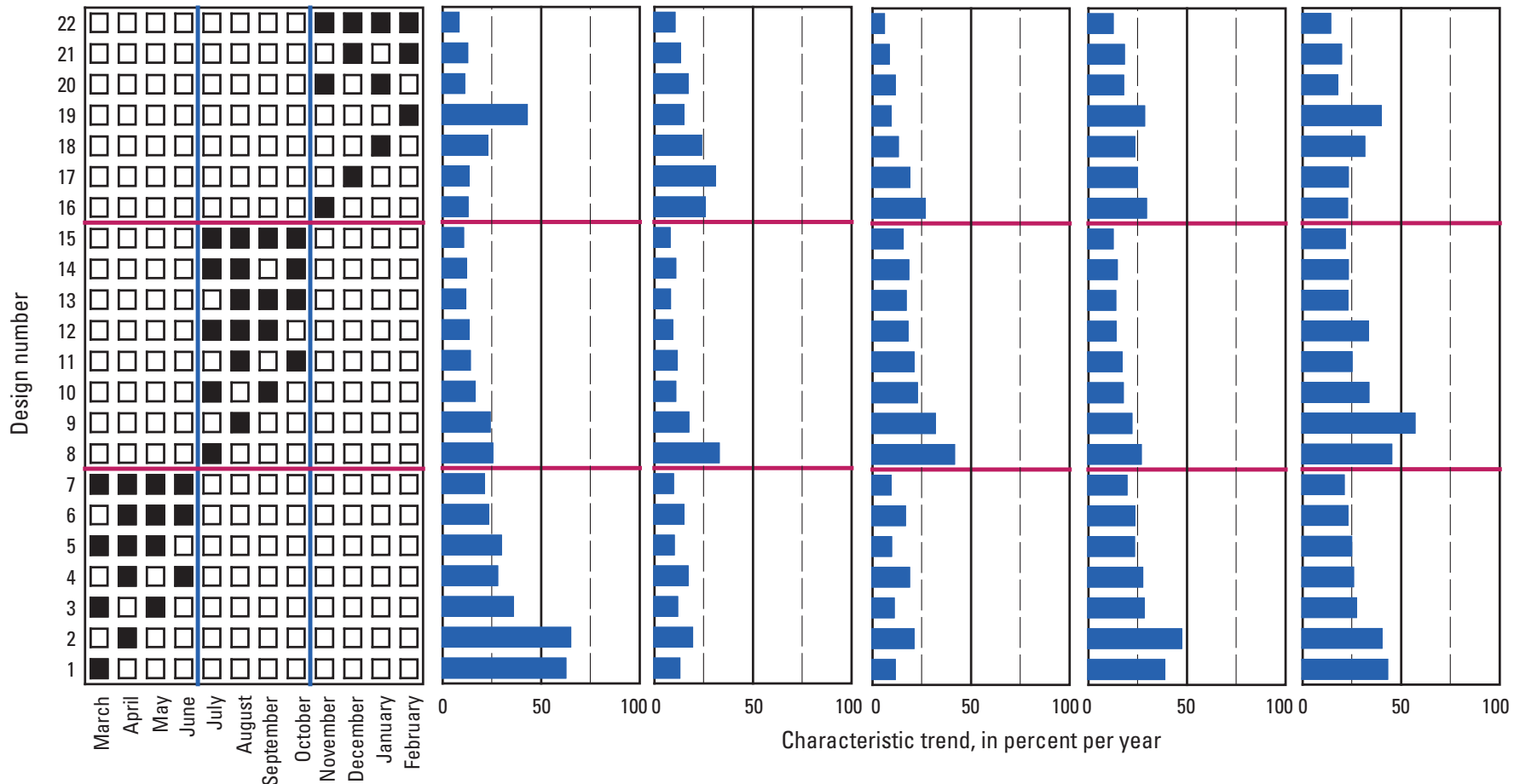

Characteristic trend, in percent per year

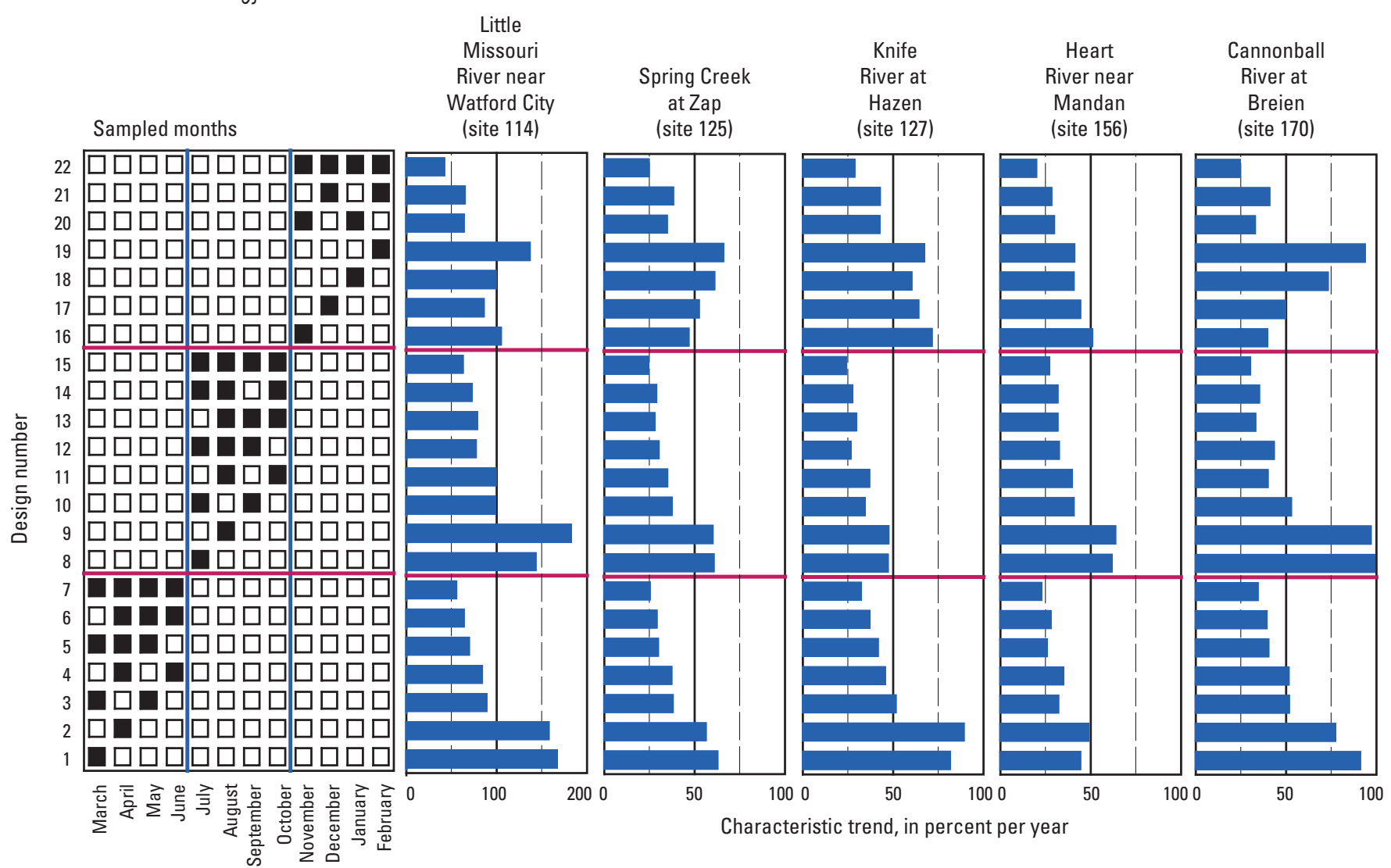

Figure 37. Characteristic seasonal trends for selected sampling designs for total phosphorus at selected sites in North Dakota. 
If an additional sample was added (designs 3 and 4), most of the sites had characteristic seasonal trends less than 40 percent for nitrate plus nitrite (fig. 36), but for total phosphorus many sites did not meet this criterion (fig. 37). Using a three-sample design (designs 5 and 6) would provide sensitivity to detect small trends that meet the criterion of a characteristic trend less than 40 percent for all sites except the Little Missouri River near Watford City, N. Dak. (site 114). The most efficient design for sampling nitrate plus nitrite and total phosphorus appears to be one where samples are collected in April, May and June (design 6), however, sample design 5, where samples are collected in March, April and May, also meets the criterion of a characteristic seasonal trend less than 40 percent for most of the tested sites.

For the summer season, similar to the spring season, the one-sample designs (designs 8 and 9) for most sites had characteristic seasonal trends greater than 40 percent and did not meet the criterion for detecting trends in nitrate plus nitrite and phosphorus (figs. 36 and 37). By adding an additional sample in the summer (designs 10 and 11), the characteristic seasonal trend is less than 40 percent at most sites. The twosample design, where samples are collected in August and October (design 11), meets the criterion for all sites for nitrate plus nitrite and for 9 out of 10 sites for total phosphorus. A three-sample design with samples in July, August, and October (design 14) provides substantial improvement in sensitivity to detect trends compared to design 11, especially for total phosphorus. Therefore, an efficient design for sampling nitrate plus nitrite and phosphorus in the summer season appears to be the three-sample design with samples in July, August, and October (design 14).

For the winter season, the one-sample designs (designs 16-19) provide poor sensitivity to detect trends in nitrate plus nitrite or total phosphorus for most sites (figs. 36 and 37). Although all of the sites had characteristic seasonal trends greater than 40 percent for all of the one-sample designs for total phosphorus, 6 out of 8 sites had characteristic seasonal trends less than 40 percent if the sample was collected in November for nitrate plus nitrite; however, as indicated previously, flow conditions in November are similar to October and thus a sample in November may not be necessary if a sample is collected in October. To reflect true winter conditions, if the November sample is excluded, design 21, with samples collected in December and February, has good sensitivity for detecting trends in nitrate plus nitrite for all of the sites and good sensitivity for detecting trends in phosphorus for 9 out of 10 sites.

Overall, the most efficient design for the detection of seasonal trends for nitrate plus nitrite and total phosphorus appears to be one that includes eight samples collected per year in the months of April, May, June, July, August, October, December, and February. Although reducing winter sampling to a single sample in December or January would result in considerable loss of sensitivity for detecting trends in that season, collecting two samples in winter may not be feasible because of safety concerns. Therefore, the best feasible design consists of seven samples per year (April, May, June, July, August, October, and December or January).

When merging the results for nitrate plus nitrite and phosphorus with the previous results for sulfate and TDS, an efficient overall design for detecting seasonal trends consists of seven samples per year, with samples in early April, May, June, July, August, October, and January. This design consists of a combination of designs 6,14 , and 18 . Maintaining a seven-sample design at all 81 sites in the spatial network (fig. 33) would result in 567 samples per year for both major ions (such as sulfate) and nutrients (such as nitrate plus nitrite and total phosphorus), compared to about 460 samples per year (for major ions) and 300 samples per year (for nutrients) currently (2010) being collected as part of the NDDH ambient network, HLSP, and other sampling programs in the Souris, James, and Red River of the North Basins. Therefore, to control sampling costs and allow extra samples for load estimation (see the following section), the sampling design for the level-3 sites were reduced from 7 to 4 samples per year. The design for the level-3 sites therefore consists of four samples per year, with samples in April, June, August, and October. This design should maintain reasonable power for detecting trends in the spring and summer seasons, and would provide considerable improvement compared to the two-sample HLSP design. Many of the level-3 sites have seasonally operated (March-October) streamgages because of negligible flows in winter, so excluding winter sampling was considered prudent for these sites. For level-2 sites, the sampling design was reduced from 7 to 6 samples per year, with samples in April, May, June, August, October, and January. Maintaining the seven-sample design for the 34 level-1 sites, the six-sample design for the 21 level-2 sites, and the four-sample design for the 26 level-3 sites would result in 468 major ion and nutrient samples per year for monitoring trends statewide.

The current (2010) sampling design for the NDDH ambient network includes eight samples collected in April, May (two samples), June, July, August, October, and January. Excluding the extra sample in May, the design would be equivalent to a combination of designs 6,14 , and 18 (figs. 34-37), which is the recommended seven-sample trend design for level-1 sites. Thus, the ambient network design has good sensitivity for detecting trends in all seasons except possibly winter (for certain sites). Although reducing sampling at these sites from 8 to 7 or perhaps even fewer samples per year would maintain nearly equivalent power for detecting trends, as indicated in the next section, maintaining at least 8 samples per year is necessary for load estimation. The NDSWC HLSP sampling design consists essentially of a combination of designs 2 and 9 . That combination has moderate sensitivity for detecting trends in sulfate and TDS for spring and summer but poor sensitivity for detecting trends in nitrate plus nitrite and phosphorus in all seasons. Therefore, increasing from 2 to 4 or 6 samples per year would provide much better sensitivity for detecting trends in nutrient concentrations. 


\section{Sample Frequency for Estimation of Constituent Loads}

One goal of the NDDH ambient network is to provide accurate estimates of loads over 5-year to 10-year periods. Unlike sample designs for detecting trends that are targeting changes in flow-adjusted concentration through time, sample designs for the estimation of constituent loads should focus on the main contributing factor that can affect the annual load - changes in concentration with streamflow. Loads are estimated by performing a regression analysis of concentration (or load) with streamflow and sometimes time and season, as discussed previously in the Load and Yield Estimation section. In general, the greatest streamflow will yield the greatest load, therefore it is important to design a sampling strategy that would target high-flow conditions such as snowmelt runoff and rainfall runoff events. Although it is important to define the constituent concentration at the full range of streamflow at a site, if the high streamflow conditions are not adequately included in the sampling, the estimated annual loads may be lower than what is actually occurring at the site.

Gilroy and others (1990) analyzed the relative efficiency of minimum variance unbiased estimates (MVUE) of loads for the simple regression model

$$
\ln (L)=\beta_{0}+\beta_{1} \ln \left(Q_{d}\right)+E
$$

where the terms in equation 5 are defined in the more detailed load model shown in equation 1 . Although this model does not include the trend and seasonality terms in equation 3 , uncertainty in load estimates generally is dominated by the flow term and thus the efficiency principles derived using equation 5 should be applicable to the more complicated model (eq. 1). Using elegant statistical theory, Gilroy and others (1990) developed a close approximation to the root-meansquared error of the estimated total load for an N-day period,

$$
R M S E=100\left(E V\left\{<L_{N}>-L_{N}\right\}^{2}\right)^{1 / 2} / L_{N}
$$

where

$$
\begin{gathered}
R M S E \quad \begin{array}{c}
\text { is the root-mean-squared error of the load } \\
\text { estimate as a percentage of the true load; }
\end{array} \\
E V \quad \text { denotes expected value; } \\
\mathrm{L}_{N} \quad \begin{array}{c}
\text { is the sum of the true daily loads in pounds } \\
\text { per day for an N-day period; and }
\end{array} \\
L_{N}>\quad \begin{array}{l}
\text { is the MVUE of } \mathrm{L}_{\mathrm{N}} \text { based on a calibration } \\
\text { data set with } \mathrm{M} \text { observations (for example, } \\
\text { the estimate obtained using S-LOADEST); }
\end{array}
\end{gathered}
$$

Assuming $\mathrm{N}$ is large (for example, for a 5-year period, $\mathrm{N}$ would be 1,826 days), the size of the calibration data set (the number of concentration samples) used to fit the model (eq. 5) is small in relation to $\mathrm{N}$, daily discharges are log-normally distributed, and the errors in equation 5 are independent and identically distributed normal random variables, Gilroy and others (1990) show that RMSE depends on two quantities,

$$
D^{*}=\left(A V E_{\mathrm{C}}\{\ln Q\}-A V E_{N}\{\ln Q\}\right) / S D_{N}\{\ln Q\}
$$

and

$$
R^{*}=S D_{C}\{\ln Q\} / S D_{N}\{\ln Q\}
$$

where

$D^{*} \quad$ is the difference between the average of the log-transformed daily flows for the calibration dataset $\left(A V E_{C}\{\ln Q\}\right)$ and the average of the log-transformed daily flows for the full dataset $\left(A V E_{N}\{\ln Q\}\right)$, divided by the standard deviation of logtransformed daily flows for the full dataset $\left(S D_{N}\{\ln Q\}\right)$; and

$R^{*} \quad$ is the ratio of the standard deviations of the log-transformed daily flows for the calibration dataset $\left(S D_{C}\{\ln Q\}\right)$ and the full dataset.

Gilroy and others (1990) show that an efficient design (one with small RMSE for a fixed number of samples) should have $R^{*}$ greater than 1 and $D^{*}$ close to $\beta_{1}$. Designs with $R^{*}$ less than 1 and/or $\operatorname{abs}\left(D^{*}-\beta_{1}\right)$ greater than 1 generally are highly inefficient. Because each site/constituent combination can have a different slope $\left(\beta_{1}\right)$, the most efficient design for load estimation, like the most efficient design for trend detection described in the previous section, depends on the site/ constituent combination. For example, for the extreme case of a constant point source $\left(\beta_{1}=0\right)$ the loads do not depend on flow and the best design would have $A V E_{C}\{\ln Q\}=A V E_{N}\{\ln Q\}$, so flows for the calibration data should have a similar distribution to flows for the full dataset. If concentration is constant with respect to flow (the slope of the $\ln (C)$ to $\ln Q$ relation is zero), then $\beta_{1}=1$ and the best design would have $A V E_{C}\{\ln Q\}$ $\left.=A V E_{N}\{\ln Q\}\right)+S D_{N}\{\ln Q\}$ and thus the calibration dataset should be weighted toward higher flows. The higher the slope, the more weight should be given to higher flows in the calibration dataset. Using the slopes from table 3 as a guide, the median slope was 0.89 (range of 0.67 to 1.17 ) for dissolved sulfate and TDS; 0.96 (range of 0.42 to 1.57 ) for ammonia; 1.33 ( 0.97 to 1.78 ) for nitrite plus nitrate; 1.18 (0.63 to 2.02) for total and dissolved phosphorus; 1.05 (0.92 to 1.28) for total organic carbon; and 1.26 (0.94 to 2.01) for suspended sediment. Overall, $D^{*}=1$ (covering a range of $\beta_{1}$ from 0 to 2 , with especially good efficiency for $\beta_{1}$ between 0.5 and 1.5 ) appears be a good target to maintain high efficiency for all of the sites and constituents. A value of $D^{*}=0.5$ (covering a range from -0.5 to 1.5 with especially good efficiency for a range of 0 to 1 ) would be relatively efficient for many cases but may be inefficient for nutrients and suspended sediment for some sites.

As an experiment to evaluate various designs for load estimation for 5-year time periods, daily flow data for 5 sites were used to compute $D^{*}$ and $R^{*}$ for moving 5-year windows centered on 1973 (1971-75) through 2005 (2003-07). In this analysis, flows from April 1 to October 31 of each year were 
used to avoid ice-cover conditions. The values of $D^{*}$ for the five sites and various designs are shown in figure 38 and the values of $R^{*}$ are shown in figure 39. Although the sites are scattered throughout North Dakota and represent a range of basin sizes (table 6), the overall results and conclusions were similar. For the level-3 design (samples in April, June, August, and October; fig. $38 A$ ), $D^{*}$ was less than 0.2 for most site/ time period combinations and less than 0.0 for many combinations, so that design is highly inefficient for load estimation. For the recommended seven-sample trend design for level-1 sites (samples in April, May, June, July, August, and October, excluding January; fig. 38B), $D^{*}$ increased by about $0.2 \mathrm{com}$ pared to the level-3 design for most site/time period combinations, a big improvement over level-3 but still well below the target of $D^{*}=1$. The current (2010) ambient network design consists of the level-1 trend design plus an extra sample in May (fig. 38C). Although this design provides some increases in $D^{*}$ compared to the design in figure $38 \mathrm{~B}$, the extra sample in May provides only small improvement in efficiency. Adding an extra sample in April rather than May (fig. 38D) results in much better overall efficiency, with $D^{*}$ between 0.2 and 0.4 for most site/time period combinations. Thus, moving the sample in mid- to late-May that is in the current (2010) ambient network design to mid-April would be a more efficient design for load estimation. Adding a third or fourth sample in April (figs. $38 E$ and $38 F$ ) provides still more improvement in efficiency. The design shown in figure $38 F$, with four samples in April (weekly beginning April 5) and one sample each in May, June, July, August, and October, for a total of nine samples during April-October, has $D^{*}$ greater than 0.5 for many of the site/time period combinations. Reaching the optimal target of $D^{*}=1$ is not feasible without a much higher sampling frequency.

The other consideration ( $R^{*}$ greater than 1$)$ can be evaluated using figure 39. It is apparent that the design with two samples in April (fig. 39D) has $\mathrm{R}^{*}$ greater than 1 much more frequently than the design with two samples in May (fig. 39C), providing additional evidence to place the extra sample in April instead of May. Designs with 3 or 4 samples in April (figs. $39 E$ and $39 F$ ) also have $R^{*}$ greater than 1 for most site/ time period combinations.

Given the previous results, augmenting the level-1 design for trends by adding an extra sample in April would provide a good alternative for improving load estimates. Adding as many as two more samples in April (weekly) would provide still more improvement for load estimation but at considerably more cost. It may be prudent to select a few of the ambient network sites as test sites where more frequent April samples would be collected for a few years to see if the improvement in load estimates justify the added cost of the extra samples.

\section{Overview of Potential Network}

The potential State-wide sampling network for efficiently accomplishing the objectives of the sampling programs is summarized in table 7 and figure 33 . Although only major ions and nutrients were used to determine the spatial distribution of sites and temporal sampling frequencies for this network, analysis of samples for trace-metal constituents for all sites and bacteria and sediment for the level-1 sites were included in the potential network because they are part of the current (2010) sampling networks and should be continued for monitoring water-quality conditions. The historical data for trace metals, bacteria, and sediment were not sufficient to do a rigorous analysis of network efficiency similar to what was done for major ions and nutrients. Therefore, the same design was assumed for trace metals for all sites. It was also assumed that bacteria and sediment would be sampled along with the other constituents at the level-1 sites.

For the 34 level-1 sites, samples would be collected for major ions, trace metals, nutrients, bacteria, and sediment eight times per year, with samples in January, April (two samples), May, June, July, August, and October (table 7). Thus, there would be a total of 272 samples collected per year for each of the constituents. These level-1 sites would essentially be a continuation of the $2010 \mathrm{NDDH}$ ambient network (34 sites) and the Souris River International Joint Board monitoring program at the Souris River near Sherwood, N. Dak. (site 82) and Westhope, N. Dak. (site 98). In 2010, there were a total of 324 samples collected at these sites. The smaller number of samples in the potential design compared to the 2010 sampling program result from elimination of replication between NDDH and USGS sampling programs at two sites in the Souris Basin (sites 85 and 87, fig. 33). NDDH currently collects samples eight times per year and USGS collects samples seven times per year at each of these two sites for the same constituents and thus eliminating the extra USGS sampling would result in 14 fewer samples.

For the 21 level-2 sites, samples would be collected for major ions, trace metals, and nutrients six times per year (January, April, May, June, August, and October) and for the 26 level-3 sites samples would be collected for these constituents four times per year (April, June, August, and October) (table 7). Thus, there would be a total of 230 major ion, trace metal, and nutrient samples collected each year at 47 level-2 and level-3 sites. These 47 sites would replace the existing HLSP sites, for which, in 2010, there were 166 samples collected at 83 sites for major ions and trace metals. Although there are 36 fewer sites in the proposed network, in 2010, 21 of these 36 sites were sampled as both HLSP and NDDH ambient network sites (fig. 1). Thus, most of the 2010 HLSP sites would continue to be sampled as part of the potential network and only 15 of the 2010 HLSP sites would not be included in the potential network. These 15 sites (sites $2,25,30,38,40,41,42,44,74,83,89,141,150,154$, and 186; fig. 1) generally are either in close proximity to sites in the potential network or on very small drainages. Although 


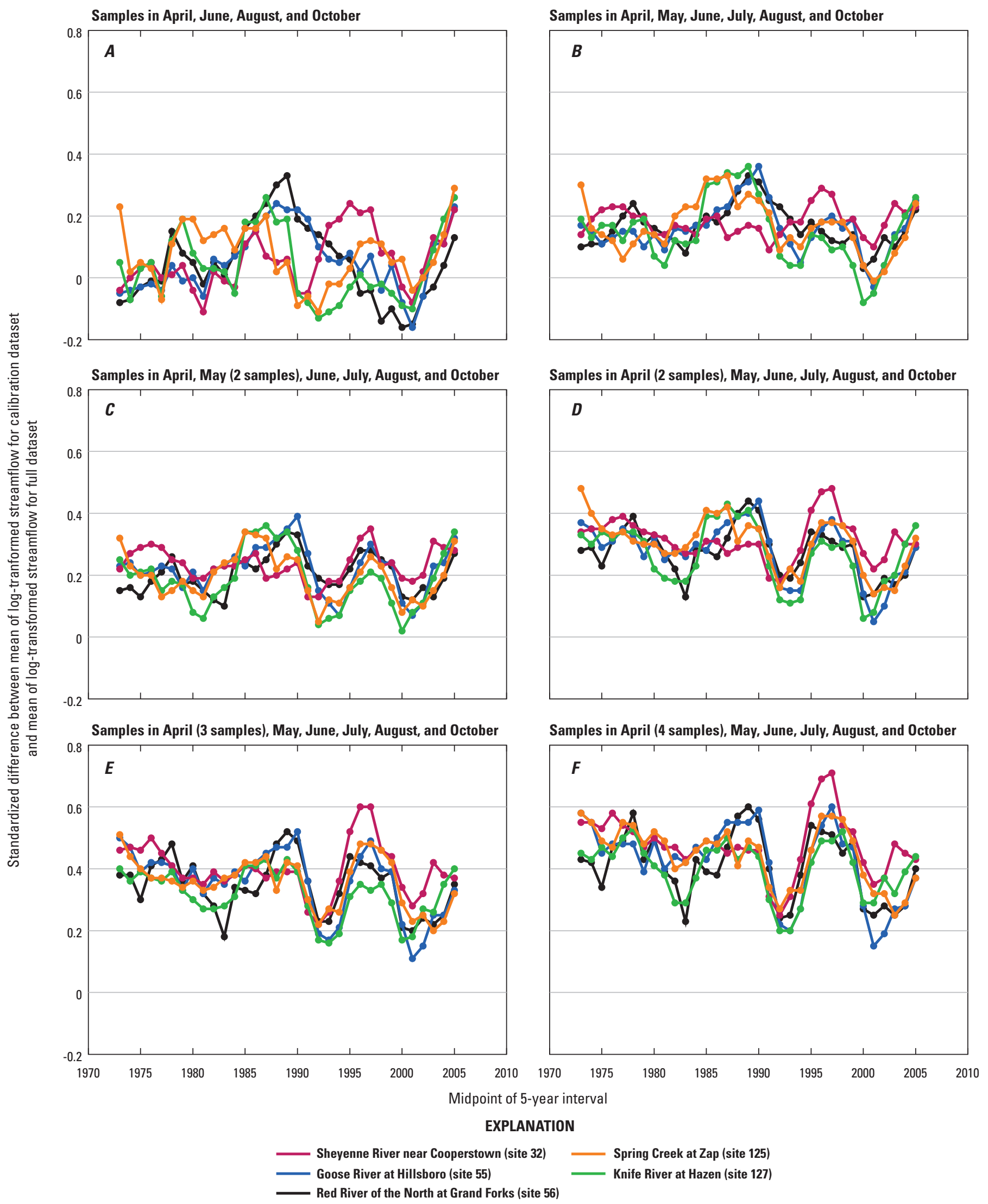

Figure 38. Efficiency of sample designs for load estimation in terms of standardized difference between the average of log-transformed flows for the calibration dataset and log-transformed flows for the full dataset for 5-year moving windows. 


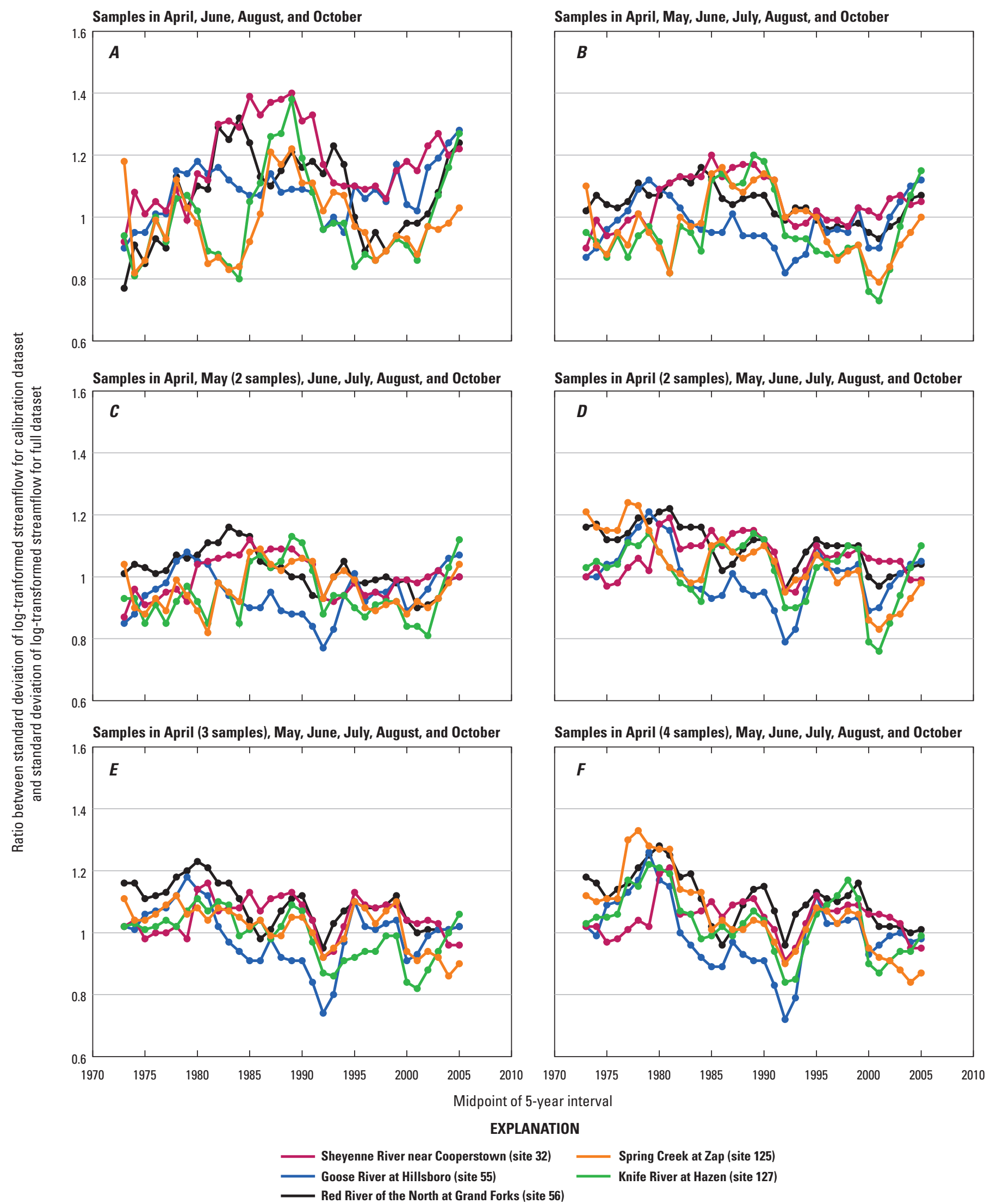

Figure 39. Efficiency of sample designs for load estimation in terms of ratio between the standard deviation of log-transformed flows for the calibration dataset and the standard deviation of log-transformed flows for the full dataset for 5-year moving windows. 
Table 7. Summary of potential state-wide water-quality sampling network for North Dakota.

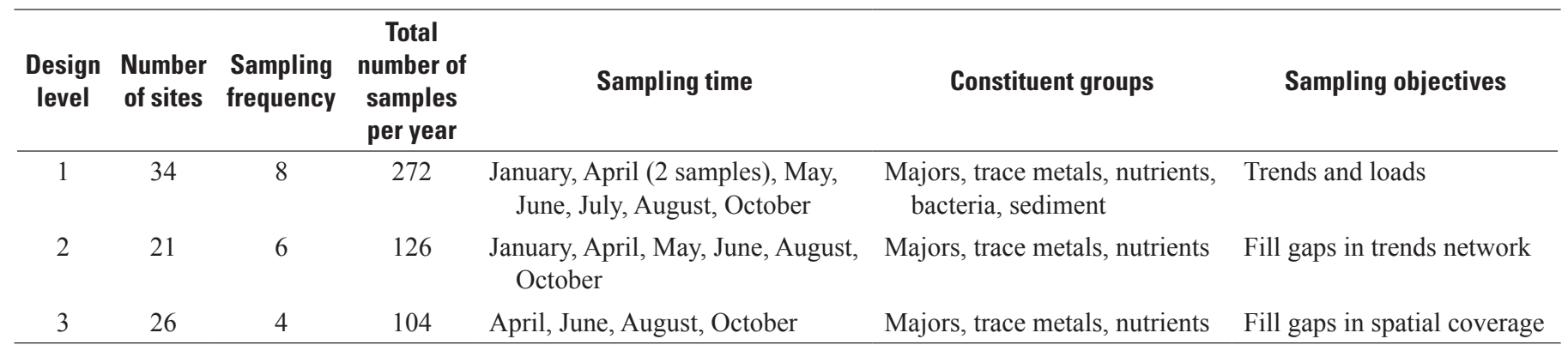

maintaining the level-2 and level-3 network at the suggested sampling frequencies would require additional resources compared to the current HLSP costs, there would be substantial benefits in terms of higher sensitivity to evaluate changes in water quality. Reducing costs from the potential level-2 and level-3 network could be achieved by lowering sampling frequencies (and losing power to detect trends) or eliminating sampling sites (and losing spatial coverage in some areas). Although nutrients are not sampled as part of the 2010 HLSP, including nutrient samples would provide substantial benefits for monitoring water-quality with negligible increase in sampling costs; however, laboratory costs would be higher as a result of adding nutrient analyses.

\section{Summary}

Although data collected for the North Dakota State Water Commission High-Low Sampling Program, the North Dakota Department of Health Ambient Water-Quality Network, and other projects and programs provide valuable information on the quality of water in streams of North Dakota, the objectives vary among the programs, some of the programs overlap spatially and temporally, and the various sampling designs may not be the most efficient or relevant to the objectives of the individual programs as they have changed through time. In response to the need to examine the large amount of historic water-quality data comprehensively across North Dakota and evaluate the efficiency of the State-wide sampling program, a study was conducted by the U.S. Geological Survey in cooperation with the North Dakota State Water Commission and the North Dakota Department of Health to describe the waterquality data collected by the various programs and determine an efficient State-wide sampling design for monitoring future water-quality conditions.

Median concentrations of water-quality constituents at sampling locations in North Dakota were determined for the period of record and for the spring (March through June), summer (July through October), and winter (November through February) seasons at each sampling location. In general, median sulfate concentrations tended to be higher in the southwest part of the State compared to the northeast part of the State in the Red River of the North Basin. Seasonal variations indicated lower median concentrations in samples collected during spring and higher concentrations from samples collected during winter at many locations. Median chloride concentrations tended to be lower in the southwest and higher in the northeast parts of the State, nearly the opposite of the pattern seen in median sulfate concentrations. Substantial differences in median chloride concentrations were not evident among samples collected during different seasons. In general, median total dissolved solid concentrations tended to be lower for sites in the Red River of the North, Souris, and James River Basins compared to sites in southwest North Dakota. Like sulfate, median total dissolved solid concentrations at many locations tended to be lower in samples collected during spring compared to samples collected during the summer and winter.

In general, overall median ammonia concentrations (total and dissolved) were spatially variable across the State. Seasonally, median ammonia concentrations generally were highest during winter and lowest in summer. The highest overall median nitrate plus nitrite concentrations (total and dissolved) tended to occur in the Red River of the North Basin. This pattern of high concentrations in the Red River of the North Basin was particularly evident during the spring season, and may reflect fertilizer application to row crops and other cropland in that area. This pattern was reversed during the winter season, with the lowest median concentration occurring in the Red River of the North Basin. Median dissolved and total phosphorus concentrations across North Dakota generally were greater for the Red, Souris, and James River Basins than river basins in the southwest part of the State. Median dissolved phosphorus concentrations tended to be similar among the seasons, whereas median total phosphorus concentrations tended to be higher in the summer than other seasons, particularly in the Souris, Devils Lake, and James River Basins. Median dissolved and total organic carbon concentrations tended to be the lowest in samples collected at sites located in the eastern part of the State compared with other median concentrations at sites throughout the remainder of the State.

Median total aluminum concentrations generally were higher in samples from sites in the southern and eastern parts of the State compared to concentrations in other parts of the State. Seasonal variations indicated higher median total aluminum concentrations during spring and summer and lower 
concentrations during winter at many locations. Median total arsenic concentrations in North Dakota indicate that the lowest values occurred mostly in the southwestern parts of the State, whereas the highest values occurred mostly in the eastern parts of the State. Seasonal variations indicated higher median total arsenic concentrations in samples collected during summer and lower concentrations from samples collected during winter at many locations. Median total chromium concentrations in water samples collected across North Dakota generally were higher in the far eastern and western areas of the State, and seasonal variations indicated higher median total chromium concentrations in samples collected during spring and lower concentrations from samples collected during winter at many locations. Median total iron concentrations in North Dakota were lowest in the Souris, Sheyenne, and James River Basins compared to sites in other parts of North Dakota. The highest median total lead concentrations in water samples were scattered across North Dakota. Seasonal variations indicated higher median total lead concentrations during spring and lower concentrations during winter at many locations, particularly in the Red River of the North Basin. Median total nickel concentrations were higher at a few sites in the southwest and eastern parts of North Dakota compared to other sites. Seasonal variations indicated higher median total nickel concentrations in samples collected during spring and lower concentrations from samples collected during winter at many locations. Median suspended-sediment concentrations and total suspended solids in water samples obtained across North Dakota were higher at sites located in parts of southwestern and eastern North Dakota compared to sites located in other areas of the State.

Normalized annual loads and yields were estimated for sulfate, total dissolved solids, nitrogen, phosphorus, total organic carbon, and suspended sediment at 34 sites across North Dakota. Normalized annual sulfate yields ranged from 9,250 to 56,200 pounds per year per square mile in the Missouri River Basin and from 4,680 to 72,500 pounds per year per square mile in the Red River of the North Basin. Total dissolved solids yields ranged from 30,400 to 119,000 pounds per year per square mile in the Missouri River Basin and from 13,000 to 169,000 pounds per year per square mile in the Red River of the North Basin. Nitrate plus nitrite yields ranged from 24 to 120 pounds per year per square mile as nitrogen in the Missouri River Basin and from 2 to 1,260 pounds per year per square mile as nitrogen in the Red River of the North Basin. Ammonia yields ranged from 6 to 22 pounds per year per square mile as nitrogen in the Missouri River Basin and from 4 to 62 pounds per year per square mile as nitrogen in the Red River of the North Basin. Normalized annual dissolved phosphorus yields ranged from less than 1 to 35 pounds per year per square mile as phosphorus in the Missouri River Basin and from 5 to 47 pounds per year per square mile as phosphorus in the Red River of the North Basin. Normalized annual total phosphorus yields ranged from less than 1 to 167 pounds per year per square mile as phosphorus in the Missouri River Basin and from 6 to 76 pounds per year per square mile as phosphorus in the Red River of the North Basin. Normalized annual total organic carbon yields ranged from 426 to 2,670 pounds per year per square mile as carbon in the Missouri River Basin and from 394 to 11,100 pounds per year per square mile as carbon in the Red River of the North Basin. Suspended-sediment yields ranged from 519 to 622,000 pounds per year per square mile in the Missouri River Basin and from 1,500 to 78,800 pounds per year per square mile in the Red River of the North Basin.

Ten sites were selected for trend analysis for sulfate, total dissolved solids, nitrate plus nitrite, and total phosphorus. The fitted trends for standardized sulfate concentrations indicated significant increases in concentration at five sites and a significant decrease in concentration at one site from 1975 through 2008. Median standardized sulfate concentrations increased from about 55 to 106 milligrams per liter for the Red River of the North at Grand Forks, North Dakota; 262 to 308 milligrams per liter for the Souris River near Sherwood, North Dakota; 455 to 506 milligrams per liter for Spring Creek at Zap, North Dakota; 440 to 520 milligrams per liter for the Knife River at Hazen, North Dakota; and 380 to 435 milligrams per liter for the Heart River near Mandan, North Dakota. The fitted trend for the Little Missouri River near Watford City, North Dakota showed a decrease in median standardized concentration from about 492 to 417 milligrams per liter. The fitted trends for standardized total dissolved solid concentrations indicated significant increases in concentration at five sites from 1975 through 2008, including the Heart River near Mandan, North Dakota, Knife River at Hazen, North Dakota, Spring Creek at Zap, North Dakota, and the Red River of the North at Grand Forks, North Dakota. The greatest increase in median standardized total dissolved solid concentrations was detected for the Wild Rice River near Abercrombie, North Dakota, where median concentrations increased from about 690 to 1,120 milligrams per liter from 1975 through 2008.

The fitted trends for standardized nitrate plus nitrite concentrations indicated no significant increases or decreases in concentration for 7 of the 8 sites analyzed for trends; however, median standardized nitrate plus nitrite concentration for the Red River of the North near Grand Forks, North Dakota increased from about 0.26 to 0.45 milligrams per liter as nitrogen during 1990 to 2008. The fitted trends for standardized total phosphorus concentrations indicated significant decreases in concentration at 4 sites and a significant increase at 1 out of the 10 sites analyzed for trends. All of the significant decreases were for sites in western North Dakota, including decreases in median standardized total phosphorus concentration for the Cannonball River at Breien, North Dakota, Heart River near Mandan, North Dakota, Knife River at Hazen, North Dakota, and Spring Creek at Zap, North Dakota. Conversely, median standardized total phosphorus concentration for the Red River of the North at Grand Forks, North Dakota increased from about 0.10 to 0.16 milligrams per liter from 1990 through 2008. 
One objective of a proposed State-wide sampling program was to describe the spatial variability of water-quality conditions across the State in the most efficient manner. To evaluate the spatial redundancy in nested (upstream/downstream) samples, two constituents - sulfate and phosphorus were selected. Weighted least-squares regression analysis was used to relate the average absolute difference between paired downstream and upstream concentrations, expressed as a percent of the average downstream concentration, to the average absolute difference in daily flow between the downstream and upstream pairs, expressed as a percent of the average downstream flow. The analysis showed that a reasonable spatial network would consist of including the most downstream sites in large basins first, followed by the next upstream site(s) that roughly bisect the downstream flows at the first sites, followed by the next upstream site(s) that roughly bisect flows for the second sites. Sampling sites to be included in a potential Statewide network were prioritized into 3 design levels: level 1 (highest priority), level 2 (second priority), and level 3 (third priority). In all, the potential network consists of 81 sites, with 34 level-1 sites, 21 level-2 sites, and 26 level-3 sites.

Given the spatial distribution and priority designation (levels 1-3) of sites in the potential spatial network, the next consideration was to determine the appropriate temporal sampling frequency to use for monitoring future water-quality conditions. The time-series model used to detect concentration trends for this report also was used to evaluate sampling designs to monitor future water-quality trends. Sampling designs were evaluated with regard to their sensitivity to detect seasonal trends that occurred during three 4-month seasons-March through June, July through October, and November through February. The most efficient design for one constituent may be an inefficient design for another constituent. Therefore, the design results for sulfate, total dissolved solids, nitrate plus nitrite, and total phosphorus were analyzed at the sites examined for trends to develop an efficient overall design. Unlike sample designs for detecting trends that are targeting flow-adjusted concentration through time, sample designs for the estimation of constituent loads should focus on the main contributing factor that can affect the annual load, changes in concentration with streamflow.

For the 34 level-1 sites, samples would be collected for major ions, trace metals, nutrients, bacteria, and sediment eight times per year, with samples in January, April (2 samples), May, June, July, August, and October. The current (2010) ambient network design consists of the level-1 trend design with an additional sample in May instead of April. Moving the sample in mid- to late-May that is in the current (2010) ambient network design to mid-April would be a more efficient design for load estimation. For the 21 level-2 sites, samples would be collected for major ions, trace metals, and nutrients six times per year (January, April, May, June, August, and October) and for the 26 level-3 sites, samples would be collected for these constituents four times per year (April,
June, August, and October). Although maintaining the level-2 and level-3 network at the suggested sampling frequencies would require additional resources compared to the current High-Low Flow Sampling Program costs, there would be substantial benefits in terms of higher sensitivity to evaluate changes in water quality.

\section{References Cited}

Allan, J.D., 1995, Stream ecology - structure and function of running waters: London, Chapman and Hall, 388 p.

Childress, C.J., Foreman, W.T., Connor, B.F., and Maloney, T.J, 1999, New reporting procedures based on long-term method detection levels and some considerations for interpretations of water-quality data provided by the U.S. Geological Survey National Water Quality Laboratory: U.S. Geological Survey Open-File Report 99-193, 19 p.

Cohn, T.A., 1995, Recent advances in statistical methods for the estimation of sediment and nutrient transport in rivers, U.S. National Report to the International Union Geodesy and Geophysics, 1991-1994: Reviews of Geophysics, Supplement 33, p. 1,117-1,123.

Cohn, T.A., Cauldes, D.L., Gilroy, E.J., Zynjuk, L.D., and Summers, R.M., 1992, The validity of a simple long-linear model for estimating fluvial constituent loads entering Chesapeake Bay: Water Resources Research, v. 28, no. 9, p. 2,353-2,363.

Cohn, T.A., DeLong, L.L., Gilroy, E.J., Hirsch, R.M., and Wells, D.K., 1989, Estimating constituent loads: Water Resources Research, v. 25, no. 5, p. 237-942.

Gilroy, E.J., Hirsch, R.M., and Cohn, T.A., 1990, Mean square error of regression-based constituent transport estimates: Water Resources Research, v. 26, no. 9, p. 2,069-2,077

Gray, J.R., Glysson, G.D., Turcios, L.M., and Schwarz, G.E., 2000, Comparability of suspended-sediment concentration and total suspended solids data: U.S. Geological WaterResources Investigations Report 00-4191, 14 p.

Guy, H.P., 1970, Fluvial sediment concepts: U.S. Geological Survey Techniques of Water-Resources Investigations, book 3 , chap. $\mathrm{C} 1,55 \mathrm{p}$.

Hirsch, R.M., Slack, J.R., and Smith, R.A., 1982, Techniques of trend analysis for monthly water-quality data: Water Resources Research, v. 18, no. 1, p. 107-121.

Hem, J.D., 1985, Study and interpretation of the chemical characteristics of natural water: U.S. Geological Survey Water-Supply Paper 2254, 263 p. 
International Souris River Board, 2009, Water quality - International Souris River Board of the International Joint Commission: accessed March 11, 2010, at http://www.ijc.org/rel/ pdf/fact_sheets/water-quality.pdf.

Jones, G., and Armstrong, N., 2001, Long-term trends in total nitrogen and total phosphorus concentrations in Manitoba streams: Water Quality Management Section, Water Branch, Manitoba Conservation, Report No. 2001-07, 154 p.

North Dakota Department of Health, 2005, North Dakota's water quality monitoring strategy for surface waters, 2005-2014, accessed August 30, 2007, at http://www.health. state.nd.us/WQ/SW/A_Publications.htm.

North Dakota Department of Health, 2010, Standards of Water Quality for the State of North Dakota, North Dakota Department of Health, Division of Water Quality, Bismarck, North Dakota, accessed March 11, 2010, at http://www.legis. nd.gov/information/acdata/pdf/33-16-02.1.pdf.

Pope, L.M., Rosner, S.M., Hoffman, D.C., and Ziegler, A.C., 2004, Summary of available State ambient stream-waterquality data, 1990-98, and limitations for national assessment: U.S. Geological Survey Water-Resources Investigations Report 03-4116, 55 p., accessed August 30, 2007, at http://pubs.water.usgs.gov/wri034316/.

Runkel, R.L., Crawford, C.G., and Cohn, T.A., 2004, Load estimator (LOADEST) -A FORTRAN program for estimating constituent loads in streams and rivers: U.S. Geological Survey Techniques and Methods, book 4, chap. A5, 69 p.

Ryberg, K.R., and Hiemenz, Gregory, 2009, Summary and analysis of water-quality data for the Arrowwood National Wildlife Refuge, east-central North Dakota, 1987-2004: U.S. Geological Survey Scientific Investigations Report 2009-5017, 92 p.

Trench, E.C., and Vecchia, A.V., 2002, Water-quality trend analysis and sampling design for streams in Connecticut, 1968-98: U.S. Geological Survey Water-Resources Investigations Report 02-4011, 94 p.
U.S. Environmental Protection Agency, 1972, Water in the challenge of the environment-A primer on EPA's statutory authority, accessed September 24, 2007, at http://www.epa. gov/history/topics/fwpca/index.html.

U.S. Environmental Protection Agency, 2009, Drinking-water Contaminants, accessed March 4, 2011, at http://water.epa. gov/drink/contaminants/index.cfm.

U.S. Geological Survey, 1992, Programs and plans-Quality of existing dissolved trace-element data: U.S. Geological Survey Office of Water-Quality Technical Memorandum 92.05, accessed May 10, 2012, at http://water.usgs.gov/ admin/memo/QW/qw92.05.html.

U.S. Geological Survey, 1993, Programs and plans-Implementation of the protocol for collecting and processing surface-water samples for low-level inorganic analyses: U.S. Geological Survey Office of Water-Quality Technical Memorandum 93.11, accessed May 10, 2012, at http:// water.usgs.gov/admin/memo/QW/qw93.11.html.

Vecchia, A.V., 2000, Water-quality trend analysis and sampling design for the Souris River, Saskatchewan, North Dakota, and Manitoba: U.S. Geological Survey WaterResources Investigations Report 00-4019, 77 p.

Vecchia, A.V., 2003, Water-Quality Trend Analysis and Sampling Design for Streams in North Dakota: U.S. Geological Survey Water-Resources Investigations Report 03-4094, $73 \mathrm{p}$.

Vecchia, A.V., 2005, Water-Quality Trend Analysis and Sampling Design for Streams in the Red River of the North Basin, Minnesota, North Dakota, and South Dakota, 1970-2001: U.S. Geological Survey Scientific Investigations Report 05-5224, 54 p.

Vecchia, A.V., 2008, Climate simulation and flood risk analysis for 2008-40 for Devils Lake, North Dakota: U.S. Geological Survey Scientific Investigations Report 2008-5011, $28 \mathrm{p}$. 


\section{Appendix 1. Summary statistics for constituents and field measurements at selected sites in North Dakota}

Table 1-1. Summary statistics for major ion constituents at selected sites in North Dakota from 1970 through 2008.

Table 1-2. Summary statistics for nutrient constituents at selected sites in North Dakota from 1970 through 2008.

Table 1-3. Summary statistics for trace-metal constituents at selected sites in North Dakota from 1993 through 2008.

Table 1-4. Summary statistics for field measurements, total suspended solids, and suspended sediment at selected sites in North Dakota from 1970 through 2008.

Table 1-5. Median seasonal concentrations for selected constituents at selected sites in North Dakota from 1970 through 2008. 
Table 1-1. Summary statistics for major ion constituents at selected sites in North Dakota from 1970 through 2008.

$\left[\mu \mathrm{g} / \mathrm{L}\right.$, micrograms per liter; <, less than; --, not available; $\mathrm{mg} / \mathrm{L}$, milligrams per liter; $\mathrm{CO}_{3}$, carbonate; $\mathrm{HCO}_{3}$, bicarbonate; $\mathrm{CaCO}_{3}$, calcium carbonate $]$

\begin{tabular}{|c|c|c|c|c|c|c|}
\hline $\begin{array}{c}\text { Site } \\
\text { identification } \\
\text { number } \\
\text { (table 1) }\end{array}$ & Site name & $\begin{array}{l}\text { Number of } \\
\text { samples }\end{array}$ & $\begin{array}{l}\text { Period of } \\
\text { record }\end{array}$ & Median & Minimum & Maximum \\
\hline 1 & Bois De Sioux River near Doran, Minn. & 40 & $2000-2007$ & 217 & $<50$ & 497 \\
\hline 2 & Red River of the North at Wahpeton, N. Dak. & 35 & $1973-2008$ & 60 & $<50$ & 280 \\
\hline 4 & Red River of the North at Brushville, Minn. & 53 & $2000-2007$ & 90 & $<50$ & 238 \\
\hline 5 & Red River of the North below Wahpeton, N. Dak. & 9 & $1970-1972$ & 70 & 60 & 110 \\
\hline 6 & Red River of the North at Hickson, N. Dak. & 91 & $1975-2008$ & 80 & $<50$ & 530 \\
\hline 7 & Wild Rice River near Rutland, N. Dak. & 31 & $1971-2008$ & 173 & $<50$ & 460 \\
\hline 8 & Wild Rice River near Cayuga, N. Dak. & 15 & $1970-1977$ & 162 & $<50$ & 3,300 \\
\hline 11 & Red River of the North at Fargo, N. Dak. & 102 & $1970-2008$ & 80 & $<50$ & 270 \\
\hline 12 & Red River of North below Fargo, N. Dak. & 87 & $1970-1986$ & 80 & $<50$ & 370 \\
\hline 13 & Red River of the North at Harwood, N. Dak. & 53 & $2000-2007$ & 117 & $<50$ & 278 \\
\hline 14 & Red River of the North near Harwood, N. Dak. & 12 & $2005-2007$ & 93 & 86 & 151 \\
\hline 15 & Sheyenne River above Harvey, N. Dak. & 180 & $1972-2008$ & 722 & $<50$ & 1,200 \\
\hline 16 & Big Coulee near Fort Totten, N. Dak. & 8 & $1970-1975$ & 80 & $<50$ & 1,100 \\
\hline 17 & Sheyenne River at Warwick, N. Dak. & 45 & $1997-2006$ & 254 & 82 & 438 \\
\hline 18 & Sheyenne River near Warwick, N. Dak. & 99 & $1970-2008$ & 150 & $<50$ & 418 \\
\hline 19 & Mauvais Coulee Tributary No. 3 near Cando, N. Dak. & 20 & $1987-2008$ & $<50$ & $<50$ & 150 \\
\hline 26 & Little Coulee near Leeds, N. Dak. & 5 & $2005-2008$ & 96 & 52 & 138 \\
\hline 27 & Little Coulee near Brinsmade, N. Dak. & 24 & $1976-1994$ & 140 & $<50$ & 360 \\
\hline 28 & Big Coulee near Churchs Ferry, N. Dak. & 113 & 1970-1994 & 100 & $<50$ & 830 \\
\hline 29 & Big Coulee at Graham Island inlet near Fort Totten, N. Dak. & 43 & $1970-1986$ & 400 & 100 & 1,500 \\
\hline 30 & Channel A near Penn, N. Dak. & 54 & $1984-2008$ & 80 & $<50$ & 480 \\
\hline 31 & Devils Lake Outlet to Stump Lake near Lakota, N. Dak. & 6 & $2005-2007$ & 530 & 377 & 619 \\
\hline 32 & Sheyenne River near Cooperstown, N. Dak. & 279 & $1970-2008$ & 190 & $<50$ & 890 \\
\hline 33 & Baldhill Creek near Dazey, N. Dak. & 73 & $1972-2008$ & 149 & $<50$ & 830 \\
\hline 34 & Sheyenne River below Baldhill Dam, N. Dak. & 135 & $1972-2008$ & 170 & $<50$ & 310 \\
\hline 35 & Sheyenne River at Valley City, N. Dak. & 14 & $1972-2005$ & 145 & $<50$ & 240 \\
\hline 36 & Sheyenne River at Lisbon, N. Dak. & 260 & $1970-2008$ & 210 & $<50$ & 400 \\
\hline 37 & Sheyenne River near Kindred, N. Dak. & 115 & $1972-2008$ & 214 & 70 & 2,600 \\
\hline 38 & $\begin{array}{l}\text { Sheyenne River above Sheyenne River Diversion near Horace, } \\
\text { N. Dak. }\end{array}$ & 21 & $1993-2008$ & 185 & 90 & 245 \\
\hline 39 & Sheyenne River near Horace, N. Dak. & 8 & $1982-1992$ & 115 & 70 & 140 \\
\hline
\end{tabular}


Table 1-1. Summary statistics for major ion constituents at selected sites in North Dakota from 1970 through 2008. - Continued $\left[\left[\mu \mathrm{g} / \mathrm{L}\right.\right.$, micrograms per liter; <, less than; --, not available; $\mathrm{mg} / \mathrm{L}$, milligrams per liter; $\mathrm{CO}_{3}$, carbonate; $\mathrm{HCO}_{3}$, bicarbonate; $\mathrm{CaCO}$, calcium carbonate $]$

\begin{tabular}{|c|c|c|c|c|c|c|}
\hline $\begin{array}{c}\text { Site } \\
\text { identification } \\
\text { number } \\
\text { (table 1) }\end{array}$ & Site name & $\begin{array}{l}\text { Number of } \\
\text { samples }\end{array}$ & $\begin{array}{c}\text { Period of } \\
\text { record }\end{array}$ & Median & Minimum & Maximum \\
\hline \multicolumn{7}{|c|}{ Boron, in $\mu \mathrm{g} / \mathrm{L}-$ Continued } \\
\hline 40 & Sheyenne River Diversion at West Fargo, N. Dak. & 5 & 1994-2007 & 131 & 91 & 168 \\
\hline 41 & Sheyenne River at West Fargo, N. Dak. & 39 & $1970-2008$ & 130 & $<50$ & 5,400 \\
\hline 43 & Maple River near Enderlin, N. Dak. & 44 & $1972-2008$ & 165 & $<50$ & 520 \\
\hline 44 & Maple River near Mapleton, N. Dak. & 16 & $1972-2008$ & 204 & $<50$ & 1,000 \\
\hline 45 & Maple River below Mapleton, N. Dak. & 56 & $1997-2008$ & 232 & $<50$ & 405 \\
\hline 46 & Sheyenne River at Harwood, N. Dak. & 1 & 2005 & 115 & 115 & 115 \\
\hline 50 & Sheyenne River near Harwood, N. Dak. & 9 & $1970-1972$ & 212 & 104 & 300 \\
\hline 51 & Elm River near Kelso, N. Dak. & 3 & 1983-1987 & $<50$ & $<50$ & 150 \\
\hline 52 & Red River of the North at Halstad, Minn. & 29 & $1972-2008$ & 106 & $<50$ & 290 \\
\hline 53 & Beaver Creek near Finley, N. Dak. & 8 & $1970-1973$ & 120 & $<50$ & 243 \\
\hline 54 & Goose River near Portland, N. Dak. & 30 & $1970-1988$ & 120 & $<50$ & 2,700 \\
\hline 55 & Goose River at Hillsboro, N. Dak. & 130 & $1970-2008$ & 200 & $<50$ & 1,100 \\
\hline 56 & Red River of the North at Grand Forks, N. Dak. & 145 & $1970-2008$ & 84 & $<50$ & 168 \\
\hline 57 & Turtle River at Turtle River State Park near Arvilla, N. Dak. & 9 & $1991-2008$ & 81 & $<50$ & 336 \\
\hline 58 & Turtle River at Manvel, N. Dak. & 104 & $1971-2008$ & 491 & $<50$ & 14,000 \\
\hline 65 & Middle Branch Park River near Edinburg, N. Dak. & 14 & $1978-1980$ & 80 & $<50$ & 120 \\
\hline 66 & Cart Creek at Mountain, N. Dak. & 17 & $1972-1984$ & 100 & $<50$ & 300 \\
\hline 67 & Park River at Grafton, N. Dak. & 119 & $1970-2008$ & 190 & $<50$ & 830 \\
\hline 68 & Red River of the North at Drayton, N. Dak. & 38 & $1972-2008$ & 85 & $<50$ & 1,100 \\
\hline 69 & Pembina County Drain 20 near Glasston, N. Dak. & 8 & 1974-1984 & 70 & $<50$ & 320 \\
\hline 70 & Hidden Island Coulee near Hansboro, N. Dak. & 21 & $1972-1994$ & $<50$ & $<50$ & 280 \\
\hline 71 & Cypress Creek near Sarles, N. Dak. & 13 & $1972-1988$ & $<50$ & $<50$ & 280 \\
\hline 72 & Pembina River near Vang, N. Dak. & 62 & 1970-1979 & 130 & $<50$ & 680 \\
\hline 73 & Little South Pembina River near Walhalla, N. Dak. & 75 & $1970-2008$ & 120 & $<50$ & 220 \\
\hline 74 & Pembina River at Walhalla, N. Dak. & 189 & 1970-2008 & 130 & $<50$ & 290 \\
\hline 75 & Pembina River at Neche, N. Dak. & 102 & $1972-2008$ & 140 & $<50$ & 380 \\
\hline 76 & Tongue River at Akra, N. Dak. & 51 & $1972-2008$ & 80 & $<50$ & 350 \\
\hline 78 & Red River of the North at Pembina, N. Dak., site 2 & 97 & $1970-2008$ & 110 & $<50$ & 396 \\
\hline 79 & Red River of the North at Emerson, Manitoba & 2 & $1982-1991$ & 145 & 130 & 160 \\
\hline 80 & Long Creek near Noonan, N. Dak. & 52 & $1972-2008$ & 82 & $<50$ & 650 \\
\hline
\end{tabular}


Table 1-1. Summary statistics for major ion constituents at selected sites in North Dakota from 1970 through 2008. - Continued $\left[\mu \mathrm{g} / \mathrm{L}\right.$, micrograms per liter; <, less than; --, not available; $\mathrm{mg} / \mathrm{L}$, milligrams per liter; $\mathrm{CO}_{3}$, carbonate; $\mathrm{HCO}_{3}$, bicarbonate; $\mathrm{CaCO}_{3}$, calcium carbonate $]$

\begin{tabular}{|c|c|c|c|c|c|c|}
\hline $\begin{array}{c}\text { Site } \\
\text { identification } \\
\text { number } \\
\text { (table 1) }\end{array}$ & Site name & $\begin{array}{c}\text { Number of } \\
\text { samples }\end{array}$ & $\begin{array}{l}\text { Period of } \\
\text { record }\end{array}$ & Median & Minimum & Maximum \\
\hline \multicolumn{7}{|c|}{ Boron, in $\mu \mathrm{g} / \mathrm{L}$-Continued } \\
\hline 81 & West Branch Short Creek near Columbus, N. Dak. & 20 & $1978-1981$ & 175 & $<50$ & 310 \\
\hline 82 & Souris River near Sherwood, N. Dak. & 302 & $1972-2008$ & 232 & $<50$ & 5,100 \\
\hline 83 & Souris River near Foxholm, N. Dak. & 142 & $1972-2008$ & 270 & $<50$ & 1,300 \\
\hline 84 & Des Lacs River at Foxholm, N. Dak. & 149 & $1972-2008$ & 148 & $<50$ & 808 \\
\hline 85 & Souris River above Minot, N. Dak. & 191 & 1970-2008 & 209 & $<50$ & 1,100 \\
\hline 86 & Bonnes Creek near Velva, N. Dak. & 8 & $1987-2005$ & 182 & 60 & 2,670 \\
\hline 87 & Souris River near Verendrye, N. Dak. & 311 & 1970-2008 & 253 & $<50$ & 980 \\
\hline 88 & Wintering River near Karlsruhe, N. Dak. & 116 & $1972-2008$ & 150 & $<50$ & 1,100 \\
\hline 89 & Souris River near Bantry, N. Dak. & 120 & 1970-2008 & 209 & $<50$ & 780 \\
\hline 90 & Willow Creek near Willow City, N. Dak. & 52 & 1972-2008 & 130 & $<50$ & 380 \\
\hline 91 & Stone Creek near Kramer, N. Dak. & 13 & $1986-1993$ & 150 & $<50$ & 10,000 \\
\hline 92 & Deep River near Upham, N. Dak. & 31 & 1972-2007 & 50 & $<50$ & 210 \\
\hline 93 & Egg Creek near Granville, N. Dak. & 14 & $1972-1981$ & 125 & $<50$ & 270 \\
\hline 94 & Cut Bank Creek at North Lake Outlet near Granville, N. Dak. & 15 & 1972-1976 & 120 & $<50$ & 320 \\
\hline 95 & Cut Bank Creek at Upham, N. Dak. & 17 & 1975-1989 & 120 & $<50$ & 470 \\
\hline 96 & Deep River below Cut Bank Creek near Upham, N. Dak. & 43 & 1975-1989 & 100 & $<50$ & 220 \\
\hline 97 & Boundary Creek near Landa, N. Dak. & 22 & 1972-1994 & 120 & $<50$ & 390 \\
\hline 98 & Souris River near Westhope, N. Dak. & 94 & 1970-2008 & 200 & 58 & 510 \\
\hline 99 & Charbonneau Creek near Charbonneau, N. Dak. & 22 & 1972-1977 & 160 & $<50$ & 760 \\
\hline 100 & Missouri River near Williston, N. Dak. & 73 & 1974-1982 & 130 & 60 & 350 \\
\hline 101 & Little Muddy River below Cow Creek near Williston, N. Dak. & 58 & $1972-2008$ & 280 & $<50$ & 860 \\
\hline 102 & Stony Creek near Williston, N. Dak. & 31 & $1977-1981$ & 280 & $<50$ & 400 \\
\hline 103 & Tobacco Garden Creek near Watford City, N. Dak. & 15 & 1976-1977 & 350 & $<50$ & 970 \\
\hline 104 & Beaver Creek near Ray, N. Dak. & 51 & $1977-1982$ & 280 & $<50$ & 370 \\
\hline 105 & White Earth River at White Earth, N. Dak. & 27 & 1970-1977 & 320 & $<50$ & 960 \\
\hline 106 & Bear Den Creek near Mandaree, N. Dak. & 23 & 1970-2008 & 230 & 70 & 354 \\
\hline 107 & Shell Creek near Parshall, N. Dak. & 21 & 1972-1977 & 380 & $<50$ & 940 \\
\hline 108 & East Fork Shell Creek near Parshall, N. Dak. & 46 & $1991-2008$ & 593 & $<50$ & 1,071 \\
\hline 109 & Deepwater Creek near Mandaree, N. Dak. & 43 & $1991-2008$ & 477 & $<50$ & 964 \\
\hline 110 & Little Missouri River at Marmarth, N. Dak. & 65 & $1971-2008$ & 260 & $<50$ & 950 \\
\hline 111 & Deep Creek near Amidon, N. Dak. & 51 & $1977-1983$ & 1,400 & 250 & 2,800 \\
\hline 112 & Little Missouri River at Medora, N. Dak. & 86 & 1972-2008 & 431 & $<50$ & 3,050 \\
\hline 113 & Beaver Creek near Trotters, N. Dak. & 79 & $1977-2008$ & 648 & 90 & 1,100 \\
\hline 114 & Little Missouri River near Watford City, N. Dak. & 113 & $1971-2008$ & 359 & $<50$ & 3,310 \\
\hline 115 & Missouri River at Garrison Dam, N. Dak. & 160 & $1971-2007$ & 118 & $<50$ & 330 \\
\hline 116 & Knife River at Manning, N. Dak. & 98 & $1972-2008$ & 395 & $<50$ & 1,500 \\
\hline 117 & Stray Creek near Manning, N. Dak. & 17 & 1975-1981 & 460 & 130 & 1,200 \\
\hline 118 & Knife River at Marshall, N. Dak. & 69 & 1972-1981 & 330 & $<50$ & 1,100 \\
\hline 119 & Elm Creek near Golden Valley, N. Dak. & 37 & 1973-1995 & 210 & $<50$ & 1,300 \\
\hline
\end{tabular}


Table 1-1. Summary statistics for major ion constituents at selected sites in North Dakota from 1970 through 2008. - Continued [ $\mu \mathrm{g} / \mathrm{L}$, micrograms per liter; <, less than; --, not available; $\mathrm{mg} / \mathrm{L}$, milligrams per liter; $\mathrm{CO}_{3}$, carbonate; $\mathrm{HCO}_{3}$, bicarbonate; $\mathrm{CaCO}_{3}$, calcium carbonate]

\begin{tabular}{|c|c|c|c|c|c|c|}
\hline $\begin{array}{c}\text { Site } \\
\text { identification } \\
\text { number } \\
\text { (table 1) }\end{array}$ & Site name & $\begin{array}{l}\text { Number of } \\
\text { samples }\end{array}$ & $\begin{array}{l}\text { Period of } \\
\text { record }\end{array}$ & Median & Minimum & Maximum \\
\hline \multicolumn{7}{|c|}{ Boron, in $\mu \mathrm{g} / \mathrm{L}$-Continued } \\
\hline 120 & Knife River near Golden Valley, N. Dak. & 111 & $1971-2008$ & 325 & $<50$ & 1,500 \\
\hline 121 & Coyote Creek near Zap, N. Dak. & 51 & 1977-1983 & 330 & $<50$ & 700 \\
\hline 123 & Spring Creek below Lake Ilo near Dunn Center, N. Dak. & 47 & 1977-1981 & 550 & 80 & 1,300 \\
\hline 124 & Spring Creek near Halliday, N. Dak. & 48 & 1977-1981 & 670 & 100 & 920 \\
\hline 125 & Spring Creek at Zap, N. Dak. & 189 & $1970-2008$ & 450 & $<50$ & 1,600 \\
\hline 126 & West Branch Otter Creek near Beulah, N. Dak. & 14 & $1972-1994$ & 120 & $<50$ & 1,200 \\
\hline 130 & West Branch Antelope Creek near Hazen, N. Dak. & 14 & $1978-1983$ & 165 & $<50$ & 460 \\
\hline 131 & Coal Creek near Stanton, N. Dak. & 28 & $1975-1981$ & 315 & 60 & 430 \\
\hline 132 & Alderin Creek near Fort Clark, N. Dak. & 35 & $1977-1982$ & 240 & $<50$ & 500 \\
\hline 133 & Coal Lake Coulee near Hensler, N. Dak. & 16 & 1978-1982 & 130 & $<50$ & 590 \\
\hline 134 & Buffalo Creek near Washburn, N. Dak. & 38 & $1978-1983$ & 635 & $<50$ & 1,100 \\
\hline 135 & Turtle Creek above Washburn, N. Dak. & 100 & $1987-2003$ & 499 & 70 & 1,400 \\
\hline 136 & Painted Woods Creek near Wilton, N. Dak. & 201 & 1970-2003 & 378 & $<50$ & 1,000 \\
\hline 137 & Square Butte Creek near Hannover, N. Dak. & 23 & $1977-1981$ & 180 & $<50$ & 310 \\
\hline 138 & Square Butte Creek above Nelson Lake near Center, N. Dak. & 55 & $1977-1982$ & 310 & $<50$ & 800 \\
\hline 145 & Heart River near South Heart, N. Dak. & 67 & 1975-2005 & 600 & 80 & 1,300 \\
\hline 146 & Heart River at Dickinson, N. Dak. & 17 & 1986-1994 & 400 & 140 & 580 \\
\hline 147 & Green River near New Hradec, N. Dak. & 97 & $1972-2008$ & 340 & $<50$ & 1,700 \\
\hline 148 & Green River near Gladstone, N. Dak. & 29 & 1970-1975 & 330 & $<50$ & 620 \\
\hline 149 & Heart River near Richardton, N. Dak. & 123 & $1972-2008$ & 378 & $<50$ & 1,700 \\
\hline 150 & Heart River above Lake Tschida near Glen Ullin, N. Dak. & 20 & 1989-2008 & 320 & 70 & 801 \\
\hline 151 & Antelope Creek near Carson, N. Dak. & 15 & $1972-2008$ & 170 & $<50$ & 650 \\
\hline 152 & Big Muddy Creek near Almont, N. Dak. & 16 & $1991-2008$ & 353 & 120 & 859 \\
\hline 153 & Heart River near Lark, N. Dak. & 43 & 1971-1994 & 210 & $<50$ & 1,700 \\
\hline 154 & Heart River at Stark Bridge near Judson, N. Dak. & 21 & $1988-2008$ & 323 & $<50$ & 548 \\
\hline 155 & Sweetbriar Creek near Judson, N. Dak. & 27 & $1972-2008$ & 302 & $<50$ & 1,200 \\
\hline 156 & Heart River near Mandan, N. Dak. & 112 & $1971-2008$ & 367 & $<50$ & 1,700 \\
\hline 157 & Long Lake Creek above Long Lake near Moffit, N. Dak. & 10 & 1990-1994 & 305 & 70 & 670 \\
\hline 158 & Apple Creek near Menoken, N. Dak. & 106 & 1972-2008 & 825 & $<50$ & 1,700 \\
\hline 159 & Missouri River near Schmidt, N. Dak. & 48 & $1976-1981$ & 120 & 90 & 150 \\
\hline
\end{tabular}


Table 1-1. Summary statistics for major ion constituents at selected sites in North Dakota from 1970 through 2008. —Continued $\left[\mu \mathrm{g} / \mathrm{L}\right.$, micrograms per liter; <, less than; --, not available; $\mathrm{mg} / \mathrm{L}$, milligrams per liter; $\mathrm{CO}_{3}$, carbonate; $\mathrm{HCO}_{3}$, bicarbonate; $\mathrm{CaCO}$, calcium carbonate $]$

\begin{tabular}{|c|c|c|c|c|c|c|}
\hline $\begin{array}{c}\text { Site } \\
\text { identification } \\
\text { number } \\
\text { (table 1) }\end{array}$ & Site name & $\begin{array}{c}\text { Number of } \\
\text { samples }\end{array}$ & $\begin{array}{l}\text { Period of } \\
\text { record }\end{array}$ & Median & Minimum & Maximum \\
\hline \multicolumn{7}{|c|}{ Boron, in $\mu \mathrm{g} / \mathrm{L}-$ Continued } \\
\hline 160 & Cannonball River at New England, N. Dak. & 37 & $1978-2005$ & 570 & 80 & 930 \\
\hline 161 & Coal Bank Creek near Havelock, N. Dak. & 63 & $1974-1983$ & 1,100 & 120 & 3,100 \\
\hline 163 & Cannonball River below Bentley, N. Dak. & 31 & $1972-1977$ & 560 & $<50$ & 1,600 \\
\hline 164 & Cannonball River near Raleigh, N. Dak. & 65 & 1997-2008 & 513 & 126 & 1,680 \\
\hline 165 & White Butte Fork Cedar Creek near Scranton, N. Dak. & 24 & $1972-1994$ & 785 & $<50$ & 2,200 \\
\hline 166 & Cedar Creek near Haynes, N. Dak. & 56 & $1971-2008$ & 545 & 60 & 3,000 \\
\hline 170 & Cannonball River at Breien, N. Dak. & 123 & 1970-2008 & 513 & $<50$ & 1,440 \\
\hline 171 & Beaver Creek near Linton, N. Dak. & 35 & $1972-1989$ & 200 & $<50$ & 660 \\
\hline 172 & Beaver Creek below Linton, N. Dak. & 18 & $1990-2008$ & 250 & 80 & 470 \\
\hline 173 & Porcupine Creek near Fort Yates, N. Dak. & 41 & 1991-1999 & 718 & $<50$ & 2,000 \\
\hline 174 & Buffalo Creek Tributary near Gascoyne, N. Dak. & 63 & 1974-1987 & 2,700 & 140 & 6,600 \\
\hline 175 & James River near Manfred, N. Dak. & 48 & $1972-1998$ & 220 & $<50$ & 586 \\
\hline 176 & James River near Grace City, N. Dak. & 137 & $1972-2008$ & 160 & $<50$ & 2,300 \\
\hline 177 & James River above Arrowwood Lake near Kensal, N. Dak. & 173 & $1985-2008$ & 150 & $<50$ & 302 \\
\hline 178 & Kelly Creek below Niccum Reservoir near Bordulac, N. Dak. & 10 & 1986-1989 & 75 & $<50$ & 110 \\
\hline 185 & James River at Oakes, N. Dak. & 107 & $1970-2008$ & 210 & $<50$ & 1,500 \\
\hline 186 & James River at N. Dak./S. Dak. State line & 91 & 1974-2008 & 200 & $<50$ & 1,100 \\
\hline \multicolumn{7}{|c|}{ Sodium, in $\mathrm{mg} / \mathrm{L}$} \\
\hline 1 & Bois De Sioux River near Doran, Minn. & 40 & $2000-2007$ & 50 & 10 & 177 \\
\hline 2 & Red River of the North at Wahpeton, N. Dak. & 74 & $1973-2008$ & 15 & 5 & 50 \\
\hline 3 & Red River of the North near Wahpeton, N. Dak. & 4 & $1993-2006$ & 23 & 16 & 268 \\
\hline 4 & Red River of the North at Brushville, Minn. & 61 & $1993-2007$ & 20 & 9 & 54 \\
\hline 5 & Red River of the North below Wahpeton, N. Dak. & 34 & 1970-1999 & 14 & 8 & 42 \\
\hline 6 & Red River of the North at Hickson, N. Dak. & 134 & $1975-2008$ & 15 & 7 & 92 \\
\hline 7 & Wild Rice River near Rutland, N. Dak. & 47 & $1971-2008$ & 86 & 6 & 433 \\
\hline 8 & Wild Rice River near Cayuga, N. Dak. & 15 & 1970-1977 & 66 & 50 & 980 \\
\hline 9 & Antelope Creek at Dwight, N. Dak. & 13 & $2001-2008$ & 43 & 25 & 80 \\
\hline 10 & Wild Rice River near Abercrombie, N. Dak. & 257 & $1970-2008$ & 100 & 8 & 420 \\
\hline 11 & Red River of the North at Fargo, N. Dak. & 186 & $1970-2008$ & 18 & 7 & 43 \\
\hline 12 & Red River of North below Fargo, N. Dak. & 146 & 1970-1986 & 19 & 7 & 110 \\
\hline
\end{tabular}


Table 1-1. Summary statistics for major ion constituents at selected sites in North Dakota from 1970 through 2008. - Continued [ $\mu \mathrm{g} / \mathrm{L}$, micrograms per liter; <, less than; --, not available; $\mathrm{mg} / \mathrm{L}$, milligrams per liter; $\mathrm{CO}_{3}$, carbonate; $\mathrm{HCO}_{3}$, bicarbonate; $\mathrm{CaCO}_{3}$, calcium carbonate]

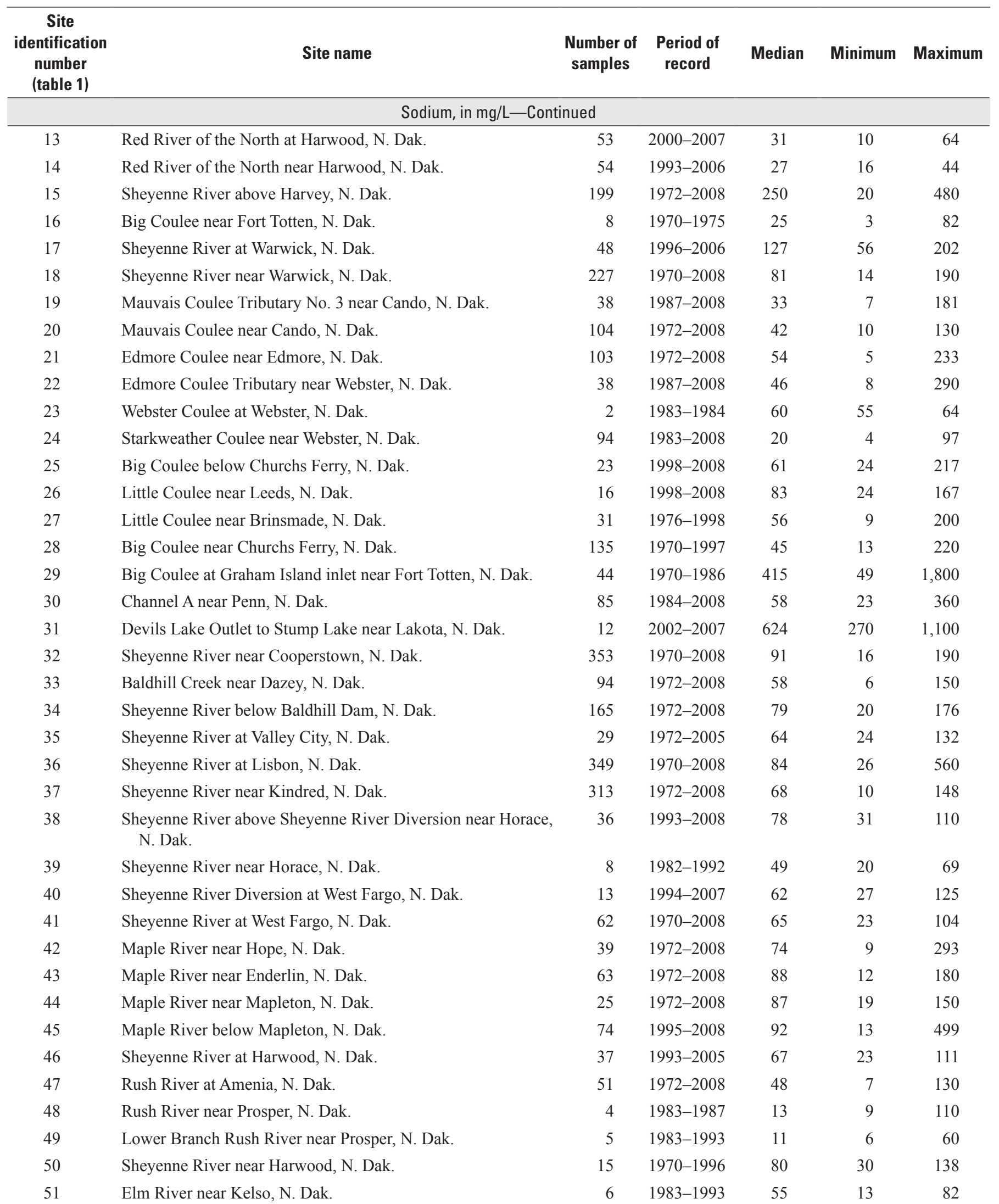


Table 1-1. Summary statistics for major ion constituents at selected sites in North Dakota from 1970 through 2008. —Continued $\left[\mu \mathrm{g} / \mathrm{L}\right.$, micrograms per liter; <, less than; --, not available; $\mathrm{mg} / \mathrm{L}$, milligrams per liter; $\mathrm{CO}_{3}$, carbonate; $\mathrm{HCO}_{3}$, bicarbonate; $\mathrm{CaCO}$, calcium carbonate $]$

\begin{tabular}{|c|c|c|c|c|c|c|}
\hline $\begin{array}{c}\text { Site } \\
\text { identification } \\
\text { number } \\
\text { (table 1) }\end{array}$ & Site name & $\begin{array}{c}\text { Number of } \\
\text { samples }\end{array}$ & $\begin{array}{l}\text { Period of } \\
\text { record }\end{array}$ & Median & Minimum & Maximum \\
\hline \multicolumn{7}{|c|}{ Sodium, in $\mathrm{mg} / \mathrm{L}-$ Continued } \\
\hline 52 & Red River of the North at Halstad, Minn. & 178 & $1972-2008$ & 31 & 8 & 78 \\
\hline 53 & Beaver Creek near Finley, N. Dak. & 117 & 1970-2003 & 82 & 7 & 290 \\
\hline 54 & Goose River near Portland, N. Dak. & 30 & 1970-1988 & 65 & 8 & 124 \\
\hline 55 & Goose River at Hillsboro, N. Dak. & 166 & 1970-2008 & 79 & 9 & 330 \\
\hline 56 & Red River of the North at Grand Forks, N. Dak. & 242 & 1970-2008 & 19 & 4 & 64 \\
\hline 57 & Turtle River at Turtle River State Park near Arvilla, N. Dak. & 99 & $1991-2008$ & 47 & 11 & 130 \\
\hline 58 & Turtle River at Manvel, N. Dak. & 105 & $1971-2008$ & 290 & 56 & 2,100 \\
\hline 59 & Red River of the North at Oslo, Minn. & 75 & $1973-2005$ & 20 & 9 & 100 \\
\hline 60 & Middle Branch Forest River near Whitman, N. Dak. & 14 & $1972-1990$ & 43 & 25 & 340 \\
\hline 61 & Forest River near Fordville, N. Dak. & 64 & 1972-2008 & 40 & 15 & 148 \\
\hline 62 & Forest River near Minto, N. Dak. & 154 & $1971-2008$ & 46 & 7 & 738 \\
\hline 63 & South Branch Park River below Homme Dam, N. Dak. & 27 & 1972-1994 & 39 & 19 & 64 \\
\hline 64 & Middle Branch Park River near Union, N. Dak. & 15 & $1972-1984$ & 52 & 18 & 83 \\
\hline 65 & Middle Branch Park River near Edinburg, N. Dak. & 14 & $1978-1980$ & 23 & 13 & 32 \\
\hline 66 & Cart Creek at Mountain, N. Dak. & 17 & $1972-1984$ & 44 & 14 & 55 \\
\hline 67 & Park River at Grafton, N. Dak. & 144 & 1970-2008 & 95 & 12 & 370 \\
\hline 68 & Red River of the North at Drayton, N. Dak. & 74 & 1972-2008 & 34 & 9 & 130 \\
\hline 69 & Pembina County Drain 20 near Glasston, N. Dak. & 8 & $1974-1984$ & 8 & $<3$ & 25 \\
\hline 70 & Hidden Island Coulee near Hansboro, N. Dak. & 22 & $1972-1995$ & 27 & 12 & 82 \\
\hline 71 & Cypress Creek near Sarles, N. Dak. & 13 & $1972-1988$ & 24 & 16 & 75 \\
\hline 72 & Pembina River near Vang, N. Dak. & 62 & $1970-1979$ & 48 & 16 & 80 \\
\hline 73 & Little South Pembina River near Walhalla, N. Dak. & 83 & $1970-2008$ & 58 & 13 & 90 \\
\hline 74 & Pembina River at Walhalla, N. Dak. & 270 & $1970-2008$ & 48 & 18 & 110 \\
\hline 75 & Pembina River at Neche, N. Dak. & 133 & 1972-2008 & 48 & 18 & 72 \\
\hline 76 & Tongue River at Akra, N. Dak. & 72 & 1972-2008 & 26 & 13 & 39 \\
\hline 77 & Red River of the North at Pembina, N. Dak., site 1 & 10 & 1993-1993 & 33 & 14 & 48 \\
\hline 78 & Red River of the North at Pembina, N. Dak., site 2 & 152 & $1970-2008$ & 31 & 8 & 130 \\
\hline 79 & Red River of the North at Emerson, Manitoba & 148 & $1977-2004$ & 34 & 8 & 190 \\
\hline 80 & Long Creek near Noonan, N. Dak. & 70 & 1972-2008 & 125 & 14 & 418 \\
\hline 81 & West Branch Short Creek near Columbus, N. Dak. & 20 & $1978-1981$ & 300 & 90 & 850 \\
\hline 82 & Souris River near Sherwood, N. Dak. & 302 & $1972-2008$ & 120 & 14 & 507 \\
\hline 83 & Souris River near Foxholm, N. Dak. & 202 & $1972-2008$ & 91 & 26 & 360 \\
\hline 84 & Des Lacs River at Foxholm, N. Dak. & 188 & 1972-2008 & 200 & 12 & 488 \\
\hline 85 & Souris River above Minot, N. Dak. & 207 & 1970-2008 & 127 & 21 & 905 \\
\hline 86 & Bonnes Creek near Velva, N. Dak. & 18 & 1987-2005 & 120 & 17 & 460 \\
\hline 87 & Souris River near Verendrye, N. Dak. & 373 & $1970-2008$ & 134 & 18 & 554 \\
\hline 88 & Wintering River near Karlsruhe, N. Dak. & 134 & $1972-2008$ & 69 & 25 & 328 \\
\hline 89 & Souris River near Bantry, N. Dak. & 152 & $1970-2008$ & 111 & 29 & 320 \\
\hline 90 & Willow Creek near Willow City, N. Dak. & 90 & 1972-2008 & 91 & $<3$ & 310 \\
\hline 91 & Stone Creek near Kramer, N. Dak. & 30 & $1986-2000$ & 81 & 12 & 500 \\
\hline
\end{tabular}


Table 1-1. Summary statistics for major ion constituents at selected sites in North Dakota from 1970 through 2008. - Continued $\left[\mu \mathrm{g} / \mathrm{L}\right.$, micrograms per liter; <, less than; --, not available; $\mathrm{mg} / \mathrm{L}$, milligrams per liter; $\mathrm{CO}_{3}$, carbonate; $\mathrm{HCO}_{3}$, bicarbonate; $\mathrm{CaCO}$, calcium carbonate $]$

\begin{tabular}{|c|c|c|c|c|c|c|}
\hline $\begin{array}{c}\text { Site } \\
\text { identification } \\
\text { number } \\
\text { (table 1) }\end{array}$ & Site name & $\begin{array}{l}\text { Number of } \\
\text { samples }\end{array}$ & $\begin{array}{l}\text { Period of } \\
\text { record }\end{array}$ & Median & Minimum & Maximum \\
\hline \multicolumn{7}{|c|}{ Sodium, in $\mathrm{mg} / \mathrm{L}$-Continued } \\
\hline 92 & Deep River near Upham, N. Dak. & 68 & $1972-2007$ & 47 & 7 & 95 \\
\hline 93 & Egg Creek near Granville, N. Dak. & 14 & $1972-1981$ & 56 & 16 & 190 \\
\hline 95 & Cut Bank Creek at Upham, N. Dak. & 33 & $1975-2000$ & 90 & 17 & 190 \\
\hline 96 & Deep River below Cut Bank Creek near Upham, N. Dak. & 43 & $1975-1989$ & 78 & 8 & 190 \\
\hline 97 & Boundary Creek near Landa, N. Dak. & 39 & $1972-2000$ & 102 & 13 & 400 \\
\hline 98 & Souris River near Westhope, N. Dak. & 214 & $1970-2008$ & 110 & 23 & 420 \\
\hline 102 & Stony Creek near Williston, N. Dak. & 32 & $1977-1981$ & 405 & 20 & 590 \\
\hline 103 & Tobacco Garden Creek near Watford City, N. Dak. & 15 & 1976-1977 & 550 & 86 & 920 \\
\hline 104 & Beaver Creek near Ray, N. Dak. & 51 & 1977-1982 & 330 & 16 & 390 \\
\hline 105 & White Earth River at White Earth, N. Dak. & 27 & $1970-1977$ & 360 & 34 & 600 \\
\hline 106 & Bear Den Creek near Mandaree, N. Dak. & 228 & $1970-2008$ & 560 & 25 & 1,000 \\
\hline 107 & Shell Creek near Parshall, N. Dak. & 21 & $1972-1977$ & 500 & 44 & 650 \\
\hline 108 & East Fork Shell Creek near Parshall, N. Dak. & 58 & $1991-2008$ & 626 & 16 & 830 \\
\hline 109 & Deepwater Creek near Mandaree, N. Dak. & 57 & $1991-2008$ & 295 & 9 & 733 \\
\hline 110 & Little Missouri River at Marmarth, N. Dak. & 86 & $1971-2008$ & 230 & 35 & 680 \\
\hline 117 & Stray Creek near Manning, N. Dak. & 17 & $1975-1981$ & 370 & 99 & 1,100 \\
\hline 118 & Knife River at Marshall, N. Dak. & 69 & 1972-1981 & 390 & 24 & 840 \\
\hline 119 & Elm Creek near Golden Valley, N. Dak. & 37 & $1973-1995$ & 180 & $<3$ & 710 \\
\hline 120 & Knife River near Golden Valley, N. Dak. & 130 & $1971-2008$ & 340 & 15 & 1,100 \\
\hline 121 & Coyote Creek near Zap, N. Dak. & 51 & $1977-1983$ & 320 & 21 & 650 \\
\hline 122 & Brush Creek near Beulah, N. Dak. & 107 & $1974-1990$ & 280 & 18 & 380 \\
\hline 123 & Spring Creek below Lake Ilo near Dunn Center, N. Dak. & 53 & $1977-1993$ & 320 & 35 & 630 \\
\hline 124 & Spring Creek near Halliday, N. Dak. & 48 & $1977-1981$ & 285 & 30 & 340 \\
\hline 125 & Spring Creek at Zap, N. Dak. & 240 & $1970-2008$ & 235 & 19 & 421 \\
\hline 126 & West Branch Otter Creek near Beulah, N. Dak. & 15 & 1972-1995 & 150 & 36 & 340 \\
\hline 127 & Knife River at Hazen, N. Dak. & 269 & 1970-2008 & 250 & 25 & 466 \\
\hline 128 & Antelope Creek above Hazen, N. Dak. & 44 & $1977-1985$ & 245 & 9 & 740 \\
\hline 129 & West Branch Antelope Creek No. 4 near Zap, N. Dak. & 21 & $1977-1985$ & 32 & $<3$ & 120 \\
\hline 130 & West Branch Antelope Creek near Hazen, N. Dak. & 14 & $1978-1983$ & 115 & 10 & 410 \\
\hline 131 & Coal Creek near Stanton, N. Dak. & 28 & $1975-1981$ & 345 & 27 & 610 \\
\hline
\end{tabular}


Table 1-1. Summary statistics for major ion constituents at selected sites in North Dakota from 1970 through 2008. —Continued $\left[\mu \mathrm{g} / \mathrm{L}\right.$, micrograms per liter; <, less than; --, not available; $\mathrm{mg} / \mathrm{L}$, milligrams per liter; $\mathrm{CO}_{3}$, carbonate; $\mathrm{HCO}_{3}$, bicarbonate; $\mathrm{CaCO}$, calcium carbonate $]$

\begin{tabular}{|c|c|c|c|c|c|c|}
\hline $\begin{array}{c}\text { Site } \\
\text { identification } \\
\text { number } \\
\text { (table 1) } \\
\end{array}$ & Site name & $\begin{array}{c}\text { Number of } \\
\text { samples }\end{array}$ & $\begin{array}{l}\text { Period of } \\
\text { record }\end{array}$ & Median & Minimum & Maximum \\
\hline \multicolumn{7}{|c|}{ Sodium, in $\mathrm{mg} / \mathrm{L}-$ Continued } \\
\hline 132 & Alderin Creek near Fort Clark, N. Dak. & 41 & 1977-1983 & 270 & 30 & 650 \\
\hline 133 & Coal Lake Coulee near Hensler, N. Dak. & 39 & $1978-1988$ & 160 & 12 & 320 \\
\hline 134 & Buffalo Creek near Washburn, N. Dak. & 38 & $1978-1983$ & 520 & 24 & 1,000 \\
\hline 135 & Turtle Creek above Washburn, N. Dak. & 100 & 1987-2003 & 229 & 36 & 1,100 \\
\hline 136 & Painted Woods Creek near Wilton, N. Dak. & 202 & 1970-2003 & 190 & $<3$ & 580 \\
\hline 137 & Square Butte Creek near Hannover, N. Dak. & 23 & 1977-1981 & 98 & 6 & 130 \\
\hline 138 & Square Butte Creek above Nelson Lake near Center, N. Dak. & 55 & 1977-1982 & 160 & 11 & 210 \\
\hline 139 & Hagel Creek near Center, N. Dak. & 38 & 1977-1982 & 205 & 16 & 400 \\
\hline 140 & Square Butte Creek below Center, N. Dak. & 81 & 1972-2008 & 190 & 59 & 291 \\
\hline 141 & Burnt Creek near Bismarck, N. Dak. & 48 & $1972-2008$ & 106 & 5 & 220 \\
\hline 142 & Missouri River at Bismarck, N. Dak. & 120 & 1974-2008 & 58 & 38 & 80 \\
\hline 143 & South Branch Heart River near South Heart, N. Dak. & 19 & $1979-1983$ & 140 & 35 & 380 \\
\hline 144 & North Creek near South Heart, N. Dak. & 16 & $1978-1981$ & 310 & 41 & 1,100 \\
\hline 145 & Heart River near South Heart, N. Dak. & 76 & $1975-2005$ & 415 & 32 & 910 \\
\hline 146 & Heart River at Dickinson, N. Dak. & 22 & 1986-1994 & 295 & 111 & 538 \\
\hline 147 & Green River near New Hradec, N. Dak. & 116 & $1972-2008$ & 150 & 15 & 520 \\
\hline 148 & Green River near Gladstone, N. Dak. & 33 & $1970-1993$ & 218 & 24 & 482 \\
\hline 149 & Heart River near Richardton, N. Dak. & 154 & $1972-2008$ & 221 & 39 & 548 \\
\hline 150 & Heart River above Lake Tschida near Glen Ullin, N. Dak. & 40 & 1989-2008 & 206 & 40 & 405 \\
\hline 151 & Antelope Creek near Carson, N. Dak. & 26 & $1972-2008$ & 76 & 34 & 220 \\
\hline 152 & Big Muddy Creek near Almont, N. Dak. & 39 & $1991-2008$ & 340 & 49 & 590 \\
\hline 153 & Heart River near Lark, N. Dak. & 45 & 1971-1995 & 140 & 30 & 230 \\
\hline 154 & Heart River at Stark Bridge near Judson, N. Dak. & 41 & 1988-2008 & 180 & 22 & 278 \\
\hline 155 & Sweetbriar Creek near Judson, N. Dak. & 33 & $1972-2008$ & 232 & 25 & 403 \\
\hline 156 & Heart River near Mandan, N. Dak. & 232 & $1971-2008$ & 206 & 19 & 453 \\
\hline 157 & Long Lake Creek above Long Lake near Moffit, N. Dak. & 33 & 1988-2004 & 140 & 15 & 230 \\
\hline 158 & Apple Creek near Menoken, N. Dak. & 129 & $1972-2008$ & 240 & 9 & 460 \\
\hline 159 & Missouri River near Schmidt, N. Dak. & 68 & 1974-1981 & 63 & 53 & 74 \\
\hline 160 & Cannonball River at New England, N. Dak. & 36 & $1978-1981$ & 420 & 45 & 810 \\
\hline 161 & Coal Bank Creek near Havelock, N. Dak. & 84 & $1974-1983$ & 270 & 35 & 660 \\
\hline 162 & Cannonball River at Regent, N. Dak. & 119 & 1970-2008 & 270 & 36 & 490 \\
\hline 163 & Cannonball River below Bentley, N. Dak. & 31 & $1972-1977$ & 290 & 55 & 480 \\
\hline 164 & Cannonball River near Raleigh, N. Dak. & 89 & 1993-2008 & 240 & 10 & 1,060 \\
\hline 165 & White Butte Fork Cedar Creek near Scranton, N. Dak. & 26 & $1972-1995$ & 365 & 44 & 920 \\
\hline 166 & Cedar Creek near Haynes, N. Dak. & 76 & 1971-2008 & 280 & 25 & 530 \\
\hline 167 & Timber Creek near Bentley, N. Dak. & 47 & $1977-1981$ & 390 & 70 & 670 \\
\hline 168 & Cedar Creek near Pretty Rock, N. Dak. & 30 & 1971-1976 & 335 & 33 & 540 \\
\hline 169 & Cedar Creek near Raleigh, N. Dak. & 120 & $1972-2008$ & 240 & 24 & 783 \\
\hline 170 & Cannonball River at Breien, N. Dak. & 260 & 1970-2008 & 243 & 3 & 630 \\
\hline 171 & Beaver Creek near Linton, N. Dak. & 39 & 1972-1993 & 77 & 10 & 140 \\
\hline
\end{tabular}


Table 1-1. Summary statistics for major ion constituents at selected sites in North Dakota from 1970 through 2008. —Continued [ $\mu \mathrm{g} / \mathrm{L}$, micrograms per liter; <, less than; --, not available; $\mathrm{mg} / \mathrm{L}$, milligrams per liter; $\mathrm{CO}_{3}$, carbonate; $\mathrm{HCO}_{3}$, bicarbonate; $\mathrm{CaCO}_{3}$, calcium carbonate $]$

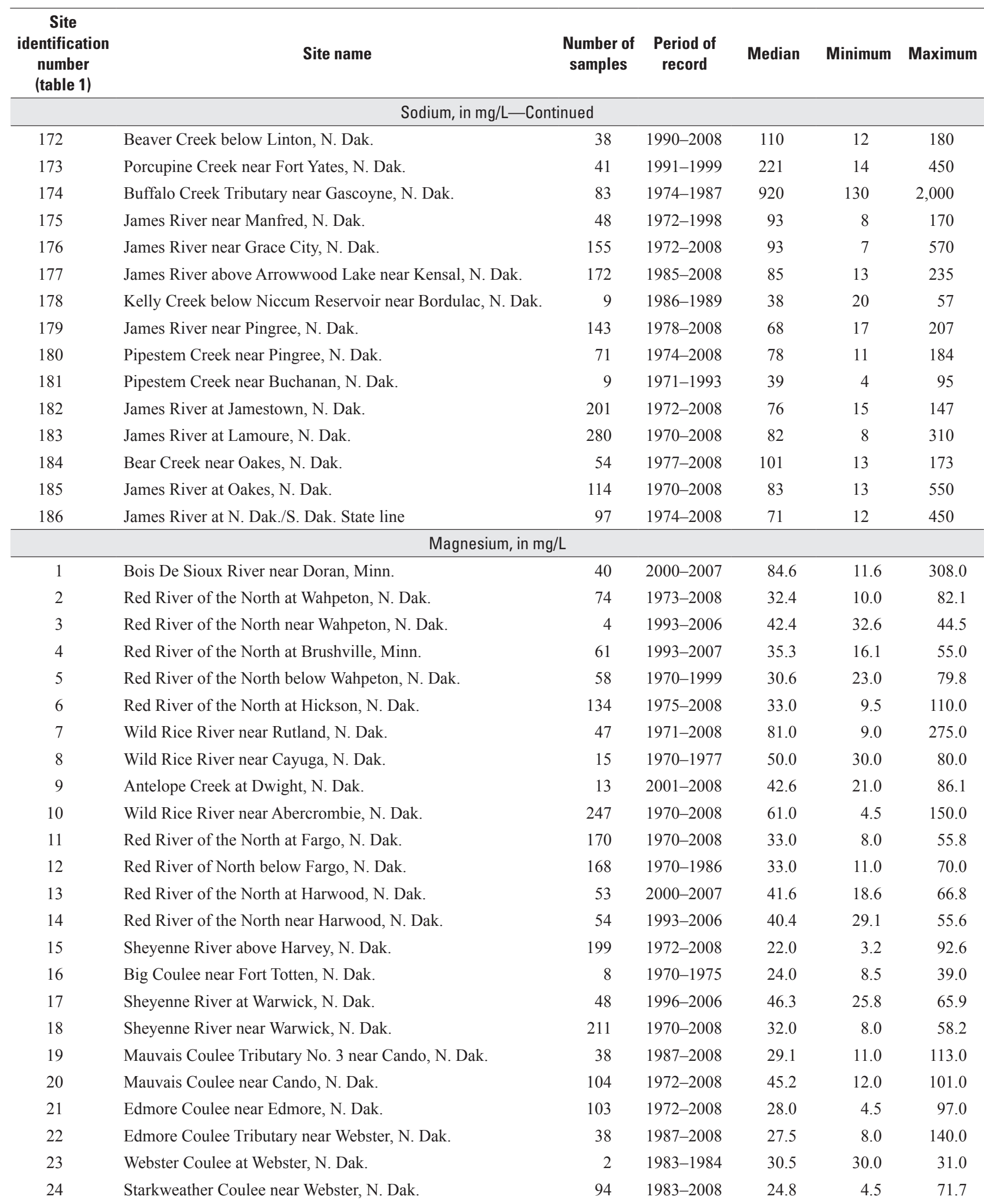


Table 1-1. Summary statistics for major ion constituents at selected sites in North Dakota from 1970 through 2008. —Continued $\left[\mu \mathrm{g} / \mathrm{L}\right.$, micrograms per liter; <, less than; --, not available; $\mathrm{mg} / \mathrm{L}$, milligrams per liter; $\mathrm{CO}_{3}$, carbonate; $\mathrm{HCO}_{3}$, bicarbonate; $\mathrm{CaCO}$, calcium carbonate $]$

\begin{tabular}{|c|c|c|c|c|c|c|}
\hline $\begin{array}{c}\text { Site } \\
\text { identification } \\
\text { number } \\
\text { (table 1) }\end{array}$ & Site name & $\begin{array}{l}\text { Number of } \\
\text { samples }\end{array}$ & $\begin{array}{l}\text { Period of } \\
\text { record }\end{array}$ & Median & Minimum & Maximum \\
\hline \multicolumn{7}{|c|}{ Magnesium, in $\mathrm{mg} / \mathrm{L}$ —Continued } \\
\hline 25 & Big Coulee below Churchs Ferry, N. Dak. & 23 & 1998-2008 & 44.6 & 23.0 & 83.9 \\
\hline 26 & Little Coulee near Leeds, N. Dak. & 16 & $1998-2008$ & 42.7 & 15.0 & 116.0 \\
\hline 27 & Little Coulee near Brinsmade, N. Dak. & 31 & 1976-1998 & 36.0 & 8.3 & 110.0 \\
\hline 28 & Big Coulee near Churchs Ferry, N. Dak. & 136 & $1970-1997$ & 32.0 & 9.8 & 130.0 \\
\hline 29 & Big Coulee at Graham Island inlet near Fort Totten, N. Dak. & 44 & $1970-1986$ & 105.0 & 26.0 & 340.0 \\
\hline 30 & Channel A near Penn, N. Dak. & 85 & 1984-2008 & 32.0 & 16.0 & 110.0 \\
\hline 31 & Devils Lake Outlet to Stump Lake near Lakota, N. Dak. & 12 & $2002-2007$ & 142.0 & 64.0 & 250.0 \\
\hline 32 & Sheyenne River near Cooperstown, N. Dak. & 337 & $1970-2008$ & 35.0 & 7.6 & 65.3 \\
\hline 33 & Baldhill Creek near Dazey, N. Dak. & 94 & 1972-2008 & 39.0 & 7.3 & 68.0 \\
\hline 34 & Sheyenne River below Baldhill Dam, N. Dak. & 165 & $1972-2008$ & 38.4 & 10.0 & 79.0 \\
\hline 35 & Sheyenne River at Valley City, N. Dak. & 29 & $1972-2005$ & 31.0 & 16.0 & 56.9 \\
\hline 36 & Sheyenne River at Lisbon, N. Dak. & 333 & $1970-2008$ & 33.0 & 11.0 & 75.0 \\
\hline 37 & Sheyenne River near Kindred, N. Dak. & 313 & $1972-2008$ & 31.0 & 11.0 & 70.0 \\
\hline 38 & $\begin{array}{l}\text { Sheyenne River above Sheyenne River Diversion near Horace, } \\
\text { N. Dak. }\end{array}$ & 36 & $1993-2008$ & 40.3 & 18.0 & 54.0 \\
\hline 39 & Sheyenne River near Horace, N. Dak. & 8 & $1982-1992$ & 22.5 & 12.0 & 29.0 \\
\hline 40 & Sheyenne River Diversion at West Fargo, N. Dak. & 13 & 1994-2007 & 32.0 & 17.0 & 50.7 \\
\hline 41 & Sheyenne River at West Fargo, N. Dak. & 62 & $1970-2008$ & 31.0 & 13.0 & 72.3 \\
\hline 42 & Maple River near Hope, N. Dak. & 39 & $1972-2008$ & 43.0 & 11.0 & 150.0 \\
\hline 43 & Maple River near Enderlin, N. Dak. & 63 & $1972-2008$ & 60.0 & 9.5 & 100.0 \\
\hline 44 & Maple River near Mapleton, N. Dak. & 25 & $1972-2008$ & 56.6 & 18.0 & 79.5 \\
\hline 45 & Maple River below Mapleton, N. Dak. & 74 & $1995-2008$ & 64.2 & 13.0 & 120.0 \\
\hline 46 & Sheyenne River at Harwood, N. Dak. & 37 & $1993-2005$ & 38.6 & 25.6 & 63.0 \\
\hline 47 & Rush River at Amenia, N. Dak. & 51 & 1972-2008 & 48.6 & 8.5 & 90.4 \\
\hline 48 & Rush River near Prosper, N. Dak. & 4 & $1983-1987$ & 16.0 & 9.5 & 79.0 \\
\hline 49 & Lower Branch Rush River near Prosper, N. Dak. & 5 & $1983-1993$ & 9.0 & 6.5 & 51.4 \\
\hline 50 & Sheyenne River near Harwood, N. Dak. & 39 & $1970-1996$ & 33.0 & 17.0 & 47.0 \\
\hline 51 & Elm River near Kelso, N. Dak. & 6 & $1983-1993$ & 45.4 & 11.0 & 58.7 \\
\hline 52 & Red River of the North at Halstad, Minn. & 178 & $1972-2008$ & 34.0 & 13.0 & 58.0 \\
\hline 53 & Beaver Creek near Finley, N. Dak. & 117 & $1970-2003$ & 45.0 & 7.5 & 130.0 \\
\hline 54 & Goose River near Portland, N. Dak. & 30 & $1970-1988$ & 65.0 & 12.0 & 130.0 \\
\hline 55 & Goose River at Hillsboro, N. Dak. & 166 & $1970-2008$ & 65.2 & 14.0 & 105.0 \\
\hline 56 & Red River of the North at Grand Forks, N. Dak. & 226 & 1970-2008 & 28.0 & 10.7 & 110.0 \\
\hline 57 & Turtle River at Turtle River State Park near Arvilla, N. Dak. & 99 & $1991-2008$ & 36.0 & 6.7 & 60.0 \\
\hline 58 & Turtle River at Manvel, N. Dak. & 105 & $1971-2008$ & 66.2 & 18.0 & 200.0 \\
\hline 59 & Red River of the North at Oslo, Minn. & 75 & $1973-2005$ & 25.0 & 11.0 & 44.0 \\
\hline 60 & Middle Branch Forest River near Whitman, N. Dak. & 14 & $1972-1990$ & 25.0 & 15.0 & 140.0 \\
\hline 61 & Forest River near Fordville, N. Dak. & 64 & $1972-2008$ & 30.0 & 16.0 & 63.3 \\
\hline 62 & Forest River near Minto, N. Dak. & 154 & $1971-2008$ & 39.0 & 7.4 & 140.0 \\
\hline 63 & South Branch Park River below Homme Dam, N. Dak. & 27 & 1972-1994 & 26.0 & 9.0 & 40.0 \\
\hline
\end{tabular}


Table 1-1. Summary statistics for major ion constituents at selected sites in North Dakota from 1970 through 2008.—Continued [ $\mu \mathrm{g} / \mathrm{L}$, micrograms per liter; <, less than; --, not available; $\mathrm{mg} / \mathrm{L}$, milligrams per liter; $\mathrm{CO}_{3}$, carbonate; $\mathrm{HCO}_{3}$, bicarbonate; $\mathrm{CaCO}_{3}$, calcium carbonate $]$

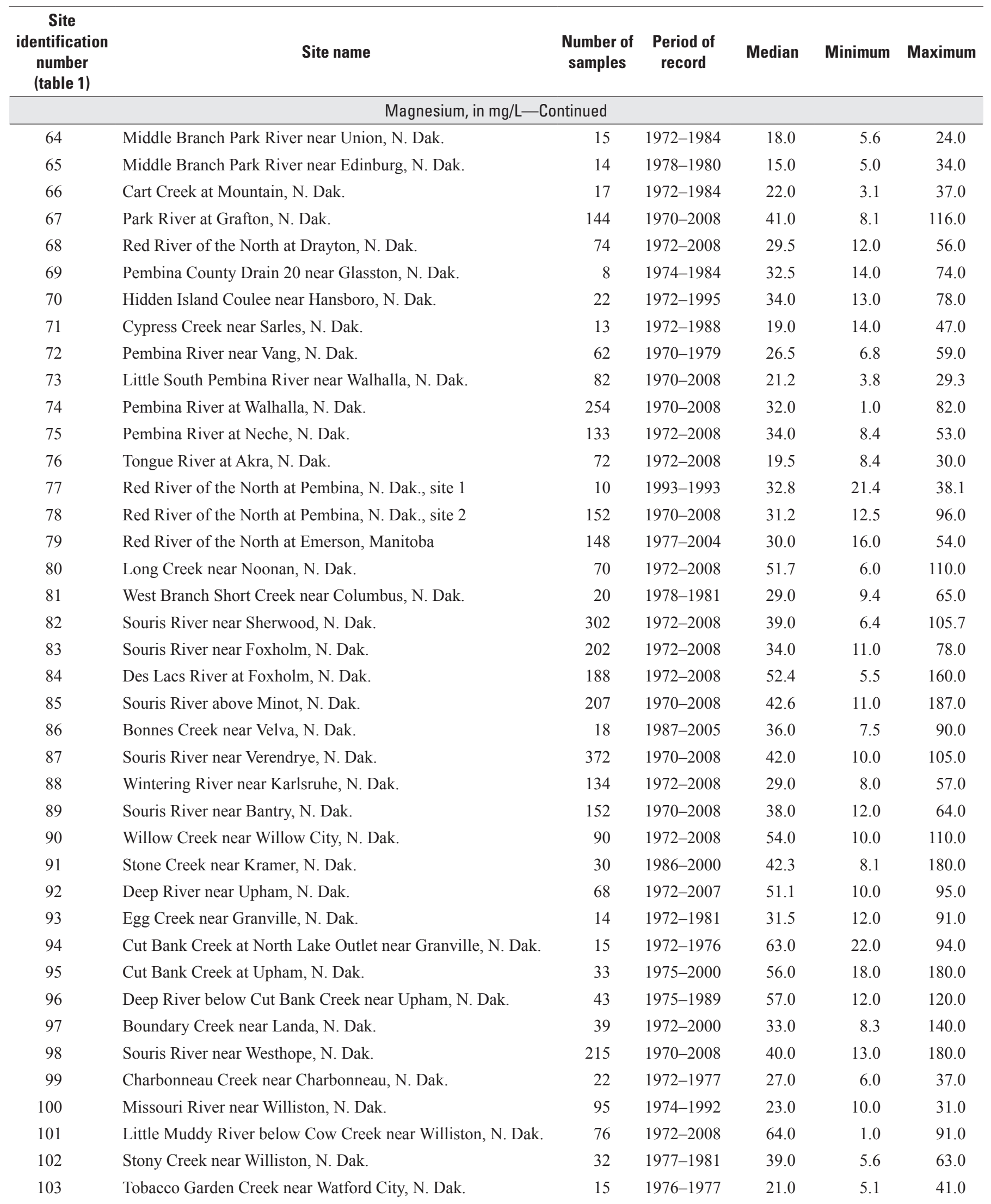


Table 1-1. Summary statistics for major ion constituents at selected sites in North Dakota from 1970 through 2008. —Continued $\left[\mu \mathrm{g} / \mathrm{L}\right.$, micrograms per liter; <, less than; --, not available; $\mathrm{mg} / \mathrm{L}$, milligrams per liter; $\mathrm{CO}_{3}$, carbonate; $\mathrm{HCO}_{3}$, bicarbonate; $\mathrm{CaCO}$, calcium carbonate $]$

\begin{tabular}{|c|c|c|c|c|c|c|}
\hline $\begin{array}{c}\text { Site } \\
\text { identification } \\
\text { number } \\
\text { (table 1) } \\
\end{array}$ & Site name & $\begin{array}{c}\text { Number of } \\
\text { samples }\end{array}$ & $\begin{array}{l}\text { Period of } \\
\text { record }\end{array}$ & Median & Minimum & Maximum \\
\hline \multicolumn{7}{|c|}{ Magnesium, in $\mathrm{mg} / \mathrm{L}$ —Continued } \\
\hline 104 & Beaver Creek near Ray, N. Dak. & 51 & 1977-1982 & 66.0 & 6.9 & 92.0 \\
\hline 105 & White Earth River at White Earth, N. Dak. & 27 & $1970-1977$ & 38.0 & 17.0 & 74.0 \\
\hline 106 & Bear Den Creek near Mandaree, N. Dak. & 228 & 1970-2008 & 25.0 & 4.6 & 230.0 \\
\hline 107 & Shell Creek near Parshall, N. Dak. & 21 & 1972-1977 & 46.0 & 9.2 & 61.0 \\
\hline 108 & East Fork Shell Creek near Parshall, N. Dak. & 58 & 1991-2008 & 64.5 & 5.6 & 127.4 \\
\hline 109 & Deepwater Creek near Mandaree, N. Dak. & 57 & 1991-2008 & 63.0 & 5.5 & 100.0 \\
\hline 110 & Little Missouri River at Marmarth, N. Dak. & 86 & 1971-2008 & 23.9 & 4.6 & 70.0 \\
\hline 111 & Deep Creek near Amidon, N. Dak. & 51 & $1977-1983$ & 120.0 & 21.0 & 250.0 \\
\hline 112 & Little Missouri River at Medora, N. Dak. & 112 & $1972-2008$ & 33.1 & 6.0 & 338.0 \\
\hline 113 & Beaver Creek near Trotters, N. Dak. & 95 & $1977-2008$ & 88.0 & 11.0 & 140.0 \\
\hline 114 & Little Missouri River near Watford City, N. Dak. & 243 & 1971-2008 & 30.0 & 3.2 & 128.0 \\
\hline 115 & Missouri River at Garrison Dam, N. Dak. & 288 & $1971-2007$ & 20.8 & 9.8 & 28.0 \\
\hline 116 & Knife River at Manning, N. Dak. & 118 & $1972-2008$ & 25.0 & 2.5 & 54.0 \\
\hline 117 & Stray Creek near Manning, N. Dak. & 17 & 1975-1981 & 53.0 & 16.0 & 180.0 \\
\hline 118 & Knife River at Marshall, N. Dak. & 69 & $1972-1981$ & 35.0 & 7.3 & 100.0 \\
\hline 119 & Elm Creek near Golden Valley, N. Dak. & 37 & 1973-1995 & 21.0 & 6.0 & 63.0 \\
\hline 120 & Knife River near Golden Valley, N. Dak. & 130 & 1971-2008 & 34.0 & 4.5 & 79.2 \\
\hline 121 & Coyote Creek near Zap, N. Dak. & 51 & $1977-1983$ & 37.0 & 7.0 & 81.0 \\
\hline 122 & Brush Creek near Beulah, N. Dak. & 107 & $1974-1990$ & 57.0 & 8.4 & 97.0 \\
\hline 123 & Spring Creek below Lake Ilo near Dunn Center, N. Dak. & 53 & $1977-1993$ & 43.0 & 5.4 & 110.0 \\
\hline 124 & Spring Creek near Halliday, N. Dak. & 48 & $1977-1981$ & 60.0 & 5.9 & 86.0 \\
\hline 125 & Spring Creek at Zap, N. Dak. & 240 & 1970-2008 & 49.0 & 5.9 & 80.0 \\
\hline 126 & West Branch Otter Creek near Beulah, N. Dak. & 15 & $1972-1995$ & 40.0 & 10.0 & 66.0 \\
\hline 127 & Knife River at Hazen, N. Dak. & 269 & 1970-2008 & 42.0 & 6.3 & 88.4 \\
\hline 128 & Antelope Creek above Hazen, N. Dak. & 44 & $1977-1985$ & 46.5 & 4.8 & 92.0 \\
\hline 129 & West Branch Antelope Creek No. 4 near Zap, N. Dak. & 21 & 1977-1985 & 27.0 & 2.9 & 65.0 \\
\hline 130 & West Branch Antelope Creek near Hazen, N. Dak. & 14 & $1978-1983$ & 27.0 & 5.5 & 81.0 \\
\hline 131 & Coal Creek near Stanton, N. Dak. & 28 & 1975-1981 & 61.0 & 7.9 & 100.0 \\
\hline 132 & Alderin Creek near Fort Clark, N. Dak. & 41 & $1977-1983$ & 34.0 & 5.8 & 73.0 \\
\hline 133 & Coal Lake Coulee near Hensler, N. Dak. & 39 & $1978-1988$ & 41.0 & 2.5 & 130.0 \\
\hline 134 & Buffalo Creek near Washburn, N. Dak. & 38 & $1978-1983$ & 24.0 & 5.6 & 55.0 \\
\hline 135 & Turtle Creek above Washburn, N. Dak. & 100 & $1987-2003$ & 60.0 & 12.0 & 130.1 \\
\hline 136 & Painted Woods Creek near Wilton, N. Dak. & 202 & 1970-2003 & 68.0 & 2.5 & 260.0 \\
\hline 137 & Square Butte Creek near Hannover, N. Dak. & 23 & 1977-1981 & 54.0 & 4.6 & 84.0 \\
\hline 138 & Square Butte Creek above Nelson Lake near Center, N. Dak. & 55 & 1977-1982 & 34.0 & 5.2 & 48.0 \\
\hline 139 & Hagel Creek near Center, N. Dak. & 38 & 1977-1982 & 26.0 & 3.9 & 52.0 \\
\hline 140 & Square Butte Creek below Center, N. Dak. & 81 & $1972-2008$ & 35.0 & 14.0 & 68.0 \\
\hline 141 & Burnt Creek near Bismarck, N. Dak. & 48 & $1972-2008$ & 51.0 & 4.0 & 76.0 \\
\hline 142 & Missouri River at Bismarck, N. Dak. & 127 & 1974-2008 & 21.0 & 13.0 & 26.0 \\
\hline 143 & South Branch Heart River near South Heart, N. Dak. & 19 & 1979-1983 & 8.9 & $<1.0$ & 56.0 \\
\hline
\end{tabular}


Table 1-1. Summary statistics for major ion constituents at selected sites in North Dakota from 1970 through 2008. - Continued [ $\mu \mathrm{g} / \mathrm{L}$, micrograms per liter; <, less than; --, not available; $\mathrm{mg} / \mathrm{L}$, milligrams per liter; $\mathrm{CO}_{3}$, carbonate; $\mathrm{HCO}_{3}$, bicarbonate; $\mathrm{CaCO}_{3}$, calcium carbonate]

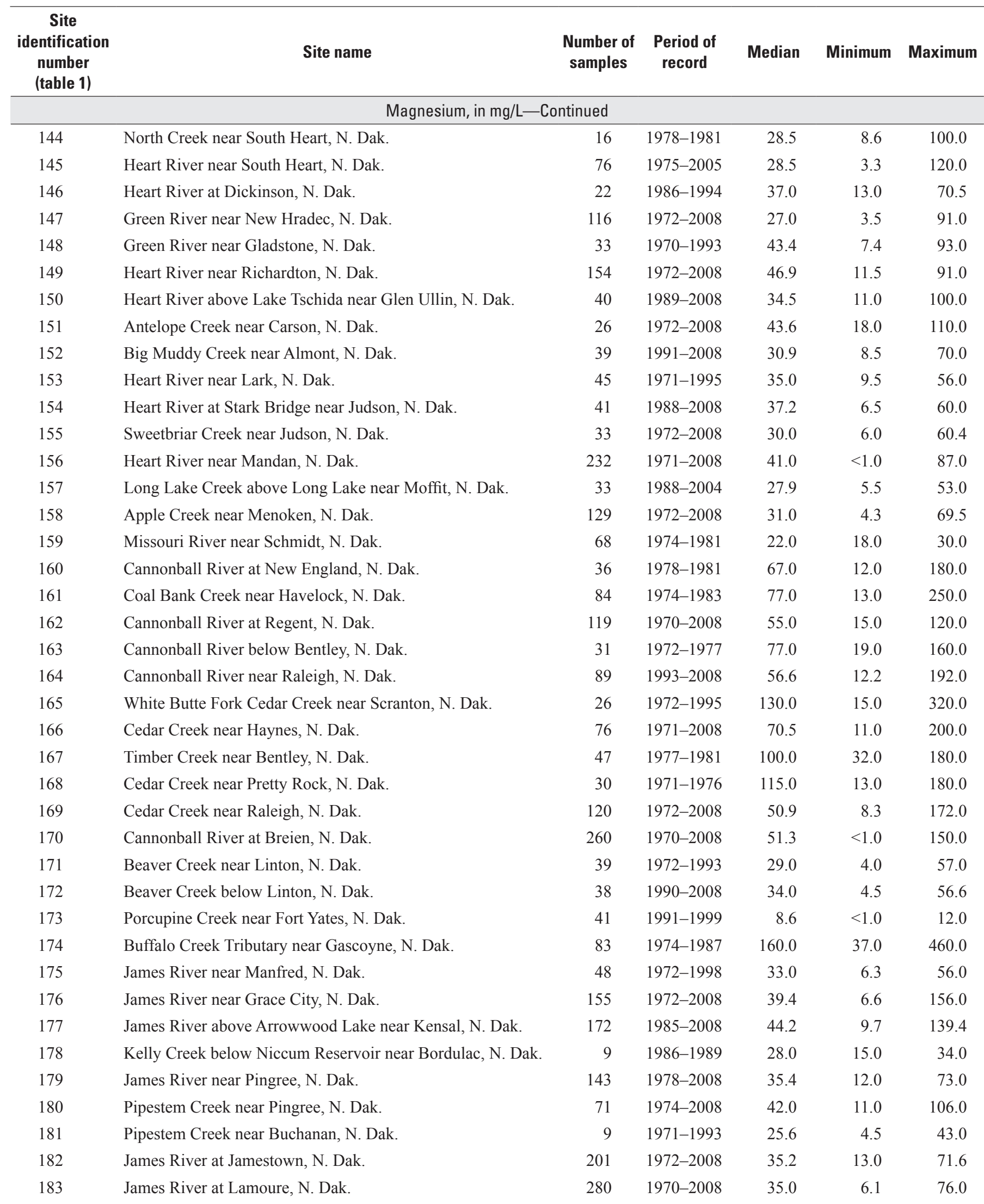


Table 1-1. Summary statistics for major ion constituents at selected sites in North Dakota from 1970 through 2008. —Continued $\left[\mu \mathrm{g} / \mathrm{L}\right.$, micrograms per liter; <, less than; --, not available; $\mathrm{mg} / \mathrm{L}$, milligrams per liter; $\mathrm{CO}_{3}$, carbonate; $\mathrm{HCO}_{3}$, bicarbonate; $\mathrm{CaCO}_{3}$, calcium carbonate $]$

\begin{tabular}{|c|c|c|c|c|c|c|}
\hline $\begin{array}{c}\text { Site } \\
\text { identification } \\
\text { number } \\
\text { (table 1) } \\
\end{array}$ & Site name & $\begin{array}{l}\text { Number of } \\
\text { samples }\end{array}$ & $\begin{array}{l}\text { Period of } \\
\text { record }\end{array}$ & Median & Minimum & Maximum \\
\hline \multicolumn{7}{|c|}{ Magnesium, in $\mathrm{mg} / \mathrm{L}$-Continued } \\
\hline 184 & Bear Creek near Oakes, N. Dak. & 54 & $1977-2008$ & 59.6 & 6.0 & 110.0 \\
\hline 185 & James River at Oakes, N. Dak. & 135 & 1970-2008 & 37.0 & 8.2 & 190.0 \\
\hline 186 & James River at N. Dak./S. Dak. State line & 97 & 1974-2008 & 33.0 & 9.1 & 190.0 \\
\hline \multicolumn{7}{|c|}{ Potassium, in $\mathrm{mg} / \mathrm{L}$} \\
\hline 1 & Bois De Sioux River near Doran, Minn. & 40 & $2000-2007$ & 10.3 & 4.9 & 25.0 \\
\hline 2 & Red River of the North at Wahpeton, N. Dak. & 74 & 1973-2008 & 5.7 & 2.5 & 15.0 \\
\hline 3 & Red River of the North near Wahpeton, N. Dak. & 4 & 1993-2006 & 7.0 & 5.2 & 8.7 \\
\hline 4 & Red River of the North at Brushville, Minn. & 61 & 1993-2007 & 5.9 & 4.0 & 20.0 \\
\hline 5 & Red River of the North below Wahpeton, N. Dak. & 23 & 1970-1999 & 4.9 & 3.6 & 14.2 \\
\hline 6 & Red River of the North at Hickson, N. Dak. & 134 & 1975-2008 & 5.5 & 1.3 & 24.0 \\
\hline 7 & Wild Rice River near Rutland, N. Dak. & 47 & 1971-2008 & 19.4 & 8.5 & 34.4 \\
\hline 8 & Wild Rice River near Cayuga, N. Dak. & 15 & $1970-1977$ & 15.0 & 9.7 & 25.0 \\
\hline 9 & Antelope Creek at Dwight, N. Dak. & 13 & $2001-2008$ & 10.1 & 7.6 & 12.4 \\
\hline 10 & Wild Rice River near Abercrombie, N. Dak. & 249 & 1970-2008 & 14.7 & 1.9 & 47.0 \\
\hline 11 & Red River of the North at Fargo, N. Dak. & 140 & 1970-2008 & 6.0 & 3.2 & 23.2 \\
\hline 12 & Red River of North below Fargo, N. Dak. & 136 & $1970-1986$ & 6.1 & 3.7 & 20.0 \\
\hline 13 & Red River of the North at Harwood, N. Dak. & 53 & $2000-2007$ & 9.6 & 5.0 & 17.5 \\
\hline 14 & Red River of the North near Harwood, N. Dak. & 54 & 1993-2006 & 7.8 & 4.8 & 17.2 \\
\hline 15 & Sheyenne River above Harvey, N. Dak. & 198 & $1972-2008$ & 8.3 & 4.2 & 20.0 \\
\hline 16 & Big Coulee near Fort Totten, N. Dak. & 8 & $1970-1975$ & 4.5 & 3.6 & 29.0 \\
\hline 17 & Sheyenne River at Warwick, N. Dak. & 48 & 1996-2006 & 10.4 & 5.8 & 12.8 \\
\hline 18 & Sheyenne River near Warwick, N. Dak. & 221 & 1970-2008 & 8.3 & 1.8 & 19.0 \\
\hline 19 & Mauvais Coulee Tributary No. 3 near Cando, N. Dak. & 38 & $1987-2008$ & 13.0 & 7.7 & 31.0 \\
\hline 20 & Mauvais Coulee near Cando, N. Dak. & 104 & 1972-2008 & 13.0 & 4.9 & 23.0 \\
\hline 21 & Edmore Coulee near Edmore, N. Dak. & 103 & $1972-2008$ & 14.0 & 5.0 & 32.0 \\
\hline 22 & Edmore Coulee Tributary near Webster, N. Dak. & 38 & 1987-2008 & 13.0 & 8.0 & 28.0 \\
\hline 23 & Webster Coulee at Webster, N. Dak. & 2 & $1983-1984$ & 19.5 & 19.0 & 20.0 \\
\hline 24 & Starkweather Coulee near Webster, N. Dak. & 94 & 1983-2008 & 14.0 & 3.0 & 28.0 \\
\hline 25 & Big Coulee below Churchs Ferry, N. Dak. & 23 & 1998-2008 & 16.0 & 9.7 & 34.5 \\
\hline 26 & Little Coulee near Leeds, N. Dak. & 16 & 1998-2008 & 20.5 & 10.0 & 26.0 \\
\hline 27 & Little Coulee near Brinsmade, N. Dak. & 31 & 1976-1998 & 19.0 & 9.1 & 40.0 \\
\hline 28 & Big Coulee near Churchs Ferry, N. Dak. & 135 & 1970-1997 & 18.0 & 5.3 & 65.0 \\
\hline 29 & Big Coulee at Graham Island inlet near Fort Totten, N. Dak. & 44 & 1970-1986 & 58.5 & 16.0 & 230.0 \\
\hline 30 & Channel A near Penn, N. Dak. & 85 & 1984-2008 & 17.0 & 7.0 & 30.0 \\
\hline 31 & Devils Lake Outlet to Stump Lake near Lakota, N. Dak. & 12 & $2002-2007$ & 74.8 & 37.0 & 125.6 \\
\hline 32 & Sheyenne River near Cooperstown, N. Dak. & 347 & $1970-2008$ & 8.8 & 6.0 & 17.0 \\
\hline 33 & Baldhill Creek near Dazey, N. Dak. & 94 & $1972-2008$ & 9.5 & 4.8 & 17.0 \\
\hline 34 & Sheyenne River below Baldhill Dam, N. Dak. & 165 & $1972-2008$ & 10.7 & 1.8 & 16.0 \\
\hline 35 & Sheyenne River at Valley City, N. Dak. & 29 & $1972-2005$ & 9.8 & 6.5 & 15.0 \\
\hline 36 & Sheyenne River at Lisbon, N. Dak. & 344 & 1970-2008 & 11.0 & 4.9 & 22.0 \\
\hline
\end{tabular}


Table 1-1. Summary statistics for major ion constituents at selected sites in North Dakota from 1970 through 2008.—Continued $\left[\mu \mathrm{g} / \mathrm{L}\right.$, micrograms per liter; <, less than; --, not available; mg/L, milligrams per liter; $\mathrm{CO}_{3}$, carbonate; $\mathrm{HCO}_{3}$, bicarbonate; $\mathrm{CaCO}$, calcium carbonate $]$

\begin{tabular}{|c|c|c|c|c|c|c|}
\hline $\begin{array}{c}\text { Site } \\
\text { identification } \\
\text { number } \\
\text { (table 1) } \\
\end{array}$ & Site name & $\begin{array}{l}\text { Number of } \\
\text { samples }\end{array}$ & $\begin{array}{l}\text { Period of } \\
\text { record }\end{array}$ & Median & Minimum & Maximum \\
\hline \multicolumn{7}{|c|}{ Potassium, in $\mathrm{mg} / \mathrm{L}-$ Continued } \\
\hline 37 & Sheyenne River near Kindred, N. Dak. & 312 & $1972-2008$ & 9.4 & 3.8 & 15.0 \\
\hline 38 & $\begin{array}{l}\text { Sheyenne River above Sheyenne River Diversion near Horace, } \\
\text { N. Dak. }\end{array}$ & 36 & 1993-2008 & 9.9 & 7.5 & 12.0 \\
\hline 39 & Sheyenne River near Horace, N. Dak. & 8 & 1982-1992 & 9.7 & 6.5 & 15.0 \\
\hline 40 & Sheyenne River Diversion at West Fargo, N. Dak. & 13 & 1994-2007 & 9.7 & 7.4 & 12.3 \\
\hline 41 & Sheyenne River at West Fargo, N. Dak. & 62 & $1970-2008$ & 9.1 & 5.7 & 14.0 \\
\hline 42 & Maple River near Hope, N. Dak. & 39 & $1972-2008$ & 9.0 & 3.5 & 22.0 \\
\hline 43 & Maple River near Enderlin, N. Dak. & 63 & $1972-2008$ & 12.0 & 8.3 & 24.0 \\
\hline 47 & Rush River at Amenia, N. Dak. & 51 & $1972-2008$ & 11.1 & 4.9 & 19.0 \\
\hline 48 & Rush River near Prosper, N. Dak. & 4 & $1983-1987$ & 12.0 & 7.9 & 17.0 \\
\hline 49 & Lower Branch Rush River near Prosper, N. Dak. & 5 & $1983-1993$ & 7.6 & 6.2 & 11.8 \\
\hline 50 & Sheyenne River near Harwood, N. Dak. & 3 & 1970-1996 & 8.9 & 7.3 & 11.0 \\
\hline 51 & Elm River near Kelso, N. Dak. & 6 & $1983-1993$ & 13.4 & 9.5 & 16.0 \\
\hline 52 & Red River of the North at Halstad, Minn. & 178 & $1972-2008$ & 7.2 & 3.9 & 18.0 \\
\hline 53 & Beaver Creek near Finley, N. Dak. & 116 & $1970-2003$ & 11.0 & 1.5 & 19.0 \\
\hline 54 & Goose River near Portland, N. Dak. & 30 & 1970-1988 & 11.5 & 4.9 & 19.0 \\
\hline 61 & Forest River near Fordville, N. Dak. & 64 & $1972-2008$ & 6.1 & 3.2 & 13.0 \\
\hline 62 & Forest River near Minto, N. Dak. & 154 & $1971-2008$ & 7.3 & 3.6 & 62.0 \\
\hline 63 & South Branch Park River below Homme Dam, N. Dak. & 27 & $1972-1994$ & 7.8 & 4.0 & 12.0 \\
\hline 64 & Middle Branch Park River near Union, N. Dak. & 15 & $1972-1984$ & 5.6 & 1.6 & 18.0 \\
\hline 65 & Middle Branch Park River near Edinburg, N. Dak. & 14 & 1978-1980 & 6.1 & 4.1 & 9.6 \\
\hline 66 & Cart Creek at Mountain, N. Dak. & 17 & $1972-1984$ & 5.1 & 1.6 & 11.0 \\
\hline 67 & Park River at Grafton, N. Dak. & 144 & $1970-2008$ & 9.1 & 3.8 & 16.0 \\
\hline 68 & Red River of the North at Drayton, N. Dak. & 74 & $1972-2008$ & 7.3 & 3.1 & 12.0 \\
\hline 69 & Pembina County Drain 20 near Glasston, N. Dak. & 8 & $1974-1984$ & 6.7 & 4.2 & 14.0 \\
\hline 70 & Hidden Island Coulee near Hansboro, N. Dak. & 22 & 1972-1995 & 11.0 & 5.2 & 20.0 \\
\hline 71 & Cypress Creek near Sarles, N. Dak. & 13 & 1972-1988 & 8.3 & 3.7 & 12.0 \\
\hline 72 & Pembina River near Vang, N. Dak. & 62 & 1970-1979 & 8.6 & 3.2 & 12.0 \\
\hline 73 & Little South Pembina River near Walhalla, N. Dak. & 82 & $1970-2008$ & 7.6 & 2.5 & 12.0 \\
\hline 74 & Pembina River at Walhalla, N. Dak. & 226 & 1970-2008 & 9.0 & $<1.0$ & 21.0 \\
\hline 75 & Pembina River at Neche, N. Dak. & 133 & $1972-2008$ & 10.4 & 3.2 & 13.9 \\
\hline
\end{tabular}


Table 1-1. Summary statistics for major ion constituents at selected sites in North Dakota from 1970 through 2008. —Continued $\left[\mu \mathrm{g} / \mathrm{L}\right.$, micrograms per liter; <, less than; --, not available; $\mathrm{mg} / \mathrm{L}$, milligrams per liter; $\mathrm{CO}_{3}$, carbonate; $\mathrm{HCO}_{3}$, bicarbonate; $\mathrm{CaCO}$, calcium carbonate $]$

\begin{tabular}{|c|c|c|c|c|c|c|}
\hline $\begin{array}{c}\text { Site } \\
\text { identification } \\
\text { number } \\
\text { (table 1) }\end{array}$ & Site name & $\begin{array}{c}\text { Number of } \\
\text { samples }\end{array}$ & $\begin{array}{l}\text { Period of } \\
\text { record }\end{array}$ & Median & Minimum & Maximum \\
\hline \multicolumn{7}{|c|}{ Potassium, in $\mathrm{mg} / \mathrm{L}-$ Continued } \\
\hline 76 & Tongue River at Akra, N. Dak. & 72 & 1972-2008 & 6.1 & 3.8 & 11.0 \\
\hline 77 & Red River of the North at Pembina, N. Dak., site 1 & 10 & $1993-1993$ & 8.0 & 4.3 & 11.2 \\
\hline 78 & Red River of the North at Pembina, N. Dak., site 2 & 144 & 1970-2008 & 7.4 & 4.3 & 11.4 \\
\hline 79 & Red River of the North at Emerson, Manitoba & 148 & 1977-2004 & 6.7 & 3.8 & 17.0 \\
\hline 80 & Long Creek near Noonan, N. Dak. & 70 & 1972-2008 & 12.2 & 5.4 & 23.0 \\
\hline 81 & West Branch Short Creek near Columbus, N. Dak. & 20 & $1978-1981$ & 11.0 & 7.0 & 17.0 \\
\hline 82 & Souris River near Sherwood, N. Dak. & 200 & $1972-2008$ & 11.0 & 6.5 & 21.7 \\
\hline 83 & Souris River near Foxholm, N. Dak. & 172 & $1972-2008$ & 13.0 & $<1.0$ & 26.0 \\
\hline 84 & Des Lacs River at Foxholm, N. Dak. & 188 & 1972-2008 & 12.0 & 4.1 & 29.6 \\
\hline 85 & Souris River above Minot, N. Dak. & 169 & 1970-2008 & 14.0 & 6.2 & 29.2 \\
\hline 86 & Bonnes Creek near Velva, N. Dak. & 18 & $1987-2005$ & 12.0 & 7.6 & 19.0 \\
\hline 87 & Souris River near Verendrye, N. Dak. & 288 & 1970-2008 & 12.9 & 6.0 & 25.0 \\
\hline 88 & Wintering River near Karlsruhe, N. Dak. & 134 & 1972-2008 & 6.0 & 1.1 & 20.0 \\
\hline 89 & Souris River near Bantry, N. Dak. & 108 & $1970-2008$ & 11.0 & 2.0 & 15.7 \\
\hline 90 & Willow Creek near Willow City, N. Dak. & 84 & 1972-2008 & 12.6 & $<1.0$ & 77.0 \\
\hline 91 & Stone Creek near Kramer, N. Dak. & 25 & $1986-2000$ & 18.0 & 9.2 & 24.0 \\
\hline 92 & Deep River near Upham, N. Dak. & 68 & 1972-2007 & 16.1 & 8.2 & 27.0 \\
\hline 93 & Egg Creek near Granville, N. Dak. & 14 & $1972-1981$ & 11.5 & 7.7 & 19.0 \\
\hline 94 & Cut Bank Creek at North Lake Outlet near Granville, N. Dak. & 15 & $1972-1976$ & 16.0 & 8.0 & 37.0 \\
\hline 95 & Cut Bank Creek at Upham, N. Dak. & 33 & $1975-2000$ & 16.5 & 10.0 & 44.0 \\
\hline 96 & Deep River below Cut Bank Creek near Upham, N. Dak. & 43 & 1975-1989 & 15.0 & 9.6 & 27.0 \\
\hline 97 & Boundary Creek near Landa, N. Dak. & 33 & $1972-2000$ & 12.0 & 6.8 & 27.0 \\
\hline 98 & Souris River near Westhope, N. Dak. & 201 & $1970-2008$ & 14.0 & 4.1 & 61.0 \\
\hline 99 & Charbonneau Creek near Charbonneau, N. Dak. & 22 & $1972-1977$ & 8.1 & 5.2 & 10.0 \\
\hline 100 & Missouri River near Williston, N. Dak. & 95 & 1974-1992 & 4.3 & 2.7 & 17.0 \\
\hline 101 & Little Muddy River below Cow Creek near Williston, N. Dak. & 77 & 1972-2008 & 9.9 & 5.9 & 16.0 \\
\hline 102 & Stony Creek near Williston, N. Dak. & 32 & $1977-1981$ & 10.0 & 5.8 & 17.0 \\
\hline 103 & Tobacco Garden Creek near Watford City, N. Dak. & 15 & $1976-1977$ & 8.0 & 6.0 & 9.4 \\
\hline 104 & Beaver Creek near Ray, N. Dak. & 51 & $1977-1982$ & 9.7 & 6.8 & 12.0 \\
\hline 105 & White Earth River at White Earth, N. Dak. & 27 & $1970-1977$ & 8.1 & 4.5 & 15.0 \\
\hline 106 & Bear Den Creek near Mandaree, N. Dak. & 228 & 1970-2008 & 7.6 & $<1.0$ & 14.0 \\
\hline 107 & Shell Creek near Parshall, N. Dak. & 21 & 1972-1977 & 8.0 & 6.4 & 11.0 \\
\hline 108 & East Fork Shell Creek near Parshall, N. Dak. & 58 & $1991-2008$ & 11.0 & 2.6 & 19.0 \\
\hline 109 & Deepwater Creek near Mandaree, N. Dak. & 57 & $1991-2008$ & 10.6 & 7.1 & 19.7 \\
\hline 110 & Little Missouri River at Marmarth, N. Dak. & 86 & 1971-2008 & 7.6 & 3.8 & 16.0 \\
\hline 111 & Deep Creek near Amidon, N. Dak. & 51 & $1977-1983$ & 14.0 & 6.4 & 22.0 \\
\hline 112 & Little Missouri River at Medora, N. Dak. & 112 & $1972-2008$ & 10.1 & 1.8 & 67.7 \\
\hline 113 & Beaver Creek near Trotters, N. Dak. & 94 & $1977-2008$ & 11.0 & 6.0 & 17.0 \\
\hline 114 & Little Missouri River near Watford City, N. Dak. & 243 & $1971-2008$ & 10.0 & 4.4 & 49.9 \\
\hline 115 & Missouri River at Garrison Dam, N. Dak. & 288 & 1971-2007 & 4.0 & 3.1 & 8.2 \\
\hline
\end{tabular}


Table 1-1. Summary statistics for major ion constituents at selected sites in North Dakota from 1970 through 2008.—Continued $\left[\mu \mathrm{g} / \mathrm{L}\right.$, micrograms per liter; <, less than; --, not available; mg/L, milligrams per liter; $\mathrm{CO}_{3}$, carbonate; $\mathrm{HCO}_{3}$, bicarbonate; $\mathrm{CaCO}$, calcium carbonate $]$

\begin{tabular}{|c|c|c|c|c|c|c|}
\hline $\begin{array}{c}\text { Site } \\
\text { identification } \\
\text { number } \\
\text { (table 1) }\end{array}$ & Site name & $\begin{array}{l}\text { Number of } \\
\text { samples }\end{array}$ & $\begin{array}{l}\text { Period of } \\
\text { record }\end{array}$ & Median & Minimum & Maximum \\
\hline \multicolumn{7}{|c|}{ Potassium, in $\mathrm{mg} / \mathrm{L}$-Continued } \\
\hline 116 & Knife River at Manning, N. Dak. & 118 & $1972-2008$ & 8.5 & 4.6 & 16.0 \\
\hline 117 & Stray Creek near Manning, N. Dak. & 17 & $1975-1981$ & 12.0 & 6.6 & 21.0 \\
\hline 119 & Elm Creek near Golden Valley, N. Dak. & 37 & $1973-1995$ & 11.0 & 6.2 & 610.0 \\
\hline 120 & Knife River near Golden Valley, N. Dak. & 130 & $1971-2008$ & 10.7 & 6.3 & 18.0 \\
\hline 121 & Coyote Creek near Zap, N. Dak. & 51 & $1977-1983$ & 9.7 & 4.7 & 15.0 \\
\hline 122 & Brush Creek near Beulah, N. Dak. & 98 & 1974-1988 & 9.0 & 6.7 & 14.0 \\
\hline 126 & West Branch Otter Creek near Beulah, N. Dak. & 15 & $1972-1995$ & 9.2 & 5.5 & 13.0 \\
\hline 127 & Knife River at Hazen, N. Dak. & 270 & $1970-2008$ & 9.0 & 1.0 & 15.0 \\
\hline 128 & Antelope Creek above Hazen, N. Dak. & 28 & $1977-1982$ & 12.0 & 6.6 & 23.0 \\
\hline 129 & West Branch Antelope Creek No. 4 near Zap, N. Dak. & 10 & $1977-1982$ & 7.1 & 5.2 & 13.0 \\
\hline 130 & West Branch Antelope Creek near Hazen, N. Dak. & 14 & $1978-1983$ & 8.5 & 6.9 & 14.0 \\
\hline 131 & Coal Creek near Stanton, N. Dak. & 28 & $1975-1981$ & 10.5 & 1.2 & 23.0 \\
\hline 132 & Alderin Creek near Fort Clark, N. Dak. & 35 & 1977-1982 & 10.0 & 5.3 & 15.0 \\
\hline 133 & Coal Lake Coulee near Hensler, N. Dak. & 17 & $1978-1982$ & 8.0 & 4.9 & 22.0 \\
\hline 134 & Buffalo Creek near Washburn, N. Dak. & 38 & $1978-1983$ & 9.6 & 6.1 & 30.0 \\
\hline 141 & Burnt Creek near Bismarck, N. Dak. & 48 & $1972-2008$ & 9.0 & 5.1 & 21.0 \\
\hline 142 & Missouri River at Bismarck, N. Dak. & 127 & 1974-2008 & 4.1 & 2.7 & 6.9 \\
\hline 143 & South Branch Heart River near South Heart, N. Dak. & 20 & $1979-1983$ & 6.4 & 2.2 & 12.0 \\
\hline 144 & North Creek near South Heart, N. Dak. & 16 & 1978-1981 & 9.7 & 4.9 & 17.0 \\
\hline 145 & Heart River near South Heart, N. Dak. & 76 & $1975-2005$ & 8.2 & 3.7 & 24.0 \\
\hline 146 & Heart River at Dickinson, N. Dak. & 22 & 1986-1994 & 8.8 & 3.5 & 16.0 \\
\hline 147 & Green River near New Hradec, N. Dak. & 116 & $1972-2008$ & 6.3 & 3.7 & 85.0 \\
\hline 148 & Green River near Gladstone, N. Dak. & 33 & $1970-1993$ & 6.7 & 4.9 & 9.5 \\
\hline 149 & Heart River near Richardton, N. Dak. & 154 & $1972-2008$ & 9.8 & 4.3 & 19.0 \\
\hline 150 & Heart River above Lake Tschida near Glen Ullin, N. Dak. & 40 & 1989-2008 & 12.0 & 4.6 & 16.0 \\
\hline 151 & Antelope Creek near Carson, N. Dak. & 26 & $1972-2008$ & 8.3 & 4.5 & 12.0 \\
\hline 152 & Big Muddy Creek near Almont, N. Dak. & 39 & 1991-2008 & 9.9 & 3.9 & 15.0 \\
\hline 153 & Heart River near Lark, N. Dak. & 45 & 1971-1995 & 8.1 & 4.4 & 14.0 \\
\hline 154 & Heart River at Stark Bridge near Judson, N. Dak. & 41 & 1988-2008 & 11.0 & 4.1 & 13.0 \\
\hline 155 & Sweetbriar Creek near Judson, N. Dak. & 33 & $1972-2008$ & 7.1 & 2.7 & 13.8 \\
\hline
\end{tabular}


Table 1-1. Summary statistics for major ion constituents at selected sites in North Dakota from 1970 through 2008. —Continued $\left[\mu \mathrm{g} / \mathrm{L}\right.$, micrograms per liter; <, less than; --, not available; $\mathrm{mg} / \mathrm{L}$, milligrams per liter; $\mathrm{CO}_{3}$, carbonate; $\mathrm{HCO}_{3}$, bicarbonate; $\mathrm{CaCO}$, calcium carbonate $]$

\begin{tabular}{|c|c|c|c|c|c|c|}
\hline $\begin{array}{c}\text { Site } \\
\text { identification } \\
\text { number } \\
\text { (table 1) }\end{array}$ & Site name & $\begin{array}{l}\text { Number of } \\
\text { samples }\end{array}$ & $\begin{array}{l}\text { Period of } \\
\text { record }\end{array}$ & Median & Minimum & Maximum \\
\hline \multicolumn{7}{|c|}{ Potassium, in $\mathrm{mg} / \mathrm{L}-$ Continued } \\
\hline 156 & Heart River near Mandan, N. Dak. & 232 & 1971-2008 & 9.1 & 2.5 & 14.1 \\
\hline 157 & Long Lake Creek above Long Lake near Moffit, N. Dak. & 33 & 1988-2004 & 12.1 & 6.7 & 43.0 \\
\hline 158 & Apple Creek near Menoken, N. Dak. & 128 & $1972-2008$ & 8.6 & 4.7 & 43.0 \\
\hline 159 & Missouri River near Schmidt, N. Dak. & 68 & 1974-1981 & 4.2 & 3.7 & 5.5 \\
\hline 160 & Cannonball River at New England, N. Dak. & 36 & $1978-1981$ & 10.0 & $<1.0$ & 15.0 \\
\hline 161 & Coal Bank Creek near Havelock, N. Dak. & 84 & $1974-1983$ & 9.1 & 4.4 & 15.0 \\
\hline 162 & Cannonball River at Regent, N. Dak. & 119 & 1970-2008 & 8.3 & 3.5 & 17.0 \\
\hline 163 & Cannonball River below Bentley, N. Dak. & 31 & $1972-1977$ & 7.8 & 5.2 & 14.0 \\
\hline 164 & Cannonball River near Raleigh, N. Dak. & 89 & 1993-2008 & 11.7 & 5.5 & 28.2 \\
\hline 165 & White Butte Fork Cedar Creek near Scranton, N. Dak. & 26 & $1972-1995$ & 11.0 & 6.4 & 30.0 \\
\hline 166 & Cedar Creek near Haynes, N. Dak. & 76 & $1971-2008$ & 11.0 & 5.0 & 17.0 \\
\hline 167 & Timber Creek near Bentley, N. Dak. & 47 & 1977-1981 & 12.0 & 1.2 & 20.0 \\
\hline 168 & Cedar Creek near Pretty Rock, N. Dak. & 30 & 1971-1976 & 10.0 & 8.5 & 14.0 \\
\hline 169 & Cedar Creek near Raleigh, N. Dak. & 120 & $1972-2008$ & 11.1 & 4.7 & 18.5 \\
\hline 170 & Cannonball River at Breien, N. Dak. & 260 & 1970-2008 & 10.0 & $<1.0$ & 23.2 \\
\hline 171 & Beaver Creek near Linton, N. Dak. & 39 & $1972-1993$ & 12.0 & 5.9 & 16.0 \\
\hline 172 & Beaver Creek below Linton, N. Dak. & 38 & 1990-2008 & 15.1 & 7.2 & 18.9 \\
\hline 173 & Porcupine Creek near Fort Yates, N. Dak. & 41 & 1991-1999 & 6.4 & $<1.0$ & 8.7 \\
\hline 174 & Buffalo Creek Tributary near Gascoyne, N. Dak. & 83 & $1974-1987$ & 14.0 & $<1.0$ & 33.0 \\
\hline 175 & James River near Manfred, N. Dak. & 48 & $1972-1998$ & 11.0 & 2.0 & 19.0 \\
\hline 176 & James River near Grace City, N. Dak. & 155 & $1972-2008$ & 14.0 & 5.6 & 25.3 \\
\hline 177 & James River above Arrowwood Lake near Kensal, N. Dak. & 172 & 1985-2008 & 13.4 & 3.6 & 26.0 \\
\hline 178 & Kelly Creek below Niccum Reservoir near Bordulac, N. Dak. & 9 & 1986-1989 & 11.0 & 6.5 & 16.0 \\
\hline 179 & James River near Pingree, N. Dak. & 143 & 1978-2008 & 14.0 & 2.7 & 35.0 \\
\hline 180 & Pipestem Creek near Pingree, N. Dak. & 71 & 1974-2008 & 12.3 & 5.9 & 22.0 \\
\hline 181 & Pipestem Creek near Buchanan, N. Dak. & 9 & 1971-1993 & 10.0 & 9.3 & 12.6 \\
\hline 182 & James River at Jamestown, N. Dak. & 201 & 1972-2008 & 12.9 & $<1.0$ & 107.0 \\
\hline 183 & James River at Lamoure, N. Dak. & 284 & 1970-2008 & 13.0 & 1.9 & 45.9 \\
\hline 184 & Bear Creek near Oakes, N. Dak. & 54 & 1977-2008 & 14.7 & 8.2 & 23.0 \\
\hline 185 & James River at Oakes, N. Dak. & 101 & $1970-2008$ & 14.0 & 5.9 & 46.0 \\
\hline 186 & James River at N. Dak./S. Dak. State line & 97 & 1974-2008 & 13.9 & 5.6 & 40.0 \\
\hline \multicolumn{7}{|c|}{ Calcium, in mg/L } \\
\hline 1 & Bois De Sioux River near Doran, Minn. & 40 & $2000-2007$ & 118.0 & 28.4 & 352.0 \\
\hline 2 & Red River of the North at Wahpeton, N. Dak. & 74 & 1973-2008 & 50.0 & 27.0 & 123.0 \\
\hline 3 & Red River of the North near Wahpeton, N. Dak. & 4 & 1993-2006 & 58.7 & 45.7 & 62.1 \\
\hline 4 & Red River of the North at Brushville, Minn. & 61 & 1993-2007 & 51.5 & 38.2 & 84.1 \\
\hline 5 & Red River of the North below Wahpeton, N. Dak. & 58 & 1970-1999 & 43.0 & 36.0 & 124.0 \\
\hline 6 & Red River of the North at Hickson, N. Dak. & 134 & 1975-2008 & 50.0 & 21.0 & 140.0 \\
\hline 7 & Wild Rice River near Rutland, N. Dak. & 47 & $1971-2008$ & 99.0 & 23.0 & 168.0 \\
\hline 8 & Wild Rice River near Cayuga, N. Dak. & 15 & 1970-1977 & 84.0 & 55.0 & 160.0 \\
\hline
\end{tabular}


Table 1-1. Summary statistics for major ion constituents at selected sites in North Dakota from 1970 through 2008.—Continued $\left[\mu \mathrm{g} / \mathrm{L}\right.$, micrograms per liter; <, less than; --, not available; mg/L, milligrams per liter; $\mathrm{CO}_{3}$, carbonate; $\mathrm{HCO}_{3}$, bicarbonate; $\mathrm{CaCO}$, calcium carbonate $]$

\begin{tabular}{|c|c|c|c|c|c|c|}
\hline $\begin{array}{c}\text { Site } \\
\text { identification } \\
\text { number } \\
\text { (table 1) }\end{array}$ & Site name & $\begin{array}{l}\text { Number of } \\
\text { samples }\end{array}$ & $\begin{array}{l}\text { Period of } \\
\text { record }\end{array}$ & Median & Minimum & Maximum \\
\hline \multicolumn{7}{|c|}{ Calcium, in $\mathrm{mg} / \mathrm{L}$-Continued } \\
\hline 9 & Antelope Creek at Dwight, N. Dak. & 13 & $2001-2008$ & 73.1 & 42.0 & 118.0 \\
\hline 10 & Wild Rice River near Abercrombie, N. Dak. & 247 & 1970-2008 & 110.0 & 13.0 & 290.0 \\
\hline 11 & Red River of the North at Fargo, N. Dak. & 170 & 1970-2008 & 47.8 & 21.0 & 84.9 \\
\hline 12 & Red River of North below Fargo, N. Dak. & 168 & 1970-1986 & 48.0 & 30.0 & 98.0 \\
\hline 13 & Red River of the North at Harwood, N. Dak. & 53 & $2000-2007$ & 62.4 & 39.4 & 96.2 \\
\hline 14 & Red River of the North near Harwood, N. Dak. & 54 & 1993-2006 & 64.9 & 42.7 & 80.5 \\
\hline 15 & Sheyenne River above Harvey, N. Dak. & 199 & $1972-2008$ & 34.0 & 13.0 & 140.0 \\
\hline 16 & Big Coulee near Fort Totten, N. Dak. & 8 & $1970-1975$ & 75.0 & 18.0 & 81.0 \\
\hline 17 & Sheyenne River at Warwick, N. Dak. & 48 & 1996-2006 & 66.5 & 40.3 & 109.0 \\
\hline 18 & Sheyenne River near Warwick, N. Dak. & 211 & 1970-2008 & 53.0 & 17.0 & 100.0 \\
\hline 19 & Mauvais Coulee Tributary No. 3 near Cando, N. Dak. & 38 & $1987-2008$ & 62.5 & 24.0 & 170.0 \\
\hline 20 & Mauvais Coulee near Cando, N. Dak. & 104 & $1972-2008$ & 77.0 & 26.0 & 170.0 \\
\hline 21 & Edmore Coulee near Edmore, N. Dak. & 103 & $1972-2008$ & 59.0 & 18.0 & 160.0 \\
\hline 22 & Edmore Coulee Tributary near Webster, N. Dak. & 38 & $1987-2008$ & 56.5 & 27.0 & 170.0 \\
\hline 23 & Webster Coulee at Webster, N. Dak. & 2 & $1983-1984$ & 49.5 & 46.0 & 53.0 \\
\hline 24 & Starkweather Coulee near Webster, N. Dak. & 94 & $1983-2008$ & 56.5 & 13.0 & 106.0 \\
\hline 25 & Big Coulee below Churchs Ferry, N. Dak. & 23 & 1998-2008 & 71.0 & 46.0 & 130.0 \\
\hline 26 & Little Coulee near Leeds, N. Dak. & 16 & 1998-2008 & 55.5 & 30.0 & 149.0 \\
\hline 27 & Little Coulee near Brinsmade, N. Dak. & 31 & 1976-1998 & 66.0 & 20.0 & 310.0 \\
\hline 28 & Big Coulee near Churchs Ferry, N. Dak. & 136 & 1970-1997 & 59.0 & 22.0 & 190.0 \\
\hline 29 & Big Coulee at Graham Island inlet near Fort Totten, N. Dak. & 44 & 1970-1986 & 72.0 & 50.0 & 150.0 \\
\hline 30 & Channel A near Penn, N. Dak. & 85 & 1984-2008 & 58.0 & 30.0 & 170.0 \\
\hline 31 & Devils Lake Outlet to Stump Lake near Lakota, N. Dak. & 12 & $2002-2007$ & 66.7 & 35.0 & 130.0 \\
\hline 32 & Sheyenne River near Cooperstown, N. Dak. & 337 & 1970-2008 & 68.6 & 19.0 & 120.0 \\
\hline 33 & Baldhill Creek near Dazey, N. Dak. & 94 & $1972-2008$ & 66.0 & 20.0 & 160.0 \\
\hline 34 & Sheyenne River below Baldhill Dam, N. Dak. & 165 & $1972-2008$ & 61.6 & 22.0 & 117.0 \\
\hline 35 & Sheyenne River at Valley City, N. Dak. & 29 & $1972-2005$ & 51.0 & 30.0 & 79.0 \\
\hline 36 & Sheyenne River at Lisbon, N. Dak. & 333 & 1970-2008 & 65.0 & 30.0 & 130.0 \\
\hline 37 & Sheyenne River near Kindred, N. Dak. & 313 & $1972-2008$ & 78.0 & 28.0 & 117.0 \\
\hline 38 & $\begin{array}{l}\text { Sheyenne River above Sheyenne River Diversion near Horace, } \\
\text { N. Dak. }\end{array}$ & 36 & 1993-2008 & 76.4 & 40.0 & 110.0 \\
\hline 39 & Sheyenne River near Horace, N. Dak. & 8 & 1982-1992 & 49.5 & 31.0 & 76.0 \\
\hline 40 & Sheyenne River Diversion at West Fargo, N. Dak. & 13 & 1994-2007 & 66.0 & 39.0 & 81.6 \\
\hline 41 & Sheyenne River at West Fargo, N. Dak. & 62 & 1970-2008 & 77.0 & 33.0 & 110.0 \\
\hline 42 & Maple River near Hope, N. Dak. & 39 & $1972-2008$ & 85.2 & 26.0 & 230.0 \\
\hline 43 & Maple River near Enderlin, N. Dak. & 63 & $1972-2008$ & 130.0 & 26.0 & 200.0 \\
\hline 44 & Maple River near Mapleton, N. Dak. & 25 & $1972-2008$ & 110.0 & 40.0 & 140.0 \\
\hline 45 & Maple River below Mapleton, N. Dak. & 74 & 1995-2008 & 118.0 & 34.0 & 309.0 \\
\hline 46 & Sheyenne River at Harwood, N. Dak. & 37 & 1993-2005 & 82.5 & 61.0 & 126.0 \\
\hline 47 & Rush River at Amenia, N. Dak. & 51 & $1972-2008$ & 110.0 & 27.0 & 180.0 \\
\hline
\end{tabular}


Table 1-1. Summary statistics for major ion constituents at selected sites in North Dakota from 1970 through 2008. —Continued $\left[\mu \mathrm{g} / \mathrm{L}\right.$, micrograms per liter; <, less than; --, not available; $\mathrm{mg} / \mathrm{L}$, milligrams per liter; $\mathrm{CO}_{3}$, carbonate; $\mathrm{HCO}_{3}$, bicarbonate; $\mathrm{CaCO}$, calcium carbonate $]$

\begin{tabular}{|c|c|c|c|c|c|c|}
\hline $\begin{array}{c}\text { Site } \\
\text { identification } \\
\text { number } \\
\text { (table 1) }\end{array}$ & Site name & $\begin{array}{c}\text { Number of } \\
\text { samples }\end{array}$ & $\begin{array}{l}\text { Period of } \\
\text { record }\end{array}$ & Median & Minimum & Maximum \\
\hline \multicolumn{7}{|c|}{ Calcium, in $\mathrm{mg} / \mathrm{L}$-Continued } \\
\hline 48 & Rush River near Prosper, N. Dak. & 4 & 1983-1987 & 45.0 & 27.0 & 170.0 \\
\hline 49 & Lower Branch Rush River near Prosper, N. Dak. & 5 & $1983-1993$ & 25.0 & 22.0 & 124.0 \\
\hline 50 & Sheyenne River near Harwood, N. Dak. & 39 & 1970-1996 & 78.0 & 41.0 & 130.0 \\
\hline 51 & Elm River near Kelso, N. Dak. & 6 & 1983-1993 & 92.1 & 28.0 & 118.0 \\
\hline 52 & Red River of the North at Halstad, Minn. & 178 & 1972-2008 & 61.4 & 28.0 & 96.0 \\
\hline 53 & Beaver Creek near Finley, N. Dak. & 116 & 1970-2003 & 95.0 & 21.0 & 240.0 \\
\hline 54 & Goose River near Portland, N. Dak. & 30 & 1970-1988 & 120.0 & 36.0 & 360.0 \\
\hline 55 & Goose River at Hillsboro, N. Dak. & 166 & 1970-2008 & 130.0 & 35.0 & 242.0 \\
\hline 56 & Red River of the North at Grand Forks, N. Dak. & 227 & 1970-2008 & 58.0 & 29.8 & 150.0 \\
\hline 57 & Turtle River at Turtle River State Park near Arvilla, N. Dak. & 99 & $1991-2008$ & 97.1 & 25.0 & 130.0 \\
\hline 58 & Turtle River at Manvel, N. Dak. & 105 & $1971-2008$ & 145.0 & 55.0 & 410.0 \\
\hline 59 & Red River of the North at Oslo, Minn. & 75 & 1973-2005 & 55.0 & 28.0 & 86.0 \\
\hline 60 & Middle Branch Forest River near Whitman, N. Dak. & 14 & $1972-1990$ & 59.0 & 36.0 & 200.0 \\
\hline 61 & Forest River near Fordville, N. Dak. & 64 & 1972-2008 & 76.3 & 36.0 & 114.0 \\
\hline 62 & Forest River near Minto, N. Dak. & 154 & $1971-2008$ & 84.8 & 33.0 & 180.0 \\
\hline 63 & South Branch Park River below Homme Dam, N. Dak. & 27 & 1972-1994 & 71.0 & 24.0 & 96.0 \\
\hline 64 & Middle Branch Park River near Union, N. Dak. & 15 & 1972-1984 & 50.0 & 16.0 & 62.0 \\
\hline 65 & Middle Branch Park River near Edinburg, N. Dak. & 14 & $1978-1980$ & 40.5 & 16.0 & 78.0 \\
\hline 66 & Cart Creek at Mountain, N. Dak. & 17 & $1972-1984$ & 79.0 & 13.0 & 130.0 \\
\hline 67 & Park River at Grafton, N. Dak. & 144 & 1970-2008 & 93.0 & 32.0 & 224.0 \\
\hline 68 & Red River of the North at Drayton, N. Dak. & 74 & $1972-2008$ & 61.0 & 30.0 & 98.0 \\
\hline 69 & Pembina County Drain 20 near Glasston, N. Dak. & 8 & 1974-1984 & 53.0 & 37.0 & 110.0 \\
\hline 70 & Hidden Island Coulee near Hansboro, N. Dak. & 22 & $1972-1995$ & 64.0 & 32.0 & 150.0 \\
\hline 71 & Cypress Creek near Sarles, N. Dak. & 13 & $1972-1988$ & 51.0 & 37.0 & 110.0 \\
\hline 72 & Pembina River near Vang, N. Dak. & 62 & $1970-1979$ & 63.5 & 26.0 & 160.0 \\
\hline 73 & Little South Pembina River near Walhalla, N. Dak. & 82 & 1970-2008 & 72.5 & 18.0 & 110.0 \\
\hline 74 & Pembina River at Walhalla, N. Dak. & 254 & $1970-2008$ & 73.5 & 26.0 & 150.0 \\
\hline 75 & Pembina River at Neche, N. Dak. & 133 & 1972-2008 & 81.1 & 27.4 & 151.0 \\
\hline 76 & Tongue River at Akra, N. Dak. & 72 & 1972-2008 & 63.4 & 32.3 & 98.0 \\
\hline 77 & Red River of the North at Pembina, N. Dak., site 1 & 10 & $1993-1993$ & 67.4 & 53.5 & 78.6 \\
\hline 78 & Red River of the North at Pembina, N. Dak., site 2 & 152 & 1970-2008 & 66.0 & 31.8 & 140.0 \\
\hline 79 & Red River of the North at Emerson, Manitoba & 148 & $1977-2004$ & 63.0 & 36.0 & 110.0 \\
\hline 80 & Long Creek near Noonan, N. Dak. & 70 & 1972-2008 & 67.5 & 11.0 & 140.0 \\
\hline 81 & West Branch Short Creek near Columbus, N. Dak. & 20 & $1978-1981$ & 41.5 & 16.0 & 85.0 \\
\hline 82 & Souris River near Sherwood, N. Dak. & 302 & 1972-2008 & 60.4 & 14.2 & 164.3 \\
\hline 83 & Souris River near Foxholm, N. Dak. & 202 & $1972-2008$ & 51.0 & 25.0 & 110.0 \\
\hline 84 & Des Lacs River at Foxholm, N. Dak. & 188 & $1972-2008$ & 74.5 & 13.0 & 180.0 \\
\hline 85 & Souris River above Minot, N. Dak. & 207 & $1970-2008$ & 57.0 & 22.0 & 217.0 \\
\hline 86 & Bonnes Creek near Velva, N. Dak. & 18 & $1987-2005$ & 70.0 & 18.0 & 140.0 \\
\hline 87 & Souris River near Verendrye, N. Dak. & 373 & 1970-2008 & 69.0 & 22.0 & 151.7 \\
\hline
\end{tabular}


Table 1-1. Summary statistics for major ion constituents at selected sites in North Dakota from 1970 through 2008.—Continued $\left[\mu \mathrm{g} / \mathrm{L}\right.$, micrograms per liter; <, less than; --, not available; mg/L, milligrams per liter; $\mathrm{CO}_{3}$, carbonate; $\mathrm{HCO}_{3}$, bicarbonate; $\mathrm{CaCO}$, calcium carbonate $]$

\begin{tabular}{|c|c|c|c|c|c|c|}
\hline $\begin{array}{c}\text { Site } \\
\text { identification } \\
\text { number } \\
\text { (table 1) }\end{array}$ & Site name & $\begin{array}{l}\text { Number of } \\
\text { samples }\end{array}$ & $\begin{array}{l}\text { Period of } \\
\text { record }\end{array}$ & Median & Minimum & Maximum \\
\hline \multicolumn{7}{|c|}{ Calcium, in $\mathrm{mg} / \mathrm{L}$-Continued } \\
\hline 88 & Wintering River near Karlsruhe, N. Dak. & 134 & $1972-2008$ & 52.5 & 12.7 & 100.0 \\
\hline 89 & Souris River near Bantry, N. Dak. & 152 & $1970-2008$ & 60.6 & 25.6 & 110.0 \\
\hline 91 & Stone Creek near Kramer, N. Dak. & 30 & $1986-2000$ & 59.0 & 17.0 & 170.0 \\
\hline 92 & Deep River near Upham, N. Dak. & 68 & $1972-2007$ & 59.1 & 19.0 & 93.0 \\
\hline 93 & Egg Creek near Granville, N. Dak. & 14 & $1972-1981$ & 41.5 & 18.0 & 86.0 \\
\hline 94 & Cut Bank Creek at North Lake Outlet near Granville, N. Dak. & 15 & $1972-1976$ & 43.0 & 20.0 & 66.0 \\
\hline 98 & Souris River near Westhope, N. Dak. & 214 & $1970-2008$ & 52.4 & 27.0 & 330.0 \\
\hline 99 & Charbonneau Creek near Charbonneau, N. Dak. & 22 & $1972-1977$ & 32.5 & 15.0 & 59.0 \\
\hline 100 & Missouri River near Williston, N. Dak. & 95 & 1974-1992 & 56.0 & 33.0 & 73.0 \\
\hline 101 & Little Muddy River below Cow Creek near Williston, N. Dak. & 77 & $1972-2008$ & 55.0 & 15.0 & 110.0 \\
\hline 102 & Stony Creek near Williston, N. Dak. & 32 & $1977-1981$ & 45.0 & 12.0 & 63.0 \\
\hline 103 & Tobacco Garden Creek near Watford City, N. Dak. & 15 & 1976-1977 & 35.0 & 17.0 & 110.0 \\
\hline 104 & Beaver Creek near Ray, N. Dak. & 51 & 1977-1982 & 64.0 & 17.0 & 90.0 \\
\hline 105 & White Earth River at White Earth, N. Dak. & 27 & $1970-1977$ & 49.0 & 22.0 & 120.0 \\
\hline 106 & Bear Den Creek near Mandaree, N. Dak. & 228 & $1970-2008$ & 34.0 & 5.5 & 70.0 \\
\hline 113 & Beaver Creek near Trotters, N. Dak. & 95 & $1977-2008$ & 83.6 & 20.0 & 160.0 \\
\hline 114 & Little Missouri River near Watford City, N. Dak. & 243 & $1971-2008$ & 65.0 & 6.0 & 160.0 \\
\hline 115 & Missouri River at Garrison Dam, N. Dak. & 288 & $1971-2007$ & 51.9 & 26.0 & 65.0 \\
\hline 116 & Knife River at Manning, N. Dak. & 118 & $1972-2008$ & 42.0 & 7.0 & 87.0 \\
\hline 117 & Stray Creek near Manning, N. Dak. & 17 & $1975-1981$ & 50.0 & 19.0 & 110.0 \\
\hline 118 & Knife River at Marshall, N. Dak. & 69 & $1972-1981$ & 52.0 & 18.0 & 140.0 \\
\hline 119 & Elm Creek near Golden Valley, N. Dak. & 37 & $1973-1995$ & 36.0 & 10.0 & 82.0 \\
\hline 120 & Knife River near Golden Valley, N. Dak. & 130 & 1971-2008 & 44.1 & 11.0 & 91.0 \\
\hline 121 & Coyote Creek near Zap, N. Dak. & 51 & $1977-1983$ & 51.0 & 15.0 & 120.0 \\
\hline 122 & Brush Creek near Beulah, N. Dak. & 107 & $1974-1990$ & 78.0 & 17.0 & 110.0 \\
\hline 123 & Spring Creek below Lake Ilo near Dunn Center, N. Dak. & 53 & $1977-1993$ & 65.0 & 11.0 & 170.0 \\
\hline 124 & Spring Creek near Halliday, N. Dak. & 48 & 1977-1981 & 76.5 & 10.0 & 110.0 \\
\hline 125 & Spring Creek at Zap, N. Dak. & 240 & 1970-2008 & 66.5 & 13.0 & 120.0 \\
\hline 126 & West Branch Otter Creek near Beulah, N. Dak. & 15 & $1972-1995$ & 53.0 & 16.0 & 90.0 \\
\hline 127 & Knife River at Hazen, N. Dak. & 269 & $1970-2008$ & 64.7 & 15.0 & 130.0 \\
\hline
\end{tabular}


Table 1-1. Summary statistics for major ion constituents at selected sites in North Dakota from 1970 through 2008. —Continued $\left[\mu \mathrm{g} / \mathrm{L}\right.$, micrograms per liter; <, less than; --, not available; $\mathrm{mg} / \mathrm{L}$, milligrams per liter; $\mathrm{CO}_{3}$, carbonate; $\mathrm{HCO}_{3}$, bicarbonate; $\mathrm{CaCO}$, calcium carbonate $]$

\begin{tabular}{|c|c|c|c|c|c|c|}
\hline $\begin{array}{c}\text { Site } \\
\text { identification } \\
\text { number } \\
\text { (table 1) }\end{array}$ & Site name & $\begin{array}{c}\text { Number of } \\
\text { samples }\end{array}$ & $\begin{array}{l}\text { Period of } \\
\text { record }\end{array}$ & Median & Minimum & Maximum \\
\hline \multicolumn{7}{|c|}{ Calcium, in $\mathrm{mg} / \mathrm{L}$-Continued } \\
\hline 128 & Antelope Creek above Hazen, N. Dak. & 44 & $1977-1985$ & 70.0 & 12.0 & 120.0 \\
\hline 129 & West Branch Antelope Creek No. 4 near Zap, N. Dak. & 21 & $1977-1985$ & 58.0 & 7.9 & 130.0 \\
\hline 130 & West Branch Antelope Creek near Hazen, N. Dak. & 14 & $1978-1983$ & 37.0 & 12.0 & 84.0 \\
\hline 131 & Coal Creek near Stanton, N. Dak. & 28 & $1975-1981$ & 60.5 & 15.0 & 110.0 \\
\hline 132 & Alderin Creek near Fort Clark, N. Dak. & 41 & $1977-1983$ & 45.0 & 12.0 & 110.0 \\
\hline 133 & Coal Lake Coulee near Hensler, N. Dak. & 39 & $1978-1988$ & 56.0 & 7.8 & 110.0 \\
\hline 134 & Buffalo Creek near Washburn, N. Dak. & 38 & $1978-1983$ & 30.5 & 15.0 & 82.0 \\
\hline 135 & Turtle Creek above Washburn, N. Dak. & 100 & $1987-2003$ & 31.7 & 13.0 & 71.4 \\
\hline 136 & Painted Woods Creek near Wilton, N. Dak. & 202 & $1970-2003$ & 60.0 & 11.0 & 280.0 \\
\hline 137 & Square Butte Creek near Hannover, N. Dak. & 23 & $1977-1981$ & 78.0 & 9.3 & 110.0 \\
\hline 138 & Square Butte Creek above Nelson Lake near Center, N. Dak. & 55 & 1977-1982 & 60.0 & 13.0 & 83.0 \\
\hline 139 & Hagel Creek near Center, N. Dak. & 38 & $1977-1982$ & 32.5 & 9.5 & 110.0 \\
\hline 140 & Square Butte Creek below Center, N. Dak. & 81 & $1972-2008$ & 70.0 & 29.0 & 101.0 \\
\hline 141 & Burnt Creek near Bismarck, N. Dak. & 48 & $1972-2008$ & 54.5 & 9.0 & 82.0 \\
\hline 142 & Missouri River at Bismarck, N. Dak. & 126 & 1974-2008 & 53.0 & 30.0 & 61.0 \\
\hline 143 & South Branch Heart River near South Heart, N. Dak. & 18 & 1979-1983 & 20.0 & 1.9 & 94.0 \\
\hline 144 & North Creek near South Heart, N. Dak. & 16 & 1978-1981 & 41.5 & 12.0 & 120.0 \\
\hline 145 & Heart River near South Heart, N. Dak. & 76 & $1975-2005$ & 48.5 & 9.4 & 120.0 \\
\hline 146 & Heart River at Dickinson, N. Dak. & 22 & 1986-1994 & 58.0 & 23.0 & 106.0 \\
\hline 147 & Green River near New Hradec, N. Dak. & 116 & $1972-2008$ & 40.0 & 7.0 & 83.0 \\
\hline 148 & Green River near Gladstone, N. Dak. & 33 & $1970-1993$ & 81.0 & 15.0 & 110.0 \\
\hline 149 & Heart River near Richardton, N. Dak. & 154 & $1972-2008$ & 67.2 & 21.1 & 160.0 \\
\hline 150 & Heart River above Lake Tschida near Glen Ullin, N. Dak. & 40 & 1989-2008 & 49.0 & 21.0 & 120.0 \\
\hline 151 & Antelope Creek near Carson, N. Dak. & 26 & $1972-2008$ & 49.8 & 22.7 & 96.0 \\
\hline 152 & Big Muddy Creek near Almont, N. Dak. & 39 & $1991-2008$ & 35.4 & 17.0 & 79.0 \\
\hline 153 & Heart River near Lark, N. Dak. & 45 & $1971-1995$ & 55.0 & 18.0 & 90.0 \\
\hline 154 & Heart River at Stark Bridge near Judson, N. Dak. & 41 & $1988-2008$ & 52.0 & 15.0 & 77.0 \\
\hline 155 & Sweetbriar Creek near Judson, N. Dak. & 33 & $1972-2008$ & 32.0 & 13.0 & 73.0 \\
\hline 156 & Heart River near Mandan, N. Dak. & 232 & 1971-2008 & 57.0 & 18.0 & 150.0 \\
\hline 157 & Long Lake Creek above Long Lake near Moffit, N. Dak. & 33 & 1988-2004 & 41.0 & 0.5 & 59.0 \\
\hline 158 & Apple Creek near Menoken, N. Dak. & 129 & $1972-2008$ & 46.0 & 11.0 & 100.0 \\
\hline 159 & Missouri River near Schmidt, N. Dak. & 68 & 1974-1981 & 54.5 & 43.0 & 61.0 \\
\hline 160 & Cannonball River at New England, N. Dak. & 36 & 1978-1981 & 91.0 & 17.0 & 170.0 \\
\hline 161 & Coal Bank Creek near Havelock, N. Dak. & 84 & 1974-1983 & 115.0 & 23.0 & 240.0 \\
\hline 162 & Cannonball River at Regent, N. Dak. & 119 & $1970-2008$ & 79.0 & 27.8 & 200.0 \\
\hline 163 & Cannonball River below Bentley, N. Dak. & 31 & 1972-1977 & 84.0 & 32.0 & 160.0 \\
\hline 164 & Cannonball River near Raleigh, N. Dak. & 89 & 1993-2008 & 59.2 & 26.3 & 167.0 \\
\hline 165 & White Butte Fork Cedar Creek near Scranton, N. Dak. & 26 & $1972-1995$ & 185.0 & 23.0 & 750.0 \\
\hline 166 & Cedar Creek near Haynes, N. Dak. & 76 & $1971-2008$ & 67.5 & 19.0 & 190.0 \\
\hline 167 & Timber Creek near Bentley, N. Dak. & 47 & $1977-1981$ & 120.0 & 39.0 & 190.0 \\
\hline
\end{tabular}


Table 1-1. Summary statistics for major ion constituents at selected sites in North Dakota from 1970 through 2008.—Continued $\left[\mu \mathrm{g} / \mathrm{L}\right.$, micrograms per liter; <, less than; --, not available; mg/L, milligrams per liter; $\mathrm{CO}_{3}$, carbonate; $\mathrm{HCO}_{3}$, bicarbonate; $\mathrm{CaCO}$, calcium carbonate $]$

\begin{tabular}{|c|c|c|c|c|c|c|}
\hline $\begin{array}{c}\text { Site } \\
\text { identification } \\
\text { number } \\
\text { (table 1) }\end{array}$ & Site name & $\begin{array}{l}\text { Number of } \\
\text { samples }\end{array}$ & $\begin{array}{l}\text { Period of } \\
\text { record }\end{array}$ & Median & Minimum & Maximum \\
\hline \multicolumn{7}{|c|}{ Calcium, in $\mathrm{mg} / \mathrm{L}$-Continued } \\
\hline 168 & Cedar Creek near Pretty Rock, N. Dak. & 30 & $1971-1976$ & 110.0 & 33.0 & 180.0 \\
\hline 169 & Cedar Creek near Raleigh, N. Dak. & 120 & $1972-2008$ & 59.4 & 18.0 & 149.0 \\
\hline 171 & Beaver Creek near Linton, N. Dak. & 39 & $1972-1993$ & 57.0 & 9.5 & 100.0 \\
\hline 172 & Beaver Creek below Linton, N. Dak. & 38 & $1990-2008$ & 63.6 & 11.0 & 93.0 \\
\hline 173 & Porcupine Creek near Fort Yates, N. Dak. & 41 & 1991-1999 & 28.2 & 2.4 & 36.6 \\
\hline 174 & Buffalo Creek Tributary near Gascoyne, N. Dak. & 83 & $1974-1987$ & 130.0 & 30.0 & 250.0 \\
\hline 178 & Kelly Creek below Niccum Reservoir near Bordulac, N. Dak. & 9 & 1986-1989 & 43.0 & 28.0 & 58.0 \\
\hline 179 & James River near Pingree, N. Dak. & 143 & $1978-2008$ & 47.8 & 18.0 & 110.7 \\
\hline 180 & Pipestem Creek near Pingree, N. Dak. & 71 & 1974-2008 & 61.0 & 21.0 & 129.0 \\
\hline 181 & Pipestem Creek near Buchanan, N. Dak. & 9 & $1972-1993$ & 50.2 & 9.6 & 68.5 \\
\hline 182 & James River at Jamestown, N. Dak. & 201 & $1972-2008$ & 69.0 & 24.0 & 142.0 \\
\hline 183 & James River at Lamoure, N. Dak. & 280 & $1970-2008$ & 67.0 & 17.0 & 190.0 \\
\hline 184 & Bear Creek near Oakes, N. Dak. & 54 & $1977-2008$ & 77.9 & 15.0 & 190.0 \\
\hline 185 & James River at Oakes, N. Dak. & 135 & $1970-2008$ & 65.0 & 21.0 & 350.0 \\
\hline 186 & James River at N. Dak./S. Dak. State line & 97 & $1974-2008$ & 58.8 & 23.0 & 380.0 \\
\hline 11 & Red River of the North at Fargo, N. Dak. & 16 & 1994-1996 & 0.11 & 0.05 & 0.56 \\
\hline 13 & Red River of the North at Harwood, N. Dak. & 53 & $2000-2007$ & 0.15 & $<0.04$ & 0.50 \\
\hline 14 & Red River of the North near Harwood, N. Dak. & 26 & 1993-1996 & 0.15 & $<0.04$ & 0.34 \\
\hline 17 & Sheyenne River at Warwick, N. Dak. & 48 & $1996-2006$ & 0.20 & $<0.04$ & 0.90 \\
\hline 18 & Sheyenne River near Warwick, N. Dak. & 16 & $2005-2007$ & 0.21 & 0.07 & 0.41 \\
\hline 32 & Sheyenne River near Cooperstown, N. Dak. & 80 & $1994-2007$ & 0.36 & 0.16 & 0.96 \\
\hline 33 & Baldhill Creek near Dazey, N. Dak. & 3 & 1993-1996 & 0.18 & 0.09 & 0.88 \\
\hline 34 & Sheyenne River below Baldhill Dam, N. Dak. & 73 & $1994-2007$ & 0.29 & 0.05 & 0.71 \\
\hline 35 & Sheyenne River at Valley City, N. Dak. & 4 & 1993 & 0.47 & 0.30 & 0.80 \\
\hline 36 & Sheyenne River at Lisbon, N. Dak. & 69 & $1997-2007$ & 0.47 & 0.09 & 2.86 \\
\hline 37 & Sheyenne River near Kindred, N. Dak. & 70 & $1996-2007$ & 0.50 & 0.12 & 3.19 \\
\hline 41 & Sheyenne River at West Fargo, N. Dak. & 13 & 1994-2006 & 0.76 & 0.16 & 1.41 \\
\hline 45 & Maple River below Mapleton, N. Dak. & 47 & $1997-2007$ & 0.33 & 0.08 & 1.12 \\
\hline 46 & Sheyenne River at Harwood, N. Dak. & 10 & 1993 & 0.41 & 0.22 & 1.34 \\
\hline 49 & Lower Branch Rush River near Prosper, N. Dak. & 1 & 1993 & 0.08 & 0.08 & 0.08 \\
\hline
\end{tabular}


Table 1-1. Summary statistics for major ion constituents at selected sites in North Dakota from 1970 through 2008. —Continued $\left[\mu \mathrm{g} / \mathrm{L}\right.$, micrograms per liter; <, less than; --, not available; $\mathrm{mg} / \mathrm{L}$, milligrams per liter; $\mathrm{CO}_{3}$, carbonate; $\mathrm{HCO}_{3}$, bicarbonate; $\mathrm{CaCO}_{3}$, calcium carbonate $]$

\begin{tabular}{|c|c|c|c|c|c|c|}
\hline $\begin{array}{c}\text { Site } \\
\text { identification } \\
\text { number } \\
\text { (table 1) }\end{array}$ & Site name & $\begin{array}{c}\text { Number of } \\
\text { samples }\end{array}$ & $\begin{array}{l}\text { Period of } \\
\text { record }\end{array}$ & Median & Minimum & Maximum \\
\hline \multicolumn{7}{|c|}{ Manganese, total, in $\mathrm{mg} / \mathrm{L}-$ Continued } \\
\hline 51 & Elm River near Kelso, N. Dak. & 3 & 1993 & 0.41 & 0.35 & 0.52 \\
\hline 55 & Goose River at Hillsboro, N. Dak. & 76 & 1994-2007 & 0.34 & 0.13 & 1.04 \\
\hline 56 & Red River of the North at Grand Forks, N. Dak. & 46 & 1997-2006 & 0.12 & $<0.04$ & 0.69 \\
\hline 58 & Turtle River at Manvel, N. Dak. & 38 & 1993-2006 & 0.28 & 0.09 & 1.13 \\
\hline 62 & Forest River near Minto, N. Dak. & 47 & 1994-2006 & 0.27 & 0.07 & 1.34 \\
\hline 67 & Park River at Grafton, N. Dak. & 40 & 1994-2006 & 0.37 & 0.10 & 2.17 \\
\hline 68 & Red River of the North at Drayton, N. Dak. & 15 & 1994-1996 & 0.18 & $<0.04$ & 0.58 \\
\hline 75 & Pembina River at Neche, N. Dak. & 55 & 1994-2006 & 0.30 & 0.06 & 5.85 \\
\hline 76 & Tongue River at Akra, N. Dak. & 1 & 1993 & 0.33 & 0.33 & 0.33 \\
\hline 77 & Red River of the North at Pembina, N. Dak., site 1 & 10 & 1993 & 0.17 & $<0.04$ & 0.44 \\
\hline 78 & Red River of the North at Pembina, N. Dak., site 2 & 52 & 1997-2006 & 0.18 & $<0.04$ & 1.16 \\
\hline 80 & Long Creek near Noonan, N. Dak. & 10 & 1997-1997 & 0.21 & 0.08 & 0.40 \\
\hline 83 & Souris River near Foxholm, N. Dak. & 11 & 1997-1998 & 0.17 & 0.06 & 3.74 \\
\hline 84 & Des Lacs River at Foxholm, N. Dak. & 97 & 1993-2007 & 0.26 & $<0.04$ & 1.99 \\
\hline 85 & Souris River above Minot, N. Dak. & 75 & 1994-2007 & 0.17 & $<0.04$ & 4.68 \\
\hline 87 & Souris River near Verendrye, N. Dak. & 76 & 1997-2007 & 0.22 & 0.05 & 0.63 \\
\hline 88 & Wintering River near Karlsruhe, N. Dak. & 11 & 1997-1998 & $<0.04$ & $<0.04$ & 0.16 \\
\hline 89 & Souris River near Bantry, N. Dak. & 10 & $1997-1998$ & 0.20 & $<0.04$ & 0.61 \\
\hline 90 & Willow Creek near Willow City, N. Dak. & 6 & 1997 & 0.09 & $<0.04$ & 0.14 \\
\hline 92 & Deep River near Upham, N. Dak. & 7 & 1997 & $<0.04$ & $<0.04$ & 0.15 \\
\hline 98 & Souris River near Westhope, N. Dak. & 7 & 1997-1998 & $<0.04$ & $<0.04$ & 0.79 \\
\hline 101 & Little Muddy River below Cow Creek near Williston, N. Dak. & 9 & 1999 & 0.08 & $<0.04$ & 0.11 \\
\hline 110 & Little Missouri River at Marmarth, N. Dak. & 8 & 1999 & 0.32 & 0.05 & 1.11 \\
\hline 112 & Little Missouri River at Medora, N. Dak. & 88 & 1993-2007 & 0.21 & $<0.04$ & 18.10 \\
\hline 113 & Beaver Creek near Trotters, N. Dak. & 9 & 1999 & $<0.04$ & $<0.04$ & 0.08 \\
\hline 114 & Little Missouri River near Watford City, N. Dak. & 81 & $1994-2007$ & 0.30 & $<0.04$ & 18.70 \\
\hline 120 & Knife River near Golden Valley, N. Dak. & 60 & $1997-2007$ & 0.15 & 0.07 & 0.50 \\
\hline 123 & Spring Creek below Lake Ilo near Dunn Center, N. Dak. & 6 & 1993 & 0.07 & $<0.04$ & 0.09 \\
\hline 125 & Spring Creek at Zap, N. Dak. & 77 & $1993-2007$ & 0.10 & $<0.04$ & 0.49 \\
\hline 127 & Knife River at Hazen, N. Dak. & 94 & $1993-2007$ & 0.14 & 0.07 & 1.16 \\
\hline 140 & Square Butte Creek below Center, N. Dak. & 4 & 1993 & 0.31 & 0.11 & 0.37 \\
\hline 142 & Missouri River at Bismarck, N. Dak. & 1 & 1994 & $<0.04$ & $<0.04$ & $<0.04$ \\
\hline 146 & Heart River at Dickinson, N. Dak. & 5 & 1993 & 0.26 & 0.09 & 0.40 \\
\hline 148 & Green River near Gladstone, N. Dak. & 4 & 1993 & 0.22 & 0.09 & 0.33 \\
\hline 149 & Heart River near Richardton, N. Dak. & 76 & $1994-2007$ & 0.19 & 0.05 & 0.46 \\
\hline 152 & Big Muddy Creek near Almont, N. Dak. & 3 & 1993 & 0.25 & 0.15 & 0.32 \\
\hline 156 & Heart River near Mandan, N. Dak. & 81 & $1994-2007$ & 0.06 & $<0.04$ & 0.58 \\
\hline 158 & Apple Creek near Menoken, N. Dak. & 4 & 1993 & 0.17 & 0.05 & 0.24 \\
\hline 164 & Cannonball River near Raleigh, N. Dak. & 76 & 1993-2007 & 0.11 & $<0.04$ & 1.03 \\
\hline
\end{tabular}


Table 1-1. Summary statistics for major ion constituents at selected sites in North Dakota from 1970 through 2008.—Continued [ $\mu \mathrm{g} / \mathrm{L}$, micrograms per liter; <, less than; --, not available; $\mathrm{mg} / \mathrm{L}$, milligrams per liter; $\mathrm{CO}_{3}$, carbonate; $\mathrm{HCO}_{3}$, bicarbonate; $\mathrm{CaCO}_{3}$, calcium carbonate $]$

\begin{tabular}{|c|c|c|c|c|c|c|}
\hline $\begin{array}{c}\text { Site } \\
\text { identification } \\
\text { number } \\
\text { (table 1) }\end{array}$ & Site name & $\begin{array}{l}\text { Number of } \\
\text { samples }\end{array}$ & $\begin{array}{l}\text { Period of } \\
\text { record }\end{array}$ & Median & Minimum & Maximum \\
\hline \multicolumn{7}{|c|}{ Manganese, total, in $\mathrm{mg} / \mathrm{L}-$ Continued } \\
\hline 169 & Cedar Creek near Raleigh, N. Dak. & 68 & $1993-2007$ & 0.16 & $<0.04$ & 1.76 \\
\hline 170 & Cannonball River at Breien, N. Dak. & 74 & 1994-2007 & 0.11 & $<0.04$ & 1.63 \\
\hline 175 & James River near Manfred, N. Dak. & 6 & 1998 & $<0.04$ & $<0.04$ & 0.05 \\
\hline 176 & James River near Grace City, N. Dak. & 60 & $1997-2007$ & 0.22 & $<0.04$ & 1.80 \\
\hline 177 & James River above Arrowwood Lake near Kensal, N. Dak. & 9 & 1998-1999 & 0.21 & $<0.04$ & 1.05 \\
\hline 180 & Pipestem Creek near Pingree, N. Dak. & 17 & 1994-1999 & 0.20 & $<0.04$ & 0.75 \\
\hline 184 & Bear Creek near Oakes, N. Dak. & 9 & 1998-1999 & 0.51 & 0.08 & 0.62 \\
\hline 185 & James River at Oakes, N. Dak. & 3 & 1993 & 0.09 & 0.09 & 0.24 \\
\hline 186 & James River at N. Dak./S. Dak. State line & 9 & 1998-1999 & 0.44 & 0.24 & 1.49 \\
\hline \multicolumn{7}{|c|}{ Manganese, dissolved, in $\mathrm{mg} / \mathrm{L}$} \\
\hline 2 & Red River of the North at Wahpeton, N. Dak. & 57 & $1973-2008$ & $<0.04$ & $<0.04$ & 0.11 \\
\hline 5 & Red River of the North below Wahpeton, N. Dak. & 9 & $1970-1972$ & $<0.04$ & $<0.04$ & 0.06 \\
\hline 6 & Red River of the North at Hickson, N. Dak. & 51 & $1975-2008$ & $<0.04$ & $<0.04$ & 0.09 \\
\hline 7 & Wild Rice River near Rutland, N. Dak. & 46 & $1971-2008$ & 0.14 & $<0.04$ & 1.70 \\
\hline 8 & Wild Rice River near Cayuga, N. Dak. & 15 & 1970-1977 & 0.21 & $<0.04$ & 0.48 \\
\hline 15 & Sheyenne River above Harvey, N. Dak. & 89 & $1972-2008$ & 0.07 & $<0.04$ & 0.38 \\
\hline 16 & Big Coulee near Fort Totten, N. Dak. & 8 & 1970-1975 & 0.13 & $<0.04$ & 0.20 \\
\hline 18 & Sheyenne River near Warwick, N. Dak. & 80 & $1970-2008$ & 0.07 & $<0.04$ & 0.66 \\
\hline 19 & Mauvais Coulee Tributary No. 3 near Cando, N. Dak. & 38 & $1987-2008$ & $<0.04$ & $<0.04$ & 0.92 \\
\hline 20 & Mauvais Coulee near Cando, N. Dak. & 104 & $1972-2008$ & 0.08 & $<0.04$ & 2.10 \\
\hline 21 & Edmore Coulee near Edmore, N. Dak. & 102 & $1972-2008$ & $<0.04$ & $<0.04$ & 1.40 \\
\hline 22 & Edmore Coulee Tributary near Webster, N. Dak. & 38 & $1987-2008$ & 0.05 & $<0.04$ & 2.00 \\
\hline 23 & Webster Coulee at Webster, N. Dak. & 2 & $1983-1984$ & $<0.04$ & $<0.04$ & $<0.04$ \\
\hline 24 & Starkweather Coulee near Webster, N. Dak. & 92 & $1983-2008$ & $<0.04$ & $<0.04$ & 0.63 \\
\hline 25 & Big Coulee below Churchs Ferry, N. Dak. & 23 & $1998-2008$ & 0.13 & $<0.04$ & 0.94 \\
\hline 26 & Little Coulee near Leeds, N. Dak. & 16 & 1998-2008 & 0.12 & $<0.04$ & 0.73 \\
\hline 27 & Little Coulee near Brinsmade, N. Dak. & 21 & 1976-1998 & 0.12 & $<0.04$ & 2.60 \\
\hline 28 & Big Coulee near Churchs Ferry, N. Dak. & 88 & $1970-1997$ & 0.07 & $<0.04$ & 1.20 \\
\hline 29 & Big Coulee at Graham Island inlet near Fort Totten, N. Dak. & 39 & $1970-1985$ & $<0.04$ & $<0.04$ & 3.40 \\
\hline 30 & Channel A near Penn, N. Dak. & 85 & 1984-2008 & 0.09 & $<0.04$ & 3.30 \\
\hline
\end{tabular}


Table 1-1. Summary statistics for major ion constituents at selected sites in North Dakota from 1970 through 2008. —Continued $\left[\mu \mathrm{g} / \mathrm{L}\right.$, micrograms per liter; <, less than; --, not available; $\mathrm{mg} / \mathrm{L}$, milligrams per liter; $\mathrm{CO}_{3}$, carbonate; $\mathrm{HCO}_{3}$, bicarbonate; $\mathrm{CaCO}$, calcium carbonate $]$

\begin{tabular}{|c|c|c|c|c|c|c|}
\hline $\begin{array}{c}\text { Site } \\
\text { identification } \\
\text { number } \\
\text { (table 1) }\end{array}$ & Site name & $\begin{array}{l}\text { Number of } \\
\text { samples }\end{array}$ & $\begin{array}{l}\text { Period of } \\
\text { record }\end{array}$ & Median & Minimum & Maximum \\
\hline \multicolumn{7}{|c|}{ Manganese, dissolved, in $\mathrm{mg} / \mathrm{L}-$ Continued } \\
\hline 31 & Devils Lake Outlet to Stump Lake near Lakota, N. Dak. & 12 & 2002-2007 & $<0.04$ & $<0.04$ & 0.32 \\
\hline 32 & Sheyenne River near Cooperstown, N. Dak. & 150 & 1970-2008 & 0.19 & $<0.04$ & 5.00 \\
\hline 33 & Baldhill Creek near Dazey, N. Dak. & 91 & $1972-2008$ & 0.16 & $<0.04$ & 2.20 \\
\hline 34 & Sheyenne River below Baldhill Dam, N. Dak. & 95 & $1972-2008$ & 0.19 & $<0.04$ & 2.10 \\
\hline 35 & Sheyenne River at Valley City, N. Dak. & 25 & $1972-2005$ & 0.23 & $<0.04$ & 0.77 \\
\hline 36 & Sheyenne River at Lisbon, N. Dak. & 92 & 1970-2008 & 0.16 & $<0.04$ & 0.88 \\
\hline 37 & Sheyenne River near Kindred, N. Dak. & 214 & $1972-2008$ & 0.07 & $<0.04$ & 0.50 \\
\hline 38 & $\begin{array}{l}\text { Sheyenne River above Sheyenne River Diversion near Horace, } \\
\text { N. Dak. }\end{array}$ & 36 & 1993-2008 & $<0.04$ & $<0.04$ & 0.21 \\
\hline 39 & Sheyenne River near Horace, N. Dak. & 8 & $1982-1992$ & 0.16 & $<0.04$ & 0.28 \\
\hline 40 & Sheyenne River Diversion at West Fargo, N. Dak. & 13 & 1994-2007 & 0.06 & $<0.04$ & 0.19 \\
\hline 41 & Sheyenne River at West Fargo, N. Dak. & 41 & 1972-2008 & $<0.04$ & $<0.04$ & 0.26 \\
\hline 42 & Maple River near Hope, N. Dak. & 39 & $1972-2008$ & 0.09 & $<0.04$ & 3.66 \\
\hline 43 & Maple River near Enderlin, N. Dak. & 63 & $1972-2008$ & 0.38 & $<0.04$ & 1.57 \\
\hline 44 & Maple River near Mapleton, N. Dak. & 25 & $1972-2008$ & 0.06 & $<0.04$ & 1.10 \\
\hline 45 & Maple River below Mapleton, N. Dak. & 28 & $1995-2008$ & 0.07 & $<0.04$ & 0.69 \\
\hline 46 & Sheyenne River at Harwood, N. Dak. & 6 & $2000-2005$ & 0.07 & $<0.04$ & 0.12 \\
\hline 47 & Rush River at Amenia, N. Dak. & 51 & $1972-2008$ & 0.20 & $<0.04$ & 1.10 \\
\hline 48 & Rush River near Prosper, N. Dak. & 4 & 1983-1987 & 0.10 & $<0.04$ & 0.22 \\
\hline 49 & Lower Branch Rush River near Prosper, N. Dak. & 4 & $1983-1987$ & $<0.04$ & $<0.04$ & 0.04 \\
\hline 50 & Sheyenne River near Harwood, N. Dak. & 10 & 1970-1996 & 0.05 & $<0.04$ & 0.55 \\
\hline 51 & Elm River near Kelso, N. Dak. & 3 & 1983-1987 & 0.15 & 0.09 & 0.28 \\
\hline 52 & Red River of the North at Halstad, Minn. & 125 & 1972-2008 & $<0.04$ & $<0.04$ & 0.20 \\
\hline 53 & Beaver Creek near Finley, N. Dak. & 72 & $1970-2003$ & 0.16 & $<0.04$ & 0.72 \\
\hline 54 & Goose River near Portland, N. Dak. & 23 & 1970-1988 & 0.07 & $<0.04$ & 1.50 \\
\hline 55 & Goose River at Hillsboro, N. Dak. & 86 & $1970-2008$ & 0.16 & $<0.04$ & 1.20 \\
\hline 56 & Red River of the North at Grand Forks, N. Dak. & 124 & $1970-2008$ & $<0.04$ & $<0.04$ & 0.25 \\
\hline 57 & Turtle River at Turtle River State Park near Arvilla, N. Dak. & 99 & $1991-2008$ & 0.13 & $<0.04$ & 0.48 \\
\hline 58 & Turtle River at Manvel, N. Dak. & 66 & $1971-2008$ & 0.28 & $<0.04$ & 1.50 \\
\hline 59 & Red River of the North at Oslo, Minn. & 40 & $1973-2005$ & $<0.04$ & $<0.04$ & 0.08 \\
\hline 60 & Middle Branch Forest River near Whitman, N. Dak. & 14 & $1972-1990$ & 0.10 & $<0.04$ & 0.46 \\
\hline 61 & Forest River near Fordville, N. Dak. & 64 & 1972-2008 & 0.18 & $<0.04$ & 0.73 \\
\hline 62 & Forest River near Minto, N. Dak. & 106 & $1971-2008$ & 0.14 & $<0.04$ & 15.00 \\
\hline 63 & South Branch Park River below Homme Dam, N. Dak. & 27 & 1972-1994 & 0.56 & $<0.04$ & 3.10 \\
\hline 64 & Middle Branch Park River near Union, N. Dak. & 15 & $1972-1984$ & 0.34 & $<0.04$ & 1.00 \\
\hline 65 & Middle Branch Park River near Edinburg, N. Dak. & 13 & $1978-1980$ & 0.05 & $<0.04$ & 0.18 \\
\hline 66 & Cart Creek at Mountain, N. Dak. & 17 & 1972-1984 & 0.09 & $<0.04$ & 0.61 \\
\hline 67 & Park River at Grafton, N. Dak. & 96 & $1970-2008$ & 0.13 & $<0.04$ & 0.85 \\
\hline 68 & Red River of the North at Drayton, N. Dak. & 59 & 1972-2008 & $<0.04$ & $<0.04$ & 0.09 \\
\hline 69 & Pembina County Drain 20 near Glasston, N. Dak. & 8 & 1974-1984 & 0.05 & $<0.04$ & 0.10 \\
\hline
\end{tabular}


Table 1-1. Summary statistics for major ion constituents at selected sites in North Dakota from 1970 through 2008.—Continued [ $\mu \mathrm{g} / \mathrm{L}$, micrograms per liter; <, less than; --, not available; $\mathrm{mg} / \mathrm{L}$, milligrams per liter; $\mathrm{CO}_{3}$, carbonate; $\mathrm{HCO}_{3}$, bicarbonate; $\mathrm{CaCO}_{3}$, calcium carbonate $]$

\begin{tabular}{|c|c|c|c|c|c|c|}
\hline $\begin{array}{c}\text { Site } \\
\text { identification } \\
\text { number } \\
\text { (table 1) } \\
\end{array}$ & Site name & $\begin{array}{c}\text { Number of } \\
\text { samples }\end{array}$ & $\begin{array}{l}\text { Period of } \\
\text { record }\end{array}$ & Median & Minimum & Maximum \\
\hline \multicolumn{7}{|c|}{ Manganese, dissolved, in $\mathrm{mg} / \mathrm{L}-$ Continued } \\
\hline 70 & Hidden Island Coulee near Hansboro, N. Dak. & 22 & $1972-1995$ & 0.08 & $<0.04$ & 0.51 \\
\hline 71 & Cypress Creek near Sarles, N. Dak. & 13 & $1972-1988$ & $<0.04$ & $<0.04$ & 0.58 \\
\hline 72 & Pembina River near Vang, N. Dak. & 60 & 1970-1979 & 0.06 & $<0.04$ & 2.10 \\
\hline 73 & Little South Pembina River near Walhalla, N. Dak. & 81 & 1970-2008 & 0.05 & $<0.04$ & 2.40 \\
\hline 74 & Pembina River at Walhalla, N. Dak. & 149 & 1970-2008 & 0.07 & $<0.04$ & 0.86 \\
\hline 75 & Pembina River at Neche, N. Dak. & 78 & $1972-2008$ & $<0.04$ & $<0.04$ & 0.78 \\
\hline 76 & Tongue River at Akra, N. Dak. & 69 & $1972-2008$ & 0.41 & $<0.04$ & 11.00 \\
\hline 78 & Red River of the North at Pembina, N. Dak., site 2 & 95 & 1970-2008 & $<0.04$ & $<0.04$ & 0.56 \\
\hline 79 & Red River of the North at Emerson, Manitoba & 95 & 1978-2004 & $<0.04$ & $<0.04$ & 0.09 \\
\hline 80 & Long Creek near Noonan, N. Dak. & 69 & $1972-2008$ & 0.05 & $<0.04$ & 0.88 \\
\hline 81 & West Branch Short Creek near Columbus, N. Dak. & 10 & 1978-1981 & $<0.04$ & $<0.04$ & 0.07 \\
\hline 82 & Souris River near Sherwood, N. Dak. & 73 & 1972-1991 & 0.08 & $<0.04$ & 4.00 \\
\hline 83 & Souris River near Foxholm, N. Dak. & 145 & $1972-2008$ & 0.12 & $<0.04$ & 4.08 \\
\hline 84 & Des Lacs River at Foxholm, N. Dak. & 76 & $1972-2008$ & 0.09 & $<0.04$ & 0.94 \\
\hline 85 & Souris River above Minot, N. Dak. & 65 & 1970-1998 & 0.05 & $<0.04$ & 6.50 \\
\hline 86 & Bonnes Creek near Velva, N. Dak. & 18 & $1987-2005$ & 0.09 & $<0.04$ & 0.17 \\
\hline 87 & Souris River near Verendrye, N. Dak. & 54 & 1970-1998 & 0.14 & $<0.04$ & 1.00 \\
\hline 88 & Wintering River near Karlsruhe, N. Dak. & 92 & $1972-2008$ & 0.08 & $<0.04$ & 1.30 \\
\hline 89 & Souris River near Bantry, N. Dak. & 79 & 1970-2008 & $<0.04$ & $<0.04$ & 2.20 \\
\hline 90 & Willow Creek near Willow City, N. Dak. & 57 & $1972-2008$ & $<0.04$ & $<0.04$ & 1.60 \\
\hline 91 & Stone Creek near Kramer, N. Dak. & 4 & 1986-1989 & $<0.04$ & $<0.04$ & 0.06 \\
\hline 92 & Deep River near Upham, N. Dak. & 46 & 1972-2007 & $<0.04$ & $<0.04$ & 0.54 \\
\hline 93 & Egg Creek near Granville, N. Dak. & 14 & $1972-1981$ & $<0.04$ & $<0.04$ & 0.06 \\
\hline 94 & Cut Bank Creek at North Lake Outlet near Granville, N. Dak. & 15 & $1972-1976$ & $<0.04$ & $<0.04$ & 0.41 \\
\hline 95 & Cut Bank Creek at Upham, N. Dak. & 15 & 1975-1989 & $<0.04$ & $<0.04$ & 2.20 \\
\hline 96 & Deep River below Cut Bank Creek near Upham, N. Dak. & 14 & 1975-1989 & $<0.04$ & $<0.04$ & 0.18 \\
\hline 97 & Boundary Creek near Landa, N. Dak. & 13 & 1972-1994 & $<0.04$ & $<0.04$ & 0.30 \\
\hline 98 & Souris River near Westhope, N. Dak. & 107 & 1970-2001 & 0.05 & $<0.04$ & 2.90 \\
\hline 99 & Charbonneau Creek near Charbonneau, N. Dak. & 22 & $1972-1977$ & $<0.04$ & $<0.04$ & 0.09 \\
\hline 100 & Missouri River near Williston, N. Dak. & 17 & 1975-1992 & $<0.04$ & $<0.04$ & 0.04 \\
\hline 101 & Little Muddy River below Cow Creek near Williston, N. Dak. & 77 & $1972-2008$ & $<0.04$ & $<0.04$ & 0.30 \\
\hline 102 & Stony Creek near Williston, N. Dak. & 16 & $1977-1981$ & $<0.04$ & $<0.04$ & 0.14 \\
\hline 103 & Tobacco Garden Creek near Watford City, N. Dak. & 14 & 1976-1977 & 0.05 & $<0.04$ & 0.46 \\
\hline 104 & Beaver Creek near Ray, N. Dak. & 28 & $1977-1982$ & $<0.04$ & $<0.04$ & 0.11 \\
\hline 105 & White Earth River at White Earth, N. Dak. & 18 & 1973-1977 & 0.08 & $<0.04$ & 0.24 \\
\hline 106 & Bear Den Creek near Mandaree, N. Dak. & 115 & 1970-2008 & $<0.04$ & $<0.04$ & 1.80 \\
\hline 107 & Shell Creek near Parshall, N. Dak. & 21 & 1972-1977 & 0.06 & $<0.04$ & 0.22 \\
\hline 108 & East Fork Shell Creek near Parshall, N. Dak. & 58 & 1991-2008 & 0.05 & $<0.04$ & 0.33 \\
\hline 109 & Deepwater Creek near Mandaree, N. Dak. & 57 & 1991-2008 & 0.05 & $<0.04$ & 0.27 \\
\hline 110 & Little Missouri River at Marmarth, N. Dak. & 85 & 1971-2008 & $<0.04$ & $<0.04$ & 0.38 \\
\hline
\end{tabular}


Table 1-1. Summary statistics for major ion constituents at selected sites in North Dakota from 1970 through 2008. —Continued $\left[\mu \mathrm{g} / \mathrm{L}\right.$, micrograms per liter; <, less than; --, not available; $\mathrm{mg} / \mathrm{L}$, milligrams per liter; $\mathrm{CO}_{3}$, carbonate; $\mathrm{HCO}_{3}$, bicarbonate; $\mathrm{CaCO}$, calcium carbonate $]$

\begin{tabular}{|c|c|c|c|c|c|c|}
\hline $\begin{array}{c}\text { Site } \\
\text { identification } \\
\text { number } \\
\text { (table 1) }\end{array}$ & Site name & $\begin{array}{c}\text { Number of } \\
\text { samples }\end{array}$ & $\begin{array}{l}\text { Period of } \\
\text { record }\end{array}$ & Median & Minimum & Maximum \\
\hline \multicolumn{7}{|c|}{ Manganese, dissolved, in $\mathrm{mg} / \mathrm{L}-$ Continued } \\
\hline 111 & Deep Creek near Amidon, N. Dak. & 26 & $1977-1983$ & 0.08 & $<0.04$ & 0.26 \\
\hline 112 & Little Missouri River at Medora, N. Dak. & 33 & $1972-2008$ & $<0.04$ & $<0.04$ & 0.06 \\
\hline 113 & Beaver Creek near Trotters, N. Dak. & 69 & $1977-2008$ & $<0.04$ & $<0.04$ & 0.14 \\
\hline 114 & Little Missouri River near Watford City, N. Dak. & 121 & 1971-2008 & $<0.04$ & $<0.04$ & 0.40 \\
\hline 115 & Missouri River at Garrison Dam, N. Dak. & 103 & 1971-1998 & $<0.04$ & $<0.04$ & 0.07 \\
\hline 116 & Knife River at Manning, N. Dak. & 92 & 1972-2008 & 0.05 & $<0.04$ & 0.36 \\
\hline 117 & Stray Creek near Manning, N. Dak. & 9 & 1979-1981 & $<0.04$ & $<0.04$ & 0.40 \\
\hline 118 & Knife River at Marshall, N. Dak. & 43 & 1972-1981 & $<0.04$ & $<0.04$ & 0.32 \\
\hline 119 & Elm Creek near Golden Valley, N. Dak. & 29 & 1973-1995 & 0.08 & $<0.04$ & 1.60 \\
\hline 120 & Knife River near Golden Valley, N. Dak. & 76 & $1971-2008$ & $<0.04$ & $<0.04$ & 0.14 \\
\hline 121 & Coyote Creek near Zap, N. Dak. & 30 & $1977-1983$ & 0.05 & $<0.04$ & 0.19 \\
\hline 122 & Brush Creek near Beulah, N. Dak. & 61 & $1974-1988$ & 0.09 & $<0.04$ & 0.40 \\
\hline 123 & Spring Creek below Lake Ilo near Dunn Center, N. Dak. & 20 & $1977-1981$ & 0.15 & $<0.04$ & 0.66 \\
\hline 124 & Spring Creek near Halliday, N. Dak. & 22 & $1977-1981$ & 0.05 & $<0.04$ & 0.18 \\
\hline 125 & Spring Creek at Zap, N. Dak. & 90 & $1972-2008$ & 0.06 & $<0.04$ & 0.17 \\
\hline 126 & West Branch Otter Creek near Beulah, N. Dak. & 15 & 1972-1995 & 0.12 & $<0.04$ & 0.35 \\
\hline 127 & Knife River at Hazen, N. Dak. & 127 & 1970-2008 & $<0.04$ & $<0.04$ & 0.36 \\
\hline 128 & Antelope Creek above Hazen, N. Dak. & 15 & $1977-1982$ & $<0.04$ & $<0.04$ & 0.13 \\
\hline 129 & West Branch Antelope Creek No. 4 near Zap, N. Dak. & 10 & 1977-1982 & 0.05 & $<0.04$ & 0.12 \\
\hline 130 & West Branch Antelope Creek near Hazen, N. Dak. & 10 & $1978-1983$ & 0.05 & $<0.04$ & 0.12 \\
\hline 131 & Coal Creek near Stanton, N. Dak. & 13 & 1977-1981 & $<0.04$ & $<0.04$ & 0.09 \\
\hline 132 & Alderin Creek near Fort Clark, N. Dak. & 21 & $1978-1982$ & $<0.04$ & $<0.04$ & 0.62 \\
\hline 133 & Coal Lake Coulee near Hensler, N. Dak. & 12 & 1978-1982 & 0.04 & $<0.04$ & 0.14 \\
\hline 134 & Buffalo Creek near Washburn, N. Dak. & 23 & $1979-1983$ & 0.05 & $<0.04$ & 0.14 \\
\hline 136 & Painted Woods Creek near Wilton, N. Dak. & 63 & 1970-1977 & 0.05 & $<0.04$ & 1.20 \\
\hline 137 & Square Butte Creek near Hannover, N. Dak. & 14 & 1977-1981 & $<0.04$ & $<0.04$ & 0.10 \\
\hline 138 & Square Butte Creek above Nelson Lake near Center, N. Dak. & 28 & $1977-1982$ & 0.11 & $<0.04$ & 0.28 \\
\hline 139 & Hagel Creek near Center, N. Dak. & 24 & $1977-1982$ & $<0.04$ & $<0.04$ & 0.24 \\
\hline 140 & Square Butte Creek below Center, N. Dak. & 79 & $1972-2008$ & 0.08 & $<0.04$ & 1.00 \\
\hline 141 & Burnt Creek near Bismarck, N. Dak. & 48 & $1972-2008$ & $<0.04$ & $<0.04$ & 0.25 \\
\hline 142 & Missouri River at Bismarck, N. Dak. & 41 & 1974-2008 & $<0.04$ & $<0.04$ & 0.04 \\
\hline 143 & South Branch Heart River near South Heart, N. Dak. & 15 & $1979-1983$ & $<0.04$ & $<0.04$ & 0.23 \\
\hline 144 & North Creek near South Heart, N. Dak. & 8 & 1979-1981 & 0.05 & $<0.04$ & 0.25 \\
\hline 145 & Heart River near South Heart, N. Dak. & 48 & $1977-2005$ & 0.06 & $<0.04$ & 0.85 \\
\hline 146 & Heart River at Dickinson, N. Dak. & 17 & 1986-1994 & 0.09 & $<0.04$ & 0.31 \\
\hline 147 & Green River near New Hradec, N. Dak. & 90 & $1972-2008$ & $<0.04$ & $<0.04$ & 0.41 \\
\hline 148 & Green River near Gladstone, N. Dak. & 29 & $1970-1975$ & $<0.04$ & $<0.04$ & 0.16 \\
\hline 149 & Heart River near Richardton, N. Dak. & 83 & $1972-2008$ & $<0.04$ & $<0.04$ & 1.10 \\
\hline 150 & Heart River above Lake Tschida near Glen Ullin, N. Dak. & 40 & 1989-2008 & $<0.04$ & $<0.04$ & 0.16 \\
\hline 151 & Antelope Creek near Carson, N. Dak. & 26 & 1972-2008 & $<0.04$ & $<0.04$ & 0.12 \\
\hline
\end{tabular}


Table 1-1. Summary statistics for major ion constituents at selected sites in North Dakota from 1970 through 2008.—Continued [ $\mu \mathrm{g} / \mathrm{L}$, micrograms per liter; <, less than; --, not available; $\mathrm{mg} / \mathrm{L}$, milligrams per liter; $\mathrm{CO}_{3}$, carbonate; $\mathrm{HCO}_{3}$, bicarbonate; $\mathrm{CaCO}_{3}$, calcium carbonate $]$

\begin{tabular}{|c|c|c|c|c|c|c|}
\hline $\begin{array}{c}\text { Site } \\
\text { identification } \\
\text { number } \\
\text { (table 1) } \\
\end{array}$ & Site name & $\begin{array}{l}\text { Number of } \\
\text { samples }\end{array}$ & $\begin{array}{l}\text { Period of } \\
\text { record }\end{array}$ & Median & Minimum & Maximum \\
\hline 152 & Big Muddy Creek near Almont, N. Dak. & 36 & $1991-2008$ & $<0.04$ & $<0.04$ & 0.22 \\
\hline 153 & Heart River near Lark, N. Dak. & 45 & $1971-1995$ & $<0.04$ & $<0.04$ & 0.18 \\
\hline 155 & Sweetbriar Creek near Judson, N. Dak. & 33 & $1972-2008$ & $<0.04$ & $<0.04$ & 0.89 \\
\hline 156 & Heart River near Mandan, N. Dak. & 114 & $1971-2008$ & $<0.04$ & $<0.04$ & 0.65 \\
\hline 157 & Long Lake Creek above Long Lake near Moffit, N. Dak. & 29 & 1990-2004 & 0.12 & $<0.04$ & 0.28 \\
\hline 158 & Apple Creek near Menoken, N. Dak. & 125 & 1972-2008 & 0.10 & $<0.04$ & 1.90 \\
\hline 159 & Missouri River near Schmidt, N. Dak. & 8 & 1975-1979 & $<0.04$ & $<0.04$ & 0.04 \\
\hline 162 & Cannonball River at Regent, N. Dak. & 93 & $1970-2008$ & 0.06 & $<0.04$ & 1.10 \\
\hline 163 & Cannonball River below Bentley, N. Dak. & 31 & $1972-1977$ & 0.05 & $<0.04$ & 0.26 \\
\hline 164 & Cannonball River near Raleigh, N. Dak. & 21 & $2001-2008$ & $<0.04$ & $<0.04$ & 0.07 \\
\hline 165 & White Butte Fork Cedar Creek near Scranton, N. Dak. & 26 & 1972-1995 & 0.22 & $<0.04$ & 0.80 \\
\hline 166 & Cedar Creek near Haynes, N. Dak. & 73 & $1972-2008$ & 0.06 & $<0.04$ & 0.44 \\
\hline 167 & Timber Creek near Bentley, N. Dak. & 23 & $1977-1981$ & 0.06 & $<0.04$ & 0.25 \\
\hline 168 & Cedar Creek near Pretty Rock, N. Dak. & 30 & $1971-1976$ & 0.06 & $<0.04$ & 0.24 \\
\hline 169 & Cedar Creek near Raleigh, N. Dak. & 60 & $1972-2008$ & $<0.04$ & $<0.04$ & 0.16 \\
\hline 170 & Cannonball River at Breien, N. Dak. & 139 & 1970-2008 & $<0.04$ & $<0.04$ & 0.41 \\
\hline 177 & James River above Arrowwood Lake near Kensal, N. Dak. & 172 & $1985-2008$ & 0.05 & $<0.04$ & 4.80 \\
\hline 178 & Kelly Creek below Niccum Reservoir near Bordulac, N. Dak. & 9 & 1986-1989 & $<0.04$ & $<0.04$ & 0.44 \\
\hline 179 & James River near Pingree, N. Dak. & 130 & $1978-2008$ & 0.11 & $<0.04$ & 5.50 \\
\hline 180 & Pipestem Creek near Pingree, N. Dak. & 61 & 1974-2008 & 0.10 & $<0.04$ & 0.98 \\
\hline 181 & Pipestem Creek near Buchanan, N. Dak. & 4 & $1972-1974$ & 0.17 & 0.05 & 0.24 \\
\hline 182 & James River at Jamestown, N. Dak. & 140 & 1972-2008 & 0.58 & $<0.04$ & 2.40 \\
\hline 183 & James River at Lamoure, N. Dak. & 149 & $1970-2008$ & 0.27 & $<0.04$ & 4.80 \\
\hline 184 & Bear Creek near Oakes, N. Dak. & 53 & 1977-2008 & 0.23 & $<0.04$ & 0.88 \\
\hline 185 & James River at Oakes, N. Dak. & 105 & $1970-2008$ & 0.14 & $<0.04$ & 2.60 \\
\hline 186 & James River at N. Dak./S. Dak. State line & 31 & $1975-2008$ & 0.22 & $<0.04$ & 1.55 \\
\hline \multicolumn{7}{|c|}{ Chloride, in $\mathrm{mg} / \mathrm{L}$} \\
\hline 1 & Bois De Sioux River near Doran, Minn. & 40 & $2000-2007$ & 16.5 & $<4.0$ & 65.0 \\
\hline 2 & Red River of the North at Wahpeton, N. Dak. & 74 & 1973-2008 & 12.0 & $<4.0$ & 37.6 \\
\hline 3 & Red River of the North near Wahpeton, N. Dak. & 4 & 1993-2006 & 14.05 & 9.3 & 16.7 \\
\hline
\end{tabular}


Table 1-1. Summary statistics for major ion constituents at selected sites in North Dakota from 1970 through 2008. —Continued $\left[\mu \mathrm{g} / \mathrm{L}\right.$, micrograms per liter; <, less than; --, not available; $\mathrm{mg} / \mathrm{L}$, milligrams per liter; $\mathrm{CO}_{3}$, carbonate; $\mathrm{HCO}_{3}$, bicarbonate; $\mathrm{CaCO}_{3}$, calcium carbonate $]$

\begin{tabular}{|c|c|c|c|c|c|c|}
\hline $\begin{array}{c}\text { Site } \\
\text { identification } \\
\text { number } \\
\text { (table 1) }\end{array}$ & Site name & $\begin{array}{c}\text { Number of } \\
\text { samples }\end{array}$ & $\begin{array}{l}\text { Period of } \\
\text { record }\end{array}$ & Median & Minimum & Maximum \\
\hline \multicolumn{7}{|c|}{ Chloride, in $\mathrm{mg} / \mathrm{L}$-Continued } \\
\hline 4 & Red River of the North at Brushville, Minn. & 61 & $1993-2007$ & 15.3 & $<4.0$ & 47.7 \\
\hline 5 & Red River of the North below Wahpeton, N. Dak. & 58 & 1970-1999 & 7.1 & $<4.0$ & 23.5 \\
\hline 6 & Red River of the North at Hickson, N. Dak. & 134 & $1975-2008$ & 11.0 & $<4.0$ & 44.0 \\
\hline 7 & Wild Rice River near Rutland, N. Dak. & 47 & $1971-2008$ & 32.0 & $<4.0$ & 156.0 \\
\hline 8 & Wild Rice River near Cayuga, N. Dak. & 15 & 1970-1977 & 30.0 & 17.0 & 408.0 \\
\hline 9 & Antelope Creek at Dwight, N. Dak. & 13 & $2001-2008$ & 24.3 & 13.2 & 40.0 \\
\hline 10 & Wild Rice River near Abercrombie, N. Dak. & 218 & 1970-2008 & 38.9 & $<4.0$ & 180.0 \\
\hline 11 & Red River of the North at Fargo, N. Dak. & 139 & 1970-2008 & 13.0 & $<4.0$ & 45.5 \\
\hline 12 & Red River of North below Fargo, N. Dak. & 167 & 1970-1986 & 11.0 & 4.4 & 96.0 \\
\hline 13 & Red River of the North at Harwood, N. Dak. & 53 & $2000-2007$ & 21.3 & 5.6 & 63.6 \\
\hline 14 & Red River of the North near Harwood, N. Dak. & 59 & 1993-2007 & 18.7 & 4.8 & 35.4 \\
\hline 15 & Sheyenne River above Harvey, N. Dak. & 199 & $1972-2008$ & 17.0 & $<4.0$ & 54.0 \\
\hline 16 & Big Coulee near Fort Totten, N. Dak. & 8 & $1970-1975$ & 8.5 & $<4.0$ & 36.0 \\
\hline 17 & Sheyenne River at Warwick, N. Dak. & 48 & 1996-2006 & 17.0 & 8.4 & 51.3 \\
\hline 18 & Sheyenne River near Warwick, N. Dak. & 181 & 1970-2008 & 14.0 & $<4.0$ & 28.0 \\
\hline 19 & Mauvais Coulee Tributary No. 3 near Cando, N. Dak. & 38 & $1987-2008$ & 17.2 & $<4.0$ & 123.0 \\
\hline 20 & Mauvais Coulee near Cando, N. Dak. & 104 & 1972-2008 & 19.0 & $<4.0$ & 60.0 \\
\hline 21 & Edmore Coulee near Edmore, N. Dak. & 103 & $1972-2008$ & 20.4 & $<4.0$ & 100.0 \\
\hline 22 & Edmore Coulee Tributary near Webster, N. Dak. & 38 & $1987-2008$ & 20.1 & 4.3 & 150.0 \\
\hline 23 & Webster Coulee at Webster, N. Dak. & 2 & $1983-1984$ & 20.0 & 19.0 & 21.0 \\
\hline 24 & Starkweather Coulee near Webster, N. Dak. & 94 & $1983-2008$ & 14.0 & $<4.0$ & 49.8 \\
\hline 25 & Big Coulee below Churchs Ferry, N. Dak. & 23 & $1998-2008$ & 24.5 & 9.3 & 98.8 \\
\hline 26 & Little Coulee near Leeds, N. Dak. & 16 & $1998-2008$ & 24.5 & 11.0 & 46.9 \\
\hline 27 & Little Coulee near Brinsmade, N. Dak. & 31 & 1976-1998 & 23.0 & $<4.0$ & 150.0 \\
\hline 28 & Big Coulee near Churchs Ferry, N. Dak. & 136 & 1970-1997 & 18.5 & 6.2 & 92.0 \\
\hline 29 & Big Coulee at Graham Island inlet near Fort Totten, N. Dak. & 44 & 1970-1986 & 195.0 & 21.0 & 900.0 \\
\hline 30 & Channel A near Penn, N. Dak. & 87 & 1984-2008 & 19.0 & 7.4 & 96.0 \\
\hline 31 & Devils Lake Outlet to Stump Lake near Lakota, N. Dak. & 12 & $2002-2007$ & 278.5 & 96.4 & 480.0 \\
\hline 32 & Sheyenne River near Cooperstown, N. Dak. & 321 & 1970-2008 & 16.0 & $<4.0$ & 39.0 \\
\hline 33 & Baldhill Creek near Dazey, N. Dak. & 104 & $1972-2008$ & 15.0 & $<4.0$ & 51.0 \\
\hline 34 & Sheyenne River below Baldhill Dam, N. Dak. & 181 & $1972-2008$ & 15.3 & 4.7 & 31.0 \\
\hline 35 & Sheyenne River at Valley City, N. Dak. & 29 & $1972-2005$ & 18.0 & 5.1 & 52.0 \\
\hline 36 & Sheyenne River at Lisbon, N. Dak. & 300 & 1970-2008 & 27.0 & 7.2 & 110.0 \\
\hline 37 & Sheyenne River near Kindred, N. Dak. & 313 & $1972-2008$ & 26.0 & 5.7 & 74.0 \\
\hline 38 & $\begin{array}{l}\text { Sheyenne River above Sheyenne River Diversion near Horace, } \\
\text { N. Dak. }\end{array}$ & 43 & $1993-2008$ & 24.0 & $<4.0$ & 40.0 \\
\hline 39 & Sheyenne River near Horace, N. Dak. & 8 & 1982-1992 & 19.5 & 7.4 & 28.0 \\
\hline 40 & Sheyenne River Diversion at West Fargo, N. Dak. & 13 & 1994-2007 & 15.7 & $<4.0$ & 26.0 \\
\hline 41 & Sheyenne River at West Fargo, N. Dak. & 62 & $1970-2008$ & 26.6 & 7.8 & 57.3 \\
\hline 42 & Maple River near Hope, N. Dak. & 39 & $1972-2008$ & 26.0 & 5.3 & 90.0 \\
\hline
\end{tabular}


Table 1-1. Summary statistics for major ion constituents at selected sites in North Dakota from 1970 through 2008.—Continued [ $\mu \mathrm{g} / \mathrm{L}$, micrograms per liter; <, less than; --, not available; $\mathrm{mg} / \mathrm{L}$, milligrams per liter; $\mathrm{CO}_{3}$, carbonate; $\mathrm{HCO}_{3}$, bicarbonate; $\mathrm{CaCO}_{3}$, calcium carbonate $]$

\begin{tabular}{|c|c|c|c|c|c|c|}
\hline $\begin{array}{c}\text { Site } \\
\text { identification } \\
\text { number } \\
\text { (table 1) }\end{array}$ & Site name & $\begin{array}{l}\text { Number of } \\
\text { samples }\end{array}$ & $\begin{array}{l}\text { Period of } \\
\text { record }\end{array}$ & Median & Minimum & Maximum \\
\hline \multicolumn{7}{|c|}{ Chloride, in $\mathrm{mg} / \mathrm{L}$-Continued } \\
\hline 43 & Maple River near Enderlin, N. Dak. & 63 & $1972-2008$ & 53.0 & $<4.0$ & 140.0 \\
\hline 44 & Maple River near Mapleton, N. Dak. & 25 & $1972-2008$ & 45.6 & 15.0 & 99.0 \\
\hline 46 & Sheyenne River at Harwood, N. Dak. & 36 & $1993-2005$ & 31.4 & 11.0 & 94.5 \\
\hline 47 & Rush River at Amenia, N. Dak. & 51 & $1972-2008$ & 17.0 & $<4.0$ & 64.0 \\
\hline 48 & Rush River near Prosper, N. Dak. & 4 & $1983-1987$ & 10.4 & $<4.0$ & 39.0 \\
\hline 49 & Lower Branch Rush River near Prosper, N. Dak. & 5 & $1983-1993$ & 4.8 & $<4.0$ & 29.7 \\
\hline 53 & Beaver Creek near Finley, N. Dak. & 116 & $1970-2003$ & 19.0 & $<4.0$ & 68.0 \\
\hline 54 & Goose River near Portland, N. Dak. & 30 & $1970-1988$ & 25.0 & 4.6 & 134.0 \\
\hline 55 & Goose River at Hillsboro, N. Dak. & 166 & $1970-2008$ & 36.5 & 6.9 & 310.0 \\
\hline 56 & Red River of the North at Grand Forks, N. Dak. & 198 & $1970-2008$ & 12.0 & $<4.0$ & 32.4 \\
\hline 57 & Turtle River at Turtle River State Park near Arvilla, N. Dak. & 99 & $1991-2008$ & 23.0 & 6.8 & 160.0 \\
\hline 58 & Turtle River at Manvel, N. Dak. & 106 & $1971-2008$ & 446.5 & 71.0 & $3,600.0$ \\
\hline 59 & Red River of the North at Oslo, Minn. & 75 & $1973-2005$ & 15.0 & 5.1 & 130.0 \\
\hline 60 & Middle Branch Forest River near Whitman, N. Dak. & 14 & $1972-1990$ & 15.0 & 4.9 & 100.0 \\
\hline 61 & Forest River near Fordville, N. Dak. & 64 & $1972-2008$ & 13.0 & 4.8 & 35.2 \\
\hline 68 & Red River of the North at Drayton, N. Dak. & 74 & $1972-2008$ & 33.5 & 5.8 & 160.0 \\
\hline 69 & Pembina County Drain 20 near Glasston, N. Dak. & 8 & 1974-1984 & 5.9 & $<4.0$ & 27.0 \\
\hline 70 & Hidden Island Coulee near Hansboro, N. Dak. & 22 & $1972-1995$ & 11.0 & $<4.0$ & 24.0 \\
\hline 71 & Cypress Creek near Sarles, N. Dak. & 13 & $1972-1988$ & 9.2 & $<4.0$ & 31.0 \\
\hline 72 & Pembina River near Vang, N. Dak. & 63 & 1970-1979 & 11.0 & $<4.0$ & 24.0 \\
\hline 73 & Little South Pembina River near Walhalla, N. Dak. & 83 & $1970-2008$ & 15.0 & $<4.0$ & 27.0 \\
\hline 74 & Pembina River at Walhalla, N. Dak. & 225 & $1970-2008$ & 13.0 & $<4.0$ & 57.0 \\
\hline 75 & Pembina River at Neche, N. Dak. & 133 & $1972-2008$ & 15.0 & $<4.0$ & 31.0 \\
\hline 76 & Tongue River at Akra, N. Dak. & 72 & $1972-2008$ & 10.0 & $<4.0$ & 40.0 \\
\hline 77 & Red River of the North at Pembina, N. Dak., site 1 & 10 & 1993 & 27.8 & 15.6 & 66.2 \\
\hline 78 & Red River of the North at Pembina, N. Dak., site 2 & 160 & $1970-2008$ & 26.0 & 5.0 & 142.0 \\
\hline 79 & Red River of the North at Emerson, Manitoba & 149 & $1977-2004$ & 34.0 & 9.8 & 240.0 \\
\hline 80 & Long Creek near Noonan, N. Dak. & 70 & $1972-2008$ & 18.0 & $<4.0$ & 48.5 \\
\hline 81 & West Branch Short Creek near Columbus, N. Dak. & 20 & $1978-1981$ & 14.5 & 4.4 & 37.0 \\
\hline 82 & Souris River near Sherwood, N. Dak. & 304 & $1972-2008$ & 47.5 & $<4.0$ & 220.0 \\
\hline
\end{tabular}


Table 1-1. Summary statistics for major ion constituents at selected sites in North Dakota from 1970 through 2008. —Continued $\left[\mu \mathrm{g} / \mathrm{L}\right.$, micrograms per liter; <, less than; --, not available; $\mathrm{mg} / \mathrm{L}$, milligrams per liter; $\mathrm{CO}_{3}$, carbonate; $\mathrm{HCO}_{3}$, bicarbonate; $\mathrm{CaCO}$, calcium carbonate $]$

\begin{tabular}{|c|c|c|c|c|c|c|}
\hline $\begin{array}{c}\text { Site } \\
\text { identification } \\
\text { number } \\
\text { (table 1) }\end{array}$ & Site name & $\begin{array}{l}\text { Number of } \\
\text { samples }\end{array}$ & $\begin{array}{l}\text { Period of } \\
\text { record }\end{array}$ & Median & Minimum & Maximum \\
\hline \multicolumn{7}{|c|}{ Chloride, in $\mathrm{mg} / \mathrm{L}$-Continued } \\
\hline 83 & Souris River near Foxholm, N. Dak. & 202 & $1972-2008$ & 22.0 & 5.3 & 160.0 \\
\hline 84 & Des Lacs River at Foxholm, N. Dak. & 188 & $1972-2008$ & 26.1 & $<4.0$ & 53.0 \\
\hline 85 & Souris River above Minot, N. Dak. & 207 & 1970-2008 & 29.8 & $<4.0$ & 161.0 \\
\hline 86 & Bonnes Creek near Velva, N. Dak. & 18 & 1987-2005 & 7.6 & $<4.0$ & 19.0 \\
\hline 87 & Souris River near Verendrye, N. Dak. & 371 & 1970-2008 & 32.5 & $<4.0$ & 247.0 \\
\hline 88 & Wintering River near Karlsruhe, N. Dak. & 134 & 1972-2008 & 11.2 & 4.9 & 38.0 \\
\hline 89 & Souris River near Bantry, N. Dak. & 152 & 1970-2008 & 26.7 & 5.8 & 110.0 \\
\hline 90 & Willow Creek near Willow City, N. Dak. & 90 & 1972-2008 & 22.2 & $<4.0$ & 220.0 \\
\hline 91 & Stone Creek near Kramer, N. Dak. & 30 & $1986-2000$ & 12.4 & $<4.0$ & 56.0 \\
\hline 92 & Deep River near Upham, N. Dak. & 68 & $1972-2007$ & 45.5 & $<4.0$ & 180.0 \\
\hline 93 & Egg Creek near Granville, N. Dak. & 14 & $1972-1981$ & 20.0 & 5.4 & 73.0 \\
\hline 94 & Cut Bank Creek at North Lake Outlet near Granville, N. Dak. & 15 & $1972-1976$ & 31.0 & 10.0 & 66.0 \\
\hline 95 & Cut Bank Creek at Upham, N. Dak. & 33 & $1975-2000$ & 26.4 & 8.4 & 58.0 \\
\hline 96 & Deep River below Cut Bank Creek near Upham, N. Dak. & 43 & 1975-1989 & 39.0 & 8.5 & 130.0 \\
\hline 97 & Boundary Creek near Landa, N. Dak. & 39 & $1972-2000$ & 14.0 & $<4.0$ & 59.0 \\
\hline 98 & Souris River near Westhope, N. Dak. & 214 & 1970-2008 & 28.0 & 6.0 & 140.0 \\
\hline 99 & Charbonneau Creek near Charbonneau, N. Dak. & 22 & $1972-1977$ & 5.4 & $<4.0$ & 7.5 \\
\hline 100 & Missouri River near Williston, N. Dak. & 99 & $1970-1992$ & 10.0 & 5.3 & 22.0 \\
\hline 101 & Little Muddy River below Cow Creek near Williston, N. Dak. & 77 & $1972-2008$ & 8.0 & $<4.0$ & 89.0 \\
\hline 102 & Stony Creek near Williston, N. Dak. & 32 & $1977-1981$ & 7.9 & $<4.0$ & 50.0 \\
\hline 103 & Tobacco Garden Creek near Watford City, N. Dak. & 15 & $1976-1977$ & 5.1 & $<4.0$ & 8.9 \\
\hline 104 & Beaver Creek near Ray, N. Dak. & 51 & $1977-1982$ & 12.0 & $<4.0$ & 23.0 \\
\hline 105 & White Earth River at White Earth, N. Dak. & 27 & $1970-1977$ & 34.0 & 14.0 & 150.0 \\
\hline 106 & Bear Den Creek near Mandaree, N. Dak. & 229 & 1970-2008 & 4.4 & $<4.0$ & 180.0 \\
\hline 107 & Shell Creek near Parshall, N. Dak. & 21 & $1972-1977$ & 8.5 & $<4.0$ & 17.0 \\
\hline 108 & East Fork Shell Creek near Parshall, N. Dak. & 58 & 1991-2008 & 19.0 & $<4.0$ & 52.0 \\
\hline 109 & Deepwater Creek near Mandaree, N. Dak. & 57 & 1991-2008 & 10.9 & $<4.0$ & 33.0 \\
\hline 110 & Little Missouri River at Marmarth, N. Dak. & 86 & 1971-2008 & 9.0 & $<4.0$ & 30.0 \\
\hline 111 & Deep Creek near Amidon, N. Dak. & 51 & $1977-1983$ & 9.8 & $<4.0$ & 28.0 \\
\hline 112 & Little Missouri River at Medora, N. Dak. & 112 & $1972-2008$ & 9.6 & $<4.0$ & 36.7 \\
\hline 113 & Beaver Creek near Trotters, N. Dak. & 94 & $1977-2008$ & 9.2 & $<4.0$ & 22.0 \\
\hline 114 & Little Missouri River near Watford City, N. Dak. & 243 & $1971-2008$ & 9.4 & $<4.0$ & 219.0 \\
\hline 115 & Missouri River at Garrison Dam, N. Dak. & 264 & $1971-2007$ & 9.5 & $<4.0$ & 19.0 \\
\hline 116 & Knife River at Manning, N. Dak. & 118 & $1972-2008$ & 6.6 & $<4.0$ & 32.0 \\
\hline 117 & Stray Creek near Manning, N. Dak. & 17 & $1975-1981$ & 7.0 & $<4.0$ & 14.0 \\
\hline 118 & Knife River at Marshall, N. Dak. & 69 & $1972-1981$ & 5.3 & $<4.0$ & 49.0 \\
\hline 119 & Elm Creek near Golden Valley, N. Dak. & 36 & 1973-1994 & 4.5 & $<4.0$ & 31.0 \\
\hline 120 & Knife River near Golden Valley, N. Dak. & 130 & 1971-2008 & 6.4 & $<4.0$ & 26.0 \\
\hline 121 & Coyote Creek near Zap, N. Dak. & 51 & $1977-1983$ & 6.9 & $<4.0$ & 17.0 \\
\hline 122 & Brush Creek near Beulah, N. Dak. & 105 & 1974-1990 & 6.7 & $<4.0$ & 14.0 \\
\hline
\end{tabular}


Table 1-1. Summary statistics for major ion constituents at selected sites in North Dakota from 1970 through 2008.—Continued [ $\mu \mathrm{g} / \mathrm{L}$, micrograms per liter; <, less than; --, not available; $\mathrm{mg} / \mathrm{L}$, milligrams per liter; $\mathrm{CO}_{3}$, carbonate; $\mathrm{HCO}_{3}$, bicarbonate; $\mathrm{CaCO}_{3}$, calcium carbonate $]$

\begin{tabular}{|c|c|c|c|c|c|c|}
\hline $\begin{array}{c}\text { Site } \\
\text { identification } \\
\text { number } \\
\text { (table 1) }\end{array}$ & Site name & $\begin{array}{c}\text { Number of } \\
\text { samples }\end{array}$ & $\begin{array}{l}\text { Period of } \\
\text { record }\end{array}$ & Median & Minimum & Maximum \\
\hline \multicolumn{7}{|c|}{ Chloride, in $\mathrm{mg} / \mathrm{L}$-Continued } \\
\hline 123 & Spring Creek below Lake Ilo near Dunn Center, N. Dak. & 53 & 1977-1993 & 6.8 & $<4.0$ & 42.0 \\
\hline 124 & Spring Creek near Halliday, N. Dak. & 48 & 1977-1981 & 6.5 & $<4.0$ & 26.0 \\
\hline 125 & Spring Creek at Zap, N. Dak. & 239 & 1970-2008 & 7.3 & $<4.0$ & 31.4 \\
\hline 126 & West Branch Otter Creek near Beulah, N. Dak. & 15 & $1972-1995$ & $<4.0$ & $<4.0$ & 30.0 \\
\hline 127 & Knife River at Hazen, N. Dak. & 270 & 1970-2008 & 7.2 & $<4.0$ & 48.0 \\
\hline 128 & Antelope Creek above Hazen, N. Dak. & 28 & $1977-1982$ & 11.0 & $<4.0$ & 24.0 \\
\hline 129 & West Branch Antelope Creek No. 4 near Zap, N. Dak. & 10 & 1977-1982 & $<4.0$ & $<4.0$ & 5.6 \\
\hline 130 & West Branch Antelope Creek near Hazen, N. Dak. & 14 & $1978-1983$ & $<4.0$ & $<4.0$ & 18.0 \\
\hline 131 & Coal Creek near Stanton, N. Dak. & 28 & 1975-1981 & 8.1 & $<4.0$ & 18.0 \\
\hline 132 & Alderin Creek near Fort Clark, N. Dak. & 35 & 1977-1982 & 5.1 & $<4.0$ & 19.0 \\
\hline 133 & Coal Lake Coulee near Hensler, N. Dak. & 17 & 1978-1987 & $<4.0$ & $<4.0$ & 14.0 \\
\hline 134 & Buffalo Creek near Washburn, N. Dak. & 38 & $1978-1983$ & 6.5 & $<4.0$ & 150.0 \\
\hline 135 & Turtle Creek above Washburn, N. Dak. & 102 & $1987-2003$ & 12.2 & $<4.0$ & 80.0 \\
\hline 136 & Painted Woods Creek near Wilton, N. Dak. & 201 & 1970-2003 & 17.0 & $<4.0$ & 82.0 \\
\hline 137 & Square Butte Creek near Hannover, N. Dak. & 23 & $1977-1981$ & 5.9 & $<4.0$ & 9.7 \\
\hline 138 & Square Butte Creek above Nelson Lake near Center, N. Dak. & 56 & 1977-1982 & 7.0 & $<4.0$ & 12.0 \\
\hline 139 & Hagel Creek near Center, N. Dak. & 38 & 1977-1982 & 4.3 & $<4.0$ & 10.0 \\
\hline 140 & Square Butte Creek below Center, N. Dak. & 81 & $1972-2008$ & 10.0 & $<4.0$ & 27.3 \\
\hline 141 & Burnt Creek near Bismarck, N. Dak. & 48 & $1972-2008$ & 7.3 & $<4.0$ & 15.0 \\
\hline 142 & Missouri River at Bismarck, N. Dak. & 128 & 1974-2008 & 9.6 & $<4.0$ & 15.0 \\
\hline 143 & South Branch Heart River near South Heart, N. Dak. & 31 & 1979-1996 & 4.8 & $<4.0$ & 47.0 \\
\hline 144 & North Creek near South Heart, N. Dak. & 24 & 1978-1996 & 5.2 & $<4.0$ & 15.2 \\
\hline 145 & Heart River near South Heart, N. Dak. & 76 & 1975-2005 & 18.0 & $<4.0$ & 100.0 \\
\hline 146 & Heart River at Dickinson, N. Dak. & 22 & 1986-1994 & 48.0 & 7.0 & 110.0 \\
\hline 147 & Green River near New Hradec, N. Dak. & 116 & $1972-2008$ & 5.9 & $<4.0$ & 20.0 \\
\hline 148 & Green River near Gladstone, N. Dak. & 33 & 1970-1993 & 6.3 & $<4.0$ & 25.0 \\
\hline 149 & Heart River near Richardton, N. Dak. & 154 & $1972-2008$ & 17.0 & $<4.0$ & 66.2 \\
\hline 150 & Heart River above Lake Tschida near Glen Ullin, N. Dak. & 40 & 1989-2008 & 13.5 & $<4.0$ & 48.0 \\
\hline 151 & Antelope Creek near Carson, N. Dak. & 26 & $1972-2008$ & 5.9 & $<4.0$ & 14.0 \\
\hline 152 & Big Muddy Creek near Almont, N. Dak. & 39 & $1991-2008$ & 7.0 & $<4.0$ & 16.4 \\
\hline 153 & Heart River near Lark, N. Dak. & 45 & 1971-1995 & 5.9 & $<4.0$ & 15.0 \\
\hline 154 & Heart River at Stark Bridge near Judson, N. Dak. & 41 & $1988-2008$ & 10.0 & $<4.0$ & 19.6 \\
\hline 155 & Sweetbriar Creek near Judson, N. Dak. & 33 & $1972-2008$ & 5.1 & $<4.0$ & 13.1 \\
\hline 156 & Heart River near Mandan, N. Dak. & 232 & 1971-2008 & 12.0 & $<4.0$ & 303.0 \\
\hline 157 & Long Lake Creek above Long Lake near Moffit, N. Dak. & 33 & 1988-2004 & 6.2 & $<4.0$ & 18.0 \\
\hline 158 & Apple Creek near Menoken, N. Dak. & 129 & $1972-2008$ & 30.0 & $<4.0$ & 77.0 \\
\hline 159 & Missouri River near Schmidt, N. Dak. & 68 & 1974-1981 & 9.4 & 7.4 & 18.0 \\
\hline 160 & Cannonball River at New England, N. Dak. & 36 & 1978-1981 & 8.1 & $<4.0$ & 42.0 \\
\hline 161 & Coal Bank Creek near Havelock, N. Dak. & 84 & $1974-1983$ & 10.0 & $<4.0$ & 89.0 \\
\hline 162 & Cannonball River at Regent, N. Dak. & 119 & 1970-2008 & 9.7 & $<4.0$ & 55.0 \\
\hline
\end{tabular}


Table 1-1. Summary statistics for major ion constituents at selected sites in North Dakota from 1970 through 2008. —Continued $\left[\mu \mathrm{g} / \mathrm{L}\right.$, micrograms per liter; <, less than; --, not available; $\mathrm{mg} / \mathrm{L}$, milligrams per liter; $\mathrm{CO}_{3}$, carbonate; $\mathrm{HCO}_{3}$, bicarbonate; $\mathrm{CaCO}$, calcium carbonate $]$

\begin{tabular}{|c|c|c|c|c|c|c|}
\hline $\begin{array}{c}\text { Site } \\
\text { identification } \\
\text { number } \\
\text { (table 1) } \\
\end{array}$ & Site name & $\begin{array}{l}\text { Number of } \\
\text { samples }\end{array}$ & $\begin{array}{l}\text { Period of } \\
\text { record }\end{array}$ & Median & Minimum & Maximum \\
\hline \multicolumn{7}{|c|}{ Chloride, in $\mathrm{mg} / \mathrm{L}$-Continued } \\
\hline 163 & Cannonball River below Bentley, N. Dak. & 31 & $1972-1977$ & 11.0 & $<4.0$ & 17.0 \\
\hline 164 & Cannonball River near Raleigh, N. Dak. & 89 & 1993-2008 & 9.9 & $<4.0$ & 41.8 \\
\hline 165 & White Butte Fork Cedar Creek near Scranton, N. Dak. & 26 & $1972-1995$ & 18.5 & 4.4 & 180.0 \\
\hline 166 & Cedar Creek near Haynes, N. Dak. & 76 & $1971-2008$ & 11.0 & $<4.0$ & 22.0 \\
\hline 167 & Timber Creek near Bentley, N. Dak. & 47 & 1977-1981 & 14.0 & $<4.0$ & 510.0 \\
\hline 168 & Cedar Creek near Pretty Rock, N. Dak. & 30 & 1971-1976 & 11.5 & $<4.0$ & 18.0 \\
\hline 169 & Cedar Creek near Raleigh, N. Dak. & 120 & $1972-2008$ & 9.3 & $<4.0$ & 34.8 \\
\hline 170 & Cannonball River at Breien, N. Dak. & 260 & 1970-2008 & 11.0 & $<4.0$ & 62.0 \\
\hline 171 & Beaver Creek near Linton, N. Dak. & 39 & $1972-1993$ & 7.8 & $<4.0$ & 19.0 \\
\hline 172 & Beaver Creek below Linton, N. Dak. & 38 & 1990-2008 & 11.0 & $<4.0$ & 55.0 \\
\hline 173 & Porcupine Creek near Fort Yates, N. Dak. & 41 & 1991-1999 & 13.7 & $<4.0$ & 62.0 \\
\hline 174 & Buffalo Creek Tributary near Gascoyne, N. Dak. & 83 & 1974-1987 & 14.0 & $<4.0$ & 89.0 \\
\hline 175 & James River near Manfred, N. Dak. & 48 & 1972-1998 & 9.8 & $<4.0$ & 20.0 \\
\hline 176 & James River near Grace City, N. Dak. & 155 & $1972-2008$ & 18.0 & $<4.0$ & 250.0 \\
\hline 177 & James River above Arrowwood Lake near Kensal, N. Dak. & 172 & 1985-2008 & 18.0 & $<4.0$ & 59.3 \\
\hline 178 & Kelly Creek below Niccum Reservoir near Bordulac, N. Dak. & 9 & 1986-1989 & 11.0 & 4.9 & 17.0 \\
\hline 179 & James River near Pingree, N. Dak. & 142 & 1978-2008 & 14.5 & $<4.0$ & 54.0 \\
\hline 180 & Pipestem Creek near Pingree, N. Dak. & 71 & 1974-2008 & 12.0 & $<4.0$ & 27.9 \\
\hline 181 & Pipestem Creek near Buchanan, N. Dak. & 9 & $1972-1993$ & 7.3 & $<4.0$ & 28.0 \\
\hline 182 & James River at Jamestown, N. Dak. & 201 & $1972-2008$ & 21.6 & 6.0 & 84.5 \\
\hline 183 & James River at Lamoure, N. Dak. & 284 & $1970-2008$ & 28.3 & $<4.0$ & 180.0 \\
\hline 184 & Bear Creek near Oakes, N. Dak. & 54 & $1977-2008$ & 50.2 & 6.8 & 110.0 \\
\hline 185 & James River at Oakes, N. Dak. & 136 & 1970-2008 & 38.7 & 7.3 & 430.0 \\
\hline 186 & James River at N. Dak./S. Dak. State line & 97 & 1974-2008 & 30.0 & 5.5 & 250.0 \\
\hline \multicolumn{7}{|c|}{ Sulfate, in $\mathrm{mg} / \mathrm{L}$} \\
\hline 1 & Bois De Sioux River near Doran, Minn. & 40 & $2000-2007$ & 462 & 41 & 1,660 \\
\hline 2 & Red River of the North at Wahpeton, N. Dak. & 74 & 1973-2008 & 86 & 15 & 520 \\
\hline 3 & Red River of the North near Wahpeton, N. Dak. & 4 & 1993-2006 & 176 & 49 & 433 \\
\hline 4 & Red River of the North at Brushville, Minn. & 61 & 1993-2007 & 87 & 21 & 273 \\
\hline 5 & Red River of the North below Wahpeton, N. Dak. & 64 & 1970-1999 & 38 & 12 & 422 \\
\hline 6 & Red River of the North at Hickson, N. Dak. & 134 & 1975-2008 & 67 & 5 & 340 \\
\hline 7 & Wild Rice River near Rutland, N. Dak. & 47 & 1971-2008 & 490 & 52 & 1,870 \\
\hline 8 & Wild Rice River near Cayuga, N. Dak. & 15 & $1970-1977$ & 320 & 230 & 1,310 \\
\hline 9 & Antelope Creek at Dwight, N. Dak. & 13 & $2001-2008$ & 235 & 140 & 419 \\
\hline 10 & Wild Rice River near Abercrombie, N. Dak. & 224 & 1970-2008 & 394 & 11 & 1,200 \\
\hline 11 & Red River of the North at Fargo, N. Dak. & 144 & 1970-2008 & 86 & 23 & 311 \\
\hline 12 & Red River of North below Fargo, N. Dak. & 174 & $1970-1986$ & 69 & 19 & 330 \\
\hline 13 & Red River of the North at Harwood, N. Dak. & 53 & $2000-2007$ & 146 & 38 & 288 \\
\hline 14 & Red River of the North near Harwood, N. Dak. & 59 & 1993-2007 & 152 & 40 & 298 \\
\hline
\end{tabular}


Table 1-1. Summary statistics for major ion constituents at selected sites in North Dakota from 1970 through 2008. - Continued $\left[\mu \mathrm{g} / \mathrm{L}\right.$, micrograms per liter; <, less than; --, not available; mg/L, milligrams per liter; $\mathrm{CO}_{3}$, carbonate; $\mathrm{HCO}_{3}$, bicarbonate; $\mathrm{CaCO}$, calcium carbonate $]$

\begin{tabular}{|c|c|c|c|c|c|c|}
\hline $\begin{array}{c}\text { Site } \\
\text { identification } \\
\text { number } \\
\text { (table 1) } \\
\end{array}$ & Site name & $\begin{array}{l}\text { Number of } \\
\text { samples }\end{array}$ & $\begin{array}{l}\text { Period of } \\
\text { record }\end{array}$ & Median & Minimum & Maximum \\
\hline \multicolumn{7}{|c|}{ Sulfate, in $\mathrm{mg} / \mathrm{L}$-Continued } \\
\hline 15 & Sheyenne River above Harvey, N. Dak. & 199 & $1972-2008$ & 210 & 37 & 610 \\
\hline 16 & Big Coulee near Fort Totten, N. Dak. & 8 & $1970-1975$ & 20 & 10 & 220 \\
\hline 17 & Sheyenne River at Warwick, N. Dak. & 48 & $1996-2006$ & 217 & 139 & 353 \\
\hline 18 & Sheyenne River near Warwick, N. Dak. & 186 & 1970-2008 & 110 & 35 & 307 \\
\hline 19 & Mauvais Coulee Tributary No. 3 near Cando, N. Dak. & 38 & $1987-2008$ & 179 & 33 & 820 \\
\hline 20 & Mauvais Coulee near Cando, N. Dak. & 104 & $1972-2008$ & 230 & 35 & 680 \\
\hline 21 & Edmore Coulee near Edmore, N. Dak. & 103 & 1972-2008 & 170 & 17 & 793 \\
\hline 22 & Edmore Coulee Tributary near Webster, N. Dak. & 38 & 1987-2008 & 190 & 25 & 1,100 \\
\hline 23 & Webster Coulee at Webster, N. Dak. & 2 & $1983-1984$ & 155 & 150 & 160 \\
\hline 24 & Starkweather Coulee near Webster, N. Dak. & 94 & $1983-2008$ & 115 & 17 & 472 \\
\hline 25 & Big Coulee below Churchs Ferry, N. Dak. & 23 & $1998-2008$ & 227 & 110 & 556 \\
\hline 26 & Little Coulee near Leeds, N. Dak. & 16 & 1998-2008 & 270 & 91 & 896 \\
\hline 27 & Little Coulee near Brinsmade, N. Dak. & 31 & $1976-1998$ & 160 & 44 & 1,100 \\
\hline 28 & Big Coulee near Churchs Ferry, N. Dak. & 136 & $1970-1997$ & 150 & 54 & 600 \\
\hline 29 & Big Coulee at Graham Island inlet near Fort Totten, N. Dak. & 44 & $1970-1986$ & 910 & 150 & 3,800 \\
\hline 30 & Channel A near Penn, N. Dak. & 87 & 1984-2008 & 172 & 51 & 1,200 \\
\hline 31 & Devils Lake Outlet to Stump Lake near Lakota, N. Dak. & 12 & $2002-2007$ & 1,385 & 527 & 2,500 \\
\hline 32 & Sheyenne River near Cooperstown, N. Dak. & 316 & $1970-2008$ & 160 & 37 & 360 \\
\hline 33 & Baldhill Creek near Dazey, N. Dak. & 94 & $1972-2008$ & 170 & 29 & 309 \\
\hline 34 & Sheyenne River below Baldhill Dam, N. Dak. & 172 & $1972-2008$ & 208 & 48 & 382 \\
\hline 35 & Sheyenne River at Valley City, N. Dak. & 29 & $1972-2005$ & 140 & 74 & 300 \\
\hline 36 & Sheyenne River at Lisbon, N. Dak. & 304 & $1970-2008$ & 200 & 64 & 437 \\
\hline 37 & Sheyenne River near Kindred, N. Dak. & 313 & $1972-2008$ & 170 & 50 & 389 \\
\hline 38 & $\begin{array}{l}\text { Sheyenne River above Sheyenne River Diversion near Horace, } \\
\text { N. Dak. }\end{array}$ & 43 & $1993-2008$ & 227 & 98 & 300 \\
\hline 39 & Sheyenne River near Horace, N. Dak. & 8 & $1982-1992$ & 115 & 58 & 150 \\
\hline 40 & Sheyenne River Diversion at West Fargo, N. Dak. & 13 & 1994-2007 & 180 & 100 & 284 \\
\hline 41 & Sheyenne River at West Fargo, N. Dak. & 62 & $1970-2008$ & 170 & 69 & 403 \\
\hline 42 & Maple River near Hope, N. Dak. & 39 & $1972-2008$ & 322 & 66 & 1,100 \\
\hline 43 & Maple River near Enderlin, N. Dak. & 63 & $1972-2008$ & 450 & 42 & 650 \\
\hline 44 & Maple River near Mapleton, N. Dak. & 25 & 1972-2008 & 370 & 100 & 589 \\
\hline 45 & Maple River below Mapleton, N. Dak. & 74 & $1995-2008$ & 419 & 73 & 1,130 \\
\hline 46 & Sheyenne River at Harwood, N. Dak. & 36 & 1993-2005 & 222 & 94 & 417 \\
\hline 47 & Rush River at Amenia, N. Dak. & 51 & $1972-2008$ & 280 & 37 & 670 \\
\hline 48 & Rush River near Prosper, N. Dak. & 4 & 1983-1987 & 75 & 41 & 550 \\
\hline 49 & Lower Branch Rush River near Prosper, N. Dak. & 5 & $1983-1993$ & 27 & 10 & 376 \\
\hline 50 & Sheyenne River near Harwood, N. Dak. & 44 & 1970-1996 & 180 & 100 & 260 \\
\hline 51 & Elm River near Kelso, N. Dak. & 6 & 1983-1993 & 208 & 45 & 326 \\
\hline 52 & Red River of the North at Halstad, Minn. & 183 & $1972-2008$ & 110 & 37 & 270 \\
\hline 53 & Beaver Creek near Finley, N. Dak. & 114 & 1970-2003 & 320 & 6 & 980 \\
\hline
\end{tabular}


Table 1-1. Summary statistics for major ion constituents at selected sites in North Dakota from 1970 through 2008. —Continued $\left[\mu \mathrm{g} / \mathrm{L}\right.$, micrograms per liter; <, less than; --, not available; $\mathrm{mg} / \mathrm{L}$, milligrams per liter; $\mathrm{CO}_{3}$, carbonate; $\mathrm{HCO}_{3}$, bicarbonate; $\mathrm{CaCO}$, calcium carbonate $]$

\begin{tabular}{|c|c|c|c|c|c|c|}
\hline $\begin{array}{c}\text { Site } \\
\text { identification } \\
\text { number } \\
\text { (table 1) }\end{array}$ & Site name & $\begin{array}{c}\text { Number of } \\
\text { samples }\end{array}$ & $\begin{array}{l}\text { Period of } \\
\text { record }\end{array}$ & Median & Minimum & Maximum \\
\hline \multicolumn{7}{|c|}{ Sulfate, in $\mathrm{mg} / \mathrm{L}-$ Continued } \\
\hline 54 & Goose River near Portland, N. Dak. & 30 & $1970-1988$ & 405 & 84 & 810 \\
\hline 55 & Goose River at Hillsboro, N. Dak. & 166 & 1970-2008 & 445 & 68 & 800 \\
\hline 56 & Red River of the North at Grand Forks, N. Dak. & 202 & 1970-2008 & 87 & 18 & 310 \\
\hline 57 & Turtle River at Turtle River State Park near Arvilla, N. Dak. & 99 & 1991-2008 & 205 & 48 & 490 \\
\hline 58 & Turtle River at Manvel, N. Dak. & 106 & $1971-2008$ & 425 & 100 & 1,600 \\
\hline 59 & Red River of the North at Oslo, Minn. & 75 & 1973-2005 & 74 & 20 & 180 \\
\hline 60 & Middle Branch Forest River near Whitman, N. Dak. & 14 & $1972-1990$ & 185 & 78 & 1,400 \\
\hline 61 & Forest River near Fordville, N. Dak. & 64 & $1972-2008$ & 174 & 84 & 569 \\
\hline 62 & Forest River near Minto, N. Dak. & 155 & $1971-2008$ & 216 & 36 & 543 \\
\hline 63 & South Branch Park River below Homme Dam, N. Dak. & 27 & 1972-1994 & 180 & 47 & 260 \\
\hline 64 & Middle Branch Park River near Union, N. Dak. & 15 & $1972-1984$ & 63 & $<4$ & 120 \\
\hline 65 & Middle Branch Park River near Edinburg, N. Dak. & 14 & $1978-1980$ & 87 & 31 & 150 \\
\hline 66 & Cart Creek at Mountain, N. Dak. & 17 & $1972-1984$ & 180 & 27 & 280 \\
\hline 67 & Park River at Grafton, N. Dak. & 145 & $1970-2008$ & 260 & 53 & 849 \\
\hline 68 & Red River of the North at Drayton, N. Dak. & 74 & 1972-2008 & 103 & 37 & 220 \\
\hline 69 & Pembina County Drain 20 near Glasston, N. Dak. & 8 & 1974-1984 & 120 & 38 & 480 \\
\hline 70 & Hidden Island Coulee near Hansboro, N. Dak. & 22 & $1972-1995$ & 200 & 51 & 430 \\
\hline 71 & Cypress Creek near Sarles, N. Dak. & 13 & $1972-1988$ & 100 & 74 & 290 \\
\hline 72 & Pembina River near Vang, N. Dak. & 63 & $1970-1979$ & 180 & 56 & 410 \\
\hline 73 & Little South Pembina River near Walhalla, N. Dak. & 83 & 1970-2008 & 180 & 36 & 287 \\
\hline 74 & Pembina River at Walhalla, N. Dak. & 230 & 1970-2008 & 180 & 49 & 310 \\
\hline 75 & Pembina River at Neche, N. Dak. & 133 & $1972-2008$ & 195 & 60 & 293 \\
\hline 76 & Tongue River at Akra, N. Dak. & 72 & 1972-2008 & 88 & 6 & 141 \\
\hline 77 & Red River of the North at Pembina, N. Dak., site 1 & 10 & 1993 & 120 & 63 & 158 \\
\hline 78 & Red River of the North at Pembina, N. Dak., site 2 & 164 & 1970-2008 & 110 & $<4$ & 448 \\
\hline 79 & Red River of the North at Emerson, Manitoba & 149 & 1977-2004 & 94 & 6 & 230 \\
\hline 80 & Long Creek near Noonan, N. Dak. & 70 & 1972-2008 & 376 & 39 & 1,110 \\
\hline 81 & West Branch Short Creek near Columbus, N. Dak. & 20 & $1978-1981$ & 495 & 160 & 1,300 \\
\hline 82 & Souris River near Sherwood, N. Dak. & 304 & 1972-2008 & 230 & 45 & 1,000 \\
\hline 83 & Souris River near Foxholm, N. Dak. & 202 & $1972-2008$ & 180 & 69 & 560 \\
\hline 84 & Des Lacs River at Foxholm, N. Dak. & 188 & $1972-2008$ & 456 & 33 & 1,100 \\
\hline 85 & Souris River above Minot, N. Dak. & 207 & $1970-2008$ & 267 & 70 & 1,850 \\
\hline 86 & Bonnes Creek near Velva, N. Dak. & 18 & $1987-2005$ & 390 & 51 & 1,200 \\
\hline 87 & Souris River near Verendrye, N. Dak. & 371 & 1970-2008 & 284 & 69 & 1,040 \\
\hline 88 & Wintering River near Karlsruhe, N. Dak. & 134 & 1972-2008 & 94 & 5 & 570 \\
\hline 89 & Souris River near Bantry, N. Dak. & 152 & $1970-2008$ & 215 & 69 & 550 \\
\hline 90 & Willow Creek near Willow City, N. Dak. & 90 & $1972-2008$ & 235 & 30 & 750 \\
\hline 91 & Stone Creek near Kramer, N. Dak. & 30 & $1986-2000$ & 227 & 30 & 1,700 \\
\hline 92 & Deep River near Upham, N. Dak. & 68 & $1972-2007$ & 141 & 32 & 421 \\
\hline 93 & Egg Creek near Granville, N. Dak. & 14 & 1972-1981 & 160 & 52 & 430 \\
\hline
\end{tabular}


Table 1-1. Summary statistics for major ion constituents at selected sites in North Dakota from 1970 through 2008.—Continued $\left[\mu \mathrm{g} / \mathrm{L}\right.$, micrograms per liter; <, less than; --, not available; mg/L, milligrams per liter; $\mathrm{CO}_{3}$, carbonate; $\mathrm{HCO}_{3}$, bicarbonate; $\mathrm{CaCO}$, calcium carbonate $]$

\begin{tabular}{|c|c|c|c|c|c|c|}
\hline $\begin{array}{c}\text { Site } \\
\text { identification } \\
\text { number } \\
\text { (table 1) }\end{array}$ & Site name & $\begin{array}{l}\text { Number of } \\
\text { samples }\end{array}$ & $\begin{array}{l}\text { Period of } \\
\text { record }\end{array}$ & Median & Minimum & Maximum \\
\hline \multicolumn{7}{|c|}{ Sulfate, in $\mathrm{mg} / \mathrm{L}$-Continued } \\
\hline 94 & Cut Bank Creek at North Lake Outlet near Granville, N. Dak. & 15 & $1972-1976$ & 250 & 97 & 470 \\
\hline 95 & Cut Bank Creek at Upham, N. Dak. & 33 & $1975-2000$ & 236 & 50 & 1,300 \\
\hline 97 & Boundary Creek near Landa, N. Dak. & 39 & $1972-2000$ & 286 & 32 & 1,200 \\
\hline 98 & Souris River near Westhope, N. Dak. & 214 & $1970-2008$ & 200 & 59 & 860 \\
\hline 99 & Charbonneau Creek near Charbonneau, N. Dak. & 22 & $1972-1977$ & 710 & 77 & 980 \\
\hline 100 & Missouri River near Williston, N. Dak. & 100 & 1970-1982 & 200 & 88 & 290 \\
\hline 104 & Beaver Creek near Ray, N. Dak. & 51 & $1977-1982$ & 610 & 8 & 730 \\
\hline 105 & White Earth River at White Earth, N. Dak. & 27 & 1970-1977 & 421 & 75 & 580 \\
\hline 106 & Bear Den Creek near Mandaree, N. Dak. & 229 & 1970-2008 & 690 & 45 & 1,200 \\
\hline 107 & Shell Creek near Parshall, N. Dak. & 21 & $1972-1977$ & 680 & 84 & 910 \\
\hline 108 & East Fork Shell Creek near Parshall, N. Dak. & 58 & 1991-2008 & 1,000 & 36 & 1,545 \\
\hline 109 & Deepwater Creek near Mandaree, N. Dak. & 57 & $1991-2008$ & 570 & 19 & 1,250 \\
\hline 110 & Little Missouri River at Marmarth, N. Dak. & 86 & $1971-2008$ & 484 & 73 & 1,200 \\
\hline 111 & Deep Creek near Amidon, N. Dak. & 51 & $1977-1983$ & 1,700 & 280 & 2,900 \\
\hline 112 & Little Missouri River at Medora, N. Dak. & 112 & $1972-2008$ & 615 & 119 & 2,000 \\
\hline 119 & Elm Creek near Golden Valley, N. Dak. & 37 & 1973-1995 & 340 & 9 & 940 \\
\hline 120 & Knife River near Golden Valley, N. Dak. & 130 & $1971-2008$ & 564 & 38 & 1,420 \\
\hline 121 & Coyote Creek near Zap, N. Dak. & 51 & $1977-1983$ & 510 & 50 & 1,000 \\
\hline 122 & Brush Creek near Beulah, N. Dak. & 107 & 1974-1990 & 490 & 35 & 790 \\
\hline 123 & Spring Creek below Lake Ilo near Dunn Center, N. Dak. & 53 & 1977-1993 & 580 & 57 & 1,500 \\
\hline 124 & Spring Creek near Halliday, N. Dak. & 48 & $1977-1981$ & 605 & 53 & 770 \\
\hline 125 & Spring Creek at Zap, N. Dak. & 238 & $1970-2008$ & 492 & 46 & 803 \\
\hline 126 & West Branch Otter Creek near Beulah, N. Dak. & 15 & $1972-1995$ & 410 & 110 & 760 \\
\hline 127 & Knife River at Hazen, N. Dak. & 270 & 1970-2008 & 459 & 57 & 890 \\
\hline 128 & Antelope Creek above Hazen, N. Dak. & 44 & 1977-1985 & 465 & $<4$ & 1,100 \\
\hline 129 & West Branch Antelope Creek No. 4 near Zap, N. Dak. & 21 & 1977-1985 & 170 & 6 & 520 \\
\hline 130 & West Branch Antelope Creek near Hazen, N. Dak. & 14 & $1978-1983$ & 190 & 27 & 900 \\
\hline 131 & Coal Creek near Stanton, N. Dak. & 28 & $1975-1981$ & 600 & 56 & 1,000 \\
\hline 132 & Alderin Creek near Fort Clark, N. Dak. & 41 & $1977-1983$ & 440 & 46 & 920 \\
\hline 133 & Coal Lake Coulee near Hensler, N. Dak. & 38 & $1978-1988$ & 315 & 6 & 780 \\
\hline
\end{tabular}


Table 1-1. Summary statistics for major ion constituents at selected sites in North Dakota from 1970 through 2008. —Continued $\left[\mu \mathrm{g} / \mathrm{L}\right.$, micrograms per liter; <, less than; --, not available; $\mathrm{mg} / \mathrm{L}$, milligrams per liter; $\mathrm{CO}_{3}$, carbonate; $\mathrm{HCO}_{3}$, bicarbonate; $\mathrm{CaCO}_{3}$, calcium carbonate $]$

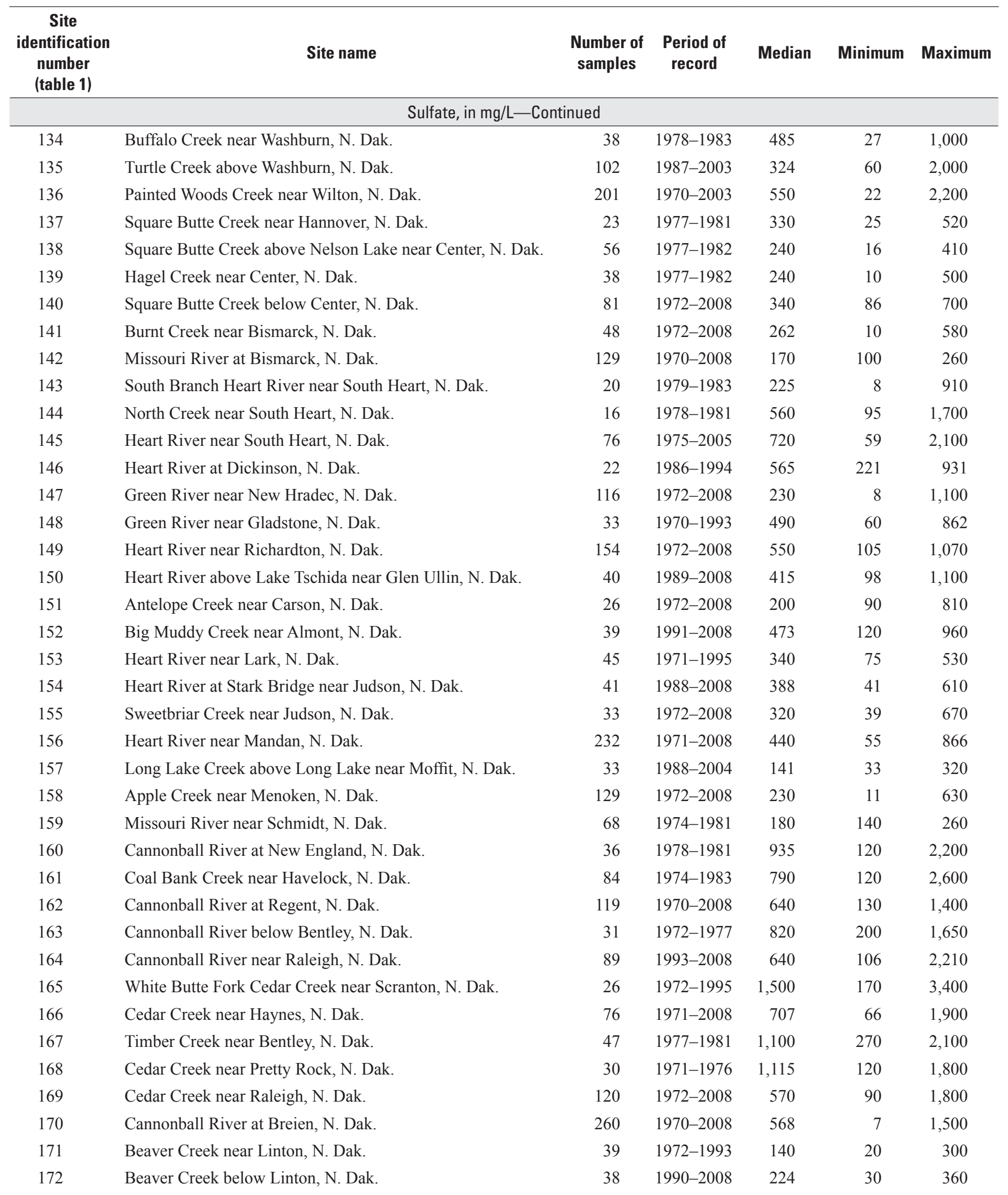


Table 1-1. Summary statistics for major ion constituents at selected sites in North Dakota from 1970 through 2008. —Continued $\left[\mu \mathrm{g} / \mathrm{L}\right.$, micrograms per liter; <, less than; --, not available; $\mathrm{mg} / \mathrm{L}$, milligrams per liter; $\mathrm{CO}_{3}$, carbonate; $\mathrm{HCO}_{3}$, bicarbonate; $\mathrm{CaCO}_{3}$, calcium carbonate $]$

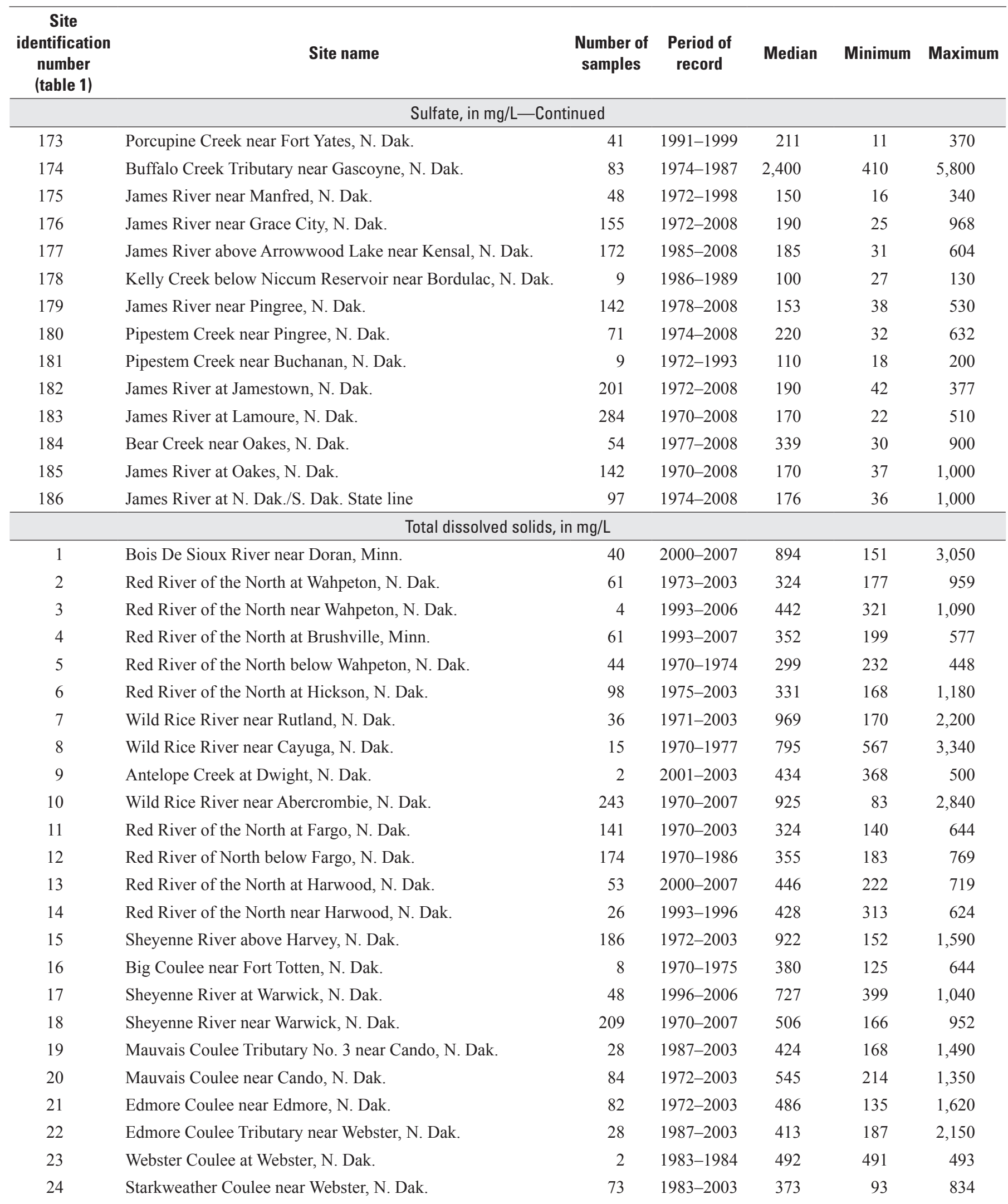


Table 1-1. Summary statistics for major ion constituents at selected sites in North Dakota from 1970 through 2008. —Continued $\left[\mu \mathrm{g} / \mathrm{L}\right.$, micrograms per liter; <, less than; --, not available; $\mathrm{mg} / \mathrm{L}$, milligrams per liter; $\mathrm{CO}_{3}$, carbonate; $\mathrm{HCO}_{3}$, bicarbonate; $\mathrm{CaCO}_{3}$, calcium carbonate $]$

\begin{tabular}{|c|c|c|c|c|c|c|}
\hline $\begin{array}{c}\text { Site } \\
\text { identification } \\
\text { number } \\
\text { (table 1) }\end{array}$ & Site name & $\begin{array}{c}\text { Number of } \\
\text { samples }\end{array}$ & $\begin{array}{l}\text { Period of } \\
\text { record }\end{array}$ & Median & Minimum & Maximum \\
\hline \multicolumn{7}{|c|}{ Total dissolved solids, in mg/L—Continued } \\
\hline 25 & Big Coulee below Churchs Ferry, N. Dak. & 13 & $1998-2003$ & 545 & 351 & 1,110 \\
\hline 26 & Little Coulee near Leeds, N. Dak. & 9 & $1998-2003$ & 578 & 269 & 821 \\
\hline 27 & Little Coulee near Brinsmade, N. Dak. & 31 & $1976-1998$ & 572 & 168 & 2,240 \\
\hline 28 & Big Coulee near Churchs Ferry, N. Dak. & 136 & $1970-1997$ & 498 & 196 & 1,910 \\
\hline 29 & Big Coulee at Graham Island inlet near Fort Totten, N. Dak. & 44 & $1970-1986$ & 2,060 & 457 & 7,610 \\
\hline 30 & Channel A near Penn, N. Dak. & 75 & 1984-2003 & 516 & 271 & 2,300 \\
\hline 31 & Devils Lake Outlet to Stump Lake near Lakota, N. Dak. & 3 & $2002-2003$ & 3,970 & 1,330 & 4,980 \\
\hline 32 & Sheyenne River near Cooperstown, N. Dak. & 331 & 1970-2007 & 622 & 169 & 1,070 \\
\hline 33 & Baldhill Creek near Dazey, N. Dak. & 83 & 1972-2003 & 555 & 131 & 991 \\
\hline 34 & Sheyenne River below Baldhill Dam, N. Dak. & 144 & 1972-2007 & 579 & 196 & 1,090 \\
\hline 35 & Sheyenne River at Valley City, N. Dak. & 27 & $1972-2003$ & 473 & 278 & 886 \\
\hline 36 & Sheyenne River at Lisbon, N. Dak. & 333 & $1970-2007$ & 605 & 220 & 1,060 \\
\hline 37 & Sheyenne River near Kindred, N. Dak. & 301 & $1972-2007$ & 562 & 200 & 991 \\
\hline 38 & $\begin{array}{l}\text { Sheyenne River above Sheyenne River Diversion near Horace, } \\
\text { N. Dak. }\end{array}$ & 18 & $1993-2003$ & 633 & 277 & 832 \\
\hline 39 & Sheyenne River near Horace, N. Dak. & 45 & $1976-1992$ & 539 & 231 & 772 \\
\hline 40 & Sheyenne River Diversion at West Fargo, N. Dak. & 8 & 1994-2001 & 499 & 273 & 743 \\
\hline 41 & Sheyenne River at West Fargo, N. Dak. & 56 & 1970-2006 & 565 & 260 & 903 \\
\hline 42 & Maple River near Hope, N. Dak. & 30 & $1972-2003$ & 654 & 209 & 2,210 \\
\hline 43 & Maple River near Enderlin, N. Dak. & 52 & $1972-2003$ & 1,070 & 182 & 1,570 \\
\hline 44 & Maple River near Mapleton, N. Dak. & 14 & $1972-2003$ & 859 & 326 & 1,260 \\
\hline 45 & Maple River below Mapleton, N. Dak. & 62 & 1995-2007 & 940 & 223 & 2,800 \\
\hline 46 & Sheyenne River at Harwood, N. Dak. & 14 & $1993-2003$ & 569 & 382 & 956 \\
\hline 47 & Rush River at Amenia, N. Dak. & 40 & 1972-2003 & 766 & 137 & 1,430 \\
\hline 48 & Rush River near Prosper, N. Dak. & 4 & $1983-1987$ & 294 & 159 & 1,270 \\
\hline 49 & Lower Branch Rush River near Prosper, N. Dak. & 5 & $1983-1993$ & 172 & 119 & 865 \\
\hline 50 & Sheyenne River near Harwood, N. Dak. & 45 & $1970-1996$ & 632 & 327 & 965 \\
\hline 51 & Elm River near Kelso, N. Dak. & 6 & $1983-1993$ & 684 & 212 & 855 \\
\hline 52 & Red River of the North at Halstad, Minn. & 158 & $1972-2003$ & 429 & 176 & 695 \\
\hline 53 & Beaver Creek near Finley, N. Dak. & 113 & $1970-2003$ & 787 & 138 & 2,110 \\
\hline 54 & Goose River near Portland, N. Dak. & 30 & $1970-1988$ & 939 & 221 & 1,790 \\
\hline 55 & Goose River at Hillsboro, N. Dak. & 146 & $1970-2007$ & 983 & 228 & 2,190 \\
\hline 56 & Red River of the North at Grand Forks, N. Dak. & 206 & 1970-2006 & 344 & 158 & 731 \\
\hline 57 & Turtle River at Turtle River State Park near Arvilla, N. Dak. & 88 & $1991-2003$ & 614 & 165 & 1,150 \\
\hline 58 & Turtle River at Manvel, N. Dak. & 74 & $1971-2006$ & 1,460 & 465 & 4,560 \\
\hline 59 & Red River of the North at Oslo, Minn. & 71 & 1973-2002 & 334 & 183 & 679 \\
\hline 60 & Middle Branch Forest River near Whitman, N. Dak. & 14 & $1972-1990$ & 430 & 312 & 2,460 \\
\hline 61 & Forest River near Fordville, N. Dak. & 53 & $1972-2003$ & 465 & 294 & 988 \\
\hline 62 & Forest River near Minto, N. Dak. & 121 & $1971-2006$ & 570 & 173 & 2,890 \\
\hline 63 & South Branch Park River below Homme Dam, N. Dak. & 27 & 1972-1994 & 486 & 203 & 647 \\
\hline
\end{tabular}


Table 1-1. Summary statistics for major ion constituents at selected sites in North Dakota from 1970 through 2008.—Continued $\left[\mu \mathrm{g} / \mathrm{L}\right.$, micrograms per liter; <, less than; --, not available; mg/L, milligrams per liter; $\mathrm{CO}_{3}$, carbonate; $\mathrm{HCO}_{3}$, bicarbonate; $\mathrm{CaCO}$, calcium carbonate $]$

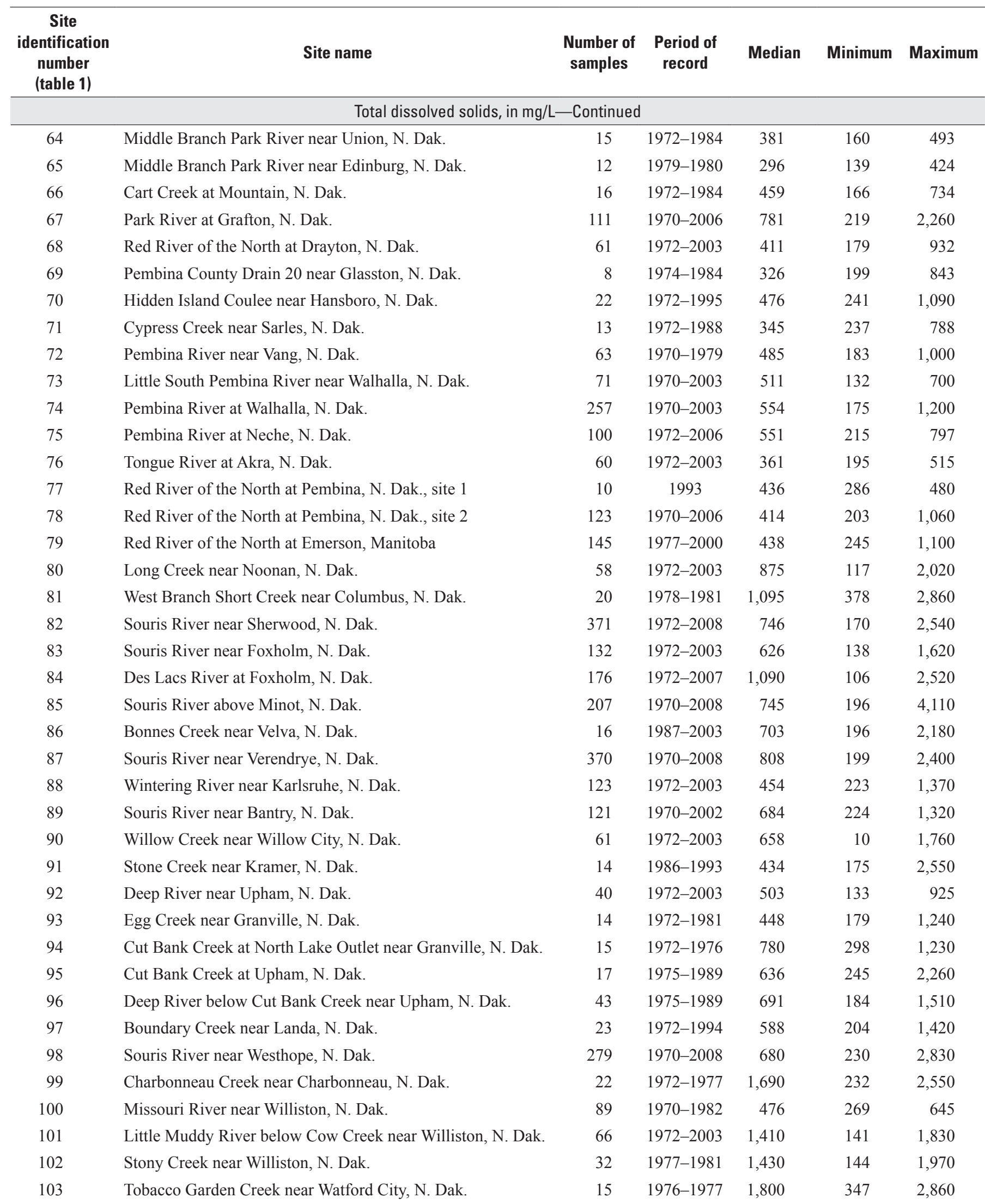


Table 1-1. Summary statistics for major ion constituents at selected sites in North Dakota from 1970 through 2008. —Continued $\left[\mu \mathrm{g} / \mathrm{L}\right.$, micrograms per liter; <, less than; --, not available; $\mathrm{mg} / \mathrm{L}$, milligrams per liter; $\mathrm{CO}_{3}$, carbonate; $\mathrm{HCO}_{3}$, bicarbonate; $\mathrm{CaCO}$, calcium carbonate $]$

\begin{tabular}{|c|c|c|c|c|c|c|}
\hline $\begin{array}{c}\text { Site } \\
\text { identification } \\
\text { number } \\
\text { (table 1) } \\
\end{array}$ & Site name & $\begin{array}{l}\text { Number of } \\
\text { samples }\end{array}$ & $\begin{array}{l}\text { Period of } \\
\text { record }\end{array}$ & Median & Minimum & Maximum \\
\hline \multicolumn{7}{|c|}{ Total dissolved solids, in $\mathrm{mg} / \mathrm{L}$-Continued } \\
\hline 104 & Beaver Creek near Ray, N. Dak. & 50 & 1977-1982 & 1,420 & 147 & 1,690 \\
\hline 105 & White Earth River at White Earth, N. Dak. & 27 & $1970-1977$ & 1,350 & 261 & 1,890 \\
\hline 106 & Bear Den Creek near Mandaree, N. Dak. & 212 & 1970-2003 & 1,815 & 171 & 3,110 \\
\hline 107 & Shell Creek near Parshall, N. Dak. & 21 & 1972-1977 & 1,760 & 256 & 2,130 \\
\hline 108 & East Fork Shell Creek near Parshall, N. Dak. & 45 & 1991-2003 & 2,230 & 160 & 3,036 \\
\hline 109 & Deepwater Creek near Mandaree, N. Dak. & 40 & 1991-2003 & 1,326 & 130 & 2,080 \\
\hline 110 & Little Missouri River at Marmarth, N. Dak. & 74 & 1971-2003 & 1,040 & 184 & 2,230 \\
\hline 111 & Deep Creek near Amidon, N. Dak. & 51 & $1977-1983$ & 3,100 & 548 & 4,450 \\
\hline 112 & Little Missouri River at Medora, N. Dak. & 100 & $1972-2007$ & 1,205 & 259 & 4,110 \\
\hline 113 & Beaver Creek near Trotters, N. Dak. & 83 & $1977-2003$ & 1,800 & 222 & 2,760 \\
\hline 114 & Little Missouri River near Watford City, N. Dak. & 230 & $1971-2007$ & 1,235 & 264 & 3,150 \\
\hline 115 & Missouri River at Garrison Dam, N. Dak. & 277 & $1971-2007$ & 424 & 350 & 641 \\
\hline 116 & Knife River at Manning, N. Dak. & 107 & $1972-2003$ & 1,160 & 81 & 2,400 \\
\hline 117 & Stray Creek near Manning, N. Dak. & 17 & 1975-1981 & 1,610 & 460 & 4,360 \\
\hline 118 & Knife River at Marshall, N. Dak. & 69 & $1972-1981$ & 1,400 & 192 & 2,560 \\
\hline 119 & Elm Creek near Golden Valley, N. Dak. & 37 & 1973-1995 & 762 & 215 & 2,450 \\
\hline 120 & Knife River near Golden Valley, N. Dak. & 119 & $1971-2007$ & 1,310 & 148 & 3,410 \\
\hline 121 & Coyote Creek near Zap, N. Dak. & 51 & $1977-1983$ & 1,220 & 159 & 2,420 \\
\hline 122 & Brush Creek near Beulah, N. Dak. & 107 & $1974-1990$ & 1,270 & 174 & 2,540 \\
\hline 123 & Spring Creek below Lake Ilo near Dunn Center, N. Dak. & 53 & $1977-1993$ & 1,300 & 164 & 2,430 \\
\hline 124 & Spring Creek near Halliday, N. Dak. & 46 & $1977-1981$ & 1,325 & 157 & 1,620 \\
\hline 125 & Spring Creek at Zap, N. Dak. & 221 & 1970-2007 & 1,120 & 145 & 1,790 \\
\hline 126 & West Branch Otter Creek near Beulah, N. Dak. & 15 & $1972-1995$ & 833 & 234 & 1,560 \\
\hline 127 & Knife River at Hazen, N. Dak. & 256 & 1970-2007 & 1,110 & 200 & 1,890 \\
\hline 128 & Antelope Creek above Hazen, N. Dak. & 44 & $1977-1985$ & 1,125 & 112 & 2,350 \\
\hline 129 & West Branch Antelope Creek No. 4 near Zap, N. Dak. & 12 & 1977-1985 & 598 & 112 & 1,020 \\
\hline 130 & West Branch Antelope Creek near Hazen, N. Dak. & 14 & $1978-1983$ & 614 & 113 & 1,930 \\
\hline 131 & Coal Creek near Stanton, N. Dak. & 28 & 1975-1981 & 1,445 & 166 & 2,510 \\
\hline 132 & Alderin Creek near Fort Clark, N. Dak. & 40 & $1977-1983$ & 1,075 & 162 & 2,370 \\
\hline 133 & Coal Lake Coulee near Hensler, N. Dak. & 39 & $1978-1988$ & 925 & 98 & 1,760 \\
\hline 134 & Buffalo Creek near Washburn, N. Dak. & 38 & $1978-1983$ & 1,655 & 164 & 3,120 \\
\hline 135 & Turtle Creek above Washburn, N. Dak. & 55 & $1987-2003$ & 1,310 & 436 & 3,800 \\
\hline 136 & Painted Woods Creek near Wilton, N. Dak. & 153 & 1970-2003 & 1,140 & 110 & 3,750 \\
\hline 137 & Square Butte Creek near Hannover, N. Dak. & 23 & 1977-1981 & 772 & 81 & 1,120 \\
\hline 138 & Square Butte Creek above Nelson Lake near Center, N. Dak. & 55 & 1977-1982 & 775 & 112 & 1,030 \\
\hline 139 & Hagel Creek near Center, N. Dak. & 38 & 1977-1982 & 762 & 112 & 1,490 \\
\hline 140 & Square Butte Creek below Center, N. Dak. & 70 & $1972-2003$ & 839 & 342 & 1,290 \\
\hline 141 & Burnt Creek near Bismarck, N. Dak. & 39 & 1972-2002 & 709 & 55 & 1,250 \\
\hline 142 & Missouri River at Bismarck, N. Dak. & 174 & 1970-2003 & 436 & 274 & 4,050 \\
\hline 143 & South Branch Heart River near South Heart, N. Dak. & 19 & 1979-1983 & 462 & 220 & 1,720 \\
\hline
\end{tabular}


Table 1-1. Summary statistics for major ion constituents at selected sites in North Dakota from 1970 through 2008. - Continued $\left[\mu \mathrm{g} / \mathrm{L}\right.$, micrograms per liter; <, less than; --, not available; $\mathrm{mg} / \mathrm{L}$, milligrams per liter; $\mathrm{CO}_{3}$, carbonate; $\mathrm{HCO}_{3}$, bicarbonate; $\mathrm{CaCO}_{3}$, calcium carbonate $]$

\begin{tabular}{|c|c|c|c|c|c|c|}
\hline $\begin{array}{c}\text { Site } \\
\text { identification } \\
\text { number } \\
\text { (table 1) } \\
\end{array}$ & Site name & $\begin{array}{l}\text { Number of } \\
\text { samples }\end{array}$ & $\begin{array}{l}\text { Period of } \\
\text { record }\end{array}$ & Median & Minimum & Maximum \\
\hline \multicolumn{7}{|c|}{ Total dissolved solids, in mg/L-Continued } \\
\hline 144 & North Creek near South Heart, N. Dak. & 16 & $1978-1981$ & 1,175 & 235 & 3,460 \\
\hline 145 & Heart River near South Heart, N. Dak. & 72 & 1975-2002 & 1,655 & 203 & 3,550 \\
\hline 146 & Heart River at Dickinson, N. Dak. & 22 & $1986-1994$ & 1,245 & 514 & 2,140 \\
\hline 147 & Green River near New Hradec, N. Dak. & 105 & $1972-2003$ & 685 & 103 & 2,140 \\
\hline 148 & Green River near Gladstone, N. Dak. & 33 & 1970-1993 & 1,080 & 177 & 1,950 \\
\hline 149 & Heart River near Richardton, N. Dak. & 144 & $1972-2007$ & 1,130 & 242 & 2,290 \\
\hline 150 & Heart River above Lake Tschida near Glen Ullin, N. Dak. & 29 & 1989-2003 & 1,010 & 257 & 1,850 \\
\hline 151 & Antelope Creek near Carson, N. Dak. & 17 & 1972-2003 & 615 & 303 & 1,550 \\
\hline 152 & Big Muddy Creek near Almont, N. Dak. & 28 & 1991-2003 & 1,130 & 276 & 2,010 \\
\hline 153 & Heart River near Lark, N. Dak. & 45 & $1971-1995$ & 750 & 193 & 1,090 \\
\hline 154 & Heart River at Stark Bridge near Judson, N. Dak. & 30 & 1988-2003 & 886 & 176 & 1,280 \\
\hline 155 & Sweetbriar Creek near Judson, N. Dak. & 23 & $1972-2003$ & 809 & 184 & 1,380 \\
\hline 156 & Heart River near Mandan, N. Dak. & 219 & $1971-2007$ & 961 & 182 & 1,980 \\
\hline 157 & Long Lake Creek above Long Lake near Moffit, N. Dak. & 29 & 1988-2002 & 595 & 150 & 1,040 \\
\hline 158 & Apple Creek near Menoken, N. Dak. & 117 & $1972-2003$ & 970 & 93 & 1,730 \\
\hline 159 & Missouri River near Schmidt, N. Dak. & 141 & 1974-1981 & 441 & 347 & 525 \\
\hline 160 & Cannonball River at New England, N. Dak. & 35 & 1978-1981 & 1,790 & 281 & 4,070 \\
\hline 161 & Coal Bank Creek near Havelock, N. Dak. & 83 & 1974-1983 & 1,550 & 309 & 4,290 \\
\hline 162 & Cannonball River at Regent, N. Dak. & 108 & 1970-2003 & 1,320 & 290 & 2,450 \\
\hline 163 & Cannonball River below Bentley, N. Dak. & 31 & $1972-1977$ & 1,530 & 366 & 2,800 \\
\hline 164 & Cannonball River near Raleigh, N. Dak. & 80 & 1993-2007 & 1,235 & 367 & 4,340 \\
\hline 165 & White Butte Fork Cedar Creek near Scranton, N. Dak. & 26 & $1972-1995$ & 2,575 & 368 & 5,200 \\
\hline 166 & Cedar Creek near Haynes, N. Dak. & 65 & $1971-2003$ & 1,520 & 232 & 3,230 \\
\hline 167 & Timber Creek near Bentley, N. Dak. & 47 & $1977-1981$ & 1,990 & 531 & 3,480 \\
\hline 168 & Cedar Creek near Pretty Rock, N. Dak. & 30 & 1971-1976 & 1,900 & 302 & 2,970 \\
\hline 169 & Cedar Creek near Raleigh, N. Dak. & 111 & 1972-2007 & 1,150 & 241 & 3,030 \\
\hline 170 & Cannonball River at Breien, N. Dak. & 246 & 1970-2007 & 1,175 & 20 & 2,960 \\
\hline 171 & Beaver Creek near Linton, N. Dak. & 39 & $1972-1993$ & 538 & 72 & 834 \\
\hline 172 & Beaver Creek below Linton, N. Dak. & 26 & 1990-2002 & 639 & 129 & 1,000 \\
\hline 173 & Porcupine Creek near Fort Yates, N. Dak. & 40 & 1992-1999 & 777 & 82 & 1,390 \\
\hline 174 & Buffalo Creek Tributary near Gascoyne, N. Dak. & 83 & 1974-1987 & 4,270 & 772 & 9,280 \\
\hline 175 & James River near Manfred, N. Dak. & 48 & $1972-1998$ & 593 & 134 & 933 \\
\hline 176 & James River near Grace City, N. Dak. & 145 & $1972-2007$ & 646 & 134 & 6,540 \\
\hline 177 & James River above Arrowwood Lake near Kensal, N. Dak. & 171 & 1985-2008 & 656 & 164 & 1,818 \\
\hline 178 & Kelly Creek below Niccum Reservoir near Bordulac, N. Dak. & 9 & $1986-1989$ & 362 & 218 & 529 \\
\hline 179 & James River near Pingree, N. Dak. & 143 & $1978-2008$ & 552 & 191 & 1,147 \\
\hline 180 & Pipestem Creek near Pingree, N. Dak. & 60 & 1974-2003 & 580 & 174 & 1,110 \\
\hline 181 & Pipestem Creek near Buchanan, N. Dak. & 9 & $1972-1993$ & 376 & 81 & 619 \\
\hline 182 & James River at Jamestown, N. Dak. & 188 & $1972-2007$ & 591 & 197 & 1,060 \\
\hline 183 & James River at Lamoure, N. Dak. & 272 & 1970-2007 & 609 & 141 & 1,790 \\
\hline
\end{tabular}


Table 1-1. Summary statistics for major ion constituents at selected sites in North Dakota from 1970 through 2008. —Continued $\left[\mu \mathrm{g} / \mathrm{L}\right.$, micrograms per liter; <, less than; --, not available; $\mathrm{mg} / \mathrm{L}$, milligrams per liter; $\mathrm{CO}_{3}$, carbonate; $\mathrm{HCO}_{3}$, bicarbonate; $\mathrm{CaCO}$, calcium carbonate $]$

\begin{tabular}{|c|c|c|c|c|c|c|}
\hline $\begin{array}{c}\text { Site } \\
\text { identification } \\
\text { number } \\
\text { (table 1) }\end{array}$ & Site name & $\begin{array}{l}\text { Number of } \\
\text { samples }\end{array}$ & $\begin{array}{l}\text { Period of } \\
\text { record }\end{array}$ & Median & Minimum & Maximum \\
\hline \multicolumn{7}{|c|}{ Total dissolved solids, in $\mathrm{mg} / \mathrm{L}$-Continued } \\
\hline 184 & Bear Creek near Oakes, N. Dak. & 43 & $1977-2003$ & 857 & 141 & 1,680 \\
\hline 185 & James River at Oakes, N. Dak. & 125 & 1970-2008 & 672 & 155 & 3,330 \\
\hline 186 & James River at N. Dak./S. Dak. State line & 85 & $1974-2003$ & 541 & 177 & 2,960 \\
\hline \multicolumn{7}{|c|}{ Fluoride, in mg/L } \\
\hline 2 & Red River of the North at Wahpeton, N. Dak. & 55 & 1973-2008 & $<1.0$ & $<1.0$ & $<1.0$ \\
\hline 5 & Red River of the North below Wahpeton, N. Dak. & 14 & 1970-1974 & $<1.0$ & $<1.0$ & $<1.0$ \\
\hline 6 & Red River of the North at Hickson, N. Dak. & 111 & 1975-2008 & $<1.0$ & $<1.0$ & $<1.0$ \\
\hline 7 & Wild Rice River near Rutland, N. Dak. & 47 & $1971-2008$ & $<1.0$ & $<1.0$ & $<1.0$ \\
\hline 8 & Wild Rice River near Cayuga, N. Dak. & 15 & $1970-1977$ & $<1.0$ & $<1.0$ & 6.7 \\
\hline 9 & Antelope Creek at Dwight, N. Dak. & 13 & $2001-2008$ & $<1.0$ & $<1.0$ & $<1.0$ \\
\hline 10 & Wild Rice River near Abercrombie, N. Dak. & 161 & $1970-2008$ & $<1.0$ & $<1.0$ & 1.5 \\
\hline 11 & Red River of the North at Fargo, N. Dak. & 119 & 1970-2008 & $<1.0$ & $<1.0$ & $<1.0$ \\
\hline 12 & Red River of North below Fargo, N. Dak. & 145 & $1970-1986$ & $<1.0$ & $<1.0$ & $<1.0$ \\
\hline 14 & Red River of the North near Harwood, N. Dak. & 13 & $2005-2007$ & $<1.0$ & $<1.0$ & $<1.0$ \\
\hline 15 & Sheyenne River above Harvey, N. Dak. & 197 & $1972-2008$ & $<1.0$ & $<1.0$ & $<1.0$ \\
\hline 16 & Big Coulee near Fort Totten, N. Dak. & 8 & $1970-1975$ & $<1.0$ & $<1.0$ & $<1.0$ \\
\hline 18 & Sheyenne River near Warwick, N. Dak. & 108 & $1970-2008$ & $<1.0$ & $<1.0$ & $<1.0$ \\
\hline 19 & Mauvais Coulee Tributary No. 3 near Cando, N. Dak. & 38 & $1987-2008$ & $<1.0$ & $<1.0$ & $<1.0$ \\
\hline 20 & Mauvais Coulee near Cando, N. Dak. & 103 & $1972-2008$ & $<1.0$ & $<1.0$ & $<1.0$ \\
\hline 21 & Edmore Coulee near Edmore, N. Dak. & 103 & $1972-2008$ & $<1.0$ & $<1.0$ & $<1.0$ \\
\hline 22 & Edmore Coulee Tributary near Webster, N. Dak. & 38 & $1987-2008$ & $<1.0$ & $<1.0$ & $<1.0$ \\
\hline 23 & Webster Coulee at Webster, N. Dak. & 2 & 1983-1984 & $<1.0$ & $<1.0$ & $<1.0$ \\
\hline 24 & Starkweather Coulee near Webster, N. Dak. & 92 & $1983-2008$ & $<1.0$ & $<1.0$ & $<1.0$ \\
\hline 25 & Big Coulee below Churchs Ferry, N. Dak. & 23 & 1998-2008 & $<1.0$ & $<1.0$ & $<1.0$ \\
\hline 26 & Little Coulee near Leeds, N. Dak. & 16 & $1998-2008$ & $<1.0$ & $<1.0$ & $<1.0$ \\
\hline 27 & Little Coulee near Brinsmade, N. Dak. & 31 & 1976-1998 & $<1.0$ & $<1.0$ & $<1.0$ \\
\hline 28 & Big Coulee near Churchs Ferry, N. Dak. & 135 & 1970-1997 & $<1.0$ & $<1.0$ & 1.2 \\
\hline 29 & Big Coulee at Graham Island inlet near Fort Totten, N. Dak. & 44 & 1970-1986 & $<1.0$ & $<1.0$ & $<1.0$ \\
\hline 30 & Channel A near Penn, N. Dak. & 84 & $1984-2008$ & $<1.0$ & $<1.0$ & $<1.0$ \\
\hline 31 & Devils Lake Outlet to Stump Lake near Lakota, N. Dak. & 11 & $2002-2007$ & $<1.0$ & $<1.0$ & $<1.0$ \\
\hline 32 & Sheyenne River near Cooperstown, N. Dak. & 230 & 1970-2008 & $<1.0$ & $<1.0$ & $<1.0$ \\
\hline 33 & Baldhill Creek near Dazey, N. Dak. & 91 & $1972-2008$ & $<1.0$ & $<1.0$ & $<1.0$ \\
\hline 34 & Sheyenne River below Baldhill Dam, N. Dak. & 95 & $1972-2008$ & $<1.0$ & $<1.0$ & $<1.0$ \\
\hline 35 & Sheyenne River at Valley City, N. Dak. & 25 & $1972-2005$ & $<1.0$ & $<1.0$ & $<1.0$ \\
\hline 36 & Sheyenne River at Lisbon, N. Dak. & 231 & $1970-2008$ & $<1.0$ & $<1.0$ & $<1.0$ \\
\hline 37 & Sheyenne River near Kindred, N. Dak. & 239 & $1972-2008$ & $<1.0$ & $<1.0$ & $<1.0$ \\
\hline 38 & $\begin{array}{l}\text { Sheyenne River above Sheyenne River Diversion near Horace, } \\
\text { N. Dak. }\end{array}$ & 41 & $1993-2008$ & $<1.0$ & $<1.0$ & $<1.0$ \\
\hline 39 & Sheyenne River near Horace, N. Dak. & 8 & $1982-1992$ & $<1.0$ & $<1.0$ & $<1.0$ \\
\hline 40 & Sheyenne River Diversion at West Fargo, N. Dak. & 13 & 1994-2007 & $<1.0$ & $<1.0$ & $<1.0$ \\
\hline
\end{tabular}


Table 1-1. Summary statistics for major ion constituents at selected sites in North Dakota from 1970 through 2008.—Continued [ $\mu \mathrm{g} / \mathrm{L}$, micrograms per liter; <, less than; --, not available; $\mathrm{mg} / \mathrm{L}$, milligrams per liter; $\mathrm{CO}_{3}$, carbonate; $\mathrm{HCO}_{3}$, bicarbonate; $\mathrm{CaCO}_{3}$, calcium carbonate $]$

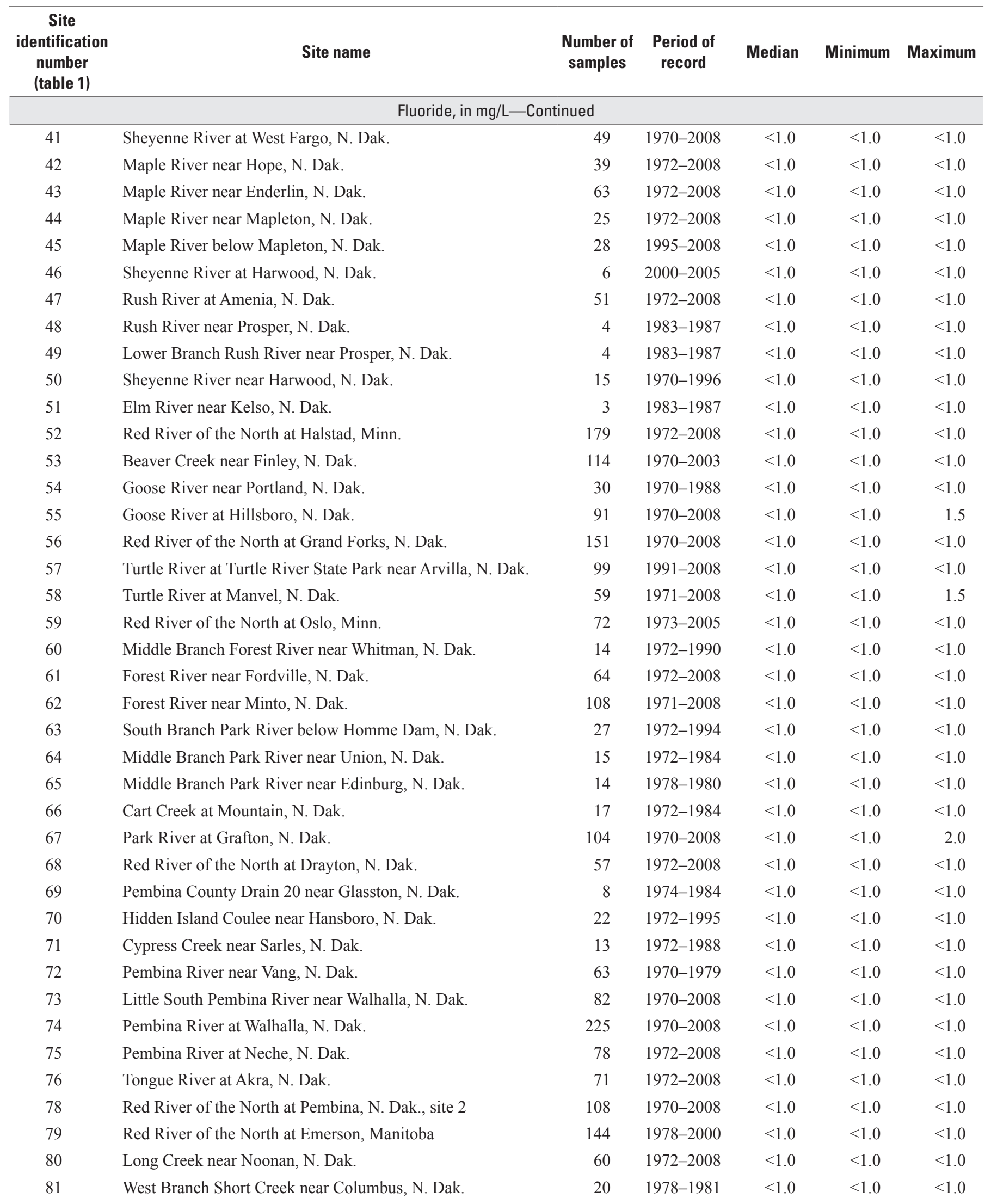


Table 1-1. Summary statistics for major ion constituents at selected sites in North Dakota from 1970 through 2008. —Continued $\left[\mu \mathrm{g} / \mathrm{L}\right.$, micrograms per liter; <, less than; --, not available; $\mathrm{mg} / \mathrm{L}$, milligrams per liter; $\mathrm{CO}_{3}$, carbonate; $\mathrm{HCO}_{3}$, bicarbonate; $\mathrm{CaCO}_{3}$, calcium carbonate $]$

\begin{tabular}{|c|c|c|c|c|c|c|}
\hline $\begin{array}{c}\text { Site } \\
\text { identification } \\
\text { number } \\
\text { (table 1) }\end{array}$ & Site name & $\begin{array}{c}\text { Number of } \\
\text { samples }\end{array}$ & $\begin{array}{l}\text { Period of } \\
\text { record }\end{array}$ & Median & Minimum & Maximum \\
\hline \multicolumn{7}{|c|}{ Fluoride, in $\mathrm{mg} / \mathrm{L}$-Continued } \\
\hline 82 & Souris River near Sherwood, N. Dak. & 297 & 1972-2008 & $<1.0$ & $<1.0$ & 1.8 \\
\hline 83 & Souris River near Foxholm, N. Dak. & 192 & $1972-2008$ & $<1.0$ & $<1.0$ & 1.2 \\
\hline 84 & Des Lacs River at Foxholm, N. Dak. & 92 & $1972-2008$ & $<1.0$ & $<1.0$ & $<1.0$ \\
\hline 85 & Souris River above Minot, N. Dak. & 137 & $1970-2008$ & $<1.0$ & $<1.0$ & $<1.0$ \\
\hline 86 & Bonnes Creek near Velva, N. Dak. & 18 & $1987-2005$ & $<1.0$ & $<1.0$ & $<1.0$ \\
\hline 87 & Souris River near Verendrye, N. Dak. & 287 & 1970-2008 & $<1.0$ & $<1.0$ & 1.2 \\
\hline 88 & Wintering River near Karlsruhe, N. Dak. & 123 & $1972-2008$ & $<1.0$ & $<1.0$ & $<1.0$ \\
\hline 89 & Souris River near Bantry, N. Dak. & 124 & 1970-2008 & $<1.0$ & $<1.0$ & $<1.0$ \\
\hline 90 & Willow Creek near Willow City, N. Dak. & 65 & 1972-2008 & $<1.0$ & $<1.0$ & $<1.0$ \\
\hline 91 & Stone Creek near Kramer, N. Dak. & 14 & $1986-1993$ & $<1.0$ & $<1.0$ & $<1.0$ \\
\hline 92 & Deep River near Upham, N. Dak. & 42 & 1972-2007 & $<1.0$ & $<1.0$ & $<1.0$ \\
\hline 93 & Egg Creek near Granville, N. Dak. & 14 & $1972-1981$ & $<1.0$ & $<1.0$ & $<1.0$ \\
\hline 94 & Cut Bank Creek at North Lake Outlet near Granville, N. Dak. & 15 & $1972-1976$ & $<1.0$ & $<1.0$ & $<1.0$ \\
\hline 95 & Cut Bank Creek at Upham, N. Dak. & 17 & $1975-1989$ & $<1.0$ & $<1.0$ & $<1.0$ \\
\hline 96 & Deep River below Cut Bank Creek near Upham, N. Dak. & 43 & $1975-1989$ & $<1.0$ & $<1.0$ & $<1.0$ \\
\hline 97 & Boundary Creek near Landa, N. Dak. & 23 & 1972-1994 & $<1.0$ & $<1.0$ & $<1.0$ \\
\hline 98 & Souris River near Westhope, N. Dak. & 193 & 1970-2008 & $<1.0$ & $<1.0$ & $<1.0$ \\
\hline 99 & Charbonneau Creek near Charbonneau, N. Dak. & 22 & 1972-1977 & $<1.0$ & $<1.0$ & $<1.0$ \\
\hline 100 & Missouri River near Williston, N. Dak. & 88 & 1974-1992 & $<1.0$ & $<1.0$ & $<1.0$ \\
\hline 101 & Little Muddy River below Cow Creek near Williston, N. Dak. & 68 & 1972-2008 & $<1.0$ & $<1.0$ & $<1.0$ \\
\hline 102 & Stony Creek near Williston, N. Dak. & 32 & $1977-1981$ & $<1.0$ & $<1.0$ & $<1.0$ \\
\hline 103 & Tobacco Garden Creek near Watford City, N. Dak. & 15 & 1976-1977 & $<1.0$ & $<1.0$ & $<1.0$ \\
\hline 104 & Beaver Creek near Ray, N. Dak. & 51 & $1977-1982$ & $<1.0$ & $<1.0$ & $<1.0$ \\
\hline 105 & White Earth River at White Earth, N. Dak. & 27 & $1970-1977$ & $<1.0$ & $<1.0$ & $<1.0$ \\
\hline 106 & Bear Den Creek near Mandaree, N. Dak. & 228 & 1970-2008 & $<1.0$ & $<1.0$ & 2.5 \\
\hline 107 & Shell Creek near Parshall, N. Dak. & 21 & 1972-1977 & $<1.0$ & $<1.0$ & $<1.0$ \\
\hline 108 & East Fork Shell Creek near Parshall, N. Dak. & 58 & $1991-2008$ & $<1.0$ & $<1.0$ & $<1.0$ \\
\hline 109 & Deepwater Creek near Mandaree, N. Dak. & 56 & $1991-2008$ & $<1.0$ & $<1.0$ & $<1.0$ \\
\hline 110 & Little Missouri River at Marmarth, N. Dak. & 78 & $1971-2008$ & $<1.0$ & $<1.0$ & 1.2 \\
\hline 111 & Deep Creek near Amidon, N. Dak. & 51 & $1977-1983$ & $<1.0$ & $<1.0$ & $<1.0$ \\
\hline 112 & Little Missouri River at Medora, N. Dak. & 24 & $1972-2008$ & $<1.0$ & $<1.0$ & $<1.0$ \\
\hline 113 & Beaver Creek near Trotters, N. Dak. & 86 & $1977-2008$ & $<1.0$ & $<1.0$ & $<1.0$ \\
\hline 114 & Little Missouri River near Watford City, N. Dak. & 162 & $1971-2008$ & $<1.0$ & $<1.0$ & $<1.0$ \\
\hline 115 & Missouri River at Garrison Dam, N. Dak. & 256 & $1971-2007$ & $<1.0$ & $<1.0$ & 4.8 \\
\hline 116 & Knife River at Manning, N. Dak. & 118 & 1972-2008 & $<1.0$ & $<1.0$ & 1.4 \\
\hline 117 & Stray Creek near Manning, N. Dak. & 17 & $1975-1981$ & $<1.0$ & $<1.0$ & $<1.0$ \\
\hline 118 & Knife River at Marshall, N. Dak. & 69 & $1972-1981$ & $<1.0$ & $<1.0$ & 1.3 \\
\hline 119 & Elm Creek near Golden Valley, N. Dak. & 36 & 1973-1994 & $<1.0$ & $<1.0$ & $<1.0$ \\
\hline 120 & Knife River near Golden Valley, N. Dak. & 70 & $1971-2008$ & $<1.0$ & $<1.0$ & $<1.0$ \\
\hline 121 & Coyote Creek near Zap, N. Dak. & 51 & $1977-1983$ & $<1.0$ & $<1.0$ & $<1.0$ \\
\hline
\end{tabular}


Table 1-1. Summary statistics for major ion constituents at selected sites in North Dakota from 1970 through 2008.—Continued [ $\mu \mathrm{g} / \mathrm{L}$, micrograms per liter; <, less than; --, not available; $\mathrm{mg} / \mathrm{L}$, milligrams per liter; $\mathrm{CO}_{3}$, carbonate; $\mathrm{HCO}_{3}$, bicarbonate; $\mathrm{CaCO}_{3}$, calcium carbonate $]$

\begin{tabular}{|c|c|c|c|c|c|c|}
\hline $\begin{array}{c}\text { Site } \\
\text { identification } \\
\text { number } \\
\text { (table 1) }\end{array}$ & Site name & $\begin{array}{c}\text { Number of } \\
\text { samples }\end{array}$ & $\begin{array}{l}\text { Period of } \\
\text { record }\end{array}$ & Median & Minimum & Maximum \\
\hline \multicolumn{7}{|c|}{ Fluoride, in $\mathrm{mg} / \mathrm{L}-$ Continued } \\
\hline 122 & Brush Creek near Beulah, N. Dak. & 99 & 1974-1990 & $<1.0$ & $<1.0$ & $<1.0$ \\
\hline 123 & Spring Creek below Lake Ilo near Dunn Center, N. Dak. & 47 & 1977-1981 & $<1.0$ & $<1.0$ & $<1.0$ \\
\hline 124 & Spring Creek near Halliday, N. Dak. & 48 & $1977-1981$ & $<1.0$ & $<1.0$ & $<1.0$ \\
\hline 125 & Spring Creek at Zap, N. Dak. & 159 & 1970-2008 & $<1.0$ & $<1.0$ & $<1.0$ \\
\hline 126 & West Branch Otter Creek near Beulah, N. Dak. & 15 & $1972-1995$ & $<1.0$ & $<1.0$ & $<1.0$ \\
\hline 127 & Knife River at Hazen, N. Dak. & 188 & 1970-2008 & $<1.0$ & $<1.0$ & $<1.0$ \\
\hline 128 & Antelope Creek above Hazen, N. Dak. & 27 & 1977-1982 & $<1.0$ & $<1.0$ & $<1.0$ \\
\hline 129 & West Branch Antelope Creek No. 4 near Zap, N. Dak. & 10 & $1977-1982$ & $<1.0$ & $<1.0$ & $<1.0$ \\
\hline 130 & West Branch Antelope Creek near Hazen, N. Dak. & 14 & $1978-1983$ & $<1.0$ & $<1.0$ & $<1.0$ \\
\hline 131 & Coal Creek near Stanton, N. Dak. & 28 & 1975-1981 & $<1.0$ & $<1.0$ & $<1.0$ \\
\hline 132 & Alderin Creek near Fort Clark, N. Dak. & 35 & 1977-1982 & $<1.0$ & $<1.0$ & $<1.0$ \\
\hline 133 & Coal Lake Coulee near Hensler, N. Dak. & 16 & 1978-1982 & $<1.0$ & $<1.0$ & $<1.0$ \\
\hline 134 & Buffalo Creek near Washburn, N. Dak. & 38 & $1978-1983$ & $<1.0$ & $<1.0$ & $<1.0$ \\
\hline 135 & Turtle Creek above Washburn, N. Dak. & 100 & $1987-2003$ & $<1.0$ & $<1.0$ & $<1.0$ \\
\hline 136 & Painted Woods Creek near Wilton, N. Dak. & 199 & $1970-2003$ & $<1.0$ & $<1.0$ & $<1.0$ \\
\hline 137 & Square Butte Creek near Hannover, N. Dak. & 23 & 1977-1981 & $<1.0$ & $<1.0$ & $<1.0$ \\
\hline 138 & Square Butte Creek above Nelson Lake near Center, N. Dak. & 56 & 1977-1982 & $<1.0$ & $<1.0$ & $<1.0$ \\
\hline 139 & Hagel Creek near Center, N. Dak. & 38 & $1977-1982$ & $<1.0$ & $<1.0$ & $<1.0$ \\
\hline 140 & Square Butte Creek below Center, N. Dak. & 79 & $1972-2008$ & $<1.0$ & $<1.0$ & $<1.0$ \\
\hline 141 & Burnt Creek near Bismarck, N. Dak. & 48 & $1972-2008$ & $<1.0$ & $<1.0$ & 2.0 \\
\hline 142 & Missouri River at Bismarck, N. Dak. & 97 & 1974-2008 & $<1.0$ & $<1.0$ & $<1.0$ \\
\hline 143 & South Branch Heart River near South Heart, N. Dak. & 19 & 1979-1983 & $<1.0$ & $<1.0$ & $<1.0$ \\
\hline 144 & North Creek near South Heart, N. Dak. & 16 & 1978-1981 & $<1.0$ & $<1.0$ & $<1.0$ \\
\hline 145 & Heart River near South Heart, N. Dak. & 76 & $1975-2005$ & $<1.0$ & $<1.0$ & 2.6 \\
\hline 146 & Heart River at Dickinson, N. Dak. & 17 & 1986-1994 & $<1.0$ & $<1.0$ & $<1.0$ \\
\hline 147 & Green River near New Hradec, N. Dak. & 116 & $1972-2008$ & $<1.0$ & $<1.0$ & 1.1 \\
\hline 148 & Green River near Gladstone, N. Dak. & 29 & 1970-1975 & $<1.0$ & $<1.0$ & 1.1 \\
\hline 149 & Heart River near Richardton, N. Dak. & 80 & $1972-2008$ & $<1.0$ & $<1.0$ & $<1.0$ \\
\hline 150 & Heart River above Lake Tschida near Glen Ullin, N. Dak. & 40 & 1989-2008 & $<1.0$ & $<1.0$ & $<1.0$ \\
\hline 151 & Antelope Creek near Carson, N. Dak. & 26 & $1972-2008$ & $<1.0$ & $<1.0$ & $<1.0$ \\
\hline 152 & Big Muddy Creek near Almont, N. Dak. & 36 & 1991-2008 & $<1.0$ & $<1.0$ & 1.5 \\
\hline 153 & Heart River near Lark, N. Dak. & 45 & 1971-1995 & $<1.0$ & $<1.0$ & 1.3 \\
\hline 154 & Heart River at Stark Bridge near Judson, N. Dak. & 41 & $1988-2008$ & $<1.0$ & $<1.0$ & $<1.0$ \\
\hline 155 & Sweetbriar Creek near Judson, N. Dak. & 33 & $1972-2008$ & $<1.0$ & $<1.0$ & $<1.0$ \\
\hline 156 & Heart River near Mandan, N. Dak. & 152 & 1971-2008 & $<1.0$ & $<1.0$ & 1.1 \\
\hline 157 & Long Lake Creek above Long Lake near Moffit, N. Dak. & 33 & 1988-2004 & $<1.0$ & $<1.0$ & $<1.0$ \\
\hline 158 & Apple Creek near Menoken, N. Dak. & 125 & $1972-2008$ & $<1.0$ & $<1.0$ & 1.5 \\
\hline 159 & Missouri River near Schmidt, N. Dak. & 63 & $1975-1981$ & $<1.0$ & $<1.0$ & $<1.0$ \\
\hline 160 & Cannonball River at New England, N. Dak. & 36 & 1978-1981 & $<1.0$ & $<1.0$ & $<1.0$ \\
\hline 161 & Coal Bank Creek near Havelock, N. Dak. & 84 & 1974-1983 & $<1.0$ & $<1.0$ & $<1.0$ \\
\hline
\end{tabular}


Table 1-1. Summary statistics for major ion constituents at selected sites in North Dakota from 1970 through 2008. —Continued $\left[\mu \mathrm{g} / \mathrm{L}\right.$, micrograms per liter; <, less than; --, not available; $\mathrm{mg} / \mathrm{L}$, milligrams per liter; $\mathrm{CO}_{3}$, carbonate; $\mathrm{HCO}_{3}$, bicarbonate; $\mathrm{CaCO}$, calcium carbonate $]$

\begin{tabular}{|c|c|c|c|c|c|c|}
\hline $\begin{array}{c}\text { Site } \\
\text { identification } \\
\text { number } \\
\text { (table 1) } \\
\end{array}$ & Site name & $\begin{array}{l}\text { Number of } \\
\text { samples }\end{array}$ & $\begin{array}{l}\text { Period of } \\
\text { record }\end{array}$ & Median & Minimum & Maximum \\
\hline \multicolumn{7}{|c|}{ Fluoride, in $\mathrm{mg} / \mathrm{L}-$ Continued } \\
\hline 162 & Cannonball River at Regent, N. Dak. & 119 & 1970-2008 & $<1.0$ & $<1.0$ & 1.1 \\
\hline 163 & Cannonball River below Bentley, N. Dak. & 31 & $1972-1977$ & $<1.0$ & $<1.0$ & $<1.0$ \\
\hline 164 & Cannonball River near Raleigh, N. Dak. & 15 & $2001-2008$ & $<1.0$ & $<1.0$ & $<1.0$ \\
\hline 165 & White Butte Fork Cedar Creek near Scranton, N. Dak. & 26 & 1972-1995 & $<1.0$ & $<1.0$ & $<1.0$ \\
\hline 166 & Cedar Creek near Haynes, N. Dak. & 76 & $1971-2008$ & $<1.0$ & $<1.0$ & 1.1 \\
\hline 167 & Timber Creek near Bentley, N. Dak. & 47 & 1977-1981 & $<1.0$ & $<1.0$ & $<1.0$ \\
\hline 168 & Cedar Creek near Pretty Rock, N. Dak. & 30 & 1971-1976 & $<1.0$ & $<1.0$ & 1.1 \\
\hline 169 & Cedar Creek near Raleigh, N. Dak. & 55 & 1972-2008 & $<1.0$ & $<1.0$ & $<1.0$ \\
\hline 170 & Cannonball River at Breien, N. Dak. & 188 & 1970-2008 & $<1.0$ & $<1.0$ & 1.4 \\
\hline 171 & Beaver Creek near Linton, N. Dak. & 35 & 1972-1989 & $<1.0$ & $<1.0$ & $<1.0$ \\
\hline 172 & Beaver Creek below Linton, N. Dak. & 38 & 1990-2008 & $<1.0$ & $<1.0$ & $<1.0$ \\
\hline 173 & Porcupine Creek near Fort Yates, N. Dak. & 41 & 1991-1999 & $<1.0$ & $<1.0$ & 1.5 \\
\hline 174 & Buffalo Creek Tributary near Gascoyne, N. Dak. & 83 & 1974-1987 & $<1.0$ & $<1.0$ & 1.8 \\
\hline 175 & James River near Manfred, N. Dak. & 24 & $1972-1995$ & $<1.0$ & $<1.0$ & $<1.0$ \\
\hline 176 & James River near Grace City, N. Dak. & 73 & $1972-2008$ & $<1.0$ & $<1.0$ & 2.8 \\
\hline 177 & James River above Arrowwood Lake near Kensal, N. Dak. & 138 & $1991-2008$ & $<1.0$ & $<1.0$ & $<1.0$ \\
\hline 179 & James River near Pingree, N. Dak. & 134 & 1978-2008 & $<1.0$ & $<1.0$ & $<1.0$ \\
\hline 180 & Pipestem Creek near Pingree, N. Dak. & 54 & 1974-2008 & $<1.0$ & $<1.0$ & $<1.0$ \\
\hline 181 & Pipestem Creek near Buchanan, N. Dak. & 4 & $1972-1974$ & $<1.0$ & $<1.0$ & $<1.0$ \\
\hline 182 & James River at Jamestown, N. Dak. & 80 & $1972-2008$ & $<1.0$ & $<1.0$ & $<1.0$ \\
\hline 183 & James River at Lamoure, N. Dak. & 213 & $1970-2008$ & $<1.0$ & $<1.0$ & 2.0 \\
\hline 184 & Bear Creek near Oakes, N. Dak. & 45 & 1977-2008 & $<1.0$ & $<1.0$ & $<1.0$ \\
\hline 185 & James River at Oakes, N. Dak. & 110 & 1970-2008 & $<1.0$ & $<1.0$ & $<1.0$ \\
\hline 186 & James River at N. Dak./S. Dak. State line & 88 & 1974-2008 & $<1.0$ & $<1.0$ & $<1.0$ \\
\hline \multicolumn{7}{|c|}{ Silica, in mg/L } \\
\hline 2 & Red River of the North at Wahpeton, N. Dak. & 35 & 1973-2008 & 14.0 & 2 & 21.0 \\
\hline 6 & Red River of the North at Hickson, N. Dak. & 92 & 1975-2008 & 13.0 & $<1.0$ & 23.0 \\
\hline 7 & Wild Rice River near Rutland, N. Dak. & 30 & 1971-2008 & 11.0 & $<1.0$ & 42.0 \\
\hline 8 & Wild Rice River near Cayuga, N. Dak. & 15 & $1970-1977$ & 14.0 & $<1.0$ & 25.0 \\
\hline 9 & Antelope Creek at Dwight, N. Dak. & 7 & $2003-2008$ & 14.8 & 9.1 & 17.8 \\
\hline 10 & Wild Rice River near Abercrombie, N. Dak. & 141 & 1970-2008 & 21.4 & 2.3 & 51.0 \\
\hline 11 & Red River of the North at Fargo, N. Dak. & 104 & 1970-2008 & 14.0 & 1.5 & 25.8 \\
\hline 12 & Red River of North below Fargo, N. Dak. & 132 & 1973-1986 & 13.0 & $<1.0$ & 46.0 \\
\hline 14 & Red River of the North near Harwood, N. Dak. & 13 & $2005-2007$ & 20.1 & 13.6 & 66.7 \\
\hline 15 & Sheyenne River above Harvey, N. Dak. & 181 & $1972-2008$ & 24.0 & 2.2 & 52.0 \\
\hline 16 & Big Coulee near Fort Totten, N. Dak. & 8 & $1970-1975$ & 23.5 & 2.5 & 32.0 \\
\hline 18 & Sheyenne River near Warwick, N. Dak. & 87 & $1970-2008$ & 16.3 & 2.8 & 40.0 \\
\hline 19 & Mauvais Coulee Tributary No. 3 near Cando, N. Dak. & 19 & $1987-2008$ & 18.0 & 2.4 & 56.0 \\
\hline 20 & Mauvais Coulee near Cando, N. Dak. & 59 & 1972-2008 & 14.0 & $<1.0$ & 54.0 \\
\hline
\end{tabular}


Table 1-1. Summary statistics for major ion constituents at selected sites in North Dakota from 1970 through 2008.—Continued $\left[\mu \mathrm{g} / \mathrm{L}\right.$, micrograms per liter; <, less than; --, not available; mg/L, milligrams per liter; $\mathrm{CO}_{3}$, carbonate; $\mathrm{HCO}_{3}$, bicarbonate; $\mathrm{CaCO}$, calcium carbonate $]$

\begin{tabular}{|c|c|c|c|c|c|c|}
\hline $\begin{array}{c}\text { Site } \\
\text { identification } \\
\text { number } \\
\text { (table 1) }\end{array}$ & Site name & $\begin{array}{l}\text { Number of } \\
\text { samples }\end{array}$ & $\begin{array}{l}\text { Period of } \\
\text { record }\end{array}$ & Median & Minimum & Maximum \\
\hline \multicolumn{7}{|c|}{ Silica, in $\mathrm{mg} / \mathrm{L}$-Continued } \\
\hline 21 & Edmore Coulee near Edmore, N. Dak. & 57 & $1972-2008$ & 15.0 & $<1.0$ & 34.8 \\
\hline 22 & Edmore Coulee Tributary near Webster, N. Dak. & 17 & $1987-2008$ & 17.0 & 2.0 & 35.5 \\
\hline 23 & Webster Coulee at Webster, N. Dak. & 2 & $1983-1984$ & 15.0 & 12.0 & 18.0 \\
\hline 24 & Starkweather Coulee near Webster, N. Dak. & 49 & $1983-2008$ & 19.0 & $<1.0$ & 35.0 \\
\hline 25 & Big Coulee below Churchs Ferry, N. Dak. & 6 & $2003-2008$ & 17.7 & 8.3 & 20.6 \\
\hline 26 & Little Coulee near Leeds, N. Dak. & 5 & 2004-2008 & 20.8 & 5.3 & 31.6 \\
\hline 27 & Little Coulee near Brinsmade, N. Dak. & 24 & $1976-1994$ & 17.5 & 1.4 & 37.0 \\
\hline 28 & Big Coulee near Churchs Ferry, N. Dak. & 113 & 1970-1994 & 15.0 & $<1.0$ & 58.0 \\
\hline 29 & Big Coulee at Graham Island inlet near Fort Totten, N. Dak. & 44 & 1970-1986 & 14.0 & 2.3 & 38.0 \\
\hline 30 & Channel A near Penn, N. Dak. & 53 & 1984-2008 & 13.0 & $<1.0$ & 33.0 \\
\hline 31 & Devils Lake Outlet to Stump Lake near Lakota, N. Dak. & 5 & $2003-2005$ & 12.8 & 2.1 & 19.5 \\
\hline 32 & Sheyenne River near Cooperstown, N. Dak. & 213 & $1970-2008$ & 21.9 & $<1.0$ & 44.4 \\
\hline 33 & Baldhill Creek near Dazey, N. Dak. & 72 & $1972-2008$ & 13.8 & $<1.0$ & 34.0 \\
\hline 34 & Sheyenne River below Baldhill Dam, N. Dak. & 78 & $1972-2008$ & 15.0 & $<1.0$ & 31.5 \\
\hline 35 & Sheyenne River at Valley City, N. Dak. & 15 & $1972-2005$ & 13.0 & 3.8 & 29.9 \\
\hline 36 & Sheyenne River at Lisbon, N. Dak. & 213 & $1970-2008$ & 13.0 & $<1.0$ & 28.0 \\
\hline 37 & Sheyenne River near Kindred, N. Dak. & 222 & $1972-2008$ & 17.0 & 3.9 & 48.0 \\
\hline 38 & $\begin{array}{l}\text { Sheyenne River above Sheyenne River Diversion near Horace, } \\
\text { N. Dak. }\end{array}$ & 23 & $1993-2008$ & 22.4 & 9.4 & 73.9 \\
\hline 39 & Sheyenne River near Horace, N. Dak. & 8 & 1982-1992 & 13.0 & 8.6 & 21.0 \\
\hline 40 & Sheyenne River Diversion at West Fargo, N. Dak. & 4 & $1994-2005$ & 17.7 & 13.3 & 26.5 \\
\hline 41 & Sheyenne River at West Fargo, N. Dak. & 39 & $1970-2008$ & 16.0 & 3.2 & 24.0 \\
\hline 42 & Maple River near Hope, N. Dak. & 25 & $1972-2008$ & 19.0 & 4.5 & 36.0 \\
\hline 43 & Maple River near Enderlin, N. Dak. & 43 & $1972-2008$ & 21.0 & 9.2 & 29.0 \\
\hline 44 & Maple River near Mapleton, N. Dak. & 15 & 1972-2008 & 19.0 & 9.3 & 29.2 \\
\hline 45 & Maple River below Mapleton, N. Dak. & 8 & $2003-2008$ & 22.0 & 11.7 & 28.7 \\
\hline 46 & Sheyenne River at Harwood, N. Dak. & 2 & $2004-2005$ & 18.0 & 13.1 & 22.8 \\
\hline 47 & Rush River at Amenia, N. Dak. & 31 & $1972-2008$ & 16.2 & 3.1 & 32.0 \\
\hline 48 & Rush River near Prosper, N. Dak. & 4 & 1983-1987 & 15.0 & 9.4 & 18.0 \\
\hline 49 & Lower Branch Rush River near Prosper, N. Dak. & 4 & 1983-1987 & 16.5 & 9.5 & 22.0 \\
\hline 51 & Elm River near Kelso, N. Dak. & 3 & 1983-1987 & 12.0 & 7.8 & 16.0 \\
\hline 52 & Red River of the North at Halstad, Minn. & 162 & $1972-2008$ & 15.0 & 3.8 & 74.1 \\
\hline 53 & Beaver Creek near Finley, N. Dak. & 105 & $1970-2003$ & 15.0 & 3.5 & 110.0 \\
\hline 54 & Goose River near Portland, N. Dak. & 30 & 1970-1988 & 15.5 & 9.3 & 28.0 \\
\hline 55 & Goose River at Hillsboro, N. Dak. & 70 & $1970-2008$ & 17.0 & 1.7 & 31.0 \\
\hline 56 & Red River of the North at Grand Forks, N. Dak. & 134 & 1970-2008 & 12.6 & 1.9 & 24.5 \\
\hline 57 & Turtle River at Turtle River State Park near Arvilla, N. Dak. & 89 & $1991-2008$ & 20.0 & 4.5 & 41.0 \\
\hline 58 & Turtle River at Manvel, N. Dak. & 66 & $1971-2008$ & 15.0 & $<1.0$ & 33.1 \\
\hline 59 & Red River of the North at Oslo, Minn. & 66 & $1973-2005$ & 11.0 & $<1.0$ & 20.0 \\
\hline 60 & Middle Branch Forest River near Whitman, N. Dak. & 14 & 1972-1990 & 13.0 & 4.6 & 27.0 \\
\hline
\end{tabular}


Table 1-1. Summary statistics for major ion constituents at selected sites in North Dakota from 1970 through 2008. —Continued $\left[\mu \mathrm{g} / \mathrm{L}\right.$, micrograms per liter; <, less than; --, not available; $\mathrm{mg} / \mathrm{L}$, milligrams per liter; $\mathrm{CO}_{3}$, carbonate; $\mathrm{HCO}_{3}$, bicarbonate; $\mathrm{CaCO}_{3}$, calcium carbonate $]$

\begin{tabular}{|c|c|c|c|c|c|c|}
\hline $\begin{array}{c}\text { Site } \\
\text { identification } \\
\text { number } \\
\text { (table 1) }\end{array}$ & Site name & $\begin{array}{c}\text { Number of } \\
\text { samples }\end{array}$ & $\begin{array}{l}\text { Period of } \\
\text { record }\end{array}$ & Median & Minimum & Maximum \\
\hline \multicolumn{7}{|c|}{ Silica, in $\mathrm{mg} / \mathrm{L}$-Continued } \\
\hline 61 & Forest River near Fordville, N. Dak. & 44 & 1972-2008 & 17.0 & 4.9 & 29.0 \\
\hline 62 & Forest River near Minto, N. Dak. & 90 & $1971-2008$ & 17.0 & 2.0 & 33.7 \\
\hline 63 & South Branch Park River below Homme Dam, N. Dak. & 27 & $1972-1994$ & 14.0 & 1.7 & 24.0 \\
\hline 64 & Middle Branch Park River near Union, N. Dak. & 15 & 1972-1984 & 18.0 & 3.6 & 34.0 \\
\hline 65 & Middle Branch Park River near Edinburg, N. Dak. & 14 & $1978-1980$ & 16.0 & $<1.0$ & 26.0 \\
\hline 66 & Cart Creek at Mountain, N. Dak. & 17 & $1972-1984$ & 19.0 & 4.6 & 28.0 \\
\hline 67 & Park River at Grafton, N. Dak. & 88 & 1970-2008 & 17.0 & 3.8 & 32.2 \\
\hline 68 & Red River of the North at Drayton, N. Dak. & 37 & $1972-2008$ & 12.0 & 2.4 & 20.9 \\
\hline 69 & Pembina County Drain 20 near Glasston, N. Dak. & 8 & 1974-1984 & 9.5 & 3.0 & 16.0 \\
\hline 70 & Hidden Island Coulee near Hansboro, N. Dak. & 21 & 1972-1994 & 15.0 & 3.5 & 28.0 \\
\hline 71 & Cypress Creek near Sarles, N. Dak. & 13 & $1972-1988$ & 18.0 & 4.9 & 42.0 \\
\hline 72 & Pembina River near Vang, N. Dak. & 63 & 1970-1979 & 18.0 & 9.4 & 58.0 \\
\hline 73 & Little South Pembina River near Walhalla, N. Dak. & 73 & 1970-2008 & 20.0 & 8.6 & 30.0 \\
\hline 74 & Pembina River at Walhalla, N. Dak. & 215 & $1970-2008$ & 21.0 & $<1.0$ & 34.0 \\
\hline 75 & Pembina River at Neche, N. Dak. & 62 & 1972-2008 & 21.0 & $<1.0$ & 34.1 \\
\hline 76 & Tongue River at Akra, N. Dak. & 50 & $1972-2008$ & 14.0 & $<1.0$ & 25.0 \\
\hline 78 & Red River of the North at Pembina, N. Dak., site 2 & 94 & 1994-2008 & 15.0 & 2.0 & 62.9 \\
\hline 79 & Red River of the North at Emerson, Manitoba & 143 & $1978-2000$ & 13.0 & 2.5 & 38.0 \\
\hline 80 & Long Creek near Noonan, N. Dak. & 41 & 1972-2008 & 6.0 & $<1.0$ & 22.0 \\
\hline 81 & West Branch Short Creek near Columbus, N. Dak. & 20 & $1978-1981$ & 11.0 & 1.2 & 19.0 \\
\hline 82 & Souris River near Sherwood, N. Dak. & 173 & $1972-1991$ & 8.4 & $<1.0$ & 580.0 \\
\hline 83 & Souris River near Foxholm, N. Dak. & 147 & $1972-2008$ & 5.9 & $<1.0$ & 20.0 \\
\hline 84 & Des Lacs River at Foxholm, N. Dak. & 71 & 1972-2008 & 10.0 & $<1.0$ & 25.0 \\
\hline 85 & Souris River above Minot, N. Dak. & 64 & $1970-1994$ & 5.5 & $<1.0$ & 25.0 \\
\hline 86 & Bonnes Creek near Velva, N. Dak. & 9 & $1987-2005$ & 12.0 & 4.8 & 21.0 \\
\hline 87 & Souris River near Verendrye, N. Dak. & 186 & 1970-1991 & 9.9 & $<1.0$ & 32.0 \\
\hline 88 & Wintering River near Karlsruhe, N. Dak. & 103 & 1972-2008 & 15.0 & 3.4 & 35.0 \\
\hline 89 & Souris River near Bantry, N. Dak. & 67 & $1970-2008$ & 9.4 & $<1.0$ & 23.0 \\
\hline 90 & Willow Creek near Willow City, N. Dak. & 38 & 1972-2008 & 11.1 & 1.4 & 56.0 \\
\hline 91 & Stone Creek near Kramer, N. Dak. & 9 & 1986-1989 & 22.0 & 8.9 & 36.0 \\
\hline 92 & Deep River near Upham, N. Dak. & 24 & $1972-2005$ & 7.2 & 1.1 & 27.3 \\
\hline 93 & Egg Creek near Granville, N. Dak. & 14 & $1972-1981$ & 7.5 & 1.9 & 12.0 \\
\hline 94 & Cut Bank Creek at North Lake Outlet near Granville, N. Dak. & 15 & $1972-1976$ & 12.0 & 6.5 & 24.0 \\
\hline 95 & Cut Bank Creek at Upham, N. Dak. & 17 & $1975-1989$ & 9.8 & $<1.0$ & 40.0 \\
\hline 96 & Deep River below Cut Bank Creek near Upham, N. Dak. & 43 & 1975-1989 & 11.0 & $<1.0$ & 34.0 \\
\hline 97 & Boundary Creek near Landa, N. Dak. & 17 & $1972-1994$ & 11.0 & 1.1 & 28.0 \\
\hline 98 & Souris River near Westhope, N. Dak. & 186 & $1970-1994$ & 8.9 & $<1.0$ & 58.0 \\
\hline 99 & Charbonneau Creek near Charbonneau, N. Dak. & 22 & 1972-1977 & 7.4 & 1.5 & 12.0 \\
\hline 100 & Missouri River near Williston, N. Dak. & 95 & 1974-1992 & 8.6 & 4.8 & 11.0 \\
\hline 101 & Little Muddy River below Cow Creek near Williston, N. Dak. & 48 & 1972-2008 & 11.0 & 3.2 & 28.0 \\
\hline
\end{tabular}


Table 1-1. Summary statistics for major ion constituents at selected sites in North Dakota from 1970 through 2008.—Continued $\left[\mu \mathrm{g} / \mathrm{L}\right.$, micrograms per liter; <, less than; --, not available; mg/L, milligrams per liter; $\mathrm{CO}_{3}$, carbonate; $\mathrm{HCO}_{3}$, bicarbonate; $\mathrm{CaCO}$, calcium carbonate $]$

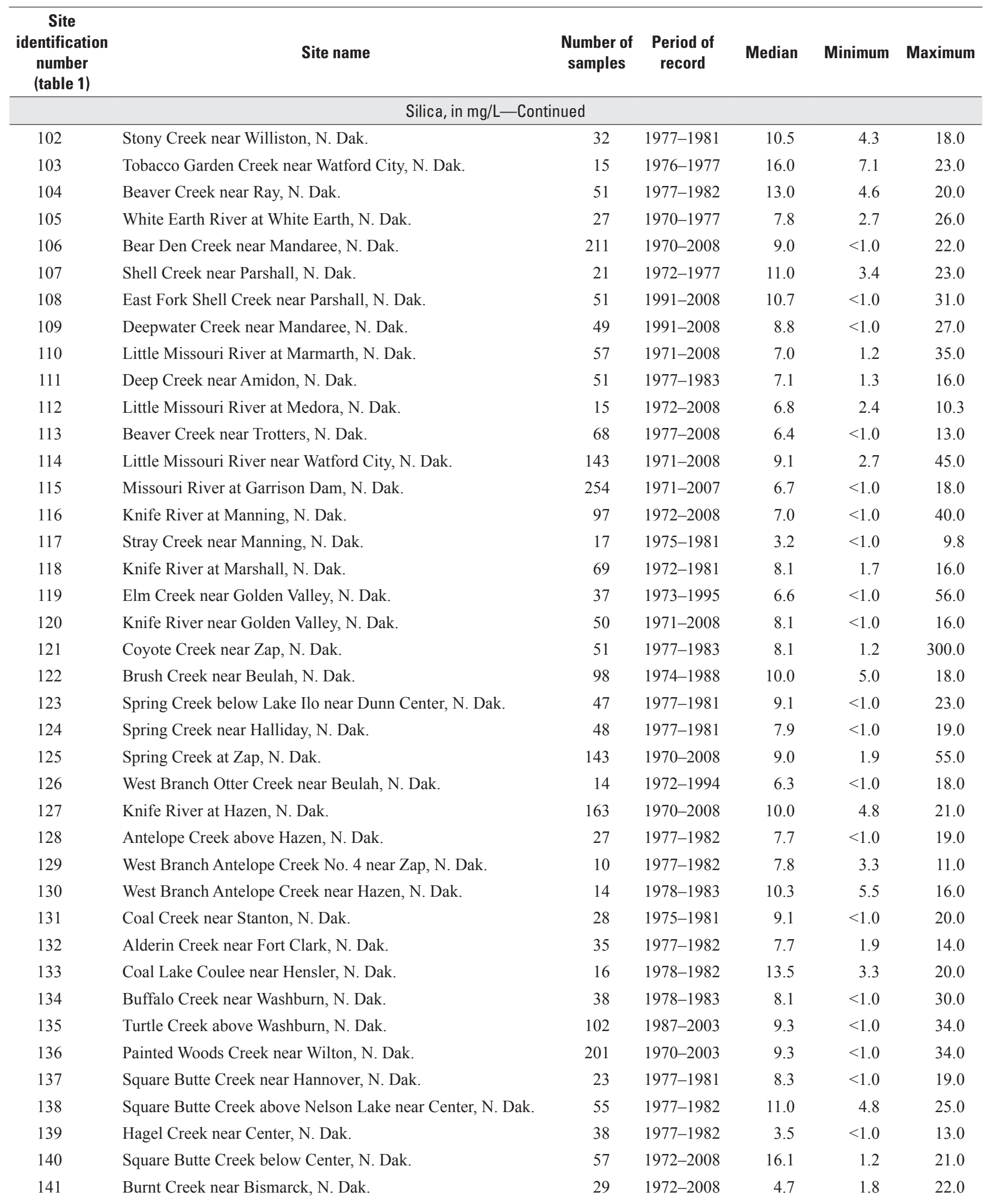


Table 1-1. Summary statistics for major ion constituents at selected sites in North Dakota from 1970 through 2008. —Continued $\left[\mu \mathrm{g} / \mathrm{L}\right.$, micrograms per liter; <, less than; --, not available; $\mathrm{mg} / \mathrm{L}$, milligrams per liter; $\mathrm{CO}_{3}$, carbonate; $\mathrm{HCO}_{3}$, bicarbonate; $\mathrm{CaCO}$, calcium carbonate $]$

\begin{tabular}{|c|c|c|c|c|c|c|}
\hline $\begin{array}{c}\text { Site } \\
\text { identification } \\
\text { number } \\
\text { (table 1) }\end{array}$ & Site name & $\begin{array}{c}\text { Number of } \\
\text { samples }\end{array}$ & $\begin{array}{l}\text { Period of } \\
\text { record }\end{array}$ & Median & Minimum & Maximum \\
\hline \multicolumn{7}{|c|}{ Silica, in $\mathrm{mg} / \mathrm{L}-$ Continued } \\
\hline 142 & Missouri River at Bismarck, N. Dak. & 91 & 1974-2008 & 7.3 & 2.7 & 21.0 \\
\hline 143 & South Branch Heart River near South Heart, N. Dak. & 19 & $1979-1983$ & 9.1 & 1.6 & 130.0 \\
\hline 144 & North Creek near South Heart, N. Dak. & 16 & 1978-1981 & 5.0 & $<1.0$ & 12.0 \\
\hline 145 & Heart River near South Heart, N. Dak. & 69 & $1975-2005$ & 8.7 & 1.5 & 49.8 \\
\hline 146 & Heart River at Dickinson, N. Dak. & 17 & 1986-1994 & 9.2 & 4.2 & 16.0 \\
\hline 147 & Green River near New Hradec, N. Dak. & 96 & $1972-2008$ & 6.2 & 1.6 & 16.0 \\
\hline 148 & Green River near Gladstone, N. Dak. & 29 & $1970-1975$ & 7.9 & 4.8 & 17.0 \\
\hline 149 & Heart River near Richardton, N. Dak. & 59 & $1972-2008$ & 5.2 & 1.3 & 13.0 \\
\hline 150 & Heart River above Lake Tschida near Glen Ullin, N. Dak. & 19 & 1989-2008 & 5.6 & 2.0 & 9.7 \\
\hline 151 & Antelope Creek near Carson, N. Dak. & 14 & $1972-2008$ & 2.9 & 2.0 & 8.8 \\
\hline 152 & Big Muddy Creek near Almont, N. Dak. & 15 & 1991-2008 & 7.6 & 2.0 & 13.0 \\
\hline 153 & Heart River near Lark, N. Dak. & 43 & 1971-1994 & 4.8 & $<1.0$ & 25.0 \\
\hline 154 & Heart River at Stark Bridge near Judson, N. Dak. & 20 & 1988-2008 & 4.8 & 2.7 & 23.0 \\
\hline 155 & Sweetbriar Creek near Judson, N. Dak. & 27 & 1972-2008 & 3.5 & $<1.0$ & 9.5 \\
\hline 156 & Heart River near Mandan, N. Dak. & 131 & 1971-2008 & 5.9 & $<1.0$ & 15.0 \\
\hline 157 & Long Lake Creek above Long Lake near Moffit, N. Dak. & 17 & 1988-2004 & 14.0 & 6.2 & 25.6 \\
\hline 158 & Apple Creek near Menoken, N. Dak. & 105 & 1972-2008 & 12.0 & $<1.0$ & 28.0 \\
\hline 159 & Missouri River near Schmidt, N. Dak. & 68 & $1974-1981$ & 7.5 & 4.8 & 8.9 \\
\hline 160 & Cannonball River at New England, N. Dak. & 36 & 1978-1981 & 6.8 & 2.7 & 11.0 \\
\hline 161 & Coal Bank Creek near Havelock, N. Dak. & 84 & $1974-1983$ & 4.6 & $<1.0$ & 15.0 \\
\hline 162 & Cannonball River at Regent, N. Dak. & 98 & 1970-2008 & 5.7 & $<1.0$ & 14.0 \\
\hline 163 & Cannonball River below Bentley, N. Dak. & 31 & $1972-1977$ & 3.7 & $<1.0$ & 8.8 \\
\hline 164 & Cannonball River near Raleigh, N. Dak. & 7 & 2004-2008 & 4.2 & 2.0 & 9.9 \\
\hline 165 & White Butte Fork Cedar Creek near Scranton, N. Dak. & 24 & 1972-1994 & 5.2 & $<1.0$ & 23.0 \\
\hline 166 & Cedar Creek near Haynes, N. Dak. & 55 & $1971-2008$ & 3.2 & $<1.0$ & 8.8 \\
\hline 167 & Timber Creek near Bentley, N. Dak. & 47 & 1977-1981 & 3.6 & $<1.0$ & 14.0 \\
\hline 168 & Cedar Creek near Pretty Rock, N. Dak. & 30 & $1971-1976$ & 4.0 & 1.3 & 6.7 \\
\hline 169 & Cedar Creek near Raleigh, N. Dak. & 35 & 1972-2008 & 4.9 & $<1.0$ & 20.0 \\
\hline 170 & Cannonball River at Breien, N. Dak. & 167 & 1970-2008 & 6.7 & $<1.0$ & 22.0 \\
\hline 171 & Beaver Creek near Linton, N. Dak. & 35 & 1972-1989 & 11.0 & 2.8 & 25.0 \\
\hline 172 & Beaver Creek below Linton, N. Dak. & 18 & 1990-2008 & 15.8 & 10.0 & 24.3 \\
\hline 173 & Porcupine Creek near Fort Yates, N. Dak. & 41 & 1991-1999 & 14.1 & 4.8 & 20.0 \\
\hline 174 & Buffalo Creek Tributary near Gascoyne, N. Dak. & 83 & 1974-1987 & 5.7 & $<1.0$ & 17.0 \\
\hline 175 & James River near Manfred, N. Dak. & 30 & $1972-1995$ & 12.0 & 2.5 & 28.0 \\
\hline 176 & James River near Grace City, N. Dak. & 47 & $1972-2008$ & 8.7 & $<1.0$ & 33.6 \\
\hline 177 & James River above Arrowwood Lake near Kensal, N. Dak. & 138 & $1985-2008$ & 13.6 & 2.2 & 47.0 \\
\hline 178 & Kelly Creek below Niccum Reservoir near Bordulac, N. Dak. & 4 & $1986-1987$ & 11.0 & 4.6 & 14.0 \\
\hline 179 & James River near Pingree, N. Dak. & 136 & 1978-2008 & 12.7 & $<1.0$ & 42.8 \\
\hline 180 & Pipestem Creek near Pingree, N. Dak. & 33 & 1974-2008 & 14.0 & 1.9 & 35.0 \\
\hline
\end{tabular}


Table 1-1. Summary statistics for major ion constituents at selected sites in North Dakota from 1970 through 2008.—Continued $\left[\mu \mathrm{g} / \mathrm{L}\right.$, micrograms per liter; <, less than; --, not available; mg/L, milligrams per liter; $\mathrm{CO}_{3}$, carbonate; $\mathrm{HCO}_{3}$, bicarbonate; $\mathrm{CaCO}$, calcium carbonate $]$

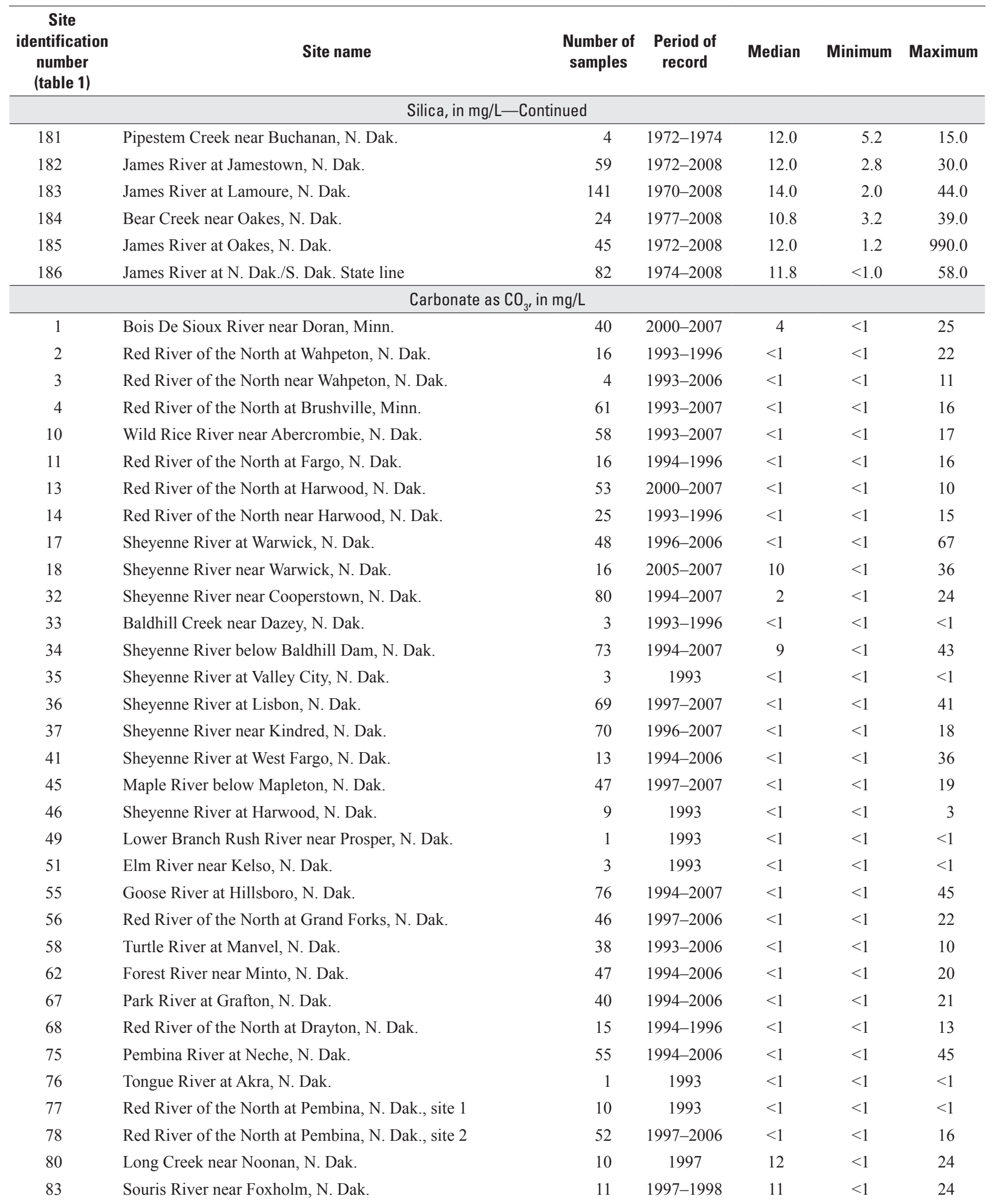


Table 1-1. Summary statistics for major ion constituents at selected sites in North Dakota from 1970 through 2008. —Continued $\left[\mu \mathrm{g} / \mathrm{L}\right.$, micrograms per liter; <, less than; --, not available; $\mathrm{mg} / \mathrm{L}$, milligrams per liter; $\mathrm{CO}_{3}$, carbonate; $\mathrm{HCO}_{3}$, bicarbonate; $\mathrm{CaCO}$, calcium carbonate $]$

\begin{tabular}{|c|c|c|c|c|c|c|}
\hline $\begin{array}{c}\text { Site } \\
\text { identification } \\
\text { number } \\
\text { (table 1) }\end{array}$ & Site name & $\begin{array}{c}\text { Number of } \\
\text { samples }\end{array}$ & $\begin{array}{l}\text { Period of } \\
\text { record }\end{array}$ & Median & Minimum & Maximum \\
\hline \multicolumn{7}{|c|}{ Carbonate as $\mathrm{CO}_{3^{\prime}}$ in $\mathrm{mg} / \mathrm{L}-$ Continued } \\
\hline 84 & Des Lacs River at Foxholm, N. Dak. & 97 & 1993-2007 & 10 & $<1$ & 89 \\
\hline 85 & Souris River above Minot, N. Dak. & 75 & 1994-2007 & 2 & $<1$ & 92 \\
\hline 87 & Souris River near Verendrye, N. Dak. & 76 & 1997-2007 & $<1$ & $<1$ & 58 \\
\hline 88 & Wintering River near Karlsruhe, N. Dak. & 11 & 1997-1998 & $<1$ & $<1$ & 36 \\
\hline 89 & Souris River near Bantry, N. Dak. & 10 & 1997-1998 & $<1$ & $<1$ & 16 \\
\hline 90 & Willow Creek near Willow City, N. Dak. & 6 & 1997 & 29 & 9 & 44 \\
\hline 92 & Deep River near Upham, N. Dak. & 7 & 1997 & 11 & $<1$ & 39 \\
\hline 98 & Souris River near Westhope, N. Dak. & 7 & 1997-1998 & 24 & $<1$ & 57 \\
\hline 101 & Little Muddy River below Cow Creek near Williston, N. Dak. & 9 & 1999 & 20 & $<1$ & 24 \\
\hline 110 & Little Missouri River at Marmarth, N. Dak. & 8 & 1999 & $<1$ & $<1$ & 23 \\
\hline 112 & Little Missouri River at Medora, N. Dak. & 88 & 1993-2007 & 7 & $<1$ & 39 \\
\hline 113 & Beaver Creek near Trotters, N. Dak. & 9 & 1999 & 20 & $<1$ & 22 \\
\hline 114 & Little Missouri River near Watford City, N. Dak. & 81 & 1994-2007 & 3 & $<1$ & 34 \\
\hline 120 & Knife River near Golden Valley, N. Dak. & 60 & $1997-2007$ & 5 & $<1$ & 60 \\
\hline 123 & Spring Creek below Lake Ilo near Dunn Center, N. Dak. & 6 & 1993 & 18 & $<1$ & 35 \\
\hline 125 & Spring Creek at Zap, N. Dak. & 77 & 1993-2007 & $<1$ & $<1$ & 38 \\
\hline 127 & Knife River at Hazen, N. Dak. & 94 & 1993-2007 & $<1$ & $<1$ & 35 \\
\hline 140 & Square Butte Creek below Center, N. Dak. & 3 & 1993 & $<1$ & $<1$ & $<1$ \\
\hline 142 & Missouri River at Bismarck, N. Dak. & 1 & 1994 & $<1$ & $<1$ & $<1$ \\
\hline 146 & Heart River at Dickinson, N. Dak. & 5 & 1993 & $<1$ & $<1$ & 19 \\
\hline 148 & Green River near Gladstone, N. Dak. & 4 & 1993 & $<1$ & $<1$ & 17 \\
\hline 149 & Heart River near Richardton, N. Dak. & 76 & 1994-2007 & 3 & $<1$ & 28 \\
\hline 152 & Big Muddy Creek near Almont, N. Dak. & 3 & 1993 & 19 & $<1$ & 34 \\
\hline 156 & Heart River near Mandan, N. Dak. & 81 & 1994-2007 & 7 & $<1$ & 26 \\
\hline 158 & Apple Creek near Menoken, N. Dak. & 2 & 1993 & $<1$ & $<1$ & $<1$ \\
\hline 164 & Cannonball River near Raleigh, N. Dak. & 76 & $1993-2007$ & 7 & $<1$ & 25 \\
\hline 169 & Cedar Creek near Raleigh, N. Dak. & 68 & 1993-2007 & 6 & $<1$ & 45 \\
\hline 170 & Cannonball River at Breien, N. Dak. & 74 & 1994-2007 & 3 & $<1$ & 34 \\
\hline 171 & Beaver Creek near Linton, N. Dak. & 3 & 1993 & $<1$ & $<1$ & $<1$ \\
\hline 175 & James River near Manfred, N. Dak. & 6 & 1998 & 5 & $<1$ & 19 \\
\hline 176 & James River near Grace City, N. Dak. & 60 & $1997-2007$ & 10 & $<1$ & 69 \\
\hline 177 & James River above Arrowwood Lake near Kensal, N. Dak. & 9 & 1998-1999 & 3 & $<1$ & 37 \\
\hline 180 & Pipestem Creek near Pingree, N. Dak. & 17 & 1994-1999 & 4 & $<1$ & 33 \\
\hline 181 & Pipestem Creek near Buchanan, N. Dak. & 4 & 1993 & $<1$ & $<1$ & $<1$ \\
\hline 182 & James River at Jamestown, N. Dak. & 75 & $1997-2007$ & $<1$ & $<1$ & 40 \\
\hline 183 & James River at Lamoure, N. Dak. & 73 & $1996-2007$ & 4 & $<1$ & 65 \\
\hline 184 & Bear Creek near Oakes, N. Dak. & 9 & 1998-1999 & $<1$ & $<1$ & 9 \\
\hline 185 & James River at Oakes, N. Dak. & 3 & 1993 & $<1$ & $<1$ & $<1$ \\
\hline 186 & James River at N. Dak./S. Dak. State line & 9 & 1998-1999 & 12 & $<1$ & 25 \\
\hline
\end{tabular}


Table 1-1. Summary statistics for major ion constituents at selected sites in North Dakota from 1970 through 2008.—Continued $\left[\mu \mathrm{g} / \mathrm{L}\right.$, micrograms per liter; <, less than; --, not available; mg/L, milligrams per liter; $\mathrm{CO}_{3}$, carbonate; $\mathrm{HCO}_{3}$, bicarbonate; $\mathrm{CaCO}$, calcium carbonate $]$

\begin{tabular}{|c|c|c|c|c|c|c|}
\hline $\begin{array}{c}\text { Site } \\
\text { identification } \\
\text { number } \\
\text { (table 1) } \\
\end{array}$ & Site name & $\begin{array}{l}\text { Number of } \\
\text { samples }\end{array}$ & $\begin{array}{l}\text { Period of } \\
\text { record }\end{array}$ & Median & Minimum & Maximum \\
\hline \multicolumn{7}{|c|}{ Bicarbonate as $\mathrm{HCO}_{3}$, in $\mathrm{mg} / \mathrm{L}$} \\
\hline 1 & Bois De Sioux River near Doran, Minn. & 40 & $2000-2007$ & 263 & 92 & 942 \\
\hline 2 & Red River of the North at Wahpeton, N. Dak. & 17 & 1993-1996 & 233 & 149 & 442 \\
\hline 4 & Red River of the North at Brushville, Minn. & 61 & 1993-2007 & 245 & 124 & 364 \\
\hline 10 & Wild Rice River near Abercrombie, N. Dak. & 58 & 1993-2007 & 368 & 123 & 838 \\
\hline 11 & Red River of the North at Fargo, N. Dak. & 16 & 1994-1996 & 258 & 178 & 290 \\
\hline 13 & Red River of the North at Harwood, N. Dak. & 53 & $2000-2007$ & 256 & 131 & 347 \\
\hline 32 & Sheyenne River near Cooperstown, N. Dak. & 80 & 1994-2007 & 439 & 159 & 604 \\
\hline 33 & Baldhill Creek near Dazey, N. Dak. & 3 & 1993-1996 & 414 & 300 & 438 \\
\hline 34 & Sheyenne River below Baldhill Dam, N. Dak. & 73 & 1994-2007 & 338 & 181 & 618 \\
\hline 35 & Sheyenne River at Valley City, N. Dak. & 4 & 1993 & 273 & 248 & 336 \\
\hline 36 & Sheyenne River at Lisbon, N. Dak. & 69 & 1997-2007 & 345 & 201 & 578 \\
\hline 37 & Sheyenne River near Kindred, N. Dak. & 70 & 1996-2007 & 357 & 229 & 556 \\
\hline 41 & Sheyenne River at West Fargo, N. Dak. & 13 & 1994-2006 & 347 & 199 & 388 \\
\hline 45 & Maple River below Mapleton, N. Dak. & 47 & 1997-2007 & 348 & 142 & 1,090 \\
\hline 46 & Sheyenne River at Harwood, N. Dak. & 10 & 1993 & 344 & 255 & 411 \\
\hline 67 & Park River at Grafton, N. Dak. & 40 & 1994-2006 & 303 & 120 & 644 \\
\hline 68 & Red River of the North at Drayton, N. Dak. & 15 & 1994-1996 & 265 & 181 & 312 \\
\hline 75 & Pembina River at Neche, N. Dak. & 55 & 1994-2006 & 299 & 125 & 518 \\
\hline 76 & Tongue River at Akra, N. Dak. & 1 & 1993 & 181 & 181 & 181 \\
\hline 77 & Red River of the North at Pembina, N. Dak., site 1 & 10 & 1993 & 274 & 162 & 320 \\
\hline 78 & Red River of the North at Pembina, N. Dak., site 2 & 52 & 1997-2006 & 266 & 131 & 422 \\
\hline 80 & Long Creek near Noonan, N. Dak. & 10 & 1997 & 333 & 176 & 388 \\
\hline 83 & Souris River near Foxholm, N. Dak. & 11 & 1997-1998 & 252 & 150 & 584 \\
\hline 84 & Des Lacs River at Foxholm, N. Dak. & 97 & $1993-2007$ & 445 & 145 & 868 \\
\hline 85 & Souris River above Minot, N. Dak. & 75 & 1994-2007 & 345 & 162 & 1,540 \\
\hline 87 & Souris River near Verendrye, N. Dak. & 76 & 1997-2007 & 371 & 164 & 654 \\
\hline 88 & Wintering River near Karlsruhe, N. Dak. & 11 & 1997-1998 & 386 & 250 & 690 \\
\hline 89 & Souris River near Bantry, N. Dak. & 10 & 1997-1998 & 416 & 235 & 581 \\
\hline 90 & Willow Creek near Willow City, N. Dak. & 6 & 1997 & 400 & 211 & 491 \\
\hline 92 & Deep River near Upham, N. Dak. & 7 & 1997 & 267 & 164 & 333 \\
\hline
\end{tabular}


Table 1-1. Summary statistics for major ion constituents at selected sites in North Dakota from 1970 through 2008. —Continued $\left[\mu \mathrm{g} / \mathrm{L}\right.$, micrograms per liter; <, less than; --, not available; $\mathrm{mg} / \mathrm{L}$, milligrams per liter; $\mathrm{CO}_{3}$, carbonate; $\mathrm{HCO}_{3}$, bicarbonate; $\mathrm{CaCO}$, calcium carbonate $]$

\begin{tabular}{|c|c|c|c|c|c|c|}
\hline $\begin{array}{c}\text { Site } \\
\text { identification } \\
\text { number } \\
\text { (table 1) }\end{array}$ & Site name & $\begin{array}{c}\text { Number of } \\
\text { samples }\end{array}$ & $\begin{array}{l}\text { Period of } \\
\text { record }\end{array}$ & Median & Minimum & Maximum \\
\hline \multicolumn{7}{|c|}{ Bicarbonate as $\mathrm{HCO}_{3}$, in $\mathrm{mg} / \mathrm{L}-$ Continued } \\
\hline 98 & Souris River near Westhope, N. Dak. & 7 & $1997-1998$ & 332 & 295 & 911 \\
\hline 101 & Little Muddy River below Cow Creek near Williston, N. Dak. & 9 & 1999 & 628 & 158 & 661 \\
\hline 112 & Little Missouri River at Medora, N. Dak. & 88 & 1993-2007 & 325 & 87 & 1,330 \\
\hline 113 & Beaver Creek near Trotters, N. Dak. & 9 & 1999 & 396 & 289 & 484 \\
\hline 114 & Little Missouri River near Watford City, N. Dak. & 81 & 1994-2007 & 339 & 121 & 875 \\
\hline 120 & Knife River near Golden Valley, N. Dak. & 60 & 1997-2007 & 588 & 172 & 1,420 \\
\hline 140 & Square Butte Creek below Center, N. Dak. & 4 & 1993 & 491 & 404 & 525 \\
\hline 142 & Missouri River at Bismarck, N. Dak. & 1 & 1994 & 192 & 192 & 192 \\
\hline 146 & Heart River at Dickinson, N. Dak. & 5 & 1993 & 378 & 221 & 890 \\
\hline 148 & Green River near Gladstone, N. Dak. & 4 & 1993 & 416 & 290 & 852 \\
\hline 149 & Heart River near Richardton, N. Dak. & 76 & 1994-2007 & 362 & 102 & 827 \\
\hline 152 & Big Muddy Creek near Almont, N. Dak. & 3 & 1993 & 665 & 335 & 703 \\
\hline 156 & Heart River near Mandan, N. Dak. & 81 & 1994-2007 & 377 & 159 & 850 \\
\hline 158 & Apple Creek near Menoken, N. Dak. & 4 & 1993 & 343 & 147 & 425 \\
\hline 164 & Cannonball River near Raleigh, N. Dak. & 76 & 1993-2007 & 340 & 121 & 1,310 \\
\hline 180 & Pipestem Creek near Pingree, N. Dak. & 17 & 1994-1999 & 391 & 228 & 571 \\
\hline 181 & Pipestem Creek near Buchanan, N. Dak. & 5 & 1993 & 299 & 59 & 448 \\
\hline 182 & James River at Jamestown, N. Dak. & 75 & $1997-2007$ & 347 & 194 & 515 \\
\hline 183 & James River at Lamoure, N. Dak. & 73 & 1996-2007 & 303 & 134 & 650 \\
\hline 184 & Bear Creek near Oakes, N. Dak. & 9 & 1998-1999 & 379 & 160 & 451 \\
\hline 185 & James River at Oakes, N. Dak. & 3 & 1993 & 272 & 121 & 284 \\
\hline 186 & James River at N. Dak./S. Dak. State line & 9 & 1998-1999 & 258 & 227 & 335 \\
\hline \multicolumn{7}{|c|}{ Alkalinity, total, as $\mathrm{CaCO}_{3^{\prime}}$ in $\mathrm{mg} / \mathrm{L}$} \\
\hline 1 & Bois De Sioux River near Doran, Minn. & 40 & 2000-2007 & 216 & 75 & 772 \\
\hline 2 & Red River of the North at Wahpeton, N. Dak. & 17 & 1993-1996 & 209 & 141 & 362 \\
\hline 3 & Red River of the North near Wahpeton, N. Dak. & 4 & 1993-2006 & 233 & 188 & 442 \\
\hline 4 & Red River of the North at Brushville, Minn. & 61 & 1993-2007 & 208 & 102 & 298 \\
\hline 10 & Wild Rice River near Abercrombie, N. Dak. & 58 & 1993-2007 & 307 & 101 & 687 \\
\hline 11 & Red River of the North at Fargo, N. Dak. & 16 & 1994-1996 & 212 & 146 & 254 \\
\hline 13 & Red River of the North at Harwood, N. Dak. & 53 & $2000-2007$ & 211 & 107 & 285 \\
\hline
\end{tabular}


Table 1-1. Summary statistics for major ion constituents at selected sites in North Dakota from 1970 through 2008.—Continued $\left[\mu \mathrm{g} / \mathrm{L}\right.$, micrograms per liter; <, less than; --, not available; mg/L, milligrams per liter; $\mathrm{CO}_{3}$, carbonate; $\mathrm{HCO}_{3}$, bicarbonate; $\mathrm{CaCO}$, calcium carbonate $]$

\begin{tabular}{|c|c|c|c|c|c|c|}
\hline $\begin{array}{c}\text { Site } \\
\text { identification } \\
\text { number } \\
\text { (table 1) }\end{array}$ & Site name & $\begin{array}{c}\text { Number of } \\
\text { samples }\end{array}$ & $\begin{array}{l}\text { Period of } \\
\text { record }\end{array}$ & Median & Minimum & Maximum \\
\hline \multicolumn{7}{|c|}{ Alkalinity, total, as $\mathrm{CaCO}_{3}$, in $\mathrm{mg} / \mathrm{L}$-Continued } \\
\hline 14 & Red River of the North near Harwood, N. Dak. & 26 & 1993-1996 & 214 & 162 & 265 \\
\hline 17 & Sheyenne River at Warwick, N. Dak. & 48 & 1996-2006 & 420 & 184 & 581 \\
\hline 32 & Sheyenne River near Cooperstown, N. Dak. & 80 & 1994-2007 & 371 & 130 & 495 \\
\hline 33 & Baldhill Creek near Dazey, N. Dak. & 3 & 1993-1996 & 339 & 246 & 359 \\
\hline 34 & Sheyenne River below Baldhill Dam, N. Dak. & 73 & 1994-2007 & 299 & 148 & 506 \\
\hline 35 & Sheyenne River at Valley City, N. Dak. & 4 & 1993 & 224 & 203 & 275 \\
\hline 45 & Maple River below Mapleton, N. Dak. & 47 & 1997-2007 & 301 & 116 & 893 \\
\hline 46 & Sheyenne River at Harwood, N. Dak. & 10 & 1993 & 284 & 209 & 337 \\
\hline 49 & Lower Branch Rush River near Prosper, N. Dak. & 1 & 1993 & 351 & 351 & 351 \\
\hline 51 & Elm River near Kelso, N. Dak. & 3 & 1993 & 290 & 263 & 348 \\
\hline 55 & Goose River at Hillsboro, N. Dak. & 76 & 1994-2007 & 292 & 141 & 498 \\
\hline 56 & Red River of the North at Grand Forks, N. Dak. & 46 & 1997-2006 & 222 & 130 & 295 \\
\hline 58 & Turtle River at Manvel, N. Dak. & 38 & 1993-2006 & 260 & 110 & 361 \\
\hline 62 & Forest River near Minto, N. Dak. & 47 & 1994-2006 & 243 & 113 & 396 \\
\hline 67 & Park River at Grafton, N. Dak. & 40 & 1994-2006 & 254 & 98 & 528 \\
\hline 83 & Souris River near Foxholm, N. Dak. & 11 & 1997-1998 & 246 & 123 & 478 \\
\hline 84 & Des Lacs River at Foxholm, N. Dak. & 97 & 1993-2007 & 393 & 119 & 712 \\
\hline 85 & Souris River above Minot, N. Dak. & 75 & 1994-2007 & 319 & 133 & 1,260 \\
\hline 87 & Souris River near Verendrye, N. Dak. & 76 & 1997-2007 & 318 & 134 & 536 \\
\hline 88 & Wintering River near Karlsruhe, N. Dak. & 11 & 1997-1998 & 324 & 205 & 565 \\
\hline 89 & Souris River near Bantry, N. Dak. & 10 & 1997-1998 & 346 & 192 & 476 \\
\hline 90 & Willow Creek near Willow City, N. Dak. & 6 & 1997 & 359 & 234 & 470 \\
\hline 92 & Deep River near Upham, N. Dak. & 7 & 1997 & 256 & 134 & 294 \\
\hline 98 & Souris River near Westhope, N. Dak. & 7 & 1997-1998 & 346 & 295 & 746 \\
\hline 101 & Little Muddy River below Cow Creek near Williston, N. Dak. & 9 & 1999 & 554 & 129 & 580 \\
\hline 110 & Little Missouri River at Marmarth, N. Dak. & 8 & 1999 & 230 & 121 & 399 \\
\hline 112 & Little Missouri River at Medora, N. Dak. & 88 & 1993-2007 & 287 & 71 & 1,090 \\
\hline 113 & Beaver Creek near Trotters, N. Dak. & 9 & 1999 & 361 & 237 & 431 \\
\hline 114 & Little Missouri River near Watford City, N. Dak. & 81 & 1994-2007 & 299 & 99 & 717 \\
\hline
\end{tabular}


Table 1-1. Summary statistics for major ion constituents at selected sites in North Dakota from 1970 through 2008. —Continued $\left[\mu \mathrm{g} / \mathrm{L}\right.$, micrograms per liter; <, less than; --, not available; $\mathrm{mg} / \mathrm{L}$, milligrams per liter; $\mathrm{CO}_{3}$, carbonate; $\mathrm{HCO}_{3}$, bicarbonate; $\mathrm{CaCO}$, calcium carbonate $]$

\begin{tabular}{|c|c|c|c|c|c|c|}
\hline $\begin{array}{c}\text { Site } \\
\text { identification } \\
\text { number } \\
\text { (table 1) }\end{array}$ & Site name & $\begin{array}{l}\text { Number of } \\
\text { samples }\end{array}$ & $\begin{array}{l}\text { Period of } \\
\text { record }\end{array}$ & Median & Minimum & Maximum \\
\hline \multicolumn{7}{|c|}{ Alkalinity, total, as $\mathrm{CaCO}_{3^{\prime}}$ in $\mathrm{mg} / \mathrm{L}$-Continued } \\
\hline 120 & Knife River near Golden Valley, N. Dak. & 60 & $1997-2007$ & 521 & 141 & 1,160 \\
\hline 123 & Spring Creek below Lake Ilo near Dunn Center, N. Dak. & 6 & 1993 & 526 & 256 & 604 \\
\hline 127 & Knife River at Hazen, N. Dak. & 94 & $1993-2007$ & 438 & 113 & 723 \\
\hline 140 & Square Butte Creek below Center, N. Dak. & 4 & 1993 & 402 & 331 & 430 \\
\hline 142 & Missouri River at Bismarck, N. Dak. & 1 & 1994 & 157 & 157 & 157 \\
\hline 146 & Heart River at Dickinson, N. Dak. & 5 & 1993 & 310 & 181 & 729 \\
\hline 156 & Heart River near Mandan, N. Dak. & 81 & 1994-2007 & 330 & 130 & 697 \\
\hline 158 & Apple Creek near Menoken, N. Dak. & 4 & 1993 & 281 & 120 & 348 \\
\hline 164 & Cannonball River near Raleigh, N. Dak. & 76 & 1993-2007 & 298 & 99 & 1,070 \\
\hline 169 & Cedar Creek near Raleigh, N. Dak. & 68 & 1993-2007 & 310 & 99 & 710 \\
\hline 170 & Cannonball River at Breien, N. Dak. & 74 & 1994-2007 & 319 & 11 & 714 \\
\hline 171 & Beaver Creek near Linton, N. Dak. & 4 & 1993 & 238 & 150 & 305 \\
\hline 175 & James River near Manfred, N. Dak. & 6 & 1998 & 400 & 224 & 449 \\
\hline 176 & James River near Grace City, N. Dak. & 60 & $1997-2007$ & 336 & 148 & 892 \\
\hline 177 & James River above Arrowwood Lake near Kensal, N. Dak. & 9 & 1998-1999 & 279 & 143 & 580 \\
\hline 186 & James River at N. Dak./S. Dak. State line & 9 & 1998-1999 & 237 & 206 & 297 \\
\hline \multicolumn{7}{|c|}{ Alkalinity, incremental titration, dissolved as $\mathrm{CaCO}_{3^{\prime}}$ in $\mathrm{mg} / \mathrm{L}$} \\
\hline 2 & Red River of the North at Wahpeton, N. Dak. & 1 & 1991 & 238 & 238 & 238 \\
\hline 6 & Red River of the North at Hickson, N. Dak. & 1 & 1991 & 208 & 208 & 208 \\
\hline 10 & Wild Rice River near Abercrombie, N. Dak. & 1 & 1994 & 295 & 295 & 295 \\
\hline 11 & Red River of the North at Fargo, N. Dak. & 1 & 1991 & 248 & 248 & 248 \\
\hline 18 & Sheyenne River near Warwick, N. Dak. & 4 & 1993 & 309 & 256 & 351 \\
\hline 32 & Sheyenne River near Cooperstown, N. Dak. & 10 & 1995 & 206 & 81 & 416 \\
\hline 33 & Baldhill Creek near Dazey, N. Dak. & 10 & 1995 & 168 & 60 & 337 \\
\hline 34 & Sheyenne River below Baldhill Dam, N. Dak. & 10 & 1995 & 219 & 139 & 371 \\
\hline 36 & Sheyenne River at Lisbon, N. Dak. & 24 & 1993-1996 & 256 & 139 & 367 \\
\hline 37 & Sheyenne River near Kindred, N. Dak. & 47 & $1988-1996$ & 262 & 140 & 358 \\
\hline 41 & Sheyenne River at West Fargo, N. Dak. & 1 & 1991 & 262 & 262 & 262 \\
\hline 52 & Red River of the North at Halstad, Minn. & 44 & 1988-1995 & 227 & 100 & 355 \\
\hline 53 & Beaver Creek near Finley, N. Dak. & 17 & 1989-1996 & 253 & 98 & 598 \\
\hline
\end{tabular}


Table 1-1. Summary statistics for major ion constituents at selected sites in North Dakota from 1970 through 2008.—Continued $\left[\mu \mathrm{g} / \mathrm{L}\right.$, micrograms per liter; <, less than; --, not available; mg/L, milligrams per liter; $\mathrm{CO}_{3}$, carbonate; $\mathrm{HCO}_{3}$, bicarbonate; $\mathrm{CaCO}$, calcium carbonate $]$

\begin{tabular}{|c|c|c|c|c|c|c|}
\hline $\begin{array}{c}\text { Site } \\
\text { identification } \\
\text { number } \\
\text { (table 1) }\end{array}$ & Site name & $\begin{array}{c}\text { Number of } \\
\text { samples }\end{array}$ & $\begin{array}{l}\text { Period of } \\
\text { record }\end{array}$ & Median & Minimum & Maximum \\
\hline \multicolumn{7}{|c|}{ Alkalinity, incremental titration, dissolved as $\mathrm{CaCO}_{3}$, in $\mathrm{mg} / \mathrm{L}$-Continued } \\
\hline 55 & Goose River at Hillsboro, N. Dak. & 1 & 1994 & 306 & 306 & 306 \\
\hline 56 & Red River of the North at Grand Forks, N. Dak. & 25 & 1991-1995 & 207 & 114 & 306 \\
\hline 59 & Red River of the North at Oslo, Minn. & 1 & 1991 & 314 & 314 & 314 \\
\hline 67 & Park River at Grafton, N. Dak. & 1 & 1991 & 286 & 286 & 286 \\
\hline 68 & Red River of the North at Drayton, N. Dak. & 2 & 1991 & 312 & 303 & 320 \\
\hline 74 & Pembina River at Walhalla, N. Dak. & 27 & 1993-1995 & 212 & 86 & 359 \\
\hline 89 & Souris River near Bantry, N. Dak. & 1 & 1992 & 396 & 396 & 396 \\
\hline 98 & Souris River near Westhope, N. Dak. & 17 & $1988-1993$ & 356 & 124 & 1090 \\
\hline 106 & Bear Den Creek near Mandaree, N. Dak. & 38 & 1988-1995 & 733 & 306 & 1,080 \\
\hline 114 & Little Missouri River near Watford City, N. Dak. & 22 & 1989-1994 & 279 & 98 & 740 \\
\hline 115 & Missouri River at Garrison Dam, N. Dak. & 93 & 1988-2007 & 156 & 119 & 196 \\
\hline 122 & Brush Creek near Beulah, N. Dak. & 9 & 1989-1990 & 492 & 260 & 568 \\
\hline 127 & Knife River at Hazen, N. Dak. & 19 & 1988-1993 & 488 & 311 & 720 \\
\hline 156 & Heart River near Mandan, N. Dak. & 28 & 1988-1993 & 433 & 256 & 844 \\
\hline 157 & Long Lake Creek above Long Lake near Moffit, N. Dak. & 1 & 1989 & 70 & 70 & 70 \\
\hline 9 & Antelope Creek at Dwight, N. Dak. & 13 & 2001-2008 & 199 & 111 & 361 \\
\hline 10 & Wild Rice River near Abercrombie, N. Dak. & 62 & 1980-2008 & 279 & 42 & 750 \\
\hline 11 & Red River of the North at Fargo, N. Dak. & 76 & 1983-2008 & 203 & 70 & 265 \\
\hline 12 & Red River of North below Fargo, N. Dak. & 46 & 1980-1986 & 209 & 105 & 270 \\
\hline 14 & Red River of the North near Harwood, N. Dak. & 33 & 1997-2007 & 229 & 92 & 261 \\
\hline 15 & Sheyenne River above Harvey, N. Dak. & 140 & 1980-2008 & 498 & 96 & 834 \\
\hline 18 & Sheyenne River near Warwick, N. Dak. & 57 & 1983-2008 & 300 & 99 & 548 \\
\hline 19 & Mauvais Coulee Tributary No. 3 near Cando, N. Dak. & 38 & 1987-2008 & 154 & 66 & 344 \\
\hline 20 & Mauvais Coulee near Cando, N. Dak. & 95 & 1983-2008 & 224 & 63 & 430 \\
\hline 21 & Edmore Coulee near Edmore, N. Dak. & 95 & 1983-2008 & 188 & 63 & 697 \\
\hline 22 & Edmore Coulee Tributary near Webster, N. Dak. & 38 & 1987-2008 & 178 & 76 & 402 \\
\hline 23 & Webster Coulee at Webster, N. Dak. & 2 & 1983-1984 & 215 & 210 & 220 \\
\hline 24 & Starkweather Coulee near Webster, N. Dak. & 94 & 1983-2008 & 158 & 34 & 336 \\
\hline 25 & Big Coulee below Churchs Ferry, N. Dak. & 23 & 1998-2008 & 235 & 150 & 325 \\
\hline 26 & Little Coulee near Leeds, N. Dak. & 16 & 1998-2008 & 220 & 105 & 335 \\
\hline
\end{tabular}


Table 1-1. Summary statistics for major ion constituents at selected sites in North Dakota from 1970 through 2008. —Continued $\left[\mu \mathrm{g} / \mathrm{L}\right.$, micrograms per liter; <, less than; --, not available; $\mathrm{mg} / \mathrm{L}$, milligrams per liter; $\mathrm{CO}_{3}$, carbonate; $\mathrm{HCO}_{3}$, bicarbonate; $\mathrm{CaCO}$, calcium carbonate $]$

\begin{tabular}{|c|c|c|c|c|c|c|}
\hline $\begin{array}{c}\text { Site } \\
\text { identification } \\
\text { number } \\
\text { (table 1) }\end{array}$ & Site name & $\begin{array}{l}\text { Number of } \\
\text { samples }\end{array}$ & $\begin{array}{l}\text { Period of } \\
\text { record }\end{array}$ & Median & Minimum & Maximum \\
\hline \multicolumn{7}{|c|}{ Acid neutralizing capacity, total, in mg/L-Continued } \\
\hline 27 & Little Coulee near Brinsmade, N. Dak. & 19 & $1980-1998$ & 253 & 140 & 380 \\
\hline 28 & Big Coulee near Churchs Ferry, N. Dak. & 62 & 1983-1997 & 201 & 110 & 463 \\
\hline 29 & Big Coulee at Graham Island inlet near Fort Totten, N. Dak. & 12 & $1980-1986$ & 374 & 322 & 457 \\
\hline 30 & Channel A near Penn, N. Dak. & 87 & $1984-2008$ & 194 & 79 & 473 \\
\hline 31 & Devils Lake Outlet to Stump Lake near Lakota, N. Dak. & 12 & $2002-2007$ & 389 & 167 & 647 \\
\hline 32 & Sheyenne River near Cooperstown, N. Dak. & 91 & $1980-2008$ & 320 & 80 & 570 \\
\hline 33 & Baldhill Creek near Dazey, N. Dak. & 64 & $1980-2008$ & 253 & 63 & 480 \\
\hline 34 & Sheyenne River below Baldhill Dam, N. Dak. & 79 & $1980-2008$ & 270 & 130 & 415 \\
\hline 35 & Sheyenne River at Valley City, N. Dak. & 18 & $1987-2005$ & 260 & 120 & 391 \\
\hline 36 & Sheyenne River at Lisbon, N. Dak. & 114 & 1980-2008 & 260 & 127 & 543 \\
\hline 37 & Sheyenne River near Kindred, N. Dak. & 126 & 1980-2008 & 267 & 137 & 385 \\
\hline 38 & $\begin{array}{l}\text { Sheyenne River above Sheyenne River Diversion near Horace, } \\
\text { N. Dak. }\end{array}$ & 43 & $1993-2008$ & 289 & 127 & 389 \\
\hline 39 & Sheyenne River near Horace, N. Dak. & 8 & 1982-1992 & 195 & 110 & 290 \\
\hline 40 & Sheyenne River Diversion at West Fargo, N. Dak. & 13 & 1994-2007 & 237 & 126 & 360 \\
\hline 41 & Sheyenne River at West Fargo, N. Dak. & 31 & $1983-2008$ & 266 & 120 & 335 \\
\hline 42 & Maple River near Hope, N. Dak. & 33 & $1983-2008$ & 185 & 64 & 510 \\
\hline 43 & Maple River near Enderlin, N. Dak. & 45 & $1982-2008$ & 272 & 83 & 390 \\
\hline 44 & Maple River near Mapleton, N. Dak. & 17 & 1995-2008 & 258 & 97 & 354 \\
\hline 45 & Maple River below Mapleton, N. Dak. & 28 & 1995-2008 & 239 & 94 & 368 \\
\hline 46 & Sheyenne River at Harwood, N. Dak. & 26 & $1997-2005$ & 265 & 151 & 371 \\
\hline 47 & Rush River at Amenia, N. Dak. & 44 & $1983-2008$ & 254 & 77 & 410 \\
\hline 48 & Rush River near Prosper, N. Dak. & 4 & 1983-1987 & 130 & 80 & 400 \\
\hline 49 & Lower Branch Rush River near Prosper, N. Dak. & 4 & 1983-1987 & 97 & 77 & 120 \\
\hline 50 & Sheyenne River near Harwood, N. Dak. & 1 & 1996 & 141 & 141 & 141 \\
\hline 51 & Elm River near Kelso, N. Dak. & 3 & 1983-1987 & 100 & 80 & 340 \\
\hline 52 & Red River of the North at Halstad, Minn. & 139 & 1980-2008 & 230 & 104 & 350 \\
\hline 53 & Beaver Creek near Finley, N. Dak. & 60 & $1981-2003$ & 262 & 73 & 633 \\
\hline 54 & Goose River near Portland, N. Dak. & 4 & 1983-1988 & 120 & 100 & 177 \\
\hline 55 & Goose River at Hillsboro, N. Dak. & 57 & $1983-2008$ & 242 & 98 & 414 \\
\hline 56 & Red River of the North at Grand Forks, N. Dak. & 100 & $1983-2008$ & 199 & 95 & 310 \\
\hline 57 & Turtle River at Turtle River State Park near Arvilla, N. Dak. & 77 & $1991-2008$ & 259 & 101 & 334 \\
\hline 58 & Turtle River at Manvel, N. Dak. & 47 & $1981-2008$ & 218 & 100 & 317 \\
\hline 59 & Red River of the North at Oslo, Minn. & 20 & $1987-2005$ & 159 & 99 & 236 \\
\hline 60 & Middle Branch Forest River near Whitman, N. Dak. & 8 & 1983-1990 & 178 & 90 & 300 \\
\hline 61 & Forest River near Fordville, N. Dak. & 47 & 1983-2008 & 220 & 106 & 290 \\
\hline 62 & Forest River near Minto, N. Dak. & 72 & 1983-2008 & 239 & 98 & 330 \\
\hline 63 & South Branch Park River below Homme Dam, N. Dak. & 19 & 1981-1994 & 190 & 93 & 254 \\
\hline 64 & Middle Branch Park River near Union, N. Dak. & 4 & $1983-1984$ & 210 & 140 & 330 \\
\hline 66 & Cart Creek at Mountain, N. Dak. & 3 & 1983-1984 & 240 & 150 & 270 \\
\hline
\end{tabular}


Table 1-1. Summary statistics for major ion constituents at selected sites in North Dakota from 1970 through 2008.—Continued $\left[\mu \mathrm{g} / \mathrm{L}\right.$, micrograms per liter; <, less than; --, not available; mg/L, milligrams per liter; $\mathrm{CO}_{3}$, carbonate; $\mathrm{HCO}_{3}$, bicarbonate; $\mathrm{CaCO}$, calcium carbonate $]$

\begin{tabular}{|c|c|c|c|c|c|c|}
\hline $\begin{array}{c}\text { Site } \\
\text { identification } \\
\text { number } \\
\text { (table 1) }\end{array}$ & Site name & $\begin{array}{c}\text { Number of } \\
\text { samples }\end{array}$ & $\begin{array}{l}\text { Period of } \\
\text { record }\end{array}$ & Median & Minimum & Maximum \\
\hline \multicolumn{7}{|c|}{ Acid neutralizing capacity, total, in $\mathrm{mg} / \mathrm{L}$-Continued } \\
\hline 67 & Park River at Grafton, N. Dak. & 70 & $1983-2008$ & 245 & 92 & 356 \\
\hline 68 & Red River of the North at Drayton, N. Dak. & 50 & $1983-2008$ & 200 & 95 & 310 \\
\hline 70 & Hidden Island Coulee near Hansboro, N. Dak. & 16 & $1983-1995$ & 157 & 110 & 420 \\
\hline 71 & Cypress Creek near Sarles, N. Dak. & 7 & $1983-1988$ & 170 & 90 & 300 \\
\hline 73 & Little South Pembina River near Walhalla, N. Dak. & 16 & $2001-2008$ & 189 & 108 & 250 \\
\hline 74 & Pembina River at Walhalla, N. Dak. & 79 & $1980-2008$ & 220 & 26 & 349 \\
\hline 79 & Red River of the North at Emerson, Manitoba & 111 & $1980-2004$ & 214 & 124 & 360 \\
\hline 80 & Long Creek near Noonan, N. Dak. & 41 & $1982-2008$ & 230 & 46 & 470 \\
\hline 82 & Souris River near Sherwood, N. Dak. & 213 & $1980-2008$ & 302 & 65 & 922 \\
\hline 83 & Souris River near Foxholm, N. Dak. & 90 & $1982-2008$ & 315 & 159 & 639 \\
\hline 84 & Des Lacs River at Foxholm, N. Dak. & 74 & $1983-2008$ & 371 & 50 & 855 \\
\hline 85 & Souris River above Minot, N. Dak. & 105 & $1981-2008$ & 308 & 76 & 826 \\
\hline 86 & Bonnes Creek near Velva, N. Dak. & 18 & $1987-2005$ & 200 & 82 & 516 \\
\hline 87 & Souris River near Verendrye, N. Dak. & 180 & $1980-2008$ & 339 & 64 & 594 \\
\hline 88 & Wintering River near Karlsruhe, N. Dak. & 78 & $1980-2008$ & 311 & 88 & 612 \\
\hline 96 & Deep River below Cut Bank Creek near Upham, N. Dak. & 13 & 1986-1989 & 284 & 84 & 436 \\
\hline 97 & Boundary Creek near Landa, N. Dak. & 31 & $1986-2000$ & 293 & 64 & 500 \\
\hline 98 & Souris River near Westhope, N. Dak. & 81 & $1980-2008$ & 340 & 126 & 1,170 \\
\hline 100 & Missouri River near Williston, N. Dak. & 20 & 1980-1992 & 160 & 98 & 200 \\
\hline 101 & Little Muddy River below Cow Creek near Williston, N. Dak. & 46 & $1983-2008$ & 530 & 76 & 660 \\
\hline 102 & Stony Creek near Williston, N. Dak. & 1 & 1981 & 57 & 57 & 57 \\
\hline 106 & Bear Den Creek near Mandaree, N. Dak. & 116 & $1980-2008$ & 697 & 75 & 1,060 \\
\hline 108 & East Fork Shell Creek near Parshall, N. Dak. & 58 & $1991-2008$ & 670 & 53 & 909 \\
\hline 109 & Deepwater Creek near Mandaree, N. Dak. & 57 & $1991-2008$ & 483 & 56 & 802 \\
\hline 110 & Little Missouri River at Marmarth, N. Dak. & 50 & $1983-2008$ & 254 & 65 & 649 \\
\hline 111 & Deep Creek near Amidon, N. Dak. & 3 & 1983 & 490 & 258 & 549 \\
\hline 112 & Little Missouri River at Medora, N. Dak. & 16 & $2001-2008$ & 253 & 80 & 427 \\
\hline 113 & Beaver Creek near Trotters, N. Dak. & 39 & $1983-2008$ & 310 & 96 & 431 \\
\hline 114 & Little Missouri River near Watford City, N. Dak. & 84 & $1980-2008$ & 277 & 97 & 713 \\
\hline 115 & Missouri River at Garrison Dam, N. Dak. & 97 & $1980-2000$ & 160 & 106 & 439 \\
\hline
\end{tabular}


Table 1-1. Summary statistics for major ion constituents at selected sites in North Dakota from 1970 through 2008. —Continued $\left[\mu \mathrm{g} / \mathrm{L}\right.$, micrograms per liter; <, less than; --, not available; $\mathrm{mg} / \mathrm{L}$, milligrams per liter; $\mathrm{CO}_{3}$, carbonate; $\mathrm{HCO}_{3}$, bicarbonate; $\mathrm{CaCO}$, calcium carbonate $]$

\begin{tabular}{|c|c|c|c|c|c|c|}
\hline $\begin{array}{c}\text { Site } \\
\text { identification } \\
\text { number } \\
\text { (table 1) }\end{array}$ & Site name & $\begin{array}{c}\text { Number of } \\
\text { samples }\end{array}$ & $\begin{array}{l}\text { Period of } \\
\text { record }\end{array}$ & Median & Minimum & Maximum \\
\hline \multicolumn{7}{|c|}{ Acid neutralizing capacity, total, in $\mathrm{mg} / \mathrm{L}$-Continued } \\
\hline 116 & Knife River at Manning, N. Dak. & 57 & 1980-2008 & 420 & 46 & 910 \\
\hline 119 & Elm Creek near Golden Valley, N. Dak. & 11 & 1981-1995 & 180 & 73 & 588 \\
\hline 120 & Knife River near Golden Valley, N. Dak. & 48 & 1983-2008 & 394 & 80 & 690 \\
\hline 121 & Coyote Creek near Zap, N. Dak. & 2 & 1981-1983 & 510 & 390 & 630 \\
\hline 122 & Brush Creek near Beulah, N. Dak. & 29 & $1983-1990$ & 495 & 204 & 601 \\
\hline 124 & Spring Creek near Halliday, N. Dak. & 1 & 1981 & 510 & 510 & 510 \\
\hline 125 & Spring Creek at Zap, N. Dak. & 69 & 1980-2008 & 357 & 67 & 610 \\
\hline 126 & West Branch Otter Creek near Beulah, N. Dak. & 10 & 1983-1995 & 172 & 63 & 350 \\
\hline 127 & Knife River at Hazen, N. Dak. & 87 & 1980-2008 & 430 & 76 & 712 \\
\hline 128 & Antelope Creek above Hazen, N. Dak. & 16 & $1982-1985$ & 282 & 68 & 503 \\
\hline 129 & West Branch Antelope Creek No. 4 near Zap, N. Dak. & 21 & $1977-1985$ & 154 & 26 & 371 \\
\hline 130 & West Branch Antelope Creek near Hazen, N. Dak. & 1 & 1983 & 536 & 536 & 536 \\
\hline 132 & Alderin Creek near Fort Clark, N. Dak. & 6 & $1982-1983$ & 319 & 142 & 485 \\
\hline 133 & Coal Lake Coulee near Hensler, N. Dak. & 21 & $1982-1988$ & 347 & 56 & 568 \\
\hline 134 & Buffalo Creek near Washburn, N. Dak. & 2 & 1983 & 850 & 759 & 941 \\
\hline 135 & Turtle Creek above Washburn, N. Dak. & 99 & 1987-2003 & 567 & 112 & 1,136 \\
\hline 136 & Painted Woods Creek near Wilton, N. Dak. & 131 & $1982-2003$ & 277 & 65 & 574 \\
\hline 140 & Square Butte Creek below Center, N. Dak. & 48 & 1983-2008 & 408 & 129 & 446 \\
\hline 141 & Burnt Creek near Bismarck, N. Dak. & 41 & 1983-2008 & 337 & 54 & 460 \\
\hline 142 & Missouri River at Bismarck, N. Dak. & 55 & 1986-2008 & 164 & 118 & 200 \\
\hline 143 & South Branch Heart River near South Heart, N. Dak. & 14 & $1981-1996$ & 133 & 18 & 270 \\
\hline 144 & North Creek near South Heart, N. Dak. & 8 & 1995-1996 & 156 & 60 & 814 \\
\hline 145 & Heart River near South Heart, N. Dak. & 23 & $1981-2005$ & 270 & 84 & 910 \\
\hline 146 & Heart River at Dickinson, N. Dak. & 17 & 1986-1994 & 380 & 185 & 499 \\
\hline 147 & Green River near New Hradec, N. Dak. & 43 & 1983-2008 & 244 & 43 & 460 \\
\hline 149 & Heart River near Richardton, N. Dak. & 48 & 1983-2008 & 267 & 86 & 440 \\
\hline 150 & Heart River above Lake Tschida near Glen Ullin, N. Dak. & 40 & 1989-2008 & 270 & 92 & 600 \\
\hline 151 & Antelope Creek near Carson, N. Dak. & 18 & 1999-2008 & 275 & 152 & 352 \\
\hline 152 & Big Muddy Creek near Almont, N. Dak. & 36 & $1991-2008$ & 470 & 91 & 873 \\
\hline 153 & Heart River near Lark, N. Dak. & 22 & $1983-1995$ & 290 & 100 & 370 \\
\hline 154 & Heart River at Stark Bridge near Judson, N. Dak. & 41 & 1988-2008 & 304 & 100 & 417 \\
\hline 155 & Sweetbriar Creek near Judson, N. Dak. & 13 & $2002-2008$ & 354 & 118 & 577 \\
\hline 156 & Heart River near Mandan, N. Dak. & 100 & 1980-2008 & 322 & 99 & 771 \\
\hline 157 & Long Lake Creek above Long Lake near Moffit, N. Dak. & 33 & 1988-2004 & 360 & 66 & 547 \\
\hline 158 & Apple Creek near Menoken, N. Dak. & 55 & 1980-2008 & 420 & 49 & 722 \\
\hline 159 & Missouri River near Schmidt, N. Dak. & 11 & $1980-1981$ & 150 & 150 & 170 \\
\hline 161 & Coal Bank Creek near Havelock, N. Dak. & 5 & $1981-1983$ & 270 & 196 & 379 \\
\hline 162 & Cannonball River at Regent, N. Dak. & 58 & 1980-2008 & 350 & 91 & 510 \\
\hline 164 & Cannonball River near Raleigh, N. Dak. & 15 & $2001-2008$ & 264 & 97 & 320 \\
\hline
\end{tabular}


Table 1-1. Summary statistics for major ion constituents at selected sites in North Dakota from 1970 through 2008.—Continued $\left[\mu \mathrm{g} / \mathrm{L}\right.$, micrograms per liter; <, less than; --, not available; mg/L, milligrams per liter; $\mathrm{CO}_{3}$, carbonate; $\mathrm{HCO}_{3}$, bicarbonate; $\mathrm{CaCO}$, calcium carbonate $]$

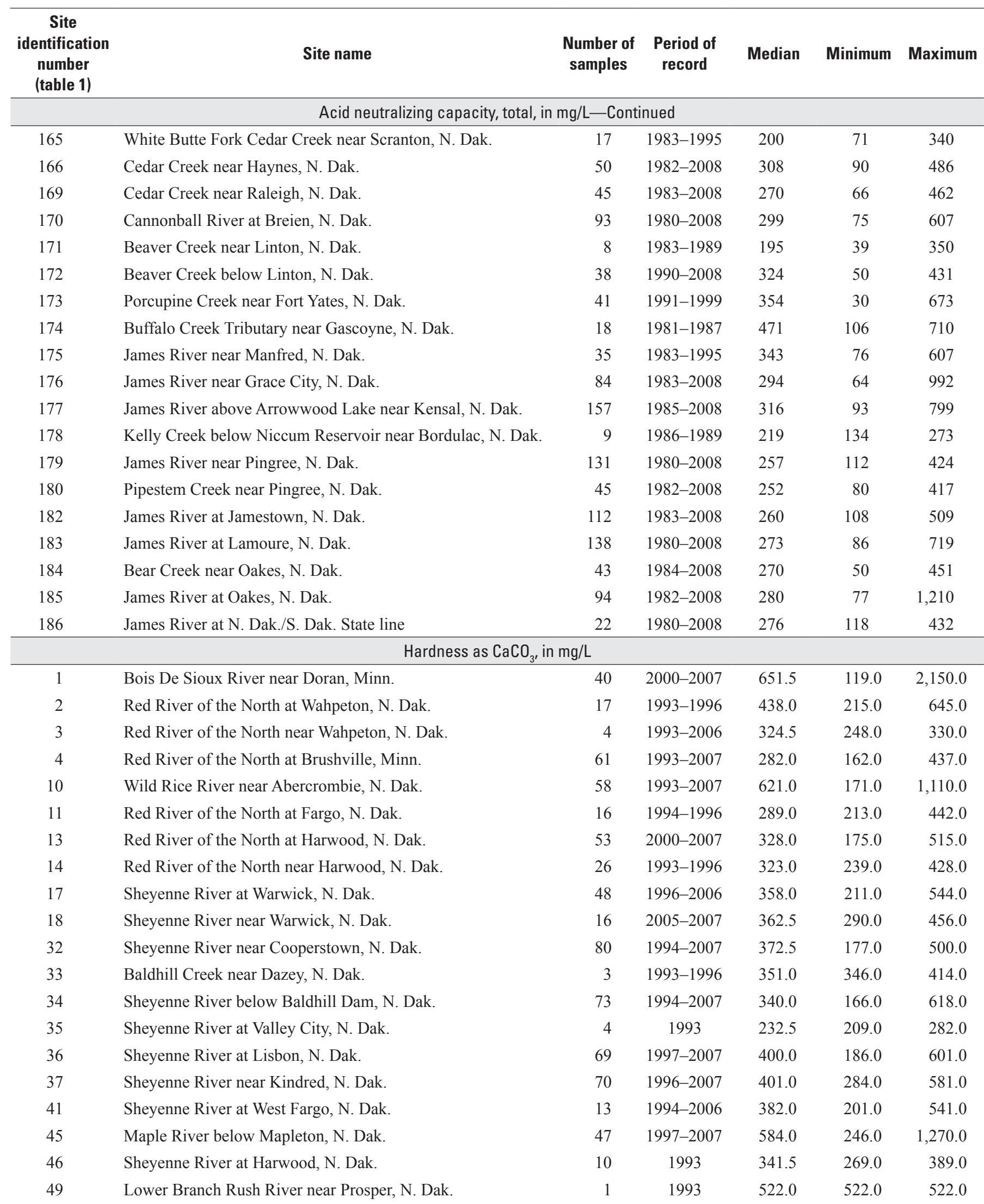


Table 1-1. Summary statistics for major ion constituents at selected sites in North Dakota from 1970 through 2008. —Continued $\left[\mu \mathrm{g} / \mathrm{L}\right.$, micrograms per liter; <, less than; --, not available; $\mathrm{mg} / \mathrm{L}$, milligrams per liter; $\mathrm{CO}_{3}$, carbonate; $\mathrm{HCO}_{3}$, bicarbonate; $\mathrm{CaCO}$, calcium carbonate $]$

\begin{tabular}{|c|c|c|c|c|c|c|}
\hline $\begin{array}{c}\text { Site } \\
\text { identification } \\
\text { number } \\
\text { (table 1) }\end{array}$ & Site name & $\begin{array}{c}\text { Number of } \\
\text { samples }\end{array}$ & $\begin{array}{l}\text { Period of } \\
\text { record }\end{array}$ & Median & Minimum & Maximum \\
\hline \multicolumn{7}{|c|}{ Hardness as $\mathrm{CaCO}_{3^{\prime}}$ in $\mathrm{mg} / \mathrm{L}$-Continued } \\
\hline 51 & Elm River near Kelso, N. Dak. & 3 & 1993 & 450.0 & 392.0 & 537.0 \\
\hline 55 & Goose River at Hillsboro, N. Dak. & 76 & 1994-2007 & 632.5 & 328.0 & $1,040.0$ \\
\hline 56 & Red River of the North at Grand Forks, N. Dak. & 46 & $1997-2006$ & 302.5 & 162.0 & 465.0 \\
\hline 58 & Turtle River at Manvel, N. Dak. & 38 & 1993-2006 & 646.0 & 304.0 & $1,110.0$ \\
\hline 62 & Forest River near Minto, N. Dak. & 47 & 1994-2006 & 413.0 & 242.0 & 688.0 \\
\hline 67 & Park River at Grafton, N. Dak. & 40 & 1994-2006 & 442.5 & 172.0 & $1,040.0$ \\
\hline 68 & Red River of the North at Drayton, N. Dak. & 15 & 1994-1996 & 319.0 & 214.0 & 422.0 \\
\hline 75 & Pembina River at Neche, N. Dak. & 55 & 1994-2006 & 376.0 & 162.0 & 589.0 \\
\hline 76 & Tongue River at Akra, N. Dak. & 1 & 1993 & 217.0 & 217.0 & 217.0 \\
\hline 77 & Red River of the North at Pembina, N. Dak., site 1 & 10 & 1993 & 310.0 & 222.0 & 345.0 \\
\hline 78 & Red River of the North at Pembina, N. Dak., site 2 & 52 & 1997-2006 & 314.0 & 173.0 & 526.0 \\
\hline 80 & Long Creek near Noonan, N. Dak. & 10 & 1997 & 429.5 & 211.0 & 510.0 \\
\hline 83 & Souris River near Foxholm, N. Dak. & 11 & $1997-1998$ & 246.0 & 124.0 & 460.0 \\
\hline 84 & Des Lacs River at Foxholm, N. Dak. & 97 & 1993-2007 & 464.0 & 167.0 & 844.0 \\
\hline 85 & Souris River above Minot, N. Dak. & 75 & 1994-2007 & 325.0 & 144.0 & $1,310.0$ \\
\hline 87 & Souris River near Verendrye, N. Dak. & 76 & 1997-2007 & 377.0 & 183.0 & 772.0 \\
\hline 88 & Wintering River near Karlsruhe, N. Dak. & 11 & $1997-1998$ & 269.0 & 138.0 & 313.0 \\
\hline 89 & Souris River near Bantry, N. Dak. & 10 & $1997-1998$ & 313.5 & 200.0 & 461.0 \\
\hline 90 & Willow Creek near Willow City, N. Dak. & 6 & 1997 & 381.5 & 287.0 & 443.0 \\
\hline 92 & Deep River near Upham, N. Dak. & 7 & 1997 & 326.0 & 188.0 & 400.0 \\
\hline 98 & Souris River near Westhope, N. Dak. & 7 & $1997-1998$ & 263.0 & 234.0 & 711.0 \\
\hline 101 & Little Muddy River below Cow Creek near Williston, N. Dak. & 9 & 1999 & 446.0 & 145.0 & 594.0 \\
\hline 110 & Little Missouri River at Marmarth, N. Dak. & 8 & 1999 & 335.5 & 253.0 & 392.0 \\
\hline 112 & Little Missouri River at Medora, N. Dak. & 88 & 1993-2007 & 275.5 & 44.0 & $2,610.0$ \\
\hline 113 & Beaver Creek near Trotters, N. Dak. & 9 & 1999 & 599.0 & 334.0 & 745.0 \\
\hline 114 & Little Missouri River near Watford City, N. Dak. & 81 & 1994-2007 & 294.0 & 28.0 & 817.0 \\
\hline 120 & Knife River near Golden Valley, N. Dak. & 60 & $1997-2007$ & 263.5 & 133.0 & 527.0 \\
\hline 123 & Spring Creek below Lake Ilo near Dunn Center, N. Dak. & 6 & 1993 & 174.0 & 83.0 & 200.0 \\
\hline 125 & Spring Creek at Zap, N. Dak. & 77 & 1993-2007 & 368.0 & 69.0 & 598.0 \\
\hline 127 & Knife River at Hazen, N. Dak. & 94 & 1993-2007 & 349.0 & 125.0 & 619.0 \\
\hline 140 & Square Butte Creek below Center, N. Dak. & 4 & 1993 & 410.0 & 369.0 & 453.0 \\
\hline 142 & Missouri River at Bismarck, N. Dak. & 1 & 1994 & 200.0 & 200.0 & 200.0 \\
\hline 146 & Heart River at Dickinson, N. Dak. & 5 & 1993 & 293.0 & 164.0 & 555.0 \\
\hline 148 & Green River near Gladstone, N. Dak. & 4 & 1993 & 339.0 & 183.0 & 512.0 \\
\hline 149 & Heart River near Richardton, N. Dak. & 76 & 1994-2007 & 369.5 & 100.0 & 647.0 \\
\hline 152 & Big Muddy Creek near Almont, N. Dak. & 3 & 1993 & 196.0 & 109.0 & 231.0 \\
\hline 156 & Heart River near Mandan, N. Dak. & 81 & 1994-2007 & 318.0 & 150.0 & 567.0 \\
\hline 158 & Apple Creek near Menoken, N. Dak. & 4 & 1993 & 203.0 & 102.0 & 221.0 \\
\hline 164 & Cannonball River near Raleigh, N. Dak. & 76 & 1993-2007 & 421.0 & 123.0 & $1,200.0$ \\
\hline 169 & Cedar Creek near Raleigh, N. Dak. & 68 & 1993-2007 & 362.0 & 98.0 & $1,080.0$ \\
\hline
\end{tabular}


Table 1-1. Summary statistics for major ion constituents at selected sites in North Dakota from 1970 through 2008.—Continued $\left[\mu \mathrm{g} / \mathrm{L}\right.$, micrograms per liter; <, less than; --, not available; mg/L, milligrams per liter; $\mathrm{CO}_{3}$, carbonate; $\mathrm{HCO}_{3}$, bicarbonate; $\mathrm{CaCO}$, calcium carbonate $]$

\begin{tabular}{|c|c|c|c|c|c|c|}
\hline $\begin{array}{c}\text { Site } \\
\text { identification } \\
\text { number } \\
\text { (table 1) }\end{array}$ & Site name & $\begin{array}{l}\text { Number of } \\
\text { samples }\end{array}$ & $\begin{array}{l}\text { Period of } \\
\text { record }\end{array}$ & Median & Minimum & Maximum \\
\hline \multicolumn{7}{|c|}{ Hardness as $\mathrm{CaCO}_{3}$, in $\mathrm{mg} / \mathrm{L}-$ Continued } \\
\hline 170 & Cannonball River at Breien, N. Dak. & 74 & 1994-2007 & 356.0 & 1.0 & 853.0 \\
\hline 171 & Beaver Creek near Linton, N. Dak. & 4 & 1993 & 185.0 & 130.0 & 243.0 \\
\hline 176 & James River near Grace City, N. Dak. & 60 & $1997-2007$ & 377.0 & 156.0 & $1,070.0$ \\
\hline 177 & James River above Arrowwood Lake near Kensal, N. Dak. & 9 & 1998-1999 & 276.0 & 146.0 & 455.0 \\
\hline 180 & Pipestem Creek near Pingree, N. Dak. & 17 & 1994-1999 & 447.0 & 233.0 & 578.0 \\
\hline 181 & Pipestem Creek near Buchanan, N. Dak. & 5 & 1993 & 240.0 & 42.0 & 326.0 \\
\hline 185 & James River at Oakes, N. Dak. & 3 & 1993 & 203.0 & 120.0 & 252.0 \\
\hline 186 & James River at N. Dak./S. Dak. State line & 9 & 1998-1999 & 286.0 & 266.0 & 333.0 \\
\hline \multicolumn{7}{|c|}{ Sodium absorption ratio, unitless } \\
\hline 1 & Bois De Sioux River near Doran, Minn. & 40 & $2000-2007$ & 0.85 & 0.39 & 1.66 \\
\hline 2 & Red River of the North at Wahpeton, N. Dak. & 17 & 1993-1996 & 0.70 & 0.30 & 1.24 \\
\hline 3 & Red River of the North near Wahpeton, N. Dak. & 4 & 1993-2006 & 0.55 & 0.43 & 6.48 \\
\hline 4 & Red River of the North at Brushville, Minn. & 61 & $1993-2007$ & 0.48 & 0.24 & 1.33 \\
\hline 10 & Wild Rice River near Abercrombie, N. Dak. & 58 & $1993-2007$ & 1.72 & 0.63 & 3.28 \\
\hline 11 & Red River of the North at Fargo, N. Dak. & 16 & 1994-1996 & 0.52 & 0.27 & 0.78 \\
\hline 33 & Baldhill Creek near Dazey, N. Dak. & 3 & 1993-1996 & 1.55 & 0.90 & 2.57 \\
\hline 34 & Sheyenne River below Baldhill Dam, N. Dak. & 73 & 1994-2007 & 2.09 & 1.03 & 3.09 \\
\hline 35 & Sheyenne River at Valley City, N. Dak. & 4 & 1993 & 1.45 & 1.19 & 1.74 \\
\hline 36 & Sheyenne River at Lisbon, N. Dak. & 69 & $1997-2007$ & 2.26 & 1.06 & 3.15 \\
\hline 37 & Sheyenne River near Kindred, N. Dak. & 70 & 1996-2007 & 1.76 & 0.58 & 2.67 \\
\hline 41 & Sheyenne River at West Fargo, N. Dak. & 13 & 1994-2006 & 1.57 & 0.91 & 2.02 \\
\hline 45 & Maple River below Mapleton, N. Dak. & 47 & $1997-2007$ & 1.71 & 0.60 & 6.10 \\
\hline 46 & Sheyenne River at Harwood, N. Dak. & 10 & 1993 & 1.36 & 0.54 & 2.67 \\
\hline 49 & Lower Branch Rush River near Prosper, N. Dak. & 1 & 1993 & 1.15 & 1.15 & 1.15 \\
\hline 51 & Elm River near Kelso, N. Dak. & 3 & 1993 & 1.48 & 1.21 & 1.80 \\
\hline 55 & Goose River at Hillsboro, N. Dak. & 76 & 1994-2007 & 1.37 & 0.65 & 2.36 \\
\hline 56 & Red River of the North at Grand Forks, N. Dak. & 46 & $1997-2006$ & 0.60 & 0.13 & 1.29 \\
\hline 58 & Turtle River at Manvel, N. Dak. & 38 & 1993-2006 & 4.60 & 1.17 & 15.10 \\
\hline 62 & Forest River near Minto, N. Dak. & 47 & 1994-2006 & 1.13 & 0.61 & 12.20 \\
\hline 67 & Park River at Grafton, N. Dak. & 40 & 1994-2006 & 1.97 & 0.85 & 4.93 \\
\hline
\end{tabular}


Table 1-1. Summary statistics for major ion constituents at selected sites in North Dakota from 1970 through 2008. —Continued $\left[\mu \mathrm{g} / \mathrm{L}\right.$, micrograms per liter; <, less than; --, not available; $\mathrm{mg} / \mathrm{L}$, milligrams per liter; $\mathrm{CO}_{3}$, carbonate; $\mathrm{HCO}_{3}$, bicarbonate; $\mathrm{CaCO}$, calcium carbonate $]$

\begin{tabular}{|c|c|c|c|c|c|c|}
\hline $\begin{array}{c}\text { Site } \\
\text { identification } \\
\text { number } \\
\text { (table 1) }\end{array}$ & Site name & $\begin{array}{c}\text { Number of } \\
\text { samples }\end{array}$ & $\begin{array}{l}\text { Period of } \\
\text { record }\end{array}$ & Median & Minimum & Maximum \\
\hline \multicolumn{7}{|c|}{ Sodium absorption ratio, unitless-Continued } \\
\hline 68 & Red River of the North at Drayton, N. Dak. & 15 & 1994-1996 & 0.86 & 0.47 & 1.16 \\
\hline 75 & Pembina River at Neche, N. Dak. & 55 & 1994-2006 & 1.12 & 0.63 & 1.53 \\
\hline 76 & Tongue River at Akra, N. Dak. & 1 & 1993 & 0.78 & 0.78 & 0.78 \\
\hline 77 & Red River of the North at Pembina, N. Dak., site 1 & 10 & 1993 & 0.78 & 0.42 & 1.21 \\
\hline 78 & Red River of the North at Pembina, N. Dak., site 2 & 52 & 1997-2006 & 0.80 & 0.38 & 2.50 \\
\hline 80 & Long Creek near Noonan, N. Dak. & 10 & 1997 & 3.29 & 2.17 & 5.20 \\
\hline 83 & Souris River near Foxholm, N. Dak. & 11 & 1997-1998 & 2.08 & 1.54 & 2.82 \\
\hline 84 & Des Lacs River at Foxholm, N. Dak. & 97 & 1993-2007 & 4.33 & 1.63 & 6.88 \\
\hline 85 & Souris River above Minot, N. Dak. & 75 & 1994-2007 & 3.32 & 1.57 & 10.90 \\
\hline 87 & Souris River near Verendrye, N. Dak. & 76 & 1997-2007 & 3.56 & 1.55 & 8.86 \\
\hline 88 & Wintering River near Karlsruhe, N. Dak. & 11 & $1997-1998$ & 4.38 & 1.04 & 7.41 \\
\hline 89 & Souris River near Bantry, N. Dak. & 10 & 1997-1998 & 2.89 & 2.09 & 3.17 \\
\hline 90 & Willow Creek near Willow City, N. Dak. & 6 & 1997 & 2.13 & 1.50 & 3.68 \\
\hline 92 & Deep River near Upham, N. Dak. & 7 & 1997 & 1.05 & 0.68 & 1.56 \\
\hline 98 & Souris River near Westhope, N. Dak. & 7 & $1997-1998$ & 3.38 & 2.65 & 4.78 \\
\hline 101 & Little Muddy River below Cow Creek near Williston, N. Dak. & 9 & 1999 & 5.77 & 1.08 & 8.84 \\
\hline 110 & Little Missouri River at Marmarth, N. Dak. & 8 & 1999 & 5.93 & 2.99 & 10.80 \\
\hline 112 & Little Missouri River at Medora, N. Dak. & 88 & $1993-2007$ & 8.89 & 1.77 & 21.50 \\
\hline 113 & Beaver Creek near Trotters, N. Dak. & 9 & 1999 & 5.92 & 4.38 & 7.43 \\
\hline 114 & Little Missouri River near Watford City, N. Dak. & 81 & 1994-2007 & 7.69 & 2.43 & 16.20 \\
\hline 120 & Knife River near Golden Valley, N. Dak. & 60 & 1997-2007 & 9.99 & 3.87 & 20.80 \\
\hline 123 & Spring Creek below Lake Ilo near Dunn Center, N. Dak. & 6 & 1993 & 9.06 & 5.97 & 10.80 \\
\hline 125 & Spring Creek at Zap, N. Dak. & 77 & 1993-2007 & 5.36 & 1.05 & 9.67 \\
\hline 127 & Knife River at Hazen, N. Dak. & 94 & $1993-2007$ & 5.91 & 2.60 & 9.34 \\
\hline 140 & Square Butte Creek below Center, N. Dak. & 4 & 1993 & 5.53 & 5.10 & 6.02 \\
\hline 142 & Missouri River at Bismarck, N. Dak. & 1 & 1994 & 1.90 & 1.90 & 1.90 \\
\hline 146 & Heart River at Dickinson, N. Dak. & 5 & 1993 & 4.67 & 3.77 & 9.93 \\
\hline 148 & Green River near Gladstone, N. Dak. & 4 & 1993 & 5.39 & 3.89 & 9.26 \\
\hline 149 & Heart River near Richardton, N. Dak. & 76 & 1994-2007 & 5.28 & 1.68 & 9.66 \\
\hline 152 & Big Muddy Creek near Almont, N. Dak. & 3 & 1993 & 9.74 & 6.13 & 12.20 \\
\hline 156 & Heart River near Mandan, N. Dak. & 81 & 1994-2007 & 5.08 & 2.29 & 8.81 \\
\hline 158 & Apple Creek near Menoken, N. Dak. & 4 & 1993 & 3.53 & 1.55 & 4.32 \\
\hline 164 & Cannonball River near Raleigh, N. Dak. & 76 & 1993-2007 & 5.36 & 1.21 & 13.30 \\
\hline 169 & Cedar Creek near Raleigh, N. Dak. & 68 & 1993-2007 & 5.57 & 1.96 & 14.00 \\
\hline 170 & Cannonball River at Breien, N. Dak. & 74 & 1994-2007 & 5.26 & 0.04 & 10.60 \\
\hline 171 & Beaver Creek near Linton, N. Dak. & 4 & 1993 & 1.96 & 1.68 & 2.07 \\
\hline 175 & James River near Manfred, N. Dak. & 6 & 1998 & 2.90 & 1.63 & 4.39 \\
\hline 176 & James River near Grace City, N. Dak. & 60 & $1997-2007$ & 2.21 & 0.57 & 8.96 \\
\hline 177 & James River above Arrowwood Lake near Kensal, N. Dak. & 9 & 1998-1999 & 1.49 & 0.53 & 2.67 \\
\hline 180 & Pipestem Creek near Pingree, N. Dak. & 17 & 1994-1999 & 1.83 & 1.08 & 2.28 \\
\hline
\end{tabular}


Table 1-1. Summary statistics for major ion constituents at selected sites in North Dakota from 1970 through 2008. —Continued $\left[\mu \mathrm{g} / \mathrm{L}\right.$, micrograms per liter; <, less than; --, not available; mg/L, milligrams per liter; $\mathrm{CO}_{3}$, carbonate; $\mathrm{HCO}_{3}$, bicarbonate; $\mathrm{CaCO}_{3}$, calcium carbonate $]$

\begin{tabular}{llrrrrr}
\hline $\begin{array}{c}\text { Site } \\
\text { identification } \\
\text { number } \\
\text { (table 1) }\end{array}$ & \multicolumn{1}{c}{ Site name } & $\begin{array}{c}\text { Number of } \\
\text { samples }\end{array}$ & $\begin{array}{c}\text { Period of } \\
\text { record }\end{array}$ & Median & Minimum Maximum \\
\hline \multicolumn{1}{c}{ Sodium absorption ratio, unitless_-Continued } \\
\hline 181 & Pipestem Creek near Buchanan, N. Dak. & 5 & 1993 & 1.05 & 0.26 & 1.43 \\
182 & James River at Jamestown, N. Dak. & 75 & $1997-2007$ & 1.98 & 0.89 & 3.07 \\
183 & James River at Lamoure, N. Dak. & 73 & $1996-2007$ & 1.89 & 0.63 & 2.93 \\
184 & Bear Creek near Oakes, N. Dak. & 9 & $1998-1999$ & 2.03 & 1.43 & 2.19 \\
185 & James River at Oakes, N. Dak. & 3 & 1993 & 1.09 & 0.94 & 1.38 \\
186 & James River at N. Dak./S. Dak. State line & 9 & $1998-1999$ & 1.35 & 1.11 & 1.61 \\
\hline
\end{tabular}


Table 1-2. Summary statistics for nutrient constituents at selected sites in North Dakota from 1970 through 2008.

[N, nitrogen; mg/L, milligrams per liter; $<$, less than; --, not available; P, phosphorus; $\mathrm{C}$, carbon]

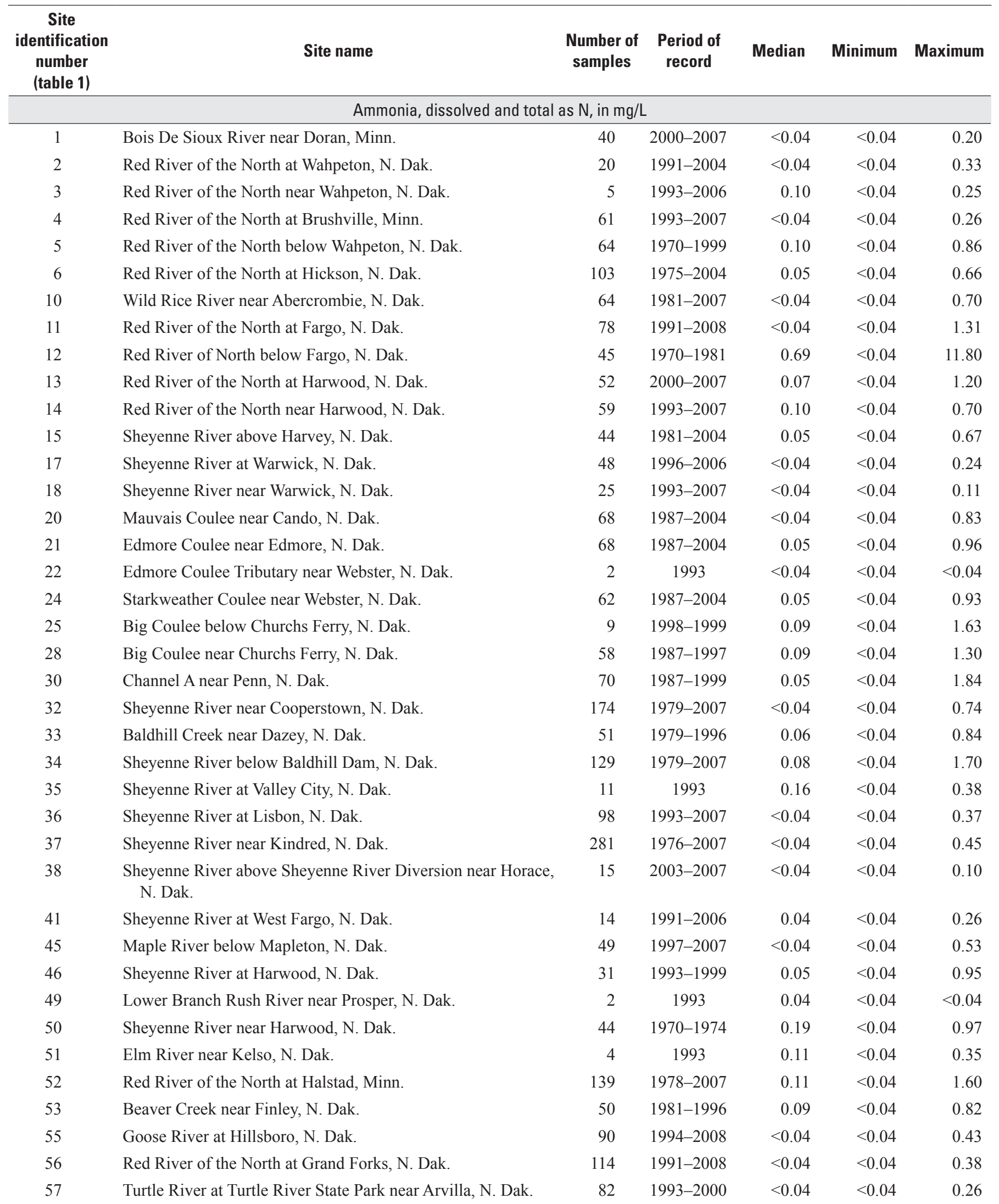


Table 1-2. Summary statistics for nutrient constituents at selected sites in North Dakota from 1970 through 2008. —Continued

[N, nitrogen; mg/L, milligrams per liter; <, less than; --, not available; $\mathrm{P}$, phosphorus; $\mathrm{C}$, carbon]

\begin{tabular}{|c|c|c|c|c|c|c|}
\hline $\begin{array}{c}\text { Site } \\
\text { identification } \\
\text { number } \\
\text { (table 1) }\end{array}$ & Site name & $\begin{array}{c}\text { Number of } \\
\text { samples }\end{array}$ & $\begin{array}{l}\text { Period of } \\
\text { record }\end{array}$ & Median & Minimum & Maximum \\
\hline \multicolumn{7}{|c|}{ Ammonia, dissolved and total as $\mathrm{N}$, in $\mathrm{mg} / \mathrm{L}$-Continued } \\
\hline 58 & Turtle River at Manvel, N. Dak. & 69 & $1993-2008$ & $<0.04$ & $<0.04$ & 1.01 \\
\hline 59 & Red River of the North at Oslo, Minn. & 6 & 1977-2004 & 0.22 & $<0.04$ & 0.53 \\
\hline 62 & Forest River near Minto, N. Dak. & 78 & 1994-2008 & $<0.04$ & $<0.04$ & 0.67 \\
\hline 67 & Park River at Grafton, N. Dak. & 71 & 1994-2008 & $<0.04$ & $<0.04$ & 0.67 \\
\hline 68 & Red River of the North at Drayton, N. Dak. & 18 & 1991-2004 & $<0.04$ & $<0.04$ & 0.23 \\
\hline 72 & Pembina River near Vang, N. Dak. & 50 & $1976-1979$ & $<0.04$ & $<0.04$ & 0.28 \\
\hline 73 & Little South Pembina River near Walhalla, N. Dak. & 63 & $1976-1995$ & $<0.04$ & $<0.04$ & 0.44 \\
\hline 74 & Pembina River at Walhalla, N. Dak. & 143 & 1976-1995 & $<0.04$ & $<0.04$ & 0.35 \\
\hline 75 & Pembina River at Neche, N. Dak. & 86 & 1994-2008 & $<0.04$ & $<0.04$ & 0.24 \\
\hline 76 & Tongue River at Akra, N. Dak. & 25 & 1979-2004 & 0.13 & $<0.04$ & 0.38 \\
\hline 77 & Red River of the North at Pembina, N. Dak., site 1 & 10 & 1993 & 0.14 & $<0.04$ & 0.93 \\
\hline 78 & Red River of the North at Pembina, N. Dak., site 2 & 167 & $1970-2008$ & $<0.04$ & $<0.04$ & 0.76 \\
\hline 79 & Red River of the North at Emerson, Manitoba & 149 & 1978-2004 & 0.09 & $<0.04$ & 2.30 \\
\hline 80 & Long Creek near Noonan, N. Dak. & 10 & 1997 & $<0.04$ & $<0.04$ & 0.44 \\
\hline 81 & West Branch Short Creek near Columbus, N. Dak. & 19 & $1978-1981$ & 0.06 & $<0.04$ & 0.45 \\
\hline 82 & Souris River near Sherwood, N. Dak. & 322 & $1974-2008$ & 0.07 & $<0.04$ & 3.30 \\
\hline 83 & Souris River near Foxholm, N. Dak. & 183 & 1972-1998 & 0.10 & $<0.04$ & 4.80 \\
\hline 84 & Des Lacs River at Foxholm, N. Dak. & 138 & $1981-2007$ & $<0.04$ & $<0.04$ & 2.50 \\
\hline 85 & Souris River above Minot, N. Dak. & 143 & $1981-2008$ & $<0.04$ & $<0.04$ & 4.72 \\
\hline 87 & Souris River near Verendrye, N. Dak. & 344 & $1970-2008$ & 0.09 & $<0.04$ & 3.20 \\
\hline 88 & Wintering River near Karlsruhe, N. Dak. & 57 & 1981-1998 & $<0.04$ & $<0.04$ & 0.25 \\
\hline 89 & Souris River near Bantry, N. Dak. & 88 & $1981-2000$ & $<0.04$ & $<0.04$ & 1.30 \\
\hline 90 & Willow Creek near Willow City, N. Dak. & 47 & $1982-2000$ & $<0.04$ & $<0.04$ & 0.25 \\
\hline 91 & Stone Creek near Kramer, N. Dak. & 29 & $1986-2000$ & $<0.04$ & $<0.04$ & 0.45 \\
\hline 92 & Deep River near Upham, N. Dak. & 27 & $1997-2000$ & $<0.04$ & $<0.04$ & 0.28 \\
\hline 95 & Cut Bank Creek at Upham, N. Dak. & 16 & $1999-2000$ & $<0.04$ & $<0.04$ & 0.22 \\
\hline 96 & Deep River below Cut Bank Creek near Upham, N. Dak. & 15 & $1982-1989$ & 0.07 & $<0.04$ & 0.38 \\
\hline 97 & Boundary Creek near Landa, N. Dak. & 30 & $1986-2000$ & $<0.04$ & $<0.04$ & 0.28 \\
\hline 98 & Souris River near Westhope, N. Dak. & 258 & $1970-2007$ & 0.14 & $<0.04$ & 3.60 \\
\hline 100 & Missouri River near Williston, N. Dak. & 100 & 1970-1982 & $<0.04$ & $<0.04$ & 0.80 \\
\hline 101 & Little Muddy River below Cow Creek near Williston, N. Dak. & 9 & 1999 & $<0.04$ & $<0.04$ & 0.05 \\
\hline 102 & Stony Creek near Williston, N. Dak. & 29 & $1977-1981$ & 0.08 & $<0.04$ & 107.00 \\
\hline 104 & Beaver Creek near Ray, N. Dak. & 44 & $1977-1982$ & 0.06 & $<0.04$ & 118.00 \\
\hline 106 & Bear Den Creek near Mandaree, N. Dak. & 95 & 1980-1996 & 0.05 & $<0.04$ & 0.86 \\
\hline 108 & East Fork Shell Creek near Parshall, N. Dak. & 47 & $1991-2000$ & $<0.04$ & $<0.04$ & 0.58 \\
\hline 109 & Deepwater Creek near Mandaree, N. Dak. & 44 & $1991-2000$ & $<0.04$ & $<0.04$ & 3.32 \\
\hline 110 & Little Missouri River at Marmarth, N. Dak. & 8 & 1999 & $<0.04$ & $<0.04$ & 0.06 \\
\hline 111 & Deep Creek near Amidon, N. Dak. & 45 & $1977-1983$ & $<0.04$ & $<0.04$ & 0.28 \\
\hline 112 & Little Missouri River at Medora, N. Dak. & 89 & 1979-2007 & $<0.04$ & $<0.04$ & 1.06 \\
\hline 113 & Beaver Creek near Trotters, N. Dak. & 52 & $1977-1999$ & $<0.04$ & $<0.04$ & 0.28 \\
\hline
\end{tabular}


Table 1-2. Summary statistics for nutrient constituents at selected sites in North Dakota from 1970 through 2008. - Continued [N, nitrogen; mg/L, milligrams per liter; <, less than; --, not available; P, phosphorus; $\mathrm{C}$, carbon]

\begin{tabular}{|c|c|c|c|c|c|c|}
\hline $\begin{array}{c}\text { Site } \\
\text { identification } \\
\text { number } \\
\text { (table 1) }\end{array}$ & Site name & $\begin{array}{c}\text { Number of } \\
\text { samples }\end{array}$ & $\begin{array}{l}\text { Period of } \\
\text { record }\end{array}$ & Median & Minimum & Maximum \\
\hline \multicolumn{7}{|c|}{ Ammonia, dissolved and total as $\mathrm{N}$, in $\mathrm{mg} / \mathrm{L}-$ Continued } \\
\hline 114 & Little Missouri River near Watford City, N. Dak. & 170 & 1977-2007 & $<0.04$ & $<0.04$ & 0.54 \\
\hline 115 & Missouri River at Garrison Dam, N. Dak. & 217 & 1974-2007 & $<0.04$ & $<0.04$ & 0.22 \\
\hline 116 & Knife River at Manning, N. Dak. & 45 & $1977-1982$ & 0.06 & $<0.04$ & 111.00 \\
\hline 117 & Stray Creek near Manning, N. Dak. & 16 & $1975-1981$ & 0.06 & $<0.04$ & 100.00 \\
\hline 118 & Knife River at Marshall, N. Dak. & 43 & $1977-1981$ & 0.07 & $<0.04$ & 126.00 \\
\hline 119 & Elm Creek near Golden Valley, N. Dak. & 15 & $1977-1981$ & 0.06 & $<0.04$ & 0.68 \\
\hline 120 & Knife River near Golden Valley, N. Dak. & 60 & $1997-2007$ & $<0.04$ & $<0.04$ & 0.40 \\
\hline 121 & Coyote Creek near Zap, N. Dak. & 44 & $1977-1983$ & 0.06 & $<0.04$ & 120.00 \\
\hline 122 & Brush Creek near Beulah, N. Dak. & 62 & $1975-1990$ & 0.07 & $<0.04$ & 76.00 \\
\hline 123 & Spring Creek below Lake Ilo near Dunn Center, N. Dak. & 46 & $1977-1993$ & 0.09 & $<0.04$ & 2.90 \\
\hline 124 & Spring Creek near Halliday, N. Dak. & 41 & $1977-1981$ & 0.06 & $<0.04$ & 104.00 \\
\hline 125 & Spring Creek at Zap, N. Dak. & 172 & 1974-2007 & $<0.04$ & $<0.04$ & 0.67 \\
\hline 127 & Knife River at Hazen, N. Dak. & 234 & 1974-2007 & 0.05 & $<0.04$ & 0.62 \\
\hline 128 & Antelope Creek above Hazen, N. Dak. & 26 & $1977-1982$ & 0.10 & $<0.04$ & 0.78 \\
\hline 129 & West Branch Antelope Creek No. 4 near Zap, N. Dak. & 1 & 1977 & $<0.04$ & $<0.04$ & $<0.04$ \\
\hline 130 & West Branch Antelope Creek near Hazen, N. Dak. & 14 & $1978-1983$ & 0.20 & $<0.04$ & 0.58 \\
\hline 131 & Coal Creek near Stanton, N. Dak. & 25 & $1975-1981$ & 0.05 & $<0.04$ & 0.28 \\
\hline 132 & Alderin Creek near Fort Clark, N. Dak. & 29 & $1977-1982$ & 0.07 & $<0.04$ & 0.71 \\
\hline 133 & Coal Lake Coulee near Hensler, N. Dak. & 16 & $1978-1982$ & 0.06 & $<0.04$ & 0.45 \\
\hline 134 & Buffalo Creek near Washburn, N. Dak. & 33 & 1978-1983 & 0.11 & $<0.04$ & 129.00 \\
\hline 135 & Turtle Creek above Washburn, N. Dak. & 76 & $1990-2003$ & $<0.04$ & $<0.04$ & 1.20 \\
\hline 136 & Painted Woods Creek near Wilton, N. Dak. & 77 & $1970-2003$ & $<0.04$ & $<0.04$ & 0.35 \\
\hline 137 & Square Butte Creek near Hannover, N. Dak. & 21 & $1977-1981$ & 0.05 & $<0.04$ & 0.85 \\
\hline 138 & Square Butte Creek above Nelson Lake near Center, N. Dak. & 49 & $1977-1982$ & 0.13 & $<0.04$ & 80.00 \\
\hline 139 & Hagel Creek near Center, N. Dak. & 32 & $1977-1982$ & 0.14 & $<0.04$ & 80.00 \\
\hline 140 & Square Butte Creek below Center, N. Dak. & 4 & 1993 & 0.59 & 0.10 & 1.00 \\
\hline 142 & Missouri River at Bismarck, N. Dak. & 205 & 1970-2001 & $<0.04$ & $<0.04$ & 0.43 \\
\hline 143 & South Branch Heart River near South Heart, N. Dak. & 27 & 1979-1996 & 0.09 & $<0.04$ & 0.45 \\
\hline 144 & North Creek near South Heart, N. Dak. & 22 & 1978-1996 & 0.07 & $<0.04$ & 43.00 \\
\hline 145 & Heart River near South Heart, N. Dak. & 48 & $1975-1983$ & 0.09 & $<0.04$ & 2.10 \\
\hline 146 & Heart River at Dickinson, N. Dak. & 5 & 1993 & 0.23 & $<0.04$ & 0.78 \\
\hline 147 & Green River near New Hradec, N. Dak. & 45 & $1977-1982$ & 0.06 & $<0.04$ & 79.00 \\
\hline 148 & Green River near Gladstone, N. Dak. & 4 & 1993 & 0.07 & $<0.04$ & 0.22 \\
\hline 149 & Heart River near Richardton, N. Dak. & 76 & 1994-2007 & $<0.04$ & $<0.04$ & 0.42 \\
\hline 152 & Big Muddy Creek near Almont, N. Dak. & 3 & 1993 & 0.06 & $<0.04$ & 0.43 \\
\hline 156 & Heart River near Mandan, N. Dak. & 187 & 1978-2007 & $<0.04$ & $<0.04$ & 0.71 \\
\hline 157 & Long Lake Creek above Long Lake near Moffit, N. Dak. & 3 & 1988-1989 & 0.09 & $<0.04$ & 0.27 \\
\hline 158 & Apple Creek near Menoken, N. Dak. & 51 & $1977-2007$ & 0.07 & $<0.04$ & 0.72 \\
\hline 159 & Missouri River near Schmidt, N. Dak. & 140 & 1974-1981 & $<0.04$ & $<0.04$ & 0.26 \\
\hline 160 & Cannonball River at New England, N. Dak. & 28 & $1978-1981$ & 0.07 & $<0.04$ & 0.94 \\
\hline
\end{tabular}


Table 1-2. Summary statistics for nutrient constituents at selected sites in North Dakota from 1970 through 2008. —Continued

[N, nitrogen; mg/L, milligrams per liter; <, less than; --, not available; $\mathrm{P}$, phosphorus; $\mathrm{C}$, carbon]

\begin{tabular}{|c|c|c|c|c|c|c|}
\hline $\begin{array}{c}\text { Site } \\
\text { identification } \\
\text { number } \\
\text { (table 1) }\end{array}$ & Site name & $\begin{array}{c}\text { Number of } \\
\text { samples }\end{array}$ & $\begin{array}{l}\text { Period of } \\
\text { record }\end{array}$ & Median & Minimum & Maximum \\
\hline 161 & Coal Bank Creek near Havelock, N. Dak. & 39 & $1975-1983$ & 0.05 & $<0.04$ & 0.74 \\
\hline 162 & Cannonball River at Regent, N. Dak. & 40 & $1977-1981$ & 0.05 & $<0.04$ & 0.44 \\
\hline 167 & Timber Creek near Bentley, N. Dak. & 39 & 1977-1981 & 0.06 & $<0.04$ & 1.10 \\
\hline 169 & Cedar Creek near Raleigh, N. Dak. & 68 & $1993-2007$ & $<0.04$ & $<0.04$ & 0.43 \\
\hline 170 & Cannonball River at Breien, N. Dak. & 169 & $1977-2007$ & $<0.04$ & $<0.04$ & 0.43 \\
\hline 171 & Beaver Creek near Linton, N. Dak. & 4 & 1993 & 0.07 & $<0.04$ & 0.20 \\
\hline 173 & Porcupine Creek near Fort Yates, N. Dak. & 36 & 1991-1999 & $<0.04$ & $<0.04$ & 0.22 \\
\hline 176 & James River near Grace City, N. Dak. & 105 & $1987-2007$ & $<0.04$ & $<0.04$ & 1.60 \\
\hline 177 & James River above Arrowwood Lake near Kensal, N. Dak. & 140 & $1987-2008$ & $<0.04$ & $<0.04$ & 2.50 \\
\hline 178 & Kelly Creek below Niccum Reservoir near Bordulac, N. Dak. & 5 & $1987-1989$ & $<0.04$ & $<0.04$ & 0.49 \\
\hline 179 & James River near Pingree, N. Dak. & 82 & $1987-2008$ & $<0.04$ & $<0.04$ & 1.72 \\
\hline 180 & Pipestem Creek near Pingree, N. Dak. & 17 & 1994-1999 & $<0.04$ & $<0.04$ & 0.41 \\
\hline 181 & Pipestem Creek near Buchanan, N. Dak. & 5 & 1993 & 0.10 & $<0.04$ & 0.95 \\
\hline 182 & James River at Jamestown, N. Dak. & 139 & $1987-2007$ & 0.09 & $<0.04$ & 1.10 \\
\hline 183 & James River at Lamoure, N. Dak. & 131 & $1981-2007$ & $<0.04$ & $<0.04$ & 1.50 \\
\hline 184 & Bear Creek near Oakes, N. Dak. & 9 & 1998-1999 & 0.14 & $<0.04$ & 0.55 \\
\hline 4 & Red River of the North at Brushville, Minn. & 54 & $1996-2007$ & 0.16 & $<0.10$ & 1.84 \\
\hline 5 & Red River of the North below Wahpeton, N. Dak. & 47 & 1971-1999 & $<0.10$ & $<0.10$ & 1.30 \\
\hline 6 & Red River of the North at Hickson, N. Dak. & 104 & $1975-2004$ & $<0.10$ & $<0.10$ & 2.30 \\
\hline 7 & Wild Rice River near Rutland, N. Dak. & 3 & $1971-1975$ & $<0.10$ & $<0.10$ & 0.44 \\
\hline 10 & Wild Rice River near Abercrombie, N. Dak. & 163 & $1971-2007$ & $<0.10$ & $<0.10$ & 3.80 \\
\hline 11 & Red River of the North at Fargo, N. Dak. & 106 & $1971-2008$ & 0.19 & $<0.10$ & 2.14 \\
\hline 12 & Red River of North below Fargo, N. Dak. & 155 & $1971-1986$ & 0.31 & $<0.10$ & 4.60 \\
\hline 13 & Red River of the North at Harwood, N. Dak. & 52 & $2000-2007$ & 0.88 & 0.20 & 5.56 \\
\hline 14 & Red River of the North near Harwood, N. Dak. & 59 & $1993-2007$ & 0.48 & $<0.10$ & 2.28 \\
\hline 15 & Sheyenne River above Harvey, N. Dak. & 154 & $1977-2004$ & $<0.10$ & $<0.10$ & 2.00 \\
\hline 17 & Sheyenne River at Warwick, N. Dak. & 48 & 1996-2006 & 0.19 & $<0.10$ & 0.96 \\
\hline 18 & Sheyenne River near Warwick, N. Dak. & 123 & $1971-2007$ & $<0.10$ & $<0.10$ & 4.20 \\
\hline 20 & Mauvais Coulee near Cando, N. Dak. & 68 & $1987-2004$ & $<0.10$ & $<0.10$ & 2.80 \\
\hline 21 & Edmore Coulee near Edmore, N. Dak. & 70 & $1983-2004$ & $<0.10$ & $<0.10$ & 4.60 \\
\hline 22 & Edmore Coulee Tributary near Webster, N. Dak. & 2 & 1993 & $<0.10$ & $<0.10$ & $<0.10$ \\
\hline
\end{tabular}


Table 1-2. Summary statistics for nutrient constituents at selected sites in North Dakota from 1970 through 2008. - Continued

[N, nitrogen; mg/L, milligrams per liter; <, less than; --, not available; P, phosphorus; $\mathrm{C}$, carbon]

\begin{tabular}{|c|c|c|c|c|c|c|}
\hline $\begin{array}{c}\text { Site } \\
\text { identification } \\
\text { number } \\
\text { (table 1) }\end{array}$ & Site name & $\begin{array}{c}\text { Number of } \\
\text { samples }\end{array}$ & $\begin{array}{l}\text { Period of } \\
\text { record }\end{array}$ & Median & Minimum & Maximum \\
\hline \multicolumn{7}{|c|}{ Nitrate + Nitrite dissolved and total as $\mathrm{N}$, in $\mathrm{mg} / \mathrm{L}-$ Continued } \\
\hline 24 & Starkweather Coulee near Webster, N. Dak. & 63 & $1987-2004$ & 0.11 & $<0.10$ & 8.30 \\
\hline 25 & Big Coulee below Churchs Ferry, N. Dak. & 9 & 1998-1999 & $<0.10$ & $<0.10$ & 0.87 \\
\hline 27 & Little Coulee near Brinsmade, N. Dak. & 16 & 1976-1981 & 0.23 & $<0.10$ & 3.40 \\
\hline 28 & Big Coulee near Churchs Ferry, N. Dak. & 119 & 1971-1997 & 0.27 & $<0.10$ & 3.10 \\
\hline 29 & Big Coulee at Graham Island inlet near Fort Totten, N. Dak. & 41 & $1971-1986$ & $<0.10$ & $<0.10$ & 9.50 \\
\hline 30 & Channel A near Penn, N. Dak. & 71 & 1987-1999 & $<0.10$ & $<0.10$ & 2.90 \\
\hline 32 & Sheyenne River near Cooperstown, N. Dak. & 263 & $1971-2007$ & 0.13 & $<0.10$ & 2.10 \\
\hline 33 & Baldhill Creek near Dazey, N. Dak. & 51 & 1979-1996 & $<0.10$ & $<0.10$ & 2.20 \\
\hline 34 & Sheyenne River below Baldhill Dam, N. Dak. & 129 & 1979-2007 & 0.13 & $<0.10$ & 1.60 \\
\hline 35 & Sheyenne River at Valley City, N. Dak. & 11 & 1993 & 0.21 & $<0.10$ & 0.30 \\
\hline 36 & Sheyenne River at Lisbon, N. Dak. & 252 & 1971-2007 & 0.21 & $<0.10$ & 1.50 \\
\hline 37 & Sheyenne River near Kindred, N. Dak. & 282 & 1976-2007 & 0.13 & $<0.10$ & 3.60 \\
\hline 38 & $\begin{array}{l}\text { Sheyenne River above Sheyenne River Diversion near Horace, } \\
\text { N. Dak. }\end{array}$ & 15 & 2003-2007 & $<0.10$ & $<0.10$ & 2.38 \\
\hline 39 & Sheyenne River near Horace, N. Dak. & 37 & 1976-1979 & 0.19 & $<0.10$ & 3.50 \\
\hline 41 & Sheyenne River at West Fargo, N. Dak. & 14 & 1991-2006 & $<0.10$ & $<0.10$ & 1.59 \\
\hline 42 & Maple River near Hope, N. Dak. & 1 & 1981 & $<0.10$ & $<0.10$ & $<0.10$ \\
\hline 45 & Maple River below Mapleton, N. Dak. & 49 & 1997-2007 & $<0.10$ & $<0.10$ & 7.70 \\
\hline 46 & Sheyenne River at Harwood, N. Dak. & 31 & 1993-1999 & 0.36 & $<0.10$ & 3.85 \\
\hline 49 & Lower Branch Rush River near Prosper, N. Dak. & 2 & 1993 & 0.30 & $<0.10$ & 0.50 \\
\hline 50 & Sheyenne River near Harwood, N. Dak. & 27 & 1971-1974 & 0.21 & $<0.10$ & 1.90 \\
\hline 51 & Elm River near Kelso, N. Dak. & 4 & 1993 & $<0.10$ & $<0.10$ & 0.91 \\
\hline 52 & Red River of the North at Halstad, Minn. & 140 & $1978-2007$ & 0.43 & $<0.10$ & 4.90 \\
\hline 53 & Beaver Creek near Finley, N. Dak. & 97 & 1971-1996 & $<0.10$ & $<0.10$ & 5.00 \\
\hline 55 & Goose River at Hillsboro, N. Dak. & 90 & 1994-2008 & 0.39 & $<0.10$ & 5.91 \\
\hline 56 & Red River of the North at Grand Forks, N. Dak. & 139 & $1971-2008$ & 0.40 & $<0.10$ & 4.60 \\
\hline 57 & Turtle River at Turtle River State Park near Arvilla, N. Dak. & 82 & $1993-2000$ & 0.32 & $<0.10$ & 2.70 \\
\hline 58 & Turtle River at Manvel, N. Dak. & 87 & 1980-2008 & 0.16 & $<0.10$ & 2.60 \\
\hline 59 & Red River of the North at Oslo, Minn. & 57 & 1973-2004 & 0.22 & $<0.10$ & 2.70 \\
\hline 62 & Forest River near Minto, N. Dak. & 78 & 1994-2008 & 0.48 & $<0.10$ & 2.86 \\
\hline 63 & South Branch Park River below Homme Dam, N. Dak. & 1 & 1981 & $<0.10$ & $<0.10$ & $<0.10$ \\
\hline 64 & Middle Branch Park River near Union, N. Dak. & 1 & 1980 & 0.92 & 0.92 & 0.92 \\
\hline 65 & Middle Branch Park River near Edinburg, N. Dak. & 13 & 1978-1980 & 0.83 & $<0.10$ & 3.30 \\
\hline 66 & Cart Creek at Mountain, N. Dak. & 6 & 1978-1979 & 1.60 & 0.14 & 2.50 \\
\hline 67 & Park River at Grafton, N. Dak. & 71 & 1994-2008 & 0.54 & $<0.10$ & 3.32 \\
\hline 68 & Red River of the North at Drayton, N. Dak. & 18 & 1991-2004 & 0.37 & 0.10 & 1.19 \\
\hline 72 & Pembina River near Vang, N. Dak. & 79 & 1971-1979 & 0.14 & $<0.10$ & 2.70 \\
\hline 73 & Little South Pembina River near Walhalla, N. Dak. & 92 & 1971-1995 & 0.64 & $<0.10$ & 4.00 \\
\hline 74 & Pembina River at Walhalla, N. Dak. & 202 & $1971-1995$ & 0.13 & $<0.10$ & 3.00 \\
\hline 75 & Pembina River at Neche, N. Dak. & 86 & 1994-2008 & 0.17 & $<0.10$ & 3.74 \\
\hline
\end{tabular}


Table 1-2. Summary statistics for nutrient constituents at selected sites in North Dakota from 1970 through 2008. —Continued

[N, nitrogen; mg/L, milligrams per liter; <, less than; --, not available; $\mathrm{P}$, phosphorus; $\mathrm{C}$, carbon]

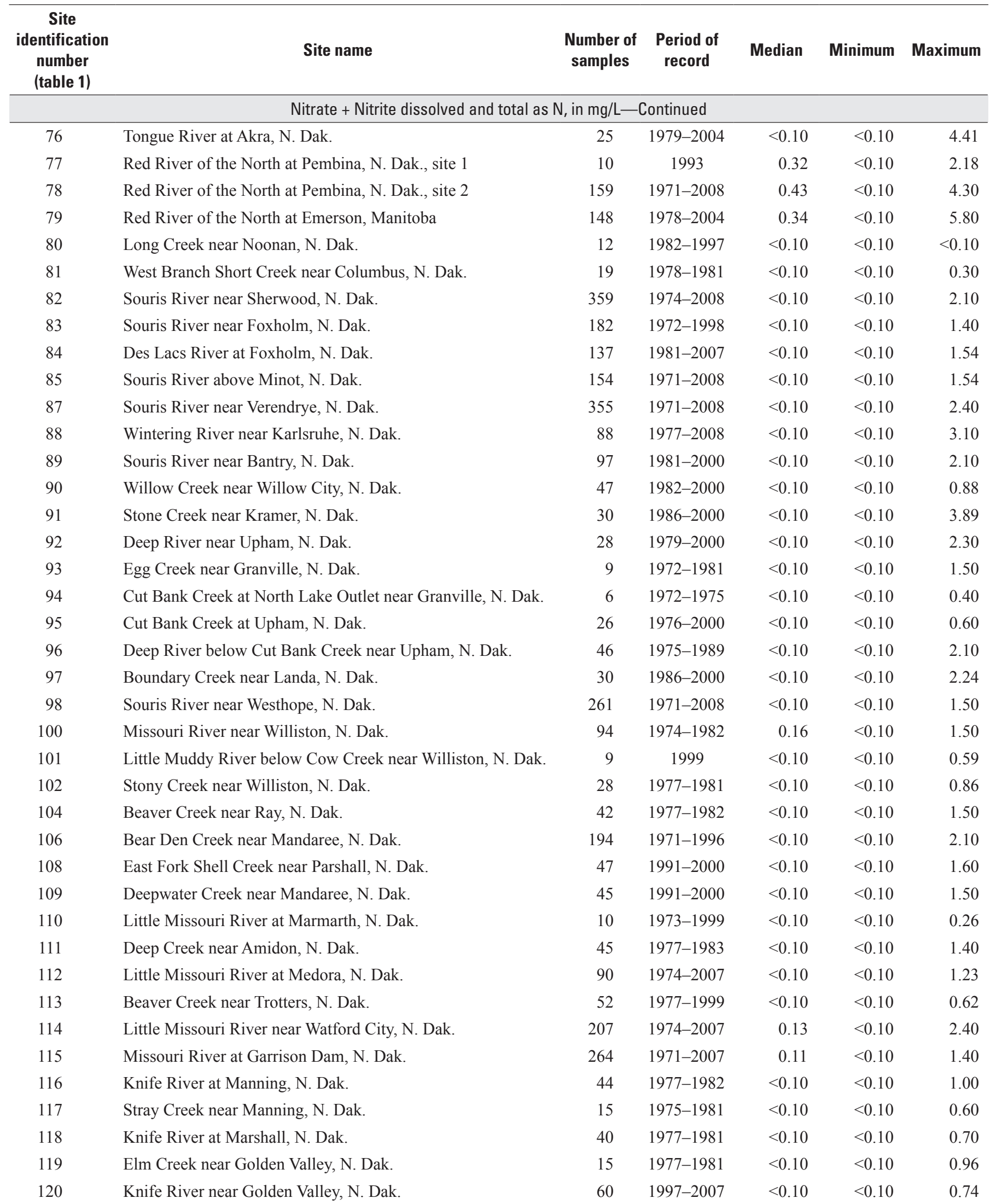


Table 1-2. Summary statistics for nutrient constituents at selected sites in North Dakota from 1970 through 2008. - Continued

[N, nitrogen; mg/L, milligrams per liter; <, less than; --, not available; P, phosphorus; $\mathrm{C}$, carbon]

\begin{tabular}{|c|c|c|c|c|c|c|}
\hline $\begin{array}{c}\text { Site } \\
\text { identification } \\
\text { number } \\
\text { (table 1) }\end{array}$ & Site name & $\begin{array}{c}\text { Number of } \\
\text { samples }\end{array}$ & $\begin{array}{l}\text { Period of } \\
\text { record }\end{array}$ & Median & Minimum & Maximum \\
\hline \multicolumn{7}{|c|}{ Nitrate + Nitrite dissolved and total as $\mathrm{N}$, in $\mathrm{mg} / \mathrm{L}-$ Continued } \\
\hline 121 & Coyote Creek near Zap, N. Dak. & 43 & $1977-1983$ & $<0.10$ & $<0.10$ & 1.60 \\
\hline 122 & Brush Creek near Beulah, N. Dak. & 99 & $1974-1990$ & $<0.10$ & $<0.10$ & 2.10 \\
\hline 123 & Spring Creek below Lake Ilo near Dunn Center, N. Dak. & 45 & $1977-1993$ & $<0.10$ & $<0.10$ & 0.72 \\
\hline 124 & Spring Creek near Halliday, N. Dak. & 41 & $1977-1981$ & $<0.10$ & $<0.10$ & 0.58 \\
\hline 125 & Spring Creek at Zap, N. Dak. & 173 & 1974-2007 & $<0.10$ & $<0.10$ & 1.20 \\
\hline 127 & Knife River at Hazen, N. Dak. & 234 & 1974-2007 & $<0.10$ & $<0.10$ & 1.20 \\
\hline 128 & Antelope Creek above Hazen, N. Dak. & 26 & $1977-1982$ & 0.18 & $<0.10$ & 1.80 \\
\hline 129 & West Branch Antelope Creek No. 4 near Zap, N. Dak. & 1 & 1977 & 0.21 & 0.21 & 0.21 \\
\hline 130 & West Branch Antelope Creek near Hazen, N. Dak. & 14 & $1978-1983$ & 0.14 & $<0.10$ & 0.83 \\
\hline 131 & Coal Creek near Stanton, N. Dak. & 25 & $1975-1981$ & $<0.10$ & $<0.10$ & 0.65 \\
\hline 132 & Alderin Creek near Fort Clark, N. Dak. & 29 & 1977-1982 & $<0.10$ & $<0.10$ & 1.60 \\
\hline 133 & Coal Lake Coulee near Hensler, N. Dak. & 16 & 1978-1982 & $<0.10$ & $<0.10$ & 0.72 \\
\hline 134 & Buffalo Creek near Washburn, N. Dak. & 32 & 1978-1983 & $<0.10$ & $<0.10$ & 1.80 \\
\hline 135 & Turtle Creek above Washburn, N. Dak. & 95 & $1987-2003$ & $<0.10$ & $<0.10$ & 1.20 \\
\hline 136 & Painted Woods Creek near Wilton, N. Dak. & 184 & $1971-2003$ & $<0.10$ & $<0.10$ & 1.60 \\
\hline 137 & Square Butte Creek near Hannover, N. Dak. & 21 & $1977-1981$ & $<0.10$ & $<0.10$ & 0.50 \\
\hline 138 & Square Butte Creek above Nelson Lake near Center, N. Dak. & 48 & 1977-1982 & 0.75 & $<0.10$ & 4.70 \\
\hline 139 & Hagel Creek near Center, N. Dak. & 31 & 1977-1982 & $<0.10$ & $<0.10$ & 0.58 \\
\hline 140 & Square Butte Creek below Center, N. Dak. & 5 & 1983-1993 & 0.23 & $<0.10$ & 0.40 \\
\hline 142 & Missouri River at Bismarck, N. Dak. & 192 & $1971-2001$ & 0.12 & $<0.10$ & 0.80 \\
\hline 143 & South Branch Heart River near South Heart, N. Dak. & 27 & 1979-1996 & 0.29 & $<0.10$ & 1.80 \\
\hline 144 & North Creek near South Heart, N. Dak. & 22 & 1978-1996 & $<0.10$ & $<0.10$ & 0.85 \\
\hline 145 & Heart River near South Heart, N. Dak. & 49 & $1975-1984$ & $<0.10$ & $<0.10$ & 1.50 \\
\hline 146 & Heart River at Dickinson, N. Dak. & 5 & 1993 & $<0.10$ & $<0.10$ & 0.18 \\
\hline 147 & Green River near New Hradec, N. Dak. & 44 & 1977-1982 & $<0.10$ & $<0.10$ & 0.69 \\
\hline 148 & Green River near Gladstone, N. Dak. & 4 & 1993 & $<0.10$ & $<0.10$ & $<0.10$ \\
\hline 149 & Heart River near Richardton, N. Dak. & 76 & 1994-2007 & $<0.10$ & $<0.10$ & 1.52 \\
\hline 152 & Big Muddy Creek near Almont, N. Dak. & 3 & 1993 & $<0.10$ & $<0.10$ & 0.12 \\
\hline 156 & Heart River near Mandan, N. Dak. & 187 & $1978-2007$ & $<0.10$ & $<0.10$ & 1.20 \\
\hline 157 & Long Lake Creek above Long Lake near Moffit, N. Dak. & 3 & 1988-1989 & $<0.10$ & $<0.10$ & 0.35 \\
\hline 158 & Apple Creek near Menoken, N. Dak. & 59 & 1977-2007 & $<0.10$ & $<0.10$ & 1.40 \\
\hline 159 & Missouri River near Schmidt, N. Dak. & 141 & 1974-1981 & 0.13 & $<0.10$ & 0.47 \\
\hline 160 & Cannonball River at New England, N. Dak. & 28 & 1978-1981 & $<0.10$ & $<0.10$ & 2.00 \\
\hline 161 & Coal Bank Creek near Havelock, N. Dak. & 76 & $1974-1983$ & $<0.10$ & $<0.10$ & 7.00 \\
\hline 162 & Cannonball River at Regent, N. Dak. & 40 & $1977-1981$ & $<0.10$ & $<0.10$ & 0.86 \\
\hline 164 & Cannonball River near Raleigh, N. Dak. & 76 & 1993-2007 & $<0.10$ & $<0.10$ & 2.77 \\
\hline 166 & Cedar Creek near Haynes, N. Dak. & 1 & 1973 & $<0.10$ & $<0.10$ & $<0.10$ \\
\hline 167 & Timber Creek near Bentley, N. Dak. & 39 & 1977-1981 & 0.38 & $<0.10$ & 4.80 \\
\hline 169 & Cedar Creek near Raleigh, N. Dak. & 68 & $1993-2007$ & $<0.10$ & $<0.10$ & 3.56 \\
\hline 170 & Cannonball River at Breien, N. Dak. & 204 & $1971-2007$ & $<0.10$ & $<0.10$ & 3.52 \\
\hline
\end{tabular}


Table 1-2. Summary statistics for nutrient constituents at selected sites in North Dakota from 1970 through 2008.—Continued

[N, nitrogen; mg/L, milligrams per liter; <, less than; --, not available; $\mathrm{P}$, phosphorus; $\mathrm{C}$, carbon]

\begin{tabular}{|c|c|c|c|c|c|c|}
\hline $\begin{array}{c}\text { Site } \\
\text { identification } \\
\text { number } \\
\text { (table 1) }\end{array}$ & Site name & $\begin{array}{l}\text { Number of } \\
\text { samples }\end{array}$ & $\begin{array}{l}\text { Period of } \\
\text { record }\end{array}$ & Median & Minimum & Maximum \\
\hline \multicolumn{7}{|c|}{ Nitrate + Nitrite dissolved and total as $\mathrm{N}$, in $\mathrm{mg} / \mathrm{L}$-Continued } \\
\hline 171 & Beaver Creek near Linton, N. Dak. & 4 & 1993 & $<0.10$ & $<0.10$ & 0.15 \\
\hline 173 & Porcupine Creek near Fort Yates, N. Dak. & 36 & 1991-1999 & $<0.10$ & $<0.10$ & 0.60 \\
\hline 175 & James River near Manfred, N. Dak. & 39 & $1985-1998$ & $<0.10$ & $<0.10$ & 1.90 \\
\hline 176 & James River near Grace City, N. Dak. & 116 & $1985-2007$ & $<0.10$ & $<0.10$ & 2.50 \\
\hline 177 & James River above Arrowwood Lake near Kensal, N. Dak. & 158 & $1985-2008$ & $<0.10$ & $<0.10$ & 2.30 \\
\hline 178 & Kelly Creek below Niccum Reservoir near Bordulac, N. Dak. & 10 & $1986-1989$ & $<0.10$ & $<0.10$ & 0.70 \\
\hline 182 & James River at Jamestown, N. Dak. & 159 & 1984-2007 & 0.22 & $<0.10$ & 29.60 \\
\hline 183 & James River at Lamoure, N. Dak. & 253 & $1971-2007$ & 0.14 & $<0.10$ & 4.72 \\
\hline 184 & Bear Creek near Oakes, N. Dak. & 9 & 1998-1999 & 0.22 & $<0.10$ & 2.42 \\
\hline 185 & James River at Oakes, N. Dak. & 123 & $1971-2008$ & $<0.10$ & $<0.10$ & 2.94 \\
\hline 186 & James River at N. Dak./S. Dak. State line & 84 & $1974-2004$ & $<0.10$ & $<0.10$ & 3.00 \\
\hline \multicolumn{7}{|c|}{ Ammonia plus organic nitrogen, dissolved as $\mathrm{N}$, in $\mathrm{mg} / \mathrm{L}$} \\
\hline 1 & Bois De Sioux River near Doran, Minn. & 11 & $2006-2007$ & 1.02 & 0.80 & 1.19 \\
\hline 2 & Red River of the North at Wahpeton, N. Dak. & 3 & $1991-2004$ & 0.50 & 0.48 & 1.10 \\
\hline 3 & Red River of the North near Wahpeton, N. Dak. & 1 & 2006 & 0.61 & 0.61 & 0.61 \\
\hline 15 & Sheyenne River above Harvey, N. Dak. & 24 & $1986-2004$ & 1.10 & 0.20 & 1.60 \\
\hline 17 & Sheyenne River at Warwick, N. Dak. & 4 & $2005-2006$ & 1.08 & 0.96 & 1.43 \\
\hline 18 & Sheyenne River near Warwick, N. Dak. & 25 & $1993-2007$ & 1.00 & 0.40 & 1.73 \\
\hline 20 & Mauvais Coulee near Cando, N. Dak. & 3 & 2004 & 1.41 & 0.63 & 1.77 \\
\hline 21 & Edmore Coulee near Edmore, N. Dak. & 3 & 2004 & 1.38 & 0.71 & 2.26 \\
\hline 24 & Starkweather Coulee near Webster, N. Dak. & 2 & 2004 & 1.14 & 1.04 & 1.24 \\
\hline 32 & Sheyenne River near Cooperstown, N. Dak. & 91 & 1979-2007 & 1.00 & $<0.20$ & 2.40 \\
\hline 33 & Baldhill Creek near Dazey, N. Dak. & 38 & $1979-1981$ & 0.88 & 0.42 & 2.30 \\
\hline 34 & Sheyenne River below Baldhill Dam, N. Dak. & 53 & 1979-2007 & 1.11 & $<0.20$ & 2.90 \\
\hline 36 & Sheyenne River at Lisbon, N. Dak. & 49 & $1986-2007$ & $<0.20$ & 0.20 & 1.90 \\
\hline 37 & Sheyenne River near Kindred, N. Dak. & 156 & $1976-2007$ & 0.63 & $<0.20$ & 1.80 \\
\hline 38 & $\begin{array}{l}\text { Sheyenne River above Sheyenne River Diversion near Horace, } \\
\text { N. Dak. }\end{array}$ & 2 & 2003-2004 & 0.49 & 0.46 & 0.52 \\
\hline 41 & Sheyenne River at West Fargo, N. Dak. & 2 & $1991-2006$ & 1.05 & 0.81 & 1.30 \\
\hline 45 & Maple River below Mapleton, N. Dak. & 13 & $2005-2007$ & 0.88 & 0.47 & 1.14 \\
\hline
\end{tabular}


Table 1-2. Summary statistics for nutrient constituents at selected sites in North Dakota from 1970 through 2008. - Continued

[N, nitrogen; mg/L, milligrams per liter; <, less than; --, not available; P, phosphorus; $\mathrm{C}$, carbon]

\begin{tabular}{|c|c|c|c|c|c|c|}
\hline $\begin{array}{c}\text { Site } \\
\text { identification } \\
\text { number } \\
\text { (table 1) }\end{array}$ & Site name & $\begin{array}{c}\text { Number of } \\
\text { samples }\end{array}$ & $\begin{array}{l}\text { Period of } \\
\text { record }\end{array}$ & Median & Minimum & Maximum \\
\hline \multicolumn{7}{|c|}{ Ammonia plus organic nitrogen, dissolved as $\mathrm{N}$, in $\mathrm{mg} / \mathrm{L}$-Continued } \\
\hline 52 & Red River of the North at Halstad, Minn. & 64 & $1978-2004$ & 0.95 & 0.50 & 2.90 \\
\hline 53 & Beaver Creek near Finley, N. Dak. & 10 & 1981-1982 & 1.50 & 1.00 & 2.90 \\
\hline 55 & Goose River at Hillsboro, N. Dak. & 25 & 1994-2008 & 0.77 & 0.57 & 1.07 \\
\hline 56 & Red River of the North at Grand Forks, N. Dak. & 66 & $1991-2008$ & 0.77 & 0.49 & 1.50 \\
\hline 57 & Turtle River at Turtle River State Park near Arvilla, N. Dak. & 82 & $1993-2000$ & 0.51 & 0.22 & 1.52 \\
\hline 58 & Turtle River at Manvel, N. Dak. & 29 & $2005-2008$ & 0.79 & 0.60 & 1.38 \\
\hline 59 & Red River of the North at Oslo, Minn. & 5 & 1977-2004 & 0.89 & 0.69 & 1.30 \\
\hline 62 & Forest River near Minto, N. Dak. & 30 & $2005-2008$ & 0.55 & 0.24 & 1.21 \\
\hline 67 & Park River at Grafton, N. Dak. & 30 & $2005-2008$ & 0.68 & 0.29 & 1.22 \\
\hline 68 & Red River of the North at Drayton, N. Dak. & 3 & $1991-2004$ & 0.71 & 0.65 & 1.10 \\
\hline 73 & Little South Pembina River near Walhalla, N. Dak. & 11 & 1994-1995 & 0.70 & 0.30 & 1.20 \\
\hline 74 & Pembina River at Walhalla, N. Dak. & 83 & 1979-1995 & 0.73 & $<0.20$ & 3.90 \\
\hline 75 & Pembina River at Neche, N. Dak. & 30 & $2005-2008$ & 0.68 & 0.30 & 1.17 \\
\hline 76 & Tongue River at Akra, N. Dak. & 8 & 1979-1980 & 0.93 & 0.30 & 1.30 \\
\hline 78 & Red River of the North at Pembina, N. Dak., site 2 & 85 & 1994-2008 & 0.76 & 0.54 & 1.61 \\
\hline 79 & Red River of the North at Emerson, Manitoba & 79 & 1978-2004 & 0.97 & $<0.20$ & 2.80 \\
\hline 81 & West Branch Short Creek near Columbus, N. Dak. & 2 & 1981 & 1.13 & 0.96 & 1.30 \\
\hline 82 & Souris River near Sherwood, N. Dak. & 9 & 1978-2007 & 1.10 & 0.85 & 2.93 \\
\hline 84 & Des Lacs River at Foxholm, N. Dak. & 14 & $2006-2007$ & 1.26 & 0.65 & 2.28 \\
\hline 85 & Souris River above Minot, N. Dak. & 24 & $1981-2007$ & 1.41 & 0.84 & 2.70 \\
\hline 87 & Souris River near Verendrye, N. Dak. & 16 & $1987-2007$ & 1.39 & 0.89 & 3.84 \\
\hline 89 & Souris River near Bantry, N. Dak. & 8 & $1982-1983$ & 1.55 & 0.80 & 2.10 \\
\hline 98 & Souris River near Westhope, N. Dak. & 46 & $1978-1983$ & 1.65 & 0.71 & 3.70 \\
\hline 101 & Little Muddy River below Cow Creek near Williston, N. Dak. & 9 & 1999 & 0.62 & $<0.20$ & 0.83 \\
\hline 102 & Stony Creek near Williston, N. Dak. & 2 & 1981 & 1.08 & 0.56 & 1.60 \\
\hline 104 & Beaver Creek near Ray, N. Dak. & 8 & $1981-1982$ & 0.76 & 0.43 & 1.20 \\
\hline 106 & Bear Den Creek near Mandaree, N. Dak. & 20 & 1980-1991 & 1.10 & 0.60 & 1.70 \\
\hline 110 & Little Missouri River at Marmarth, N. Dak. & 8 & 1999 & 0.37 & 0.28 & 0.59 \\
\hline 111 & Deep Creek near Amidon, N. Dak. & 9 & $1981-1983$ & 0.92 & 0.66 & 1.40 \\
\hline 112 & Little Missouri River at Medora, N. Dak. & 20 & 1999-2007 & 0.38 & $<0.20$ & 0.70 \\
\hline 113 & Beaver Creek near Trotters, N. Dak. & 12 & 1981-1999 & 0.53 & 0.36 & 1.10 \\
\hline 114 & Little Missouri River near Watford City, N. Dak. & 62 & $1977-2007$ & 0.62 & 0.24 & 3.10 \\
\hline 115 & Missouri River at Garrison Dam, N. Dak. & 111 & 1977-2007 & 0.23 & $<0.20$ & 0.88 \\
\hline 116 & Knife River at Manning, N. Dak. & 9 & 1980-1982 & 1.40 & 0.82 & 2.70 \\
\hline 117 & Stray Creek near Manning, N. Dak. & 3 & $1980-1981$ & 1.40 & 0.86 & 2.10 \\
\hline 118 & Knife River at Marshall, N. Dak. & 5 & $1980-1981$ & 1.30 & 1.00 & 3.50 \\
\hline 119 & Elm Creek near Golden Valley, N. Dak. & 2 & 1981 & 0.90 & 0.50 & 1.30 \\
\hline 120 & Knife River near Golden Valley, N. Dak. & 20 & $2005-2007$ & 0.92 & 0.50 & 1.44 \\
\hline 121 & Coyote Creek near Zap, N. Dak. & 13 & 1980-1983 & 1.00 & 0.72 & 2.70 \\
\hline 122 & Brush Creek near Beulah, N. Dak. & 59 & $1976-1988$ & 0.80 & $<0.20$ & 4.40 \\
\hline
\end{tabular}


Table 1-2. Summary statistics for nutrient constituents at selected sites in North Dakota from 1970 through 2008. —Continued

[N, nitrogen; mg/L, milligrams per liter; <, less than; --, not available; $\mathrm{P}$, phosphorus; $\mathrm{C}$, carbon]

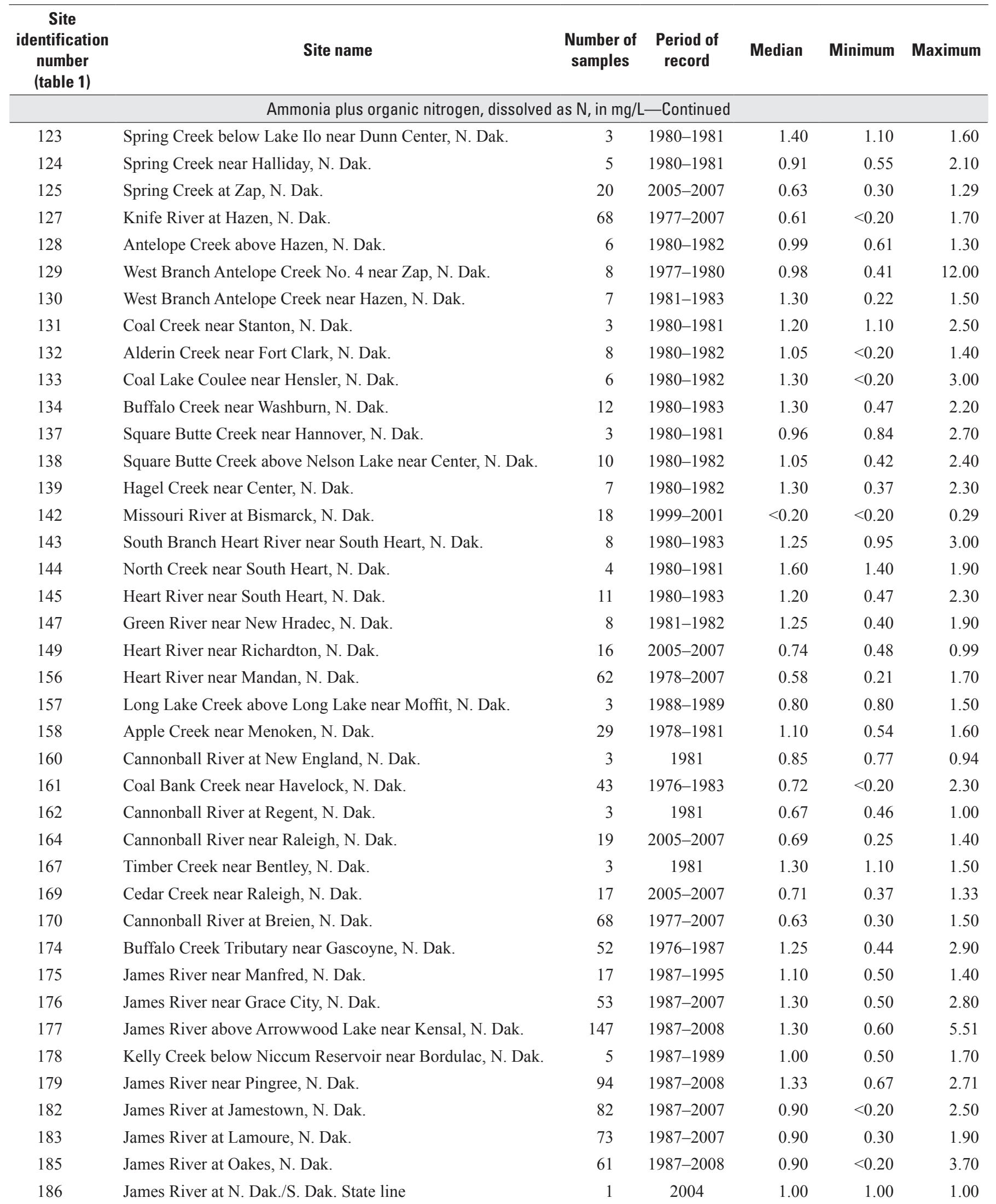


Table 1-2. Summary statistics for nutrient constituents at selected sites in North Dakota from 1970 through 2008. - Continued

[N, nitrogen; mg/L, milligrams per liter; <, less than; --, not available; P, phosphorus; $\mathrm{C}$, carbon]

\begin{tabular}{|c|c|c|c|c|c|c|}
\hline $\begin{array}{c}\text { Site } \\
\text { identification } \\
\text { number } \\
\text { (table 1) }\end{array}$ & Site name & $\begin{array}{c}\text { Number of } \\
\text { samples }\end{array}$ & $\begin{array}{l}\text { Period of } \\
\text { record }\end{array}$ & Median & Minimum & Maximum \\
\hline \multicolumn{7}{|c|}{ Total nitrogen, dissolved as $\mathrm{N}$, in $\mathrm{mg} / \mathrm{L}$} \\
\hline 1 & Bois De Sioux River near Doran, Minn. & 11 & 2006-2007 & 1.20 & 0.90 & 1.90 \\
\hline 3 & Red River of the North near Wahpeton, N. Dak. & 1 & 2006 & 0.63 & 0.63 & 0.63 \\
\hline 4 & Red River of the North at Brushville, Minn. & 12 & 2000-2007 & 0.94 & 0.53 & 1.51 \\
\hline 10 & Wild Rice River near Abercrombie, N. Dak. & 12 & 2006-2007 & 1.05 & 0.75 & 1.69 \\
\hline 11 & Red River of the North at Fargo, N. Dak. & 28 & $2006-2008$ & 1.14 & 0.60 & 2.77 \\
\hline 13 & Red River of the North at Harwood, N. Dak. & 12 & 2006-2007 & 1.92 & 1.20 & 3.19 \\
\hline 17 & Sheyenne River at Warwick, N. Dak. & 4 & 2005-2006 & 1.13 & 1.04 & 1.78 \\
\hline 18 & Sheyenne River near Warwick, N. Dak. & 16 & 2005-2007 & 0.97 & 0.53 & 1.47 \\
\hline 32 & Sheyenne River near Cooperstown, N. Dak. & 19 & 2005-2007 & 0.80 & 0.51 & 1.48 \\
\hline 34 & Sheyenne River below Baldhill Dam, N. Dak. & 19 & 2005-2007 & 1.16 & 0.86 & 2.30 \\
\hline 36 & Sheyenne River at Lisbon, N. Dak. & 19 & 2005-2007 & 0.99 & 0.48 & 1.77 \\
\hline 37 & Sheyenne River near Kindred, N. Dak. & 11 & 2006-2007 & 0.55 & 0.37 & 1.38 \\
\hline 41 & Sheyenne River at West Fargo, N. Dak. & 1 & 2006 & 0.83 & 0.83 & 0.83 \\
\hline 45 & Maple River below Mapleton, N. Dak. & 11 & 2006-2007 & 0.90 & 0.57 & 3.08 \\
\hline 55 & Goose River at Hillsboro, N. Dak. & 19 & $2006-2008$ & 0.86 & 0.59 & 2.49 \\
\hline 56 & Red River of the North at Grand Forks, N. Dak. & 25 & 2006-2008 & 1.28 & 0.55 & 4.40 \\
\hline 58 & Turtle River at Manvel, N. Dak. & 22 & 2006-2008 & 0.85 & 0.63 & 2.16 \\
\hline 62 & Forest River near Minto, N. Dak. & 23 & 2006-2008 & 0.92 & 0.52 & 2.61 \\
\hline 67 & Park River at Grafton, N. Dak. & 23 & 2006-2008 & 1.27 & 0.55 & 4.08 \\
\hline 75 & Pembina River at Neche, N. Dak. & 23 & 2006-2008 & 0.76 & 0.35 & 4.81 \\
\hline 78 & Red River of the North at Pembina, N. Dak., site 2 & 24 & $2006-2008$ & 1.32 & 0.64 & 4.47 \\
\hline 84 & Des Lacs River at Foxholm, N. Dak. & 14 & 2006-2007 & 1.28 & 0.67 & 2.32 \\
\hline 85 & Souris River above Minot, N. Dak. & 13 & 2006-2007 & 1.43 & 1.08 & 2.16 \\
\hline 87 & Souris River near Verendrye, N. Dak. & 14 & $2006-2007$ & 1.52 & 1.07 & 4.37 \\
\hline 101 & Little Muddy River below Cow Creek near Williston, N. Dak. & 9 & 1999 & 0.75 & 0.02 & 1.22 \\
\hline 110 & Little Missouri River at Marmarth, N. Dak. & 8 & 1999 & 0.44 & 0.29 & 0.91 \\
\hline 112 & Little Missouri River at Medora, N. Dak. & 20 & 1999-2007 & 0.43 & 0.22 & 1.64 \\
\hline 113 & Beaver Creek near Trotters, N. Dak. & 9 & 1999 & 0.57 & 0.37 & 1.37 \\
\hline 114 & Little Missouri River near Watford City, N. Dak. & 20 & 1999-2007 & 0.55 & 0.27 & 1.67 \\
\hline 120 & Knife River near Golden Valley, N. Dak. & 20 & $2005-2007$ & 0.97 & 0.52 & 1.65 \\
\hline 125 & Spring Creek at Zap, N. Dak. & 20 & 2005-2007 & 0.69 & 0.33 & 1.58 \\
\hline 127 & Knife River at Hazen, N. Dak. & 19 & 2005-2007 & 0.70 & 0.38 & 1.46 \\
\hline 149 & Heart River near Richardton, N. Dak. & 16 & $2005-2007$ & 0.78 & 0.50 & 1.02 \\
\hline 156 & Heart River near Mandan, N. Dak. & 20 & 2005-2007 & 0.50 & 0.34 & 1.14 \\
\hline 164 & Cannonball River near Raleigh, N. Dak. & 19 & 2005-2007 & 0.82 & 0.29 & 4.15 \\
\hline 169 & Cedar Creek near Raleigh, N. Dak. & 17 & 2005-2007 & 0.79 & 0.39 & 4.98 \\
\hline 170 & Cannonball River at Breien, N. Dak. & 18 & 2005-2007 & 0.61 & 0.39 & 4.72 \\
\hline 176 & James River near Grace City, N. Dak. & 20 & 2005-2007 & 1.37 & 0.54 & 2.18 \\
\hline 182 & James River at Jamestown, N. Dak. & 20 & $2005-2007$ & 1.39 & 0.36 & 3.75 \\
\hline 183 & James River at Lamoure, N. Dak. & 19 & 2005-2007 & 1.04 & 0.57 & 4.62 \\
\hline
\end{tabular}


Table 1-2. Summary statistics for nutrient constituents at selected sites in North Dakota from 1970 through 2008. —Continued

[N, nitrogen; mg/L, milligrams per liter; <, less than; --, not available; $\mathrm{P}$, phosphorus; $\mathrm{C}$, carbon]

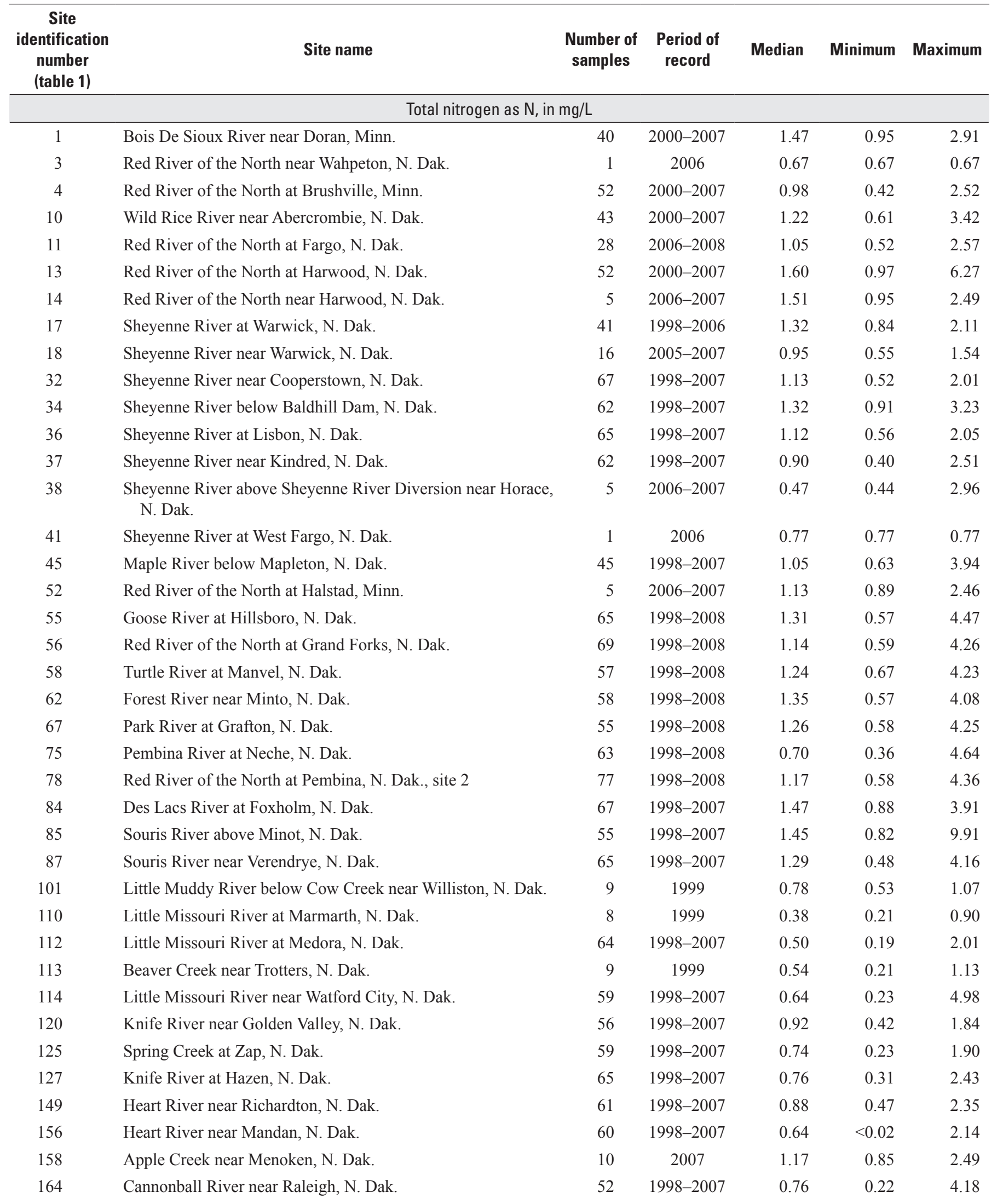


Table 1-2. Summary statistics for nutrient constituents at selected sites in North Dakota from 1970 through 2008. - Continued

$[\mathrm{N}$, nitrogen; mg/L, milligrams per liter; <, less than; --, not available; $\mathrm{P}$, phosphorus; $\mathrm{C}$, carbon]

\begin{tabular}{|c|c|c|c|c|c|c|}
\hline $\begin{array}{c}\text { Site } \\
\text { identification } \\
\text { number } \\
\text { (table 1) } \\
\end{array}$ & Site name & $\begin{array}{c}\text { Number of } \\
\text { samples }\end{array}$ & $\begin{array}{l}\text { Period of } \\
\text { record }\end{array}$ & Median & Minimum & Maximum \\
\hline \multicolumn{7}{|c|}{ Total nitrogen as $\mathrm{N}$, in $\mathrm{mg} / \mathrm{L}$-Continued } \\
\hline 169 & Cedar Creek near Raleigh, N. Dak. & 45 & 1998-2007 & 0.84 & $<0.02$ & 4.81 \\
\hline 170 & Cannonball River at Breien, N. Dak. & 54 & 1998-2007 & 0.69 & 0.34 & 4.73 \\
\hline 175 & James River near Manfred, N. Dak. & 6 & 1998 & 1.21 & 1.08 & 1.63 \\
\hline 176 & James River near Grace City, N. Dak. & 56 & 1998-2007 & 1.64 & 0.55 & 3.02 \\
\hline 177 & James River above Arrowwood Lake near Kensal, N. Dak. & 9 & 1998-1999 & 1.22 & 0.98 & 1.90 \\
\hline 180 & Pipestem Creek near Pingree, N. Dak. & 9 & 1998-1999 & 1.51 & 0.92 & 2.32 \\
\hline 182 & James River at Jamestown, N. Dak. & 70 & 1998-2007 & 1.61 & 0.44 & 30.50 \\
\hline 183 & James River at Lamoure, N. Dak. & 65 & 1998-2007 & 1.57 & 0.83 & 6.48 \\
\hline 184 & Bear Creek near Oakes, N. Dak. & 9 & 1998-1999 & 1.69 & 1.22 & 3.74 \\
\hline 186 & James River at N. Dak./S. Dak. State line & 9 & 1998-1999 & 1.31 & 0.91 & 2.16 \\
\hline \multicolumn{7}{|c|}{ Phosphorus, dissolved as $\mathrm{P}$, in $\mathrm{mg} / \mathrm{L}$} \\
\hline 1 & Bois De Sioux River near Doran, Minn. & 11 & $2006-2007$ & 0.16 & $<0.01$ & 0.27 \\
\hline 2 & Red River of the North at Wahpeton, N. Dak. & 3 & 1991-2004 & 0.02 & $<0.01$ & 0.06 \\
\hline 3 & Red River of the North near Wahpeton, N. Dak. & 1 & 2006 & $<0.01$ & $<0.01$ & $<0.01$ \\
\hline 4 & Red River of the North at Brushville, Minn. & 12 & $2000-2007$ & 0.09 & 0.02 & 0.18 \\
\hline 5 & Red River of the North below Wahpeton, N. Dak. & 26 & 1970-1999 & 0.09 & $<0.01$ & 0.28 \\
\hline 6 & Red River of the North at Hickson, N. Dak. & 93 & 1975-2004 & 0.08 & $<0.01$ & 0.84 \\
\hline 7 & Wild Rice River near Rutland, N. Dak. & 7 & $1971-1975$ & 0.10 & $<0.01$ & 0.39 \\
\hline 8 & Wild Rice River near Cayuga, N. Dak. & 3 & 1970 & 0.12 & 0.10 & 0.41 \\
\hline 10 & Wild Rice River near Abercrombie, N. Dak. & 126 & 1970-2007 & 0.25 & $<0.01$ & 2.00 \\
\hline 11 & Red River of the North at Fargo, N. Dak. & 94 & $1970-2008$ & 0.11 & $<0.01$ & 2.40 \\
\hline 12 & Red River of North below Fargo, N. Dak. & 87 & 1970-1986 & 0.15 & $<0.01$ & 1.20 \\
\hline 13 & Red River of the North at Harwood, N. Dak. & 12 & $2006-2007$ & 0.22 & 0.11 & 0.48 \\
\hline 14 & Red River of the North near Harwood, N. Dak. & 30 & $1997-2007$ & 0.17 & $<0.01$ & 0.35 \\
\hline 15 & Sheyenne River above Harvey, N. Dak. & 135 & $1977-2004$ & 0.20 & $<0.01$ & 0.75 \\
\hline 16 & Big Coulee near Fort Totten, N. Dak. & 1 & 1970 & 0.04 & 0.04 & 0.04 \\
\hline 17 & Sheyenne River at Warwick, N. Dak. & 4 & 2005-2006 & 0.21 & 0.15 & 0.23 \\
\hline 18 & Sheyenne River near Warwick, N. Dak. & 129 & 1970-2007 & 0.13 & $<0.01$ & 0.98 \\
\hline 20 & Mauvais Coulee near Cando, N. Dak. & 4 & 1993-2004 & 0.28 & 0.23 & 0.29 \\
\hline 21 & Edmore Coulee near Edmore, N. Dak. & 5 & 1983-2004 & 0.21 & 0.04 & 0.53 \\
\hline 24 & Starkweather Coulee near Webster, N. Dak. & 2 & 2004 & 0.29 & 0.14 & 0.45 \\
\hline 27 & Little Coulee near Brinsmade, N. Dak. & 16 & 1976-1981 & 0.36 & 0.10 & 1.00 \\
\hline 28 & Big Coulee near Churchs Ferry, N. Dak. & 74 & 1970-1979 & 0.26 & $<0.01$ & 7.30 \\
\hline 29 & Big Coulee at Graham Island inlet near Fort Totten, N. Dak. & 43 & 1970-1986 & 0.28 & 0.04 & 1.50 \\
\hline 30 & Channel A near Penn, N. Dak. & 4 & $1992-1993$ & 0.55 & 0.04 & 0.75 \\
\hline 32 & Sheyenne River near Cooperstown, N. Dak. & 200 & 1970-2007 & 0.16 & $<0.01$ & 0.78 \\
\hline 33 & Baldhill Creek near Dazey, N. Dak. & 38 & 1979-1981 & 0.04 & $<0.01$ & 0.36 \\
\hline 34 & Sheyenne River below Baldhill Dam, N. Dak. & 63 & 1979-2007 & 0.22 & 0.04 & 0.43 \\
\hline 36 & Sheyenne River at Lisbon, N. Dak. & 213 & $1970-2007$ & 0.11 & $<0.01$ & 0.49 \\
\hline 37 & Sheyenne River near Kindred, N. Dak. & 223 & $1976-2007$ & 0.07 & $<0.01$ & 0.30 \\
\hline
\end{tabular}


Table 1-2. Summary statistics for nutrient constituents at selected sites in North Dakota from 1970 through 2008. —Continued

[N, nitrogen; mg/L, milligrams per liter; <, less than; --, not available; $\mathrm{P}$, phosphorus; $\mathrm{C}$, carbon]

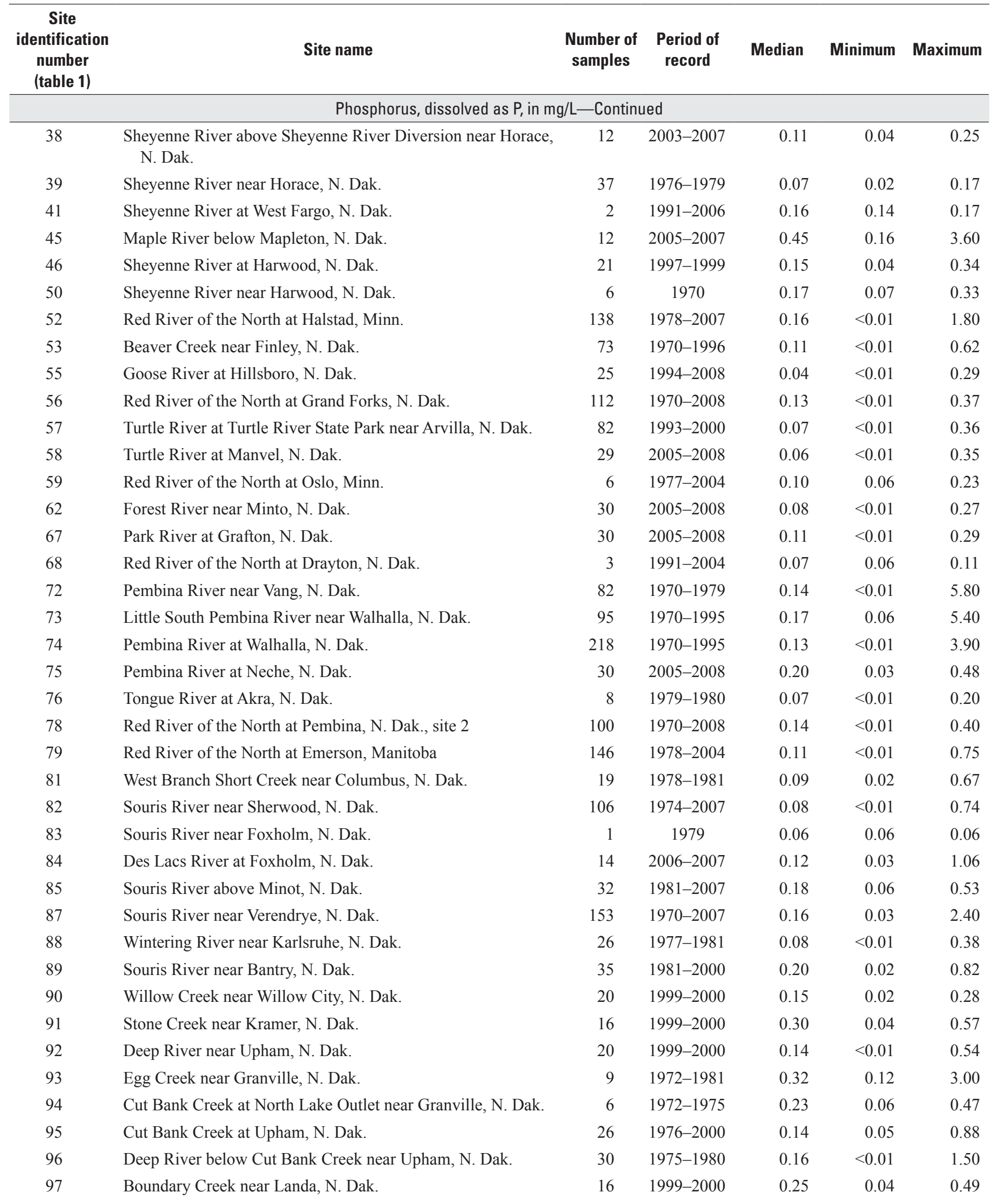


Table 1-2. Summary statistics for nutrient constituents at selected sites in North Dakota from 1970 through 2008. - Continued

[N, nitrogen; mg/L, milligrams per liter; <, less than; --, not available; P, phosphorus; $\mathrm{C}$, carbon]

\begin{tabular}{|c|c|c|c|c|c|c|}
\hline $\begin{array}{c}\text { Site } \\
\text { identification } \\
\text { number } \\
\text { (table 1) }\end{array}$ & Site name & $\begin{array}{l}\text { Number of } \\
\text { samples }\end{array}$ & $\begin{array}{l}\text { Period of } \\
\text { record }\end{array}$ & Median & Minimum & Maximum \\
\hline \multicolumn{7}{|c|}{ Phosphorus, dissolved as $\mathrm{P}$, in $\mathrm{mg} / \mathrm{L}$-Continued } \\
\hline 98 & Souris River near Westhope, N. Dak. & 188 & $1970-1994$ & 0.18 & $<0.01$ & 3.00 \\
\hline 100 & Missouri River near Williston, N. Dak. & 6 & 1970 & 0.06 & 0.03 & 0.10 \\
\hline 101 & Little Muddy River below Cow Creek near Williston, N. Dak. & 9 & 1999 & 0.05 & 0.02 & 0.31 \\
\hline 102 & Stony Creek near Williston, N. Dak. & 28 & $1977-1981$ & 0.03 & $<0.01$ & 0.82 \\
\hline 104 & Beaver Creek near Ray, N. Dak. & 42 & $1977-1982$ & 0.02 & $<0.01$ & 0.21 \\
\hline 106 & Bear Den Creek near Mandaree, N. Dak. & 130 & $1970-1996$ & 0.02 & $<0.01$ & 0.88 \\
\hline 110 & Little Missouri River at Marmarth, N. Dak. & 10 & 1973-1999 & 0.05 & $<0.01$ & 0.17 \\
\hline 111 & Deep Creek near Amidon, N. Dak. & 46 & $1977-1983$ & $<0.01$ & $<0.01$ & 0.11 \\
\hline 112 & Little Missouri River at Medora, N. Dak. & 22 & 1974-2007 & 0.02 & $<0.01$ & 0.56 \\
\hline 113 & Beaver Creek near Trotters, N. Dak. & 49 & 1977-1999 & $<0.01$ & $<0.01$ & 0.16 \\
\hline 114 & Little Missouri River near Watford City, N. Dak. & 110 & 1977-2007 & $<0.01$ & $<0.01$ & 0.45 \\
\hline 115 & Missouri River at Garrison Dam, N. Dak. & 241 & 1971-2007 & $<0.01$ & $<0.01$ & 0.21 \\
\hline 116 & Knife River at Manning, N. Dak. & 45 & $1977-1982$ & 0.03 & $<0.01$ & 0.17 \\
\hline 117 & Stray Creek near Manning, N. Dak. & 14 & $1978-1981$ & 0.06 & 0.02 & 0.47 \\
\hline 118 & Knife River at Marshall, N. Dak. & 41 & $1977-1981$ & 0.02 & $<0.01$ & 0.14 \\
\hline 119 & Elm Creek near Golden Valley, N. Dak. & 15 & $1977-1981$ & 0.10 & 0.03 & 0.44 \\
\hline 120 & Knife River near Golden Valley, N. Dak. & 20 & $2005-2007$ & 0.03 & $<0.01$ & 0.12 \\
\hline 121 & Coyote Creek near Zap, N. Dak. & 43 & $1977-1983$ & 0.02 & $<0.01$ & 0.34 \\
\hline 122 & Brush Creek near Beulah, N. Dak. & 71 & $1976-1988$ & 0.03 & $<0.01$ & 0.29 \\
\hline 123 & Spring Creek below Lake Ilo near Dunn Center, N. Dak. & 39 & $1977-1981$ & 0.05 & $<0.01$ & 0.34 \\
\hline 124 & Spring Creek near Halliday, N. Dak. & 41 & $1977-1981$ & 0.02 & $<0.01$ & 0.34 \\
\hline 125 & Spring Creek at Zap, N. Dak. & 21 & 1977-2007 & $<0.01$ & $<0.01$ & 0.13 \\
\hline 127 & Knife River at Hazen, N. Dak. & 116 & $1977-2007$ & 0.02 & $<0.01$ & 0.37 \\
\hline 128 & Antelope Creek above Hazen, N. Dak. & 26 & $1977-1982$ & 0.07 & $<0.01$ & 0.63 \\
\hline 129 & West Branch Antelope Creek No. 4 near Zap, N. Dak. & 1 & 1977 & 0.10 & 0.10 & 0.10 \\
\hline 130 & West Branch Antelope Creek near Hazen, N. Dak. & 14 & $1978-1983$ & 0.19 & 0.06 & 0.63 \\
\hline 131 & Coal Creek near Stanton, N. Dak. & 24 & 1977-1981 & 0.06 & $<0.01$ & 0.36 \\
\hline 132 & Alderin Creek near Fort Clark, N. Dak. & 29 & 1977-1982 & 0.03 & $<0.01$ & 0.26 \\
\hline 133 & Coal Lake Coulee near Hensler, N. Dak. & 16 & 1978-1982 & 0.07 & 0.04 & 0.43 \\
\hline 134 & Buffalo Creek near Washburn, N. Dak. & 32 & $1978-1983$ & 0.05 & $<0.01$ & 0.51 \\
\hline 136 & Painted Woods Creek near Wilton, N. Dak. & 63 & $1970-1982$ & 0.07 & $<0.01$ & 2.10 \\
\hline 137 & Square Butte Creek near Hannover, N. Dak. & 21 & $1977-1981$ & 0.05 & $<0.01$ & 0.41 \\
\hline 138 & Square Butte Creek above Nelson Lake near Center, N. Dak. & 48 & 1977-1982 & 0.01 & $<0.01$ & 0.43 \\
\hline 139 & Hagel Creek near Center, N. Dak. & 31 & $1977-1982$ & 0.06 & $<0.01$ & 0.27 \\
\hline 142 & Missouri River at Bismarck, N. Dak. & 30 & $1970-2001$ & $<0.01$ & $<0.01$ & 1.00 \\
\hline 143 & South Branch Heart River near South Heart, N. Dak. & 17 & $1979-1983$ & 0.10 & $<0.01$ & 0.52 \\
\hline 144 & North Creek near South Heart, N. Dak. & 14 & $1978-1981$ & 0.06 & 0.02 & 0.34 \\
\hline 145 & Heart River near South Heart, N. Dak. & 47 & $1977-1983$ & 0.10 & $<0.01$ & 0.35 \\
\hline 147 & Green River near New Hradec, N. Dak. & 44 & $1977-1982$ & 0.02 & $<0.01$ & 0.17 \\
\hline 149 & Heart River near Richardton, N. Dak. & 16 & $2005-2007$ & $<0.01$ & $<0.01$ & 0.07 \\
\hline
\end{tabular}


Table 1-2. Summary statistics for nutrient constituents at selected sites in North Dakota from 1970 through 2008. —Continued

[N, nitrogen; mg/L, milligrams per liter; <, less than; --, not available; $\mathrm{P}$, phosphorus; $\mathrm{C}$, carbon]

\begin{tabular}{|c|c|c|c|c|c|c|}
\hline $\begin{array}{c}\text { Site } \\
\text { identification } \\
\text { number } \\
\text { (table 1) } \\
\end{array}$ & Site name & $\begin{array}{l}\text { Number of } \\
\text { samples }\end{array}$ & $\begin{array}{l}\text { Period of } \\
\text { record }\end{array}$ & Median & Minimum & Maximum \\
\hline 156 & Heart River near Mandan, N. Dak. & 126 & $1978-2007$ & $<0.01$ & $<0.01$ & 0.10 \\
\hline 157 & Long Lake Creek above Long Lake near Moffit, N. Dak. & 3 & $1988-1989$ & 0.22 & 0.09 & 0.71 \\
\hline 159 & Missouri River near Schmidt, N. Dak. & 1 & 1978 & 0.03 & 0.03 & 0.03 \\
\hline 160 & Cannonball River at New England, N. Dak. & 28 & $1978-1981$ & 0.02 & $<0.01$ & 0.16 \\
\hline 161 & Coal Bank Creek near Havelock, N. Dak. & 54 & $1976-1983$ & 0.02 & $<0.01$ & 0.10 \\
\hline 162 & Cannonball River at Regent, N. Dak. & 41 & 1977-1981 & $<0.01$ & $<0.01$ & 0.69 \\
\hline 164 & Cannonball River near Raleigh, N. Dak. & 19 & $2005-2007$ & $<0.01$ & $<0.01$ & 0.08 \\
\hline 169 & Cedar Creek near Raleigh, N. Dak. & 17 & $2005-2007$ & 0.02 & $<0.01$ & 0.09 \\
\hline 170 & Cannonball River at Breien, N. Dak. & 113 & $1977-2007$ & $<0.01$ & $<0.01$ & 0.17 \\
\hline 174 & Buffalo Creek Tributary near Gascoyne, N. Dak. & 60 & $1976-1987$ & 0.03 & $<0.01$ & 0.27 \\
\hline 175 & James River near Manfred, N. Dak. & 33 & $1985-1995$ & 0.23 & 0.02 & 0.84 \\
\hline 176 & James River near Grace City, N. Dak. & 76 & $1985-2007$ & 0.18 & $<0.01$ & 0.69 \\
\hline 177 & James River above Arrowwood Lake near Kensal, N. Dak. & 150 & $1985-2008$ & 0.13 & $<0.01$ & 1.36 \\
\hline 178 & Kelly Creek below Niccum Reservoir near Bordulac, N. Dak. & 9 & 1986-1989 & 0.07 & 0.02 & 0.41 \\
\hline 179 & James River near Pingree, N. Dak. & 132 & $1978-2008$ & 0.11 & $<0.01$ & 1.08 \\
\hline 182 & James River at Jamestown, N. Dak. & 104 & 1984-2007 & 0.09 & $<0.01$ & 2.91 \\
\hline 3 & Red River of the North near Wahpeton, N. Dak. & 5 & $1993-2006$ & 0.09 & 0.02 & 0.33 \\
\hline 4 & Red River of the North at Brushville, Minn. & 61 & $1993-2007$ & 0.16 & 0.03 & 0.54 \\
\hline 5 & Red River of the North below Wahpeton, N. Dak. & 64 & 1970-1999 & 0.15 & 0.03 & 0.40 \\
\hline 6 & Red River of the North at Hickson, N. Dak. & 102 & $1975-2004$ & 0.18 & 0.03 & 1.20 \\
\hline 10 & Wild Rice River near Abercrombie, N. Dak. & 63 & $1993-2007$ & 0.21 & 0.03 & 0.68 \\
\hline 11 & Red River of the North at Fargo, N. Dak. & 77 & 1994-2008 & 0.19 & 0.04 & 1.28 \\
\hline 12 & Red River of North below Fargo, N. Dak. & 96 & $1970-1977$ & 0.47 & 0.11 & 11.00 \\
\hline 13 & Red River of the North at Harwood, N. Dak. & 52 & $2000-2007$ & 0.30 & 0.11 & 1.41 \\
\hline 14 & Red River of the North near Harwood, N. Dak. & 59 & $1993-2007$ & 0.27 & 0.05 & 0.48 \\
\hline 15 & Sheyenne River above Harvey, N. Dak. & 2 & $2003-2004$ & 0.22 & 0.21 & 0.23 \\
\hline 17 & Sheyenne River at Warwick, N. Dak. & 48 & 1996-2006 & 0.20 & 0.03 & 0.50 \\
\hline 18 & Sheyenne River near Warwick, N. Dak. & 25 & $1993-2007$ & 0.21 & 0.03 & 0.51 \\
\hline 20 & Mauvais Coulee near Cando, N. Dak. & 60 & 1989-2004 & 0.33 & 0.06 & 0.78 \\
\hline 21 & Edmore Coulee near Edmore, N. Dak. & 62 & 1989-2004 & 0.40 & 0.08 & 1.12 \\
\hline 22 & Edmore Coulee Tributary near Webster, N. Dak. & 2 & 1993 & 0.31 & 0.27 & 0.34 \\
\hline
\end{tabular}


Table 1-2. Summary statistics for nutrient constituents at selected sites in North Dakota from 1970 through 2008. - Continued

[N, nitrogen; mg/L, milligrams per liter; <, less than; --, not available; P, phosphorus; $\mathrm{C}$, carbon]

\begin{tabular}{|c|c|c|c|c|c|c|}
\hline $\begin{array}{c}\text { Site } \\
\text { identification } \\
\text { number } \\
\text { (table 1) }\end{array}$ & Site name & $\begin{array}{c}\text { Number of } \\
\text { samples }\end{array}$ & $\begin{array}{l}\text { Period of } \\
\text { record }\end{array}$ & Median & Minimum & Maximum \\
\hline \multicolumn{7}{|c|}{ Phosphorus, total as $\mathrm{P}$, in $\mathrm{mg} / \mathrm{L}-$ Continued } \\
\hline 24 & Starkweather Coulee near Webster, N. Dak. & 56 & 1989-2004 & 0.29 & 0.02 & 1.19 \\
\hline 25 & Big Coulee below Churchs Ferry, N. Dak. & 9 & 1998-1999 & 0.38 & 0.22 & 0.99 \\
\hline 28 & Big Coulee near Churchs Ferry, N. Dak. & 50 & 1989-1997 & 0.40 & 0.15 & 0.92 \\
\hline 30 & Channel A near Penn, N. Dak. & 59 & 1989-1999 & 0.30 & 0.06 & 0.98 \\
\hline 32 & Sheyenne River near Cooperstown, N. Dak. & 173 & 1979-2007 & 0.21 & $<0.01$ & 0.57 \\
\hline 33 & Baldhill Creek near Dazey, N. Dak. & 51 & 1979-1996 & 0.14 & 0.03 & 0.69 \\
\hline 34 & Sheyenne River below Baldhill Dam, N. Dak. & 129 & 1979-2007 & 0.24 & 0.05 & 0.46 \\
\hline 35 & Sheyenne River at Valley City, N. Dak. & 11 & 1993 & 0.28 & 0.12 & 0.46 \\
\hline 36 & Sheyenne River at Lisbon, N. Dak. & 99 & 1993-2007 & 0.22 & 0.06 & 1.20 \\
\hline 37 & Sheyenne River near Kindred, N. Dak. & 283 & $1976-2007$ & 0.18 & 0.02 & 1.80 \\
\hline 38 & $\begin{array}{l}\text { Sheyenne River above Sheyenne River Diversion near Horace, } \\
\text { N. Dak. }\end{array}$ & 15 & 2003-2007 & 0.22 & 0.08 & 0.72 \\
\hline 41 & Sheyenne River at West Fargo, N. Dak. & 13 & 1994-2006 & 0.24 & 0.04 & 0.48 \\
\hline 45 & Maple River below Mapleton, N. Dak. & 49 & $1997-2007$ & 0.26 & 0.04 & 11.10 \\
\hline 46 & Sheyenne River at Harwood, N. Dak. & 31 & 1993-1999 & 0.38 & 0.07 & 0.89 \\
\hline 49 & Lower Branch Rush River near Prosper, N. Dak. & 2 & 1993 & 0.25 & 0.23 & 0.27 \\
\hline 50 & Sheyenne River near Harwood, N. Dak. & 44 & 1970-1974 & 0.30 & 0.12 & 0.81 \\
\hline 51 & Elm River near Kelso, N. Dak. & 4 & 1993 & 0.51 & 0.37 & 0.80 \\
\hline 52 & Red River of the North at Halstad, Minn. & 140 & $1978-2007$ & 0.28 & 0.07 & 2.70 \\
\hline 53 & Beaver Creek near Finley, N. Dak. & 82 & 1974-1996 & 0.18 & 0.04 & 0.67 \\
\hline 55 & Goose River at Hillsboro, N. Dak. & 90 & 1994-2008 & 0.09 & 0.02 & 0.45 \\
\hline 56 & Red River of the North at Grand Forks, N. Dak. & 114 & 1970-2008 & 0.18 & 0.03 & 0.68 \\
\hline 57 & Turtle River at Turtle River State Park near Arvilla, N. Dak. & 83 & $1993-2000$ & 0.09 & 0.02 & 1.27 \\
\hline 58 & Turtle River at Manvel, N. Dak. & 69 & $1993-2008$ & 0.15 & $<0.01$ & 0.50 \\
\hline 59 & Red River of the North at Oslo, Minn. & 56 & 1973-2004 & 0.22 & 0.08 & 1.50 \\
\hline 62 & Forest River near Minto, N. Dak. & 78 & 1994-2008 & 0.11 & $<0.01$ & 0.44 \\
\hline 67 & Park River at Grafton, N. Dak. & 71 & 1994-2008 & 0.14 & 0.02 & 0.58 \\
\hline 68 & Red River of the North at Drayton, N. Dak. & 17 & 1994-2004 & 0.17 & 0.08 & 0.52 \\
\hline 73 & Little South Pembina River near Walhalla, N. Dak. & 11 & 1994-1995 & 0.29 & 0.16 & 0.46 \\
\hline 74 & Pembina River at Walhalla, N. Dak. & 85 & 1979-1995 & 0.23 & $<0.01$ & 1.40 \\
\hline 75 & Pembina River at Neche, N. Dak. & 86 & 1994-2008 & 0.26 & 0.04 & 1.34 \\
\hline 76 & Tongue River at Akra, N. Dak. & 25 & 1979-2004 & 0.14 & 0.04 & 0.43 \\
\hline 77 & Red River of the North at Pembina, N. Dak., site 1 & 10 & 1993 & 0.25 & 0.08 & 0.51 \\
\hline 78 & Red River of the North at Pembina, N. Dak., site 2 & 169 & $1970-2008$ & 0.25 & 0.02 & 1.14 \\
\hline 79 & Red River of the North at Emerson, Manitoba & 149 & 1978-2004 & 0.19 & 0.02 & 0.88 \\
\hline 80 & Long Creek near Noonan, N. Dak. & 10 & 1997 & 0.17 & 0.08 & 0.51 \\
\hline 81 & West Branch Short Creek near Columbus, N. Dak. & 18 & $1978-1981$ & 0.17 & 0.07 & 0.68 \\
\hline 82 & Souris River near Sherwood, N. Dak. & 341 & 1974-2008 & 0.18 & 0.02 & 1.90 \\
\hline 83 & Souris River near Foxholm, N. Dak. & 184 & 1972-1998 & 0.24 & $<0.01$ & 2.20 \\
\hline 84 & Des Lacs River at Foxholm, N. Dak. & 138 & 1981-2007 & 0.27 & 0.05 & 1.70 \\
\hline
\end{tabular}


Table 1-2. Summary statistics for nutrient constituents at selected sites in North Dakota from 1970 through 2008.—Continued

[N, nitrogen; mg/L, milligrams per liter; <, less than; --, not available; $\mathrm{P}$, phosphorus; $\mathrm{C}$, carbon]

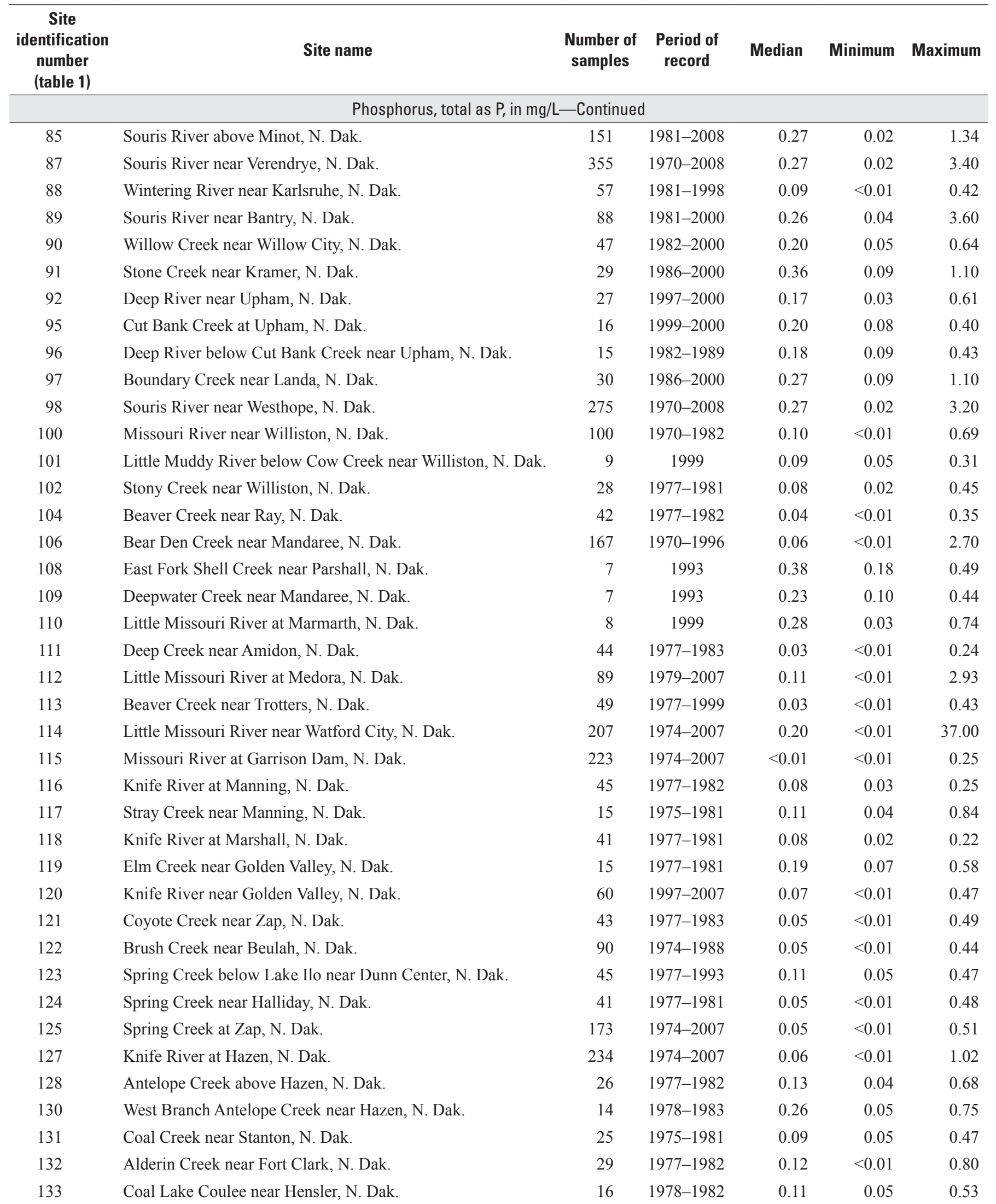


Table 1-2. Summary statistics for nutrient constituents at selected sites in North Dakota from 1970 through 2008. - Continued

$[\mathrm{N}$, nitrogen; mg/L, milligrams per liter; <, less than; --, not available; $\mathrm{P}$, phosphorus; $\mathrm{C}$, carbon]

\begin{tabular}{|c|c|c|c|c|c|c|}
\hline $\begin{array}{c}\text { Site } \\
\text { identification } \\
\text { number } \\
\text { (table 1) }\end{array}$ & Site name & $\begin{array}{l}\text { Number of } \\
\text { samples }\end{array}$ & $\begin{array}{l}\text { Period of } \\
\text { record }\end{array}$ & Median & Minimum & Maximum \\
\hline \multicolumn{7}{|c|}{ Phosphorus, total as $\mathrm{P}$, in $\mathrm{mg} / \mathrm{L}-$ Continued } \\
\hline 134 & Buffalo Creek near Washburn, N. Dak. & 31 & $1978-1983$ & 0.10 & 0.04 & 0.56 \\
\hline 136 & Painted Woods Creek near Wilton, N. Dak. & 1 & 1970 & 0.07 & 0.07 & 0.07 \\
\hline 137 & Square Butte Creek near Hannover, N. Dak. & 21 & $1977-1981$ & 0.05 & 0.03 & 0.47 \\
\hline 138 & Square Butte Creek above Nelson Lake near Center, N. Dak. & 48 & $1977-1982$ & 0.05 & $<0.01$ & 0.52 \\
\hline 139 & Hagel Creek near Center, N. Dak. & 31 & $1977-1982$ & 0.11 & 0.03 & 0.83 \\
\hline 140 & Square Butte Creek below Center, N. Dak. & 4 & 1993 & 0.03 & $<0.01$ & 0.10 \\
\hline 142 & Missouri River at Bismarck, N. Dak. & 197 & 1970-2001 & 0.03 & $<0.01$ & 1.50 \\
\hline 143 & South Branch Heart River near South Heart, N. Dak. & 28 & 1979-1996 & 0.28 & 0.06 & 7.40 \\
\hline 144 & North Creek near South Heart, N. Dak. & 22 & 1978-1996 & 0.25 & 0.05 & 0.91 \\
\hline 145 & Heart River near South Heart, N. Dak. & 48 & $1975-1983$ & 0.20 & 0.06 & 1.90 \\
\hline 146 & Heart River at Dickinson, N. Dak. & 5 & 1993 & 0.10 & 0.03 & 0.26 \\
\hline 147 & Green River near New Hradec, N. Dak. & 44 & 1977-1982 & 0.07 & $<0.01$ & 0.34 \\
\hline 148 & Green River near Gladstone, N. Dak. & 4 & 1993 & 0.06 & $<0.01$ & 0.27 \\
\hline 149 & Heart River near Richardton, N. Dak. & 76 & 1994-2007 & 0.06 & $<0.01$ & 0.22 \\
\hline 152 & Big Muddy Creek near Almont, N. Dak. & 3 & 1993 & 0.32 & 0.29 & 0.36 \\
\hline 156 & Heart River near Mandan, N. Dak. & 187 & $1978-2007$ & 0.03 & $<0.01$ & 0.84 \\
\hline 157 & Long Lake Creek above Long Lake near Moffit, N. Dak. & 3 & 1988-1989 & 0.34 & 0.15 & 0.96 \\
\hline 158 & Apple Creek near Menoken, N. Dak. & 56 & $1977-2007$ & 0.36 & 0.13 & 2.00 \\
\hline 159 & Missouri River near Schmidt, N. Dak. & 140 & 1974-1981 & 0.04 & $<0.01$ & 0.70 \\
\hline 160 & Cannonball River at New England, N. Dak. & 28 & $1978-1981$ & 0.05 & $<0.01$ & 0.42 \\
\hline 161 & Coal Bank Creek near Havelock, N. Dak. & 76 & $1974-1983$ & 0.04 & $<0.01$ & 0.47 \\
\hline 162 & Cannonball River at Regent, N. Dak. & 40 & 1977-1981 & 0.04 & $<0.01$ & 0.14 \\
\hline 164 & Cannonball River near Raleigh, N. Dak. & 76 & 1993-2007 & 0.03 & $<0.01$ & 0.51 \\
\hline 167 & Timber Creek near Bentley, N. Dak. & 39 & $1977-1981$ & 0.04 & $<0.01$ & 1.30 \\
\hline 169 & Cedar Creek near Raleigh, N. Dak. & 68 & $1993-2007$ & 0.04 & $<0.01$ & 0.42 \\
\hline 170 & Cannonball River at Breien, N. Dak. & 204 & 1974-2007 & 0.05 & $<0.01$ & 1.10 \\
\hline 171 & Beaver Creek near Linton, N. Dak. & 4 & 1993 & 0.50 & 0.30 & 0.77 \\
\hline 174 & Buffalo Creek Tributary near Gascoyne, N. Dak. & 77 & 1974-1987 & 0.09 & 0.03 & 0.66 \\
\hline 175 & James River near Manfred, N. Dak. & 20 & $1986-1998$ & 0.28 & 0.09 & 0.62 \\
\hline 176 & James River near Grace City, N. Dak. & 84 & $1986-2007$ & 0.26 & 0.06 & 0.73 \\
\hline 177 & James River above Arrowwood Lake near Kensal, N. Dak. & 82 & 1986-2008 & 0.25 & 0.06 & 1.11 \\
\hline 178 & Kelly Creek below Niccum Reservoir near Bordulac, N. Dak. & 8 & $1986-1989$ & 0.13 & 0.08 & 0.48 \\
\hline 179 & James River near Pingree, N. Dak. & 59 & $1986-2008$ & 0.34 & 0.08 & 1.46 \\
\hline 180 & Pipestem Creek near Pingree, N. Dak. & 17 & 1994-1999 & 0.30 & 0.12 & 0.72 \\
\hline 181 & Pipestem Creek near Buchanan, N. Dak. & 5 & 1993 & 0.47 & 0.29 & 0.71 \\
\hline 182 & James River at Jamestown, N. Dak. & 104 & 1986-2007 & 0.33 & 0.06 & 14.10 \\
\hline 183 & James River at Lamoure, N. Dak. & 103 & 1984-2007 & 0.50 & 0.09 & 4.08 \\
\hline 184 & Bear Creek near Oakes, N. Dak. & 9 & 1998-1999 & 0.35 & 0.16 & 1.01 \\
\hline 185 & James River at Oakes, N. Dak. & 79 & $1970-2008$ & 0.33 & 0.08 & 1.53 \\
\hline 186 & James River at N. Dak./S. Dak. State line & 10 & 1998-2004 & 0.33 & 0.19 & 0.79 \\
\hline
\end{tabular}


Table 1-2. Summary statistics for nutrient constituents at selected sites in North Dakota from 1970 through 2008. —Continued

[N, nitrogen; mg/L, milligrams per liter; <, less than; --, not available; $\mathrm{P}$, phosphorus; $\mathrm{C}$, carbon]

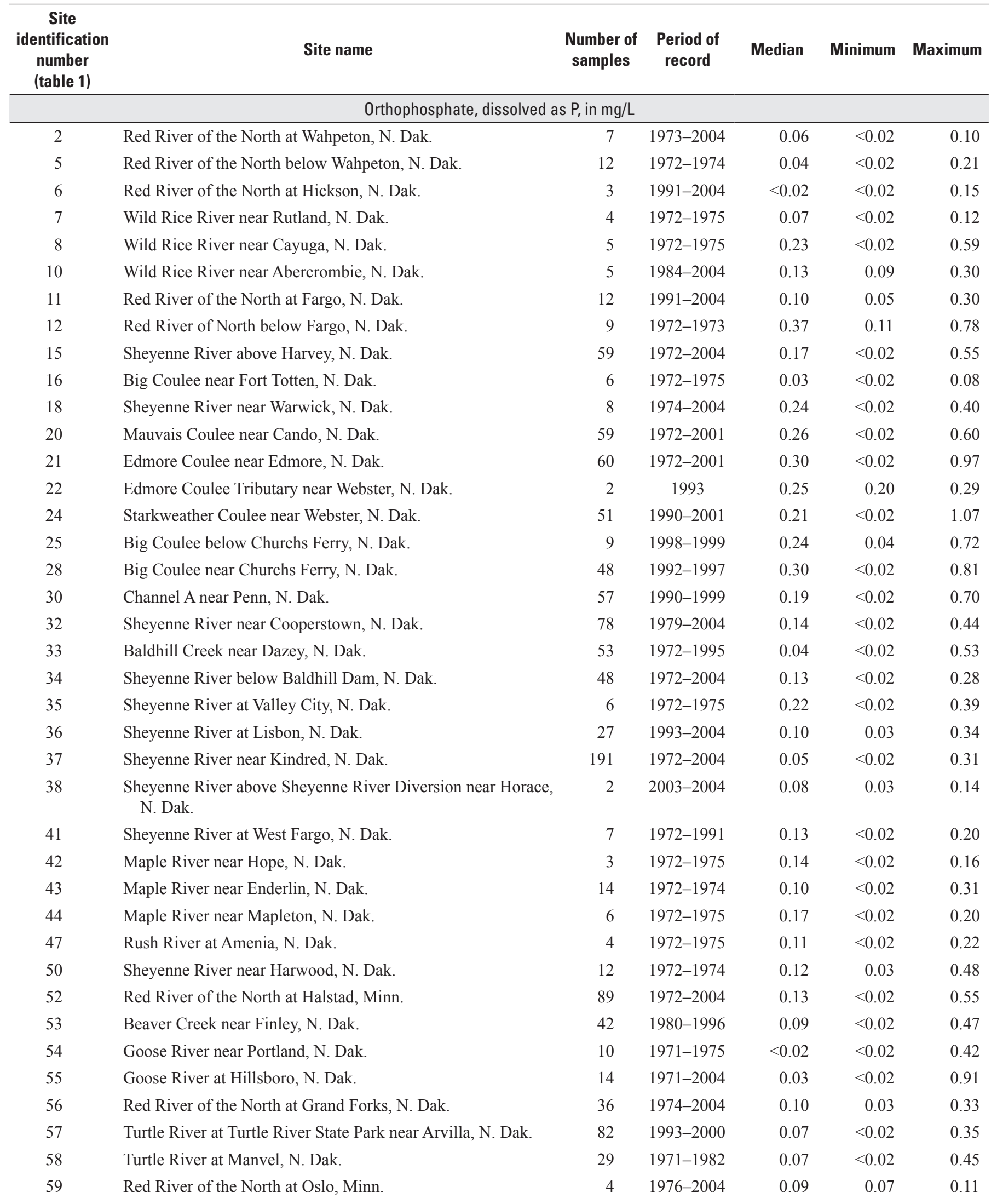


Table 1-2. Summary statistics for nutrient constituents at selected sites in North Dakota from 1970 through 2008. - Continued

[N, nitrogen; mg/L, milligrams per liter; <, less than; --, not available; P, phosphorus; $\mathrm{C}$, carbon]

\begin{tabular}{|c|c|c|c|c|c|c|}
\hline $\begin{array}{c}\text { Site } \\
\text { identification } \\
\text { number } \\
\text { (table 1) } \\
\end{array}$ & Site name & $\begin{array}{l}\text { Number of } \\
\text { samples }\end{array}$ & $\begin{array}{l}\text { Period of } \\
\text { record }\end{array}$ & Median & Minimum & Maximum \\
\hline \multicolumn{7}{|c|}{ Orthophosphate, dissolved as $\mathrm{P}$, in $\mathrm{mg} / \mathrm{L}$-Continued } \\
\hline 60 & Middle Branch Forest River near Whitman, N. Dak. & 3 & $1972-1975$ & 0.14 & $<0.02$ & 0.23 \\
\hline 61 & Forest River near Fordville, N. Dak. & 12 & $1972-1975$ & 0.04 & $<0.02$ & 0.16 \\
\hline 62 & Forest River near Minto, N. Dak. & 25 & $1971-1975$ & $<0.02$ & $<0.02$ & 0.13 \\
\hline 63 & South Branch Park River below Homme Dam, N. Dak. & 5 & 1972-1981 & 0.19 & $<0.02$ & 0.22 \\
\hline 64 & Middle Branch Park River near Union, N. Dak. & 6 & $1972-1980$ & 0.11 & $<0.02$ & 0.36 \\
\hline 65 & Middle Branch Park River near Edinburg, N. Dak. & 12 & $1978-1980$ & 0.20 & 0.03 & 0.47 \\
\hline 66 & Cart Creek at Mountain, N. Dak. & 11 & $1972-1979$ & 0.14 & $<0.02$ & 0.28 \\
\hline 67 & Park River at Grafton, N. Dak. & 7 & $1971-1975$ & 0.04 & $<0.02$ & 0.25 \\
\hline 68 & Red River of the North at Drayton, N. Dak. & 7 & 1972-2004 & 0.11 & $<0.02$ & 0.17 \\
\hline 69 & Pembina County Drain 20 near Glasston, N. Dak. & 2 & 1974-1975 & 0.13 & 0.05 & 0.22 \\
\hline 70 & Hidden Island Coulee near Hansboro, N. Dak. & 4 & $1972-1975$ & 0.08 & $<0.02$ & 0.39 \\
\hline 71 & Cypress Creek near Sarles, N. Dak. & 3 & $1972-1975$ & 0.11 & $<0.02$ & 0.39 \\
\hline 72 & Pembina River near Vang, N. Dak. & 51 & 1976-1979 & 0.11 & 0.03 & 0.49 \\
\hline 73 & Little South Pembina River near Walhalla, N. Dak. & 65 & 1973-1995 & 0.13 & 0.03 & 0.58 \\
\hline 74 & Pembina River at Walhalla, N. Dak. & 143 & $1976-1995$ & 0.10 & $<0.02$ & 0.57 \\
\hline 75 & Pembina River at Neche, N. Dak. & 5 & $1972-1975$ & 0.13 & $<0.02$ & 0.20 \\
\hline 76 & Tongue River at Akra, N. Dak. & 20 & $1972-1980$ & 0.06 & $<0.02$ & 0.16 \\
\hline 78 & Red River of the North at Pembina, N. Dak., site 2 & 60 & $1972-2000$ & 0.12 & $<0.02$ & 0.34 \\
\hline 79 & Red River of the North at Emerson, Manitoba & 104 & 1981-2004 & 0.10 & $<0.02$ & 0.35 \\
\hline 80 & Long Creek near Noonan, N. Dak. & 16 & 1972-1984 & 0.06 & $<0.02$ & 0.29 \\
\hline 82 & Souris River near Sherwood, N. Dak. & 76 & $1972-2007$ & 0.08 & $<0.02$ & 0.90 \\
\hline 83 & Souris River near Foxholm, N. Dak. & 136 & 1972-1991 & 0.10 & $<0.02$ & 1.20 \\
\hline 84 & Des Lacs River at Foxholm, N. Dak. & 43 & 1972-1991 & 0.12 & $<0.02$ & 1.20 \\
\hline 85 & Souris River above Minot, N. Dak. & 21 & 1971-2007 & 0.11 & $<0.02$ & 0.48 \\
\hline 87 & Souris River near Verendrye, N. Dak. & 133 & $1972-2007$ & 0.15 & $<0.02$ & 2.70 \\
\hline 88 & Wintering River near Karlsruhe, N. Dak. & 57 & $1972-1991$ & 0.04 & $<0.02$ & 0.33 \\
\hline 89 & Souris River near Bantry, N. Dak. & 17 & 1971-1983 & 0.08 & $<0.02$ & 0.82 \\
\hline 90 & Willow Creek near Willow City, N. Dak. & 23 & 1972-1989 & 0.10 & $<0.02$ & 0.29 \\
\hline 91 & Stone Creek near Kramer, N. Dak. & 8 & $1986-1989$ & 0.37 & 0.19 & 1.00 \\
\hline 92 & Deep River near Upham, N. Dak. & 6 & $1972-1975$ & 0.04 & $<0.02$ & 0.07 \\
\hline 93 & Egg Creek near Granville, N. Dak. & 4 & 1972-1974 & 0.88 & $<0.02$ & 1.11 \\
\hline 94 & Cut Bank Creek at North Lake Outlet near Granville, N. Dak. & 3 & $1972-1973$ & 0.25 & $<0.02$ & 0.91 \\
\hline 95 & Cut Bank Creek at Upham, N. Dak. & 1 & 1975 & 0.07 & 0.07 & 0.07 \\
\hline 96 & Deep River below Cut Bank Creek near Upham, N. Dak. & 12 & $1986-1989$ & 0.08 & 0.04 & 0.33 \\
\hline 97 & Boundary Creek near Landa, N. Dak. & 14 & $1972-1990$ & 0.11 & $<0.02$ & 0.46 \\
\hline 98 & Souris River near Westhope, N. Dak. & 119 & 1972-1994 & 0.11 & $<0.02$ & 2.90 \\
\hline 99 & Charbonneau Creek near Charbonneau, N. Dak. & 6 & $1972-1975$ & 0.03 & $<0.02$ & 0.07 \\
\hline 100 & Missouri River near Williston, N. Dak. & 16 & 1975-1976 & $<0.02$ & $<0.02$ & 0.06 \\
\hline 101 & Little Muddy River below Cow Creek near Williston, N. Dak. & 16 & $1972-1975$ & $<0.02$ & $<0.02$ & 0.09 \\
\hline 105 & White Earth River at White Earth, N. Dak. & 5 & $1973-1975$ & 0.18 & 0.10 & 0.30 \\
\hline
\end{tabular}


Table 1-2. Summary statistics for nutrient constituents at selected sites in North Dakota from 1970 through 2008. —Continued

[N, nitrogen; mg/L, milligrams per liter; <, less than; --, not available; $\mathrm{P}$, phosphorus; $\mathrm{C}$, carbon]

\begin{tabular}{|c|c|c|c|c|c|c|}
\hline $\begin{array}{c}\text { Site } \\
\text { identification } \\
\text { number } \\
\text { (table 1) }\end{array}$ & Site name & $\begin{array}{c}\text { Number of } \\
\text { samples }\end{array}$ & $\begin{array}{l}\text { Period of } \\
\text { record }\end{array}$ & Median & Minimum & Maximum \\
\hline \multicolumn{7}{|c|}{ Orthophosphate, dissolved as $\mathrm{P}$, in $\mathrm{mg} / \mathrm{L}$-Continued } \\
\hline 106 & Bear Den Creek near Mandaree, N. Dak. & 82 & $1979-1996$ & $<0.02$ & $<0.02$ & 0.42 \\
\hline 107 & Shell Creek near Parshall, N. Dak. & 6 & $1972-1975$ & 0.05 & $<0.02$ & 0.15 \\
\hline 108 & East Fork Shell Creek near Parshall, N. Dak. & 47 & $1991-2000$ & 0.21 & $<0.02$ & 0.64 \\
\hline 109 & Deepwater Creek near Mandaree, N. Dak. & 44 & $1991-2000$ & 0.08 & $<0.02$ & 0.39 \\
\hline 110 & Little Missouri River at Marmarth, N. Dak. & 15 & $1972-1975$ & 0.04 & $<0.02$ & 0.16 \\
\hline 112 & Little Missouri River at Medora, N. Dak. & 4 & $1972-1974$ & 0.04 & $<0.02$ & 0.13 \\
\hline 114 & Little Missouri River near Watford City, N. Dak. & 57 & 1971-1994 & $<0.02$ & $<0.02$ & 0.11 \\
\hline 115 & Missouri River at Garrison Dam, N. Dak. & 132 & $1977-2007$ & $<0.02$ & $<0.02$ & 0.06 \\
\hline 116 & Knife River at Manning, N. Dak. & 16 & $1972-1975$ & 0.06 & $<0.02$ & 0.22 \\
\hline 118 & Knife River at Marshall, N. Dak. & 14 & $1972-1975$ & 0.04 & $<0.02$ & 0.11 \\
\hline 119 & Elm Creek near Golden Valley, N. Dak. & 4 & $1973-1975$ & 0.07 & 0.06 & 0.10 \\
\hline 120 & Knife River near Golden Valley, N. Dak. & 17 & $1971-1975$ & $<0.02$ & $<0.02$ & 0.10 \\
\hline 122 & Brush Creek near Beulah, N. Dak. & 16 & $1975-1990$ & $<0.02$ & $<0.02$ & 0.08 \\
\hline 125 & Spring Creek at Zap, N. Dak. & 30 & $1972-1976$ & $<0.02$ & $<0.02$ & 0.08 \\
\hline 126 & West Branch Otter Creek near Beulah, N. Dak. & 3 & $1972-1974$ & $<0.02$ & $<0.02$ & 0.03 \\
\hline 127 & Knife River at Hazen, N. Dak. & 58 & $1971-1993$ & $<0.02$ & $<0.02$ & 0.32 \\
\hline 134 & Buffalo Creek near Washburn, N. Dak. & 1 & 1981 & 0.08 & 0.08 & 0.08 \\
\hline 135 & Turtle Creek above Washburn, N. Dak. & 96 & $1987-2003$ & 0.08 & $<0.02$ & 0.59 \\
\hline 136 & Painted Woods Creek near Wilton, N. Dak. & 129 & $1982-2003$ & $<0.02$ & $<0.02$ & 0.39 \\
\hline 140 & Square Butte Creek below Center, N. Dak. & 16 & $1972-1983$ & $<0.02$ & $<0.02$ & 0.04 \\
\hline 141 & Burnt Creek near Bismarck, N. Dak. & 4 & $1972-1975$ & 0.04 & $<0.02$ & 0.07 \\
\hline 142 & Missouri River at Bismarck, N. Dak. & 22 & 1973-1976 & $<0.02$ & $<0.02$ & 0.09 \\
\hline 145 & Heart River near South Heart, N. Dak. & 1 & 1984 & 0.07 & 0.07 & 0.07 \\
\hline 147 & Green River near New Hradec, N. Dak. & 17 & $1972-1975$ & 0.03 & $<0.02$ & 0.36 \\
\hline 148 & Green River near Gladstone, N. Dak. & 19 & 1971-1974 & $<0.02$ & $<0.02$ & 0.05 \\
\hline 149 & Heart River near Richardton, N. Dak. & 17 & $1972-1975$ & 0.03 & $<0.02$ & 0.11 \\
\hline 151 & Antelope Creek near Carson, N. Dak. & 7 & $1972-1975$ & $<0.02$ & $<0.02$ & 0.04 \\
\hline 153 & Heart River near Lark, N. Dak. & 18 & $1971-1975$ & $<0.02$ & $<0.02$ & 0.03 \\
\hline 155 & Sweetbriar Creek near Judson, N. Dak. & 6 & $1972-1975$ & $<0.02$ & $<0.02$ & 0.09 \\
\hline 156 & Heart River near Mandan, N. Dak. & 80 & $1971-1993$ & $<0.02$ & $<0.02$ & 0.03 \\
\hline 158 & Apple Creek near Menoken, N. Dak. & 63 & 1972-1984 & 0.24 & $<0.02$ & 1.20 \\
\hline 159 & Missouri River near Schmidt, N. Dak. & 14 & $1975-1977$ & $<0.02$ & $<0.02$ & 0.05 \\
\hline 161 & Coal Bank Creek near Havelock, N. Dak. & 17 & 1975-1976 & $<0.02$ & $<0.02$ & 0.10 \\
\hline 162 & Cannonball River at Regent, N. Dak. & 10 & $1971-1975$ & $<0.02$ & $<0.02$ & 0.08 \\
\hline 163 & Cannonball River below Bentley, N. Dak. & 26 & $1972-1975$ & $<0.02$ & $<0.02$ & 0.07 \\
\hline 165 & White Butte Fork Cedar Creek near Scranton, N. Dak. & 5 & $1972-1975$ & $<0.02$ & $<0.02$ & 0.39 \\
\hline 166 & Cedar Creek near Haynes, N. Dak. & 17 & $1972-1975$ & $<0.02$ & $<0.02$ & 0.08 \\
\hline 168 & Cedar Creek near Pretty Rock, N. Dak. & 29 & 1971-1975 & $<0.02$ & $<0.02$ & 0.12 \\
\hline 169 & Cedar Creek near Raleigh, N. Dak. & 6 & $1972-1975$ & 0.03 & $<0.02$ & 0.15 \\
\hline 170 & Cannonball River at Breien, N. Dak. & 59 & 1971-1992 & $<0.02$ & $<0.02$ & 0.10 \\
\hline
\end{tabular}


Table 1-2. Summary statistics for nutrient constituents at selected sites in North Dakota from 1970 through 2008. - Continued

[N, nitrogen; mg/L, milligrams per liter; <, less than; --, not available; P, phosphorus; $\mathrm{C}$, carbon]

\begin{tabular}{|c|c|c|c|c|c|c|}
\hline $\begin{array}{c}\text { Site } \\
\text { identification } \\
\text { number } \\
\text { (table 1) }\end{array}$ & Site name & $\begin{array}{l}\text { Number of } \\
\text { samples }\end{array}$ & $\begin{array}{l}\text { Period of } \\
\text { record }\end{array}$ & Median & Minimum & Maximum \\
\hline 171 & Beaver Creek near Linton, N. Dak. & 16 & 1972-1975 & 0.18 & $<0.02$ & 0.43 \\
\hline 173 & Porcupine Creek near Fort Yates, N. Dak. & 36 & 1991-1999 & $<0.02$ & $<0.02$ & 0.14 \\
\hline 175 & James River near Manfred, N. Dak. & 33 & $1972-1995$ & 0.20 & $<0.02$ & 0.88 \\
\hline 176 & James River near Grace City, N. Dak. & 59 & $1972-1995$ & 0.11 & $<0.02$ & 0.66 \\
\hline 177 & James River above Arrowwood Lake near Kensal, N. Dak. & 155 & $1985-2008$ & 0.09 & $<0.02$ & 1.20 \\
\hline 178 & Kelly Creek below Niccum Reservoir near Bordulac, N. Dak. & 9 & 1986-1989 & 0.04 & $<0.02$ & 0.35 \\
\hline 179 & James River near Pingree, N. Dak. & 103 & $1986-2008$ & 0.10 & $<0.02$ & 0.99 \\
\hline 183 & James River at Lamoure, N. Dak. & 69 & $1981-1995$ & 0.11 & $<0.02$ & 0.41 \\
\hline 185 & James River at Oakes, N. Dak. & 105 & $1972-2008$ & 0.10 & $<0.02$ & 1.47 \\
\hline 186 & James River at N. Dak./S. Dak. State line & 1 & 1981 & 0.28 & 0.28 & 0.28 \\
\hline \multicolumn{7}{|c|}{ Organic carbon, dissolved as $\mathrm{C}$, in $\mathrm{mg} / \mathrm{L}$} \\
\hline 1 & Bois De Sioux River near Doran, Minn. & 11 & 2006-2007 & 12.8 & 7.0 & 13.9 \\
\hline 2 & Red River of the North at Wahpeton, N. Dak. & 1 & 1991 & 8.8 & 8.8 & 8.8 \\
\hline 3 & Red River of the North near Wahpeton, N. Dak. & 1 & 2006 & 8.6 & 8.6 & 8.6 \\
\hline 4 & Red River of the North at Brushville, Minn. & 12 & 2000-2007 & 8.0 & 6.2 & 9.9 \\
\hline 18 & Sheyenne River near Warwick, N. Dak. & 16 & 1993-2007 & 13.3 & 5.9 & 17.0 \\
\hline 32 & Sheyenne River near Cooperstown, N. Dak. & 83 & 1979-2007 & 14.0 & 5.0 & 34.0 \\
\hline 33 & Baldhill Creek near Dazey, N. Dak. & 37 & 1979-1981 & 12.0 & 3.3 & 41.0 \\
\hline 34 & Sheyenne River below Baldhill Dam, N. Dak. & 42 & 1979-2007 & 11.7 & 6.8 & 28.0 \\
\hline 36 & Sheyenne River at Lisbon, N. Dak. & 23 & 1993-2007 & 9.1 & 6.7 & 12.0 \\
\hline 37 & Sheyenne River near Kindred, N. Dak. & 67 & 1976-2007 & 8.9 & 4.7 & 25.0 \\
\hline 41 & Sheyenne River at West Fargo, N. Dak. & 1 & 2006 & 8.7 & 8.7 & 8.7 \\
\hline 45 & Maple River below Mapleton, N. Dak. & 11 & 2006-2007 & 10.5 & 8.1 & 15.0 \\
\hline 52 & Red River of the North at Halstad, Minn. & 37 & 1978-1995 & 10.0 & 7.4 & 39.0 \\
\hline 53 & Beaver Creek near Finley, N. Dak. & 4 & $1981-1982$ & 19.0 & 14.0 & 23.0 \\
\hline 55 & Goose River at Hillsboro, N. Dak. & 22 & 1994-2008 & 8.6 & 6.6 & 13.0 \\
\hline 56 & Red River of the North at Grand Forks, N. Dak. & 33 & $1991-2008$ & 8.8 & 6.3 & 14.0 \\
\hline 57 & Turtle River at Turtle River State Park near Arvilla, N. Dak. & 37 & 1993-1997 & 8.2 & 4.3 & 18.0 \\
\hline 58 & Turtle River at Manvel, N. Dak. & 22 & 2006-2008 & 8.5 & 5.1 & 14.7 \\
\hline 59 & Red River of the North at Oslo, Minn. & 2 & 1977 & 10.9 & 9.7 & 12.0 \\
\hline
\end{tabular}


Table 1-2. Summary statistics for nutrient constituents at selected sites in North Dakota from 1970 through 2008. —Continued

[N, nitrogen; mg/L, milligrams per liter; <, less than; --, not available; $\mathrm{P}$, phosphorus; $\mathrm{C}$, carbon]

\begin{tabular}{|c|c|c|c|c|c|c|}
\hline $\begin{array}{c}\text { Site } \\
\text { identification } \\
\text { number } \\
\text { (table 1) }\end{array}$ & Site name & $\begin{array}{l}\text { Number of } \\
\text { samples }\end{array}$ & $\begin{array}{l}\text { Period of } \\
\text { record }\end{array}$ & Median & Minimum & Maximum \\
\hline 62 & Forest River near Minto, N. Dak. & 33 & 1974-2008 & 6.2 & 3.2 & 19.0 \\
\hline 67 & Park River at Grafton, N. Dak. & 23 & $2006-2008$ & 7.8 & 6.7 & 10.4 \\
\hline 74 & Pembina River at Walhalla, N. Dak. & 66 & 1979-1995 & 11.0 & 2.6 & 36.0 \\
\hline 75 & Pembina River at Neche, N. Dak. & 23 & $2006-2008$ & 7.2 & 4.5 & 11.7 \\
\hline 76 & Tongue River at Akra, N. Dak. & 8 & $1979-1980$ & 16.0 & 8.0 & 150.0 \\
\hline 78 & Red River of the North at Pembina, N. Dak., site 2 & 42 & 1994-2008 & 10.0 & 6.8 & 14.0 \\
\hline 79 & Red River of the North at Emerson, Manitoba & 52 & 1978-1996 & 12.0 & 8.7 & 41.0 \\
\hline 84 & Des Lacs River at Foxholm, N. Dak. & 13 & $2006-2007$ & 16.4 & 10.3 & 24.7 \\
\hline 85 & Souris River above Minot, N. Dak. & 18 & $1981-2007$ & 15.0 & 10.0 & 22.6 \\
\hline 87 & Souris River near Verendrye, N. Dak. & 13 & $2006-2007$ & 14.4 & 11.7 & 21.0 \\
\hline 89 & Souris River near Bantry, N. Dak. & 4 & 1983 & 13.0 & 12.0 & 14.0 \\
\hline 98 & Souris River near Westhope, N. Dak. & 51 & $1978-1983$ & 16.0 & 7.1 & 35.0 \\
\hline 100 & Missouri River near Williston, N. Dak. & 82 & $1975-1982$ & 4.9 & 2.8 & 37.0 \\
\hline 102 & Stony Creek near Williston, N. Dak. & 28 & $1977-1981$ & 13.0 & 8.3 & 97.0 \\
\hline 104 & Beaver Creek near Ray, N. Dak. & 42 & $1977-1982$ & 11.0 & 5.2 & 40.0 \\
\hline 116 & Knife River at Manning, N. Dak. & 44 & $1977-1982$ & 15.0 & 8.4 & 110.0 \\
\hline 117 & Stray Creek near Manning, N. Dak. & 15 & $1975-1981$ & 22.0 & 15.0 & 46.0 \\
\hline 118 & Knife River at Marshall, N. Dak. & 41 & $1977-1981$ & 13.0 & 7.5 & 42.0 \\
\hline 119 & Elm Creek near Golden Valley, N. Dak. & 15 & 1977-1981 & 17.0 & 9.6 & 31.0 \\
\hline 120 & Knife River near Golden Valley, N. Dak. & 14 & $2006-2007$ & 17.0 & 14.0 & 20.6 \\
\hline 121 & Coyote Creek near Zap, N. Dak. & 43 & $1977-1983$ & 12.0 & 0.8 & 43.0 \\
\hline 122 & Brush Creek near Beulah, N. Dak. & 87 & $1974-1988$ & 11.0 & 5.0 & 40.0 \\
\hline 123 & Spring Creek below Lake Ilo near Dunn Center, N. Dak. & 38 & $1977-1981$ & 18.0 & 3.8 & 39.0 \\
\hline 124 & Spring Creek near Halliday, N. Dak. & 40 & $1977-1981$ & 13.5 & 2.1 & 40.0 \\
\hline 125 & Spring Creek at Zap, N. Dak. & 94 & $1975-2007$ & 11.0 & 4.5 & 42.0 \\
\hline 127 & Knife River at Hazen, N. Dak. & 73 & $1975-2007$ & 10.0 & 1.0 & 45.0 \\
\hline 128 & Antelope Creek above Hazen, N. Dak. & 26 & $1977-1982$ & 16.5 & 8.7 & 33.0 \\
\hline 129 & West Branch Antelope Creek No. 4 near Zap, N. Dak. & 1 & 1977 & 9.5 & 9.5 & 9.5 \\
\hline 130 & West Branch Antelope Creek near Hazen, N. Dak. & 14 & $1978-1983$ & 17.0 & 10.0 & 26.0 \\
\hline 131 & Coal Creek near Stanton, N. Dak. & 25 & 1975-1981 & 23.0 & 10.0 & 41.0 \\
\hline
\end{tabular}


Table 1-2. Summary statistics for nutrient constituents at selected sites in North Dakota from 1970 through 2008. - Continued [N, nitrogen; mg/L, milligrams per liter; <, less than; --, not available; P, phosphorus; $\mathrm{C}$, carbon]

\begin{tabular}{|c|c|c|c|c|c|c|}
\hline $\begin{array}{c}\text { Site } \\
\text { identification } \\
\text { number } \\
\text { (table 1) }\end{array}$ & Site name & $\begin{array}{c}\text { Number of } \\
\text { samples }\end{array}$ & $\begin{array}{l}\text { Period of } \\
\text { record }\end{array}$ & Median & Minimum & Maximum \\
\hline \multicolumn{7}{|c|}{ Organic carbon, dissolved as C, in $\mathrm{mg} / \mathrm{L}-$ Continued } \\
\hline 132 & Alderin Creek near Fort Clark, N. Dak. & 28 & $1977-1982$ & 23.5 & 7.6 & 50.0 \\
\hline 133 & Coal Lake Coulee near Hensler, N. Dak. & 16 & 1978-1982 & 18.0 & 7.7 & 29.0 \\
\hline 134 & Buffalo Creek near Washburn, N. Dak. & 32 & $1978-1983$ & 20.0 & 11.0 & 65.0 \\
\hline 137 & Square Butte Creek near Hannover, N. Dak. & 21 & $1977-1981$ & 14.0 & 7.8 & 39.0 \\
\hline 138 & Square Butte Creek above Nelson Lake near Center, N. Dak. & 47 & $1977-1982$ & 10.0 & 5.2 & 47.0 \\
\hline 139 & Hagel Creek near Center, N. Dak. & 30 & $1977-1982$ & 18.0 & 7.6 & 39.0 \\
\hline 142 & Missouri River at Bismarck, N. Dak. & 69 & $1975-1980$ & 3.7 & 2.4 & 15.0 \\
\hline 143 & South Branch Heart River near South Heart, N. Dak. & 17 & $1979-1983$ & 14.0 & 3.8 & 39.0 \\
\hline 144 & North Creek near South Heart, N. Dak. & 14 & 1978-1981 & 21.5 & 8.9 & 42.0 \\
\hline 145 & Heart River near South Heart, N. Dak. & 48 & $1975-1983$ & 17.0 & 1.0 & 44.0 \\
\hline 147 & Green River near New Hradec, N. Dak. & 46 & $1977-1982$ & 12.0 & 5.4 & 28.0 \\
\hline 149 & Heart River near Richardton, N. Dak. & 13 & $2006-2007$ & 11.4 & 9.6 & 15.7 \\
\hline 156 & Heart River near Mandan, N. Dak. & 39 & 1978-2007 & 7.9 & 3.7 & 24.0 \\
\hline 158 & Apple Creek near Menoken, N. Dak. & 11 & $1977-1979$ & 13.0 & 7.3 & 20.0 \\
\hline 159 & Missouri River near Schmidt, N. Dak. & 57 & $1975-1979$ & 3.7 & 2.5 & 10.0 \\
\hline 160 & Cannonball River at New England, N. Dak. & 27 & $1978-1981$ & 17.0 & 7.5 & 66.0 \\
\hline 161 & Coal Bank Creek near Havelock, N. Dak. & 74 & $1974-1983$ & 16.0 & 8.3 & 37.0 \\
\hline 162 & Cannonball River at Regent, N. Dak. & 40 & 1977-1981 & 12.5 & 8.7 & 120.0 \\
\hline 164 & Cannonball River near Raleigh, N. Dak. & 13 & $2006-2007$ & 12.3 & 8.3 & 25.9 \\
\hline 167 & Timber Creek near Bentley, N. Dak. & 37 & $1977-1981$ & 15.0 & 5.5 & 41.0 \\
\hline 169 & Cedar Creek near Raleigh, N. Dak. & 12 & $2006-2007$ & 12.8 & 8.0 & 18.0 \\
\hline 170 & Cannonball River at Breien, N. Dak. & 42 & 1975-2007 & 9.6 & 7.1 & 28.0 \\
\hline 174 & Buffalo Creek Tributary near Gascoyne, N. Dak. & 75 & 1974-1987 & 30.0 & 13.0 & 77.0 \\
\hline 176 & James River near Grace City, N. Dak. & 14 & $2006-2007$ & 17.9 & 5.3 & 26.2 \\
\hline 182 & James River at Jamestown, N. Dak. & 14 & $2006-2007$ & 12.7 & 5.3 & 16.4 \\
\hline 183 & James River at Lamoure, N. Dak. & 14 & $2006-2007$ & 11.4 & 6.3 & 14.8 \\
\hline \multicolumn{7}{|c|}{ Organic carbon, total as $\mathrm{C}$, in $\mathrm{mg} / \mathrm{L}$} \\
\hline 1 & Bois De Sioux River near Doran, Minn. & 13 & 2006-2007 & 11.5 & 6.9 & 14.4 \\
\hline 3 & Red River of the North near Wahpeton, N. Dak. & 1 & 2006 & 8.6 & 8.6 & 8.6 \\
\hline 4 & Red River of the North at Brushville, Minn. & 14 & $2000-2007$ & 7.9 & 6.3 & 9.0 \\
\hline 5 & Red River of the North below Wahpeton, N. Dak. & 2 & 1970 & 9.0 & 9.0 & 9.0 \\
\hline 10 & Wild Rice River near Abercrombie, N. Dak. & 14 & $2006-2007$ & 12.0 & 8.1 & 13.7 \\
\hline 11 & Red River of the North at Fargo, N. Dak. & 27 & $2006-2008$ & 7.8 & 5.6 & 9.6 \\
\hline 12 & Red River of North below Fargo, N. Dak. & 17 & $1970-1977$ & 12.0 & 7.0 & 22.0 \\
\hline 13 & Red River of the North at Harwood, N. Dak. & 14 & $2006-2007$ & 7.7 & 6.7 & 10.2 \\
\hline 17 & Sheyenne River at Warwick, N. Dak. & 2 & 2006 & 11.3 & 10.1 & 12.4 \\
\hline 18 & Sheyenne River near Warwick, N. Dak. & 12 & $2006-2007$ & 11.6 & 5.8 & 16.3 \\
\hline 32 & Sheyenne River near Cooperstown, N. Dak. & 14 & 2006-2007 & 9.6 & 5.0 & 15.2 \\
\hline 34 & Sheyenne River below Baldhill Dam, N. Dak. & 14 & $2006-2007$ & 10.6 & 9.1 & 12.6 \\
\hline
\end{tabular}


Table 1-2. Summary statistics for nutrient constituents at selected sites in North Dakota from 1970 through 2008. —Continued

[N, nitrogen; mg/L, milligrams per liter; <, less than; --, not available; $\mathrm{P}$, phosphorus; $\mathrm{C}$, carbon]

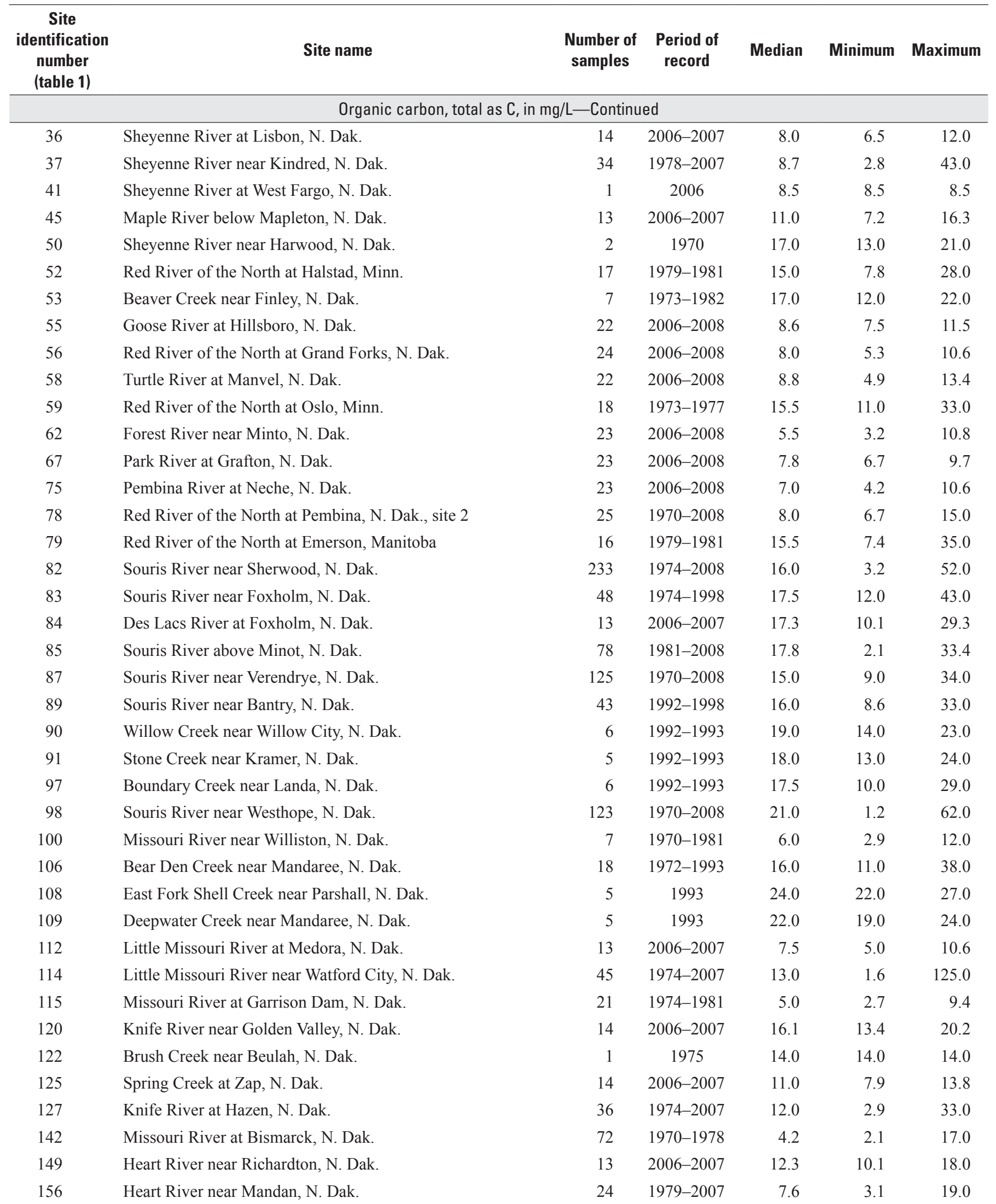




\begin{tabular}{|c|c|c|c|c|c|c|}
\hline $\begin{array}{c}\text { Site } \\
\text { identification } \\
\text { number } \\
\text { (table 1) }\end{array}$ & Site name & $\begin{array}{c}\text { Number of } \\
\text { samples }\end{array}$ & $\begin{array}{l}\text { Period of } \\
\text { record }\end{array}$ & Median & Minimum & Maximum \\
\hline \multicolumn{7}{|c|}{ Organic carbon, total as $\mathrm{C}$, in $\mathrm{mg} / \mathrm{L}-$ Continued } \\
\hline 159 & Missouri River near Schmidt, N. Dak. & 67 & $1974-1978$ & 4.0 & 2.0 & 58.0 \\
\hline 164 & Cannonball River near Raleigh, N. Dak. & 13 & $2006-2007$ & 10.2 & 4.6 & 22.8 \\
\hline 170 & Cannonball River at Breien, N. Dak. & 45 & 1974-2007 & 12.0 & 5.0 & 35.0 \\
\hline 175 & James River near Manfred, N. Dak. & 4 & $1987-1989$ & 14.5 & 14.0 & 17.0 \\
\hline 176 & James River near Grace City, N. Dak. & 22 & $1987-2007$ & 17.6 & 5.5 & 28.4 \\
\hline 177 & James River above Arrowwood Lake near Kensal, N. Dak. & 13 & 1987-1993 & 15.0 & 11.0 & 23.0 \\
\hline 183 & James River at Lamoure, N. Dak. & 36 & 1980-2007 & 13.0 & 5.6 & 27.0 \\
\hline 185 & James River at Oakes, N. Dak. & 22 & 1970-1994 & 14.5 & 4.6 & 22.0 \\
\hline
\end{tabular}


Table 1-3. Summary statistics for trace-metal constituents at selected sites in North Dakota from 1993 through 2008.

[ $\mu \mathrm{g} / \mathrm{L}$, micrograms per liter; --, not available; $<$, less than]

\begin{tabular}{|c|c|c|c|c|c|c|}
\hline $\begin{array}{c}\text { Site } \\
\text { identification } \\
\text { number } \\
\text { (table 1) }\end{array}$ & Site name & $\begin{array}{l}\text { Number of } \\
\text { samples }\end{array}$ & $\begin{array}{l}\text { Period of } \\
\text { record }\end{array}$ & Median & Minimum & Maximum \\
\hline \multicolumn{7}{|c|}{ Aluminum, total, in $\mu \mathrm{g} / \mathrm{L}$} \\
\hline 1 & Bois De Sioux River near Doran, Minn. & 40 & $2000-2007$ & 705 & 113 & 5,090 \\
\hline 3 & Red River of the North near Wahpeton, N. Dak. & 1 & 2006 & 238 & 238 & 238 \\
\hline 10 & Wild Rice River near Abercrombie, N. Dak. & 43 & $2000-2007$ & 1,530 & 138 & 6,640 \\
\hline 11 & Red River of the North at Fargo, N. Dak. & 1 & 2006 & $<100$ & $<100$ & $<100$ \\
\hline 13 & Red River of the North at Harwood, N. Dak. & 53 & $2000-2007$ & 2,800 & $<100$ & 15,400 \\
\hline 14 & Red River of the North near Harwood, N. Dak. & 5 & 2006-2007 & 3,380 & 1,750 & 11,300 \\
\hline 34 & Sheyenne River below Baldhill Dam, N. Dak. & 65 & $1997-2007$ & 103 & $<100$ & 2,150 \\
\hline 36 & Sheyenne River at Lisbon, N. Dak. & 69 & 1997-2007 & 870 & $<100$ & 9,090 \\
\hline 37 & Sheyenne River near Kindred, N. Dak. & 65 & 1997-2007 & 1,190 & 117 & 15,900 \\
\hline 38 & $\begin{array}{l}\text { Sheyenne River above Sheyenne River Diversion near Horace, } \\
\text { N. Dak. }\end{array}$ & 5 & 2006-2007 & 3,140 & 752 & 12,600 \\
\hline 41 & Sheyenne River at West Fargo, N. Dak. & 1 & 2006 & 1,720 & 1,720 & 1,720 \\
\hline 45 & Maple River below Mapleton, N. Dak. & 47 & $1997-2007$ & 1,570 & 153 & 12,000 \\
\hline 52 & Red River of the North at Halstad, Minn. & 5 & $2006-2007$ & 4,720 & 2,600 & 13,100 \\
\hline 55 & Goose River at Hillsboro, N. Dak. & 61 & $1997-2007$ & 777 & $<100$ & 8,690 \\
\hline 80 & Long Creek near Noonan, N. Dak. & 10 & 1997 & 160 & $<100$ & 606 \\
\hline 82 & Souris River near Sherwood, N. Dak. & 97 & 1993-2008 & 154 & $<100$ & 1,084 \\
\hline 83 & Souris River near Foxholm, N. Dak. & 28 & 1993-1998 & $<100$ & $<100$ & 384 \\
\hline 84 & Des Lacs River at Foxholm, N. Dak. & 78 & $1997-2007$ & 515 & 194 & 3,920 \\
\hline 85 & Souris River above Minot, N. Dak. & 122 & 1997-2008 & $<100$ & $<100$ & 1,013 \\
\hline 87 & Souris River near Verendrye, N. Dak. & 162 & 1993-2008 & 313 & $<100$ & 13,000 \\
\hline 88 & Wintering River near Karlsruhe, N. Dak. & 10 & 1997-1998 & 101 & $<100$ & 114 \\
\hline 89 & Souris River near Bantry, N. Dak. & 32 & 1993-1998 & 235 & $<100$ & 743 \\
\hline 90 & Willow Creek near Willow City, N. Dak. & 8 & 1993-1997 & 204 & $<100$ & 332 \\
\hline 91 & Stone Creek near Kramer, N. Dak. & 3 & 1993 & $<100$ & $<100$ & 170 \\
\hline 92 & Deep River near Upham, N. Dak. & 6 & 1997 & $<100$ & $<100$ & 181 \\
\hline 97 & Boundary Creek near Landa, N. Dak. & 3 & 1993 & $<100$ & $<100$ & 160 \\
\hline 98 & Souris River near Westhope, N. Dak. & 19 & $1995-2008$ & 326 & $<100$ & 1,361 \\
\hline 101 & Little Muddy River below Cow Creek near Williston, N. Dak. & 9 & 1999 & 369 & 261 & 2,700 \\
\hline 110 & Little Missouri River at Marmarth, N. Dak. & 8 & 1999 & 20,050 & 1,090 & 80,000 \\
\hline
\end{tabular}


Table 1-3. Summary statistics for trace-metal constituents at selected sites in North Dakota from 1993 through 2008. -Continued [ $\mu \mathrm{g} / \mathrm{L}$, micrograms per liter; --, not available; <, less than]

\begin{tabular}{|c|c|c|c|c|c|c|}
\hline $\begin{array}{c}\text { Site } \\
\text { identification } \\
\text { number } \\
\text { (table 1) } \\
\end{array}$ & Site name & $\begin{array}{l}\text { Number of } \\
\text { samples }\end{array}$ & $\begin{array}{l}\text { Period of } \\
\text { record }\end{array}$ & Median & Minimum & Maximum \\
\hline \multicolumn{7}{|c|}{ Aluminum, total, in $\mu \mathrm{g} / \mathrm{L}$-Continued } \\
\hline 112 & Little Missouri River at Medora, N. Dak. & 70 & 1997-2007 & 6,430 & $<100$ & 509,000 \\
\hline 113 & Beaver Creek near Trotters, N. Dak. & 9 & 1999 & 185 & $<100$ & 1,840 \\
\hline 114 & Little Missouri River near Watford City, N. Dak. & 64 & 1997-2007 & 12,350 & 150 & 657,000 \\
\hline 120 & Knife River near Golden Valley, N. Dak. & 60 & 1997-2007 & 1,415 & 160 & 19,000 \\
\hline 125 & Spring Creek at Zap, N. Dak. & 64 & 1997-2007 & 215 & $<100$ & 7,550 \\
\hline 127 & Knife River at Hazen, N. Dak. & 71 & 1997-2007 & 484 & $<100$ & 29,600 \\
\hline 149 & Heart River near Richardton, N. Dak. & 64 & 1997-2007 & 528 & $<100$ & 6,000 \\
\hline 156 & Heart River near Mandan, N. Dak. & 65 & 1997-2007 & 272 & $<100$ & 24,400 \\
\hline 164 & Cannonball River near Raleigh, N. Dak. & 57 & 1997-2007 & 1,430 & 116 & 25,300 \\
\hline 169 & Cedar Creek near Raleigh, N. Dak. & 49 & 1997-2007 & 780 & 171 & 25,600 \\
\hline 170 & Cannonball River at Breien, N. Dak. & 59 & 1997-2007 & 1,230 & $<100$ & 99,000 \\
\hline 175 & James River near Manfred, N. Dak. & 6 & 1998 & $<100$ & $<100$ & 207 \\
\hline 176 & James River near Grace City, N. Dak. & 60 & 1997-2007 & 269 & $<100$ & 1,520 \\
\hline 177 & James River above Arrowwood Lake near Kensal, N. Dak. & 9 & 1998-1999 & 239 & 107 & 642 \\
\hline 180 & Pipestem Creek near Pingree, N. Dak. & 9 & 1998-1999 & 210 & 103 & 421 \\
\hline 182 & James River at Jamestown, N. Dak. & 75 & 1997-2007 & 299 & $<100$ & 1,300 \\
\hline 183 & James River at Lamoure, N. Dak. & 68 & 1997-2007 & 767 & $<100$ & 5,900 \\
\hline 184 & Bear Creek near Oakes, N. Dak. & 9 & 1998-1999 & 451 & 138 & 1,050 \\
\hline 186 & James River at N. Dak./S. Dak. State line & 9 & 1998-1999 & 786 & 177 & 1,130 \\
\hline \multicolumn{7}{|c|}{ Arsenic, total, in $\mu \mathrm{g} / \mathrm{L}$} \\
\hline 1 & Bois De Sioux River near Doran, Minn. & 40 & $2000-2007$ & 6.1 & 2.9 & 30.0 \\
\hline 2 & Red River of the North at Wahpeton, N. Dak. & 2 & 1996 & 7.2 & 4.1 & 10.4 \\
\hline 3 & Red River of the North near Wahpeton, N. Dak. & 1 & 2006 & 24.9 & 24.9 & 24.9 \\
\hline 4 & Red River of the North at Brushville, Minn. & 54 & 1996-2007 & 3.3 & 1.5 & 9.6 \\
\hline 5 & Red River of the North below Wahpeton, N. Dak. & 20 & 1997-1999 & 3.0 & 1.5 & 7.3 \\
\hline 6 & Red River of the North at Hickson, N. Dak. & 21 & 1997-1999 & 4.2 & $<1.0$ & 7.0 \\
\hline 10 & Wild Rice River near Abercrombie, N. Dak. & 45 & 1996-2007 & 7.4 & 3.3 & 19.7 \\
\hline 11 & Red River of the North at Fargo, N. Dak. & 6 & 1996-2006 & 3.8 & 2.5 & 4.7 \\
\hline 13 & Red River of the North at Harwood, N. Dak. & 53 & $2000-2007$ & 5.2 & 1.8 & 8.2 \\
\hline 14 & Red River of the North near Harwood, N. Dak. & 31 & 1996-2007 & 4.8 & 2.0 & 7.0 \\
\hline 17 & Sheyenne River at Warwick, N. Dak. & 48 & 1996-2006 & 5.1 & 2.4 & 14.7 \\
\hline 18 & Sheyenne River near Warwick, N. Dak. & 16 & 2005-2007 & 6.0 & 2.6 & 13.8 \\
\hline 32 & Sheyenne River near Cooperstown, N. Dak. & 74 & 1996-2007 & 5.0 & $<1.0$ & 12.8 \\
\hline 33 & Baldhill Creek near Dazey, N. Dak. & 1 & 1996 & 3.1 & 3.1 & 3.1 \\
\hline 34 & Sheyenne River below Baldhill Dam, N. Dak. & 67 & 1996-2007 & 4.8 & 1.9 & 14.7 \\
\hline 36 & Sheyenne River at Lisbon, N. Dak. & 69 & 1997-2007 & 5.9 & 2.0 & 9.9 \\
\hline 37 & Sheyenne River near Kindred, N. Dak. & 70 & 1996-2007 & 6.8 & 3.0 & 15.9 \\
\hline 38 & $\begin{array}{l}\text { Sheyenne River above Sheyenne River Diversion near Horace, } \\
\text { N. Dak. }\end{array}$ & 5 & 2006-2007 & 8.1 & $<1.0$ & 10.4 \\
\hline 41 & Sheyenne River at West Fargo, N. Dak. & 6 & 1996-2006 & 6.4 & 3.5 & 11.7 \\
\hline
\end{tabular}


Table 1-3. Summary statistics for trace-metal constituents at selected sites in North Dakota from 1993 through 2008.-Continued [ $\mu \mathrm{g} / \mathrm{L}$, micrograms per liter; --, not available; $<$, less than]

\begin{tabular}{|c|c|c|c|c|c|c|}
\hline $\begin{array}{c}\text { Site } \\
\text { identification } \\
\text { number } \\
\text { (table 1) }\end{array}$ & Site name & $\begin{array}{c}\text { Number of } \\
\text { samples }\end{array}$ & $\begin{array}{l}\text { Period of } \\
\text { record }\end{array}$ & Median & Minimum & Maximum \\
\hline \multicolumn{7}{|c|}{ Arsenic, total, in $\mu \mathrm{g} / \mathrm{L}$-Continued } \\
\hline 45 & Maple River below Mapleton, N. Dak. & 47 & $1997-2007$ & 7.9 & $<1.0$ & 27.0 \\
\hline 46 & Sheyenne River at Harwood, N. Dak. & 21 & 1997-1999 & 7.2 & 3.9 & 12.0 \\
\hline 52 & Red River of the North at Halstad, Minn. & 5 & 2006-2007 & 6.2 & 2.4 & 8.5 \\
\hline 55 & Goose River at Hillsboro, N. Dak. & 64 & 1996-2007 & 5.1 & 2.5 & 12.2 \\
\hline 56 & Red River of the North at Grand Forks, N. Dak. & 47 & 1997-2006 & 4.3 & 1.3 & 10.3 \\
\hline 58 & Turtle River at Manvel, N. Dak. & 38 & 1997-2006 & 4.6 & $<1.0$ & 16.2 \\
\hline 62 & Forest River near Minto, N. Dak. & 43 & 1996-2006 & 3.7 & 1.4 & 11.3 \\
\hline 67 & Park River at Grafton, N. Dak. & 37 & 1996-2006 & 3.4 & 1.5 & 13.3 \\
\hline 68 & Red River of the North at Drayton, N. Dak. & 5 & 1996 & 3.8 & 3.1 & 5.6 \\
\hline 75 & Pembina River at Neche, N. Dak. & 47 & 1996-2006 & 5.4 & $<1.0$ & 27.7 \\
\hline 78 & Red River of the North at Pembina, N. Dak., site 2 & 58 & 1997-2007 & 5.5 & 1.4 & 12.7 \\
\hline 80 & Long Creek near Noonan, N. Dak. & 10 & 1997 & 6.0 & 3.2 & 11.8 \\
\hline 82 & Souris River near Sherwood, N. Dak. & 112 & 1993-2008 & 3.3 & $<1.0$ & 28.3 \\
\hline 83 & Souris River near Foxholm, N. Dak. & 35 & 1993-1998 & 6.3 & 1.6 & 15.0 \\
\hline 84 & Des Lacs River at Foxholm, N. Dak. & 83 & 1996-2007 & 5.4 & $<1.0$ & 27.4 \\
\hline 85 & Souris River above Minot, N. Dak. & 128 & 1996-2008 & 6.0 & 1.8 & 18.8 \\
\hline 87 & Souris River near Verendrye, N. Dak. & 174 & 1993-2008 & 4.7 & $<1.0$ & 24.4 \\
\hline 88 & Wintering River near Karlsruhe, N. Dak. & 10 & 1997-1998 & 2.9 & $<1.0$ & 6.9 \\
\hline 89 & Souris River near Bantry, N. Dak. & 65 & $1993-2000$ & 4.0 & $<1.0$ & 13.0 \\
\hline 90 & Willow Creek near Willow City, N. Dak. & 29 & 1993-2000 & 5.0 & 1.6 & 8.8 \\
\hline 91 & Stone Creek near Kramer, N. Dak. & 19 & 1993-2000 & 5.2 & 2.0 & 11.2 \\
\hline 92 & Deep River near Upham, N. Dak. & 26 & $1997-2000$ & 4.1 & $<1.0$ & 5.7 \\
\hline 95 & Cut Bank Creek at Upham, N. Dak. & 16 & 1999-2000 & 3.4 & 1.2 & 5.0 \\
\hline 97 & Boundary Creek near Landa, N. Dak. & 19 & $1993-2000$ & 5.4 & 2.0 & 11.0 \\
\hline 98 & Souris River near Westhope, N. Dak. & 20 & 1995-2008 & 5.1 & 2.0 & 12.0 \\
\hline 101 & Little Muddy River below Cow Creek near Williston, N. Dak. & 9 & 1999 & 5.5 & 1.5 & 7.4 \\
\hline 110 & Little Missouri River at Marmarth, N. Dak. & 8 & 1999 & 8.2 & 1.3 & 28.4 \\
\hline 112 & Little Missouri River at Medora, N. Dak. & 74 & 1996-2007 & 4.0 & $<1.0$ & 226.0 \\
\hline 113 & Beaver Creek near Trotters, N. Dak. & 9 & 1999 & 1.6 & $<1.0$ & 3.0 \\
\hline 114 & Little Missouri River near Watford City, N. Dak. & 69 & 1996-2007 & 6.9 & $<1.0$ & 266.0 \\
\hline 115 & Missouri River at Garrison Dam, N. Dak. & 7 & 1999-2000 & 2.0 & 1.7 & 2.7 \\
\hline 120 & Knife River near Golden Valley, N. Dak. & 60 & $1997-2007$ & 3.0 & $<1.0$ & 14.3 \\
\hline 125 & Spring Creek at Zap, N. Dak. & 64 & 1997-2007 & 1.9 & $<1.0$ & 7.0 \\
\hline 127 & Knife River at Hazen, N. Dak. & 76 & 1996-2007 & 2.2 & $<1.0$ & 13.0 \\
\hline 142 & Missouri River at Bismarck, N. Dak. & 23 & 1999-2001 & 2.0 & $<1.0$ & 2.5 \\
\hline 149 & Heart River near Richardton, N. Dak. & 68 & 1996-2007 & 2.0 & $<1.0$ & 8.2 \\
\hline 156 & Heart River near Mandan, N. Dak. & 68 & 1996-2007 & 1.3 & $<1.0$ & 7.9 \\
\hline 164 & Cannonball River near Raleigh, N. Dak. & 62 & $1996-2007$ & 2.1 & $<1.0$ & 23.1 \\
\hline 169 & Cedar Creek near Raleigh, N. Dak. & 54 & 1996-2007 & 2.5 & $<1.0$ & 7.2 \\
\hline 170 & Cannonball River at Breien, N. Dak. & 64 & 1996-2007 & 2.2 & $<1.0$ & 26.7 \\
\hline
\end{tabular}


Table 1-3. Summary statistics for trace-metal constituents at selected sites in North Dakota from 1993 through 2008. - Continued [ $\mu \mathrm{g} / \mathrm{L}$, micrograms per liter; --, not available; <, less than]

\begin{tabular}{|c|c|c|c|c|c|c|}
\hline $\begin{array}{c}\text { Site } \\
\text { identification } \\
\text { number } \\
\text { (table 1) }\end{array}$ & Site name & $\begin{array}{c}\text { Number of } \\
\text { samples }\end{array}$ & $\begin{array}{l}\text { Period of } \\
\text { record }\end{array}$ & Median & Minimum & Maximum \\
\hline \multicolumn{7}{|c|}{ Arsenic, total, in $\mu \mathrm{g} / \mathrm{L}$-Continued } \\
\hline 175 & James River near Manfred, N. Dak. & 14 & $1993-1998$ & 4.2 & 2.0 & 6.0 \\
\hline 176 & James River near Grace City, N. Dak. & 69 & 1993-2007 & 4.3 & 2.0 & 18.0 \\
\hline 177 & James River above Arrowwood Lake near Kensal, N. Dak. & 47 & 1993-2006 & 3.7 & 1.4 & 9.5 \\
\hline 179 & James River near Pingree, N. Dak. & 36 & 1993-2006 & 4.0 & $<1.0$ & 19.0 \\
\hline 180 & Pipestem Creek near Pingree, N. Dak. & 9 & $1998-1999$ & 5.3 & 1.5 & 11.7 \\
\hline 182 & James River at Jamestown, N. Dak. & 84 & 1993-2007 & 3.9 & $<1.0$ & 9.6 \\
\hline 183 & James River at Lamoure, N. Dak. & 81 & 1993-2007 & 4.9 & 1.7 & 13.8 \\
\hline 184 & Bear Creek near Oakes, N. Dak. & 9 & 1998-1999 & 5.0 & 2.0 & 8.6 \\
\hline 185 & James River at Oakes, N. Dak. & 9 & $1993-1995$ & 3.0 & 2.0 & 5.0 \\
\hline 186 & James River at N. Dak./S. Dak. State line & 9 & $1998-1999$ & 4.1 & 2.0 & 6.1 \\
\hline \multicolumn{7}{|c|}{ Arsenic, dissolved, in $\mu \mathrm{g} / \mathrm{L}$} \\
\hline 2 & Red River of the North at Wahpeton, N. Dak. & 31 & 1993-2008 & 4.0 & 2.0 & 7.0 \\
\hline 4 & Red River of the North at Brushville, Minn. & 1 & 2005 & 2.9 & 2.9 & 2.9 \\
\hline 6 & Red River of the North at Hickson, N. Dak. & 29 & 1993-2008 & 4.0 & 1.9 & 6.0 \\
\hline 7 & Wild Rice River near Rutland, N. Dak. & 28 & 1993-2008 & 5.0 & $<1.0$ & 13.0 \\
\hline 9 & Antelope Creek at Dwight, N. Dak. & 13 & $2001-2008$ & 4.5 & 2.7 & 16.5 \\
\hline 10 & Wild Rice River near Abercrombie, N. Dak. & 32 & 1993-2008 & 5.6 & 3.0 & 20.9 \\
\hline 11 & Red River of the North at Fargo, N. Dak. & 56 & 1993-2008 & 4.5 & $<1.0$ & 13.3 \\
\hline 13 & Red River of the North at Harwood, N. Dak. & 1 & 2005 & 3.6 & 3.6 & 3.6 \\
\hline 14 & Red River of the North near Harwood, N. Dak. & 7 & $2005-2006$ & 3.4 & 3.2 & 6.9 \\
\hline 15 & Sheyenne River above Harvey, N. Dak. & 35 & 1993-2008 & 4.0 & $<1.0$ & 12.5 \\
\hline 17 & Sheyenne River at Warwick, N. Dak. & 2 & 2005 & 5.2 & 4.6 & 5.8 \\
\hline 18 & Sheyenne River near Warwick, N. Dak. & 37 & $1993-2008$ & 5.3 & $<1.0$ & 14.1 \\
\hline 19 & Mauvais Coulee Tributary No. 3 near Cando, N. Dak. & 31 & 1993-2008 & 4.6 & $<1.0$ & 12.0 \\
\hline 20 & Mauvais Coulee near Cando, N. Dak. & 73 & 1993-2008 & 4.0 & $<1.0$ & 9.0 \\
\hline 21 & Edmore Coulee near Edmore, N. Dak. & 75 & 1993-2008 & 4.1 & $<1.0$ & 13.0 \\
\hline 22 & Edmore Coulee Tributary near Webster, N. Dak. & 32 & 1993-2008 & 4.2 & 2.0 & 11.0 \\
\hline 24 & Starkweather Coulee near Webster, N. Dak. & 67 & 1993-2008 & 6.0 & 2.0 & 12.0 \\
\hline 25 & Big Coulee below Churchs Ferry, N. Dak. & 23 & $1998-2008$ & 5.0 & 2.0 & 12.3 \\
\hline 26 & Little Coulee near Leeds, N. Dak. & 16 & 1998-2008 & 6.1 & 1.5 & 17.0 \\
\hline 27 & Little Coulee near Brinsmade, N. Dak. & 11 & 1993-1998 & 4.0 & 2.0 & 8.0 \\
\hline 28 & Big Coulee near Churchs Ferry, N. Dak. & 43 & 1993-1997 & 4.0 & $<1.0$ & 7.0 \\
\hline 30 & Channel A near Penn, N. Dak. & 63 & $1993-2008$ & 5.0 & 2.0 & 14.0 \\
\hline 31 & Devils Lake Outlet to Stump Lake near Lakota, N. Dak. & 12 & $2002-2007$ & 20.1 & 6.0 & 28.7 \\
\hline 32 & Sheyenne River near Cooperstown, N. Dak. & 39 & 1993-2008 & 5.1 & $<1.0$ & 19.3 \\
\hline 33 & Baldhill Creek near Dazey, N. Dak. & 29 & $1993-2008$ & 3.5 & $<1.0$ & 7.0 \\
\hline 34 & Sheyenne River below Baldhill Dam, N. Dak. & 40 & 1993-2008 & 5.0 & $<1.0$ & 19.4 \\
\hline 35 & Sheyenne River at Valley City, N. Dak. & 13 & 1994-2005 & 3.0 & $<1.0$ & 8.0 \\
\hline 36 & Sheyenne River at Lisbon, N. Dak. & 31 & 1996-2008 & 5.0 & $<1.0$ & 9.0 \\
\hline 37 & Sheyenne River near Kindred, N. Dak. & 25 & 1996-2008 & 5.0 & 2.0 & 10.0 \\
\hline
\end{tabular}


Table 1-3. Summary statistics for trace-metal constituents at selected sites in North Dakota from 1993 through 2008.-Continued [ $\mu \mathrm{g} / \mathrm{L}$, micrograms per liter; --, not available; <, less than]

\begin{tabular}{|c|c|c|c|c|c|c|}
\hline $\begin{array}{c}\text { Site } \\
\text { identification } \\
\text { number } \\
\text { (table 1) }\end{array}$ & Site name & $\begin{array}{c}\text { Number of } \\
\text { samples }\end{array}$ & $\begin{array}{l}\text { Period of } \\
\text { record }\end{array}$ & Median & Minimum & Maximum \\
\hline \multicolumn{7}{|c|}{ Arsenic, dissolved, in $\mu \mathrm{g} / \mathrm{L}$-Continued } \\
\hline 38 & $\begin{array}{l}\text { Sheyenne River above Sheyenne River Diversion near Horace, } \\
\text { N. Dak. }\end{array}$ & 35 & $1993-2008$ & 5.4 & $<1.0$ & 11.0 \\
\hline 40 & Sheyenne River Diversion at West Fargo, N. Dak. & 13 & 1994-2007 & 4.4 & 2.0 & 8.5 \\
\hline 41 & Sheyenne River at West Fargo, N. Dak. & 16 & 1993-2008 & 6.3 & 3.0 & 10.0 \\
\hline 42 & Maple River near Hope, N. Dak. & 22 & 1993-2008 & 3.4 & $<1.0$ & 13.1 \\
\hline 43 & Maple River near Enderlin, N. Dak. & 31 & 1993-2008 & 5.0 & 3.0 & 10.5 \\
\hline 44 & Maple River near Mapleton, N. Dak. & 17 & 1995-2008 & 8.0 & 3.0 & 15.3 \\
\hline 45 & Maple River below Mapleton, N. Dak. & 28 & 1995-2008 & 6.1 & 3.0 & 17.7 \\
\hline 46 & Sheyenne River at Harwood, N. Dak. & 6 & $2000-2005$ & 4.0 & 3.0 & 5.0 \\
\hline 47 & Rush River at Amenia, N. Dak. & 32 & 1993-2008 & 6.5 & 2.0 & 21.9 \\
\hline 52 & Red River of the North at Halstad, Minn. & 31 & 1996-2008 & 5.0 & 2.0 & 19.6 \\
\hline 53 & Beaver Creek near Finley, N. Dak. & 11 & $1998-2003$ & 5.0 & $<1.0$ & 18.5 \\
\hline 55 & Goose River at Hillsboro, N. Dak. & 39 & 1993-2008 & 4.2 & 2.0 & 11.9 \\
\hline 56 & Red River of the North at Grand Forks, N. Dak. & 47 & 1996-2008 & 4.0 & $<1.0$ & 9.0 \\
\hline 57 & Turtle River at Turtle River State Park near Arvilla, N. Dak. & 17 & 1996-2008 & 4.0 & 2.0 & 11.4 \\
\hline 58 & Turtle River at Manvel, N. Dak. & 30 & $2005-2008$ & 6.8 & 3.2 & 22.4 \\
\hline 59 & Red River of the North at Oslo, Minn. & 12 & 1993-2005 & 3.5 & 2.0 & 7.0 \\
\hline 61 & Forest River near Fordville, N. Dak. & 31 & 1993-2008 & 3.0 & $<1.0$ & 7.0 \\
\hline 62 & Forest River near Minto, N. Dak. & 54 & 1993-2008 & 3.2 & $<1.0$ & 20.0 \\
\hline 63 & South Branch Park River below Homme Dam, N. Dak. & 4 & 1993-1994 & 3.0 & $<1.0$ & 7.0 \\
\hline 67 & Park River at Grafton, N. Dak. & 53 & 1993-2008 & 3.6 & $<1.0$ & 11.0 \\
\hline 68 & Red River of the North at Drayton, N. Dak. & 30 & 1993-2008 & 4.0 & $<1.0$ & 7.2 \\
\hline 70 & Hidden Island Coulee near Hansboro, N. Dak. & 4 & 1993-1995 & 3.0 & $<1.0$ & 6.0 \\
\hline 73 & Little South Pembina River near Walhalla, N. Dak. & 16 & $2001-2008$ & 3.5 & $<1.0$ & 5.8 \\
\hline 74 & Pembina River at Walhalla, N. Dak. & 16 & $2001-2008$ & 4.3 & 2.0 & 6.0 \\
\hline 75 & Pembina River at Neche, N. Dak. & 52 & 1993-2008 & 4.0 & $<1.0$ & 11.1 \\
\hline 76 & Tongue River at Akra, N. Dak. & 32 & 1993-2008 & 3.0 & $<1.0$ & 12.0 \\
\hline 78 & Red River of the North at Pembina, N. Dak., site 2 & 37 & $2001-2008$ & 4.0 & 2.0 & 7.8 \\
\hline 80 & Long Creek near Noonan, N. Dak. & 38 & 1993-2008 & 4.4 & $<1.0$ & 21.6 \\
\hline 83 & Souris River near Foxholm, N. Dak. & 29 & $1997-2008$ & 6.5 & $<1.0$ & 21.0 \\
\hline 84 & Des Lacs River at Foxholm, N. Dak. & 41 & 1993-2008 & 5.0 & $<1.0$ & 28.4 \\
\hline 85 & Souris River above Minot, N. Dak. & 21 & 1993-1998 & 7.0 & $<1.0$ & 15.0 \\
\hline 86 & Bonnes Creek near Velva, N. Dak. & 13 & 1993-2005 & 2.0 & $<1.0$ & 7.0 \\
\hline 87 & Souris River near Verendrye, N. Dak. & 9 & 1997-1998 & 6.2 & 2.2 & 8.3 \\
\hline 88 & Wintering River near Karlsruhe, N. Dak. & 40 & 1993-2008 & 3.5 & $<1.0$ & 11.9 \\
\hline 89 & Souris River near Bantry, N. Dak. & 27 & 1997-2008 & 6.1 & $<1.0$ & 21.5 \\
\hline 90 & Willow Creek near Willow City, N. Dak. & 32 & 1994-2008 & 5.0 & $<1.0$ & 13.0 \\
\hline 92 & Deep River near Upham, N. Dak. & 30 & 1993-2007 & 3.3 & $<1.0$ & 9.3 \\
\hline 97 & Boundary Creek near Landa, N. Dak. & 1 & 1994 & 2.0 & 2.0 & 2.0 \\
\hline 98 & Souris River near Westhope, N. Dak. & 11 & 1997-2001 & 5.2 & $<1.0$ & 6.8 \\
\hline
\end{tabular}


Table 1-3. Summary statistics for trace-metal constituents at selected sites in North Dakota from 1993 through 2008. - Continued [ $\mu \mathrm{g} / \mathrm{L}$, micrograms per liter; --, not available; <, less than]

\begin{tabular}{|c|c|c|c|c|c|c|}
\hline $\begin{array}{c}\text { Site } \\
\text { identification } \\
\text { number } \\
\text { (table 1) }\end{array}$ & Site name & $\begin{array}{c}\text { Number of } \\
\text { samples }\end{array}$ & $\begin{array}{l}\text { Period of } \\
\text { record }\end{array}$ & Median & Minimum & Maximum \\
\hline \multicolumn{7}{|c|}{ Arsenic, dissolved, in $\mu \mathrm{g} / \mathrm{L}$-Continued } \\
\hline 101 & Little Muddy River below Cow Creek near Williston, N. Dak. & 39 & 1993-2008 & 4.0 & $<1.0$ & 12.6 \\
\hline 106 & Bear Den Creek near Mandaree, N. Dak. & 21 & 1997-2008 & 2.2 & $<1.0$ & 8.2 \\
\hline 108 & East Fork Shell Creek near Parshall, N. Dak. & 43 & 1993-2008 & 4.0 & $<1.0$ & 9.0 \\
\hline 109 & Deepwater Creek near Mandaree, N. Dak. & 44 & 1993-2008 & 4.9 & $<1.0$ & 441.0 \\
\hline 110 & Little Missouri River at Marmarth, N. Dak. & 41 & 1993-2008 & 1.9 & $<1.0$ & 6.0 \\
\hline 112 & Little Missouri River at Medora, N. Dak. & 26 & 1999-2008 & 1.7 & $<1.0$ & 3.3 \\
\hline 113 & Beaver Creek near Trotters, N. Dak. & 36 & 1993-2008 & 1.1 & $<1.0$ & 3.5 \\
\hline 114 & Little Missouri River near Watford City, N. Dak. & 36 & 1995-2008 & 1.8 & $<1.0$ & 8.3 \\
\hline 115 & Missouri River at Garrison Dam, N. Dak. & 57 & 1996-2007 & 2.0 & $<1.0$ & 3.0 \\
\hline 116 & Knife River at Manning, N. Dak. & 31 & 1993-2008 & 1.3 & $<1.0$ & 9.0 \\
\hline 120 & Knife River near Golden Valley, N. Dak. & 37 & 1993-2008 & 1.7 & $<1.0$ & 13.7 \\
\hline 125 & Spring Creek at Zap, N. Dak. & 37 & 1993-2008 & 1.1 & $<1.0$ & 6.7 \\
\hline 127 & Knife River at Hazen, N. Dak. & 36 & 1994-2008 & 1.4 & $<1.0$ & 4.7 \\
\hline 140 & Square Butte Creek below Center, N. Dak. & 30 & 1993-2008 & 2.0 & $<1.0$ & 5.8 \\
\hline 141 & Burnt Creek near Bismarck, N. Dak. & 29 & $1993-2008$ & 1.7 & $<1.0$ & 5.6 \\
\hline 142 & Missouri River at Bismarck, N. Dak. & 23 & 1993-2008 & 2.0 & $<1.0$ & 3.7 \\
\hline 145 & Heart River near South Heart, N. Dak. & 12 & 1993-2005 & 2.7 & $<1.0$ & 15.0 \\
\hline 146 & Heart River at Dickinson, N. Dak. & 4 & 1993-1994 & 1.5 & $<1.0$ & 2.0 \\
\hline 147 & Green River near New Hradec, N. Dak. & 29 & 1993-2008 & $<1.0$ & $<1.0$ & 6.0 \\
\hline 149 & Heart River near Richardton, N. Dak. & 35 & 1993-2008 & $<1.0$ & $<1.0$ & 5.4 \\
\hline 150 & Heart River above Lake Tschida near Glen Ullin, N. Dak. & 32 & 1993-2008 & $<1.0$ & $<1.0$ & 6.5 \\
\hline 151 & Antelope Creek near Carson, N. Dak. & 18 & 1999-2008 & $<1.0$ & $<1.0$ & 3.8 \\
\hline 152 & Big Muddy Creek near Almont, N. Dak. & 32 & 1993-2008 & 3.0 & $<1.0$ & 18.5 \\
\hline 153 & Heart River near Lark, N. Dak. & 6 & 1993-1995 & $<1.0$ & $<1.0$ & 2.0 \\
\hline 154 & Heart River at Stark Bridge near Judson, N. Dak. & 32 & 1993-2008 & $<1.0$ & $<1.0$ & 3.8 \\
\hline 155 & Sweetbriar Creek near Judson, N. Dak. & 13 & $2002-2008$ & 2.0 & $<1.0$ & 14.0 \\
\hline 156 & Heart River near Mandan, N. Dak. & 38 & 1994-2008 & $<1.0$ & $<1.0$ & 6.3 \\
\hline 157 & Long Lake Creek above Long Lake near Moffit, N. Dak. & 24 & 1993-2004 & 4.5 & $<1.0$ & 17.3 \\
\hline 158 & Apple Creek near Menoken, N. Dak. & 31 & 1993-2008 & 5.0 & $<1.0$ & 30.0 \\
\hline 162 & Cannonball River at Regent, N. Dak. & 31 & 1993-2008 & $<1.0$ & $<1.0$ & 5.3 \\
\hline 164 & Cannonball River near Raleigh, N. Dak. & 21 & $2001-2008$ & 1.4 & $<1.0$ & 5.1 \\
\hline 165 & White Butte Fork Cedar Creek near Scranton, N. Dak. & 5 & 1993-1995 & 2.0 & $<1.0$ & 5.0 \\
\hline 166 & Cedar Creek near Haynes, N. Dak. & 32 & 1993-2008 & 2.0 & $<1.0$ & 16.3 \\
\hline 169 & Cedar Creek near Raleigh, N. Dak. & 35 & 1993-2008 & 1.2 & $<1.0$ & 4.6 \\
\hline 170 & Cannonball River at Breien, N. Dak. & 40 & 1993-2008 & 1.1 & $<1.0$ & 8.9 \\
\hline 172 & Beaver Creek below Linton, N. Dak. & 32 & 1993-2008 & 6.0 & $<1.0$ & 14.4 \\
\hline 173 & Porcupine Creek near Fort Yates, N. Dak. & 35 & 1993-1999 & $<1.0$ & $<1.0$ & 5.2 \\
\hline 175 & James River near Manfred, N. Dak. & 22 & 1993-1998 & 5.0 & $<1.0$ & 7.0 \\
\hline 176 & James River near Grace City, N. Dak. & 61 & 1993-2008 & 4.0 & $<1.0$ & 17.8 \\
\hline 177 & James River above Arrowwood Lake near Kensal, N. Dak. & 129 & 1993-2008 & 3.6 & $<1.0$ & 10.0 \\
\hline
\end{tabular}


Table 1-3. Summary statistics for trace-metal constituents at selected sites in North Dakota from 1993 through 2008.-Continued [ $\mu \mathrm{g} / \mathrm{L}$, micrograms per liter; --, not available; <, less than]

\begin{tabular}{|c|c|c|c|c|c|c|}
\hline $\begin{array}{c}\text { Site } \\
\text { identification } \\
\text { number } \\
\text { (table 1) }\end{array}$ & Site name & $\begin{array}{c}\text { Number of } \\
\text { samples }\end{array}$ & $\begin{array}{l}\text { Period of } \\
\text { record }\end{array}$ & Median & Minimum & Maximum \\
\hline \multicolumn{7}{|c|}{ Arsenic, dissolved, in $\mu \mathrm{g} / \mathrm{L}$-Continued } \\
\hline 179 & James River near Pingree, N. Dak. & 87 & 1993-2008 & 4.4 & 1.8 & 17.0 \\
\hline 180 & Pipestem Creek near Pingree, N. Dak. & 40 & 1993-2008 & 5.0 & $<1.0$ & 16.4 \\
\hline 182 & James River at Jamestown, N. Dak. & 63 & 1993-2008 & 3.0 & $<1.0$ & 10.8 \\
\hline 183 & James River at Lamoure, N. Dak. & 59 & $1993-2008$ & 4.0 & $<1.0$ & 12.2 \\
\hline 184 & Bear Creek near Oakes, N. Dak. & 40 & 1993-2008 & 4.7 & $<1.0$ & 16.1 \\
\hline 185 & James River at Oakes, N. Dak. & 28 & 1993-2008 & 3.0 & 2.0 & 10.7 \\
\hline 186 & James River at N. Dak./S. Dak. State line & 22 & 1998-2008 & 4.3 & $<1.0$ & 23.0 \\
\hline \multicolumn{7}{|c|}{ Barium, total, in $\mu \mathrm{g} / \mathrm{L}$} \\
\hline 1 & Bois De Sioux River near Doran, Minn. & 40 & $2000-2007$ & $<100$ & $<100$ & 160 \\
\hline 2 & Red River of the North at Wahpeton, N. Dak. & 2 & 1996 & $<100$ & $<100$ & $<100$ \\
\hline 3 & Red River of the North near Wahpeton, N. Dak. & 1 & 2006 & $<100$ & $<100$ & $<100$ \\
\hline 4 & Red River of the North at Brushville, Minn. & 54 & $1996-2007$ & $<100$ & $<100$ & 116 \\
\hline 10 & Wild Rice River near Abercrombie, N. Dak. & 45 & $1996-2007$ & 102 & $<100$ & 188 \\
\hline 11 & Red River of the North at Fargo, N. Dak. & 5 & 1996 & $<100$ & $<100$ & $<100$ \\
\hline 13 & Red River of the North at Harwood, N. Dak. & 53 & $2000-2007$ & $<100$ & $<100$ & 163 \\
\hline 14 & Red River of the North near Harwood, N. Dak. & 5 & 1996 & $<100$ & $<100$ & 110 \\
\hline 17 & Sheyenne River at Warwick, N. Dak. & 48 & $1996-2006$ & $<100$ & $<100$ & 126 \\
\hline 18 & Sheyenne River near Warwick, N. Dak. & 16 & 2005-2007 & $<100$ & $<100$ & 148 \\
\hline 32 & Sheyenne River near Cooperstown, N. Dak. & 69 & $1996-2007$ & $<100$ & $<100$ & 114 \\
\hline 33 & Baldhill Creek near Dazey, N. Dak. & 1 & 1996 & $<100$ & $<100$ & $<100$ \\
\hline 34 & Sheyenne River below Baldhill Dam, N. Dak. & 62 & $1996-2007$ & $<100$ & $<100$ & $<100$ \\
\hline 36 & Sheyenne River at Lisbon, N. Dak. & 69 & $1997-2007$ & $<100$ & $<100$ & 154 \\
\hline 37 & Sheyenne River near Kindred, N. Dak. & 70 & $1996-2007$ & 102 & $<100$ & 256 \\
\hline 38 & $\begin{array}{l}\text { Sheyenne River above Sheyenne River Diversion near Horace, } \\
\text { N. Dak. }\end{array}$ & 5 & $2006-2007$ & 144 & 114 & 211 \\
\hline 41 & Sheyenne River at West Fargo, N. Dak. & 6 & 1996-2006 & 103 & $<100$ & 134 \\
\hline 45 & Maple River below Mapleton, N. Dak. & 47 & $1997-2007$ & $<100$ & $<100$ & 161 \\
\hline 52 & Red River of the North at Halstad, Minn. & 5 & $2006-2007$ & 105 & $<100$ & 136 \\
\hline 55 & Goose River at Hillsboro, N. Dak. & 64 & $1996-2007$ & $<100$ & $<100$ & 167 \\
\hline 56 & Red River of the North at Grand Forks, N. Dak. & 46 & $1997-2006$ & $<100$ & $<100$ & 164 \\
\hline 58 & Turtle River at Manvel, N. Dak. & 37 & $1997-2006$ & $<100$ & $<100$ & 122 \\
\hline 62 & Forest River near Minto, N. Dak. & 42 & 1996-2006 & $<100$ & $<100$ & 153 \\
\hline 67 & Park River at Grafton, N. Dak. & 36 & $1996-2006$ & $<100$ & $<100$ & 204 \\
\hline 68 & Red River of the North at Drayton, N. Dak. & 5 & 1996 & $<100$ & $<100$ & 103 \\
\hline 75 & Pembina River at Neche, N. Dak. & 46 & $1996-2006$ & $<100$ & $<100$ & 618 \\
\hline 78 & Red River of the North at Pembina, N. Dak., site 2 & 52 & $1997-2006$ & $<100$ & $<100$ & 238 \\
\hline 80 & Long Creek near Noonan, N. Dak. & 10 & 1997 & $<100$ & $<100$ & $<100$ \\
\hline 82 & Souris River near Sherwood, N. Dak. & 121 & $1993-2008$ & $<100$ & $<100$ & 225 \\
\hline 83 & Souris River near Foxholm, N. Dak. & 11 & $1997-1998$ & $<100$ & $<100$ & 146 \\
\hline 84 & Des Lacs River at Foxholm, N. Dak. & 83 & $1996-2007$ & $<100$ & $<100$ & 171 \\
\hline
\end{tabular}


Table 1-3. Summary statistics for trace-metal constituents at selected sites in North Dakota from 1993 through 2008. - Continued [ $\mu \mathrm{g} / \mathrm{L}$, micrograms per liter; --, not available; $<$, less than]

\begin{tabular}{|c|c|c|c|c|c|c|}
\hline $\begin{array}{c}\text { Site } \\
\text { identification } \\
\text { number } \\
\text { (table 1) }\end{array}$ & Site name & $\begin{array}{c}\text { Number of } \\
\text { samples }\end{array}$ & $\begin{array}{l}\text { Period of } \\
\text { record }\end{array}$ & Median & Minimum & Maximum \\
\hline \multicolumn{7}{|c|}{ Barium, total, in $\mu \mathrm{g} / \mathrm{L}$-Continued } \\
\hline 85 & Souris River above Minot, N. Dak. & 69 & $1996-2007$ & $<100$ & $<100$ & 304 \\
\hline 87 & Souris River near Verendrye, N. Dak. & 75 & 1997-2007 & $<100$ & $<100$ & 144 \\
\hline 88 & Wintering River near Karlsruhe, N. Dak. & 10 & 1997-1998 & 117 & $<100$ & 178 \\
\hline 89 & Souris River near Bantry, N. Dak. & 9 & $1997-1998$ & 107 & $<100$ & 154 \\
\hline 90 & Willow Creek near Willow City, N. Dak. & 5 & 1997 & $<100$ & $<100$ & $<100$ \\
\hline 91 & Stone Creek near Kramer, N. Dak. & 3 & 1993 & $<100$ & $<100$ & $<100$ \\
\hline 92 & Deep River near Upham, N. Dak. & 6 & 1997 & $<100$ & $<100$ & $<100$ \\
\hline 97 & Boundary Creek near Landa, N. Dak. & 3 & 1993 & $<100$ & $<100$ & $<100$ \\
\hline 98 & Souris River near Westhope, N. Dak. & 7 & 1997-1998 & $<100$ & $<100$ & 249 \\
\hline 101 & Little Muddy River below Cow Creek near Williston, N. Dak. & 9 & 1999 & $<100$ & $<100$ & $<100$ \\
\hline 110 & Little Missouri River at Marmarth, N. Dak. & 8 & 1999 & 241 & $<100$ & 699 \\
\hline 112 & Little Missouri River at Medora, N. Dak. & 73 & 1996-2007 & 114 & $<100$ & 3,600 \\
\hline 113 & Beaver Creek near Trotters, N. Dak. & 9 & 1999 & $<100$ & $<100$ & $<100$ \\
\hline 114 & Little Missouri River near Watford City, N. Dak. & 69 & 1996-2007 & 185 & $<100$ & 4,320 \\
\hline 120 & Knife River near Golden Valley, N. Dak. & 60 & $1997-2007$ & $<100$ & $<100$ & 207 \\
\hline 125 & Spring Creek at Zap, N. Dak. & 64 & 1997-2007 & $<100$ & $<100$ & 185 \\
\hline 127 & Knife River at Hazen, N. Dak. & 76 & 1996-2007 & $<100$ & $<100$ & 405 \\
\hline 149 & Heart River near Richardton, N. Dak. & 68 & 1996-2007 & $<100$ & $<100$ & 141 \\
\hline 156 & Heart River near Mandan, N. Dak. & 68 & 1996-2007 & $<100$ & $<100$ & 208 \\
\hline 164 & Cannonball River near Raleigh, N. Dak. & 62 & $1996-2007$ & $<100$ & $<100$ & 375 \\
\hline 169 & Cedar Creek near Raleigh, N. Dak. & 54 & 1996-2007 & $<100$ & $<100$ & 199 \\
\hline 170 & Cannonball River at Breien, N. Dak. & 64 & 1996-2007 & $<100$ & $<100$ & 731 \\
\hline 175 & James River near Manfred, N. Dak. & 6 & 1998 & $<100$ & $<100$ & $<100$ \\
\hline 176 & James River near Grace City, N. Dak. & 60 & $1997-2007$ & $<100$ & $<100$ & 201 \\
\hline 177 & James River above Arrowwood Lake near Kensal, N. Dak. & 9 & 1998-1999 & $<100$ & $<100$ & 138 \\
\hline 180 & Pipestem Creek near Pingree, N. Dak. & 9 & 1998-1999 & $<100$ & $<100$ & 122 \\
\hline 182 & James River at Jamestown, N. Dak. & 75 & $1997-2007$ & $<100$ & $<100$ & 149 \\
\hline 183 & James River at Lamoure, N. Dak. & 73 & 1996-2007 & $<100$ & $<100$ & 188 \\
\hline 184 & Bear Creek near Oakes, N. Dak. & 9 & 1998-1999 & $<100$ & $<100$ & 134 \\
\hline 186 & James River at N. Dak./S. Dak. State line & 9 & 1998-1999 & $<100$ & $<100$ & 126 \\
\hline \multicolumn{7}{|c|}{ Chromium, total, in $\mu \mathrm{g} / \mathrm{L}$} \\
\hline 1 & Bois De Sioux River near Doran, Minn. & 40 & $2000-2007$ & $<1.2$ & $<1.2$ & 6.7 \\
\hline 2 & Red River of the North at Wahpeton, N. Dak. & 2 & 1996 & 1.8 & $<1.2$ & 2.5 \\
\hline 3 & Red River of the North near Wahpeton, N. Dak. & 1 & 2006 & 1.3 & 1.3 & 1.3 \\
\hline 4 & Red River of the North at Brushville, Minn. & 54 & 1996-2007 & $<1.2$ & $<1.2$ & 7.5 \\
\hline 10 & Wild Rice River near Abercrombie, N. Dak. & 45 & 1996-2007 & 2.2 & $<1.2$ & 8.6 \\
\hline 11 & Red River of the North at Fargo, N. Dak. & 6 & 1996-2006 & 1.9 & $<1.2$ & 3.7 \\
\hline 13 & Red River of the North at Harwood, N. Dak. & 53 & 2000-2007 & 4.1 & $<1.2$ & 21.2 \\
\hline 14 & Red River of the North near Harwood, N. Dak. & 10 & 1996-2007 & 5.1 & 1.5 & 13.6 \\
\hline 17 & Sheyenne River at Warwick, N. Dak. & 48 & 1996-2006 & $<1.2$ & $<1.2$ & 3.2 \\
\hline
\end{tabular}


Table 1-3. Summary statistics for trace-metal constituents at selected sites in North Dakota from 1993 through 2008.-Continued [ $\mu \mathrm{g} / \mathrm{L}$, micrograms per liter; --, not available; <, less than]

\begin{tabular}{|c|c|c|c|c|c|c|}
\hline $\begin{array}{c}\text { Site } \\
\text { identification } \\
\text { number } \\
\text { (table 1) }\end{array}$ & Site name & $\begin{array}{c}\text { Number of } \\
\text { samples }\end{array}$ & $\begin{array}{l}\text { Period of } \\
\text { record }\end{array}$ & Median & Minimum & Maximum \\
\hline \multicolumn{7}{|c|}{ Chromium, total, in $\mu \mathrm{g} / \mathrm{L}$-Continued } \\
\hline 18 & Sheyenne River near Warwick, N. Dak. & 16 & 2005-2007 & $<1.2$ & $<1.2$ & 3.8 \\
\hline 32 & Sheyenne River near Cooperstown, N. Dak. & 74 & $1996-2007$ & 1.3 & $<1.2$ & 6.4 \\
\hline 33 & Baldhill Creek near Dazey, N. Dak. & 1 & 1996 & $<1.2$ & $<1.2$ & $<1.2$ \\
\hline 34 & Sheyenne River below Baldhill Dam, N. Dak. & 67 & $1996-2007$ & $<1.2$ & $<1.2$ & 5.7 \\
\hline 36 & Sheyenne River at Lisbon, N. Dak. & 69 & $1997-2007$ & 1.5 & $<1.2$ & 13.1 \\
\hline 37 & Sheyenne River near Kindred, N. Dak. & 70 & $1996-2007$ & 1.8 & $<1.2$ & 23.1 \\
\hline 38 & $\begin{array}{l}\text { Sheyenne River above Sheyenne River Diversion near Horace, } \\
\text { N. Dak. }\end{array}$ & 5 & 2006-2007 & 4.9 & 3.2 & 15.8 \\
\hline 41 & Sheyenne River at West Fargo, N. Dak. & 6 & 1996-2006 & 4.9 & $<1.2$ & 6.6 \\
\hline 45 & Maple River below Mapleton, N. Dak. & 47 & $1997-2007$ & 2.3 & $<1.2$ & 16.4 \\
\hline 52 & Red River of the North at Halstad, Minn. & 5 & $2006-2007$ & 6.8 & 3.3 & 15.8 \\
\hline 55 & Goose River at Hillsboro, N. Dak. & 64 & $1996-2007$ & 1.4 & $<1.2$ & 13.9 \\
\hline 56 & Red River of the North at Grand Forks, N. Dak. & 47 & $1997-2006$ & 2.3 & $<1.2$ & 21.1 \\
\hline 58 & Turtle River at Manvel, N. Dak. & 38 & $1997-2006$ & $<1.2$ & $<1.2$ & 9.9 \\
\hline 62 & Forest River near Minto, N. Dak. & 43 & 1996-2006 & $<1.2$ & $<1.2$ & 11.1 \\
\hline 67 & Park River at Grafton, N. Dak. & 37 & 1996-2006 & $<1.2$ & $<1.2$ & 23.0 \\
\hline 68 & Red River of the North at Drayton, N. Dak. & 5 & 1996 & 3.6 & $<1.2$ & 6.3 \\
\hline 75 & Pembina River at Neche, N. Dak. & 47 & 1996-2006 & 2.4 & $<1.2$ & 79.0 \\
\hline 78 & Red River of the North at Pembina, N. Dak., site 2 & 58 & $1997-2007$ & 4.5 & $<1.2$ & 36.3 \\
\hline 80 & Long Creek near Noonan, N. Dak. & 10 & 1997 & $<1.2$ & $<1.2$ & 1.8 \\
\hline 82 & Souris River near Sherwood, N. Dak. & 98 & $1993-2008$ & $<1.2$ & $<1.2$ & 2.3 \\
\hline 83 & Souris River near Foxholm, N. Dak. & 35 & 1993-1998 & $<1.2$ & $<1.2$ & $<1.2$ \\
\hline 84 & Des Lacs River at Foxholm, N. Dak. & 83 & 1996-2007 & $<1.2$ & $<1.2$ & 4.6 \\
\hline 85 & Souris River above Minot, N. Dak. & 117 & 1996-2008 & $<1.2$ & $<1.2$ & 7.4 \\
\hline 87 & Souris River near Verendrye, N. Dak. & 154 & 1993-2008 & $<1.2$ & $<1.2$ & 20.0 \\
\hline 88 & Wintering River near Karlsruhe, N. Dak. & 10 & 1997-1998 & $<1.2$ & $<1.2$ & 1.8 \\
\hline 89 & Souris River near Bantry, N. Dak. & 45 & 1993-1998 & $<1.2$ & $<1.2$ & 14.0 \\
\hline 90 & Willow Creek near Willow City, N. Dak. & 8 & 1993-1997 & $<1.2$ & $<1.2$ & 3.6 \\
\hline 91 & Stone Creek near Kramer, N. Dak. & 3 & 1993 & $<1.2$ & $<1.2$ & $<1.2$ \\
\hline 92 & Deep River near Upham, N. Dak. & 6 & 1997 & $<1.2$ & $<1.2$ & 1.6 \\
\hline 97 & Boundary Creek near Landa, N. Dak. & 3 & 1993 & $<1.2$ & $<1.2$ & $<1.2$ \\
\hline 98 & Souris River near Westhope, N. Dak. & 18 & 1995-2008 & $<1.2$ & $<1.2$ & 2.3 \\
\hline 101 & Little Muddy River below Cow Creek near Williston, N. Dak. & 9 & 1999 & $<1.2$ & $<1.2$ & 3.5 \\
\hline 110 & Little Missouri River at Marmarth, N. Dak. & 8 & 1999 & 21.0 & 1.4 & 84.3 \\
\hline 112 & Little Missouri River at Medora, N. Dak. & 74 & $1996-2007$ & 8.8 & $<1.2$ & 708.0 \\
\hline 113 & Beaver Creek near Trotters, N. Dak. & 9 & 1999 & $<1.2$ & $<1.2$ & 2.3 \\
\hline 114 & Little Missouri River near Watford City, N. Dak. & 69 & $1996-2007$ & 15.1 & $<1.2$ & 845.0 \\
\hline 120 & Knife River near Golden Valley, N. Dak. & 60 & $1997-2007$ & 2.2 & $<1.2$ & 19.2 \\
\hline 125 & Spring Creek at Zap, N. Dak. & 64 & $1997-2007$ & $<1.2$ & $<1.2$ & 9.5 \\
\hline 127 & Knife River at Hazen, N. Dak. & 76 & $1996-2007$ & $<1.2$ & $<1.2$ & 32.3 \\
\hline
\end{tabular}


Table 1-3. Summary statistics for trace-metal constituents at selected sites in North Dakota from 1993 through 2008.—Continued [ $\mu \mathrm{g} / \mathrm{L}$, micrograms per liter; --, not available; <, less than]

\begin{tabular}{|c|c|c|c|c|c|c|}
\hline $\begin{array}{c}\text { Site } \\
\text { identification } \\
\text { number } \\
\text { (table 1) }\end{array}$ & Site name & $\begin{array}{l}\text { Number of } \\
\text { samples }\end{array}$ & $\begin{array}{l}\text { Period of } \\
\text { record }\end{array}$ & Median & Minimum & Maximum \\
\hline \multicolumn{7}{|c|}{ Chromium, total, in $\mu \mathrm{g} / \mathrm{L}$-Continued } \\
\hline 149 & Heart River near Richardton, N. Dak. & 68 & 1996-2007 & $<1.2$ & $<1.2$ & 9.5 \\
\hline 156 & Heart River near Mandan, N. Dak. & 68 & 1996-2007 & $<1.2$ & $<1.2$ & 22.0 \\
\hline 164 & Cannonball River near Raleigh, N. Dak. & 62 & 1996-2007 & 1.8 & $<1.2$ & 30.8 \\
\hline 169 & Cedar Creek near Raleigh, N. Dak. & 54 & 1996-2007 & 1.4 & $<1.2$ & 29.0 \\
\hline 170 & Cannonball River at Breien, N. Dak. & 64 & 1996-2007 & 2.0 & $<1.2$ & 111.0 \\
\hline 175 & James River near Manfred, N. Dak. & 6 & 1998 & $<1.2$ & $<1.2$ & $<1.2$ \\
\hline 176 & James River near Grace City, N. Dak. & 60 & 1997-2007 & $<1.2$ & $<1.2$ & 6.3 \\
\hline 177 & James River above Arrowwood Lake near Kensal, N. Dak. & 9 & 1998-1999 & $<1.2$ & $<1.2$ & $<1.2$ \\
\hline 180 & Pipestem Creek near Pingree, N. Dak. & 9 & 1998-1999 & $<1.2$ & $<1.2$ & $<1.2$ \\
\hline 182 & James River at Jamestown, N. Dak. & 75 & 1997-2007 & $<1.2$ & $<1.2$ & 49.8 \\
\hline 183 & James River at Lamoure, N. Dak. & 73 & 1996-2007 & 1.4 & $<1.2$ & 162.0 \\
\hline 184 & Bear Creek near Oakes, N. Dak. & 9 & 1998-1999 & $<1.2$ & $<1.2$ & 1.3 \\
\hline 186 & James River at N. Dak./S. Dak. State line & 9 & 1998-1999 & 1.3 & $<1.2$ & 1.9 \\
\hline \multicolumn{7}{|c|}{ Copper, total, in $\mu \mathrm{g} / \mathrm{L}$} \\
\hline 1 & Bois De Sioux River near Doran, Minn. & 40 & 2000-2007 & 2.5 & $<1.0$ & 7.3 \\
\hline 4 & Red River of the North at Brushville, Minn. & 54 & 1996-2007 & 1.9 & $<1.0$ & 6.0 \\
\hline 11 & Red River of the North at Fargo, N. Dak. & 1 & 2006 & 1.0 & 1.0 & 1.0 \\
\hline 14 & Red River of the North near Harwood, N. Dak. & 5 & 2006-2007 & 7.8 & 5.5 & 9.8 \\
\hline 17 & Sheyenne River at Warwick, N. Dak. & 48 & 1996-2006 & 3.2 & $<1.0$ & 11.9 \\
\hline 32 & Sheyenne River near Cooperstown, N. Dak. & 5 & 2006-2007 & 4.0 & 3.2 & 114.0 \\
\hline 34 & Sheyenne River below Baldhill Dam, N. Dak. & 5 & 2006-2007 & 2.0 & 1.6 & 3.0 \\
\hline 38 & $\begin{array}{l}\text { Sheyenne River above Sheyenne River Diversion near Horace, } \\
\text { N. Dak. }\end{array}$ & 5 & 2006-2007 & 5.4 & 4.2 & 14.9 \\
\hline 52 & Red River of the North at Halstad, Minn. & 5 & 2006-2007 & 6.5 & 5.1 & 12.3 \\
\hline 56 & Red River of the North at Grand Forks, N. Dak. & 1 & 2006 & $<1.0$ & $<1.0$ & $<1.0$ \\
\hline 58 & Turtle River at Manvel, N. Dak. & 1 & 2006 & 5.8 & 5.8 & 5.8 \\
\hline 62 & Forest River near Minto, N. Dak. & 1 & 2006 & $<1.0$ & $<1.0$ & 1.0 \\
\hline 67 & Park River at Grafton, N. Dak. & 1 & 2006 & 2.1 & 2.1 & 2.1 \\
\hline 75 & Pembina River at Neche, N. Dak. & 1 & 2006 & 1.7 & 1.7 & 1.7 \\
\hline 78 & Red River of the North at Pembina, N. Dak., site 2 & 6 & 2006-2007 & 7.0 & 1.0 & 15.3 \\
\hline 82 & Souris River near Sherwood, N. Dak. & 114 & 1993-2008 & 2.6 & $<1.0$ & 18.6 \\
\hline 83 & Souris River near Foxholm, N. Dak. & 25 & 1993-1998 & 2.0 & $<1.0$ & 39.0 \\
\hline 85 & Souris River above Minot, N. Dak. & 51 & 1999-2008 & 2.5 & $<1.0$ & 11.8 \\
\hline 87 & Souris River near Verendrye, N. Dak. & 104 & 1993-2008 & 3.1 & $<1.0$ & 17.0 \\
\hline 89 & Souris River near Bantry, N. Dak. & 37 & 1993-1998 & 2.0 & $<1.0$ & 5.0 \\
\hline 90 & Willow Creek near Willow City, N. Dak. & 3 & 1993 & 2.0 & 2.0 & 3.0 \\
\hline 91 & Stone Creek near Kramer, N. Dak. & 3 & 1993 & 2.0 & $<1.0$ & 3.0 \\
\hline 97 & Boundary Creek near Landa, N. Dak. & 3 & 1993 & 2.0 & $<1.0$ & 3.0 \\
\hline 98 & Souris River near Westhope, N. Dak. & 13 & 1995-2008 & 3.8 & $<1.0$ & 7.2 \\
\hline
\end{tabular}


Table 1-3. Summary statistics for trace-metal constituents at selected sites in North Dakota from 1993 through 2008.—Continued [ $\mu \mathrm{g} / \mathrm{L}$, micrograms per liter; --, not available; <, less than]

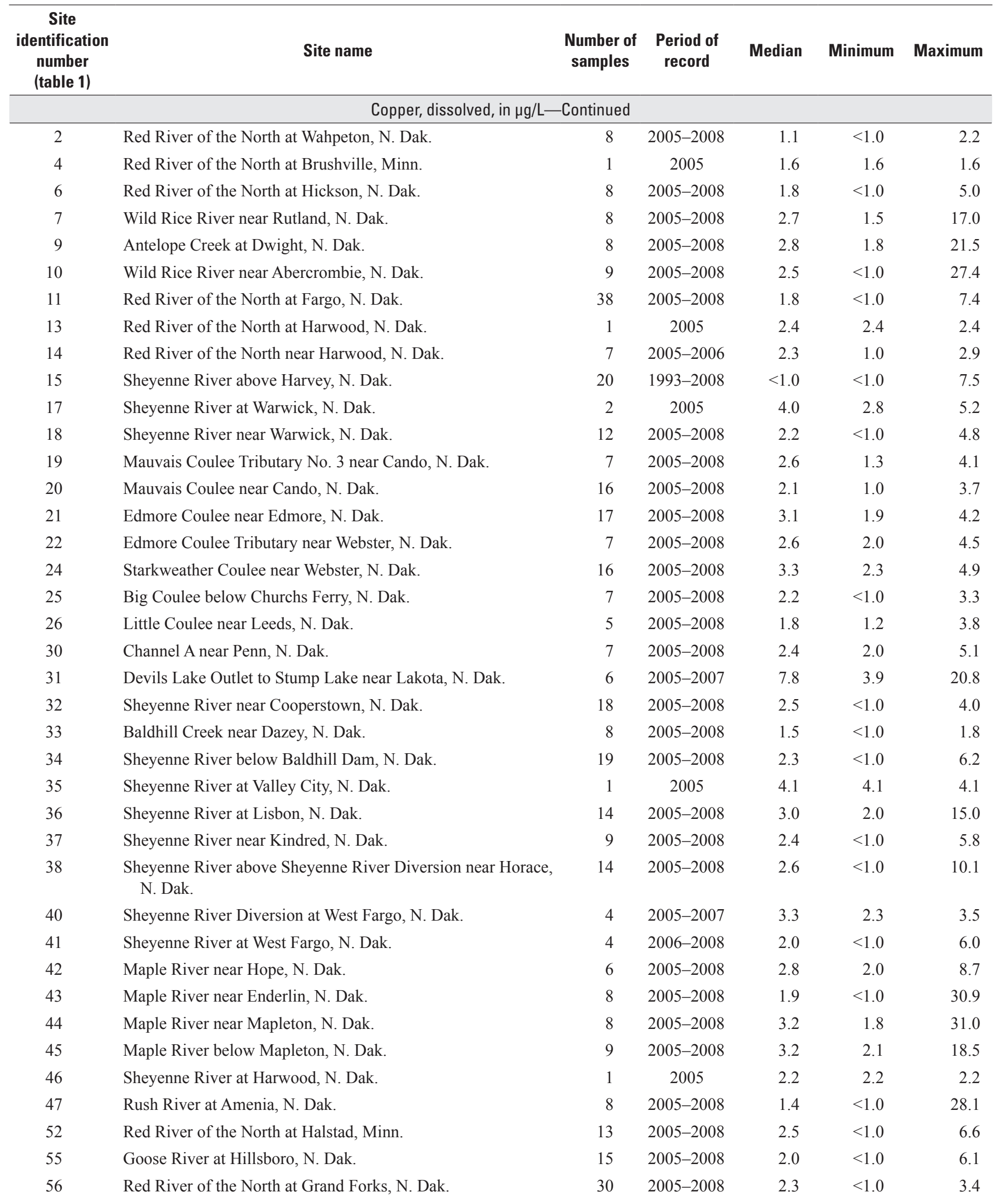


Table 1-3. Summary statistics for trace-metal constituents at selected sites in North Dakota from 1993 through 2008. - Continued [ $\mu \mathrm{g} / \mathrm{L}$, micrograms per liter; --, not available; $<$, less than]

\begin{tabular}{|c|c|c|c|c|c|c|}
\hline $\begin{array}{c}\text { Site } \\
\text { identification } \\
\text { number } \\
\text { (table 1) }\end{array}$ & Site name & $\begin{array}{l}\text { Number of } \\
\text { samples }\end{array}$ & $\begin{array}{l}\text { Period of } \\
\text { record }\end{array}$ & Median & Minimum & Maximum \\
\hline \multicolumn{7}{|c|}{ Copper, dissolved, in $\mu \mathrm{g} / \mathrm{L}$-Continued } \\
\hline 57 & Turtle River at Turtle River State Park near Arvilla, N. Dak. & 8 & 2005-2008 & 1.5 & $<1.0$ & 2.1 \\
\hline 58 & Turtle River at Manvel, N. Dak. & 30 & $2005-2008$ & 3.6 & $<1.0$ & 13.2 \\
\hline 59 & Red River of the North at Oslo, Minn. & 1 & 2005 & 2.3 & 2.3 & 2.3 \\
\hline 61 & Forest River near Fordville, N. Dak. & 8 & 2005-2008 & 1.6 & $<1.0$ & 4.2 \\
\hline 62 & Forest River near Minto, N. Dak. & 30 & 2005-2008 & 1.5 & $<1.0$ & 3.3 \\
\hline 67 & Park River at Grafton, N. Dak. & 30 & $2005-2008$ & 2.4 & $<1.0$ & 4.3 \\
\hline 68 & Red River of the North at Drayton, N. Dak. & 8 & $2005-2008$ & 3.0 & 2.1 & 4.4 \\
\hline 73 & Little South Pembina River near Walhalla, N. Dak. & 8 & $2005-2008$ & 2.5 & 1.9 & 4.6 \\
\hline 74 & Pembina River at Walhalla, N. Dak. & 8 & 2005-2008 & 2.3 & $<1.0$ & 4.9 \\
\hline 75 & Pembina River at Neche, N. Dak. & 30 & $2005-2008$ & 2.5 & $<1.0$ & 4.3 \\
\hline 76 & Tongue River at Akra, N. Dak. & 8 & $2005-2008$ & 1.6 & $<1.0$ & 2.8 \\
\hline 78 & Red River of the North at Pembina, N. Dak., site 2 & 33 & $2005-2008$ & 2.7 & 1.3 & 5.0 \\
\hline 80 & Long Creek near Noonan, N. Dak. & 17 & $1997-2008$ & 4.9 & 1.1 & 8.8 \\
\hline 83 & Souris River near Foxholm, N. Dak. & 17 & 1997-2008 & 2.6 & 1.7 & 3.9 \\
\hline 84 & Des Lacs River at Foxholm, N. Dak. & 17 & 1997-2008 & 4.7 & 2.2 & 7.6 \\
\hline 85 & Souris River above Minot, N. Dak. & 9 & 1997-1998 & 4.5 & 2.2 & 5.6 \\
\hline 86 & Bonnes Creek near Velva, N. Dak. & 1 & 2005 & 4.0 & 4.0 & 4.0 \\
\hline 87 & Souris River near Verendrye, N. Dak. & 9 & 1997-1998 & 4.4 & 2.7 & 5.5 \\
\hline 88 & Wintering River near Karlsruhe, N. Dak. & 17 & 1997-2008 & 2.8 & $<1.0$ & 8.8 \\
\hline 89 & Souris River near Bantry, N. Dak. & 17 & $1997-2008$ & 3.2 & $<1.0$ & 4.7 \\
\hline 90 & Willow Creek near Willow City, N. Dak. & 13 & $1997-2008$ & 3.0 & 1.3 & 5.9 \\
\hline 92 & Deep River near Upham, N. Dak. & 11 & $1997-2007$ & 2.0 & 1.5 & 3.6 \\
\hline 98 & Souris River near Westhope, N. Dak. & 7 & 1997-1998 & 3.9 & 3.2 & 6.8 \\
\hline 101 & Little Muddy River below Cow Creek near Williston, N. Dak. & 17 & 1999-2008 & 3.2 & 1.2 & 7.6 \\
\hline 106 & Bear Den Creek near Mandaree, N. Dak. & 8 & 2005-2008 & 7.2 & 4.0 & 20.5 \\
\hline 108 & East Fork Shell Creek near Parshall, N. Dak. & 5 & 2005-2008 & 7.2 & 3.6 & 8.0 \\
\hline 109 & Deepwater Creek near Mandaree, N. Dak. & 7 & $2005-2008$ & 3.8 & 2.2 & 10.0 \\
\hline 110 & Little Missouri River at Marmarth, N. Dak. & 17 & 1999-2008 & 6.2 & 3.1 & 26.8 \\
\hline 112 & Little Missouri River at Medora, N. Dak. & 18 & 1999-2008 & 4.7 & 3.3 & 13.0 \\
\hline 113 & Beaver Creek near Trotters, N. Dak. & 17 & 1999-2008 & 4.0 & 2.8 & 7.8 \\
\hline 114 & Little Missouri River near Watford City, N. Dak. & 18 & 1999-2008 & 5.6 & 3.1 & 16.3 \\
\hline 115 & Missouri River at Garrison Dam, N. Dak. & 10 & 1997-1998 & 1.9 & 1.2 & 2.4 \\
\hline 116 & Knife River at Manning, N. Dak. & 8 & $2005-2008$ & 5.6 & 3.2 & 9.0 \\
\hline 120 & Knife River near Golden Valley, N. Dak. & 14 & 2005-2008 & 6.2 & 4.2 & 10.1 \\
\hline 125 & Spring Creek at Zap, N. Dak. & 14 & $2005-2008$ & 3.7 & 2.2 & 5.4 \\
\hline 127 & Knife River at Hazen, N. Dak. & 14 & $2005-2008$ & 3.7 & 2.8 & 7.5 \\
\hline 140 & Square Butte Creek below Center, N. Dak. & 8 & $2005-2008$ & 3.1 & 2.1 & 5.5 \\
\hline 141 & Burnt Creek near Bismarck, N. Dak. & 6 & $2005-2008$ & 2.3 & 1.4 & 25.0 \\
\hline 142 & Missouri River at Bismarck, N. Dak. & 8 & $2005-2008$ & 1.8 & 1.3 & 3.1 \\
\hline 145 & Heart River near South Heart, N. Dak. & 1 & 2005 & 6.7 & 6.7 & 6.7 \\
\hline
\end{tabular}


Table 1-3. Summary statistics for trace-metal constituents at selected sites in North Dakota from 1993 through 2008.-Continued [ $\mu \mathrm{g} / \mathrm{L}$, micrograms per liter; --, not available; <, less than]

\begin{tabular}{|c|c|c|c|c|c|c|}
\hline $\begin{array}{c}\text { Site } \\
\text { identification } \\
\text { number } \\
\text { (table 1) } \\
\end{array}$ & Site name & $\begin{array}{l}\text { Number of } \\
\text { samples }\end{array}$ & $\begin{array}{l}\text { Period of } \\
\text { record }\end{array}$ & Median & Minimum & Maximum \\
\hline \multicolumn{7}{|c|}{ Copper, dissolved, in $\mu \mathrm{g} / \mathrm{L}$-Continued } \\
\hline 147 & Green River near New Hradec, N. Dak. & 7 & $2005-2008$ & 3.7 & 1.2 & 16.3 \\
\hline 149 & Heart River near Richardton, N. Dak. & 11 & $2005-2008$ & 3.7 & 2.3 & 19.2 \\
\hline 151 & Antelope Creek near Carson, N. Dak. & 7 & $2005-2008$ & 1.9 & $<1.0$ & 38.6 \\
\hline 152 & Big Muddy Creek near Almont, N. Dak. & 8 & $2005-2008$ & 6.4 & 2.9 & 12.5 \\
\hline 154 & Heart River at Stark Bridge near Judson, N. Dak. & 8 & $2005-2008$ & 4.2 & 1.8 & 4.9 \\
\hline 155 & Sweetbriar Creek near Judson, N. Dak. & 7 & $2005-2008$ & 4.4 & 2.0 & 42.9 \\
\hline 164 & Cannonball River near Raleigh, N. Dak. & 15 & $2005-2008$ & 4.5 & $<1.0$ & 27.4 \\
\hline 166 & Cedar Creek near Haynes, N. Dak. & 8 & $2005-2008$ & 4.9 & 2.1 & 18.1 \\
\hline 169 & Cedar Creek near Raleigh, N. Dak. & 14 & $2005-2008$ & 5.4 & 2.5 & 11.6 \\
\hline 170 & Cannonball River at Breien, N. Dak. & 15 & $2005-2008$ & 5.0 & 2.8 & 16.1 \\
\hline 172 & Beaver Creek below Linton, N. Dak. & 8 & $2005-2008$ & 3.9 & $<1.0$ & 8.8 \\
\hline 175 & James River near Manfred, N. Dak. & 6 & 1998 & 1.9 & 1.0 & 2.2 \\
\hline 176 & James River near Grace City, N. Dak. & 21 & $1998-2008$ & 1.8 & $<1.0$ & 4.4 \\
\hline 177 & James River above Arrowwood Lake near Kensal, N. Dak. & 32 & 1993-2008 & $<1.0$ & $<1.0$ & 4.0 \\
\hline 179 & James River near Pingree, N. Dak. & 17 & 1993-2008 & $<1.0$ & $<1.0$ & 2.2 \\
\hline \multicolumn{7}{|c|}{ Iron, total, in $\mu \mathrm{g} / \mathrm{L}$} \\
\hline 1 & Bois De Sioux River near Doran, Minn. & 40 & $2000-2007$ & 955 & 230 & 5,940 \\
\hline 2 & Red River of the North at Wahpeton, N. Dak. & 17 & 1993-1996 & 400 & $<10$ & 2,150 \\
\hline 3 & Red River of the North near Wahpeton, N. Dak. & 4 & 1993-2006 & 505 & 302 & 1,510 \\
\hline 4 & Red River of the North at Brushville, Minn. & 61 & 1993-2007 & 806 & 168 & 6,190 \\
\hline 5 & Red River of the North below Wahpeton, N. Dak. & 20 & 1997-1999 & 1,011 & 159 & 2,090 \\
\hline 6 & Red River of the North at Hickson, N. Dak. & 21 & 1997-1999 & 2,540 & 204 & 11,900 \\
\hline 10 & Wild Rice River near Abercrombie, N. Dak. & 58 & $1993-2007$ & 1,605 & 251 & 7,510 \\
\hline 11 & Red River of the North at Fargo, N. Dak. & 19 & 1994-2006 & 1,410 & $<10$ & 4,630 \\
\hline 13 & Red River of the North at Harwood, N. Dak. & 53 & $2000-2007$ & 2,960 & 180 & 17,700 \\
\hline 14 & Red River of the North near Harwood, N. Dak. & 52 & 1993-2007 & 2,725 & $<10$ & 8,760 \\
\hline 17 & Sheyenne River at Warwick, N. Dak. & 48 & 1996-2006 & 701 & 142 & 3,010 \\
\hline 18 & Sheyenne River near Warwick, N. Dak. & 18 & $2001-2007$ & 585 & $<10$ & 2,530 \\
\hline 24 & Starkweather Coulee near Webster, N. Dak. & 2 & 2001 & 1,455 & $<10$ & 2,900 \\
\hline 32 & Sheyenne River near Cooperstown, N. Dak. & 85 & 1994-2007 & 857 & 145 & 5,520 \\
\hline
\end{tabular}


Table 1-3. Summary statistics for trace-metal constituents at selected sites in North Dakota from 1993 through 2008. - Continued [ $\mu \mathrm{g} / \mathrm{L}$, micrograms per liter; --, not available; $<$, less than]

\begin{tabular}{|c|c|c|c|c|c|c|}
\hline $\begin{array}{c}\text { Site } \\
\text { identification } \\
\text { number } \\
\text { (table 1) } \\
\end{array}$ & Site name & $\begin{array}{l}\text { Number of } \\
\text { samples }\end{array}$ & $\begin{array}{l}\text { Period of } \\
\text { record }\end{array}$ & Median & Minimum & Maximum \\
\hline \multicolumn{7}{|c|}{ Iron, total, in $\mu \mathrm{g} / \mathrm{L}$-Continued } \\
\hline 33 & Baldhill Creek near Dazey, N. Dak. & 3 & 1993-1996 & 161 & 64 & 291 \\
\hline 34 & Sheyenne River below Baldhill Dam, N. Dak. & 80 & 1994-2007 & 134 & $<10$ & 2,620 \\
\hline 35 & Sheyenne River at Valley City, N. Dak. & 4 & 1993 & 691 & 83 & 1,210 \\
\hline 36 & Sheyenne River at Lisbon, N. Dak. & 69 & $1997-2007$ & 1,010 & 68 & 11,400 \\
\hline 37 & Sheyenne River near Kindred, N. Dak. & 72 & $1996-2007$ & 1,730 & 322 & 20,700 \\
\hline 38 & $\begin{array}{l}\text { Sheyenne River above Sheyenne River Diversion near Horace, } \\
\text { N. Dak. }\end{array}$ & 5 & 2006-2007 & 2,800 & 481 & 5,020 \\
\hline 41 & Sheyenne River at West Fargo, N. Dak. & 13 & $1994-2006$ & 1,620 & 377 & 5,750 \\
\hline 45 & Maple River below Mapleton, N. Dak. & 47 & $1997-2007$ & 1,680 & 165 & 13,800 \\
\hline 46 & Sheyenne River at Harwood, N. Dak. & 31 & 1993-1999 & 3,160 & $<10$ & 9,760 \\
\hline 52 & Red River of the North at Halstad, Minn. & 7 & $2001-2007$ & 5,100 & 2,850 & 16,000 \\
\hline 55 & Goose River at Hillsboro, N. Dak. & 76 & 1994-2007 & 1,030 & 66 & 12,100 \\
\hline 56 & Red River of the North at Grand Forks, N. Dak. & 48 & $1997-2006$ & 1,830 & 18 & 18,100 \\
\hline 58 & Turtle River at Manvel, N. Dak. & 39 & 1993-2006 & 742 & 55 & 9,870 \\
\hline 62 & Forest River near Minto, N. Dak. & 48 & 1994-2006 & 522 & $<10$ & 10,500 \\
\hline 67 & Park River at Grafton, N. Dak. & 41 & 1994-2006 & 578 & $<10$ & 22,600 \\
\hline 68 & Red River of the North at Drayton, N. Dak. & 15 & 1994-1996 & 1,810 & 671 & 4,810 \\
\hline 75 & Pembina River at Neche, N. Dak. & 56 & $1994-2006$ & 1,200 & $<10$ & 50,200 \\
\hline 76 & Tongue River at Akra, N. Dak. & 1 & 1993 & 649 & 649 & 649 \\
\hline 78 & Red River of the North at Pembina, N. Dak., site 2 & 59 & $1997-2007$ & 3,220 & $<10$ & 26,900 \\
\hline 79 & Red River of the North at Emerson, Manitoba & 2 & 2001 & 11 & $<10$ & 11 \\
\hline 80 & Long Creek near Noonan, N. Dak. & 10 & 1997 & 481 & 236 & 1,010 \\
\hline 82 & Souris River near Sherwood, N. Dak. & 120 & $1993-2008$ & 554 & 16 & 4,717 \\
\hline 83 & Souris River near Foxholm, N. Dak. & 35 & 1993-1998 & 170 & 32 & 579 \\
\hline 84 & Des Lacs River at Foxholm, N. Dak. & 97 & 1993-2007 & 858 & 68 & 4,180 \\
\hline 85 & Souris River above Minot, N. Dak. & 136 & 1994-2008 & 305 & 33 & 1,726 \\
\hline 87 & Souris River near Verendrye, N. Dak. & 177 & $1993-2008$ & 709 & 147 & 21,000 \\
\hline 88 & Wintering River near Karlsruhe, N. Dak. & 11 & $1997-1998$ & 353 & 201 & 426 \\
\hline 89 & Souris River near Bantry, N. Dak. & 64 & $1993-2000$ & 790 & 140 & 4,380 \\
\hline 90 & Willow Creek near Willow City, N. Dak. & 30 & $1993-2000$ & 497 & 19 & 1,600 \\
\hline 91 & Stone Creek near Kramer, N. Dak. & 19 & $1993-2000$ & 226 & 42 & 2,490 \\
\hline 92 & Deep River near Upham, N. Dak. & 28 & $1997-2000$ & 195 & 55 & 1,740 \\
\hline 95 & Cut Bank Creek at Upham, N. Dak. & 16 & $1999-2000$ & 50 & $<10$ & 4,660 \\
\hline 97 & Boundary Creek near Landa, N. Dak. & 19 & $1993-2000$ & 272 & 61 & 4,320 \\
\hline 98 & Souris River near Westhope, N. Dak. & 21 & $1995-2008$ & 500 & 121 & 2,130 \\
\hline 101 & Little Muddy River below Cow Creek near Williston, N. Dak. & 9 & 1999 & 783 & 384 & 3,470 \\
\hline 110 & Little Missouri River at Marmarth, N. Dak. & 8 & 1999 & 20,150 & 984 & 82,900 \\
\hline 112 & Little Missouri River at Medora, N. Dak. & 88 & $1993-2007$ & 5,650 & $<10$ & 892,000 \\
\hline 113 & Beaver Creek near Trotters, N. Dak. & 9 & 1999 & 273 & 113 & 2,420 \\
\hline 114 & Little Missouri River near Watford City, N. Dak. & 81 & 1994-2007 & 10,800 & $<10$ & 960,000 \\
\hline
\end{tabular}


Table 1-3. Summary statistics for trace-metal constituents at selected sites in North Dakota from 1993 through 2008.-Continued [ $\mu \mathrm{g} / \mathrm{L}$, micrograms per liter; --, not available; <, less than]

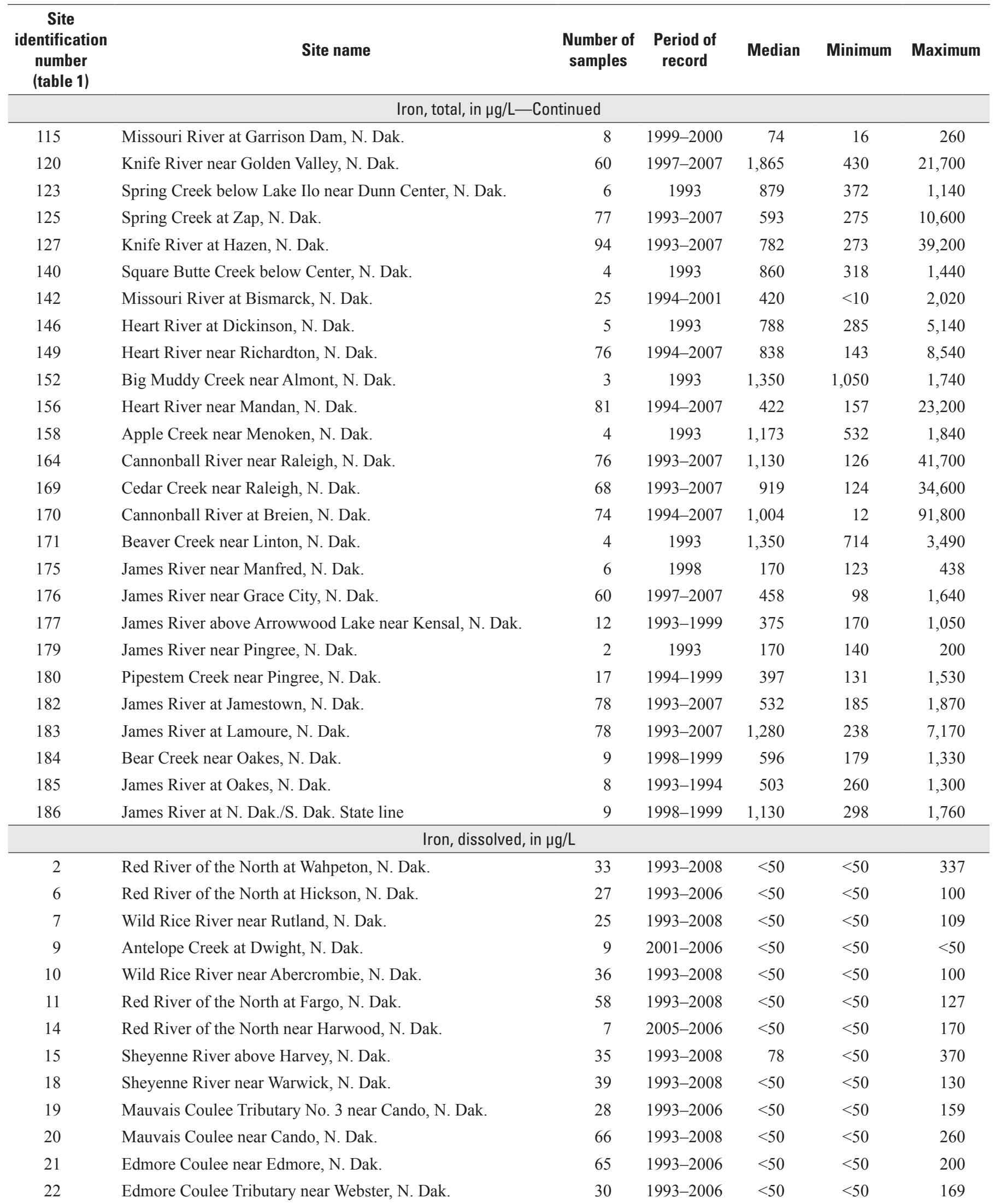


Table 1-3. Summary statistics for trace-metal constituents at selected sites in North Dakota from 1993 through 2008. -Continued [ $\mu \mathrm{g} / \mathrm{L}$, micrograms per liter; --, not available; <, less than]

\begin{tabular}{|c|c|c|c|c|c|c|}
\hline $\begin{array}{c}\text { Site } \\
\text { identification } \\
\text { number } \\
\text { (table 1) } \\
\end{array}$ & Site name & $\begin{array}{l}\text { Number of } \\
\text { samples }\end{array}$ & $\begin{array}{l}\text { Period of } \\
\text { record }\end{array}$ & Median & Minimum & Maximum \\
\hline \multicolumn{7}{|c|}{ Iron, dissolved, in $\mu \mathrm{g} / \mathrm{L}$-Continued } \\
\hline 24 & Starkweather Coulee near Webster, N. Dak. & 57 & 1993-2006 & $<50$ & $<50$ & 147 \\
\hline 25 & Big Coulee below Churchs Ferry, N. Dak. & 21 & 1998-2008 & $<50$ & $<50$ & 141 \\
\hline 26 & Little Coulee near Leeds, N. Dak. & 16 & 1998-2008 & 55 & $<50$ & 222 \\
\hline 27 & Little Coulee near Brinsmade, N. Dak. & 11 & 1993-1998 & $<50$ & $<50$ & 100 \\
\hline 28 & Big Coulee near Churchs Ferry, N. Dak. & 43 & 1993-1997 & $<50$ & $<50$ & 140 \\
\hline 30 & Channel A near Penn, N. Dak. & 59 & 1993-2006 & $<50$ & $<50$ & 210 \\
\hline 31 & Devils Lake Outlet to Stump Lake near Lakota, N. Dak. & 10 & $2002-2006$ & 70 & $<50$ & 115 \\
\hline 32 & Sheyenne River near Cooperstown, N. Dak. & 35 & 1993-2008 & $<50$ & $<50$ & 477 \\
\hline 33 & Baldhill Creek near Dazey, N. Dak. & 29 & 1993-2008 & $<50$ & $<50$ & 140 \\
\hline 34 & Sheyenne River below Baldhill Dam, N. Dak. & 36 & 1993-2008 & $<50$ & $<50$ & 146 \\
\hline 35 & Sheyenne River at Valley City, N. Dak. & 13 & 1994-2005 & $<50$ & $<50$ & 90 \\
\hline 36 & Sheyenne River at Lisbon, N. Dak. & 52 & 1993-2008 & $<50$ & $<50$ & 490 \\
\hline 37 & Sheyenne River near Kindred, N. Dak. & 56 & 1993-2008 & $<50$ & $<50$ & 130 \\
\hline 38 & $\begin{array}{l}\text { Sheyenne River above Sheyenne River Diversion near Horace, } \\
\text { N. Dak. }\end{array}$ & 33 & 1993-2006 & $<50$ & $<50$ & 160 \\
\hline 40 & Sheyenne River Diversion at West Fargo, N. Dak. & 12 & 1994-2006 & $<50$ & $<50$ & 110 \\
\hline 41 & Sheyenne River at West Fargo, N. Dak. & 15 & 1993-2008 & $<50$ & $<50$ & 120 \\
\hline 42 & Maple River near Hope, N. Dak. & 19 & 1993-2006 & $<50$ & $<50$ & 90 \\
\hline 43 & Maple River near Enderlin, N. Dak. & 29 & 1993-2008 & $<50$ & $<50$ & 120 \\
\hline 44 & Maple River near Mapleton, N. Dak. & 14 & 1995-2007 & $<50$ & $<50$ & 91 \\
\hline 45 & Maple River below Mapleton, N. Dak. & 27 & 1995-2008 & $<50$ & $<50$ & 150 \\
\hline 46 & Sheyenne River at Harwood, N. Dak. & 6 & $2000-2005$ & $<50$ & $<50$ & $<50$ \\
\hline 47 & Rush River at Amenia, N. Dak. & 27 & 1993-2006 & $<50$ & $<50$ & 161 \\
\hline 52 & Red River of the North at Halstad, Minn. & 54 & 1993-2006 & $<50$ & $<50$ & 1,710 \\
\hline 53 & Beaver Creek near Finley, N. Dak. & 24 & 1993-2003 & $<50$ & $<50$ & 210 \\
\hline 55 & Goose River at Hillsboro, N. Dak. & 42 & 1993-2008 & $<50$ & $<50$ & 140 \\
\hline 56 & Red River of the North at Grand Forks, N. Dak. & 80 & 1993-2008 & $<50$ & $<50$ & 500 \\
\hline 57 & Turtle River at Turtle River State Park near Arvilla, N. Dak. & 86 & 1993-2006 & $<50$ & $<50$ & 146 \\
\hline 58 & Turtle River at Manvel, N. Dak. & 30 & 2005-2008 & $<50$ & $<50$ & 213 \\
\hline 59 & Red River of the North at Oslo, Minn. & 14 & 1993-2005 & $<50$ & $<50$ & 6,000 \\
\hline 61 & Forest River near Fordville, N. Dak. & 27 & 1993-2006 & $<50$ & $<50$ & 150 \\
\hline 62 & Forest River near Minto, N. Dak. & 54 & 1993-2008 & $<50$ & $<50$ & 150 \\
\hline 63 & South Branch Park River below Homme Dam, N. Dak. & 4 & 1993-1994 & $<50$ & $<50$ & $<50$ \\
\hline 67 & Park River at Grafton, N. Dak. & 53 & 1993-2008 & $<50$ & $<50$ & 130 \\
\hline 68 & Red River of the North at Drayton, N. Dak. & 32 & $1993-2008$ & $<50$ & $<50$ & 160 \\
\hline 70 & Hidden Island Coulee near Hansboro, N. Dak. & 4 & 1993-1995 & $<50$ & $<50$ & 80 \\
\hline 73 & Little South Pembina River near Walhalla, N. Dak. & 13 & $2001-2007$ & $<50$ & $<50$ & 110 \\
\hline 74 & Pembina River at Walhalla, N. Dak. & 40 & 1993-2006 & $<50$ & $<50$ & 440 \\
\hline 75 & Pembina River at Neche, N. Dak. & 53 & 1993-2008 & $<50$ & $<50$ & 160 \\
\hline 76 & Tongue River at Akra, N. Dak. & 32 & 1993-2008 & $<50$ & $<50$ & 240 \\
\hline
\end{tabular}


Table 1-3. Summary statistics for trace-metal constituents at selected sites in North Dakota from 1993 through 2008.—Continued [ $\mu \mathrm{g} / \mathrm{L}$, micrograms per liter; --, not available; $<$, less than]

\begin{tabular}{|c|c|c|c|c|c|c|}
\hline $\begin{array}{c}\text { Site } \\
\text { identification } \\
\text { number } \\
\text { (table 1) }\end{array}$ & Site name & $\begin{array}{l}\text { Number of } \\
\text { samples }\end{array}$ & $\begin{array}{c}\text { Period of } \\
\text { record }\end{array}$ & Median & Minimum & Maximum \\
\hline 78 & Red River of the North at Pembina, N. Dak., site 2 & 85 & 1994-2008 & $<50$ & $<50$ & 146 \\
\hline 79 & Red River of the North at Emerson, Manitoba & 37 & 1993-2004 & $<50$ & $<50$ & 260 \\
\hline 83 & Souris River near Foxholm, N. Dak. & 20 & 1999-2008 & $<50$ & $<50$ & 60 \\
\hline 84 & Des Lacs River at Foxholm, N. Dak. & 32 & $1993-2008$ & 65 & $<50$ & 350 \\
\hline 85 & Souris River above Minot, N. Dak. & 12 & 1993-1998 & $<50$ & $<50$ & 170 \\
\hline 86 & Bonnes Creek near Velva, N. Dak. & 14 & $1993-2005$ & 194 & 60 & 310 \\
\hline 88 & Wintering River near Karlsruhe, N. Dak. & 32 & $1993-2008$ & 184 & $<50$ & 550 \\
\hline 97 & Boundary Creek near Landa, N. Dak. & 1 & 1994 & 130 & 130 & 130 \\
\hline 98 & Souris River near Westhope, N. Dak. & 10 & $1993-2001$ & 56 & $<50$ & 250 \\
\hline 101 & Little Muddy River below Cow Creek near Williston, N. Dak. & 31 & $1993-2008$ & 67 & $<50$ & 340 \\
\hline 106 & Bear Den Creek near Mandaree, N. Dak. & 35 & $1993-2007$ & 81 & $<50$ & 780 \\
\hline 108 & East Fork Shell Creek near Parshall, N. Dak. & 48 & $1993-2007$ & 69 & $<50$ & 520 \\
\hline 109 & Deepwater Creek near Mandaree, N. Dak. & 49 & $1993-2008$ & 51 & $<50$ & 480 \\
\hline 110 & Little Missouri River at Marmarth, N. Dak. & 32 & $1993-2008$ & $<50$ & $<50$ & 10,000 \\
\hline 112 & Little Missouri River at Medora, N. Dak. & 16 & $2001-2008$ & $<50$ & $<50$ & 363 \\
\hline 127 & Knife River at Hazen, N. Dak. & 35 & $1993-2008$ & $<50$ & $<50$ & 5,200 \\
\hline 135 & Turtle Creek above Washburn, N. Dak. & 63 & $1993-2003$ & $<50$ & $<50$ & 1,500 \\
\hline 136 & Painted Woods Creek near Wilton, N. Dak. & 61 & $1993-2003$ & $<50$ & $<50$ & 480 \\
\hline 140 & Square Butte Creek below Center, N. Dak. & 31 & $1993-2008$ & $<50$ & $<50$ & 400 \\
\hline 141 & Burnt Creek near Bismarck, N. Dak. & 28 & $1993-2008$ & 94 & $<50$ & 280 \\
\hline 142 & Missouri River at Bismarck, N. Dak. & 23 & $1993-2008$ & $<50$ & $<50$ & 140 \\
\hline 145 & Heart River near South Heart, N. Dak. & 12 & $1993-2005$ & 100 & $<50$ & 3,870 \\
\hline 146 & Heart River at Dickinson, N. Dak. & 4 & 1993-1994 & 75 & $<50$ & 140 \\
\hline 147 & Green River near New Hradec, N. Dak. & 29 & $1993-2008$ & 130 & $<50$ & 760 \\
\hline 149 & Heart River near Richardton, N. Dak. & 32 & $1993-2008$ & $<50$ & $<50$ & 319 \\
\hline 150 & Heart River above Lake Tschida near Glen Ullin, N. Dak. & 29 & $1993-2007$ & 57 & $<50$ & 409 \\
\hline 151 & Antelope Creek near Carson, N. Dak. & 17 & $1999-2008$ & 70 & $<50$ & 271 \\
\hline 152 & Big Muddy Creek near Almont, N. Dak. & 32 & $1993-2008$ & 70 & $<50$ & 2,600 \\
\hline 153 & Heart River near Lark, N. Dak. & 6 & $1993-1995$ & 55 & $<50$ & 270 \\
\hline 154 & Heart River at Stark Bridge near Judson, N. Dak. & 29 & $1993-2007$ & $<50$ & $<50$ & 390 \\
\hline
\end{tabular}


Table 1-3. Summary statistics for trace-metal constituents at selected sites in North Dakota from 1993 through 2008. - Continued [ $\mu \mathrm{g} / \mathrm{L}$, micrograms per liter; --, not available; <, less than]

\begin{tabular}{|c|c|c|c|c|c|c|}
\hline $\begin{array}{c}\text { Site } \\
\text { identification } \\
\text { number } \\
\text { (table 1) }\end{array}$ & Site name & $\begin{array}{l}\text { Number of } \\
\text { samples }\end{array}$ & $\begin{array}{l}\text { Period of } \\
\text { record }\end{array}$ & Median & Minimum & Maximum \\
\hline \multicolumn{7}{|c|}{ Iron, dissolved, in $\mu \mathrm{g} / \mathrm{L}$-Continued } \\
\hline 155 & Sweetbriar Creek near Judson, N. Dak. & 12 & $2002-2008$ & 54 & $<50$ & 287 \\
\hline 156 & Heart River near Mandan, N. Dak. & 35 & 1993-2008 & $<50$ & $<50$ & 360 \\
\hline 157 & Long Lake Creek above Long Lake near Moffit, N. Dak. & 24 & 1993-2004 & 110 & $<50$ & 850 \\
\hline 158 & Apple Creek near Menoken, N. Dak. & 31 & $1993-2008$ & 63 & $<50$ & 1,100 \\
\hline 162 & Cannonball River at Regent, N. Dak. & 29 & $1993-2008$ & 60 & $<50$ & 540 \\
\hline 164 & Cannonball River near Raleigh, N. Dak. & 15 & $2001-2008$ & $<50$ & $<50$ & 392 \\
\hline 165 & White Butte Fork Cedar Creek near Scranton, N. Dak. & 5 & 1993-1995 & 70 & $<50$ & 150 \\
\hline 166 & Cedar Creek near Haynes, N. Dak. & 31 & 1993-2008 & 60 & $<50$ & 364 \\
\hline 169 & Cedar Creek near Raleigh, N. Dak. & 29 & 1993-2008 & $<50$ & $<50$ & 386 \\
\hline 170 & Cannonball River at Breien, N. Dak. & 34 & 1993-2008 & $<50$ & $<50$ & 410 \\
\hline 172 & Beaver Creek below Linton, N. Dak. & 32 & 1993-2008 & $<50$ & $<50$ & 590 \\
\hline 173 & Porcupine Creek near Fort Yates, N. Dak. & 35 & 1993-1999 & $<50$ & $<50$ & 6,180 \\
\hline 175 & James River near Manfred, N. Dak. & 16 & 1993-1995 & 67 & $<50$ & 170 \\
\hline 176 & James River near Grace City, N. Dak. & 48 & 1993-2008 & $<50$ & $<50$ & 180 \\
\hline 177 & James River above Arrowwood Lake near Kensal, N. Dak. & 124 & $1993-2008$ & $<50$ & $<50$ & 280 \\
\hline 179 & James River near Pingree, N. Dak. & 75 & $1993-2008$ & $<50$ & $<50$ & 140 \\
\hline 180 & Pipestem Creek near Pingree, N. Dak. & 32 & 1993-2008 & 60 & $<50$ & 190 \\
\hline 182 & James River at Jamestown, N. Dak. & 49 & $1993-2008$ & $<50$ & $<50$ & 152 \\
\hline 183 & James River at Lamoure, N. Dak. & 45 & $1993-2008$ & $<50$ & $<50$ & 190 \\
\hline 184 & Bear Creek near Oakes, N. Dak. & 32 & 1993-2008 & $<50$ & $<50$ & 230 \\
\hline 185 & James River at Oakes, N. Dak. & 23 & 1993-2008 & $<50$ & $<50$ & 110 \\
\hline 186 & James River at N. Dak./S. Dak. State line & 14 & $2002-2008$ & $<50$ & $<50$ & 160 \\
\hline \multicolumn{7}{|c|}{ Lead, total, in $\mu \mathrm{g} / \mathrm{L}$} \\
\hline 1 & Bois De Sioux River near Doran, Minn. & 40 & $2000-2007$ & $<1.0$ & $<1.0$ & 4.7 \\
\hline 2 & Red River of the North at Wahpeton, N. Dak. & 2 & 1996 & 6.4 & 4.0 & 8.7 \\
\hline 3 & Red River of the North near Wahpeton, N. Dak. & 1 & 2006 & 1.1 & 1.1 & 1.1 \\
\hline 4 & Red River of the North at Brushville, Minn. & 54 & 1996-2007 & $<1.0$ & $<1.0$ & 3.1 \\
\hline 10 & Wild Rice River near Abercrombie, N. Dak. & 45 & $1996-2007$ & 1.6 & $<1.0$ & 5.2 \\
\hline 11 & Red River of the North at Fargo, N. Dak. & 6 & $1996-2006$ & 1.8 & $<1.0$ & 6.6 \\
\hline 13 & Red River of the North at Harwood, N. Dak. & 53 & $2000-2007$ & 1.6 & $<1.0$ & 6.7 \\
\hline 14 & Red River of the North near Harwood, N. Dak. & 10 & $1996-2007$ & 3.4 & $<1.0$ & 9.0 \\
\hline 17 & Sheyenne River at Warwick, N. Dak. & 48 & 1996-2006 & $<1.0$ & $<1.0$ & 7.9 \\
\hline 18 & Sheyenne River near Warwick, N. Dak. & 16 & $2005-2007$ & $<1.0$ & $<1.0$ & 1.5 \\
\hline 32 & Sheyenne River near Cooperstown, N. Dak. & 74 & 1996-2007 & $<1.0$ & $<1.0$ & 6.2 \\
\hline 33 & Baldhill Creek near Dazey, N. Dak. & 1 & 1996 & 6.8 & 6.8 & 6.8 \\
\hline 34 & Sheyenne River below Baldhill Dam, N. Dak. & 67 & $1996-2007$ & $<1.0$ & $<1.0$ & 7.6 \\
\hline 36 & Sheyenne River at Lisbon, N. Dak. & 69 & 1997-2007 & $<1.0$ & $<1.0$ & 8.4 \\
\hline 37 & Sheyenne River near Kindred, N. Dak. & 70 & $1996-2007$ & 1.4 & $<1.0$ & 14.4 \\
\hline 38 & Sheyenne River above Sheyenne River Diversion near Horace, & 5 & $2006-2007$ & 2.2 & 1.2 & 9.4 \\
\hline
\end{tabular}


Table 1-3. Summary statistics for trace-metal constituents at selected sites in North Dakota from 1993 through 2008.-Continued [ $\mu \mathrm{g} / \mathrm{L}$, micrograms per liter; --, not available; <, less than]

\begin{tabular}{|c|c|c|c|c|c|c|}
\hline $\begin{array}{c}\text { Site } \\
\text { identification } \\
\text { number } \\
\text { (table 1) }\end{array}$ & Site name & $\begin{array}{c}\text { Number of } \\
\text { samples }\end{array}$ & $\begin{array}{l}\text { Period of } \\
\text { record }\end{array}$ & Median & Minimum & Maximum \\
\hline \multicolumn{7}{|c|}{ Lead, total, in $\mu \mathrm{g} / \mathrm{L}$-Continued } \\
\hline 45 & Maple River below Mapleton, N. Dak. & 47 & $1997-2007$ & 1.5 & $<1.0$ & 7.3 \\
\hline 52 & Red River of the North at Halstad, Minn. & 5 & $2006-2007$ & 3.4 & $<1.0$ & 7.6 \\
\hline 55 & Goose River at Hillsboro, N. Dak. & 64 & $1996-2007$ & $<1.0$ & $<1.0$ & 8.0 \\
\hline 56 & Red River of the North at Grand Forks, N. Dak. & 47 & $1997-2006$ & $<1.0$ & $<1.0$ & 15.3 \\
\hline 58 & Turtle River at Manvel, N. Dak. & 38 & 1997-2006 & $<1.0$ & $<1.0$ & 44.3 \\
\hline 62 & Forest River near Minto, N. Dak. & 43 & 1996-2006 & $<1.0$ & $<1.0$ & 8.3 \\
\hline 67 & Park River at Grafton, N. Dak. & 37 & 1996-2006 & $<1.0$ & $<1.0$ & 12.7 \\
\hline 68 & Red River of the North at Drayton, N. Dak. & 5 & 1996 & 2.5 & $<1.0$ & 5.6 \\
\hline 75 & Pembina River at Neche, N. Dak. & 47 & 1996-2006 & $<1.0$ & $<1.0$ & 35.4 \\
\hline 78 & Red River of the North at Pembina, N. Dak., site 2 & 58 & $1997-2007$ & 1.4 & $<1.0$ & 13.7 \\
\hline 80 & Long Creek near Noonan, N. Dak. & 10 & 1997 & 1.9 & $<1.0$ & 8.2 \\
\hline 82 & Souris River near Sherwood, N. Dak. & 115 & 1993-2008 & $<1.0$ & $<1.0$ & 3.0 \\
\hline 83 & Souris River near Foxholm, N. Dak. & 35 & 1993-1998 & $<1.0$ & $<1.0$ & 11.2 \\
\hline 84 & Des Lacs River at Foxholm, N. Dak. & 83 & $1996-2007$ & $<1.0$ & $<1.0$ & 356.0 \\
\hline 85 & Souris River above Minot, N. Dak. & 121 & $1996-2008$ & $<1.0$ & $<1.0$ & 55.1 \\
\hline 87 & Souris River near Verendrye, N. Dak. & 169 & 1993-2008 & $<1.0$ & $<1.0$ & 14.0 \\
\hline 88 & Wintering River near Karlsruhe, N. Dak. & 10 & $1997-1998$ & 2.8 & $<1.0$ & 4.9 \\
\hline 89 & Souris River near Bantry, N. Dak. & 45 & 1993-1998 & $<1.0$ & $<1.0$ & 7.9 \\
\hline 90 & Willow Creek near Willow City, N. Dak. & 8 & 1993-1997 & $<1.0$ & $<1.0$ & 2.9 \\
\hline 91 & Stone Creek near Kramer, N. Dak. & 3 & 1993 & $<1.0$ & $<1.0$ & $<1.0$ \\
\hline 92 & Deep River near Upham, N. Dak. & 6 & 1997 & 1.3 & $<1.0$ & 5.4 \\
\hline 97 & Boundary Creek near Landa, N. Dak. & 3 & 1993 & $<1.0$ & $<1.0$ & $<1.0$ \\
\hline 98 & Souris River near Westhope, N. Dak. & 19 & $1995-2008$ & 1.1 & $<1.0$ & 7.0 \\
\hline 101 & Little Muddy River below Cow Creek near Williston, N. Dak. & 9 & 1999 & $<1.0$ & $<1.0$ & $<1.0$ \\
\hline 110 & Little Missouri River at Marmarth, N. Dak. & 8 & 1999 & 12.8 & $<1.0$ & 57.0 \\
\hline 112 & Little Missouri River at Medora, N. Dak. & 74 & 1996-2007 & 6.6 & $<1.0$ & 535.0 \\
\hline 113 & Beaver Creek near Trotters, N. Dak. & 9 & 1999 & $<1.0$ & $<1.0$ & 1.8 \\
\hline 114 & Little Missouri River near Watford City, N. Dak. & 69 & $1996-2007$ & 12.4 & $<1.0$ & 498.0 \\
\hline 120 & Knife River near Golden Valley, N. Dak. & 60 & $1997-2007$ & 1.4 & $<1.0$ & 17.3 \\
\hline 125 & Spring Creek at Zap, N. Dak. & 64 & $1997-2007$ & $<1.0$ & $<1.0$ & 11.3 \\
\hline 127 & Knife River at Hazen, N. Dak. & 76 & $1996-2007$ & $<1.0$ & $<1.0$ & 20.3 \\
\hline 149 & Heart River near Richardton, N. Dak. & 68 & $1996-2007$ & $<1.0$ & $<1.0$ & 8.6 \\
\hline 156 & Heart River near Mandan, N. Dak. & 68 & $1996-2007$ & $<1.0$ & $<1.0$ & 11.8 \\
\hline 164 & Cannonball River near Raleigh, N. Dak. & 62 & $1996-2007$ & 1.3 & $<1.0$ & 23.2 \\
\hline 169 & Cedar Creek near Raleigh, N. Dak. & 54 & 1996-2007 & 1.1 & $<1.0$ & 13.8 \\
\hline 170 & Cannonball River at Breien, N. Dak. & 64 & 1996-2007 & 1.4 & $<1.0$ & 76.6 \\
\hline 175 & James River near Manfred, N. Dak. & 6 & 1998 & $<1.0$ & $<1.0$ & $<1.0$ \\
\hline 176 & James River near Grace City, N. Dak. & 60 & $1997-2007$ & $<1.0$ & $<1.0$ & 16.4 \\
\hline 177 & James River above Arrowwood Lake near Kensal, N. Dak. & 9 & 1998-1999 & $<1.0$ & $<1.0$ & 8.0 \\
\hline 180 & Pipestem Creek near Pingree, N. Dak. & 9 & 1998-1999 & $<1.0$ & $<1.0$ & $<1.0$ \\
\hline
\end{tabular}


Table 1-3. Summary statistics for trace-metal constituents at selected sites in North Dakota from 1993 through 2008. - Continued [ $\mu \mathrm{g} / \mathrm{L}$, micrograms per liter; --, not available; $<$, less than]

\begin{tabular}{|c|c|c|c|c|c|c|}
\hline $\begin{array}{c}\text { Site } \\
\text { identification } \\
\text { number } \\
\text { (table 1) }\end{array}$ & Site name & $\begin{array}{c}\text { Number of } \\
\text { samples }\end{array}$ & $\begin{array}{l}\text { Period of } \\
\text { record }\end{array}$ & Median & Minimum & Maximum \\
\hline \multicolumn{7}{|c|}{ Lead, total, in $\mu \mathrm{g} / \mathrm{L}-$ Continued } \\
\hline 182 & James River at Jamestown, N. Dak. & 75 & 1997-2007 & $<1.0$ & $<1.0$ & 11.7 \\
\hline 183 & James River at Lamoure, N. Dak. & 73 & 1996-2007 & $<1.0$ & $<1.0$ & 6.2 \\
\hline 184 & Bear Creek near Oakes, N. Dak. & 9 & 1998-1999 & $<1.0$ & $<1.0$ & 2.6 \\
\hline 186 & James River at N. Dak./S. Dak. State line & 9 & 1998-1999 & $<1.0$ & $<1.0$ & 1.3 \\
\hline \multicolumn{7}{|c|}{ Lithium, dissolved, in $\mu \mathrm{g} / \mathrm{L}$} \\
\hline 2 & Red River of the North at Wahpeton, N. Dak. & 23 & 1993-2004 & 20 & $<10$ & 100 \\
\hline 6 & Red River of the North at Hickson, N. Dak. & 21 & 1993-2004 & 24 & $<10$ & 100 \\
\hline 7 & Wild Rice River near Rutland, N. Dak. & 20 & 1993-2004 & 120 & 40 & 293 \\
\hline 9 & Antelope Creek at Dwight, N. Dak. & 5 & 2001-2004 & 71 & 38 & 100 \\
\hline 10 & Wild Rice River near Abercrombie, N. Dak. & 23 & 1993-2004 & 90 & 20 & 160 \\
\hline 11 & Red River of the North at Fargo, N. Dak. & 18 & 1993-2004 & 30 & 20 & 100 \\
\hline 15 & Sheyenne River above Harvey, N. Dak. & 27 & 1993-2004 & 90 & 17 & 167 \\
\hline 18 & Sheyenne River near Warwick, N. Dak. & 25 & 1993-2004 & 80 & $<10$ & 112 \\
\hline 19 & Mauvais Coulee Tributary No. 3 near Cando, N. Dak. & 24 & 1993-2004 & 40 & $<10$ & 107 \\
\hline 20 & Mauvais Coulee near Cando, N. Dak. & 57 & 1993-2004 & 50 & $<10$ & 100 \\
\hline 21 & Edmore Coulee near Edmore, N. Dak. & 58 & 1993-2004 & 40 & $<10$ & 103 \\
\hline 22 & Edmore Coulee Tributary near Webster, N. Dak. & 25 & 1993-2004 & 50 & $<10$ & 130 \\
\hline 24 & Starkweather Coulee near Webster, N. Dak. & 51 & 1993-2004 & 30 & $<10$ & 100 \\
\hline 25 & Big Coulee below Churchs Ferry, N. Dak. & 16 & 1998-2004 & 40 & 30 & 100 \\
\hline 26 & Little Coulee near Leeds, N. Dak. & 11 & 1998-2004 & 70 & 20 & 100 \\
\hline 27 & Little Coulee near Brinsmade, N. Dak. & 11 & 1993-1998 & 50 & 30 & 60 \\
\hline 28 & Big Coulee near Churchs Ferry, N. Dak. & 43 & 1993-1997 & 30 & 20 & 60 \\
\hline 30 & Channel A near Penn, N. Dak. & 56 & 1993-2004 & 30 & 20 & 100 \\
\hline 31 & Devils Lake Outlet to Stump Lake near Lakota, N. Dak. & 6 & 2002-2004 & 342 & 130 & 400 \\
\hline 32 & Sheyenne River near Cooperstown, N. Dak. & 21 & 1993-2004 & 70 & 19 & 100 \\
\hline 33 & Baldhill Creek near Dazey, N. Dak. & 21 & 1993-2004 & 50 & $<10$ & 100 \\
\hline 34 & Sheyenne River below Baldhill Dam, N. Dak. & 21 & 1993-2004 & 69 & 20 & 100 \\
\hline 35 & Sheyenne River at Valley City, N. Dak. & 12 & 1994-2004 & 65 & 20 & 100 \\
\hline 36 & Sheyenne River at Lisbon, N. Dak. & 17 & 1996-2004 & 70 & 30 & 100 \\
\hline 37 & Sheyenne River near Kindred, N. Dak. & 23 & 1993-2004 & 60 & 20 & 100 \\
\hline 38 & $\begin{array}{l}\text { Sheyenne River above Sheyenne River Diversion near Horace, } \\
\text { N. Dak. }\end{array}$ & 21 & 1993-2004 & 70 & 20 & 100 \\
\hline 40 & Sheyenne River Diversion at West Fargo, N. Dak. & 9 & 1994-2004 & 50 & 20 & 100 \\
\hline 41 & Sheyenne River at West Fargo, N. Dak. & 12 & 1993-2004 & 70 & 50 & 78 \\
\hline 42 & Maple River near Hope, N. Dak. & 16 & 1993-2004 & 55 & 20 & 200 \\
\hline 43 & Maple River near Enderlin, N. Dak. & 23 & 1993-2004 & 70 & 20 & 130 \\
\hline 44 & Maple River near Mapleton, N. Dak. & 9 & 1995-2004 & 60 & 20 & 106 \\
\hline 45 & Maple River below Mapleton, N. Dak. & 19 & 1995-2004 & 62 & 20 & 110 \\
\hline 46 & Sheyenne River at Harwood, N. Dak. & 5 & $2000-2004$ & 62 & 60 & 100 \\
\hline 47 & Rush River at Amenia, N. Dak. & 24 & 1993-2004 & 80 & $<10$ & 130 \\
\hline
\end{tabular}


Table 1-3. Summary statistics for trace-metal constituents at selected sites in North Dakota from 1993 through 2008.—Continued [ $\mu \mathrm{g} / \mathrm{L}$, micrograms per liter; --, not available; $<$, less than]

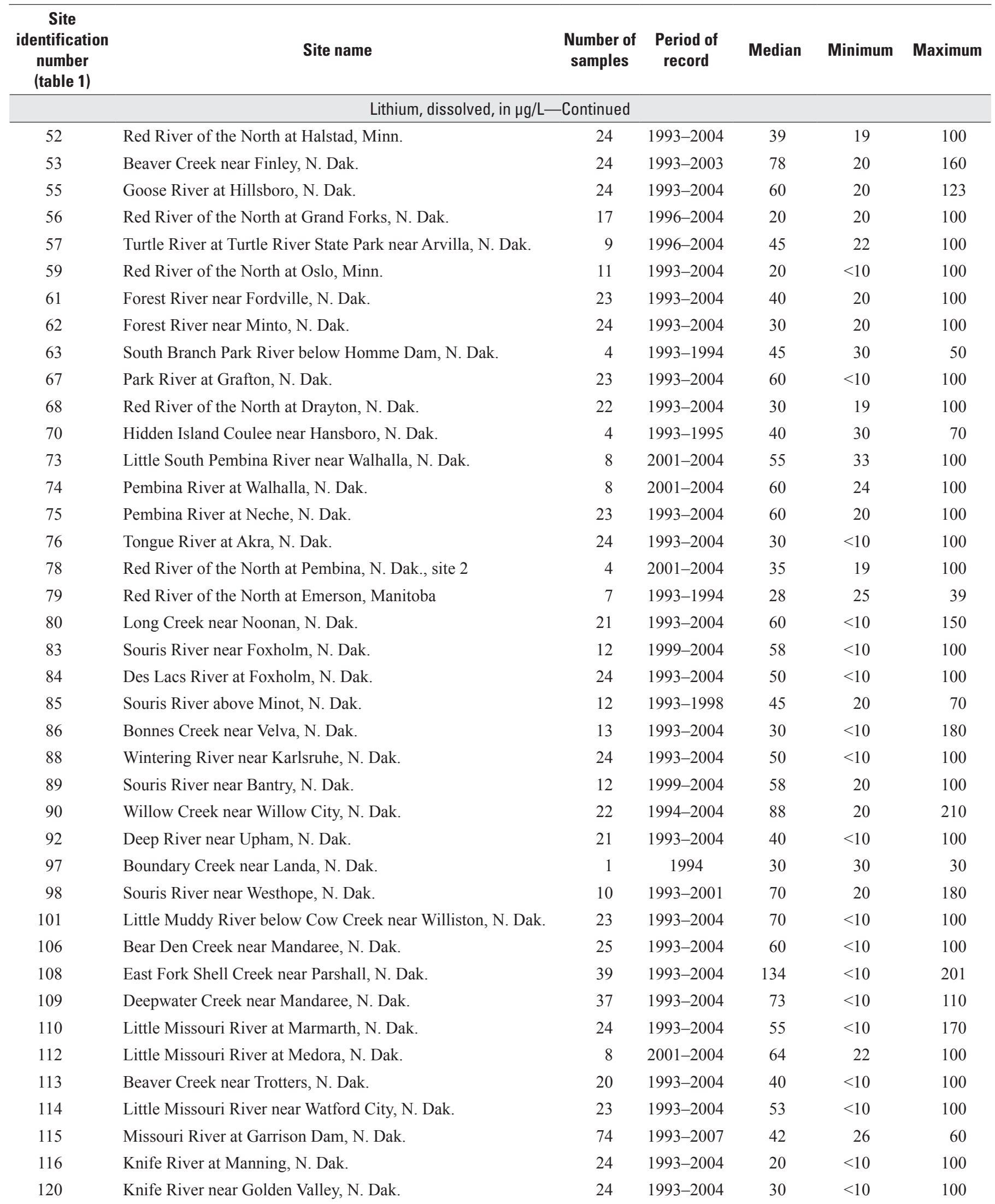


Table 1-3. Summary statistics for trace-metal constituents at selected sites in North Dakota from 1993 through 2008. - Continued [ $\mu \mathrm{g} / \mathrm{L}$, micrograms per liter; --, not available; $<$, less than]

\begin{tabular}{|c|c|c|c|c|c|c|}
\hline $\begin{array}{c}\text { Site } \\
\text { identification } \\
\text { number } \\
\text { (table 1) }\end{array}$ & Site name & $\begin{array}{c}\text { Number of } \\
\text { samples }\end{array}$ & $\begin{array}{l}\text { Period of } \\
\text { record }\end{array}$ & Median & Minimum & Maximum \\
\hline \multicolumn{7}{|c|}{ Lithium, dissolved, in $\mu \mathrm{g} / \mathrm{L}$-Continued } \\
\hline 125 & Spring Creek at Zap, N. Dak. & 24 & 1993-2004 & 45 & $<10$ & 100 \\
\hline 127 & Knife River at Hazen, N. Dak. & 27 & 1993-2004 & 36 & $<10$ & 100 \\
\hline 140 & Square Butte Creek below Center, N. Dak. & 23 & 1993-2004 & 46 & 16 & 100 \\
\hline 141 & Burnt Creek near Bismarck, N. Dak. & 23 & 1993-2004 & 70 & $<10$ & 120 \\
\hline 142 & Missouri River at Bismarck, N. Dak. & 15 & 1993-2004 & 40 & 20 & 50 \\
\hline 145 & Heart River near South Heart, N. Dak. & 11 & 1993-2004 & 13 & $<10$ & 100 \\
\hline 146 & Heart River at Dickinson, N. Dak. & 4 & 1993-1994 & 35 & 20 & 50 \\
\hline 147 & Green River near New Hradec, N. Dak. & 22 & 1993-2004 & 15 & $<10$ & 100 \\
\hline 149 & Heart River near Richardton, N. Dak. & 24 & 1993-2004 & 30 & $<10$ & 100 \\
\hline 150 & Heart River above Lake Tschida near Glen Ullin, N. Dak. & 24 & 1993-2004 & 33 & $<10$ & 100 \\
\hline 151 & Antelope Creek near Carson, N. Dak. & 11 & 1999-2004 & 60 & 26 & 100 \\
\hline 152 & Big Muddy Creek near Almont, N. Dak. & 24 & 1993-2004 & 40 & $<10$ & 100 \\
\hline 153 & Heart River near Lark, N. Dak. & 6 & 1993-1995 & 35 & $<10$ & 40 \\
\hline 154 & Heart River at Stark Bridge near Judson, N. Dak. & 24 & 1993-2004 & 40 & $<10$ & 100 \\
\hline 155 & Sweetbriar Creek near Judson, N. Dak. & 6 & $2002-2004$ & 43 & 17 & 63 \\
\hline 156 & Heart River near Mandan, N. Dak. & 27 & 1993-2004 & 49 & $<10$ & 120 \\
\hline 157 & Long Lake Creek above Long Lake near Moffit, N. Dak. & 24 & 1993-2004 & 160 & $<10$ & 270 \\
\hline 158 & Apple Creek near Menoken, N. Dak. & 23 & 1993-2004 & 150 & $<10$ & 290 \\
\hline 162 & Cannonball River at Regent, N. Dak. & 23 & 1993-2004 & 40 & $<10$ & 100 \\
\hline 164 & Cannonball River near Raleigh, N. Dak. & 6 & 2001-2004 & 50 & 12 & 100 \\
\hline 165 & White Butte Fork Cedar Creek near Scranton, N. Dak. & 5 & 1993-1995 & 30 & $<10$ & 50 \\
\hline 166 & Cedar Creek near Haynes, N. Dak. & 24 & 1993-2004 & 55 & $<10$ & 100 \\
\hline 169 & Cedar Creek near Raleigh, N. Dak. & 21 & 1993-2004 & 60 & $<10$ & 170 \\
\hline 170 & Cannonball River at Breien, N. Dak. & 25 & 1993-2004 & 50 & $<10$ & 110 \\
\hline 172 & Beaver Creek below Linton, N. Dak. & 24 & 1993-2004 & 115 & $<10$ & 243 \\
\hline 173 & Porcupine Creek near Fort Yates, N. Dak. & 35 & 1993-1999 & 73 & $<10$ & 130 \\
\hline 176 & James River near Grace City, N. Dak. & 18 & 1996-2004 & 50 & $<10$ & 100 \\
\hline 179 & James River near Pingree, N. Dak. & 2 & 1993 & 26 & 23 & 28 \\
\hline 180 & Pipestem Creek near Pingree, N. Dak. & 24 & 1993-2004 & 60 & $<10$ & 126 \\
\hline 182 & James River at Jamestown, N. Dak. & 17 & 1996-2004 & 50 & 20 & 100 \\
\hline 183 & James River at Lamoure, N. Dak. & 18 & 1996-2004 & 45 & 20 & 100 \\
\hline 184 & Bear Creek near Oakes, N. Dak. & 24 & 1993-2004 & 85 & 20 & 137 \\
\hline 186 & James River at N. Dak./S. Dak. State line & 6 & $2002-2004$ & 66 & 42 & 90 \\
\hline \multicolumn{7}{|c|}{ Molybedium, total, in $\mu \mathrm{g} / \mathrm{L}$} \\
\hline 82 & Souris River near Sherwood, N. Dak. & 116 & $1993-2008$ & 2.5 & $<1.0$ & 32.0 \\
\hline 83 & Souris River near Foxholm, N. Dak. & 25 & 1993-1998 & 2.1 & $<1.0$ & 6.0 \\
\hline 85 & Souris River above Minot, N. Dak. & 65 & 1999-2008 & 3.2 & 1.1 & 5.8 \\
\hline 87 & Souris River near Verendrye, N. Dak. & 104 & 1993-2008 & 3.3 & $<1.0$ & 12.2 \\
\hline 89 & Souris River near Bantry, N. Dak. & 37 & 1993-1998 & 1.9 & $<1.0$ & 6.0 \\
\hline 90 & Willow Creek near Willow City, N. Dak. & 3 & 1993 & $<1.0$ & $<1.0$ & $<1.0$ \\
\hline
\end{tabular}


Table 1-3. Summary statistics for trace-metal constituents at selected sites in North Dakota from 1993 through 2008.-Continued [ $\mu \mathrm{g} / \mathrm{L}$, micrograms per liter; --, not available; <, less than]

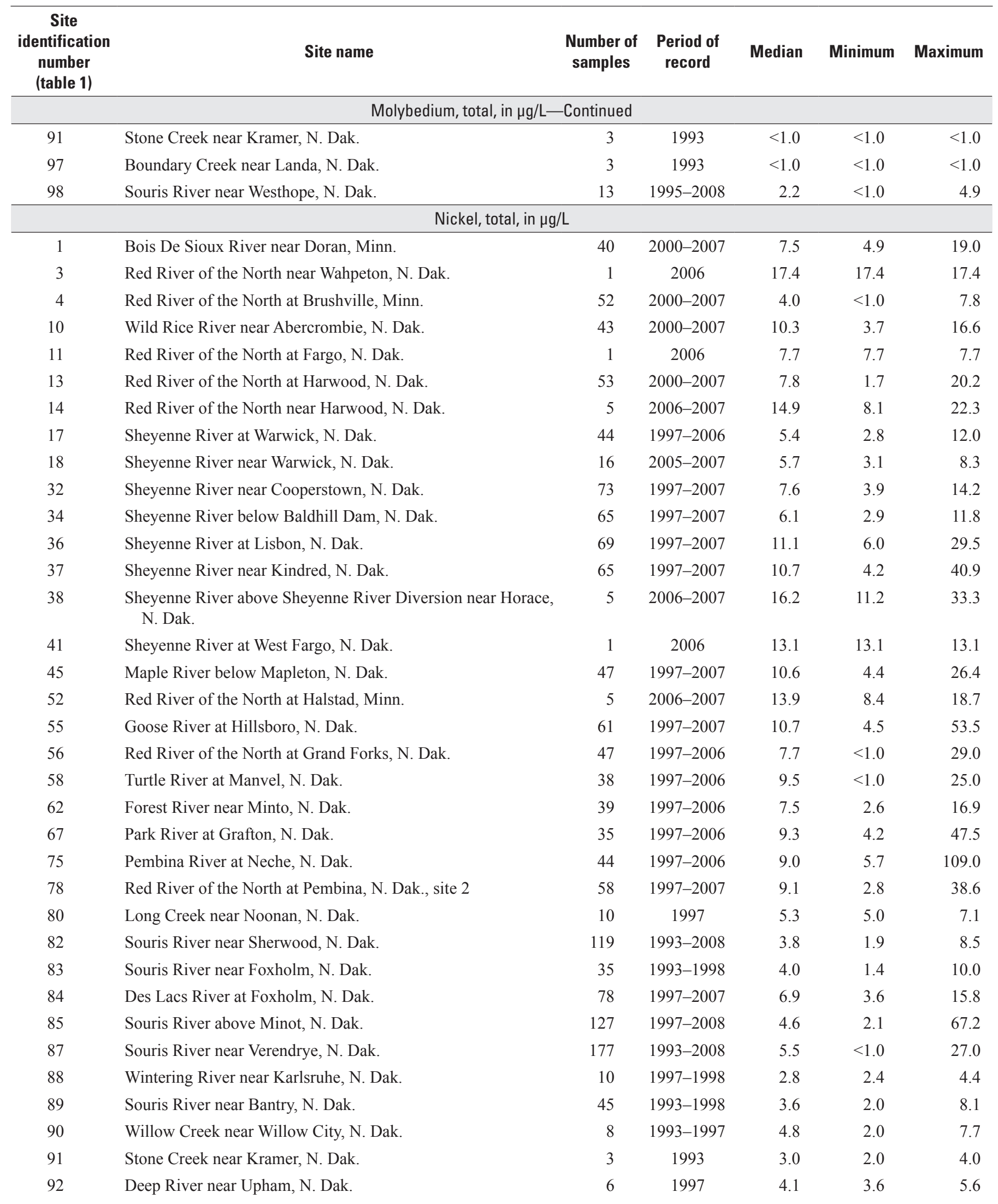


Table 1-3. Summary statistics for trace-metal constituents at selected sites in North Dakota from 1993 through 2008. - Continued [ $\mu \mathrm{g} / \mathrm{L}$, micrograms per liter; --, not available; $<$, less than]

\begin{tabular}{|c|c|c|c|c|c|c|}
\hline $\begin{array}{c}\text { Site } \\
\text { identification } \\
\text { number } \\
\text { (table 1) } \\
\end{array}$ & Site name & $\begin{array}{c}\text { Number of } \\
\text { samples }\end{array}$ & $\begin{array}{l}\text { Period of } \\
\text { record }\end{array}$ & Median & Minimum & Maximum \\
\hline \multicolumn{7}{|c|}{ Nickel, total, in $\mu \mathrm{g} / \mathrm{L}$-Continued } \\
\hline 97 & Boundary Creek near Landa, N. Dak. & 3 & 1993 & 2.0 & 2.0 & 5.0 \\
\hline 98 & Souris River near Westhope, N. Dak. & 21 & 1995-2008 & 4.5 & 2.0 & 8.1 \\
\hline 101 & Little Muddy River below Cow Creek near Williston, N. Dak. & 9 & 1999 & 4.7 & 3.5 & 6.8 \\
\hline 110 & Little Missouri River at Marmarth, N. Dak. & 8 & 1999 & 26.0 & 4.6 & 104.0 \\
\hline 112 & Little Missouri River at Medora, N. Dak. & 70 & $1997-2007$ & 13.6 & 4.5 & 994.0 \\
\hline 113 & Beaver Creek near Trotters, N. Dak. & 9 & 1999 & 4.2 & 3.4 & 11.4 \\
\hline 114 & Little Missouri River near Watford City, N. Dak. & 64 & $1997-2007$ & 21.7 & 3.3 & $1,210.0$ \\
\hline 120 & Knife River near Golden Valley, N. Dak. & 60 & 1997-2007 & 9.0 & $<1.0$ & 46.7 \\
\hline 125 & Spring Creek at Zap, N. Dak. & 64 & $1997-2007$ & 5.4 & 3.0 & 13.9 \\
\hline 127 & Knife River at Hazen, N. Dak. & 71 & 1997-2007 & 7.0 & 3.5 & 54.4 \\
\hline 149 & Heart River near Richardton, N. Dak. & 64 & 1997-2007 & 7.6 & 4.6 & 33.9 \\
\hline 156 & Heart River near Mandan, N. Dak. & 65 & $1997-2007$ & 5.3 & 2.8 & 23.4 \\
\hline 164 & Cannonball River near Raleigh, N. Dak. & 57 & $1997-2007$ & 9.9 & 4.4 & 119.0 \\
\hline 169 & Cedar Creek near Raleigh, N. Dak. & 49 & $1997-2007$ & 9.6 & 4.8 & 31.9 \\
\hline 170 & Cannonball River at Breien, N. Dak. & 59 & $1997-2007$ & 9.2 & 4.6 & 116.0 \\
\hline 175 & James River near Manfred, N. Dak. & 6 & 1998 & 4.7 & 3.3 & 9.0 \\
\hline 176 & James River near Grace City, N. Dak. & 60 & $1997-2007$ & 5.2 & 2.5 & 22.3 \\
\hline 177 & James River above Arrowwood Lake near Kensal, N. Dak. & 9 & 1998-1999 & 3.9 & 3.2 & 8.7 \\
\hline 180 & Pipestem Creek near Pingree, N. Dak. & 9 & 1998-1999 & 5.9 & 4.1 & 14.4 \\
\hline 182 & James River at Jamestown, N. Dak. & 75 & 1997-2007 & 6.1 & 2.1 & 68.9 \\
\hline 183 & James River at Lamoure, N. Dak. & 68 & $1997-2007$ & 7.0 & 3.7 & 33.5 \\
\hline 184 & Bear Creek near Oakes, N. Dak. & 9 & 1998-1999 & 6.0 & 3.9 & 16.1 \\
\hline 186 & James River at N. Dak./S. Dak. State line & 9 & 1998-1999 & 6.3 & 3.8 & 10.9 \\
\hline \multicolumn{7}{|c|}{ Nickel, dissolved, in $\mu \mathrm{g} / \mathrm{L}$} \\
\hline 2 & Red River of the North at Wahpeton, N. Dak. & 8 & $2005-2008$ & $<10.0$ & $<10.0$ & $<10.0$ \\
\hline 4 & Red River of the North at Brushville, Minn. & 1 & 2005 & $<10.0$ & $<10.0$ & $<10.0$ \\
\hline 6 & Red River of the North at Hickson, N. Dak. & 8 & $2005-2008$ & $<10.0$ & $<10.0$ & $<10.0$ \\
\hline 7 & Wild Rice River near Rutland, N. Dak. & 8 & $2005-2008$ & $<10.0$ & $<10.0$ & 19.4 \\
\hline 9 & Antelope Creek at Dwight, N. Dak. & 8 & $2005-2008$ & $<10.0$ & $<10.0$ & 18.0 \\
\hline 10 & Wild Rice River near Abercrombie, N. Dak. & 9 & $2005-2008$ & $<10.0$ & $<10.0$ & 15.5 \\
\hline 11 & Red River of the North at Fargo, N. Dak. & 38 & $2005-2008$ & $<10.0$ & $<10.0$ & 10.6 \\
\hline 14 & Red River of the North near Harwood, N. Dak. & 7 & $2005-2006$ & $<10.0$ & $<10.0$ & $<10.0$ \\
\hline 15 & Sheyenne River above Harvey, N. Dak. & 20 & $1993-2008$ & $<10.0$ & $<10.0$ & $<10.0$ \\
\hline 17 & Sheyenne River at Warwick, N. Dak. & 2 & 2005 & $<10.0$ & $<10.0$ & $<10.0$ \\
\hline 18 & Sheyenne River near Warwick, N. Dak. & 9 & 1993-2008 & $<10.0$ & $<10.0$ & $<10.0$ \\
\hline 19 & Mauvais Coulee Tributary No. 3 near Cando, N. Dak. & 7 & $2005-2008$ & $<10.0$ & $<10.0$ & $<10.0$ \\
\hline 20 & Mauvais Coulee near Cando, N. Dak. & 16 & $2005-2008$ & $<10.0$ & $<10.0$ & 11.8 \\
\hline 21 & Edmore Coulee near Edmore, N. Dak. & 17 & $2005-2008$ & $<10.0$ & $<10.0$ & 10.3 \\
\hline 22 & Edmore Coulee Tributary near Webster, N. Dak. & 7 & $2005-2008$ & $<10.0$ & $<10.0$ & 13.0 \\
\hline 24 & Starkweather Coulee near Webster, N. Dak. & 16 & $2005-2008$ & $<10.0$ & $<10.0$ & 13.8 \\
\hline
\end{tabular}


Table 1-3. Summary statistics for trace-metal constituents at selected sites in North Dakota from 1993 through 2008.-Continued [ $\mu \mathrm{g} / \mathrm{L}$, micrograms per liter; --, not available; <, less than]

\begin{tabular}{|c|c|c|c|c|c|c|}
\hline $\begin{array}{c}\text { Site } \\
\text { identification } \\
\text { number } \\
\text { (table 1) }\end{array}$ & Site name & $\begin{array}{c}\text { Number of } \\
\text { samples }\end{array}$ & $\begin{array}{l}\text { Period of } \\
\text { record }\end{array}$ & Median & Minimum & Maximum \\
\hline \multicolumn{7}{|c|}{ Nickel, dissolved, in $\mu \mathrm{g} / \mathrm{L}$-Continued } \\
\hline 25 & Big Coulee below Churchs Ferry, N. Dak. & 7 & $2005-2008$ & $<10.0$ & $<10.0$ & $<10.0$ \\
\hline 26 & Little Coulee near Leeds, N. Dak. & 5 & $2005-2008$ & $<10.0$ & $<10.0$ & $<10.0$ \\
\hline 30 & Channel A near Penn, N. Dak. & 7 & $2005-2008$ & $<10.0$ & $<10.0$ & $<10.0$ \\
\hline 31 & Devils Lake Outlet to Stump Lake near Lakota, N. Dak. & 6 & 2005-2007 & $<10.0$ & $<10.0$ & $<10.0$ \\
\hline 32 & Sheyenne River near Cooperstown, N. Dak. & 13 & $2005-2008$ & $<10.0$ & $<10.0$ & $<10.0$ \\
\hline 33 & Baldhill Creek near Dazey, N. Dak. & 8 & $2005-2008$ & $<10.0$ & $<10.0$ & $<10.0$ \\
\hline 34 & Sheyenne River below Baldhill Dam, N. Dak. & 14 & $2005-2008$ & $<10.0$ & $<10.0$ & 11.3 \\
\hline 35 & Sheyenne River at Valley City, N. Dak. & 1 & 2005 & $<10.0$ & $<10.0$ & $<10.0$ \\
\hline 36 & Sheyenne River at Lisbon, N. Dak. & 9 & $2005-2008$ & $<10.0$ & $<10.0$ & 16.4 \\
\hline 37 & Sheyenne River near Kindred, N. Dak. & 16 & 1993-2008 & $<10.0$ & $<10.0$ & 15.4 \\
\hline 38 & $\begin{array}{l}\text { Sheyenne River above Sheyenne River Diversion near Horace, } \\
\text { N. Dak. }\end{array}$ & 14 & $2005-2008$ & $<10.0$ & $<10.0$ & 18.1 \\
\hline 40 & Sheyenne River Diversion at West Fargo, N. Dak. & 4 & $2005-2007$ & $<10.0$ & $<10.0$ & 12.2 \\
\hline 41 & Sheyenne River at West Fargo, N. Dak. & 4 & 2006-2008 & $<10.0$ & $<10.0$ & 10.6 \\
\hline 42 & Maple River near Hope, N. Dak. & 6 & $2005-2008$ & $<10.0$ & $<10.0$ & 11.8 \\
\hline 43 & Maple River near Enderlin, N. Dak. & 8 & 2005-2008 & $<10.0$ & $<10.0$ & 20.5 \\
\hline 44 & Maple River near Mapleton, N. Dak. & 8 & $2005-2008$ & $<10.0$ & $<10.0$ & 35.0 \\
\hline 45 & Maple River below Mapleton, N. Dak. & 9 & $2005-2008$ & $<10.0$ & $<10.0$ & 17.7 \\
\hline 46 & Sheyenne River at Harwood, N. Dak. & 1 & 2005 & $<10.0$ & $<10.0$ & $<10.0$ \\
\hline 47 & Rush River at Amenia, N. Dak. & 8 & $2005-2008$ & $<10.0$ & $<10.0$ & 14.8 \\
\hline 52 & Red River of the North at Halstad, Minn. & 19 & 1993-2008 & $<10.0$ & $<10.0$ & 11.0 \\
\hline 53 & Beaver Creek near Finley, N. Dak. & 13 & 1993-1996 & $<10.0$ & $<10.0$ & $<10.0$ \\
\hline 55 & Goose River at Hillsboro, N. Dak. & 15 & $2005-2008$ & $<10.0$ & $<10.0$ & 11.3 \\
\hline 56 & Red River of the North at Grand Forks, N. Dak. & 30 & $2005-2008$ & $<10.0$ & $<10.0$ & 11.6 \\
\hline 57 & Turtle River at Turtle River State Park near Arvilla, N. Dak. & 8 & 2005-2008 & $<10.0$ & $<10.0$ & 10.8 \\
\hline 58 & Turtle River at Manvel, N. Dak. & 30 & $2005-2008$ & 10.1 & $<10.0$ & 25.2 \\
\hline 59 & Red River of the North at Oslo, Minn. & 1 & 2005 & $<10.0$ & $<10.0$ & $<10.0$ \\
\hline 61 & Forest River near Fordville, N. Dak. & 8 & $2005-2008$ & $<10.0$ & $<10.0$ & $<10.0$ \\
\hline 62 & Forest River near Minto, N. Dak. & 30 & $2005-2008$ & $<10.0$ & $<10.0$ & 15.2 \\
\hline 67 & Park River at Grafton, N. Dak. & 30 & $2005-2008$ & $<10.0$ & $<10.0$ & 16.9 \\
\hline 68 & Red River of the North at Drayton, N. Dak. & 8 & $2005-2008$ & $<10.0$ & $<10.0$ & $<10.0$ \\
\hline 73 & Little South Pembina River near Walhalla, N. Dak. & 8 & $2005-2008$ & $<10.0$ & $<10.0$ & 14.4 \\
\hline 74 & Pembina River at Walhalla, N. Dak. & 8 & $2005-2008$ & $<10.0$ & $<10.0$ & 16.8 \\
\hline 75 & Pembina River at Neche, N. Dak. & 30 & $2005-2008$ & $<10.0$ & $<10.0$ & 16.8 \\
\hline 76 & Tongue River at Akra, N. Dak. & 8 & $2005-2008$ & $<10.0$ & $<10.0$ & 11.1 \\
\hline 78 & Red River of the North at Pembina, N. Dak., site 2 & 33 & $2005-2008$ & $<10.0$ & $<10.0$ & $<10.0$ \\
\hline 79 & Red River of the North at Emerson, Manitoba & 7 & 1993-1994 & $<10.0$ & $<10.0$ & $<10.0$ \\
\hline 80 & Long Creek near Noonan, N. Dak. & 8 & $2005-2008$ & $<10.0$ & $<10.0$ & $<10.0$ \\
\hline 83 & Souris River near Foxholm, N. Dak. & 8 & $2005-2008$ & $<10.0$ & $<10.0$ & $<10.0$ \\
\hline 84 & Des Lacs River at Foxholm, N. Dak. & 8 & 2005-2008 & $<10.0$ & $<10.0$ & 12.9 \\
\hline
\end{tabular}


Table 1-3. Summary statistics for trace-metal constituents at selected sites in North Dakota from 1993 through 2008. - Continued [ $\mu \mathrm{g} / \mathrm{L}$, micrograms per liter; --, not available; <, less than]

\begin{tabular}{|c|c|c|c|c|c|c|}
\hline $\begin{array}{c}\text { Site } \\
\text { identification } \\
\text { number } \\
\text { (table 1) }\end{array}$ & Site name & $\begin{array}{c}\text { Number of } \\
\text { samples }\end{array}$ & $\begin{array}{l}\text { Period of } \\
\text { record }\end{array}$ & Median & Minimum & Maximum \\
\hline \multicolumn{7}{|c|}{ Nickel, dissolved, in $\mu \mathrm{g} / \mathrm{L}$-Continued } \\
\hline 86 & Bonnes Creek near Velva, N. Dak. & 1 & 2005 & $<10.0$ & $<10.0$ & $<10.0$ \\
\hline 88 & Wintering River near Karlsruhe, N. Dak. & 8 & 2005-2008 & $<10.0$ & $<10.0$ & 10.9 \\
\hline 89 & Souris River near Bantry, N. Dak. & 8 & 2005-2008 & $<10.0$ & $<10.0$ & $<10.0$ \\
\hline 90 & Willow Creek near Willow City, N. Dak. & 7 & $2005-2008$ & $<10.0$ & $<10.0$ & $<10.0$ \\
\hline 92 & Deep River near Upham, N. Dak. & 5 & 2005-2007 & $<10.0$ & $<10.0$ & $<10.0$ \\
\hline 98 & Souris River near Westhope, N. Dak. & 6 & 1993-1994 & $<10.0$ & $<10.0$ & $<10.0$ \\
\hline 101 & Little Muddy River below Cow Creek near Williston, N. Dak. & 8 & 2005-2008 & $<10.0$ & $<10.0$ & $<10.0$ \\
\hline 106 & Bear Den Creek near Mandaree, N. Dak. & 19 & 1993-2008 & $<10.0$ & $<10.0$ & 10.2 \\
\hline 108 & East Fork Shell Creek near Parshall, N. Dak. & 5 & 2005-2008 & $<10.0$ & $<10.0$ & $<10.0$ \\
\hline 109 & Deepwater Creek near Mandaree, N. Dak. & 7 & 2005-2008 & $<10.0$ & $<10.0$ & 120.0 \\
\hline 110 & Little Missouri River at Marmarth, N. Dak. & 9 & 2005-2008 & $<10.0$ & $<10.0$ & $<10.0$ \\
\hline 112 & Little Missouri River at Medora, N. Dak. & 8 & 2005-2008 & $<10.0$ & $<10.0$ & $<10.0$ \\
\hline 113 & Beaver Creek near Trotters, N. Dak. & 8 & $2005-2008$ & $<10.0$ & $<10.0$ & 11.3 \\
\hline 114 & Little Missouri River near Watford City, N. Dak. & 13 & 1993-2008 & $<10.0$ & $<10.0$ & 18.4 \\
\hline 115 & Missouri River at Garrison Dam, N. Dak. & 21 & 1993-1998 & $<10.0$ & $<10.0$ & $<10.0$ \\
\hline 116 & Knife River at Manning, N. Dak. & 8 & 2005-2008 & $<10.0$ & $<10.0$ & $<10.0$ \\
\hline 120 & Knife River near Golden Valley, N. Dak. & 8 & 2005-2008 & $<10.0$ & $<10.0$ & 16.2 \\
\hline 125 & Spring Creek at Zap, N. Dak. & 8 & 2005-2008 & $<10.0$ & $<10.0$ & $<10.0$ \\
\hline 127 & Knife River at Hazen, N. Dak. & 12 & 1993-2008 & $<10.0$ & $<10.0$ & $<10.0$ \\
\hline 140 & Square Butte Creek below Center, N. Dak. & 8 & 2005-2008 & $<10.0$ & $<10.0$ & $<10.0$ \\
\hline 141 & Burnt Creek near Bismarck, N. Dak. & 6 & 2005-2008 & $<10.0$ & $<10.0$ & $<10.0$ \\
\hline 142 & Missouri River at Bismarck, N. Dak. & 8 & 2005-2008 & $<10.0$ & $<10.0$ & $<10.0$ \\
\hline 145 & Heart River near South Heart, N. Dak. & 1 & 2005 & $<10.0$ & $<10.0$ & $<10.0$ \\
\hline 147 & Green River near New Hradec, N. Dak. & 7 & 2005-2008 & $<10.0$ & $<10.0$ & $<10.0$ \\
\hline 149 & Heart River near Richardton, N. Dak. & 8 & 2005-2008 & $<10.0$ & $<10.0$ & 12.1 \\
\hline 150 & Heart River above Lake Tschida near Glen Ullin, N. Dak. & 8 & 2005-2008 & $<10.0$ & $<10.0$ & 12.4 \\
\hline 151 & Antelope Creek near Carson, N. Dak. & 7 & 2005-2008 & $<10.0$ & $<10.0$ & $<10.0$ \\
\hline 152 & Big Muddy Creek near Almont, N. Dak. & 8 & 2005-2008 & $<10.0$ & $<10.0$ & $<10.0$ \\
\hline 154 & Heart River at Stark Bridge near Judson, N. Dak. & 8 & 2005-2008 & $<10.0$ & $<10.0$ & $<10.0$ \\
\hline 155 & Sweetbriar Creek near Judson, N. Dak. & 7 & 2005-2008 & $<10.0$ & $<10.0$ & $<10.0$ \\
\hline 156 & Heart River near Mandan, N. Dak. & 11 & 1993-2008 & $<10.0$ & $<10.0$ & $<10.0$ \\
\hline 158 & Apple Creek near Menoken, N. Dak. & 8 & 2005-2008 & $<10.0$ & $<10.0$ & $<10.0$ \\
\hline 162 & Cannonball River at Regent, N. Dak. & 8 & 2005-2008 & $<10.0$ & $<10.0$ & $<10.0$ \\
\hline 164 & Cannonball River near Raleigh, N. Dak. & 9 & 2005-2008 & $<10.0$ & $<10.0$ & $<10.0$ \\
\hline 166 & Cedar Creek near Haynes, N. Dak. & 8 & 2005-2008 & $<10.0$ & $<10.0$ & 24.7 \\
\hline 169 & Cedar Creek near Raleigh, N. Dak. & 9 & 2005-2008 & $<10.0$ & $<10.0$ & 10.3 \\
\hline 170 & Cannonball River at Breien, N. Dak. & 9 & 2005-2008 & $<10.0$ & $<10.0$ & 11.5 \\
\hline 172 & Beaver Creek below Linton, N. Dak. & 8 & $2005-2008$ & $<10.0$ & $<10.0$ & $<10.0$ \\
\hline 176 & James River near Grace City, N. Dak. & 8 & 2005-2008 & $<10.0$ & $<10.0$ & $<10.0$ \\
\hline 177 & James River above Arrowwood Lake near Kensal, N. Dak. & 24 & 2005-2008 & $<10.0$ & $<10.0$ & $<10.0$ \\
\hline
\end{tabular}


Table 1-3. Summary statistics for trace-metal constituents at selected sites in North Dakota from 1993 through 2008.-Continued [ $\mu \mathrm{g} / \mathrm{L}$, micrograms per liter; --, not available; <, less than]

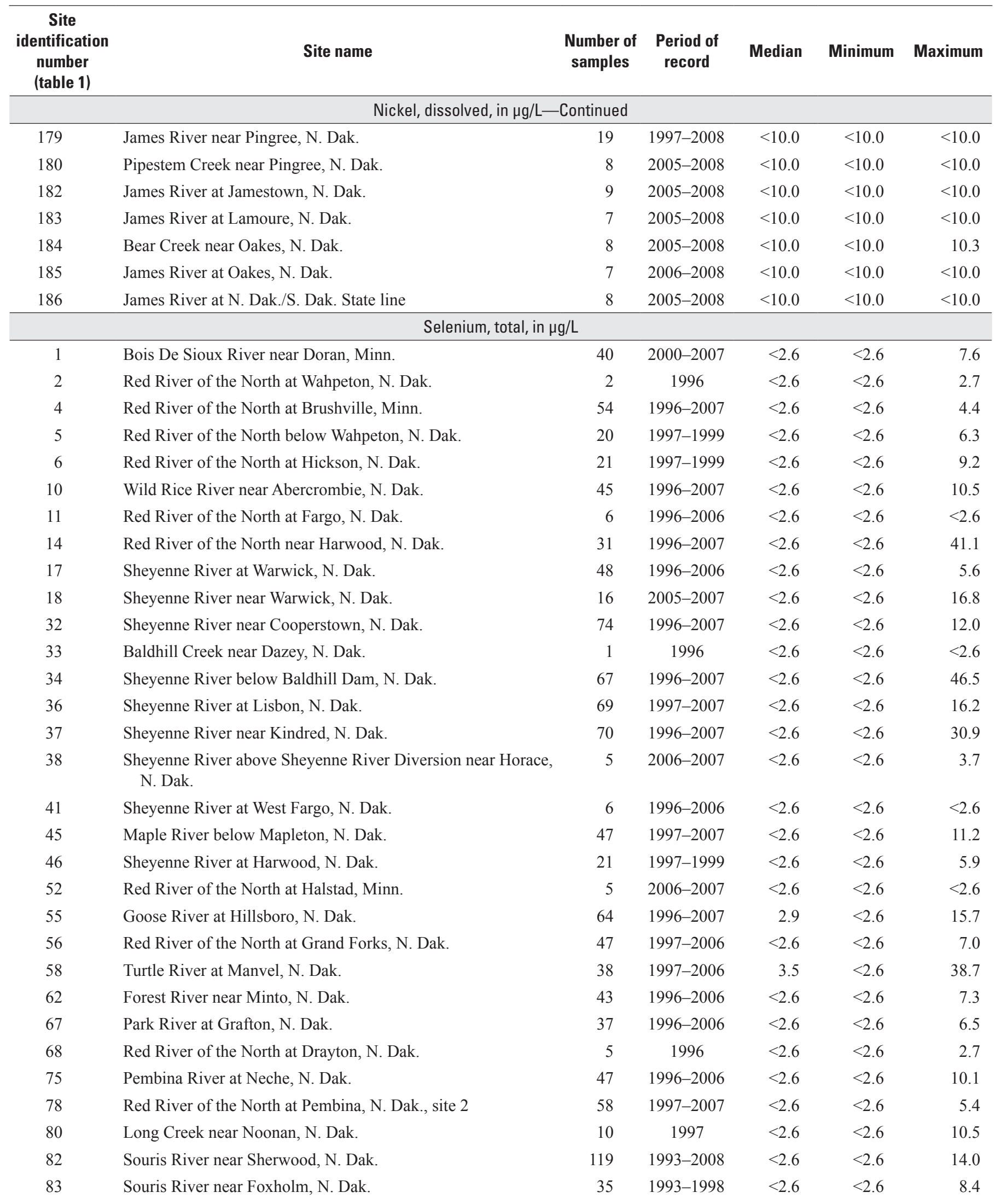


Table 1-3. Summary statistics for trace-metal constituents at selected sites in North Dakota from 1993 through 2008. - Continued [ $\mu \mathrm{g} / \mathrm{L}$, micrograms per liter; --, not available; $<$, less than]

\begin{tabular}{|c|c|c|c|c|c|c|}
\hline $\begin{array}{c}\text { Site } \\
\text { identification } \\
\text { number } \\
\text { (table 1) }\end{array}$ & Site name & $\begin{array}{l}\text { Number of } \\
\text { samples }\end{array}$ & $\begin{array}{l}\text { Period of } \\
\text { record }\end{array}$ & Median & Minimum & Maximum \\
\hline \multicolumn{7}{|c|}{ Selenium, total, in $\mu \mathrm{g} / \mathrm{L}$-Continued } \\
\hline 84 & Des Lacs River at Foxholm, N. Dak. & 83 & 1996-2007 & $<2.6$ & $<2.6$ & 127.0 \\
\hline 85 & Souris River above Minot, N. Dak. & 128 & 1996-2008 & $<2.6$ & $<2.6$ & 18.8 \\
\hline 87 & Souris River near Verendrye, N. Dak. & 175 & $1993-2008$ & $<2.6$ & $<2.6$ & 31.5 \\
\hline 88 & Wintering River near Karlsruhe, N. Dak. & 10 & 1997-1998 & $<2.6$ & $<2.6$ & 7.3 \\
\hline 89 & Souris River near Bantry, N. Dak. & 64 & $1993-2000$ & $<2.6$ & $<2.6$ & 10.0 \\
\hline 90 & Willow Creek near Willow City, N. Dak. & 28 & $1993-2000$ & $<2.6$ & $<2.6$ & 8.7 \\
\hline 91 & Stone Creek near Kramer, N. Dak. & 19 & $1993-2000$ & $<2.6$ & $<2.6$ & 10.0 \\
\hline 92 & Deep River near Upham, N. Dak. & 26 & $1997-2000$ & $<2.6$ & $<2.6$ & 9.6 \\
\hline 95 & Cut Bank Creek at Upham, N. Dak. & 16 & 1999-2000 & $<2.6$ & $<2.6$ & $<2.6$ \\
\hline 97 & Boundary Creek near Landa, N. Dak. & 19 & $1993-2000$ & $<2.6$ & $<2.6$ & 4.0 \\
\hline 98 & Souris River near Westhope, N. Dak. & 20 & 1995-2008 & $<2.6$ & $<2.6$ & 14.0 \\
\hline 101 & Little Muddy River below Cow Creek near Williston, N. Dak. & 9 & 1999 & $<2.6$ & $<2.6$ & 4.5 \\
\hline 110 & Little Missouri River at Marmarth, N. Dak. & 8 & 1999 & $<2.6$ & $<2.6$ & 7.7 \\
\hline 112 & Little Missouri River at Medora, N. Dak. & 74 & 1996-2007 & $<2.6$ & $<2.6$ & 30.5 \\
\hline 113 & Beaver Creek near Trotters, N. Dak. & 9 & 1999 & $<2.6$ & $<2.6$ & 7.8 \\
\hline 114 & Little Missouri River near Watford City, N. Dak. & 69 & $1996-2007$ & 2.8 & $<2.6$ & 173.0 \\
\hline 115 & Missouri River at Garrison Dam, N. Dak. & 7 & $1999-2000$ & $<2.6$ & $<2.6$ & 3.8 \\
\hline 120 & Knife River near Golden Valley, N. Dak. & 60 & 1997-2007 & $<2.6$ & $<2.6$ & 21.4 \\
\hline 125 & Spring Creek at Zap, N. Dak. & 64 & $1997-2007$ & $<2.6$ & $<2.6$ & 16.3 \\
\hline 127 & Knife River at Hazen, N. Dak. & 76 & 1996-2007 & $<2.6$ & $<2.6$ & 21.4 \\
\hline 142 & Missouri River at Bismarck, N. Dak. & 23 & $1999-2001$ & $<2.6$ & $<2.6$ & $<2.6$ \\
\hline 149 & Heart River near Richardton, N. Dak. & 68 & 1996-2007 & $<2.6$ & $<2.6$ & 19.5 \\
\hline 156 & Heart River near Mandan, N. Dak. & 68 & 1996-2007 & $<2.6$ & $<2.6$ & 26.1 \\
\hline 164 & Cannonball River near Raleigh, N. Dak. & 62 & 1996-2007 & $<2.6$ & $<2.6$ & 67.0 \\
\hline 169 & Cedar Creek near Raleigh, N. Dak. & 54 & $1996-2007$ & $<2.6$ & $<2.6$ & 56.5 \\
\hline 170 & Cannonball River at Breien, N. Dak. & 64 & 1996-2007 & $<2.6$ & $<2.6$ & 48.5 \\
\hline 175 & James River near Manfred, N. Dak. & 14 & 1993-1998 & $<2.6$ & $<2.6$ & $<2.6$ \\
\hline 176 & James River near Grace City, N. Dak. & 69 & $1993-2007$ & $<2.6$ & $<2.6$ & 13.4 \\
\hline 177 & James River above Arrowwood Lake near Kensal, N. Dak. & 50 & 1993-2006 & $<2.6$ & $<2.6$ & 3.0 \\
\hline 179 & James River near Pingree, N. Dak. & 37 & 1993-2006 & $<2.6$ & $<2.6$ & 3.0 \\
\hline 180 & Pipestem Creek near Pingree, N. Dak. & 9 & 1998-1999 & $<2.6$ & $<2.6$ & 5.7 \\
\hline 182 & James River at Jamestown, N. Dak. & 84 & $1993-2007$ & $<2.6$ & $<2.6$ & 8.3 \\
\hline 183 & James River at Lamoure, N. Dak. & 81 & $1993-2007$ & $<2.6$ & $<2.6$ & 14.3 \\
\hline 184 & Bear Creek near Oakes, N. Dak. & 9 & 1998-1999 & $<2.6$ & $<2.6$ & 3.7 \\
\hline 185 & James River at Oakes, N. Dak. & 9 & 1993-1995 & $<2.6$ & $<2.6$ & $<2.6$ \\
\hline 186 & James River at N. Dak./S. Dak. State line & 9 & 1998-1999 & $<2.6$ & $<2.6$ & $<2.6$ \\
\hline \multicolumn{7}{|c|}{ Selenium, dissolved, in $\mu \mathrm{g} / \mathrm{L}$} \\
\hline 2 & Red River of the North at Wahpeton, N. Dak. & 31 & $1993-2008$ & $<2.6$ & $<2.6$ & 3.4 \\
\hline 4 & Red River of the North at Brushville, Minn. & 1 & 2005 & $<2.6$ & $<2.6$ & $<2.6$ \\
\hline 6 & Red River of the North at Hickson, N. Dak. & 29 & $1993-2008$ & $<2.6$ & $<2.6$ & 4.1 \\
\hline
\end{tabular}


Table 1-3. Summary statistics for trace-metal constituents at selected sites in North Dakota from 1993 through 2008. - Continued [ $\mu \mathrm{g} / \mathrm{L}$, micrograms per liter; --, not available; $<$, less than]

\begin{tabular}{|c|c|c|c|c|c|c|}
\hline $\begin{array}{c}\text { Site } \\
\text { identification } \\
\text { number } \\
\text { (table 1) }\end{array}$ & Site name & $\begin{array}{c}\text { Number of } \\
\text { samples }\end{array}$ & $\begin{array}{l}\text { Period of } \\
\text { record }\end{array}$ & Median & Minimum & Maximum \\
\hline \multicolumn{7}{|c|}{ Selenium, dissolved, in $\mu \mathrm{g} / \mathrm{L}-$ Continued } \\
\hline 7 & Wild Rice River near Rutland, N. Dak. & 28 & $1993-2008$ & $<2.6$ & $<2.6$ & 10.4 \\
\hline 9 & Antelope Creek at Dwight, N. Dak. & 13 & $2001-2008$ & $<2.6$ & $<2.6$ & 7.6 \\
\hline 10 & Wild Rice River near Abercrombie, N. Dak. & 32 & $1993-2008$ & $<2.6$ & $<2.6$ & 9.4 \\
\hline 11 & Red River of the North at Fargo, N. Dak. & 56 & $1993-2008$ & $<2.6$ & $<2.6$ & 7.0 \\
\hline 14 & Red River of the North near Harwood, N. Dak. & 7 & $2005-2006$ & $<2.6$ & $<2.6$ & 4.2 \\
\hline 15 & Sheyenne River above Harvey, N. Dak. & 35 & $1993-2008$ & $<2.6$ & $<2.6$ & 27.5 \\
\hline 17 & Sheyenne River at Warwick, N. Dak. & 2 & 2005 & $<2.6$ & $<2.6$ & $<2.6$ \\
\hline 18 & Sheyenne River near Warwick, N. Dak. & 33 & $1993-2008$ & $<2.6$ & $<2.6$ & 6.4 \\
\hline 19 & Mauvais Coulee Tributary No. 3 near Cando, N. Dak. & 31 & $1993-2008$ & $<2.6$ & $<2.6$ & 16.3 \\
\hline 20 & Mauvais Coulee near Cando, N. Dak. & 73 & 1993-2008 & $<2.6$ & $<2.6$ & 5.2 \\
\hline 21 & Edmore Coulee near Edmore, N. Dak. & 75 & $1993-2008$ & $<2.6$ & $<2.6$ & 3.7 \\
\hline 22 & Edmore Coulee Tributary near Webster, N. Dak. & 32 & $1993-2008$ & $<2.6$ & $<2.6$ & 3.0 \\
\hline 24 & Starkweather Coulee near Webster, N. Dak. & 67 & 1993-2008 & $<2.6$ & $<2.6$ & 5.2 \\
\hline 25 & Big Coulee below Churchs Ferry, N. Dak. & 23 & $1998-2008$ & $<2.6$ & $<2.6$ & 3.0 \\
\hline 26 & Little Coulee near Leeds, N. Dak. & 16 & $1998-2008$ & $<2.6$ & $<2.6$ & 3.0 \\
\hline 27 & Little Coulee near Brinsmade, N. Dak. & 11 & 1993-1998 & $<2.6$ & $<2.6$ & $<2.6$ \\
\hline 28 & Big Coulee near Churchs Ferry, N. Dak. & 43 & 1993-1997 & $<2.6$ & $<2.6$ & $<2.6$ \\
\hline 30 & Channel A near Penn, N. Dak. & 63 & $1993-2008$ & $<2.6$ & $<2.6$ & 3.0 \\
\hline 31 & Devils Lake Outlet to Stump Lake near Lakota, N. Dak. & 12 & $2002-2007$ & 9.4 & $<2.6$ & 21.4 \\
\hline 32 & Sheyenne River near Cooperstown, N. Dak. & 34 & $1993-2008$ & $<2.6$ & $<2.6$ & 57.1 \\
\hline 33 & Baldhill Creek near Dazey, N. Dak. & 29 & $1993-2008$ & $<2.6$ & $<2.6$ & 7.7 \\
\hline 34 & Sheyenne River below Baldhill Dam, N. Dak. & 35 & $1993-2008$ & $<2.6$ & $<2.6$ & 57.9 \\
\hline 35 & Sheyenne River at Valley City, N. Dak. & 13 & $1994-2005$ & $<2.6$ & $<2.6$ & 4.8 \\
\hline 36 & Sheyenne River at Lisbon, N. Dak. & 26 & $1996-2008$ & $<2.6$ & $<2.6$ & 4.4 \\
\hline 37 & Sheyenne River near Kindred, N. Dak. & 32 & $1993-2008$ & $<2.6$ & $<2.6$ & 4.6 \\
\hline 38 & $\begin{array}{l}\text { Sheyenne River above Sheyenne River Diversion near Horace, } \\
\text { N. Dak. }\end{array}$ & 35 & $1993-2008$ & $<2.6$ & $<2.6$ & 7.7 \\
\hline 40 & Sheyenne River Diversion at West Fargo, N. Dak. & 13 & $1994-2007$ & $<2.6$ & $<2.6$ & 5.3 \\
\hline 41 & Sheyenne River at West Fargo, N. Dak. & 16 & 1993-2008 & $<2.6$ & $<2.6$ & $<2.6$ \\
\hline 42 & Maple River near Hope, N. Dak. & 22 & 1993-2008 & $<2.6$ & $<2.6$ & 4.4 \\
\hline 43 & Maple River near Enderlin, N. Dak. & 31 & $1993-2008$ & $<2.6$ & $<2.6$ & 7.0 \\
\hline 44 & Maple River near Mapleton, N. Dak. & 17 & $1995-2008$ & $<2.6$ & $<2.6$ & 7.1 \\
\hline 45 & Maple River below Mapleton, N. Dak. & 28 & $1995-2008$ & $<2.6$ & $<2.6$ & 7.1 \\
\hline 46 & Sheyenne River at Harwood, N. Dak. & 6 & $2000-2005$ & $<2.6$ & $<2.6$ & 8.0 \\
\hline 47 & Rush River at Amenia, N. Dak. & 32 & 1993-2008 & $<2.6$ & $<2.6$ & 5.6 \\
\hline 52 & Red River of the North at Halstad, Minn. & 37 & 1993-2008 & $<2.6$ & $<2.6$ & 57.1 \\
\hline 53 & Beaver Creek near Finley, N. Dak. & 24 & $1993-2003$ & $<2.6$ & $<2.6$ & 7.7 \\
\hline 55 & Goose River at Hillsboro, N. Dak. & 39 & $1993-2008$ & $<2.6$ & $<2.6$ & 9.0 \\
\hline 56 & Red River of the North at Grand Forks, N. Dak. & 47 & $1996-2008$ & $<2.6$ & $<2.6$ & 10.5 \\
\hline 57 & Turtle River at Turtle River State Park near Arvilla, N. Dak. & 17 & $1996-2008$ & $<2.6$ & $<2.6$ & 5.5 \\
\hline
\end{tabular}


Table 1-3. Summary statistics for trace-metal constituents at selected sites in North Dakota from 1993 through 2008. - Continued [ $\mu \mathrm{g} / \mathrm{L}$, micrograms per liter; --, not available; $<$, less than]

\begin{tabular}{|c|c|c|c|c|c|c|}
\hline $\begin{array}{c}\text { Site } \\
\text { identification } \\
\text { number } \\
\text { (table 1) }\end{array}$ & Site name & $\begin{array}{l}\text { Number of } \\
\text { samples }\end{array}$ & $\begin{array}{l}\text { Period of } \\
\text { record }\end{array}$ & Median & Minimum & Maximum \\
\hline \multicolumn{7}{|c|}{ Selenium, dissolved, in $\mu \mathrm{g} / \mathrm{L}$-Continued } \\
\hline 58 & Turtle River at Manvel, N. Dak. & 30 & 2005-2008 & 9.4 & 3.5 & 39.2 \\
\hline 59 & Red River of the North at Oslo, Minn. & 12 & $1993-2005$ & $<2.6$ & $<2.6$ & 4.1 \\
\hline 61 & Forest River near Fordville, N. Dak. & 31 & 1993-2008 & $<2.6$ & $<2.6$ & 3.5 \\
\hline 62 & Forest River near Minto, N. Dak. & 54 & 1993-2008 & $<2.6$ & $<2.6$ & 12.0 \\
\hline 63 & South Branch Park River below Homme Dam, N. Dak. & 4 & $1993-1994$ & $<2.6$ & $<2.6$ & $<2.6$ \\
\hline 67 & Park River at Grafton, N. Dak. & 53 & 1993-2008 & $<2.6$ & $<2.6$ & 17.2 \\
\hline 68 & Red River of the North at Drayton, N. Dak. & 30 & 1993-2008 & $<2.6$ & $<2.6$ & 4.4 \\
\hline 70 & Hidden Island Coulee near Hansboro, N. Dak. & 4 & 1993-1995 & $<2.6$ & $<2.6$ & $<2.6$ \\
\hline 73 & Little South Pembina River near Walhalla, N. Dak. & 16 & $2001-2008$ & 2.7 & $<2.6$ & 6.9 \\
\hline 74 & Pembina River at Walhalla, N. Dak. & 16 & $2001-2008$ & 2.7 & $<2.6$ & 4.8 \\
\hline 75 & Pembina River at Neche, N. Dak. & 53 & 1993-2008 & $<2.6$ & $<2.6$ & 6.7 \\
\hline 76 & Tongue River at Akra, N. Dak. & 32 & 1993-2008 & $<2.6$ & $<2.6$ & 3.0 \\
\hline 78 & Red River of the North at Pembina, N. Dak., site 2 & 37 & 2001-2008 & $<2.6$ & $<2.6$ & 10.9 \\
\hline 79 & Red River of the North at Emerson, Manitoba & 7 & 1993-1994 & $<2.6$ & $<2.6$ & $<2.6$ \\
\hline 80 & Long Creek near Noonan, N. Dak. & 29 & $1993-2008$ & $<2.6$ & $<2.6$ & 6.1 \\
\hline 83 & Souris River near Foxholm, N. Dak. & 20 & 1999-2008 & $<2.6$ & $<2.6$ & 27.5 \\
\hline 84 & Des Lacs River at Foxholm, N. Dak. & 32 & 1993-2008 & $<2.6$ & $<2.6$ & 7.3 \\
\hline 85 & Souris River above Minot, N. Dak. & 12 & $1993-1998$ & $<2.6$ & $<2.6$ & $<2.6$ \\
\hline 86 & Bonnes Creek near Velva, N. Dak. & 13 & $1993-2005$ & $<2.6$ & $<2.6$ & $<2.6$ \\
\hline 88 & Wintering River near Karlsruhe, N. Dak. & 31 & 1993-2008 & $<2.6$ & $<2.6$ & 280.0 \\
\hline 89 & Souris River near Bantry, N. Dak. & 19 & 1999-2008 & $<2.6$ & $<2.6$ & 8.2 \\
\hline 90 & Willow Creek near Willow City, N. Dak. & 27 & 1994-2008 & $<2.6$ & $<2.6$ & 4.3 \\
\hline 92 & Deep River near Upham, N. Dak. & 25 & 1993-2007 & $<2.6$ & $<2.6$ & 5.0 \\
\hline 97 & Boundary Creek near Landa, N. Dak. & 1 & 1994 & $<2.6$ & $<2.6$ & $<2.6$ \\
\hline 98 & Souris River near Westhope, N. Dak. & 10 & 1993-2001 & $<2.6$ & $<2.6$ & 3.0 \\
\hline 101 & Little Muddy River below Cow Creek near Williston, N. Dak. & 30 & 1993-2008 & $<2.6$ & $<2.6$ & 13.6 \\
\hline 106 & Bear Den Creek near Mandaree, N. Dak. & 32 & 1993-2008 & $<2.6$ & $<2.6$ & 8.9 \\
\hline 108 & East Fork Shell Creek near Parshall, N. Dak. & 43 & $1993-2008$ & $<2.6$ & $<2.6$ & 3.7 \\
\hline 109 & Deepwater Creek near Mandaree, N. Dak. & 43 & 1993-2008 & $<2.6$ & $<2.6$ & 47.4 \\
\hline 110 & Little Missouri River at Marmarth, N. Dak. & 33 & 1993-2008 & $<2.6$ & $<2.6$ & 3.6 \\
\hline 112 & Little Missouri River at Medora, N. Dak. & 16 & $2001-2008$ & $<2.6$ & $<2.6$ & 9.6 \\
\hline 113 & Beaver Creek near Trotters, N. Dak. & 28 & 1993-2008 & $<2.6$ & $<2.6$ & 6.9 \\
\hline 114 & Little Missouri River near Watford City, N. Dak. & 31 & $1993-2008$ & $<2.6$ & $<2.6$ & 31.7 \\
\hline 115 & Missouri River at Garrison Dam, N. Dak. & 74 & $1993-2007$ & $<2.6$ & $<2.6$ & $<2.6$ \\
\hline 116 & Knife River at Manning, N. Dak. & 31 & 1993-2008 & $<2.6$ & $<2.6$ & 8.1 \\
\hline 120 & Knife River near Golden Valley, N. Dak. & 31 & 1993-2008 & $<2.6$ & $<2.6$ & 5.3 \\
\hline 125 & Spring Creek at Zap, N. Dak. & 31 & 1993-2008 & $<2.6$ & $<2.6$ & 12.0 \\
\hline 127 & Knife River at Hazen, N. Dak. & 34 & 1993-2008 & $<2.6$ & $<2.6$ & 9.5 \\
\hline 140 & Square Butte Creek below Center, N. Dak. & 30 & 1993-2008 & $<2.6$ & $<2.6$ & 16.9 \\
\hline 141 & Burnt Creek near Bismarck, N. Dak. & 29 & 1993-2008 & $<2.6$ & $<2.6$ & 3.0 \\
\hline
\end{tabular}


Table 1-3. Summary statistics for trace-metal constituents at selected sites in North Dakota from 1993 through 2008.—Continued [ $\mu \mathrm{g} / \mathrm{L}$, micrograms per liter; --, not available; <, less than]

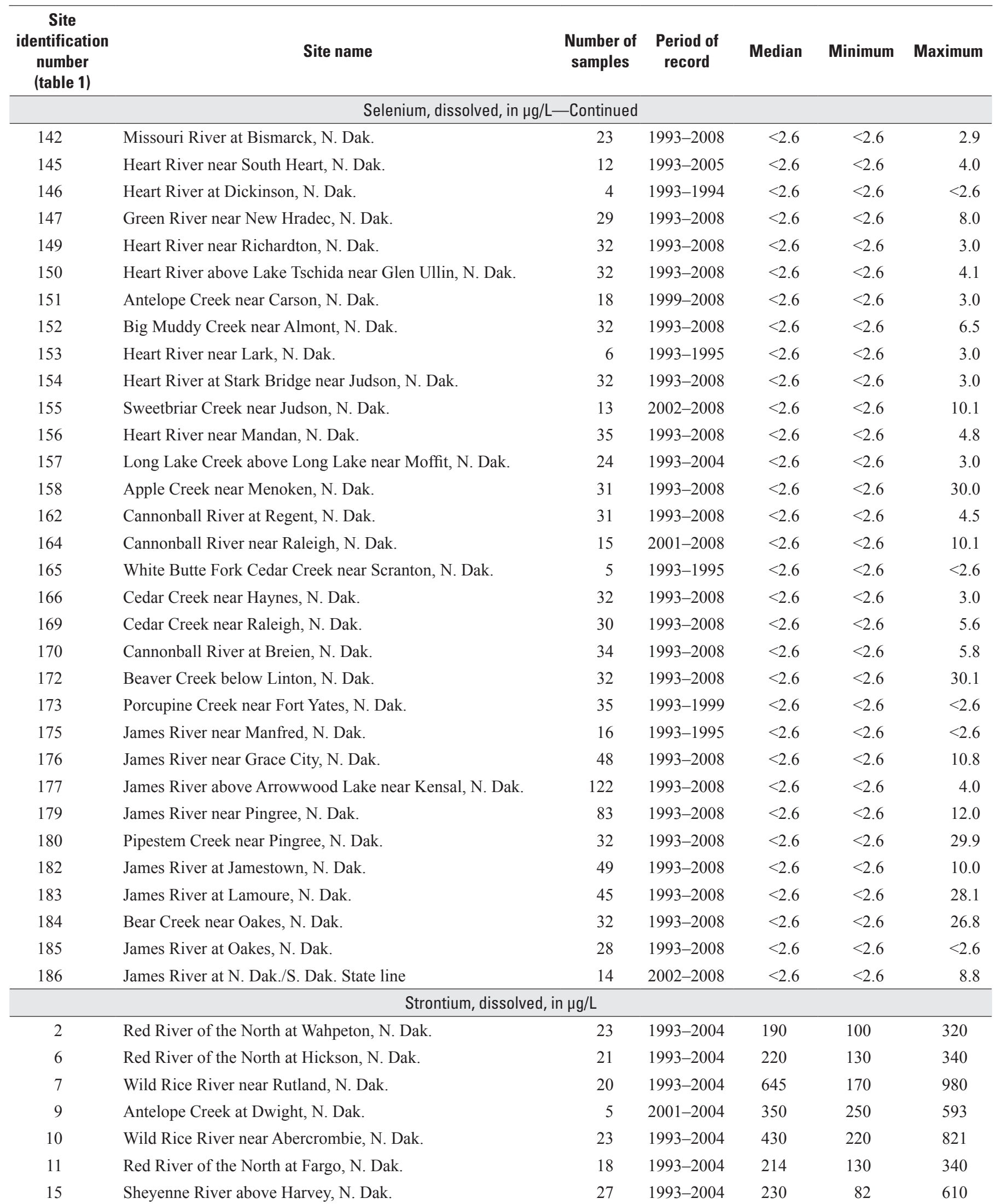


Table 1-3. Summary statistics for trace-metal constituents at selected sites in North Dakota from 1993 through 2008. - Continued [ $\mu \mathrm{g} / \mathrm{L}$, micrograms per liter; --, not available; $<$, less than]

\begin{tabular}{|c|c|c|c|c|c|c|}
\hline $\begin{array}{c}\text { Site } \\
\text { identification } \\
\text { number } \\
\text { (table 1) } \\
\end{array}$ & Site name & $\begin{array}{c}\text { Number of } \\
\text { samples }\end{array}$ & $\begin{array}{l}\text { Period of } \\
\text { record }\end{array}$ & Median & Minimum & Maximum \\
\hline \multicolumn{7}{|c|}{ Strontium, dissolved, in $\mu \mathrm{g} / \mathrm{L}$-Continued } \\
\hline 18 & Sheyenne River near Warwick, N. Dak. & 25 & 1993-2004 & 290 & 130 & 400 \\
\hline 19 & Mauvais Coulee Tributary No. 3 near Cando, N. Dak. & 24 & 1993-2004 & 295 & 130 & 630 \\
\hline 20 & Mauvais Coulee near Cando, N. Dak. & 57 & 1993-2004 & 320 & 140 & 640 \\
\hline 21 & Edmore Coulee near Edmore, N. Dak. & 58 & 1993-2004 & 290 & 100 & 610 \\
\hline 22 & Edmore Coulee Tributary near Webster, N. Dak. & 25 & 1993-2004 & 290 & 120 & 760 \\
\hline 24 & Starkweather Coulee near Webster, N. Dak. & 51 & 1993-2004 & 270 & 100 & 440 \\
\hline 25 & Big Coulee below Churchs Ferry, N. Dak. & 16 & 1998-2004 & 292 & 210 & 540 \\
\hline 26 & Little Coulee near Leeds, N. Dak. & 11 & 1998-2004 & 320 & 150 & 400 \\
\hline 27 & Little Coulee near Brinsmade, N. Dak. & 11 & 1993-1998 & 250 & 140 & 330 \\
\hline 28 & Big Coulee near Churchs Ferry, N. Dak. & 43 & 1993-1997 & 260 & 120 & 460 \\
\hline 30 & Channel A near Penn, N. Dak. & 56 & 1993-2004 & 250 & 100 & 620 \\
\hline 31 & Devils Lake Outlet to Stump Lake near Lakota, N. Dak. & 6 & 2002-2004 & 427 & 220 & 547 \\
\hline 32 & Sheyenne River near Cooperstown, N. Dak. & 21 & 1993-2004 & 300 & 92 & 480 \\
\hline 33 & Baldhill Creek near Dazey, N. Dak. & 21 & 1993-2004 & 380 & 147 & 580 \\
\hline 34 & Sheyenne River below Baldhill Dam, N. Dak. & 21 & 1993-2004 & 345 & 180 & 420 \\
\hline 35 & Sheyenne River at Valley City, N. Dak. & 12 & 1994-2004 & 340 & 180 & 500 \\
\hline 36 & Sheyenne River at Lisbon, N. Dak. & 17 & 1996-2004 & 380 & 210 & 560 \\
\hline 37 & Sheyenne River near Kindred, N. Dak. & 23 & 1993-2004 & 363 & 200 & 550 \\
\hline 38 & $\begin{array}{l}\text { Sheyenne River above Sheyenne River Diversion near Horace, } \\
\text { N. Dak. }\end{array}$ & 21 & 1993-2004 & 360 & 150 & 540 \\
\hline 40 & Sheyenne River Diversion at West Fargo, N. Dak. & 9 & 1994-2004 & 230 & 150 & 460 \\
\hline 41 & Sheyenne River at West Fargo, N. Dak. & 12 & 1993-2004 & 411 & 330 & 550 \\
\hline 42 & Maple River near Hope, N. Dak. & 16 & 1993-2004 & 365 & 200 & 824 \\
\hline 43 & Maple River near Enderlin, N. Dak. & 23 & 1993-2004 & 490 & 210 & 875 \\
\hline 44 & Maple River near Mapleton, N. Dak. & 9 & 1995-2004 & 400 & 210 & 574 \\
\hline 45 & Maple River below Mapleton, N. Dak. & 19 & 1995-2004 & 400 & 160 & 690 \\
\hline 46 & Sheyenne River at Harwood, N. Dak. & 5 & 2000-2004 & 320 & 280 & 550 \\
\hline 47 & Rush River at Amenia, N. Dak. & 24 & 1993-2004 & 495 & 150 & 770 \\
\hline 52 & Red River of the North at Halstad, Minn. & 24 & 1993-2004 & 275 & 131 & 380 \\
\hline 53 & Beaver Creek near Finley, N. Dak. & 24 & 1993-2003 & 500 & 190 & 1,100 \\
\hline 55 & Goose River at Hillsboro, N. Dak. & 24 & 1993-2004 & 415 & 140 & 753 \\
\hline 56 & Red River of the North at Grand Forks, N. Dak. & 17 & 1996-2004 & 210 & 150 & 375 \\
\hline 57 & Turtle River at Turtle River State Park near Arvilla, N. Dak. & 9 & 1996-2004 & 328 & 141 & 420 \\
\hline 59 & Red River of the North at Oslo, Minn. & 11 & 1993-2004 & 199 & 110 & 310 \\
\hline 61 & Forest River near Fordville, N. Dak. & 22 & 1993-2004 & 300 & 130 & 410 \\
\hline 62 & Forest River near Minto, N. Dak. & 24 & 1993-2004 & 310 & 120 & 410 \\
\hline 63 & South Branch Park River below Homme Dam, N. Dak. & 4 & 1993-1994 & 335 & 120 & 420 \\
\hline 67 & Park River at Grafton, N. Dak. & 23 & 1993-2004 & 370 & 120 & 590 \\
\hline 68 & Red River of the North at Drayton, N. Dak. & 22 & 1993-2004 & 230 & 130 & 345 \\
\hline 70 & Hidden Island Coulee near Hansboro, N. Dak. & 4 & 1993-1995 & 320 & 120 & 470 \\
\hline
\end{tabular}


Table 1-3. Summary statistics for trace-metal constituents at selected sites in North Dakota from 1993 through 2008.—Continued [ $\mu \mathrm{g} / \mathrm{L}$, micrograms per liter; --, not available; $<$, less than]

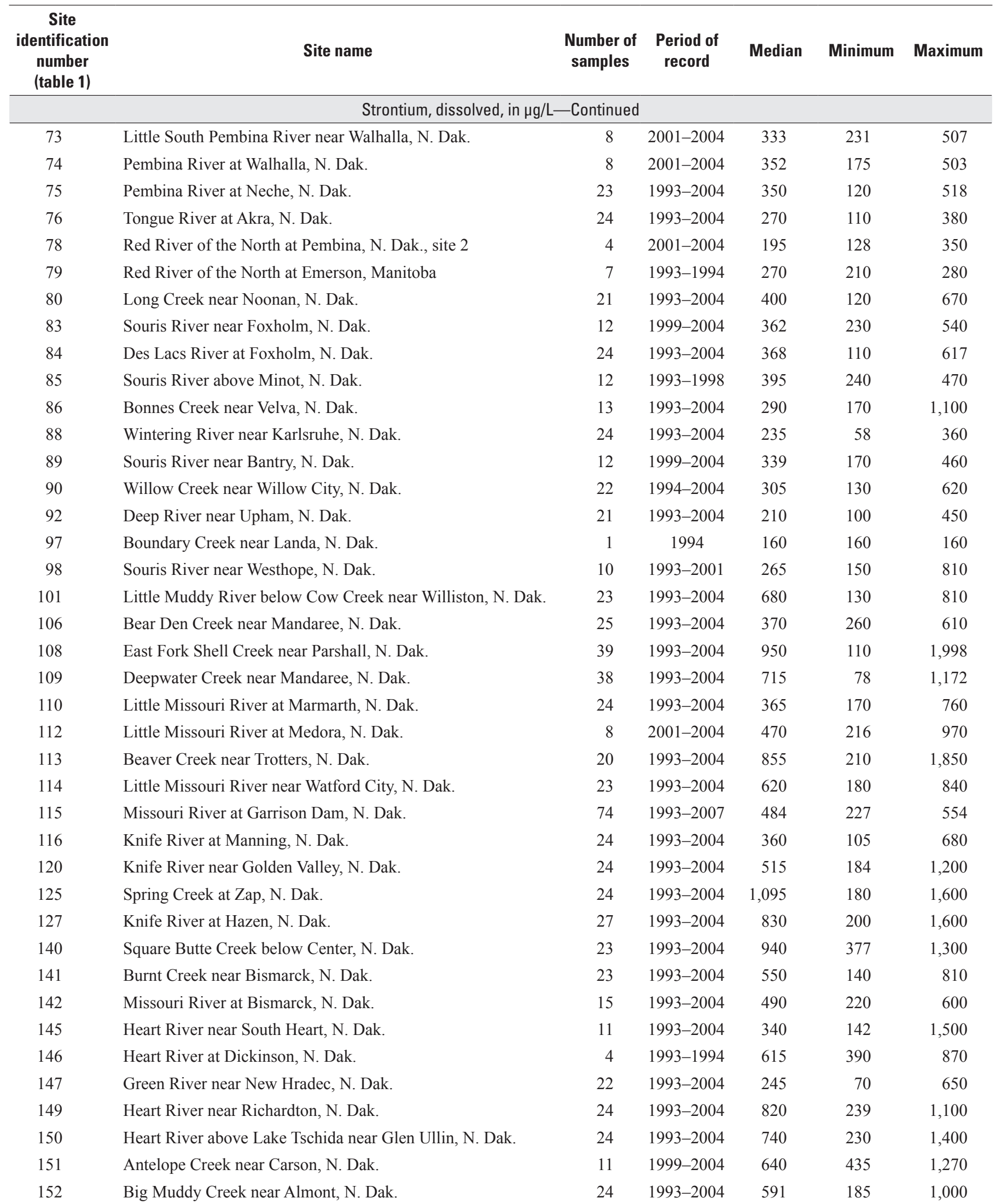


Table 1-3. Summary statistics for trace-metal constituents at selected sites in North Dakota from 1993 through 2008. - Continued [ $\mu \mathrm{g} / \mathrm{L}$, micrograms per liter; --, not available; $<$, less than]

\begin{tabular}{|c|c|c|c|c|c|c|}
\hline $\begin{array}{c}\text { Site } \\
\text { identification } \\
\text { number } \\
\text { (table 1) }\end{array}$ & Site name & $\begin{array}{c}\text { Number of } \\
\text { samples }\end{array}$ & $\begin{array}{l}\text { Period of } \\
\text { record }\end{array}$ & Median & Minimum & Maximum \\
\hline \multicolumn{7}{|c|}{ Strontium, dissolved, in $\mu \mathrm{g} / \mathrm{L}$-Continued } \\
\hline 153 & Heart River near Lark, N. Dak. & 6 & $1993-1995$ & 550 & 300 & 810 \\
\hline 154 & Heart River at Stark Bridge near Judson, N. Dak. & 24 & 1993-2004 & 705 & 160 & 950 \\
\hline 155 & Sweetbriar Creek near Judson, N. Dak. & 6 & 2002-2004 & 556 & 305 & 885 \\
\hline 156 & Heart River near Mandan, N. Dak. & 27 & 1993-2004 & 720 & 221 & 1,400 \\
\hline 157 & Long Lake Creek above Long Lake near Moffit, N. Dak. & 24 & 1993-2004 & 320 & 90 & 470 \\
\hline 158 & Apple Creek near Menoken, N. Dak. & 23 & 1993-2004 & 350 & 70 & 731 \\
\hline 162 & Cannonball River at Regent, N. Dak. & 23 & 1993-2004 & 1,100 & 296 & 1,700 \\
\hline 164 & Cannonball River near Raleigh, N. Dak. & 6 & 2001-2004 & 781 & 266 & 1,100 \\
\hline 165 & White Butte Fork Cedar Creek near Scranton, N. Dak. & 5 & 1993-1995 & 1,000 & 580 & 2,700 \\
\hline 166 & Cedar Creek near Haynes, N. Dak. & 24 & 1993-2004 & 960 & 210 & 2,000 \\
\hline 169 & Cedar Creek near Raleigh, N. Dak. & 21 & 1993-2004 & 850 & 250 & 1,500 \\
\hline 170 & Cannonball River at Breien, N. Dak. & 25 & 1993-2004 & 727 & 170 & 1,200 \\
\hline 172 & Beaver Creek below Linton, N. Dak. & 24 & 1993-2004 & 398 & 130 & 630 \\
\hline 173 & Porcupine Creek near Fort Yates, N. Dak. & 35 & 1993-1999 & 220 & 18 & 288 \\
\hline 176 & James River near Grace City, N. Dak. & 18 & 1996-2004 & 280 & 144 & 430 \\
\hline 179 & James River near Pingree, N. Dak. & 2 & 1993 & 175 & 140 & 210 \\
\hline 180 & Pipestem Creek near Pingree, N. Dak. & 24 & 1993-2004 & 340 & 160 & 579 \\
\hline 182 & James River at Jamestown, N. Dak. & 17 & 1996-2004 & 340 & 190 & 490 \\
\hline 183 & James River at Lamoure, N. Dak. & 18 & 1996-2004 & 295 & 214 & 480 \\
\hline 184 & Bear Creek near Oakes, N. Dak. & 24 & 1993-2004 & 485 & 210 & 732 \\
\hline 186 & James River at N. Dak./S. Dak. State line & 6 & 2002-2004 & 383 & 260 & 470 \\
\hline \multicolumn{7}{|c|}{ Zinc, total, in $\mu \mathrm{g} / \mathrm{L}$} \\
\hline 1 & Bois De Sioux River near Doran, Minn. & 40 & $2000-2007$ & $<40$ & $<40$ & 47 \\
\hline 2 & Red River of the North at Wahpeton, N. Dak. & 2 & 1996 & $<40$ & $<40$ & $<40$ \\
\hline 3 & Red River of the North near Wahpeton, N. Dak. & 1 & 2006 & $<40$ & $<40$ & $<40$ \\
\hline 4 & Red River of the North at Brushville, Minn. & 54 & 1996-2007 & $<40$ & $<40$ & 94 \\
\hline 10 & Wild Rice River near Abercrombie, N. Dak. & 45 & 1996-2007 & $<40$ & $<40$ & 70 \\
\hline 11 & Red River of the North at Fargo, N. Dak. & 5 & 1996 & $<40$ & $<40$ & 50 \\
\hline 13 & Red River of the North at Harwood, N. Dak. & 53 & $2000-2007$ & $<40$ & $<40$ & 237 \\
\hline 14 & Red River of the North near Harwood, N. Dak. & 5 & 1996 & $<40$ & $<40$ & $<40$ \\
\hline 17 & Sheyenne River at Warwick, N. Dak. & 48 & 1996-2006 & $<40$ & $<40$ & 42 \\
\hline 18 & Sheyenne River near Warwick, N. Dak. & 16 & 2005-2007 & $<40$ & $<40$ & $<40$ \\
\hline 32 & Sheyenne River near Cooperstown, N. Dak. & 69 & 1996-2007 & $<40$ & $<40$ & 202 \\
\hline 33 & Baldhill Creek near Dazey, N. Dak. & 1 & 1996 & $<40$ & $<40$ & $<40$ \\
\hline 34 & Sheyenne River below Baldhill Dam, N. Dak. & 62 & 1996-2007 & $<40$ & $<40$ & $<40$ \\
\hline 36 & Sheyenne River at Lisbon, N. Dak. & 69 & 1997-2007 & $<40$ & $<40$ & 54 \\
\hline 37 & Sheyenne River near Kindred, N. Dak. & 70 & 1996-2007 & $<40$ & $<40$ & 83 \\
\hline 41 & Sheyenne River at West Fargo, N. Dak. & 6 & 1996-2006 & $<40$ & $<40$ & 63 \\
\hline 45 & Maple River below Mapleton, N. Dak. & 47 & 1997-2007 & $<40$ & $<40$ & 54 \\
\hline 55 & Goose River at Hillsboro, N. Dak. & 64 & 1996-2007 & $<40$ & $<40$ & 67 \\
\hline
\end{tabular}


Table 1-3. Summary statistics for trace-metal constituents at selected sites in North Dakota from 1993 through 2008.—Continued [ $\mu \mathrm{g} / \mathrm{L}$, micrograms per liter; --, not available; $<$, less than]

\begin{tabular}{|c|c|c|c|c|c|c|}
\hline $\begin{array}{c}\text { Site } \\
\text { identification } \\
\text { number } \\
\text { (table 1) }\end{array}$ & Site name & $\begin{array}{c}\text { Number of } \\
\text { samples }\end{array}$ & $\begin{array}{l}\text { Period of } \\
\text { record }\end{array}$ & Median & Minimum & Maximum \\
\hline \multicolumn{7}{|c|}{ Zinc, total, in $\mu \mathrm{g} / \mathrm{L}$-Continued } \\
\hline 56 & Red River of the North at Grand Forks, N. Dak. & 46 & 1997-2006 & $<40$ & $<40$ & 73 \\
\hline 58 & Turtle River at Manvel, N. Dak. & 37 & $1997-2006$ & $<40$ & $<40$ & 86 \\
\hline 67 & Park River at Grafton, N. Dak. & 36 & 1996-2006 & $<40$ & $<40$ & 89 \\
\hline 68 & Red River of the North at Drayton, N. Dak. & 5 & 1996 & $<40$ & $<40$ & 42 \\
\hline 75 & Pembina River at Neche, N. Dak. & 46 & 1996-2006 & $<40$ & $<40$ & 238 \\
\hline 78 & Red River of the North at Pembina, N. Dak., site 2 & 52 & 1997-2006 & $<40$ & $<40$ & 158 \\
\hline 85 & Souris River above Minot, N. Dak. & 68 & 1996-2007 & $<40$ & $<40$ & 41 \\
\hline 87 & Souris River near Verendrye, N. Dak. & 75 & 1997-2007 & $<40$ & $<40$ & 187 \\
\hline 88 & Wintering River near Karlsruhe, N. Dak. & 10 & 1997-1998 & $<40$ & $<40$ & $<40$ \\
\hline 89 & Souris River near Bantry, N. Dak. & 9 & 1997-1998 & $<40$ & $<40$ & $<40$ \\
\hline 90 & Willow Creek near Willow City, N. Dak. & 5 & 1997 & $<40$ & $<40$ & $<40$ \\
\hline 92 & Deep River near Upham, N. Dak. & 6 & 1997 & $<40$ & $<40$ & $<40$ \\
\hline 98 & Souris River near Westhope, N. Dak. & 7 & 1997-1998 & $<40$ & $<40$ & $<40$ \\
\hline 101 & Little Muddy River below Cow Creek near Williston, N. Dak. & 9 & 1999 & $<40$ & $<40$ & $<40$ \\
\hline 110 & Little Missouri River at Marmarth, N. Dak. & 8 & 1999 & 53 & $<40$ & 261 \\
\hline 149 & Heart River near Richardton, N. Dak. & 67 & 1996-2007 & $<40$ & $<40$ & 49 \\
\hline 156 & Heart River near Mandan, N. Dak. & 67 & 1996-2007 & $<40$ & $<40$ & 59 \\
\hline 164 & Cannonball River near Raleigh, N. Dak. & 61 & 1996-2007 & $<40$ & $<40$ & 471 \\
\hline 169 & Cedar Creek near Raleigh, N. Dak. & 53 & 1996-2007 & $<40$ & $<40$ & 81 \\
\hline 170 & Cannonball River at Breien, N. Dak. & 63 & 1996-2007 & $<40$ & $<40$ & 266 \\
\hline 175 & James River near Manfred, N. Dak. & 6 & 1998 & $<40$ & $<40$ & $<40$ \\
\hline 176 & James River near Grace City, N. Dak. & 60 & 1997-2007 & $<40$ & $<40$ & 41 \\
\hline 177 & James River above Arrowwood Lake near Kensal, N. Dak. & 9 & 1998-1999 & $<40$ & $<40$ & $<40$ \\
\hline 180 & Pipestem Creek near Pingree, N. Dak. & 9 & 1998-1999 & $<40$ & $<40$ & $<40$ \\
\hline 182 & James River at Jamestown, N. Dak. & 75 & $1997-2007$ & $<40$ & $<40$ & 42 \\
\hline 183 & James River at Lamoure, N. Dak. & 73 & 1996-2007 & $<40$ & $<40$ & 45 \\
\hline 184 & Bear Creek near Oakes, N. Dak. & 9 & 1998-1999 & $<40$ & $<40$ & $<40$ \\
\hline 186 & James River at N. Dak./S. Dak. State line & 9 & 1998-1999 & $<40$ & $<40$ & $<40$ \\
\hline
\end{tabular}


Table 1-5. Median seasonal concentrations for selected constituents at selected sites in North Dakota from 1970 through 2008.

[Winter, January-February, November-December; Spring, March-June; Summer, July-October; mg/L, milligrams per liter; --, not available; N, nitrogen; <. less than; P, phosphorus; $\mathrm{C}$, carbon; $\mu \mathrm{g} / \mathrm{L}$, micrograms per liter]

\begin{tabular}{|c|c|c|c|c|c|}
\hline $\begin{array}{c}\text { Site } \\
\text { identification } \\
\text { number } \\
\text { (table 1) }\end{array}$ & Site name & Winter & Spring & Summer & $\begin{array}{c}\text { Period of } \\
\text { record }\end{array}$ \\
\hline \multicolumn{6}{|c|}{ Sulfate, in $\mathrm{mg} / \mathrm{L}$} \\
\hline 1 & Bois De Sioux River near Doran, Minn. & 623 & 403 & 420 & 462 \\
\hline 2 & Red River of the North at Wahpeton, N. Dak. & 416 & 96 & 42 & 86 \\
\hline 4 & Red River of the North at Brushville, Minn. & 43 & 147 & 73 & 87 \\
\hline 5 & Red River of the North below Wahpeton, N. Dak. & 32 & 63 & 37 & 38 \\
\hline 6 & Red River of the North at Hickson, N. Dak. & 38 & 91 & 50 & 67 \\
\hline 7 & Wild Rice River near Rutland, N. Dak. & -- & 475 & 600 & 490 \\
\hline 11 & Red River of the North at Fargo, N. Dak. & 47 & 110 & 79 & 86 \\
\hline 12 & Red River of North below Fargo, N. Dak. & 54 & 84 & 92 & 69 \\
\hline 13 & Red River of the North at Harwood, N. Dak. & 97 & 198 & 146 & 146 \\
\hline 14 & Red River of the North near Harwood, N. Dak. & 91 & 180 & 145 & 152 \\
\hline 15 & Sheyenne River above Harvey, N. Dak. & 240 & 210 & 200 & 210 \\
\hline 16 & Big Coulee near Fort Totten, N. Dak. & -- & 21 & 19 & 20 \\
\hline 17 & Sheyenne River at Warwick, N. Dak. & 264 & 222 & 207 & 217 \\
\hline 18 & Sheyenne River near Warwick, N. Dak. & 95 & 125 & 120 & 110 \\
\hline 19 & Mauvais Coulee Tributary No. 3 near Cando, N. Dak. & -- & 108 & 247 & 179 \\
\hline 26 & Little Coulee near Leeds, N. Dak. & -- & 269 & 276 & 270 \\
\hline 27 & Little Coulee near Brinsmade, N. Dak. & -- & 160 & 160 & 160 \\
\hline 28 & Big Coulee near Churchs Ferry, N. Dak. & 310 & 136 & 170 & 150 \\
\hline 29 & Big Coulee at Graham Island inlet near Fort Totten, N. Dak. & 1,350 & 640 & 1,100 & 910 \\
\hline 30 & Channel A near Penn, N. Dak. & 180 & 185 & 150 & 172 \\
\hline 31 & Devils Lake Outlet to Stump Lake near Lakota, N. Dak. & -- & 1,165 & 1,835 & 1,385 \\
\hline 32 & Sheyenne River near Cooperstown, N. Dak. & 160 & 142 & 180 & 160 \\
\hline 33 & Baldhill Creek near Dazey, N. Dak. & 235 & 140 & 200 & 170 \\
\hline 34 & Sheyenne River below Baldhill Dam, N. Dak. & 238 & 167 & 220 & 208 \\
\hline 35 & Sheyenne River at Valley City, N. Dak. & -- & 155 & 120 & 140 \\
\hline 36 & Sheyenne River at Lisbon, N. Dak. & 200 & 160 & 210 & 200 \\
\hline 37 & Sheyenne River near Kindred, N. Dak. & 200 & 160 & 170 & 170 \\
\hline 38 & Sheyenne River above Sheyenne River Diversion near Horace, N. Dak. & 270 & 214 & 227 & 227 \\
\hline 39 & Sheyenne River near Horace, N. Dak. & -- & 120 & 95 & 115 \\
\hline
\end{tabular}


Table 1-5. Median seasonal concentrations for selected constituents at selected sites in North Dakota from 1970 through 2008.Continued

[Winter, January-February, November-December; Spring, March-June; Summer, July-October; mg/L, milligrams per liter; --, not available; N, nitrogen; <. less than; P, phosphorus; $\mathrm{C}$, carbon; $\mu \mathrm{g} / \mathrm{L}$, micrograms per liter]

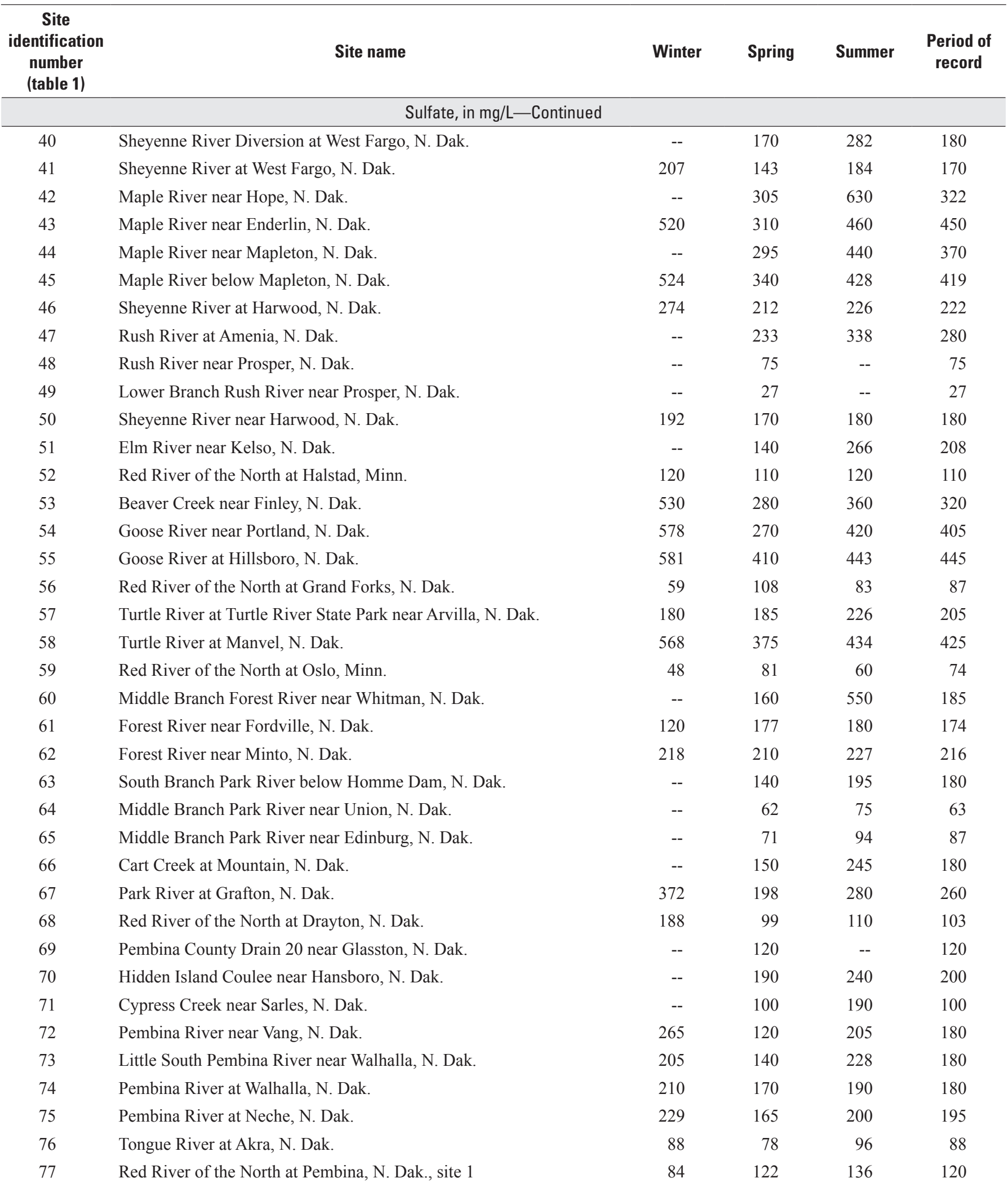


Table 1-5. Median seasonal concentrations for selected constituents at selected sites in North Dakota from 1970 through 2008.Continued

[Winter, January-February, November-December; Spring, March-June; Summer, July-October; mg/L, milligrams per liter; --, not available; N, nitrogen; <. less than; P, phosphorus; C, carbon; $\mu \mathrm{g} / \mathrm{L}$, micrograms per liter]

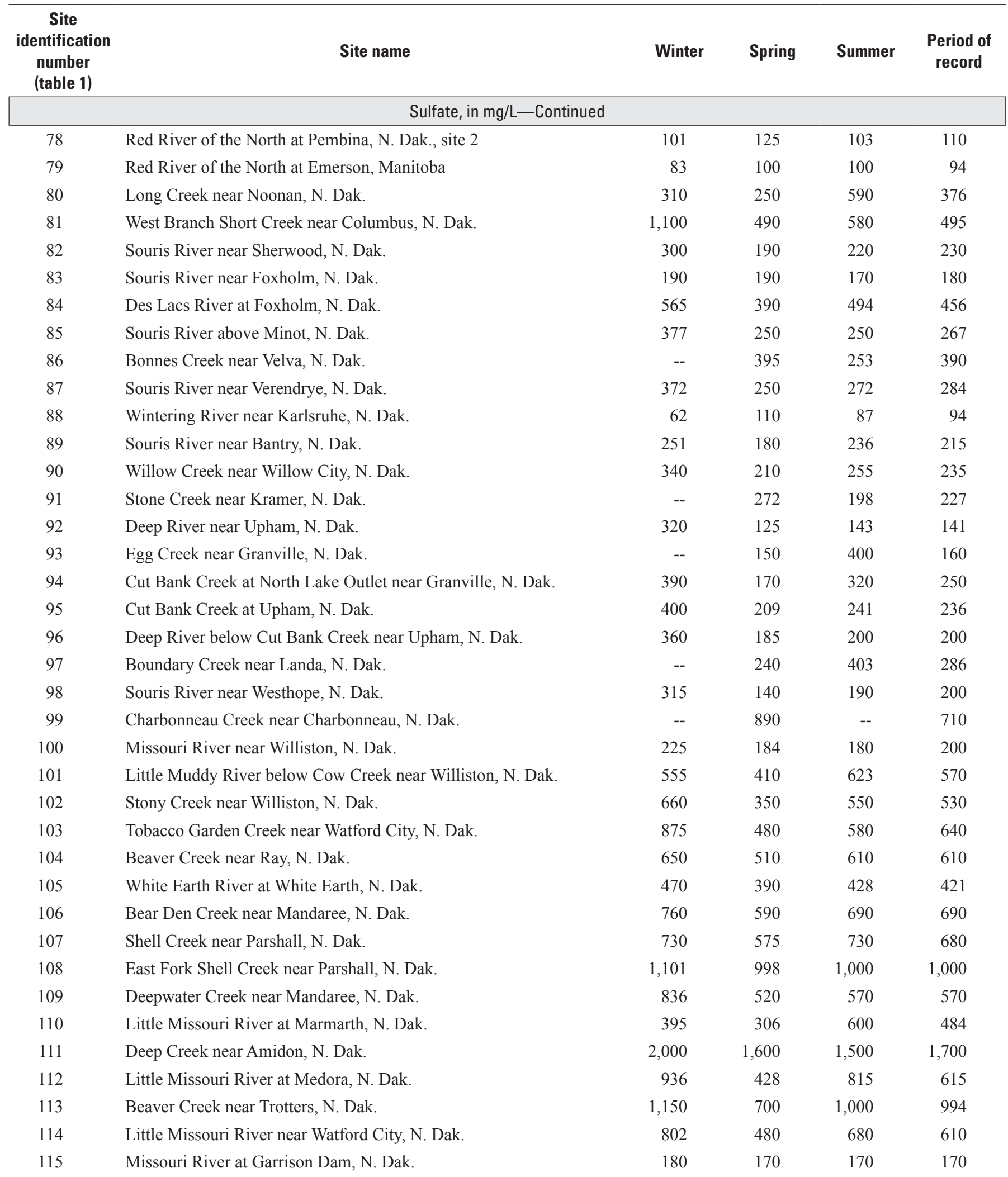


Table 1-5. Median seasonal concentrations for selected constituents at selected sites in North Dakota from 1970 through 2008.Continued

[Winter, January-February, November-December; Spring, March-June; Summer, July-October; mg/L, milligrams per liter; --, not available; N, nitrogen; <. less than; P, phosphorus; $\mathrm{C}$, carbon; $\mu \mathrm{g} / \mathrm{L}$, micrograms per liter]

\begin{tabular}{|c|c|c|c|c|c|}
\hline $\begin{array}{c}\text { Site } \\
\text { identification } \\
\text { number } \\
\text { (table 1) }\end{array}$ & Site name & Winter & Spring & Summer & $\begin{array}{c}\text { Period of } \\
\text { record }\end{array}$ \\
\hline \multicolumn{6}{|c|}{ Sulfate, in $\mathrm{mg} / \mathrm{L}$-Continued } \\
\hline 116 & Knife River at Manning, N. Dak. & 665 & 336 & 455 & 440 \\
\hline 117 & Stray Creek near Manning, N. Dak. & 700 & 800 & 1,115 & 760 \\
\hline 118 & Knife River at Marshall, N. Dak. & 825 & 575 & 540 & 570 \\
\hline 119 & Elm Creek near Golden Valley, N. Dak. & 290 & 375 & 220 & 340 \\
\hline 120 & Knife River near Golden Valley, N. Dak. & 851 & 459 & 575 & 564 \\
\hline 121 & Coyote Creek near Zap, N. Dak. & 605 & 490 & 550 & 510 \\
\hline 122 & Brush Creek near Beulah, N. Dak. & 590 & 465 & 470 & 490 \\
\hline 123 & Spring Creek below Lake Ilo near Dunn Center, N. Dak. & 600 & 460 & 770 & 580 \\
\hline 124 & Spring Creek near Halliday, N. Dak. & 660 & 400 & 635 & 605 \\
\hline 125 & Spring Creek at Zap, N. Dak. & 600 & 400 & 500 & 492 \\
\hline 126 & West Branch Otter Creek near Beulah, N. Dak. & 110 & 420 & 410 & 410 \\
\hline 127 & Knife River at Hazen, N. Dak. & 560 & 428 & 420 & 459 \\
\hline 128 & Antelope Creek above Hazen, N. Dak. & 560 & 450 & 380 & 465 \\
\hline 129 & West Branch Antelope Creek No. 4 near Zap, N. Dak. & 111 & 165 & 450 & 170 \\
\hline 130 & West Branch Antelope Creek near Hazen, N. Dak. & 54 & 210 & -- & 190 \\
\hline 131 & Coal Creek near Stanton, N. Dak. & 720 & 530 & 300 & 600 \\
\hline 132 & Alderin Creek near Fort Clark, N. Dak. & 665 & 360 & 505 & 440 \\
\hline 133 & Coal Lake Coulee near Hensler, N. Dak. & 170 & 320 & 460 & 315 \\
\hline 134 & Buffalo Creek near Washburn, N. Dak. & 510 & 390 & 490 & 485 \\
\hline 135 & Turtle Creek above Washburn, N. Dak. & 510 & 296 & 315 & 324 \\
\hline 136 & Painted Woods Creek near Wilton, N. Dak. & 640 & 425 & 635 & 550 \\
\hline 137 & Square Butte Creek near Hannover, N. Dak. & 340 & 290 & 335 & 330 \\
\hline 138 & Square Butte Creek above Nelson Lake near Center, N. Dak. & 250 & 220 & 250 & 240 \\
\hline 139 & Hagel Creek near Center, N. Dak. & 310 & 170 & 210 & 240 \\
\hline 140 & Square Butte Creek below Center, N. Dak. & 185 & 340 & 370 & 340 \\
\hline 141 & Burnt Creek near Bismarck, N. Dak. & 105 & 190 & 393 & 262 \\
\hline 142 & Missouri River at Bismarck, N. Dak. & 190 & 170 & 170 & 170 \\
\hline 143 & South Branch Heart River near South Heart, N. Dak. & 180 & 250 & 230 & 225 \\
\hline 144 & North Creek near South Heart, N. Dak. & 1,400 & 500 & 248 & 560 \\
\hline 145 & Heart River near South Heart, N. Dak. & 1,000 & 510 & 705 & 720 \\
\hline 146 & Heart River at Dickinson, N. Dak. & 758 & 425 & 610 & 565 \\
\hline 147 & Green River near New Hradec, N. Dak. & 250 & 200 & 250 & 230 \\
\hline 148 & Green River near Gladstone, N. Dak. & 540 & 433 & 510 & 490 \\
\hline 149 & Heart River near Richardton, N. Dak. & 764 & 446 & 587 & 550 \\
\hline 150 & Heart River above Lake Tschida near Glen Ullin, N. Dak. & -- & 316 & 520 & 415 \\
\hline 151 & Antelope Creek near Carson, N. Dak. & -- & 190 & 240 & 200 \\
\hline 152 & Big Muddy Creek near Almont, N. Dak. & -- & 324 & 542 & 473 \\
\hline
\end{tabular}


Table 1-5. Median seasonal concentrations for selected constituents at selected sites in North Dakota from 1970 through 2008 . Continued

[Winter, January-February, November-December; Spring, March-June; Summer, July-October; mg/L, milligrams per liter; --, not available; N, nitrogen; <. less than; P, phosphorus; $\mathrm{C}$, carbon; $\mu \mathrm{g} / \mathrm{L}$, micrograms per liter]

\begin{tabular}{|c|c|c|c|c|c|}
\hline $\begin{array}{c}\text { Site } \\
\text { identification } \\
\text { number } \\
\text { (table 1) }\end{array}$ & Site name & Winter & Spring & Summer & $\begin{array}{l}\text { Period of } \\
\text { record }\end{array}$ \\
\hline \multicolumn{6}{|c|}{ Sulfate, in $\mathrm{mg} / \mathrm{L}$-Continued } \\
\hline 153 & Heart River near Lark, N. Dak. & 360 & 250 & 410 & 340 \\
\hline 154 & Heart River at Stark Bridge near Judson, N. Dak. & -- & 290 & 448 & 388 \\
\hline 156 & Heart River near Mandan, N. Dak. & 520 & 354 & 448 & 440 \\
\hline 157 & Long Lake Creek above Long Lake near Moffit, N. Dak. & 160 & 130 & 180 & 141 \\
\hline 158 & Apple Creek near Menoken, N. Dak. & 315 & 186 & 240 & 230 \\
\hline 159 & Missouri River near Schmidt, N. Dak. & 185 & 180 & 185 & 180 \\
\hline 163 & Cannonball River below Bentley, N. Dak. & 880 & 620 & 885 & 820 \\
\hline 164 & Cannonball River near Raleigh, N. Dak. & 932 & 590 & 640 & 640 \\
\hline 165 & White Butte Fork Cedar Creek near Scranton, N. Dak. & 2,300 & 1,310 & 1,600 & 1,500 \\
\hline 166 & Cedar Creek near Haynes, N. Dak. & 1,250 & 548 & 800 & 707 \\
\hline 167 & Timber Creek near Bentley, N. Dak. & 1,600 & 1,100 & 875 & 1,100 \\
\hline 168 & Cedar Creek near Pretty Rock, N. Dak. & 1,270 & 920 & 1,200 & 1,115 \\
\hline 169 & Cedar Creek near Raleigh, N. Dak. & 742 & 570 & 532 & 570 \\
\hline 170 & Cannonball River at Breien, N. Dak. & 885 & 467 & 544 & 568 \\
\hline 171 & Beaver Creek near Linton, N. Dak. & 280 & 120 & 140 & 140 \\
\hline 178 & Kelly Creek below Niccum Reservoir near Bordulac, N. Dak. & -- & 100 & 103 & 100 \\
\hline 179 & James River near Pingree, N. Dak. & 170 & 135 & 169 & 153 \\
\hline 180 & Pipestem Creek near Pingree, N. Dak. & 338 & 180 & 244 & 220 \\
\hline 181 & Pipestem Creek near Buchanan, N. Dak. & -- & 98 & 112 & 110 \\
\hline 182 & James River at Jamestown, N. Dak. & 220 & 160 & 185 & 190 \\
\hline 183 & James River at Lamoure, N. Dak. & 242 & 147 & 160 & 170 \\
\hline 184 & Bear Creek near Oakes, N. Dak. & -- & 260 & 349 & 339 \\
\hline 185 & James River at Oakes, N. Dak. & 270 & 150 & 160 & 170 \\
\hline 186 & James River at N. Dak./S. Dak. State line & 225 & 153 & 181 & 176 \\
\hline \multicolumn{6}{|c|}{ Chloride, in $\mathrm{mg} / \mathrm{L}$} \\
\hline 1 & Bois De Sioux River near Doran, Minn. & 24.7 & 13.9 & 15.6 & 16.5 \\
\hline 2 & Red River of the North at Wahpeton, N. Dak. & 29.2 & 12.8 & 11.0 & 11.9 \\
\hline 3 & Red River of the North near Wahpeton, N. Dak. & 16.3 & 14.2 & 9.3 & 14.1 \\
\hline
\end{tabular}


Table 1-5. Median seasonal concentrations for selected constituents at selected sites in North Dakota from 1970 through 2008.Continued

[Winter, January-February, November-December; Spring, March-June; Summer, July-October; mg/L, milligrams per liter; --, not available; N, nitrogen; <. less than; P, phosphorus; $\mathrm{C}$, carbon; $\mu \mathrm{g} / \mathrm{L}$, micrograms per liter]

\begin{tabular}{|c|c|c|c|c|c|}
\hline $\begin{array}{c}\text { Site } \\
\text { identification } \\
\text { number } \\
\text { (table 1) }\end{array}$ & Site name & Winter & Spring & Summer & $\begin{array}{l}\text { Period of } \\
\text { record }\end{array}$ \\
\hline \multicolumn{6}{|c|}{ Chloride, in $\mathrm{mg} / \mathrm{L}$-Continued } \\
\hline 4 & Red River of the North at Brushville, Minn. & 18.0 & 13.3 & 18.3 & 15.3 \\
\hline 5 & Red River of the North below Wahpeton, N. Dak. & 7.8 & 7.2 & 6.9 & 7.1 \\
\hline 6 & Red River of the North at Hickson, N. Dak. & 9.6 & 11.0 & 11.5 & 11.0 \\
\hline 7 & Wild Rice River near Rutland, N. Dak. & -- & 27.5 & 46.0 & 32.0 \\
\hline 8 & Wild Rice River near Cayuga, N. Dak. & 408.0 & 35.0 & 20.0 & 30.0 \\
\hline 9 & Antelope Creek at Dwight, N. Dak. & -- & 24.1 & 27.1 & 24.3 \\
\hline 10 & Wild Rice River near Abercrombie, N. Dak. & 61.9 & 29.8 & 36.0 & 38.9 \\
\hline 11 & Red River of the North at Fargo, N. Dak. & 9.6 & 12.7 & 14.0 & 13.0 \\
\hline 12 & Red River of North below Fargo, N. Dak. & 12.0 & 11.0 & 13.4 & 11.0 \\
\hline 13 & Red River of the North at Harwood, N. Dak. & 25.5 & 18.4 & 23.2 & 21.3 \\
\hline 14 & Red River of the North near Harwood, N. Dak. & 20.6 & 17.2 & 19.3 & 18.7 \\
\hline 15 & Sheyenne River above Harvey, N. Dak. & 19.0 & 14.0 & 17.0 & 17.0 \\
\hline 16 & Big Coulee near Fort Totten, N. Dak. & -- & $<4.0$ & 9.5 & 8.5 \\
\hline 17 & Sheyenne River at Warwick, N. Dak. & 21.6 & 16.8 & 15.6 & 17.0 \\
\hline 18 & Sheyenne River near Warwick, N. Dak. & 14.0 & 14.0 & 13.1 & 14.0 \\
\hline 19 & Mauvais Coulee Tributary No. 3 near Cando, N. Dak. & -- & 9.8 & 35.2 & 17.2 \\
\hline 20 & Mauvais Coulee near Cando, N. Dak. & 31.0 & 16.0 & 25.0 & 19.0 \\
\hline 21 & Edmore Coulee near Edmore, N. Dak. & 30.0 & 18.5 & 26.0 & 20.4 \\
\hline 22 & Edmore Coulee Tributary near Webster, N. Dak. & -- & 15.4 & 26.5 & 20.1 \\
\hline 23 & Webster Coulee at Webster, N. Dak. & -- & 20.0 & -- & 20.0 \\
\hline 24 & Starkweather Coulee near Webster, N. Dak. & -- & 13.9 & 19.0 & 14.0 \\
\hline 25 & Big Coulee below Churchs Ferry, N. Dak. & -- & 25.5 & 24.5 & 24.5 \\
\hline 26 & Little Coulee near Leeds, N. Dak. & -- & 24.5 & 23.5 & 24.5 \\
\hline 27 & Little Coulee near Brinsmade, N. Dak. & -- & 23.0 & 17.5 & 23.0 \\
\hline 28 & Big Coulee near Churchs Ferry, N. Dak. & 45.0 & 15.0 & 20.0 & 18.5 \\
\hline 29 & Big Coulee at Graham Island inlet near Fort Totten, N. Dak. & 280.0 & 130.0 & 230.0 & 195.0 \\
\hline 30 & Channel A near Penn, N. Dak. & 21.5 & 19.0 & 17.0 & 19.0 \\
\hline 31 & Devils Lake Outlet to Stump Lake near Lakota, N. Dak. & -- & 227.5 & 376.0 & 278.5 \\
\hline 32 & Sheyenne River near Cooperstown, N. Dak. & 20.0 & 14.3 & 16.0 & 16.0 \\
\hline 33 & Baldhill Creek near Dazey, N. Dak. & 23.5 & 10.9 & 17.6 & 15.0 \\
\hline 34 & Sheyenne River below Baldhill Dam, N. Dak. & 18.0 & 14.1 & 15.0 & 15.3 \\
\hline 35 & Sheyenne River at Valley City, N. Dak. & -- & 19.0 & 13.0 & 18.0 \\
\hline 36 & Sheyenne River at Lisbon, N. Dak. & 32.0 & 20.5 & 29.6 & 27.0 \\
\hline 37 & Sheyenne River near Kindred, N. Dak. & 28.4 & 22.4 & 29.6 & 26.0 \\
\hline 38 & Sheyenne River above Sheyenne River Diversion near Horace, N. Dak. & 25.2 & 22.1 & 27.0 & 24.0 \\
\hline 39 & Sheyenne River near Horace, N. Dak. & -- & 18.0 & 22.0 & 19.5 \\
\hline 40 & Sheyenne River Diversion at West Fargo, N. Dak. & -- & 15.7 & 12.2 & 15.7 \\
\hline 41 & Sheyenne River at West Fargo, N. Dak. & 31.3 & 22.6 & 28.6 & 26.6 \\
\hline
\end{tabular}


Table 1-5. Median seasonal concentrations for selected constituents at selected sites in North Dakota from 1970 through 2008 . Continued

[Winter, January-February, November-December; Spring, March-June; Summer, July-October; mg/L, milligrams per liter; --, not available; N, nitrogen; <. less than; P, phosphorus; $\mathrm{C}$, carbon; $\mu \mathrm{g} / \mathrm{L}$, micrograms per liter]

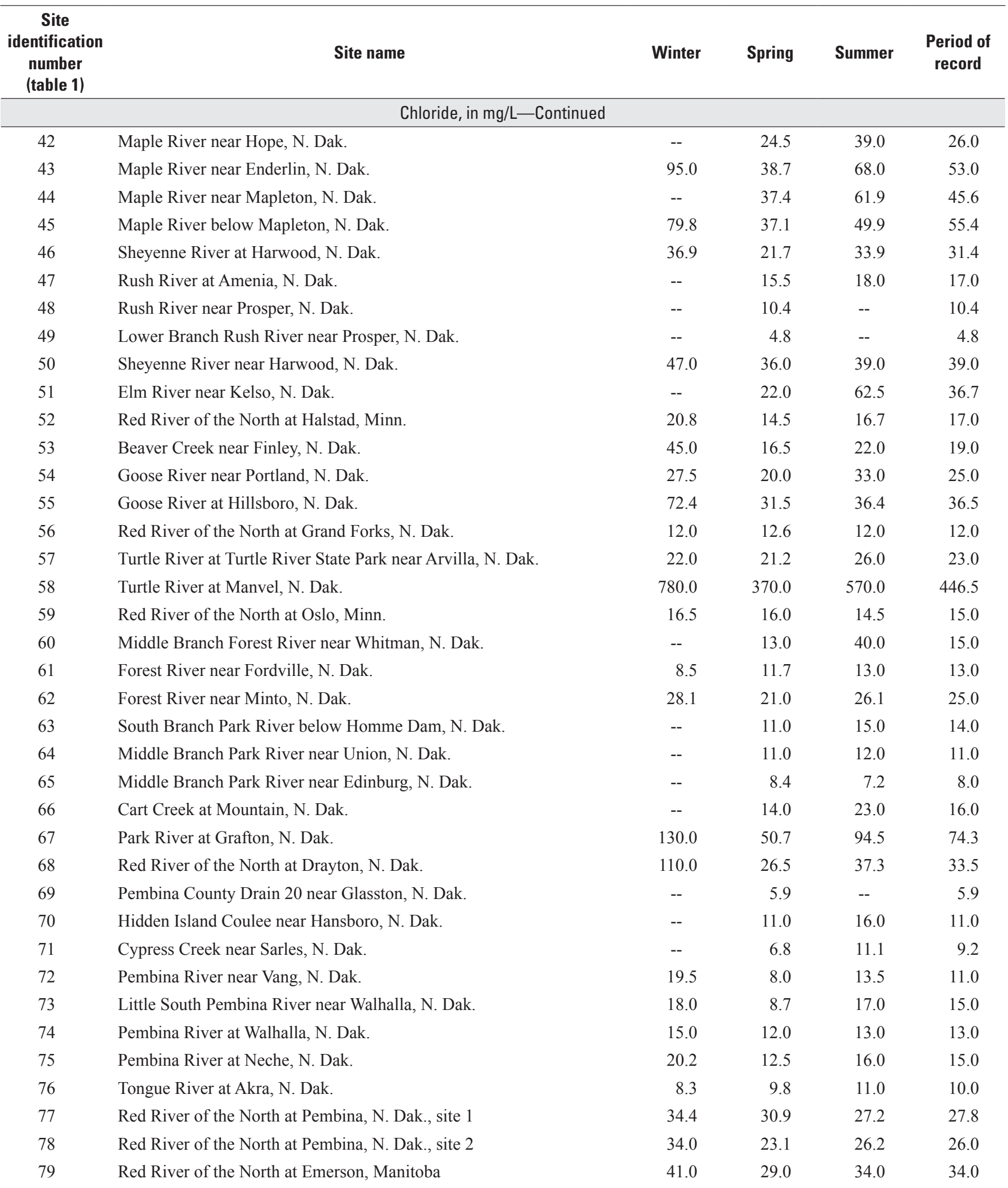


Table 1-5. Median seasonal concentrations for selected constituents at selected sites in North Dakota from 1970 through 2008.Continued

[Winter, January-February, November-December; Spring, March-June; Summer, July-October; mg/L, milligrams per liter; --, not available; N, nitrogen; <. less than; P, phosphorus; $\mathrm{C}$, carbon; $\mu \mathrm{g} / \mathrm{L}$, micrograms per liter]

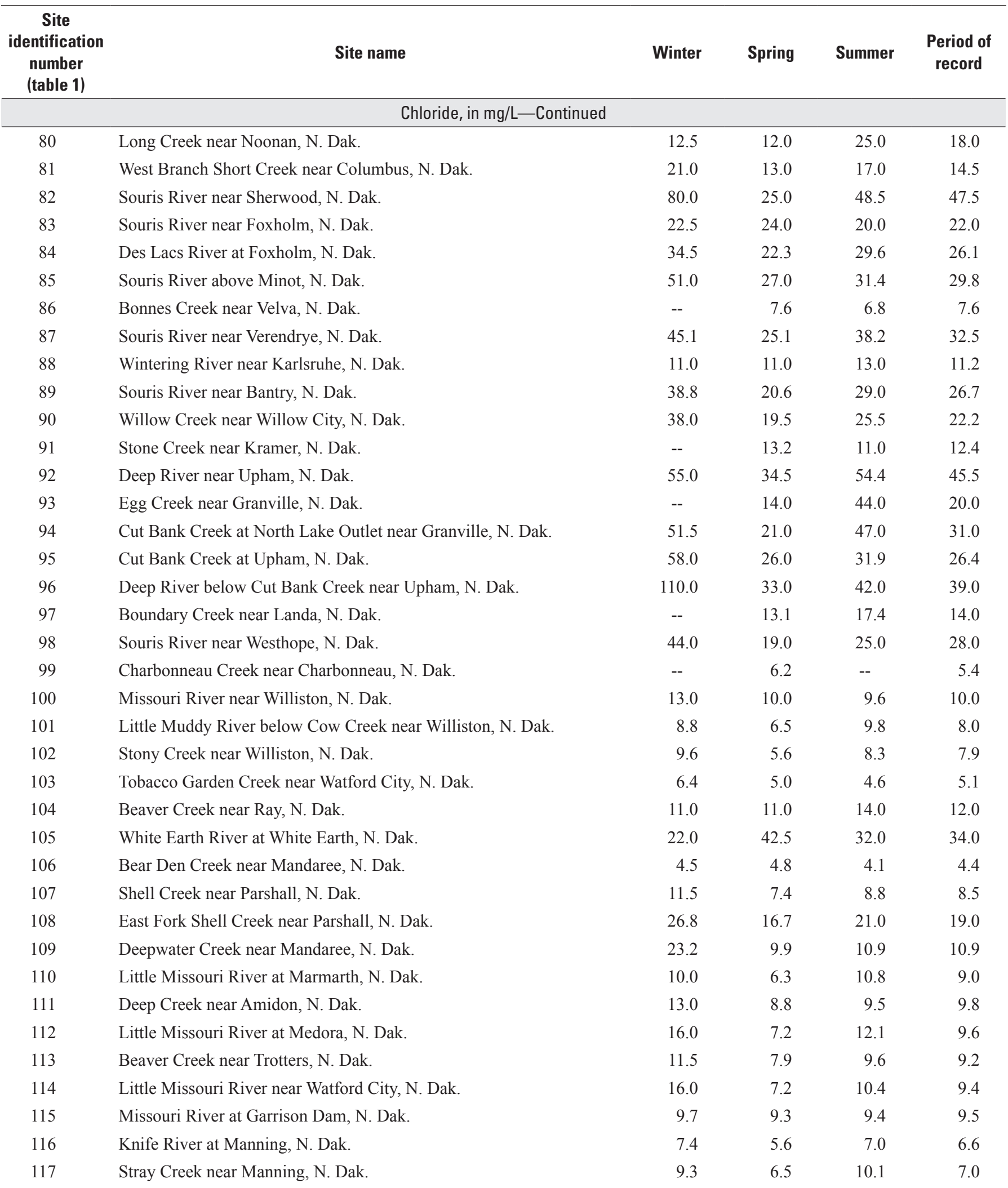


Table 1-5. Median seasonal concentrations for selected constituents at selected sites in North Dakota from 1970 through 2008 . Continued

[Winter, January-February, November-December; Spring, March-June; Summer, July-October; mg/L, milligrams per liter; --, not available; N, nitrogen; <. less than; $\mathrm{P}$, phosphorus; $\mathrm{C}$, carbon; $\mu \mathrm{g} / \mathrm{L}$, micrograms per liter]

\begin{tabular}{|c|c|c|c|c|c|}
\hline $\begin{array}{c}\text { Site } \\
\text { identification } \\
\text { number } \\
\text { (table 1) } \\
\end{array}$ & Site name & Winter & Spring & Summer & $\begin{array}{c}\text { Period of } \\
\text { record }\end{array}$ \\
\hline 118 & Knife River at Marshall, N. Dak. & 7.3 & 5.1 & 4.8 & 5.3 \\
\hline 119 & Elm Creek near Golden Valley, N. Dak. & 11.0 & 4.4 & 4.5 & 4.5 \\
\hline 121 & Coyote Creek near Zap, N. Dak. & 9.5 & 6.2 & 6.5 & 6.9 \\
\hline 122 & Brush Creek near Beulah, N. Dak. & 7.4 & 5.5 & 7.0 & 6.7 \\
\hline 123 & Spring Creek below Lake Ilo near Dunn Center, N. Dak. & 6.5 & 5.9 & 8.1 & 6.8 \\
\hline 124 & Spring Creek near Halliday, N. Dak. & 6.8 & 5.0 & 6.9 & 6.5 \\
\hline 128 & Antelope Creek above Hazen, N. Dak. & 15.0 & 8.8 & 9.6 & 11.0 \\
\hline 129 & West Branch Antelope Creek No. 4 near Zap, N. Dak. & 4.2 & $<4.0$ & -- & $<4.0$ \\
\hline 130 & West Branch Antelope Creek near Hazen, N. Dak. & $<4.0$ & $<4.0$ & -- & $<4.0$ \\
\hline 131 & Coal Creek near Stanton, N. Dak. & 12.0 & 6.1 & 8.3 & 8.1 \\
\hline 132 & Alderin Creek near Fort Clark, N. Dak. & 6.5 & 4.4 & 6.0 & 5.1 \\
\hline 133 & Coal Lake Coulee near Hensler, N. Dak. & 4.1 & $<4.0$ & 5.8 & $<4.0$ \\
\hline 134 & Buffalo Creek near Washburn, N. Dak. & 6.6 & 4.7 & 7.0 & 6.5 \\
\hline 135 & Turtle Creek above Washburn, N. Dak. & 18.0 & 10.2 & 13.9 & 12.2 \\
\hline 136 & Painted Woods Creek near Wilton, N. Dak. & 19.0 & 12.0 & 18.0 & 17.0 \\
\hline 143 & South Branch Heart River near South Heart, N. Dak. & $<4.0$ & 4.8 & 5.0 & 4.8 \\
\hline 144 & North Creek near South Heart, N. Dak. & 9.5 & 5.1 & $<4.0$ & 5.2 \\
\hline 145 & Heart River near South Heart, N. Dak. & 34.0 & 12.0 & 17.5 & 18.0 \\
\hline 146 & Heart River at Dickinson, N. Dak. & 43.2 & 33.0 & 50.0 & 48.0 \\
\hline 147 & Green River near New Hradec, N. Dak. & 6.8 & 4.9 & 6.2 & 5.9 \\
\hline 148 & Green River near Gladstone, N. Dak. & 6.7 & 5.0 & 7.9 & 6.3 \\
\hline 149 & Heart River near Richardton, N. Dak. & 19.7 & 12.0 & 20.1 & 17.0 \\
\hline 150 & Heart River above Lake Tschida near Glen Ullin, N. Dak. & -- & 12.0 & 17.0 & 13.5 \\
\hline 151 & Antelope Creek near Carson, N. Dak. & -- & 5.1 & 9.3 & 5.9 \\
\hline 152 & Big Muddy Creek near Almont, N. Dak. & -- & 5.8 & 7.5 & 7.0 \\
\hline 153 & Heart River near Lark, N. Dak. & 5.0 & 5.4 & 8.9 & 5.9 \\
\hline 154 & Heart River at Stark Bridge near Judson, N. Dak. & -- & 8.1 & 12.0 & 10.0 \\
\hline 155 & Sweetbriar Creek near Judson, N. Dak. & 4.1 & 4.9 & 6.3 & 5.1 \\
\hline
\end{tabular}


Table 1-5. Median seasonal concentrations for selected constituents at selected sites in North Dakota from 1970 through 2008.Continued

[Winter, January-February, November-December; Spring, March-June; Summer, July-October; mg/L, milligrams per liter; --, not available; N, nitrogen; <. less than; P, phosphorus; $\mathrm{C}$, carbon; $\mu \mathrm{g} / \mathrm{L}$, micrograms per liter]

\begin{tabular}{|c|c|c|c|c|c|}
\hline $\begin{array}{c}\text { Site } \\
\text { identification } \\
\text { number } \\
\text { (table 1) } \\
\end{array}$ & Site name & Winter & Spring & Summer & $\begin{array}{l}\text { Period of } \\
\text { record }\end{array}$ \\
\hline \multicolumn{6}{|c|}{ Chloride, in $\mathrm{mg} / \mathrm{L}$-Continued } \\
\hline 156 & Heart River near Mandan, N. Dak. & 16.3 & 8.7 & 13.1 & 12.0 \\
\hline 157 & Long Lake Creek above Long Lake near Moffit, N. Dak. & 4.5 & 4.2 & 9.1 & 6.2 \\
\hline 159 & Missouri River near Schmidt, N. Dak. & 9.9 & 9.1 & 9.7 & 9.4 \\
\hline 160 & Cannonball River at New England, N. Dak. & 8.9 & 9.4 & 6.8 & 8.1 \\
\hline 161 & Coal Bank Creek near Havelock, N. Dak. & 11.0 & 8.3 & 11.0 & 10.0 \\
\hline 162 & Cannonball River at Regent, N. Dak. & 11.0 & 8.2 & 10.0 & 9.7 \\
\hline 166 & Cedar Creek near Haynes, N. Dak. & 17.0 & 9.6 & 12.0 & 11.0 \\
\hline 167 & Timber Creek near Bentley, N. Dak. & 16.0 & 12.5 & 10.5 & 14.0 \\
\hline 168 & Cedar Creek near Pretty Rock, N. Dak. & 12.0 & 9.3 & 13.0 & 11.5 \\
\hline 169 & Cedar Creek near Raleigh, N. Dak. & 13.2 & 9.0 & 9.2 & 9.3 \\
\hline 170 & Cannonball River at Breien, N. Dak. & 19.0 & 7.6 & 12.0 & 11.0 \\
\hline 171 & Beaver Creek near Linton, N. Dak. & 11.0 & 6.0 & 9.0 & 7.8 \\
\hline 172 & Beaver Creek below Linton, N. Dak. & 6.2 & 8.7 & 13.0 & 11.0 \\
\hline 173 & Porcupine Creek near Fort Yates, N. Dak. & -- & 12.5 & 16.0 & 13.7 \\
\hline 174 & Buffalo Creek Tributary near Gascoyne, N. Dak. & 18.0 & 13.0 & 13.5 & 14.0 \\
\hline 181 & Pipestem Creek near Buchanan, N. Dak. & -- & 6.3 & 14.5 & 7.3 \\
\hline 182 & James River at Jamestown, N. Dak. & 44.0 & 14.8 & 17.5 & 21.6 \\
\hline 183 & James River at Lamoure, N. Dak. & 50.2 & 19.9 & 22.9 & 28.3 \\
\hline 184 & Bear Creek near Oakes, N. Dak. & -- & 41.0 & 56.9 & 50.2 \\
\hline 185 & James River at Oakes, N. Dak. & 73.0 & 22.0 & 33.0 & 38.7 \\
\hline 186 & James River at N. Dak./S. Dak. State line & 44.5 & 26.7 & 28.9 & 30.0 \\
\hline \multicolumn{6}{|c|}{ Total dissolved solids, in mg/L } \\
\hline 1 & Bois De Sioux River near Doran, Minn. & 1,145 & 795 & 874 & 894 \\
\hline 2 & Red River of the North at Wahpeton, N. Dak. & 875 & 344 & 284 & 324 \\
\hline 3 & Red River of the North near Wahpeton, N. Dak. & 321 & 442 & 1,090 & 442 \\
\hline 4 & Red River of the North at Brushville, Minn. & 339 & 392 & 350 & 352 \\
\hline 5 & Red River of the North below Wahpeton, N. Dak. & 318 & 301 & 278 & 299 \\
\hline 6 & Red River of the North at Hickson, N. Dak. & 320 & 324 & 334 & 331 \\
\hline
\end{tabular}


Table 1-5. Median seasonal concentrations for selected constituents at selected sites in North Dakota from 1970 through 2008.Continued

[Winter, January-February, November-December; Spring, March-June; Summer, July-October; mg/L, milligrams per liter; --, not available; N, nitrogen; <. less than; P, phosphorus; C, carbon; $\mu \mathrm{g} / \mathrm{L}$, micrograms per liter]

\begin{tabular}{|c|c|c|c|c|c|}
\hline $\begin{array}{c}\text { Site } \\
\text { identification } \\
\text { number } \\
\text { (table 1) }\end{array}$ & Site name & Winter & Spring & Summer & $\begin{array}{l}\text { Period of } \\
\text { record }\end{array}$ \\
\hline \multicolumn{6}{|c|}{ Total dissolved solids, in mg/L-Continued } \\
\hline 7 & Wild Rice River near Rutland, N. Dak. & -- & 859 & 1,160 & 969 \\
\hline 8 & Wild Rice River near Cayuga, N. Dak. & 3,340 & 795 & 764 & 795 \\
\hline 10 & Wild Rice River near Abercrombie, N. Dak. & 1,370 & 827 & 876 & 925 \\
\hline 11 & Red River of the North at Fargo, N. Dak. & 331 & 332 & 317 & 324 \\
\hline 12 & Red River of North below Fargo, N. Dak. & 345 & 366 & 354 & 355 \\
\hline 13 & Red River of the North at Harwood, N. Dak. & 416 & 504 & 435 & 446 \\
\hline 17 & Sheyenne River at Warwick, N. Dak. & 853 & 725 & 720 & 727 \\
\hline 18 & Sheyenne River near Warwick, N. Dak. & 490 & 492 & 561 & 506 \\
\hline 19 & Mauvais Coulee Tributary No. 3 near Cando, N. Dak. & -- & 316 & 672 & 424 \\
\hline 20 & Mauvais Coulee near Cando, N. Dak. & 771 & 446 & 754 & 545 \\
\hline 21 & Edmore Coulee near Edmore, N. Dak. & 754 & 401 & 608 & 486 \\
\hline 22 & Edmore Coulee Tributary near Webster, N. Dak. & -- & 275 & 676 & 413 \\
\hline 23 & Webster Coulee at Webster, N. Dak. & -- & 492 & -- & 492 \\
\hline 24 & Starkweather Coulee near Webster, N. Dak. & -- & 323 & 510 & 373 \\
\hline 25 & Big Coulee below Churchs Ferry, N. Dak. & -- & 460 & 555 & 545 \\
\hline 32 & Sheyenne River near Cooperstown, N. Dak. & 698 & 576 & 628 & 622 \\
\hline 33 & Baldhill Creek near Dazey, N. Dak. & 809 & 423 & 613 & 555 \\
\hline 34 & Sheyenne River below Baldhill Dam, N. Dak. & 694 & 543 & 574 & 579 \\
\hline 35 & Sheyenne River at Valley City, N. Dak. & -- & 509 & 403 & 473 \\
\hline 36 & Sheyenne River at Lisbon, N. Dak. & 654 & 485 & 630 & 605 \\
\hline 37 & Sheyenne River near Kindred, N. Dak. & 668 & 523 & 562 & 562 \\
\hline 38 & Sheyenne River above Sheyenne River Diversion near Horace, N. Dak. & -- & 505 & 679 & 633 \\
\hline 39 & Sheyenne River near Horace, N. Dak. & 687 & 508 & 539 & 539 \\
\hline 40 & Sheyenne River Diversion at West Fargo, N. Dak. & -- & 472 & 743 & 499 \\
\hline 41 & Sheyenne River at West Fargo, N. Dak. & 628 & 430 & 576 & 565 \\
\hline 42 & Maple River near Hope, N. Dak. & -- & 616 & 1,330 & 654 \\
\hline 43 & Maple River near Enderlin, N. Dak. & 1,260 & 752 & 1,140 & 1,070 \\
\hline 44 & Maple River near Mapleton, N. Dak. & -- & 691 & 1,050 & 859 \\
\hline
\end{tabular}


Table 1-5. Median seasonal concentrations for selected constituents at selected sites in North Dakota from 1970 through 2008.Continued

[Winter, January-February, November-December; Spring, March-June; Summer, July-October; mg/L, milligrams per liter; --, not available; N, nitrogen; <. less than; P, phosphorus; $\mathrm{C}$, carbon; $\mu \mathrm{g} / \mathrm{L}$, micrograms per liter]

\begin{tabular}{|c|c|c|c|c|c|}
\hline $\begin{array}{c}\text { Site } \\
\text { identification } \\
\text { number } \\
\text { (table 1) } \\
\end{array}$ & Total dissolved solids, in $\mathrm{mg}$ & Winter & Spring & Summer & $\begin{array}{c}\text { Period of } \\
\text { record }\end{array}$ \\
\hline 45 & Maple River below Mapleton, N. Dak. & 1,280 & 817 & 940 & 940 \\
\hline 46 & Sheyenne River at Harwood, N. Dak. & 598 & 581 & 548 & 569 \\
\hline 47 & Rush River at Amenia, N. Dak. & -- & 589 & 868 & 766 \\
\hline 48 & Rush River near Prosper, N. Dak. & -- & 294 & -- & 294 \\
\hline 49 & Lower Branch Rush River near Prosper, N. Dak. & -- & 172 & -- & 172 \\
\hline 50 & Sheyenne River near Harwood, N. Dak. & 681 & 632 & 560 & 632 \\
\hline 51 & Elm River near Kelso, N. Dak. & -- & 466 & 761 & 684 \\
\hline 52 & Red River of the North at Halstad, Minn. & 514 & 404 & 420 & 429 \\
\hline 53 & Beaver Creek near Finley, N. Dak. & 1,200 & 691 & 919 & 787 \\
\hline 54 & Goose River near Portland, N. Dak. & 1,430 & 726 & 941 & 939 \\
\hline 55 & Goose River at Hillsboro, N. Dak. & 1,345 & 855 & 988 & 983 \\
\hline 56 & Red River of the North at Grand Forks, N. Dak. & 349 & 354 & 331 & 344 \\
\hline 57 & Turtle River at Turtle River State Park near Arvilla, N. Dak. & 618 & 558 & 667 & 614 \\
\hline 58 & Turtle River at Manvel, N. Dak. & 2,405 & 1,280 & 1,810 & 1,460 \\
\hline 59 & Red River of the North at Oslo, Minn. & 321 & 340 & 321 & 334 \\
\hline 60 & Middle Branch Forest River near Whitman, N. Dak. & -- & 409 & 1,130 & 430 \\
\hline 61 & Forest River near Fordville, N. Dak. & 493 & 443 & 518 & 465 \\
\hline 62 & Forest River near Minto, N. Dak. & 649 & 516 & 582 & 570 \\
\hline 63 & South Branch Park River below Homme Dam, N. Dak. & -- & 404 & 542 & 486 \\
\hline 64 & Middle Branch Park River near Union, N. Dak. & -- & 293 & 419 & 381 \\
\hline 65 & Middle Branch Park River near Edinburg, N. Dak. & -- & 267 & 379 & 296 \\
\hline 66 & Cart Creek at Mountain, N. Dak. & -- & 402 & 706 & 459 \\
\hline 67 & Park River at Grafton, N. Dak. & 975 & 552 & 808 & 781 \\
\hline 68 & Red River of the North at Drayton, N. Dak. & 718 & 395 & 436 & 411 \\
\hline 69 & Pembina County Drain 20 near Glasston, N. Dak. & -- & 326 & -- & 326 \\
\hline 70 & Hidden Island Coulee near Hansboro, N. Dak. & -- & 472 & 809 & 476 \\
\hline 71 & Cypress Creek near Sarles, N. Dak. & -- & 327 & 573 & 345 \\
\hline 72 & Pembina River near Vang, N. Dak. & 774 & 336 & 570 & 485 \\
\hline 73 & Little South Pembina River near Walhalla, N. Dak. & 616 & 354 & 609 & 511 \\
\hline 74 & Pembina River at Walhalla, N. Dak. & 637 & 491 & 559 & 554 \\
\hline 75 & Pembina River at Neche, N. Dak. & 696 & 421 & 584 & 551 \\
\hline 76 & Tongue River at Akra, N. Dak. & 425 & 329 & 387 & 361 \\
\hline 77 & Red River of the North at Pembina, N. Dak., site 1 & 403 & 452 & 443 & 436 \\
\hline 78 & Red River of the North at Pembina, N. Dak., site 2 & 441 & 401 & 413 & 414 \\
\hline 79 & Red River of the North at Emerson, Manitoba & 460 & 419 & 428 & 438 \\
\hline 80 & Long Creek near Noonan, N. Dak. & 897 & 598 & 1,230 & 875 \\
\hline 81 & West Branch Short Creek near Columbus, N. Dak. & 2,270 & 1,060 & 1,390 & 1,095 \\
\hline 82 & Souris River near Sherwood, N. Dak. & 1,035 & 598 & 721 & 746 \\
\hline
\end{tabular}


Table 1-5. Median seasonal concentrations for selected constituents at selected sites in North Dakota from 1970 through 2008.Continued

[Winter, January-February, November-December; Spring, March-June; Summer, July-October; mg/L, milligrams per liter; --, not available; N, nitrogen; <. less than; P, phosphorus; C, carbon; $\mu \mathrm{g} / \mathrm{L}$, micrograms per liter]

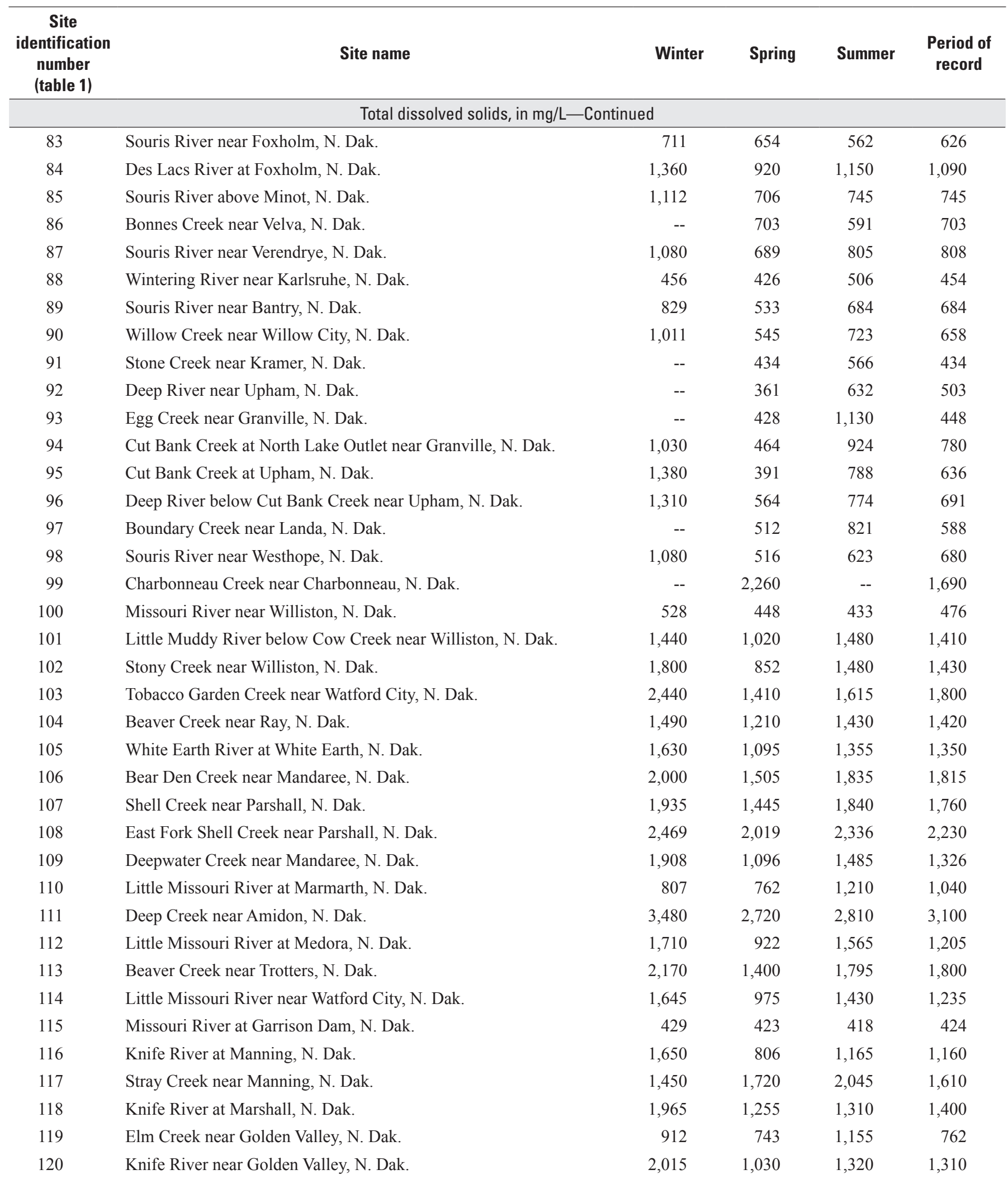


Table 1-5. Median seasonal concentrations for selected constituents at selected sites in North Dakota from 1970 through 2008.Continued

[Winter, January-February, November-December; Spring, March-June; Summer, July-October; mg/L, milligrams per liter; --, not available; N, nitrogen; <. less than; P, phosphorus; $\mathrm{C}$, carbon; $\mu \mathrm{g} / \mathrm{L}$, micrograms per liter]

\begin{tabular}{|c|c|c|c|c|c|}
\hline $\begin{array}{c}\text { Site } \\
\text { identification } \\
\text { number } \\
\text { (table 1) } \\
\end{array}$ & Site name & Winter & Spring & Summer & $\begin{array}{c}\text { Period of } \\
\text { record }\end{array}$ \\
\hline \multicolumn{6}{|c|}{ Total dissolved solids, in $\mathrm{mg} / \mathrm{L}$ —Continued } \\
\hline 121 & Coyote Creek near Zap, N. Dak. & 1,495 & 1,050 & 1,165 & 1,220 \\
\hline 122 & Brush Creek near Beulah, N. Dak. & 1,375 & 1,165 & 1,240 & 1,270 \\
\hline 123 & Spring Creek below Lake Ilo near Dunn Center, N. Dak. & 1,330 & 1,090 & 1,760 & 1,300 \\
\hline 124 & Spring Creek near Halliday, N. Dak. & 1,455 & 916 & 1,330 & 1,325 \\
\hline 125 & Spring Creek at Zap, N. Dak. & 1,380 & 880 & 1,130 & 1,120 \\
\hline 126 & West Branch Otter Creek near Beulah, N. Dak. & 236 & 833 & 867 & 833 \\
\hline 127 & Knife River at Hazen, N. Dak. & 1,410 & 998 & 1,040 & 1,110 \\
\hline 128 & Antelope Creek above Hazen, N. Dak. & 1,510 & 1,050 & 920 & 1,125 \\
\hline 129 & West Branch Antelope Creek No. 4 near Zap, N. Dak. & 457 & 596 & 999 & 598 \\
\hline 130 & West Branch Antelope Creek near Hazen, N. Dak. & 187 & 755 & -- & 614 \\
\hline 131 & Coal Creek near Stanton, N. Dak. & 1,855 & 1,285 & 915 & 1,445 \\
\hline 132 & Alderin Creek near Fort Clark, N. Dak. & 1,615 & 902 & 1,240 & 1,075 \\
\hline 133 & Coal Lake Coulee near Hensler, N. Dak. & 479 & 925 & 1,110 & 925 \\
\hline 134 & Buffalo Creek near Washburn, N. Dak. & 1,820 & 1,056 & 1,670 & 1,655 \\
\hline 135 & Turtle Creek above Washburn, N. Dak. & 1,408 & 1,190 & 1,329 & 1,310 \\
\hline 136 & Painted Woods Creek near Wilton, N. Dak. & 1,530 & 953 & 1,250 & 1,140 \\
\hline 137 & Square Butte Creek near Hannover, N. Dak. & 799 & 756 & 733 & 772 \\
\hline 138 & Square Butte Creek above Nelson Lake near Center, N. Dak. & 824 & 702 & 806 & 775 \\
\hline 139 & Hagel Creek near Center, N. Dak. & 1,050 & 476 & 746 & 762 \\
\hline 140 & Square Butte Creek below Center, N. Dak. & 729 & 793 & 903 & 839 \\
\hline 141 & Burnt Creek near Bismarck, N. Dak. & 335 & 424 & 972 & 709 \\
\hline 142 & Missouri River at Bismarck, N. Dak. & 433 & 436 & 436 & 436 \\
\hline 143 & South Branch Heart River near South Heart, N. Dak. & 460 & 589 & 512 & 462 \\
\hline 144 & North Creek near South Heart, N. Dak. & 2,730 & 959 & 566 & 1,175 \\
\hline 145 & Heart River near South Heart, N. Dak. & 2,350 & 1,220 & 1,540 & 1,655 \\
\hline 146 & Heart River at Dickinson, N. Dak. & 1,675 & 915 & 1,400 & 1,245 \\
\hline 147 & Green River near New Hradec, N. Dak. & 720 & 559 & 712 & 685 \\
\hline 148 & Green River near Gladstone, N. Dak. & 1,205 & 935 & 1,080 & 1,080 \\
\hline 149 & Heart River near Richardton, N. Dak. & 1,575 & 941 & 1,230 & 1,130 \\
\hline 150 & Heart River above Lake Tschida near Glen Ullin, N. Dak. & -- & 751 & 1,150 & 1,010 \\
\hline 151 & Antelope Creek near Carson, N. Dak. & -- & 559 & 812 & 615 \\
\hline 152 & Big Muddy Creek near Almont, N. Dak. & -- & 748 & 1,515 & 1,130 \\
\hline 153 & Heart River near Lark, N. Dak. & 897 & 626 & 877 & 750 \\
\hline 154 & Heart River at Stark Bridge near Judson, N. Dak. & -- & 728 & 991 & 886 \\
\hline 155 & Sweetbriar Creek near Judson, N. Dak. & 1,080 & 573 & 1,040 & 809 \\
\hline 156 & Heart River near Mandan, N. Dak. & 1,255 & 816 & 959 & 961 \\
\hline 157 & Long Lake Creek above Long Lake near Moffit, N. Dak. & 458 & 410 & 697 & 595 \\
\hline 158 & Apple Creek near Menoken, N. Dak. & 1,195 & 601 & 999 & 970 \\
\hline
\end{tabular}


Table 1-5. Median seasonal concentrations for selected constituents at selected sites in North Dakota from 1970 through 2008 . Continued

[Winter, January-February, November-December; Spring, March-June; Summer, July-October; mg/L, milligrams per liter; --, not available; N, nitrogen; <. less than; P, phosphorus; C, carbon; $\mu \mathrm{g} / \mathrm{L}$, micrograms per liter]

\begin{tabular}{|c|c|c|c|c|c|}
\hline $\begin{array}{c}\text { Site } \\
\text { identification } \\
\text { number } \\
\text { (table 1) } \\
\end{array}$ & Site name & Winter & Spring & Summer & $\begin{array}{l}\text { Period of } \\
\text { record }\end{array}$ \\
\hline \multicolumn{6}{|c|}{ Total dissolved solids, in $\mathrm{mg} / \mathrm{L}-$ Continued } \\
\hline 159 & Missouri River near Schmidt, N. Dak. & 441 & 441 & 442 & 441 \\
\hline 160 & Cannonball River at New England, N. Dak. & 2,100 & 1,930 & 1,410 & 1,790 \\
\hline 162 & Cannonball River at Regent, N. Dak. & 1,460 & 1,120 & 1,335 & 1,320 \\
\hline 163 & Cannonball River below Bentley, N. Dak. & 1,750 & 1,115 & 1,670 & 1,530 \\
\hline 164 & Cannonball River near Raleigh, N. Dak. & 2,100 & 1,150 & 1,240 & 1,235 \\
\hline 165 & White Butte Fork Cedar Creek near Scranton, N. Dak. & 3,970 & 2,125 & 2,650 & 2,575 \\
\hline 169 & Cedar Creek near Raleigh, N. Dak. & 1,510 & 1,045 & 1,175 & 1,150 \\
\hline 170 & Cannonball River at Breien, N. Dak. & 1,810 & 976 & 1,140 & 1,175 \\
\hline 171 & Beaver Creek near Linton, N. Dak. & 819 & 364 & 563 & 538 \\
\hline 172 & Beaver Creek below Linton, N. Dak. & 184 & 375 & 767 & 639 \\
\hline 173 & Porcupine Creek near Fort Yates, N. Dak. & -- & 766 & 788 & 777 \\
\hline 174 & Buffalo Creek Tributary near Gascoyne, N. Dak. & 4,780 & 3,900 & 3,650 & 4,270 \\
\hline 175 & James River near Manfred, N. Dak. & 707 & 480 & 649 & 593 \\
\hline 176 & James River near Grace City, N. Dak. & 1,040 & 476 & 660 & 646 \\
\hline 177 & James River above Arrowwood Lake near Kensal, N. Dak. & 857 & 559 & 620 & 656 \\
\hline 184 & Bear Creek near Oakes, N. Dak. & -- & 557 & 989 & 857 \\
\hline 185 & James River at Oakes, N. Dak. & 920 & 510 & 620 & 672 \\
\hline 186 & James River at N. Dak./S. Dak. State line & 734 & 457 & 541 & 541 \\
\hline \multicolumn{6}{|c|}{ Ammonia, dissolved and total as $\mathrm{N}$, in $\mathrm{mg} / \mathrm{L}$} \\
\hline 1 & Bois De Sioux River near Doran, Minn. & $<0.04$ & 0.06 & $<0.04$ & $<0.04$ \\
\hline 2 & Red River of the North at Wahpeton, N. Dak. & 0.18 & $<0.04$ & 0.06 & $<0.04$ \\
\hline 3 & Red River of the North near Wahpeton, N. Dak. & 0.25 & 0.10 & $<0.04$ & 0.10 \\
\hline 4 & Red River of the North at Brushville, Minn. & 0.08 & $<0.04$ & $<0.04$ & $<0.04$ \\
\hline 5 & Red River of the North below Wahpeton, N. Dak. & 0.25 & 0.10 & 0.07 & 0.10 \\
\hline 6 & Red River of the North at Hickson, N. Dak. & 0.12 & 0.06 & $<0.04$ & 0.05 \\
\hline 10 & Wild Rice River near Abercrombie, N. Dak. & $<0.04$ & 0.05 & $<0.04$ & $<0.04$ \\
\hline 11 & Red River of the North at Fargo, N. Dak. & 0.11 & 0.06 & $<0.04$ & $<0.04$ \\
\hline 12 & Red River of North below Fargo, N. Dak. & 0.98 & 0.44 & 0.81 & 0.69 \\
\hline
\end{tabular}


Table 1-5. Median seasonal concentrations for selected constituents at selected sites in North Dakota from 1970 through 2008.Continued

[Winter, January-February, November-December; Spring, March-June; Summer, July-October; mg/L, milligrams per liter; --, not available; N, nitrogen; <. less than; P, phosphorus; $\mathrm{C}$, carbon; $\mu \mathrm{g} / \mathrm{L}$, micrograms per liter]

\begin{tabular}{|c|c|c|c|c|c|}
\hline $\begin{array}{c}\text { Site } \\
\text { identification } \\
\text { number } \\
\text { (table 1) }\end{array}$ & Site name & Winter & Spring & Summer & $\begin{array}{c}\text { Period of } \\
\text { record }\end{array}$ \\
\hline \multicolumn{6}{|c|}{ Ammonia, dissolved and total as $\mathrm{N}$, in $\mathrm{mg} / \mathrm{L}$-Continued } \\
\hline 13 & Red River of the North at Harwood, N. Dak. & 0.13 & 0.06 & $<0.04$ & 0.07 \\
\hline 14 & Red River of the North near Harwood, N. Dak. & 0.27 & 0.11 & 0.09 & 0.10 \\
\hline 15 & Sheyenne River above Harvey, N. Dak. & 0.25 & $<0.04$ & $<0.04$ & 0.05 \\
\hline 17 & Sheyenne River at Warwick, N. Dak. & $<0.04$ & $<0.04$ & $<0.04$ & $<0.04$ \\
\hline 18 & Sheyenne River near Warwick, N. Dak. & $<0.04$ & $<0.04$ & 0.07 & $<0.04$ \\
\hline 20 & Mauvais Coulee near Cando, N. Dak. & $<0.04$ & $<0.04$ & $<0.04$ & $<0.04$ \\
\hline 21 & Edmore Coulee near Edmore, N. Dak. & 0.05 & 0.05 & $<0.04$ & 0.05 \\
\hline 22 & Edmore Coulee Tributary near Webster, N. Dak. & -- & -- & $<0.04$ & $<0.04$ \\
\hline 24 & Starkweather Coulee near Webster, N. Dak. & -- & 0.08 & $<0.04$ & 0.05 \\
\hline 25 & Big Coulee below Churchs Ferry, N. Dak. & -- & 0.06 & 0.81 & 0.09 \\
\hline 28 & Big Coulee near Churchs Ferry, N. Dak. & 0.17 & $<0.04$ & 0.10 & 0.09 \\
\hline 30 & Channel A near Penn, N. Dak. & 0.06 & 0.05 & 0.06 & 0.05 \\
\hline 32 & Sheyenne River near Cooperstown, N. Dak. & $<0.04$ & 0.06 & $<0.04$ & $<0.04$ \\
\hline 33 & Baldhill Creek near Dazey, N. Dak. & 0.13 & 0.06 & $<0.04$ & 0.06 \\
\hline 34 & Sheyenne River below Baldhill Dam, N. Dak. & 0.20 & 0.05 & 0.07 & 0.08 \\
\hline 35 & Sheyenne River at Valley City, N. Dak. & 0.19 & 0.18 & 0.09 & 0.16 \\
\hline 36 & Sheyenne River at Lisbon, N. Dak. & 0.06 & $<0.04$ & $<0.04$ & $<0.04$ \\
\hline 37 & Sheyenne River near Kindred, N. Dak. & 0.10 & $<0.04$ & $<0.04$ & $<0.04$ \\
\hline 38 & Sheyenne River above Sheyenne River Diversion near Horace, N. Dak. & 0.07 & $<0.04$ & $<0.04$ & $<0.04$ \\
\hline 41 & Sheyenne River at West Fargo, N. Dak. & $<0.04$ & $<0.04$ & $<0.04$ & $<0.04$ \\
\hline 45 & Maple River below Mapleton, N. Dak. & $<0.04$ & $<0.04$ & $<0.04$ & $<0.04$ \\
\hline 46 & Sheyenne River at Harwood, N. Dak. & 0.10 & 0.05 & $<0.04$ & 0.05 \\
\hline 49 & Lower Branch Rush River near Prosper, N. Dak. & -- & $<0.04$ & -- & $<0.04$ \\
\hline 50 & Sheyenne River near Harwood, N. Dak. & 0.26 & 0.22 & 0.19 & 0.19 \\
\hline 51 & Elm River near Kelso, N. Dak. & -- & $<0.04$ & 0.27 & 0.11 \\
\hline 52 & Red River of the North at Halstad, Minn. & 0.31 & 0.12 & $<0.04$ & 0.11 \\
\hline 53 & Beaver Creek near Finley, N. Dak. & 0.50 & 0.10 & 0.06 & 0.09 \\
\hline 55 & Goose River at Hillsboro, N. Dak. & 0.16 & $<0.04$ & $<0.04$ & $<0.04$ \\
\hline 56 & Red River of the North at Grand Forks, N. Dak. & 0.10 & $<0.04$ & $<0.04$ & $<0.04$ \\
\hline 57 & Turtle River at Turtle River State Park near Arvilla, N. Dak. & 0.10 & $<0.04$ & $<0.04$ & $<0.04$ \\
\hline 58 & Turtle River at Manvel, N. Dak. & 0.37 & $<0.04$ & $<0.04$ & $<0.04$ \\
\hline 59 & Red River of the North at Oslo, Minn. & 0.53 & 0.29 & $<0.04$ & 0.22 \\
\hline 62 & Forest River near Minto, N. Dak. & 0.10 & $<0.04$ & $<0.04$ & $<0.04$ \\
\hline 67 & Park River at Grafton, N. Dak. & 0.12 & $<0.04$ & $<0.04$ & $<0.04$ \\
\hline 68 & Red River of the North at Drayton, N. Dak. & $<0.04$ & 0.05 & $<0.04$ & $<0.04$ \\
\hline 72 & Pembina River near Vang, N. Dak. & 0.05 & $<0.04$ & $<0.04$ & $<0.04$ \\
\hline 73 & Little South Pembina River near Walhalla, N. Dak. & $<0.04$ & $<0.04$ & $<0.04$ & $<0.04$ \\
\hline 74 & Pembina River at Walhalla, N. Dak. & 0.08 & 0.07 & $<0.04$ & $<0.04$ \\
\hline
\end{tabular}


Table 1-5. Median seasonal concentrations for selected constituents at selected sites in North Dakota from 1970 through 2008.Continued

[Winter, January-February, November-December; Spring, March-June; Summer, July-October; mg/L, milligrams per liter; --, not available; N, nitrogen; <. less than; P, phosphorus; C, carbon; $\mu \mathrm{g} / \mathrm{L}$, micrograms per liter]

\begin{tabular}{|c|c|c|c|c|c|}
\hline $\begin{array}{c}\text { Site } \\
\text { identification } \\
\text { number } \\
\text { (table 1) }\end{array}$ & Site name & Winter & Spring & Summer & $\begin{array}{c}\text { Period of } \\
\text { record }\end{array}$ \\
\hline \multicolumn{6}{|c|}{ Ammonia, dissolved and total as $\mathrm{N}$, in $\mathrm{mg} / \mathrm{L}$-Continued } \\
\hline 75 & Pembina River at Neche, N. Dak. & $<0.04$ & $<0.04$ & $<0.04$ & $<0.04$ \\
\hline 76 & Tongue River at Akra, N. Dak. & 0.17 & 0.15 & 0.08 & 0.13 \\
\hline 78 & Red River of the North at Pembina, N. Dak., site 2 & 0.11 & 0.05 & $<0.04$ & $<0.04$ \\
\hline 79 & Red River of the North at Emerson, Manitoba & 0.16 & 0.10 & $<0.04$ & 0.09 \\
\hline 80 & Long Creek near Noonan, N. Dak. & $<0.04$ & $<0.04$ & $<0.04$ & $<0.04$ \\
\hline 81 & West Branch Short Creek near Columbus, N. Dak. & 0.13 & 0.06 & $<0.04$ & 0.06 \\
\hline 85 & Souris River above Minot, N. Dak. & 0.17 & $<0.04$ & $<0.04$ & $<0.04$ \\
\hline 87 & Souris River near Verendrye, N. Dak. & 0.21 & 0.11 & $<0.04$ & 0.09 \\
\hline 88 & Wintering River near Karlsruhe, N. Dak. & 0.07 & $<0.04$ & $<0.04$ & $<0.04$ \\
\hline 89 & Souris River near Bantry, N. Dak. & 0.12 & $<0.04$ & $<0.04$ & $<0.04$ \\
\hline 90 & Willow Creek near Willow City, N. Dak. & 0.14 & $<0.04$ & $<0.04$ & $<0.04$ \\
\hline 91 & Stone Creek near Kramer, N. Dak. & -- & 0.05 & $<0.04$ & $<0.04$ \\
\hline 92 & Deep River near Upham, N. Dak. & 0.06 & $<0.04$ & $<0.04$ & $<0.04$ \\
\hline 95 & Cut Bank Creek at Upham, N. Dak. & -- & $<0.04$ & $<0.04$ & $<0.04$ \\
\hline 96 & Deep River below Cut Bank Creek near Upham, N. Dak. & -- & 0.05 & 0.09 & 0.07 \\
\hline 106 & Bear Den Creek near Mandaree, N. Dak. & 0.14 & 0.06 & $<0.04$ & 0.05 \\
\hline 108 & East Fork Shell Creek near Parshall, N. Dak. & 0.31 & 0.05 & $<0.04$ & $<0.04$ \\
\hline 109 & Deepwater Creek near Mandaree, N. Dak. & 0.15 & $<0.04$ & $<0.04$ & $<0.04$ \\
\hline 110 & Little Missouri River at Marmarth, N. Dak. & -- & $<0.04$ & $<0.04$ & $<0.04$ \\
\hline 111 & Deep Creek near Amidon, N. Dak. & 0.07 & 0.05 & $<0.04$ & $<0.04$ \\
\hline 112 & Little Missouri River at Medora, N. Dak. & $<0.04$ & $<0.04$ & $<0.04$ & $<0.04$ \\
\hline 113 & Beaver Creek near Trotters, N. Dak. & 0.07 & $<0.04$ & $<0.04$ & $<0.04$ \\
\hline 114 & Little Missouri River near Watford City, N. Dak. & 0.10 & $<0.04$ & $<0.04$ & $<0.04$ \\
\hline 115 & Missouri River at Garrison Dam, N. Dak. & $<0.04$ & $<0.04$ & $<0.04$ & $<0.04$ \\
\hline 116 & Knife River at Manning, N. Dak. & 0.08 & 0.06 & 0.06 & 0.06 \\
\hline 117 & Stray Creek near Manning, N. Dak. & $<0.04$ & 0.08 & 0.05 & 0.06 \\
\hline 118 & Knife River at Marshall, N. Dak. & 0.10 & 0.07 & $<0.04$ & 0.07 \\
\hline 119 & Elm Creek near Golden Valley, N. Dak. & 0.41 & $<0.04$ & 0.08 & 0.06 \\
\hline
\end{tabular}


Table 1-5. Median seasonal concentrations for selected constituents at selected sites in North Dakota from 1970 through 2008.Continued

[Winter, January-February, November-December; Spring, March-June; Summer, July-October; mg/L, milligrams per liter; --, not available; N, nitrogen; <. less than; P, phosphorus; $\mathrm{C}$, carbon; $\mu \mathrm{g} / \mathrm{L}$, micrograms per liter]

\begin{tabular}{|c|c|c|c|c|c|}
\hline $\begin{array}{c}\text { Site } \\
\text { identification } \\
\text { number } \\
\text { (table 1) }\end{array}$ & Site name & Winter & Spring & Summer & $\begin{array}{c}\text { Period of } \\
\text { record }\end{array}$ \\
\hline \multicolumn{6}{|c|}{ Ammonia, dissolved and total as $\mathrm{N}$, in $\mathrm{mg} / \mathrm{L}$-Continued } \\
\hline 120 & Knife River near Golden Valley, N. Dak. & $<0.04$ & $<0.04$ & $<0.04$ & $<0.04$ \\
\hline 121 & Coyote Creek near Zap, N. Dak. & 0.13 & 0.06 & $<0.04$ & 0.06 \\
\hline 122 & Brush Creek near Beulah, N. Dak. & 0.16 & 0.08 & $<0.04$ & 0.07 \\
\hline 123 & Spring Creek below Lake Ilo near Dunn Center, N. Dak. & 0.33 & 0.07 & 0.07 & 0.09 \\
\hline 124 & Spring Creek near Halliday, N. Dak. & 0.14 & 0.06 & $<0.04$ & 0.06 \\
\hline 125 & Spring Creek at Zap, N. Dak. & 0.09 & 0.05 & $<0.04$ & $<0.04$ \\
\hline 127 & Knife River at Hazen, N. Dak. & 0.13 & $<0.04$ & $<0.04$ & 0.05 \\
\hline 128 & Antelope Creek above Hazen, N. Dak. & 0.13 & 0.10 & $<0.04$ & 0.10 \\
\hline 129 & West Branch Antelope Creek No. 4 near Zap, N. Dak. & -- & $<0.04$ & -- & $<0.04$ \\
\hline 130 & West Branch Antelope Creek near Hazen, N. Dak. & 0.40 & 0.19 & -- & 0.20 \\
\hline 131 & Coal Creek near Stanton, N. Dak. & 0.08 & $<0.04$ & 0.10 & 0.05 \\
\hline 132 & Alderin Creek near Fort Clark, N. Dak. & 0.11 & 0.15 & $<0.04$ & 0.07 \\
\hline 133 & Coal Lake Coulee near Hensler, N. Dak. & 0.12 & 0.07 & 0.05 & 0.06 \\
\hline 134 & Buffalo Creek near Washburn, N. Dak. & 0.16 & 0.15 & $<0.04$ & 0.11 \\
\hline 135 & Turtle Creek above Washburn, N. Dak. & 0.17 & $<0.04$ & $<0.04$ & $<0.04$ \\
\hline 136 & Painted Woods Creek near Wilton, N. Dak. & $<0.04$ & $<0.04$ & $<0.04$ & $<0.04$ \\
\hline 137 & Square Butte Creek near Hannover, N. Dak. & 0.05 & 0.05 & 0.06 & 0.05 \\
\hline 138 & Square Butte Creek above Nelson Lake near Center, N. Dak. & 0.21 & 0.23 & 0.10 & 0.13 \\
\hline 139 & Hagel Creek near Center, N. Dak. & 0.19 & 0.17 & 0.06 & 0.14 \\
\hline 140 & Square Butte Creek below Center, N. Dak. & 1.00 & 0.42 & 0.43 & 0.59 \\
\hline 142 & Missouri River at Bismarck, N. Dak. & $<0.04$ & $<0.04$ & $<0.04$ & $<0.04$ \\
\hline 143 & South Branch Heart River near South Heart, N. Dak. & 0.12 & 0.12 & 0.05 & 0.09 \\
\hline 144 & North Creek near South Heart, N. Dak. & $<0.04$ & 0.10 & 0.07 & 0.07 \\
\hline 145 & Heart River near South Heart, N. Dak. & 0.13 & 0.12 & 0.05 & 0.09 \\
\hline 146 & Heart River at Dickinson, N. Dak. & 0.41 & 0.14 & 0.48 & 0.23 \\
\hline 147 & Green River near New Hradec, N. Dak. & 0.07 & 0.10 & $<0.04$ & 0.06 \\
\hline 148 & Green River near Gladstone, N. Dak. & 0.13 & $<0.04$ & 0.09 & 0.07 \\
\hline 149 & Heart River near Richardton, N. Dak. & $<0.04$ & $<0.04$ & $<0.04$ & $<0.04$ \\
\hline 152 & Big Muddy Creek near Almont, N. Dak. & -- & 0.06 & -- & 0.06 \\
\hline 156 & Heart River near Mandan, N. Dak. & 0.08 & $<0.04$ & $<0.04$ & $<0.04$ \\
\hline 157 & Long Lake Creek above Long Lake near Moffit, N. Dak. & -- & 0.16 & 0.09 & 0.09 \\
\hline 158 & Apple Creek near Menoken, N. Dak. & 0.12 & 0.08 & $<0.04$ & 0.07 \\
\hline 159 & Missouri River near Schmidt, N. Dak. & $<0.04$ & $<0.04$ & $<0.04$ & $<0.04$ \\
\hline 160 & Cannonball River at New England, N. Dak. & 0.13 & 0.09 & $<0.04$ & 0.07 \\
\hline 161 & Coal Bank Creek near Havelock, N. Dak. & 0.10 & 0.05 & 0.05 & 0.05 \\
\hline 162 & Cannonball River at Regent, N. Dak. & 0.08 & $<0.04$ & 0.05 & 0.05 \\
\hline 164 & Cannonball River near Raleigh, N. Dak. & $<0.04$ & $<0.04$ & $<0.04$ & $<0.04$ \\
\hline 167 & Timber Creek near Bentley, N. Dak. & 0.14 & 0.08 & $<0.04$ & 0.06 \\
\hline
\end{tabular}


Table 1-5. Median seasonal concentrations for selected constituents at selected sites in North Dakota from 1970 through 2008 . Continued

[Winter, January-February, November-December; Spring, March-June; Summer, July-October; mg/L, milligrams per liter; --, not available; N, nitrogen; <. less than; P, phosphorus; $\mathrm{C}$, carbon; $\mu \mathrm{g} / \mathrm{L}$, micrograms per liter]

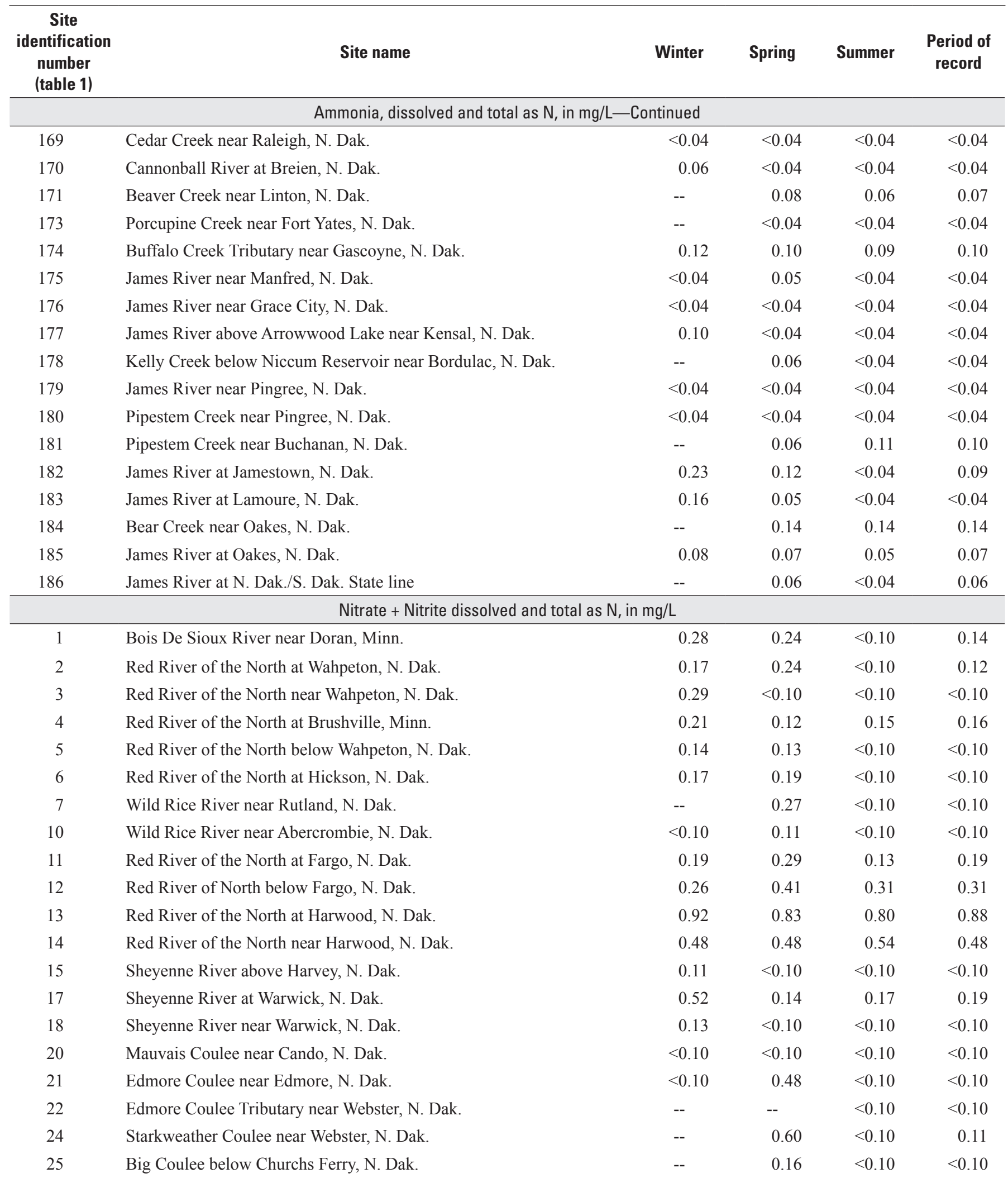


Table 1-5. Median seasonal concentrations for selected constituents at selected sites in North Dakota from 1970 through 2008.Continued

[Winter, January-February, November-December; Spring, March-June; Summer, July-October; mg/L, milligrams per liter; --, not available; N, nitrogen; <. less than; P, phosphorus; C, carbon; $\mu \mathrm{g} / \mathrm{L}$, micrograms per liter]

\begin{tabular}{|c|c|c|c|c|c|}
\hline $\begin{array}{c}\text { Site } \\
\text { identification } \\
\text { number } \\
\text { (table 1) }\end{array}$ & Site name & Winter & Spring & Summer & $\begin{array}{c}\text { Period of } \\
\text { record }\end{array}$ \\
\hline \multicolumn{6}{|c|}{ Nitrate + Nitrite dissolved and total as $\mathrm{N}$, in $\mathrm{mg} / \mathrm{L}$-Continued } \\
\hline 27 & Little Coulee near Brinsmade, N. Dak. & -- & 0.41 & 0.16 & 0.23 \\
\hline 28 & Big Coulee near Churchs Ferry, N. Dak. & 0.68 & 0.27 & 0.22 & 0.27 \\
\hline 29 & Big Coulee at Graham Island inlet near Fort Totten, N. Dak. & 0.15 & $<0.10$ & $<0.10$ & $<0.10$ \\
\hline 30 & Channel A near Penn, N. Dak. & $<0.10$ & $<0.10$ & $<0.10$ & $<0.10$ \\
\hline 32 & Sheyenne River near Cooperstown, N. Dak. & 0.17 & $<0.10$ & 0.15 & 0.13 \\
\hline 33 & Baldhill Creek near Dazey, N. Dak. & $<0.10$ & 0.26 & $<0.10$ & $<0.10$ \\
\hline 34 & Sheyenne River below Baldhill Dam, N. Dak. & 0.20 & 0.27 & $<0.10$ & 0.13 \\
\hline 35 & Sheyenne River at Valley City, N. Dak. & 0.21 & 0.23 & 0.15 & 0.21 \\
\hline 36 & Sheyenne River at Lisbon, N. Dak. & 0.37 & 0.37 & $<0.10$ & 0.21 \\
\hline 37 & Sheyenne River near Kindred, N. Dak. & 0.40 & 0.22 & $<0.10$ & 0.13 \\
\hline 38 & Sheyenne River above Sheyenne River Diversion near Horace, N. Dak. & 0.48 & 0.27 & $<0.10$ & $<0.10$ \\
\hline 39 & Sheyenne River near Horace, N. Dak. & 0.33 & 0.37 & $<0.10$ & 0.19 \\
\hline 41 & Sheyenne River at West Fargo, N. Dak. & 0.29 & 0.30 & $<0.10$ & $<0.10$ \\
\hline 42 & Maple River near Hope, N. Dak. & -- & -- & $<0.10$ & $<0.10$ \\
\hline 45 & Maple River below Mapleton, N. Dak. & 1.28 & 0.11 & $<0.10$ & $<0.10$ \\
\hline 46 & Sheyenne River at Harwood, N. Dak. & 0.61 & 0.41 & 0.29 & 0.36 \\
\hline 49 & Lower Branch Rush River near Prosper, N. Dak. & -- & 0.30 & -- & 0.30 \\
\hline 50 & Sheyenne River near Harwood, N. Dak. & 0.20 & 0.49 & 0.16 & 0.21 \\
\hline 51 & Elm River near Kelso, N. Dak. & -- & 0.50 & $<0.10$ & $<0.10$ \\
\hline 52 & Red River of the North at Halstad, Minn. & 0.37 & 0.63 & 0.36 & 0.43 \\
\hline 53 & Beaver Creek near Finley, N. Dak. & $<0.10$ & $<0.10$ & $<0.10$ & $<0.10$ \\
\hline 55 & Goose River at Hillsboro, N. Dak. & 2.11 & 0.25 & 0.30 & 0.39 \\
\hline 56 & Red River of the North at Grand Forks, N. Dak. & 0.44 & 0.44 & 0.37 & 0.40 \\
\hline 57 & Turtle River at Turtle River State Park near Arvilla, N. Dak. & 0.69 & 0.33 & 0.15 & 0.32 \\
\hline 58 & Turtle River at Manvel, N. Dak. & 0.89 & 0.15 & $<0.10$ & 0.16 \\
\hline 59 & Red River of the North at Oslo, Minn. & 0.23 & 0.45 & $<0.10$ & 0.22 \\
\hline 62 & Forest River near Minto, N. Dak. & 2.00 & 0.39 & 0.37 & 0.48 \\
\hline 63 & South Branch Park River below Homme Dam, N. Dak. & -- & $<0.10$ & -- & $<0.10$ \\
\hline 64 & Middle Branch Park River near Union, N. Dak. & -- & -- & 0.92 & 0.92 \\
\hline 65 & Middle Branch Park River near Edinburg, N. Dak. & -- & 1.55 & 0.23 & 0.83 \\
\hline 66 & Cart Creek at Mountain, N. Dak. & -- & 1.60 & -- & 1.60 \\
\hline 67 & Park River at Grafton, N. Dak. & 1.29 & 0.96 & 0.28 & 0.54 \\
\hline 68 & Red River of the North at Drayton, N. Dak. & 0.23 & 0.50 & 0.26 & 0.37 \\
\hline 72 & Pembina River near Vang, N. Dak. & $<0.10$ & 0.57 & $<0.10$ & 0.14 \\
\hline 73 & Little South Pembina River near Walhalla, N. Dak. & 0.75 & 1.00 & $<0.10$ & 0.64 \\
\hline 74 & Pembina River at Walhalla, N. Dak. & 0.12 & 0.34 & $<0.10$ & 0.13 \\
\hline 75 & Pembina River at Neche, N. Dak. & 0.69 & 0.23 & $<0.10$ & 0.17 \\
\hline 76 & Tongue River at Akra, N. Dak. & 0.12 & 0.23 & $<0.10$ & $<0.10$ \\
\hline
\end{tabular}


Table 1-5. Median seasonal concentrations for selected constituents at selected sites in North Dakota from 1970 through 2008.Continued

[Winter, January-February, November-December; Spring, March-June; Summer, July-October; mg/L, milligrams per liter; --, not available; N, nitrogen; <. less than; P, phosphorus; C, carbon; $\mu \mathrm{g} / \mathrm{L}$, micrograms per liter]

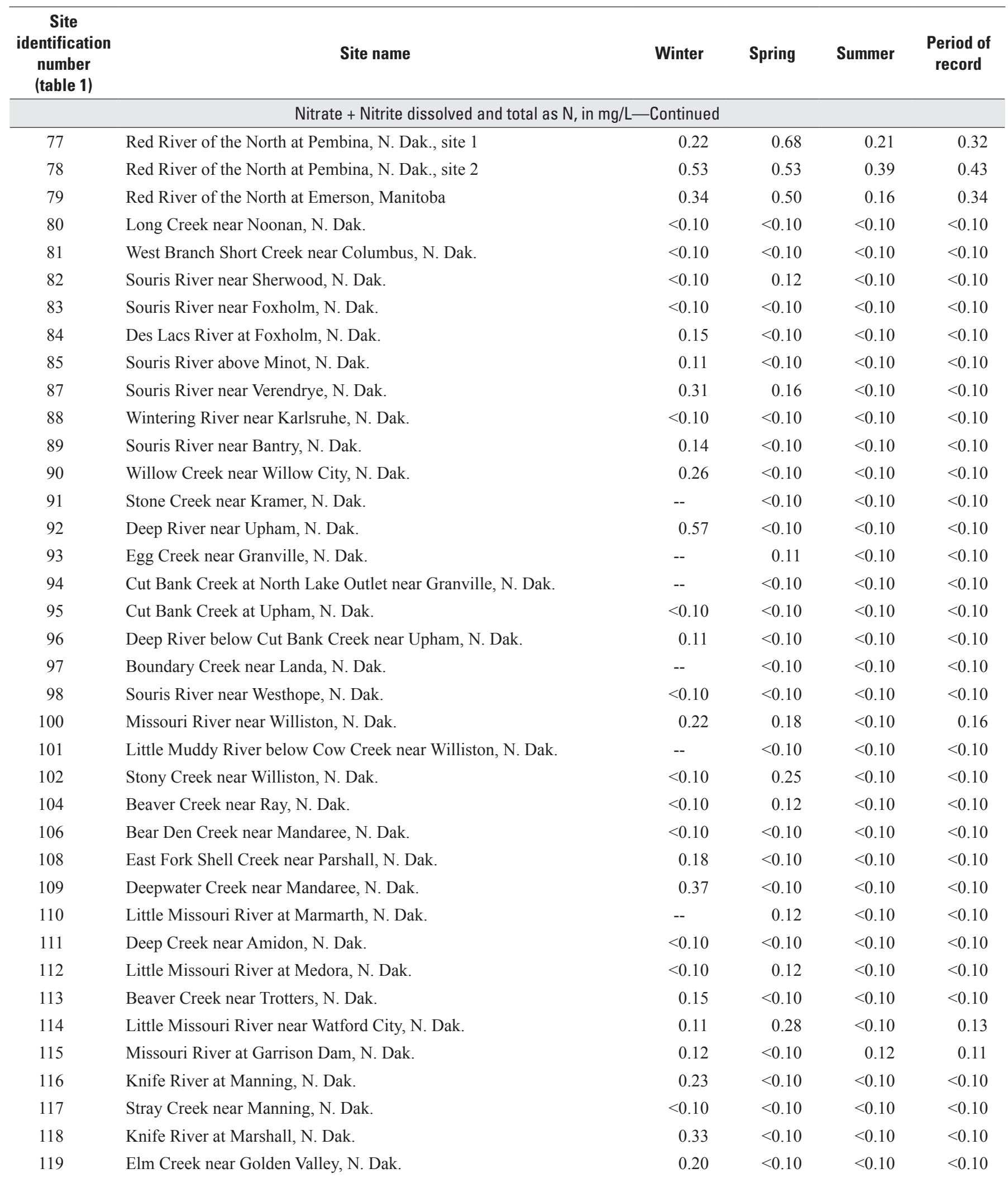


Table 1-5. Median seasonal concentrations for selected constituents at selected sites in North Dakota from 1970 through 2008.Continued

[Winter, January-February, November-December; Spring, March-June; Summer, July-October; mg/L, milligrams per liter; --, not available; N, nitrogen; <. less than; P, phosphorus; $\mathrm{C}$, carbon; $\mu \mathrm{g} / \mathrm{L}$, micrograms per liter]

\begin{tabular}{|c|c|c|c|c|c|}
\hline $\begin{array}{c}\text { Site } \\
\text { identification } \\
\text { number } \\
\text { (table 1) }\end{array}$ & Site name & Winter & Spring & Summer & $\begin{array}{c}\text { Period of } \\
\text { record }\end{array}$ \\
\hline \multicolumn{6}{|c|}{ Nitrate + Nitrite dissolved and total as $\mathrm{N}$, in $\mathrm{mg} / \mathrm{L}$-Continued } \\
\hline 120 & Knife River near Golden Valley, N. Dak. & 0.13 & $<0.10$ & $<0.10$ & $<0.10$ \\
\hline 121 & Coyote Creek near Zap, N. Dak. & 0.12 & $<0.10$ & $<0.10$ & $<0.10$ \\
\hline 122 & Brush Creek near Beulah, N. Dak. & $<0.10$ & $<0.10$ & $<0.10$ & $<0.10$ \\
\hline 123 & Spring Creek below Lake Ilo near Dunn Center, N. Dak. & 0.19 & $<0.10$ & $<0.10$ & $<0.10$ \\
\hline 124 & Spring Creek near Halliday, N. Dak. & 0.18 & $<0.10$ & $<0.10$ & $<0.10$ \\
\hline 125 & Spring Creek at Zap, N. Dak. & 0.18 & $<0.10$ & $<0.10$ & $<0.10$ \\
\hline 127 & Knife River at Hazen, N. Dak. & 0.25 & $<0.10$ & $<0.10$ & $<0.10$ \\
\hline 128 & Antelope Creek above Hazen, N. Dak. & 0.35 & 0.14 & $<0.10$ & 0.18 \\
\hline 129 & West Branch Antelope Creek No. 4 near Zap, N. Dak. & -- & 0.21 & -- & 0.21 \\
\hline 130 & West Branch Antelope Creek near Hazen, N. Dak. & 0.78 & $<0.10$ & -- & 0.14 \\
\hline 131 & Coal Creek near Stanton, N. Dak. & $<0.10$ & $<0.10$ & $<0.10$ & $<0.10$ \\
\hline 132 & Alderin Creek near Fort Clark, N. Dak. & 0.11 & 0.15 & $<0.10$ & $<0.10$ \\
\hline 133 & Coal Lake Coulee near Hensler, N. Dak. & 0.19 & 0.16 & $<0.10$ & $<0.10$ \\
\hline 134 & Buffalo Creek near Washburn, N. Dak. & 0.12 & $<0.10$ & $<0.10$ & $<0.10$ \\
\hline 135 & Turtle Creek above Washburn, N. Dak. & $<0.10$ & $<0.10$ & $<0.10$ & $<0.10$ \\
\hline 136 & Painted Woods Creek near Wilton, N. Dak. & $<0.10$ & $<0.10$ & $<0.10$ & $<0.10$ \\
\hline 137 & Square Butte Creek near Hannover, N. Dak. & $<0.10$ & $<0.10$ & $<0.10$ & $<0.10$ \\
\hline 138 & Square Butte Creek above Nelson Lake near Center, N. Dak. & 1.95 & 0.60 & 0.51 & 0.75 \\
\hline 139 & Hagel Creek near Center, N. Dak. & $<0.10$ & $<0.10$ & $<0.10$ & $<0.10$ \\
\hline 140 & Square Butte Creek below Center, N. Dak. & 0.20 & 0.32 & 0.20 & 0.23 \\
\hline 142 & Missouri River at Bismarck, N. Dak. & 0.15 & 0.11 & 0.13 & 0.12 \\
\hline 143 & South Branch Heart River near South Heart, N. Dak. & 0.72 & 0.17 & 0.26 & 0.29 \\
\hline 144 & North Creek near South Heart, N. Dak. & $<0.10$ & $<0.10$ & 0.16 & $<0.10$ \\
\hline 145 & Heart River near South Heart, N. Dak. & 0.11 & $<0.10$ & $<0.10$ & $<0.10$ \\
\hline 146 & Heart River at Dickinson, N. Dak. & 0.14 & 0.13 & $<0.10$ & $<0.10$ \\
\hline 147 & Green River near New Hradec, N. Dak. & 0.20 & $<0.10$ & $<0.10$ & $<0.10$ \\
\hline 148 & Green River near Gladstone, N. Dak. & $<0.10$ & $<0.10$ & $<0.10$ & $<0.10$ \\
\hline 149 & Heart River near Richardton, N. Dak. & 0.18 & $<0.10$ & $<0.10$ & $<0.10$ \\
\hline 152 & Big Muddy Creek near Almont, N. Dak. & -- & $<0.10$ & -- & $<0.10$ \\
\hline 156 & Heart River near Mandan, N. Dak. & 0.20 & $<0.10$ & $<0.10$ & $<0.10$ \\
\hline 157 & Long Lake Creek above Long Lake near Moffit, N. Dak. & -- & 0.23 & $<0.10$ & $<0.10$ \\
\hline 158 & Apple Creek near Menoken, N. Dak. & 0.11 & $<0.10$ & $<0.10$ & $<0.10$ \\
\hline 159 & Missouri River near Schmidt, N. Dak. & 0.14 & 0.11 & 0.14 & 0.13 \\
\hline 160 & Cannonball River at New England, N. Dak. & $<0.10$ & $<0.10$ & $<0.10$ & $<0.10$ \\
\hline 161 & Coal Bank Creek near Havelock, N. Dak. & $<0.10$ & 0.17 & $<0.10$ & $<0.10$ \\
\hline 162 & Cannonball River at Regent, N. Dak. & 0.31 & $<0.10$ & $<0.10$ & $<0.10$ \\
\hline 164 & Cannonball River near Raleigh, N. Dak. & $<0.10$ & $<0.10$ & $<0.10$ & $<0.10$ \\
\hline 166 & Cedar Creek near Haynes, N. Dak. & -- & $<0.10$ & -- & $<0.10$ \\
\hline
\end{tabular}


Table 1-5. Median seasonal concentrations for selected constituents at selected sites in North Dakota from 1970 through 2008 . Continued

[Winter, January-February, November-December; Spring, March-June; Summer, July-October; mg/L, milligrams per liter; --, not available; N, nitrogen; <. less than; P, phosphorus; C, carbon; $\mu \mathrm{g} / \mathrm{L}$, micrograms per liter]

\begin{tabular}{|c|c|c|c|c|c|}
\hline $\begin{array}{c}\text { Site } \\
\text { identification } \\
\text { number } \\
\text { (table 1) }\end{array}$ & Site name & Winter & Spring & Summer & $\begin{array}{c}\text { Period of } \\
\text { record }\end{array}$ \\
\hline \multicolumn{6}{|c|}{ Nitrate + Nitrite dissolved and total as $\mathrm{N}$, in $\mathrm{mg} / \mathrm{L}$-Continued } \\
\hline 167 & Timber Creek near Bentley, N. Dak. & 2.40 & 0.27 & $<0.10$ & 0.38 \\
\hline 169 & Cedar Creek near Raleigh, N. Dak. & $<0.10$ & $<0.10$ & $<0.10$ & $<0.10$ \\
\hline 171 & Beaver Creek near Linton, N. Dak. & -- & 0.15 & $<0.10$ & $<0.10$ \\
\hline 173 & Porcupine Creek near Fort Yates, N. Dak. & -- & $<0.10$ & $<0.10$ & $<0.10$ \\
\hline 174 & Buffalo Creek Tributary near Gascoyne, N. Dak. & $<0.10$ & $<0.10$ & $<0.10$ & $<0.10$ \\
\hline 175 & James River near Manfred, N. Dak. & $<0.10$ & $<0.10$ & $<0.10$ & $<0.10$ \\
\hline 179 & James River near Pingree, N. Dak. & $<0.10$ & $<0.10$ & $<0.10$ & $<0.10$ \\
\hline 180 & Pipestem Creek near Pingree, N. Dak. & $<0.10$ & $<0.10$ & $<0.10$ & $<0.10$ \\
\hline 181 & Pipestem Creek near Buchanan, N. Dak. & -- & $<0.10$ & $<0.10$ & $<0.10$ \\
\hline 182 & James River at Jamestown, N. Dak. & 0.23 & 0.30 & 0.20 & 0.22 \\
\hline 183 & James River at Lamoure, N. Dak. & 0.22 & 0.19 & $<0.10$ & 0.14 \\
\hline 184 & Bear Creek near Oakes, N. Dak. & -- & 0.19 & 0.29 & 0.22 \\
\hline 185 & James River at Oakes, N. Dak. & $<0.10$ & $<0.10$ & $<0.10$ & $<0.10$ \\
\hline 186 & James River at N. Dak./S. Dak. State line & $<0.10$ & $<0.10$ & $<0.10$ & $<0.10$ \\
\hline \multicolumn{6}{|c|}{ Phosphorus, dissolved as $\mathrm{P}$, in $\mathrm{mg} / \mathrm{L}$} \\
\hline 7 & Wild Rice River near Rutland, N. Dak. & -- & 0.09 & 0.10 & 0.10 \\
\hline 8 & Wild Rice River near Cayuga, N. Dak. & 0.12 & 0.26 & -- & 0.12 \\
\hline 10 & Wild Rice River near Abercrombie, N. Dak. & 0.25 & 0.21 & 0.29 & 0.25 \\
\hline 11 & Red River of the North at Fargo, N. Dak. & 0.10 & 0.11 & 0.12 & 0.11 \\
\hline 12 & Red River of North below Fargo, N. Dak. & 0.16 & 0.14 & 0.16 & 0.15 \\
\hline 13 & Red River of the North at Harwood, N. Dak. & 0.35 & 0.16 & 0.24 & 0.22 \\
\hline 14 & Red River of the North near Harwood, N. Dak. & 0.17 & 0.14 & 0.20 & 0.17 \\
\hline 15 & Sheyenne River above Harvey, N. Dak. & 0.25 & 0.22 & 0.16 & 0.20 \\
\hline 16 & Big Coulee near Fort Totten, N. Dak. & -- & 0.04 & -- & 0.04 \\
\hline 17 & Sheyenne River at Warwick, N. Dak. & -- & 0.18 & 0.22 & 0.21 \\
\hline 18 & Sheyenne River near Warwick, N. Dak. & 0.08 & 0.15 & 0.16 & 0.13 \\
\hline 20 & Mauvais Coulee near Cando, N. Dak. & 0.28 & 0.26 & 0.29 & 0.28 \\
\hline 21 & Edmore Coulee near Edmore, N. Dak. & 0.20 & 0.21 & 0.53 & 0.21 \\
\hline
\end{tabular}


Table 1-5. Median seasonal concentrations for selected constituents at selected sites in North Dakota from 1970 through 2008.Continued

[Winter, January-February, November-December; Spring, March-June; Summer, July-October; mg/L, milligrams per liter; --, not available; N, nitrogen; <. less than; P, phosphorus; $\mathrm{C}$, carbon; $\mu \mathrm{g} / \mathrm{L}$, micrograms per liter]

\begin{tabular}{|c|c|c|c|c|c|}
\hline $\begin{array}{c}\text { Site } \\
\text { identification } \\
\text { number } \\
\text { (table 1) } \\
\end{array}$ & Site name & Winter & Spring & Summer & $\begin{array}{c}\text { Period of } \\
\text { record }\end{array}$ \\
\hline \multicolumn{6}{|c|}{ Phosphorus, dissolved as $\mathrm{P}$, in $\mathrm{mg} / \mathrm{L}-$ Continued } \\
\hline 24 & Starkweather Coulee near Webster, N. Dak. & -- & 0.29 & -- & 0.29 \\
\hline 27 & Little Coulee near Brinsmade, N. Dak. & -- & 0.36 & 0.35 & 0.36 \\
\hline 28 & Big Coulee near Churchs Ferry, N. Dak. & 0.19 & 0.16 & 0.36 & 0.26 \\
\hline 29 & Big Coulee at Graham Island inlet near Fort Totten, N. Dak. & 0.40 & 0.21 & 0.32 & 0.28 \\
\hline 30 & Channel A near Penn, N. Dak. & 0.04 & -- & 0.62 & 0.55 \\
\hline 32 & Sheyenne River near Cooperstown, N. Dak. & 0.12 & 0.17 & 0.19 & 0.16 \\
\hline 33 & Baldhill Creek near Dazey, N. Dak. & 0.02 & 0.12 & 0.03 & 0.04 \\
\hline 34 & Sheyenne River below Baldhill Dam, N. Dak. & 0.24 & 0.18 & 0.23 & 0.22 \\
\hline 36 & Sheyenne River at Lisbon, N. Dak. & 0.16 & 0.13 & 0.07 & 0.11 \\
\hline 37 & Sheyenne River near Kindred, N. Dak. & 0.08 & 0.07 & 0.06 & 0.07 \\
\hline 38 & Sheyenne River above Sheyenne River Diversion near Horace, N. Dak. & 0.20 & 0.11 & 0.11 & 0.11 \\
\hline 39 & Sheyenne River near Horace, N. Dak. & 0.06 & 0.09 & 0.08 & 0.07 \\
\hline 41 & Sheyenne River at West Fargo, N. Dak. & -- & 0.17 & 0.14 & 0.16 \\
\hline 45 & Maple River below Mapleton, N. Dak. & 2.10 & 0.47 & 0.19 & 0.45 \\
\hline 46 & Sheyenne River at Harwood, N. Dak. & 0.13 & 0.17 & 0.15 & 0.15 \\
\hline 50 & Sheyenne River near Harwood, N. Dak. & 0.17 & 0.20 & -- & 0.17 \\
\hline 52 & Red River of the North at Halstad, Minn. & 0.14 & 0.15 & 0.19 & 0.16 \\
\hline 53 & Beaver Creek near Finley, N. Dak. & 0.10 & 0.10 & 0.17 & 0.11 \\
\hline 55 & Goose River at Hillsboro, N. Dak. & 0.03 & 0.03 & 0.05 & 0.04 \\
\hline 56 & Red River of the North at Grand Forks, N. Dak. & 0.15 & 0.10 & 0.14 & 0.13 \\
\hline 57 & Turtle River at Turtle River State Park near Arvilla, N. Dak. & 0.04 & 0.07 & 0.14 & 0.07 \\
\hline 58 & Turtle River at Manvel, N. Dak. & 0.06 & 0.07 & 0.07 & 0.06 \\
\hline 59 & Red River of the North at Oslo, Minn. & 0.23 & 0.10 & 0.08 & 0.10 \\
\hline 62 & Forest River near Minto, N. Dak. & 0.05 & 0.07 & 0.10 & 0.08 \\
\hline 67 & Park River at Grafton, N. Dak. & 0.07 & 0.13 & 0.11 & 0.11 \\
\hline 68 & Red River of the North at Drayton, N. Dak. & -- & 0.09 & 0.07 & 0.07 \\
\hline 72 & Pembina River near Vang, N. Dak. & 0.10 & 0.17 & 0.13 & 0.14 \\
\hline 73 & Little South Pembina River near Walhalla, N. Dak. & 0.11 & 0.20 & 0.15 & 0.17 \\
\hline 74 & Pembina River at Walhalla, N. Dak. & 0.06 & 0.15 & 0.14 & 0.13 \\
\hline 75 & Pembina River at Neche, N. Dak. & 0.10 & 0.22 & 0.21 & 0.20 \\
\hline 76 & Tongue River at Akra, N. Dak. & 0.02 & 0.07 & 0.09 & 0.07 \\
\hline 78 & Red River of the North at Pembina, N. Dak., site 2 & 0.13 & 0.16 & 0.14 & 0.14 \\
\hline 79 & Red River of the North at Emerson, Manitoba & 0.09 & 0.10 & 0.12 & 0.11 \\
\hline 81 & West Branch Short Creek near Columbus, N. Dak. & 0.05 & 0.09 & 0.36 & 0.09 \\
\hline 82 & Souris River near Sherwood, N. Dak. & 0.06 & 0.08 & 0.09 & 0.08 \\
\hline 83 & Souris River near Foxholm, N. Dak. & -- & 0.06 & -- & 0.06 \\
\hline 84 & Des Lacs River at Foxholm, N. Dak. & 0.05 & 0.12 & 0.25 & 0.12 \\
\hline 85 & Souris River above Minot, N. Dak. & 0.17 & 0.15 & 0.33 & 0.18 \\
\hline
\end{tabular}


Table 1-5. Median seasonal concentrations for selected constituents at selected sites in North Dakota from 1970 through 2008 . Continued

[Winter, January-February, November-December; Spring, March-June; Summer, July-October; mg/L, milligrams per liter; --, not available; N, nitrogen; <. less than; P, phosphorus; C, carbon; $\mu \mathrm{g} / \mathrm{L}$, micrograms per liter]

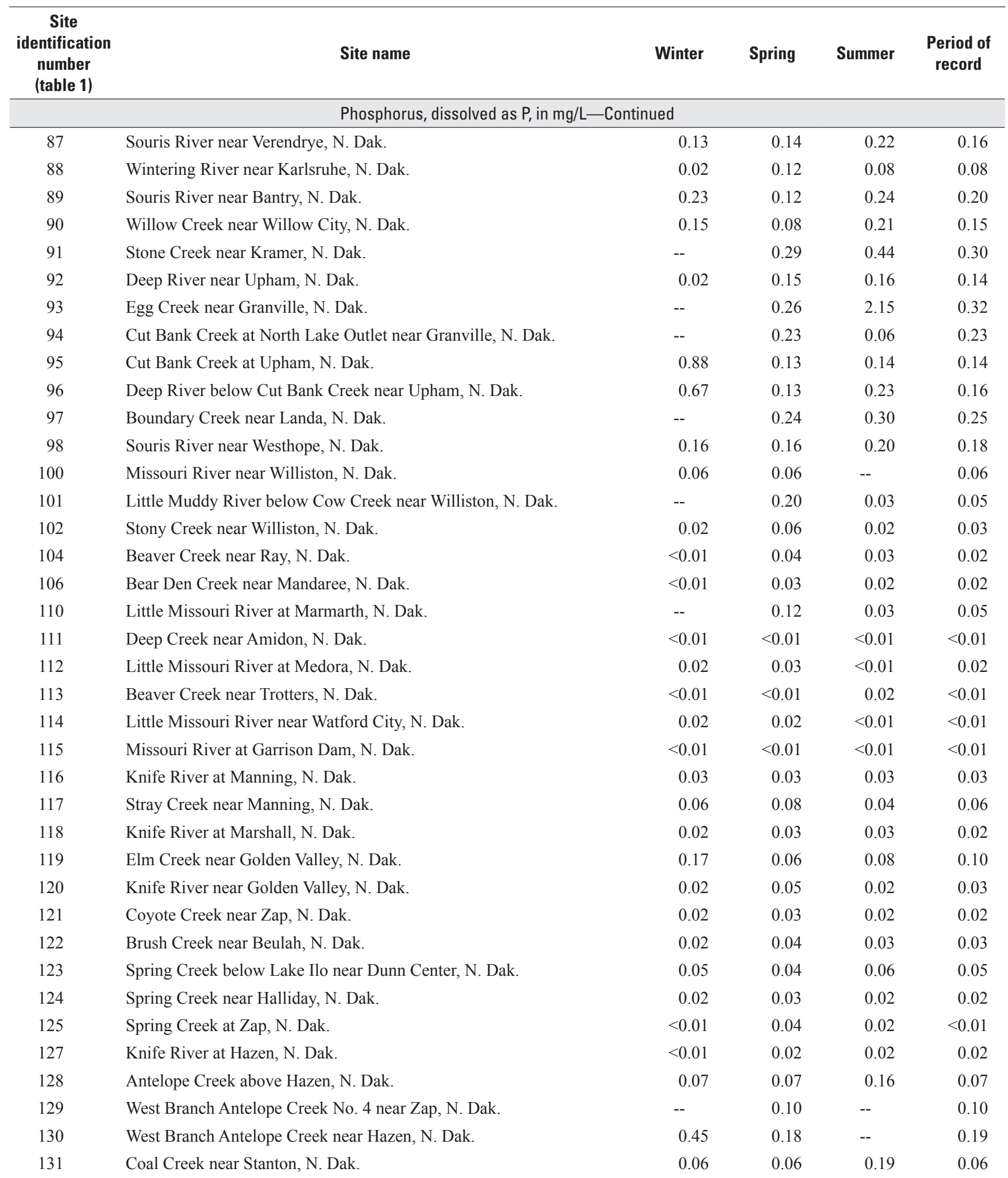


Table 1-5. Median seasonal concentrations for selected constituents at selected sites in North Dakota from 1970 through 2008.Continued

[Winter, January-February, November-December; Spring, March-June; Summer, July-October; mg/L, milligrams per liter; --, not available; N, nitrogen; <. less than; P, phosphorus; $\mathrm{C}$, carbon; $\mu \mathrm{g} / \mathrm{L}$, micrograms per liter]

\begin{tabular}{|c|c|c|c|c|c|}
\hline $\begin{array}{c}\text { Site } \\
\text { identification } \\
\text { number } \\
\text { (table 1) }\end{array}$ & Site name & Winter & Spring & Summer & $\begin{array}{c}\text { Period of } \\
\text { record }\end{array}$ \\
\hline \multicolumn{6}{|c|}{ Phosphorus, dissolved as $\mathrm{P}$, in $\mathrm{mg} / \mathrm{L}-$ Continued } \\
\hline 132 & Alderin Creek near Fort Clark, N. Dak. & 0.04 & 0.04 & 0.02 & 0.03 \\
\hline 133 & Coal Lake Coulee near Hensler, N. Dak. & 0.11 & 0.05 & 0.12 & 0.07 \\
\hline 136 & Painted Woods Creek near Wilton, N. Dak. & 0.07 & 0.10 & 0.05 & 0.07 \\
\hline 137 & Square Butte Creek near Hannover, N. Dak. & 0.03 & 0.05 & 0.08 & 0.05 \\
\hline 138 & Square Butte Creek above Nelson Lake near Center, N. Dak. & $<0.01$ & 0.02 & 0.02 & $<0.01$ \\
\hline 139 & Hagel Creek near Center, N. Dak. & 0.04 & 0.06 & 0.08 & 0.06 \\
\hline 145 & Heart River near South Heart, N. Dak. & 0.11 & 0.06 & 0.10 & 0.10 \\
\hline 147 & Green River near New Hradec, N. Dak. & 0.02 & 0.03 & 0.02 & 0.02 \\
\hline 149 & Heart River near Richardton, N. Dak. & $<0.01$ & 0.03 & $<0.01$ & $<0.01$ \\
\hline 156 & Heart River near Mandan, N. Dak. & $<0.01$ & $<0.01$ & $<0.01$ & $<0.01$ \\
\hline 157 & Long Lake Creek above Long Lake near Moffit, N. Dak. & -- & 0.16 & 0.71 & 0.22 \\
\hline 158 & Apple Creek near Menoken, N. Dak. & 0.25 & 0.22 & 0.58 & 0.28 \\
\hline 159 & Missouri River near Schmidt, N. Dak. & -- & & 0.03 & 0.03 \\
\hline 160 & Cannonball River at New England, N. Dak. & 0.02 & 0.02 & 0.02 & 0.02 \\
\hline 161 & Coal Bank Creek near Havelock, N. Dak. & 0.03 & 0.02 & 0.02 & 0.02 \\
\hline 174 & Buffalo Creek Tributary near Gascoyne, N. Dak. & 0.02 & 0.04 & 0.04 & 0.03 \\
\hline 175 & James River near Manfred, N. Dak. & 0.11 & 0.23 & 0.33 & 0.23 \\
\hline 176 & James River near Grace City, N. Dak. & 0.12 & 0.19 & 0.20 & 0.18 \\
\hline 177 & James River above Arrowwood Lake near Kensal, N. Dak. & 0.08 & 0.15 & 0.15 & 0.13 \\
\hline 178 & Kelly Creek below Niccum Reservoir near Bordulac, N. Dak. & -- & 0.04 & 0.26 & 0.07 \\
\hline 179 & James River near Pingree, N. Dak. & 0.05 & 0.09 & 0.17 & 0.11 \\
\hline 182 & James River at Jamestown, N. Dak. & 0.06 & 0.14 & 0.10 & 0.09 \\
\hline 183 & James River at Lamoure, N. Dak. & 0.09 & 0.13 & 0.15 & 0.13 \\
\hline 185 & James River at Oakes, N. Dak. & 0.10 & 0.10 & 0.14 & 0.12 \\
\hline 186 & James River at N. Dak./S. Dak. State line & 0.13 & 0.11 & 0.14 & 0.13 \\
\hline \multicolumn{6}{|c|}{ Phosphorus, total as $\mathrm{P}$, in $\mathrm{mg} / \mathrm{L}$} \\
\hline 1 & Bois De Sioux River near Doran, Minn. & 0.12 & 0.17 & 0.29 & 0.23 \\
\hline 2 & Red River of the North at Wahpeton, N. Dak. & 0.11 & 0.25 & 0.14 & 0.15 \\
\hline
\end{tabular}


Table 1-5. Median seasonal concentrations for selected constituents at selected sites in North Dakota from 1970 through 2008.Continued

[Winter, January-February, November-December; Spring, March-June; Summer, July-October; mg/L, milligrams per liter; --, not available; N, nitrogen; <. less than; P, phosphorus; C, carbon; $\mu \mathrm{g} / \mathrm{L}$, micrograms per liter]

\begin{tabular}{|c|c|c|c|c|c|}
\hline $\begin{array}{c}\text { Site } \\
\text { identification } \\
\text { number } \\
\text { (table 1) }\end{array}$ & Site name & Winter & Spring & Summer & $\begin{array}{c}\text { Period of } \\
\text { record }\end{array}$ \\
\hline \multicolumn{6}{|c|}{ Phosphorus, total as $\mathrm{P}$, in $\mathrm{mg} / \mathrm{L}$-Continued } \\
\hline 3 & Red River of the North near Wahpeton, N. Dak. & 0.04 & 0.14 & 0.02 & 0.09 \\
\hline 4 & Red River of the North at Brushville, Minn. & 0.09 & 0.13 & 0.17 & 0.16 \\
\hline 6 & Red River of the North at Hickson, N. Dak. & 0.09 & 0.19 & 0.18 & 0.18 \\
\hline 10 & Wild Rice River near Abercrombie, N. Dak. & 0.17 & 0.21 & 0.22 & 0.21 \\
\hline 11 & Red River of the North at Fargo, N. Dak. & 0.15 & 0.21 & 0.19 & 0.19 \\
\hline 12 & Red River of North below Fargo, N. Dak. & 0.42 & 0.47 & 0.62 & 0.47 \\
\hline 17 & Sheyenne River at Warwick, N. Dak. & 0.14 & 0.20 & 0.29 & 0.20 \\
\hline 18 & Sheyenne River near Warwick, N. Dak. & 0.08 & 0.16 & 0.32 & 0.21 \\
\hline 20 & Mauvais Coulee near Cando, N. Dak. & 0.32 & 0.31 & 0.46 & 0.33 \\
\hline 21 & Edmore Coulee near Edmore, N. Dak. & 0.26 & 0.37 & 0.44 & 0.40 \\
\hline 22 & Edmore Coulee Tributary near Webster, N. Dak. & -- & -- & 0.31 & 0.31 \\
\hline 24 & Starkweather Coulee near Webster, N. Dak. & -- & 0.33 & 0.24 & 0.29 \\
\hline 25 & Big Coulee below Churchs Ferry, N. Dak. & -- & 0.30 & 0.74 & 0.38 \\
\hline 28 & Big Coulee near Churchs Ferry, N. Dak. & 0.44 & 0.33 & 0.46 & 0.40 \\
\hline 30 & Channel A near Penn, N. Dak. & 0.10 & 0.29 & 0.33 & 0.30 \\
\hline 38 & Sheyenne River above Sheyenne River Diversion near Horace, N. Dak. & 0.26 & 0.26 & 0.15 & 0.22 \\
\hline 41 & Sheyenne River at West Fargo, N. Dak. & 0.14 & 0.24 & 0.29 & 0.24 \\
\hline 45 & Maple River below Mapleton, N. Dak. & 0.51 & 0.29 & 0.25 & 0.26 \\
\hline 46 & Sheyenne River at Harwood, N. Dak. & 0.16 & 0.39 & 0.42 & 0.38 \\
\hline 49 & Lower Branch Rush River near Prosper, N. Dak. & -- & 0.25 & -- & 0.25 \\
\hline 50 & Sheyenne River near Harwood, N. Dak. & 0.20 & 0.35 & 0.34 & 0.30 \\
\hline 51 & Elm River near Kelso, N. Dak. & -- & 0.39 & 0.70 & 0.51 \\
\hline 52 & Red River of the North at Halstad, Minn. & 0.18 & 0.30 & 0.31 & 0.28 \\
\hline 53 & Beaver Creek near Finley, N. Dak. & 0.11 & 0.17 & 0.24 & 0.18 \\
\hline 55 & Goose River at Hillsboro, N. Dak. & 0.09 & 0.08 & 0.13 & 0.09 \\
\hline 56 & Red River of the North at Grand Forks, N. Dak. & 0.13 & 0.21 & 0.18 & 0.18 \\
\hline 57 & Turtle River at Turtle River State Park near Arvilla, N. Dak. & 0.05 & 0.10 & 0.21 & 0.09 \\
\hline 58 & Turtle River at Manvel, N. Dak. & 0.07 & 0.15 & 0.17 & 0.15 \\
\hline
\end{tabular}


Table 1-5. Median seasonal concentrations for selected constituents at selected sites in North Dakota from 1970 through 2008.Continued

[Winter, January-February, November-December; Spring, March-June; Summer, July-October; mg/L, milligrams per liter; --, not available; N, nitrogen; <. less than; P, phosphorus; $\mathrm{C}$, carbon; $\mu \mathrm{g} / \mathrm{L}$, micrograms per liter]

\begin{tabular}{|c|c|c|c|c|c|}
\hline $\begin{array}{c}\text { Site } \\
\text { identification } \\
\text { number } \\
\text { (table 1) } \\
\end{array}$ & Site name & Winter & Spring & Summer & $\begin{array}{c}\text { Period of } \\
\text { record }\end{array}$ \\
\hline \multicolumn{6}{|c|}{ Phosphorus, total as $\mathrm{P}$, in $\mathrm{mg} / \mathrm{L}$-Continued } \\
\hline 59 & Red River of the North at Oslo, Minn. & 0.21 & 0.27 & 0.20 & 0.22 \\
\hline 62 & Forest River near Minto, N. Dak. & 0.08 & 0.12 & 0.13 & 0.11 \\
\hline 67 & Park River at Grafton, N. Dak. & 0.10 & 0.14 & 0.20 & 0.14 \\
\hline 68 & Red River of the North at Drayton, N. Dak. & 0.18 & 0.17 & 0.16 & 0.17 \\
\hline 73 & Little South Pembina River near Walhalla, N. Dak. & -- & 0.34 & 0.24 & 0.29 \\
\hline 74 & Pembina River at Walhalla, N. Dak. & 0.13 & 0.26 & 0.23 & 0.23 \\
\hline 75 & Pembina River at Neche, N. Dak. & 0.10 & 0.52 & 0.25 & 0.26 \\
\hline 76 & Tongue River at Akra, N. Dak. & 0.05 & 0.12 & 0.18 & 0.14 \\
\hline 77 & Red River of the North at Pembina, N. Dak., site 1 & 0.10 & 0.25 & 0.39 & 0.25 \\
\hline 78 & Red River of the North at Pembina, N. Dak., site 2 & 0.17 & 0.28 & 0.24 & 0.25 \\
\hline 79 & Red River of the North at Emerson, Manitoba & 0.12 & 0.23 & 0.20 & 0.19 \\
\hline 80 & Long Creek near Noonan, N. Dak. & 0.08 & 0.17 & 0.37 & 0.17 \\
\hline 81 & West Branch Short Creek near Columbus, N. Dak. & 0.15 & 0.12 & 0.49 & 0.17 \\
\hline 82 & Souris River near Sherwood, N. Dak. & 0.09 & 0.22 & 0.19 & 0.18 \\
\hline 83 & Souris River near Foxholm, N. Dak. & 0.11 & 0.27 & 0.36 & 0.24 \\
\hline 84 & Des Lacs River at Foxholm, N. Dak. & 0.16 & 0.24 & 0.40 & 0.27 \\
\hline 85 & Souris River above Minot, N. Dak. & 0.21 & 0.20 & 0.36 & 0.27 \\
\hline 87 & Souris River near Verendrye, N. Dak. & 0.17 & 0.26 & 0.36 & 0.27 \\
\hline 88 & Wintering River near Karlsruhe, N. Dak. & 0.04 & 0.11 & 0.13 & 0.09 \\
\hline 89 & Souris River near Bantry, N. Dak. & 0.16 & 0.19 & 0.34 & 0.26 \\
\hline 90 & Willow Creek near Willow City, N. Dak. & 0.20 & 0.16 & 0.25 & 0.20 \\
\hline 91 & Stone Creek near Kramer, N. Dak. & -- & 0.35 & 0.56 & 0.36 \\
\hline 92 & Deep River near Upham, N. Dak. & 0.06 & 0.13 & 0.23 & 0.17 \\
\hline 95 & Cut Bank Creek at Upham, N. Dak. & -- & 0.25 & 0.16 & 0.20 \\
\hline 96 & Deep River below Cut Bank Creek near Upham, N. Dak. & -- & 0.15 & 0.20 & 0.18 \\
\hline 97 & Boundary Creek near Landa, N. Dak. & -- & 0.25 & 0.48 & 0.27 \\
\hline 98 & Souris River near Westhope, N. Dak. & 0.21 & 0.25 & 0.31 & 0.27 \\
\hline 100 & Missouri River near Williston, N. Dak. & 0.04 & 0.20 & 0.11 & 0.10 \\
\hline 101 & Little Muddy River below Cow Creek near Williston, N. Dak. & -- & 0.26 & 0.06 & 0.09 \\
\hline 102 & Stony Creek near Williston, N. Dak. & 0.06 & 0.12 & 0.05 & 0.08 \\
\hline 104 & Beaver Creek near Ray, N. Dak. & 0.02 & 0.07 & 0.03 & 0.04 \\
\hline 106 & Bear Den Creek near Mandaree, N. Dak. & 0.03 & 0.08 & 0.07 & 0.06 \\
\hline 108 & East Fork Shell Creek near Parshall, N. Dak. & -- & 0.32 & 0.45 & 0.38 \\
\hline 109 & Deepwater Creek near Mandaree, N. Dak. & -- & 0.12 & 0.40 & 0.23 \\
\hline 110 & Little Missouri River at Marmarth, N. Dak. & -- & 0.58 & 0.05 & 0.28 \\
\hline 111 & Deep Creek near Amidon, N. Dak. & 0.03 & 0.05 & 0.02 & 0.03 \\
\hline 112 & Little Missouri River at Medora, N. Dak. & 0.04 & 0.31 & 0.06 & 0.11 \\
\hline 113 & Beaver Creek near Trotters, N. Dak. & 0.02 & 0.08 & 0.03 & 0.03 \\
\hline
\end{tabular}


Table 1-5. Median seasonal concentrations for selected constituents at selected sites in North Dakota from 1970 through 2008.Continued

[Winter, January-February, November-December; Spring, March-June; Summer, July-October; mg/L, milligrams per liter; --, not available; N, nitrogen; <. less than; P, phosphorus; C, carbon; $\mu \mathrm{g} / \mathrm{L}$, micrograms per liter]

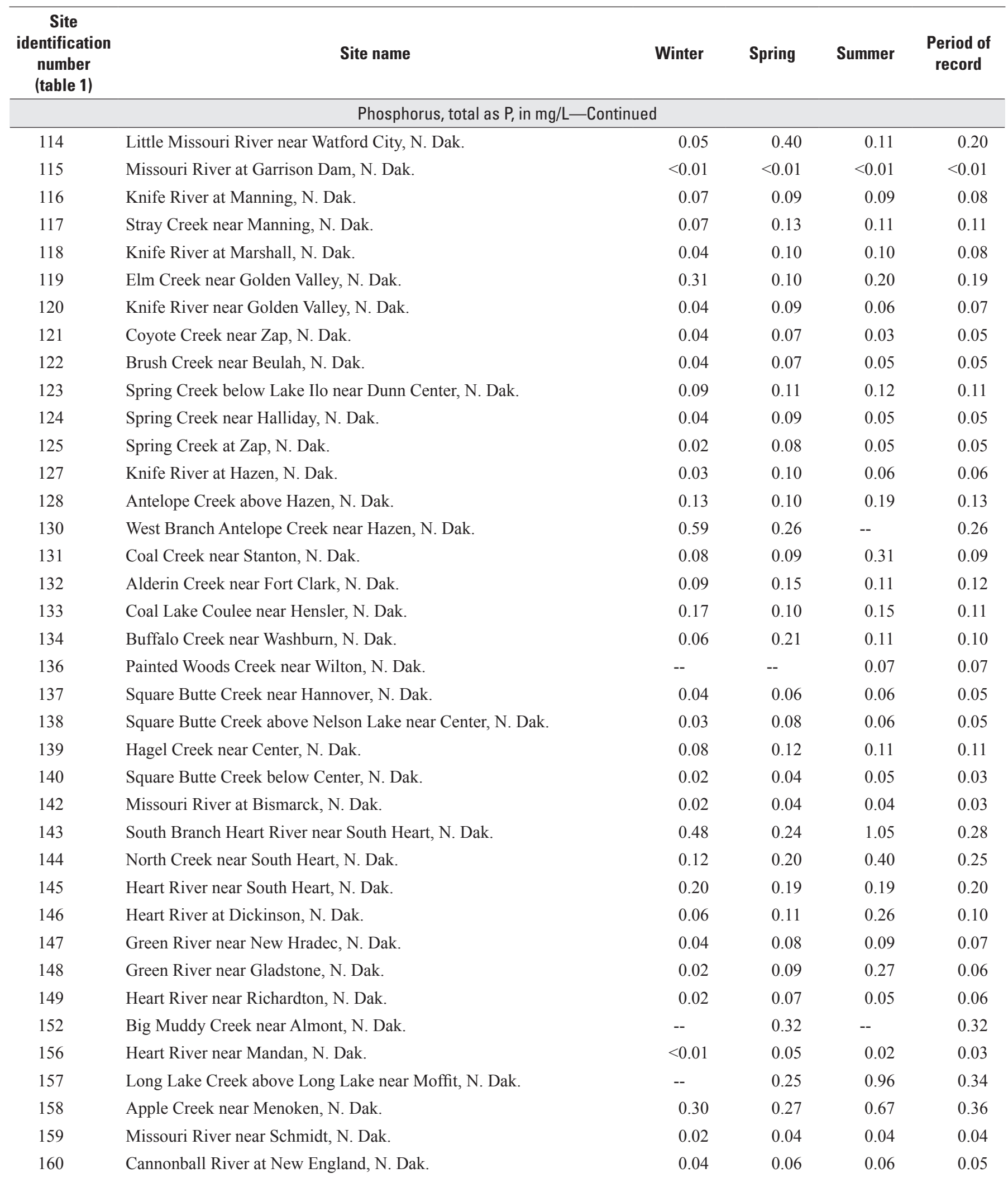


Table 1-5. Median seasonal concentrations for selected constituents at selected sites in North Dakota from 1970 through 2008.Continued

[Winter, January-February, November-December; Spring, March-June; Summer, July-October; mg/L, milligrams per liter; --, not available; N, nitrogen; <. less than; P, phosphorus; $\mathrm{C}$, carbon; $\mu \mathrm{g} / \mathrm{L}$, micrograms per liter]

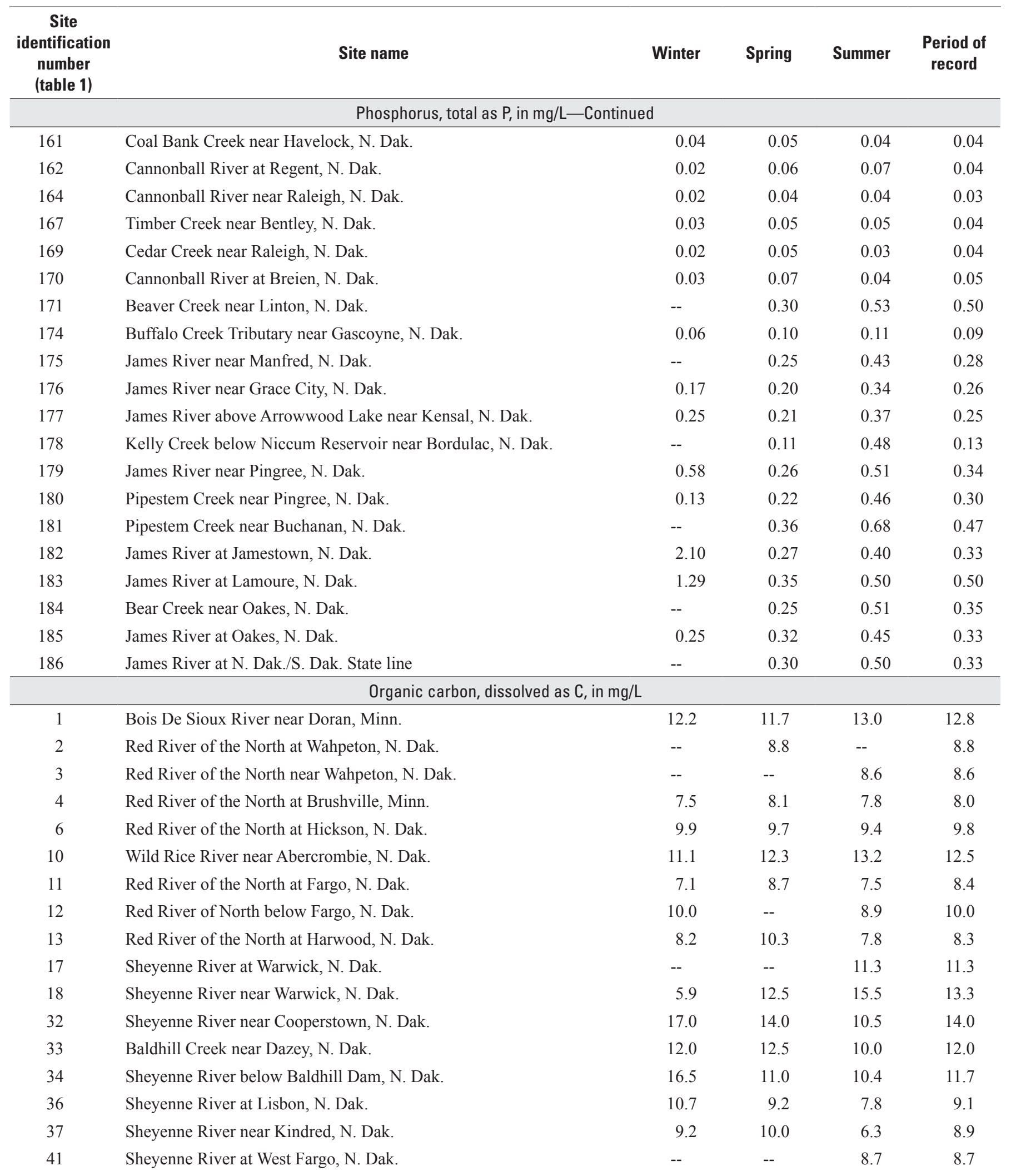


Table 1-5. Median seasonal concentrations for selected constituents at selected sites in North Dakota from 1970 through 2008.Continued

[Winter, January-February, November-December; Spring, March-June; Summer, July-October; mg/L, milligrams per liter; --, not available; N, nitrogen; <. less than; P, phosphorus; C, carbon; $\mu \mathrm{g} / \mathrm{L}$, micrograms per liter]

\begin{tabular}{|c|c|c|c|c|c|}
\hline $\begin{array}{c}\text { Site } \\
\text { identification } \\
\text { number } \\
\text { (table 1) } \\
\end{array}$ & Site name & Winter & Spring & Summer & $\begin{array}{c}\text { Period of } \\
\text { record }\end{array}$ \\
\hline \multicolumn{6}{|c|}{ Organic carbon, dissolved as $\mathrm{C}$, in $\mathrm{mg} / \mathrm{L}$-Continued } \\
\hline 45 & Maple River below Mapleton, N. Dak. & 12.3 & 12.0 & 10.2 & 10.5 \\
\hline 52 & Red River of the North at Halstad, Minn. & 10.0 & 10.4 & 10.0 & 10.0 \\
\hline 55 & Goose River at Hillsboro, N. Dak. & 8.0 & 10.3 & 8.5 & 8.6 \\
\hline 56 & Red River of the North at Grand Forks, N. Dak. & 8.0 & 9.0 & 8.8 & 8.8 \\
\hline 57 & Turtle River at Turtle River State Park near Arvilla, N. Dak. & 5.1 & 7.9 & 9.8 & 8.2 \\
\hline 58 & Turtle River at Manvel, N. Dak. & 5.5 & 8.6 & 8.7 & 8.5 \\
\hline 68 & Red River of the North at Drayton, N. Dak. & -- & 9.1 & -- & 9.1 \\
\hline 74 & Pembina River at Walhalla, N. Dak. & 8.4 & 12.0 & 9.6 & 11.0 \\
\hline 75 & Pembina River at Neche, N. Dak. & 4.7 & 8.1 & 6.6 & 7.2 \\
\hline 76 & Tongue River at Akra, N. Dak. & 12.2 & 20.5 & 14.1 & 16.0 \\
\hline 78 & Red River of the North at Pembina, N. Dak., site 2 & 9.1 & 8.9 & 11.0 & 10.0 \\
\hline 79 & Red River of the North at Emerson, Manitoba & 13.5 & 11.0 & 12.5 & 12.0 \\
\hline 81 & West Branch Short Creek near Columbus, N. Dak. & 40.0 & 24.0 & 23.0 & 24.0 \\
\hline 82 & Souris River near Sherwood, N. Dak. & 11.0 & 13.0 & 10.5 & 11.0 \\
\hline 83 & Souris River near Foxholm, N. Dak. & 41.5 & 16.0 & 14.5 & 16.0 \\
\hline 102 & Stony Creek near Williston, N. Dak. & 14.0 & 12.5 & 11.0 & 13.0 \\
\hline 104 & Beaver Creek near Ray, N. Dak. & 12.0 & 11.0 & 10.5 & 11.0 \\
\hline 106 & Bear Den Creek near Mandaree, N. Dak. & 13.0 & 24.0 & -- & 21.0 \\
\hline 111 & Deep Creek near Amidon, N. Dak. & 17.0 & 20.5 & 15.0 & 18.0 \\
\hline 112 & Little Missouri River at Medora, N. Dak. & 10.8 & 8.9 & 8.0 & 8.8 \\
\hline 113 & Beaver Creek near Trotters, N. Dak. & 11.5 & 13.0 & 11.0 & 12.0 \\
\hline 114 & Little Missouri River near Watford City, N. Dak. & 9.4 & 8.9 & 10.9 & 9.7 \\
\hline 115 & Missouri River at Garrison Dam, N. Dak. & 3.4 & 3.2 & 3.2 & 3.3 \\
\hline 116 & Knife River at Manning, N. Dak. & 16.5 & 14.5 & 15.5 & 15.0 \\
\hline 117 & Stray Creek near Manning, N. Dak. & 36.0 & 20.5 & 23.5 & 22.0 \\
\hline 118 & Knife River at Marshall, N. Dak. & 14.0 & 12.0 & 13.0 & 13.0 \\
\hline 119 & Elm Creek near Golden Valley, N. Dak. & 15.0 & 17.0 & 18.0 & 17.0 \\
\hline 120 & Knife River near Golden Valley, N. Dak. & 17.6 & 15.8 & 17.5 & 17.0 \\
\hline
\end{tabular}


Table 1-5. Median seasonal concentrations for selected constituents at selected sites in North Dakota from 1970 through 2008.Continued

[Winter, January-February, November-December; Spring, March-June; Summer, July-October; mg/L, milligrams per liter; --, not available; N, nitrogen; <. less than; P, phosphorus; $\mathrm{C}$, carbon; $\mu \mathrm{g} / \mathrm{L}$, micrograms per liter]

\begin{tabular}{|c|c|c|c|c|c|}
\hline $\begin{array}{c}\text { Site } \\
\text { identification } \\
\text { number } \\
\text { (table 1) }\end{array}$ & Site name & Winter & Spring & Summer & $\begin{array}{c}\text { Period of } \\
\text { record }\end{array}$ \\
\hline \multicolumn{6}{|c|}{ Organic carbon, dissolved as $\mathrm{C}$, in $\mathrm{mg} / \mathrm{L}$-Continued } \\
\hline 121 & Coyote Creek near Zap, N. Dak. & 13.0 & 13.0 & 12.0 & 12.0 \\
\hline 122 & Brush Creek near Beulah, N. Dak. & 12.0 & 12.0 & 9.9 & 11.0 \\
\hline 123 & Spring Creek below Lake Ilo near Dunn Center, N. Dak. & 22.0 & 12.5 & 18.0 & 18.0 \\
\hline 124 & Spring Creek near Halliday, N. Dak. & 12.5 & 15.0 & 14.0 & 13.5 \\
\hline 125 & Spring Creek at Zap, N. Dak. & 10.0 & 12.0 & 11.1 & 11.0 \\
\hline 127 & Knife River at Hazen, N. Dak. & 8.5 & 12.0 & 10.0 & 10.0 \\
\hline 128 & Antelope Creek above Hazen, N. Dak. & 18.0 & 16.5 & 13.0 & 16.5 \\
\hline 129 & West Branch Antelope Creek No. 4 near Zap, N. Dak. & -- & 9.5 & -- & 9.5 \\
\hline 130 & West Branch Antelope Creek near Hazen, N. Dak. & 26.0 & 17.0 & -- & 17.0 \\
\hline 131 & Coal Creek near Stanton, N. Dak. & 20.0 & 23.5 & 21.0 & 23.0 \\
\hline 132 & Alderin Creek near Fort Clark, N. Dak. & 27.5 & 18.5 & 22.5 & 23.5 \\
\hline 133 & Coal Lake Coulee near Hensler, N. Dak. & 22.5 & 16.0 & 28.0 & 18.0 \\
\hline 134 & Buffalo Creek near Washburn, N. Dak. & 20.0 & 19.0 & 21.0 & 20.0 \\
\hline 137 & Square Butte Creek near Hannover, N. Dak. & 15.0 & 12.5 & 18.0 & 14.0 \\
\hline 138 & Square Butte Creek above Nelson Lake near Center, N. Dak. & 9.7 & 10.0 & 11.5 & 10.0 \\
\hline 139 & Hagel Creek near Center, N. Dak. & 20.0 & 14.5 & 21.0 & 18.0 \\
\hline 142 & Missouri River at Bismarck, N. Dak. & 3.8 & 3.7 & 3.7 & 3.7 \\
\hline 143 & South Branch Heart River near South Heart, N. Dak. & 26.0 & 14.5 & 13.0 & 14.0 \\
\hline 144 & North Creek near South Heart, N. Dak. & 40.0 & 19.0 & 10.5 & 21.5 \\
\hline 145 & Heart River near South Heart, N. Dak. & 18.0 & 15.5 & 17.0 & 17.0 \\
\hline 147 & Green River near New Hradec, N. Dak. & 8.4 & 12.5 & 14.0 & 12.0 \\
\hline 149 & Heart River near Richardton, N. Dak. & 11.2 & 11.4 & 13.5 & 11.4 \\
\hline 156 & Heart River near Mandan, N. Dak. & 8.4 & 9.4 & 7.4 & 7.9 \\
\hline 158 & Apple Creek near Menoken, N. Dak. & 14.0 & 10.3 & 15.0 & 13.0 \\
\hline 159 & Missouri River near Schmidt, N. Dak. & 3.7 & 3.7 & 3.6 & 3.7 \\
\hline 160 & Cannonball River at New England, N. Dak. & 19.0 & 16.0 & 13.0 & 17.0 \\
\hline 161 & Coal Bank Creek near Havelock, N. Dak. & 15.0 & 16.0 & 17.0 & 16.0 \\
\hline 162 & Cannonball River at Regent, N. Dak. & 11.0 & 14.0 & 12.0 & 12.5 \\
\hline 164 & Cannonball River near Raleigh, N. Dak. & 23.3 & 15.0 & 9.3 & 12.3 \\
\hline 167 & Timber Creek near Bentley, N. Dak. & 16.0 & 21.0 & 13.0 & 15.0 \\
\hline 169 & Cedar Creek near Raleigh, N. Dak. & -- & 15.2 & 9.5 & 12.8 \\
\hline 170 & Cannonball River at Breien, N. Dak. & 9.9 & 9.6 & 9.3 & 9.6 \\
\hline 174 & Buffalo Creek Tributary near Gascoyne, N. Dak. & 36.5 & 28.0 & 25.0 & 30.0 \\
\hline 176 & James River near Grace City, N. Dak. & 12.2 & 19.1 & 19.5 & 17.9 \\
\hline 182 & James River at Jamestown, N. Dak. & 5.4 & 13.9 & 11.6 & 12.7 \\
\hline 183 & James River at Lamoure, N. Dak. & 7.5 & 12.2 & 10.8 & 11.4 \\
\hline \multicolumn{6}{|c|}{ Organic carbon, total as $\mathrm{C}$, in $\mathrm{mg} / \mathrm{L}$} \\
\hline 1 & Bois De Sioux River near Doran, Minn. & 12.2 & 11.0 & 13.0 & 11.5 \\
\hline
\end{tabular}


Table 1-5. Median seasonal concentrations for selected constituents at selected sites in North Dakota from 1970 through 2008.Continued

[Winter, January-February, November-December; Spring, March-June; Summer, July-October; mg/L, milligrams per liter; --, not available; N, nitrogen; <. less than; P, phosphorus; C, carbon; $\mu \mathrm{g} / \mathrm{L}$, micrograms per liter]

\begin{tabular}{|c|c|c|c|c|c|}
\hline $\begin{array}{c}\text { Site } \\
\text { identification } \\
\text { number } \\
\text { (table 1) } \\
\end{array}$ & Site name & Winter & Spring & Summer & $\begin{array}{c}\text { Period of } \\
\text { record }\end{array}$ \\
\hline \multicolumn{6}{|c|}{ Organic carbon, total as $\mathrm{C}$, in $\mathrm{mg} / \mathrm{L}-$ Continued } \\
\hline 3 & Red River of the North near Wahpeton, N. Dak. & -- & -- & 8.6 & 8.6 \\
\hline 4 & Red River of the North at Brushville, Minn. & 7.2 & 8.1 & 7.7 & 7.9 \\
\hline 10 & Wild Rice River near Abercrombie, N. Dak. & 10.8 & 11.7 & 12.8 & 12.0 \\
\hline 11 & Red River of the North at Fargo, N. Dak. & 6.8 & 8.0 & 7.5 & 7.8 \\
\hline 12 & Red River of North below Fargo, N. Dak. & 10.0 & 15.5 & 10.0 & 12.0 \\
\hline 13 & Red River of the North at Harwood, N. Dak. & 8.4 & 8.4 & 7.2 & 7.7 \\
\hline 34 & Sheyenne River below Baldhill Dam, N. Dak. & 12.3 & 10.7 & 10.3 & 10.6 \\
\hline 36 & Sheyenne River at Lisbon, N. Dak. & 11.5 & 9.3 & 7.3 & 8.0 \\
\hline 37 & Sheyenne River near Kindred, N. Dak. & 9.1 & 8.8 & 6.4 & 8.7 \\
\hline 41 & Sheyenne River at West Fargo, N. Dak. & -- & -- & 8.5 & 8.5 \\
\hline 45 & Maple River below Mapleton, N. Dak. & 12.8 & 11.4 & 10.0 & 11.0 \\
\hline 50 & Sheyenne River near Harwood, N. Dak. & -- & 17.0 & -- & 17.0 \\
\hline 52 & Red River of the North at Halstad, Minn. & 16.0 & 14.0 & 14.5 & 15.0 \\
\hline 53 & Beaver Creek near Finley, N. Dak. & -- & 18.0 & 17.0 & 17.0 \\
\hline 55 & Goose River at Hillsboro, N. Dak. & 8.0 & 9.6 & 8.6 & 8.6 \\
\hline 78 & Red River of the North at Pembina, N. Dak., site 2 & 8.4 & 8.4 & 7.9 & 8.0 \\
\hline 79 & Red River of the North at Emerson, Manitoba & 19.0 & 13.5 & 14.5 & 15.5 \\
\hline 82 & Souris River near Sherwood, N. Dak. & 15.3 & 16.6 & 15.7 & 16.0 \\
\hline 83 & Souris River near Foxholm, N. Dak. & 19.0 & 22.0 & 16.0 & 17.5 \\
\hline 84 & Des Lacs River at Foxholm, N. Dak. & 11.2 & 15.3 & 18.2 & 17.3 \\
\hline 85 & Souris River above Minot, N. Dak. & 21.9 & 16.0 & 18.5 & 17.8 \\
\hline 87 & Souris River near Verendrye, N. Dak. & 14.0 & 15.0 & 15.9 & 15.0 \\
\hline 89 & Souris River near Bantry, N. Dak. & 14.0 & 17.5 & 20.0 & 16.0 \\
\hline 90 & Willow Creek near Willow City, N. Dak. & -- & 17.0 & 23.0 & 19.0 \\
\hline 91 & Stone Creek near Kramer, N. Dak. & -- & 16.0 & 18.0 & 18.0 \\
\hline 97 & Boundary Creek near Landa, N. Dak. & -- & 17.0 & 22.0 & 17.5 \\
\hline 98 & Souris River near Westhope, N. Dak. & 22.0 & 16.0 & 22.1 & 21.0 \\
\hline 100 & Missouri River near Williston, N. Dak. & 4.5 & 7.0 & 2.9 & 6.0 \\
\hline
\end{tabular}


Table 1-5. Median seasonal concentrations for selected constituents at selected sites in North Dakota from 1970 through 2008. Continued

[Winter, January-February, November-December; Spring, March-June; Summer, July-October; mg/L, milligrams per liter; --, not available; N, nitrogen; <. less than; P, phosphorus; $\mathrm{C}$, carbon; $\mu \mathrm{g} / \mathrm{L}$, micrograms per liter]

\begin{tabular}{|c|c|c|c|c|c|}
\hline $\begin{array}{c}\text { Site } \\
\text { identification } \\
\text { number } \\
\text { (table 1) } \\
\end{array}$ & Site name & Winter & Spring & Summer & $\begin{array}{c}\text { Period of } \\
\text { record }\end{array}$ \\
\hline \multicolumn{6}{|c|}{ Organic carbon, total as $\mathrm{C}$, in $\mathrm{mg} / \mathrm{L}-$ Continued } \\
\hline 106 & Bear Den Creek near Mandaree, N. Dak. & 25.5 & 18.0 & 16.0 & 16.0 \\
\hline 108 & East Fork Shell Creek near Parshall, N. Dak. & -- & 25.0 & 24.0 & 24.0 \\
\hline 112 & Little Missouri River at Medora, N. Dak. & 10.6 & 7.1 & 7.8 & 7.5 \\
\hline 114 & Little Missouri River near Watford City, N. Dak. & 20.0 & 11.5 & 13.0 & 13.0 \\
\hline 115 & Missouri River at Garrison Dam, N. Dak. & 6.6 & 5.0 & 4.2 & 5.0 \\
\hline 120 & Knife River near Golden Valley, N. Dak. & 17.3 & 15.9 & 16.1 & 16.1 \\
\hline 142 & Missouri River at Bismarck, N. Dak. & 4.1 & 4.3 & 4.1 & 4.2 \\
\hline 149 & Heart River near Richardton, N. Dak. & 18.0 & 11.8 & 13.3 & 12.3 \\
\hline 156 & Heart River near Mandan, N. Dak. & 8.8 & 8.7 & 6.6 & 7.6 \\
\hline 159 & Missouri River near Schmidt, N. Dak. & 3.9 & 3.8 & 4.4 & 4.0 \\
\hline 164 & Cannonball River near Raleigh, N. Dak. & 22.7 & 11.7 & 8.4 & 10.2 \\
\hline 169 & Cedar Creek near Raleigh, N. Dak. & -- & 12.7 & 8.9 & 11.6 \\
\hline 170 & Cannonball River at Breien, N. Dak. & 14.0 & 11.0 & 10.5 & 12.0 \\
\hline 175 & James River near Manfred, N. Dak. & -- & 14.0 & 17.0 & 14.5 \\
\hline 176 & James River near Grace City, N. Dak. & 12.5 & 17.6 & 20.2 & 17.6 \\
\hline \multicolumn{6}{|c|}{ Aluminum, total, in $\mu \mathrm{g} / \mathrm{L}$} \\
\hline 1 & Bois De Sioux River near Doran, Minn. & 202 & 1,080 & 695 & 705 \\
\hline 3 & Red River of the North near Wahpeton, N. Dak. & -- & -- & 238 & 238 \\
\hline 4 & Red River of the North at Brushville, Minn. & 144 & 733 & 836 & 659 \\
\hline 10 & Wild Rice River near Abercrombie, N. Dak. & 369 & 2,180 & 1,745 & 1,530 \\
\hline 11 & Red River of the North at Fargo, N. Dak. & $<100$ & -- & -- & $<100$ \\
\hline 13 & Red River of the North at Harwood, N. Dak. & 250 & 3,250 & 3,010 & 2,800 \\
\hline 14 & Red River of the North near Harwood, N. Dak. & -- & 7,730 & 2,970 & 3,380 \\
\hline 17 & Sheyenne River at Warwick, N. Dak. & 234 & 961 & 853 & 688 \\
\hline 18 & Sheyenne River near Warwick, N. Dak. & 232 & 1,030 & 714 & 577 \\
\hline 32 & Sheyenne River near Cooperstown, N. Dak. & 264 & 1,370 & 906 & 831 \\
\hline 34 & Sheyenne River below Baldhill Dam, N. Dak. & $<100$ & 157 & 101 & 103 \\
\hline 36 & Sheyenne River at Lisbon, N. Dak. & 253 & 1,470 & 962 & 870 \\
\hline
\end{tabular}


Table 1-5. Median seasonal concentrations for selected constituents at selected sites in North Dakota from 1970 through 2008.Continued

[Winter, January-February, November-December; Spring, March-June; Summer, July-October; mg/L, milligrams per liter; --, not available; N, nitrogen; <. less than; P, phosphorus; C, carbon; $\mu \mathrm{g} / \mathrm{L}$, micrograms per liter]

\begin{tabular}{|c|c|c|c|c|c|}
\hline $\begin{array}{c}\text { Site } \\
\text { identification } \\
\text { number } \\
\text { (table 1) } \\
\end{array}$ & Site name & Winter & Spring & Summer & $\begin{array}{l}\text { Period of } \\
\text { record }\end{array}$ \\
\hline \multicolumn{6}{|c|}{ Aluminum, total, in $\mu \mathrm{g} / \mathrm{L}-$ Continued } \\
\hline 37 & Sheyenne River near Kindred, N. Dak. & 358 & 3,040 & 1,170 & 1,190 \\
\hline 38 & Sheyenne River above Sheyenne River Diversion near Horace, N. Dak. & -- & 8,470 & 2,560 & 3,140 \\
\hline 45 & Maple River below Mapleton, N. Dak. & 443 & 2,635 & 1,850 & 1,570 \\
\hline 52 & Red River of the North at Halstad, Minn. & -- & 8,910 & 2,910 & 4,720 \\
\hline 55 & Goose River at Hillsboro, N. Dak. & 161 & 914 & 1,004 & 777 \\
\hline 56 & Red River of the North at Grand Forks, N. Dak. & 228 & 2,410 & 1,660 & 1,440 \\
\hline 75 & Pembina River at Neche, N. Dak. & 156 & 6,840 & 1,330 & 1,255 \\
\hline 78 & Red River of the North at Pembina, N. Dak., site 2 & 304 & 5,135 & 2,565 & 2,565 \\
\hline 80 & Long Creek near Noonan, N. Dak. & 134 & 153 & 215 & 160 \\
\hline 82 & Souris River near Sherwood, N. Dak. & $<100$ & 179 & 346 & 154 \\
\hline 83 & Souris River near Foxholm, N. Dak. & 115 & $<100$ & $<100$ & $<100$ \\
\hline 84 & Des Lacs River at Foxholm, N. Dak. & 332 & 742 & 440 & 515 \\
\hline 85 & Souris River above Minot, N. Dak. & $<100$ & 125 & $<100$ & $<100$ \\
\hline 87 & Souris River near Verendrye, N. Dak. & $<100$ & 641 & 400 & 313 \\
\hline 88 & Wintering River near Karlsruhe, N. Dak. & $<100$ & 103 & 104 & 101 \\
\hline 101 & Little Muddy River below Cow Creek near Williston, N. Dak. & -- & 501 & 362 & 369 \\
\hline 110 & Little Missouri River at Marmarth, N. Dak. & -- & 52,500 & 2,680 & 20,050 \\
\hline 112 & Little Missouri River at Medora, N. Dak. & 1,575 & 18,200 & 3,055 & 6,430 \\
\hline 113 & Beaver Creek near Trotters, N. Dak. & -- & 185 & 150 & 185 \\
\hline 114 & Little Missouri River near Watford City, N. Dak. & 1,770 & 40,300 & 3,335 & 12,350 \\
\hline 120 & Knife River near Golden Valley, N. Dak. & 328 & 3,010 & 1,415 & 1,415 \\
\hline 125 & Spring Creek at Zap, N. Dak. & 129 & 433 & 242 & 215 \\
\hline 127 & Knife River at Hazen, N. Dak. & 145 & 1,410 & 484 & 484 \\
\hline 149 & Heart River near Richardton, N. Dak. & 107 & 645 & 590 & 528 \\
\hline 156 & Heart River near Mandan, N. Dak. & 115 & 547 & 237 & 272 \\
\hline 164 & Cannonball River near Raleigh, N. Dak. & 401 & 1,800 & 1,230 & 1,430 \\
\hline 169 & Cedar Creek near Raleigh, N. Dak. & 276 & 1,405 & 560 & 780 \\
\hline 170 & Cannonball River at Breien, N. Dak. & 437 & 2,200 & 902 & 1,230 \\
\hline
\end{tabular}


Table 1-5. Median seasonal concentrations for selected constituents at selected sites in North Dakota from 1970 through 2008. Continued

[Winter, January-February, November-December; Spring, March-June; Summer, July-October; mg/L, milligrams per liter; --, not available; N, nitrogen; <. less than; P, phosphorus; $\mathrm{C}$, carbon; $\mu \mathrm{g} / \mathrm{L}$, micrograms per liter]

\begin{tabular}{|c|c|c|c|c|c|}
\hline $\begin{array}{c}\text { Site } \\
\text { identification } \\
\text { number } \\
\text { (table 1) }\end{array}$ & Site name & Winter & Spring & Summer & $\begin{array}{c}\text { Period of } \\
\text { record }\end{array}$ \\
\hline \multicolumn{6}{|c|}{ Aluminum, total, in $\mu \mathrm{g} / \mathrm{L}-$ Continued } \\
\hline 175 & James River near Manfred, N. Dak. & -- & $<100$ & 207 & 100 \\
\hline 176 & James River near Grace City, N. Dak. & 138 & 286 & 375 & 269 \\
\hline 177 & James River above Arrowwood Lake near Kensal, N. Dak. & -- & 182 & 403 & 239 \\
\hline 180 & Pipestem Creek near Pingree, N. Dak. & -- & 186 & 282 & 210 \\
\hline 182 & James River at Jamestown, N. Dak. & 115 & 290 & 360 & 299 \\
\hline 183 & James River at Lamoure, N. Dak. & 207 & 1,015 & 961 & 767 \\
\hline 184 & Bear Creek near Oakes, N. Dak. & -- & 277 & 628 & 451 \\
\hline 186 & James River at N. Dak./S. Dak. State line & -- & 753 & 1,070 & 786 \\
\hline \multicolumn{6}{|c|}{ Arsenic, total, in $\mu \mathrm{g} / \mathrm{L}$} \\
\hline 1 & Bois De Sioux River near Doran, Minn. & 5.8 & 5.3 & 9.2 & 6.1 \\
\hline 2 & Red River of the North at Wahpeton, N. Dak. & -- & -- & 7.2 & 7.2 \\
\hline 3 & Red River of the North near Wahpeton, N. Dak. & -- & -- & 24.9 & 24.9 \\
\hline 4 & Red River of the North at Brushville, Minn. & 2.2 & 2.8 & 4.6 & 3.3 \\
\hline 5 & Red River of the North below Wahpeton, N. Dak. & 2.1 & 2.8 & 3.3 & 3.0 \\
\hline 6 & Red River of the North at Hickson, N. Dak. & 2.6 & 4.3 & 4.5 & 4.2 \\
\hline 10 & Wild Rice River near Abercrombie, N. Dak. & 6.1 & 6.9 & 11.0 & 7.4 \\
\hline 11 & Red River of the North at Fargo, N. Dak. & 3.6 & 4.2 & 3.9 & 3.8 \\
\hline 13 & Red River of the North at Harwood, N. Dak. & 2.5 & 4.1 & 5.9 & 5.2 \\
\hline 14 & Red River of the North near Harwood, N. Dak. & 2.4 & 4.9 & 5.1 & 5.1 \\
\hline 17 & Sheyenne River at Warwick, N. Dak. & 3.3 & 4.7 & 8.0 & 5.1 \\
\hline 18 & Sheyenne River near Warwick, N. Dak. & 4.1 & 5.6 & 8.4 & 6.0 \\
\hline 32 & Sheyenne River near Cooperstown, N. Dak. & 3.2 & 4.1 & 6.6 & 5.0 \\
\hline 33 & Baldhill Creek near Dazey, N. Dak. & -- & 3.1 & -- & 3.1 \\
\hline 34 & Sheyenne River below Baldhill Dam, N. Dak. & 5.9 & 3.3 & 5.4 & 4.8 \\
\hline 36 & Sheyenne River at Lisbon, N. Dak. & 5.4 & 4.6 & 6.5 & 5.9 \\
\hline 37 & Sheyenne River near Kindred, N. Dak. & 5.3 & 6.8 & 7.6 & 6.8 \\
\hline 38 & Sheyenne River above Sheyenne River Diversion near Horace, N. Dak. & -- & 9.5 & 7.7 & 8.1 \\
\hline 41 & Sheyenne River at West Fargo, N. Dak. & -- & 6.4 & 6.6 & 6.4 \\
\hline 45 & Maple River below Mapleton, N. Dak. & 5.6 & 6.8 & 10.1 & 7.9 \\
\hline 46 & Sheyenne River at Harwood, N. Dak. & 4.7 & 7.3 & 7.7 & 7.2 \\
\hline 52 & Red River of the North at Halstad, Minn. & -- & 5.6 & 6.3 & 6.2 \\
\hline 55 & Goose River at Hillsboro, N. Dak. & 3.2 & 4.6 & 7.5 & 5.1 \\
\hline 56 & Red River of the North at Grand Forks, N. Dak. & 2.1 & 4.4 & 5.3 & 4.3 \\
\hline 58 & Turtle River at Manvel, N. Dak. & 2.6 & 4.4 & 8.1 & 4.6 \\
\hline 62 & Forest River near Minto, N. Dak. & 2.2 & 3.6 & 5.7 & 3.7 \\
\hline 67 & Park River at Grafton, N. Dak. & 2.2 & 3.3 & 7.2 & 3.4 \\
\hline 68 & Red River of the North at Drayton, N. Dak. & -- & 4.7 & 3.1 & 3.8 \\
\hline 75 & Pembina River at Neche, N. Dak. & 1.8 & 8.6 & 5.7 & 5.4 \\
\hline
\end{tabular}


Table 1-5. Median seasonal concentrations for selected constituents at selected sites in North Dakota from 1970 through 2008.Continued

[Winter, January-February, November-December; Spring, March-June; Summer, July-October; mg/L, milligrams per liter; --, not available; N, nitrogen; <. less than; P, phosphorus; C, carbon; $\mu \mathrm{g} / \mathrm{L}$, micrograms per liter]

\begin{tabular}{|c|c|c|c|c|c|}
\hline $\begin{array}{c}\text { Site } \\
\text { identification } \\
\text { number } \\
\text { (table 1) }\end{array}$ & Site name & Winter & Spring & Summer & $\begin{array}{c}\text { Period of } \\
\text { record }\end{array}$ \\
\hline \multicolumn{6}{|c|}{ Arsenic, total, in $\mu \mathrm{g} / \mathrm{L}$-Continued } \\
\hline 78 & Red River of the North at Pembina, N. Dak., site 2 & 2.5 & 5.6 & 6.2 & 5.5 \\
\hline 80 & Long Creek near Noonan, N. Dak. & 3.2 & 4.7 & 8.7 & 6.0 \\
\hline 83 & Souris River near Foxholm, N. Dak. & 4.2 & 6.0 & 7.6 & 6.3 \\
\hline 84 & Des Lacs River at Foxholm, N. Dak. & 2.6 & 4.8 & 7.4 & 5.4 \\
\hline 85 & Souris River above Minot, N. Dak. & 4.6 & 4.8 & 8.3 & 6.0 \\
\hline 87 & Souris River near Verendrye, N. Dak. & 3.0 & 3.9 & 8.5 & 4.7 \\
\hline 91 & Stone Creek near Kramer, N. Dak. & -- & 5.0 & 7.2 & 5.2 \\
\hline 92 & Deep River near Upham, N. Dak. & 2.0 & 3.3 & 4.7 & 4.1 \\
\hline 95 & Cut Bank Creek at Upham, N. Dak. & -- & 3.1 & 3.4 & 3.4 \\
\hline 97 & Boundary Creek near Landa, N. Dak. & -- & 4.1 & 7.8 & 5.4 \\
\hline 98 & Souris River near Westhope, N. Dak. & 5.0 & 2.8 & 6.1 & 5.1 \\
\hline 101 & Little Muddy River below Cow Creek near Williston, N. Dak. & -- & 5.5 & 6.0 & 5.5 \\
\hline 110 & Little Missouri River at Marmarth, N. Dak. & -- & 19.8 & 2.6 & 8.2 \\
\hline 112 & Little Missouri River at Medora, N. Dak. & 2.5 & 8.7 & 3.3 & 4.0 \\
\hline 113 & Beaver Creek near Trotters, N. Dak. & -- & 1.9 & 1.2 & 1.6 \\
\hline 149 & Heart River near Richardton, N. Dak. & $<1.0$ & 2.2 & 2.4 & 2.0 \\
\hline 156 & Heart River near Mandan, N. Dak. & 1.1 & 1.5 & 1.2 & 1.3 \\
\hline 164 & Cannonball River near Raleigh, N. Dak. & $<1.0$ & 2.1 & 2.8 & 2.1 \\
\hline 169 & Cedar Creek near Raleigh, N. Dak. & 1.3 & 2.8 & 2.5 & 2.5 \\
\hline 170 & Cannonball River at Breien, N. Dak. & 1.4 & 2.6 & 2.1 & 2.2 \\
\hline 175 & James River near Manfred, N. Dak. & -- & 3.0 & 5.7 & 4.2 \\
\hline 176 & James River near Grace City, N. Dak. & 3.1 & 3.6 & 5.9 & 4.3 \\
\hline 177 & James River above Arrowwood Lake near Kensal, N. Dak. & -- & 2.9 & 5.2 & 3.7 \\
\hline 179 & James River near Pingree, N. Dak. & -- & 2.9 & 8.9 & 4.0 \\
\hline 180 & Pipestem Creek near Pingree, N. Dak. & -- & 4.0 & 8.8 & 5.3 \\
\hline 182 & James River at Jamestown, N. Dak. & 2.9 & 3.3 & 5.4 & 3.9 \\
\hline 183 & James River at Lamoure, N. Dak. & 3.5 & 4.0 & 6.3 & 4.9 \\
\hline 184 & Bear Creek near Oakes, N. Dak. & -- & 3.2 & 8.6 & 5.0 \\
\hline
\end{tabular}


Table 1-5. Median seasonal concentrations for selected constituents at selected sites in North Dakota from 1970 through 2008.Continued

[Winter, January-February, November-December; Spring, March-June; Summer, July-October; mg/L, milligrams per liter; --, not available; N, nitrogen; <. less than; P, phosphorus; $\mathrm{C}$, carbon; $\mu \mathrm{g} / \mathrm{L}$, micrograms per liter]

\begin{tabular}{|c|c|c|c|c|c|}
\hline $\begin{array}{c}\text { Site } \\
\text { identification } \\
\text { number } \\
\text { (table 1) }\end{array}$ & Site name & Winter & Spring & Summer & $\begin{array}{c}\text { Period of } \\
\text { record }\end{array}$ \\
\hline \multicolumn{6}{|c|}{ Arsenic, total, in $\mu \mathrm{g} / \mathrm{L}$-Continued } \\
\hline 185 & James River at Oakes, N. Dak. & -- & 2.0 & 5.0 & 3.0 \\
\hline 186 & James River at N. Dak./S. Dak. State line & -- & 3.5 & 6.0 & 4.1 \\
\hline 1 & Bois De Sioux River near Doran, Minn. & $<1.2$ & 1.4 & 1.3 & $<1.2$ \\
\hline 2 & Red River of the North at Wahpeton, N. Dak. & -- & -- & 1.8 & 1.8 \\
\hline 3 & Red River of the North near Wahpeton, N. Dak. & -- & -- & 1.3 & 1.3 \\
\hline 4 & Red River of the North at Brushville, Minn. & $<1.2$ & $<1.2$ & 1.4 & $<1.2$ \\
\hline 14 & Red River of the North near Harwood, N. Dak. & -- & 6.4 & 4.9 & 5.1 \\
\hline 17 & Sheyenne River at Warwick, N. Dak. & $<1.2$ & 1.5 & $<1.2$ & $<1.2$ \\
\hline 18 & Sheyenne River near Warwick, N. Dak. & $<1.2$ & 1.3 & $<1.2$ & $<1.2$ \\
\hline 32 & Sheyenne River near Cooperstown, N. Dak. & $<1.2$ & 1.8 & 1.4 & 1.3 \\
\hline 33 & Baldhill Creek near Dazey, N. Dak. & -- & $<1.2$ & -- & $<1.2$ \\
\hline 34 & Sheyenne River below Baldhill Dam, N. Dak. & $<1.2$ & $<1.2$ & $<1.2$ & $<1.2$ \\
\hline 36 & Sheyenne River at Lisbon, N. Dak. & $<1.2$ & 2.4 & 1.6 & 1.5 \\
\hline 37 & Sheyenne River near Kindred, N. Dak. & $<1.2$ & 4.6 & 1.8 & 1.8 \\
\hline 38 & Sheyenne River above Sheyenne River Diversion near Horace, N. Dak. & -- & 11.9 & 3.8 & 4.9 \\
\hline 62 & Forest River near Minto, N. Dak. & $<1.2$ & $<1.2$ & $<1.2$ & $<1.2$ \\
\hline 67 & Park River at Grafton, N. Dak. & $<1.2$ & 1.6 & $<1.2$ & $<1.2$ \\
\hline 68 & Red River of the North at Drayton, N. Dak. & -- & 4.2 & 3.6 & 3.6 \\
\hline 75 & Pembina River at Neche, N. Dak. & $<1.2$ & 15.8 & 2.4 & 2.4 \\
\hline 78 & Red River of the North at Pembina, N. Dak., site 2 & $<1.2$ & 8.7 & 4.5 & 4.5 \\
\hline 80 & Long Creek near Noonan, N. Dak. & $<1.2$ & $<1.2$ & $<1.2$ & $<1.2$ \\
\hline 82 & Souris River near Sherwood, N. Dak. & $<1.2$ & $<1.2$ & $<1.2$ & $<1.2$ \\
\hline 83 & Souris River near Foxholm, N. Dak. & $<1.2$ & $<1.2$ & $<1.2$ & $<1.2$ \\
\hline 84 & Des Lacs River at Foxholm, N. Dak. & $<1.2$ & $<1.2$ & $<1.2$ & $<1.2$ \\
\hline 85 & Souris River above Minot, N. Dak. & $<1.2$ & $<1.2$ & $<1.2$ & $<1.2$ \\
\hline 87 & Souris River near Verendrye, N. Dak. & $<1.2$ & $<1.2$ & $<1.2$ & $<1.2$ \\
\hline 88 & Wintering River near Karlsruhe, N. Dak. & $<1.2$ & $<1.2$ & $<1.2$ & $<1.2$ \\
\hline 89 & Souris River near Bantry, N. Dak. & $<1.2$ & $<1.2$ & $<1.2$ & $<1.2$ \\
\hline
\end{tabular}


Table 1-5. Median seasonal concentrations for selected constituents at selected sites in North Dakota from 1970 through 2008 . Continued

[Winter, January-February, November-December; Spring, March-June; Summer, July-October; mg/L, milligrams per liter; --, not available; N, nitrogen; <. less than; P, phosphorus; $\mathrm{C}$, carbon; $\mu \mathrm{g} / \mathrm{L}$, micrograms per liter]

\begin{tabular}{|c|c|c|c|c|c|}
\hline $\begin{array}{c}\text { Site } \\
\text { identification } \\
\text { number } \\
\text { (table 1) }\end{array}$ & Site name & Winter & Spring & Summer & $\begin{array}{c}\text { Period of } \\
\text { record }\end{array}$ \\
\hline \multicolumn{6}{|c|}{ Chromium, total, in $\mu \mathrm{g} / \mathrm{L}-$ Continued } \\
\hline 90 & Willow Creek near Willow City, N. Dak. & -- & $<1.2$ & $<1.2$ & $<1.2$ \\
\hline 91 & Stone Creek near Kramer, N. Dak. & -- & $<1.2$ & $<1.2$ & $<1.2$ \\
\hline 97 & Boundary Creek near Landa, N. Dak. & -- & $<1.2$ & $<1.2$ & $<1.2$ \\
\hline 98 & Souris River near Westhope, N. Dak. & 1.7 & 1.5 & $<1.2$ & $<1.2$ \\
\hline 101 & Little Muddy River below Cow Creek near Williston, N. Dak. & -- & $<1.2$ & $<1.2$ & $<1.2$ \\
\hline 110 & Little Missouri River at Marmarth, N. Dak. & -- & 54.9 & 3.4 & 21.0 \\
\hline 120 & Knife River near Golden Valley, N. Dak. & $<1.2$ & 3.4 & 2.2 & 2.2 \\
\hline 125 & Spring Creek at Zap, N. Dak. & $<1.2$ & $<1.2$ & $<1.2$ & $<1.2$ \\
\hline 127 & Knife River at Hazen, N. Dak. & $<1.2$ & 2.0 & $<1.2$ & $<1.2$ \\
\hline 149 & Heart River near Richardton, N. Dak. & $<1.2$ & $<1.2$ & $<1.2$ & $<1.2$ \\
\hline 156 & Heart River near Mandan, N. Dak. & $<1.2$ & $<1.2$ & $<1.2$ & $<1.2$ \\
\hline 164 & Cannonball River near Raleigh, N. Dak. & $<1.2$ & 2.3 & 1.7 & 1.8 \\
\hline 169 & Cedar Creek near Raleigh, N. Dak. & $<1.2$ & 2.2 & $<1.2$ & 1.4 \\
\hline 170 & Cannonball River at Breien, N. Dak. & $<1.2$ & 3.1 & 1.8 & 2.0 \\
\hline 175 & James River near Manfred, N. Dak. & -- & $<1.2$ & $<1.2$ & $<1.2$ \\
\hline 186 & James River at N. Dak./S. Dak. State line & -- & $<1.2$ & 1.6 & 1.3 \\
\hline \multicolumn{6}{|c|}{ Iron, total, in $\mu \mathrm{g} / \mathrm{L}$} \\
\hline 1 & Bois De Sioux River near Doran, Minn. & 356 & 1,200 & 1,020 & 955 \\
\hline 2 & Red River of the North at Wahpeton, N. Dak. & 310 & 657 & 421 & 400 \\
\hline 3 & Red River of the North near Wahpeton, N. Dak. & 302 & 1,038 & 444 & 505 \\
\hline 4 & Red River of the North at Brushville, Minn. & 288 & 908 & 1,110 & 806 \\
\hline 5 & Red River of the North below Wahpeton, N. Dak. & 262 & 1,370 & 1,170 & 1,011 \\
\hline 6 & Red River of the North at Hickson, N. Dak. & 320 & 3,885 & 2,420 & 2,540 \\
\hline 10 & Wild Rice River near Abercrombie, N. Dak. & 549 & 2,265 & 2,090 & 1,605 \\
\hline 11 & Red River of the North at Fargo, N. Dak. & 306 & 2,160 & 810 & 1,410 \\
\hline 13 & Red River of the North at Harwood, N. Dak. & 392 & 4,040 & 3,210 & 2,960 \\
\hline 14 & Red River of the North near Harwood, N. Dak. & 287 & 3,405 & 2,410 & 2,725 \\
\hline 17 & Sheyenne River at Warwick, N. Dak. & 366 & 932 & 987 & 701 \\
\hline
\end{tabular}


Table 1-5. Median seasonal concentrations for selected constituents at selected sites in North Dakota from 1970 through 2008.Continued

[Winter, January-February, November-December; Spring, March-June; Summer, July-October; mg/L, milligrams per liter; --, not available; N, nitrogen; <. less than; P, phosphorus; $\mathrm{C}$, carbon; $\mu \mathrm{g} / \mathrm{L}$, micrograms per liter]

\begin{tabular}{|c|c|c|c|c|c|}
\hline $\begin{array}{c}\text { Site } \\
\text { identification } \\
\text { number } \\
\text { (table 1) }\end{array}$ & Site name & Winter & Spring & Summer & $\begin{array}{c}\text { Period of } \\
\text { record }\end{array}$ \\
\hline \multicolumn{6}{|c|}{ Iron, total, in $\mu \mathrm{g} / \mathrm{L}$-Continued } \\
\hline 18 & Sheyenne River near Warwick, N. Dak. & 266 & 1,021 & 553 & 585 \\
\hline 24 & Starkweather Coulee near Webster, N. Dak. & -- & 2,900 & $<10$ & 1,455 \\
\hline 32 & Sheyenne River near Cooperstown, N. Dak. & 309 & 1,340 & 911 & 857 \\
\hline 33 & Baldhill Creek near Dazey, N. Dak. & -- & 161 & -- & 161 \\
\hline 34 & Sheyenne River below Baldhill Dam, N. Dak. & 91 & 202 & 113 & 134 \\
\hline 35 & Sheyenne River at Valley City, N. Dak. & -- & 647 & 690 & 691 \\
\hline 36 & Sheyenne River at Lisbon, N. Dak. & 332 & 1,855 & 991 & 1,010 \\
\hline 37 & Sheyenne River near Kindred, N. Dak. & 511 & 3,915 & 1,535 & 1,730 \\
\hline 38 & Sheyenne River above Sheyenne River Diversion near Horace, N. Dak. & -- & 2,751 & 2,800 & 2,800 \\
\hline 41 & Sheyenne River at West Fargo, N. Dak. & 558 & 3,625 & 1,620 & 1,620 \\
\hline 45 & Maple River below Mapleton, N. Dak. & 439 & 2,865 & 1,880 & 1,680 \\
\hline 46 & Sheyenne River at Harwood, N. Dak. & 502 & 4,940 & 3,920 & 3,160 \\
\hline 52 & Red River of the North at Halstad, Minn. & -- & 5,100 & 5,500 & 5,100 \\
\hline 55 & Goose River at Hillsboro, N. Dak. & 326 & 1,115 & 1,130 & 1,030 \\
\hline 56 & Red River of the North at Grand Forks, N. Dak. & 339 & 3,520 & 1,840 & 1,830 \\
\hline 58 & Turtle River at Manvel, N. Dak. & 243 & 1,395 & 702 & 742 \\
\hline 62 & Forest River near Minto, N. Dak. & 202 & 574 & 768 & 522 \\
\hline 67 & Park River at Grafton, N. Dak. & 146 & 798 & 624 & 578 \\
\hline 68 & Red River of the North at Drayton, N. Dak. & 1,210 & 2,920 & 1,405 & 1,810 \\
\hline 75 & Pembina River at Neche, N. Dak. & 179 & 5,850 & 1,130 & 1,200 \\
\hline 76 & Tongue River at Akra, N. Dak. & -- & 649 & -- & 649 \\
\hline 78 & Red River of the North at Pembina, N. Dak., site 2 & 375 & 6,790 & 3,275 & 3,220 \\
\hline 79 & Red River of the North at Emerson, Manitoba & -- & $<10$ & 11 & 11 \\
\hline 80 & Long Creek near Noonan, N. Dak. & 236 & 375 & 595 & 481 \\
\hline 82 & Souris River near Sherwood, N. Dak. & 327 & 713 & 672 & 554 \\
\hline 83 & Souris River near Foxholm, N. Dak. & 150 & 210 & 100 & 170 \\
\hline 84 & Des Lacs River at Foxholm, N. Dak. & 994 & 1,115 & 783 & 858 \\
\hline 85 & Souris River above Minot, N. Dak. & 427 & 332 & 213 & 305 \\
\hline 87 & Souris River near Verendrye, N. Dak. & 429 & 1,300 & 700 & 709 \\
\hline 88 & Wintering River near Karlsruhe, N. Dak. & 390 & 206 & 326 & 353 \\
\hline 89 & Souris River near Bantry, N. Dak. & 490 & 560 & 1,100 & 790 \\
\hline 90 & Willow Creek near Willow City, N. Dak. & 708 & 510 & 461 & 497 \\
\hline 91 & Stone Creek near Kramer, N. Dak. & -- & 250 & 152 & 226 \\
\hline 92 & Deep River near Upham, N. Dak. & 140 & 181 & 220 & 195 \\
\hline 95 & Cut Bank Creek at Upham, N. Dak. & -- & 50 & 44 & 50 \\
\hline 97 & Boundary Creek near Landa, N. Dak. & -- & 272 & 284 & 272 \\
\hline 98 & Souris River near Westhope, N. Dak. & 797 & 204 & 532 & 500 \\
\hline 101 & Little Muddy River below Cow Creek near Williston, N. Dak. & -- & 1,000 & 619 & 783 \\
\hline
\end{tabular}


Table 1-5. Median seasonal concentrations for selected constituents at selected sites in North Dakota from 1970 through 2008.Continued

[Winter, January-February, November-December; Spring, March-June; Summer, July-October; mg/L, milligrams per liter; --, not available; N, nitrogen; <. less than; P, phosphorus; C, carbon; $\mu \mathrm{g} / \mathrm{L}$, micrograms per liter]

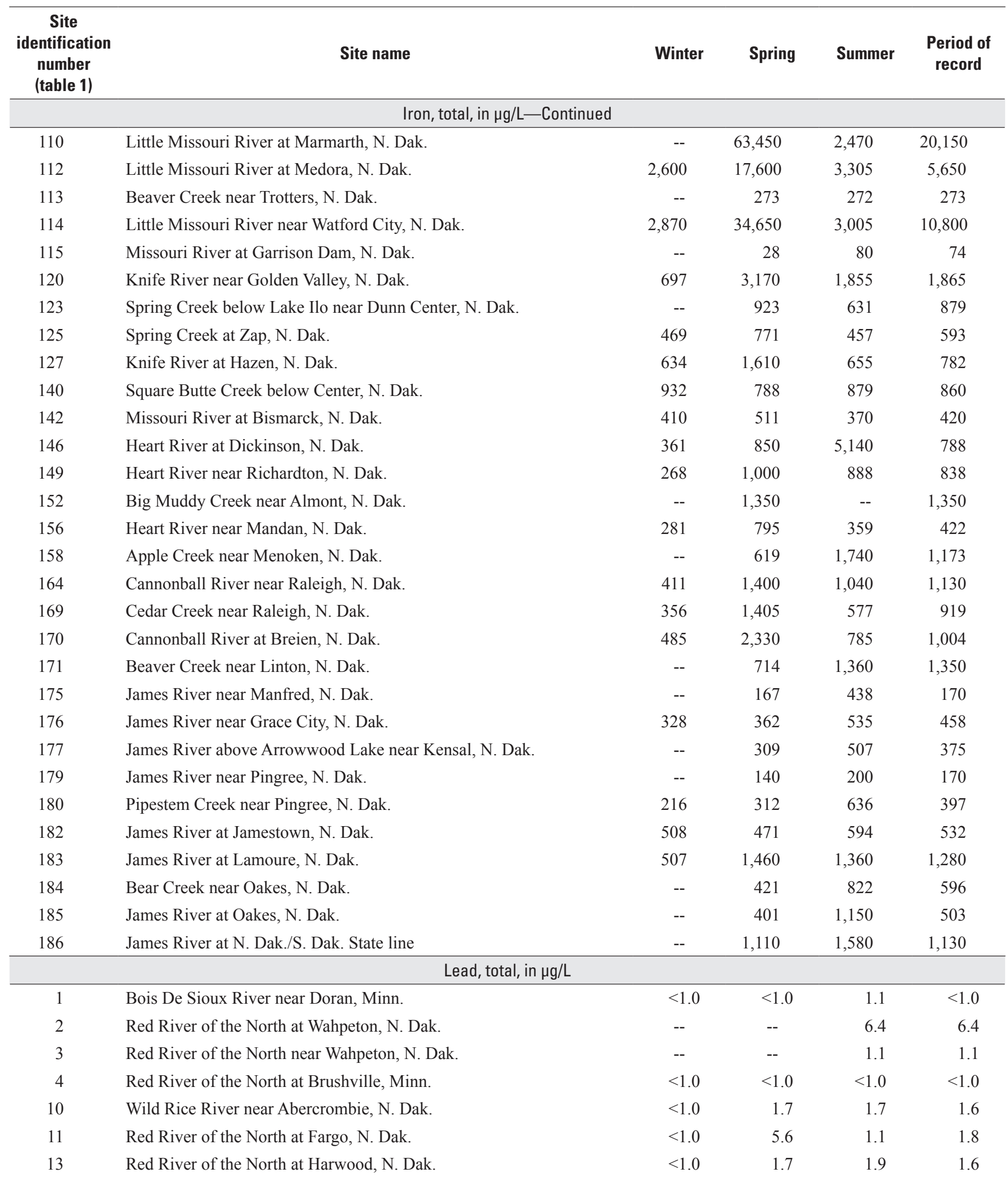


Table 1-5. Median seasonal concentrations for selected constituents at selected sites in North Dakota from 1970 through 2008.Continued

[Winter, January-February, November-December; Spring, March-June; Summer, July-October; mg/L, milligrams per liter; --, not available; N, nitrogen; <. less than; P, phosphorus; $\mathrm{C}$, carbon; $\mu \mathrm{g} / \mathrm{L}$, micrograms per liter]

\begin{tabular}{|c|c|c|c|c|c|}
\hline $\begin{array}{c}\text { Site } \\
\text { identification } \\
\text { number } \\
\text { (table 1) }\end{array}$ & Site name & Winter & Spring & Summer & $\begin{array}{c}\text { Period of } \\
\text { record }\end{array}$ \\
\hline \multicolumn{6}{|c|}{ Lead, total, in $\mu \mathrm{g} / \mathrm{L}$-Continued } \\
\hline 14 & Red River of the North near Harwood, N. Dak. & -- & 4.8 & 2.8 & 3.4 \\
\hline 17 & Sheyenne River at Warwick, N. Dak. & $<1.0$ & $<1.0$ & $<1.0$ & $<1.0$ \\
\hline 18 & Sheyenne River near Warwick, N. Dak. & $<1.0$ & $<1.0$ & $<1.0$ & $<1.0$ \\
\hline 32 & Sheyenne River near Cooperstown, N. Dak. & $<1.0$ & $<1.0$ & $<1.0$ & $<1.0$ \\
\hline 33 & Baldhill Creek near Dazey, N. Dak. & -- & 6.8 & -- & 6.8 \\
\hline 34 & Sheyenne River below Baldhill Dam, N. Dak. & $<1.0$ & $<1.0$ & $<1.0$ & $<1.0$ \\
\hline 36 & Sheyenne River at Lisbon, N. Dak. & $<1.0$ & 1.1 & $<1.0$ & $<1.0$ \\
\hline 37 & Sheyenne River near Kindred, N. Dak. & $<1.0$ & 3.4 & 1.3 & 1.4 \\
\hline 38 & Sheyenne River above Sheyenne River Diversion near Horace, N. Dak. & -- & 6.7 & 2.1 & 2.2 \\
\hline 41 & Sheyenne River at West Fargo, N. Dak. & -- & 8.3 & 2.8 & 4.8 \\
\hline 45 & Maple River below Mapleton, N. Dak. & $<1.0$ & 1.8 & 2.0 & 1.5 \\
\hline 52 & Red River of the North at Halstad, Minn. & -- & 5.3 & 3.4 & 3.4 \\
\hline 55 & Goose River at Hillsboro, N. Dak. & $<1.0$ & $<1.0$ & $<1.0$ & $<1.0$ \\
\hline 56 & Red River of the North at Grand Forks, N. Dak. & $<1.0$ & 1.6 & 1.1 & $<1.0$ \\
\hline 58 & Turtle River at Manvel, N. Dak. & $<1.0$ & 1.3 & $<1.0$ & $<1.0$ \\
\hline 62 & Forest River near Minto, N. Dak. & $<1.0$ & $<1.0$ & $<1.0$ & $<1.0$ \\
\hline 67 & Park River at Grafton, N. Dak. & $<1.0$ & 1.6 & $<1.0$ & $<1.0$ \\
\hline 68 & Red River of the North at Drayton, N. Dak. & -- & 4.5 & $<1.0$ & 2.5 \\
\hline 75 & Pembina River at Neche, N. Dak. & $<1.0$ & 7.7 & $<1.0$ & $<1.0$ \\
\hline 78 & Red River of the North at Pembina, N. Dak., site 2 & $<1.0$ & 3.3 & 1.7 & 1.4 \\
\hline 80 & Long Creek near Noonan, N. Dak. & 8.0 & 1.9 & 2.3 & 1.9 \\
\hline 82 & Souris River near Sherwood, N. Dak. & $<1.0$ & $<1.0$ & $<1.0$ & $<1.0$ \\
\hline 83 & Souris River near Foxholm, N. Dak. & 6.0 & $<1.0$ & $<1.0$ & $<1.0$ \\
\hline 84 & Des Lacs River at Foxholm, N. Dak. & $<1.0$ & 1.3 & $<1.0$ & $<1.0$ \\
\hline 85 & Souris River above Minot, N. Dak. & $<1.0$ & $<1.0$ & $<1.0$ & $<1.0$ \\
\hline 87 & Souris River near Verendrye, N. Dak. & $<1.0$ & $<1.0$ & $<1.0$ & $<1.0$ \\
\hline 88 & Wintering River near Karlsruhe, N. Dak. & 3.0 & 1.2 & 3.1 & 2.8 \\
\hline 89 & Souris River near Bantry, N. Dak. & $<1.0$ & $<1.0$ & 1.2 & $<1.0$ \\
\hline 90 & Willow Creek near Willow City, N. Dak. & -- & $<1.0$ & 1.6 & $<1.0$ \\
\hline 91 & Stone Creek near Kramer, N. Dak. & -- & $<1.0$ & $<1.0$ & $<1.0$ \\
\hline 92 & Deep River near Upham, N. Dak. & -- & 1.3 & 3.3 & 1.3 \\
\hline 97 & Boundary Creek near Landa, N. Dak. & -- & $<1.0$ & $<1.0$ & $<1.0$ \\
\hline 98 & Souris River near Westhope, N. Dak. & 2.0 & 1.9 & $<1.0$ & 1.1 \\
\hline 101 & Little Muddy River below Cow Creek near Williston, N. Dak. & -- & $<1.0$ & $<1.0$ & $<1.0$ \\
\hline 110 & Little Missouri River at Marmarth, N. Dak. & -- & 40.7 & 1.1 & 12.8 \\
\hline 112 & Little Missouri River at Medora, N. Dak. & 3.0 & 13.1 & 2.3 & 6.6 \\
\hline 113 & Beaver Creek near Trotters, N. Dak. & -- & $<1.0$ & $<1.0$ & $<1.0$ \\
\hline 114 & Little Missouri River near Watford City, N. Dak. & 4.0 & 32.0 & 3.3 & 12.4 \\
\hline
\end{tabular}


Table 1-5. Median seasonal concentrations for selected constituents at selected sites in North Dakota from 1970 through 2008.Continued

[Winter, January-February, November-December; Spring, March-June; Summer, July-October; mg/L, milligrams per liter; --, not available; N, nitrogen; <. less than; P, phosphorus; C, carbon; $\mu \mathrm{g} / \mathrm{L}$, micrograms per liter]

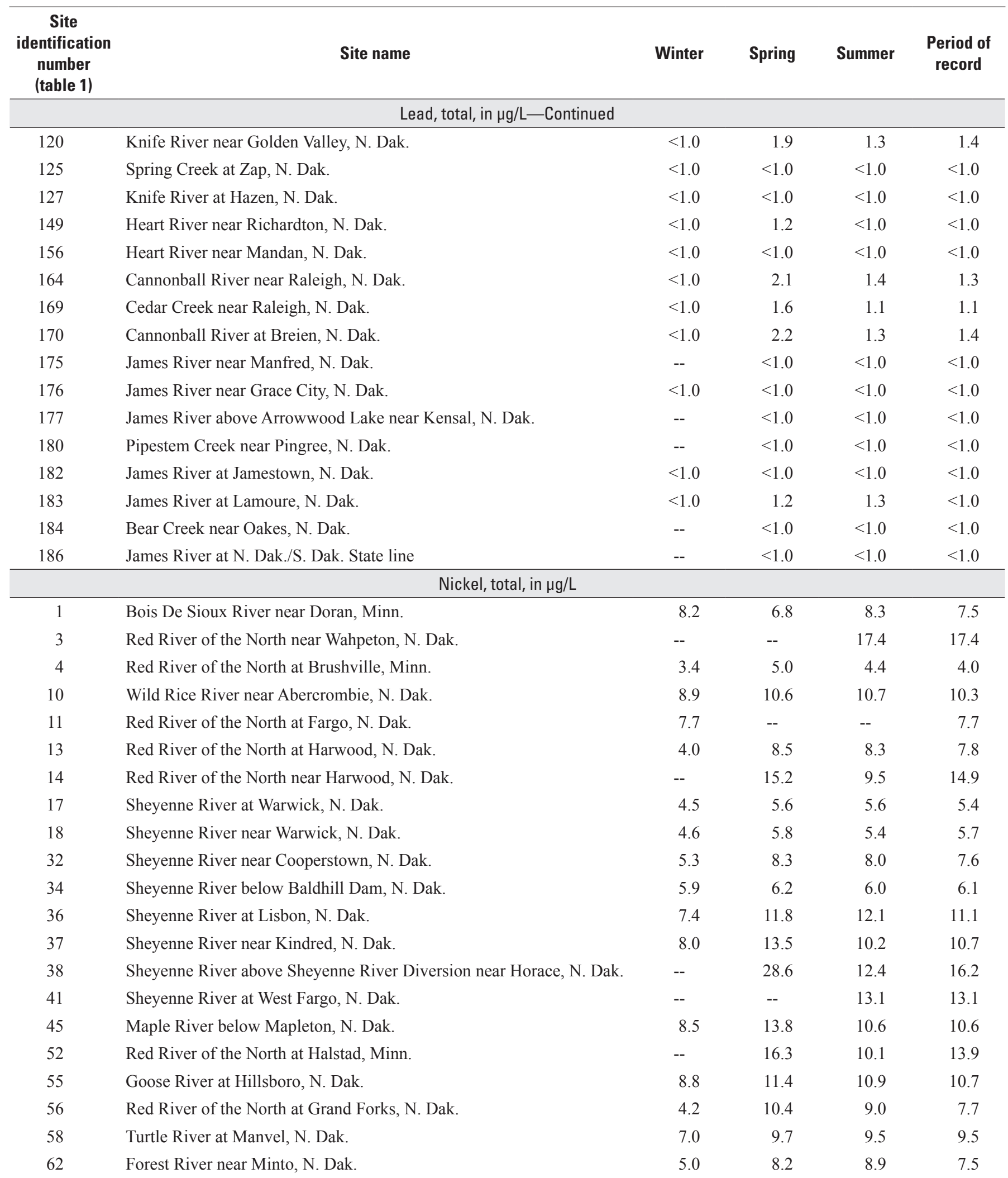


Table 1-5. Median seasonal concentrations for selected constituents at selected sites in North Dakota from 1970 through 2008.Continued

[Winter, January-February, November-December; Spring, March-June; Summer, July-October; mg/L, milligrams per liter; --, not available; N, nitrogen; <. less than; P, phosphorus; $\mathrm{C}$, carbon; $\mu \mathrm{g} / \mathrm{L}$, micrograms per liter]

\begin{tabular}{|c|c|c|c|c|c|}
\hline $\begin{array}{c}\text { Site } \\
\text { identification } \\
\text { number } \\
\text { (table 1) } \\
\end{array}$ & Site name & Winter & Spring & Summer & $\begin{array}{l}\text { Period of } \\
\text { record }\end{array}$ \\
\hline \multicolumn{6}{|c|}{ Nickel, total, in $\mu \mathrm{g} / \mathrm{L}$-Continued } \\
\hline 67 & Park River at Grafton, N. Dak. & 9.1 & 8.7 & 10.0 & 9.3 \\
\hline 75 & Pembina River at Neche, N. Dak. & 6.8 & 21.4 & 8.6 & 9.0 \\
\hline 78 & Red River of the North at Pembina, N. Dak., site 2 & 3.6 & 13.9 & 9.2 & 9.1 \\
\hline 80 & Long Creek near Noonan, N. Dak. & 5.1 & 5.6 & 5.2 & 5.3 \\
\hline 82 & Souris River near Sherwood, N. Dak. & 3.4 & 3.7 & 4.1 & 3.8 \\
\hline 83 & Souris River near Foxholm, N. Dak. & 9.1 & 3.7 & 3.4 & 4.0 \\
\hline 84 & Des Lacs River at Foxholm, N. Dak. & 7.7 & 6.8 & 6.5 & 6.9 \\
\hline 85 & Souris River above Minot, N. Dak. & 6.2 & 4.5 & 4.6 & 4.6 \\
\hline 87 & Souris River near Verendrye, N. Dak. & 5.1 & 5.8 & 5.5 & 5.5 \\
\hline 88 & Wintering River near Karlsruhe, N. Dak. & 2.8 & 3.2 & 2.9 & 2.8 \\
\hline 89 & Souris River near Bantry, N. Dak. & 3.0 & 3.5 & 5.0 & 3.6 \\
\hline 90 & Willow Creek near Willow City, N. Dak. & -- & 3.9 & 4.9 & 4.8 \\
\hline 91 & Stone Creek near Kramer, N. Dak. & -- & 2.5 & 4.0 & 3.0 \\
\hline 92 & Deep River near Upham, N. Dak. & -- & 4.0 & 4.2 & 4.1 \\
\hline 97 & Boundary Creek near Landa, N. Dak. & -- & 2.0 & 5.0 & 2.0 \\
\hline 98 & Souris River near Westhope, N. Dak. & 6.9 & 3.9 & 3.8 & 4.5 \\
\hline 101 & Little Muddy River below Cow Creek near Williston, N. Dak. & -- & 4.5 & 5.1 & 4.7 \\
\hline 110 & Little Missouri River at Marmarth, N. Dak. & -- & 66.5 & 8.8 & 26.0 \\
\hline 112 & Little Missouri River at Medora, N. Dak. & 10.7 & 27.3 & 11.1 & 13.6 \\
\hline 113 & Beaver Creek near Trotters, N. Dak. & -- & 6.3 & 3.9 & 4.2 \\
\hline 114 & Little Missouri River near Watford City, N. Dak. & 10.0 & 47.0 & 11.3 & 21.7 \\
\hline 120 & Knife River near Golden Valley, N. Dak. & 6.3 & 10.1 & 8.1 & 9.0 \\
\hline 125 & Spring Creek at Zap, N. Dak. & 4.8 & 6.0 & 5.1 & 5.4 \\
\hline 127 & Knife River at Hazen, N. Dak. & 5.6 & 8.7 & 6.4 & 7.0 \\
\hline 149 & Heart River near Richardton, N. Dak. & 6.8 & 7.6 & 7.9 & 7.6 \\
\hline 156 & Heart River near Mandan, N. Dak. & 5.3 & 6.6 & 4.8 & 5.3 \\
\hline 164 & Cannonball River near Raleigh, N. Dak. & 8.2 & 10.7 & 9.8 & 9.9 \\
\hline 169 & Cedar Creek near Raleigh, N. Dak. & 7.4 & 10.4 & 9.6 & 9.6 \\
\hline 170 & Cannonball River at Breien, N. Dak. & 7.4 & 13.1 & 7.6 & 9.2 \\
\hline 175 & James River near Manfred, N. Dak. & -- & 4.8 & 3.3 & 4.7 \\
\hline 176 & James River near Grace City, N. Dak. & 5.3 & 5.3 & 5.2 & 5.2 \\
\hline 177 & James River above Arrowwood Lake near Kensal, N. Dak. & -- & 4.1 & 3.9 & 3.9 \\
\hline 180 & Pipestem Creek near Pingree, N. Dak. & -- & 6.0 & 4.8 & 5.9 \\
\hline 182 & James River at Jamestown, N. Dak. & 6.0 & 6.2 & 6.0 & 6.1 \\
\hline 183 & James River at Lamoure, N. Dak. & 5.5 & 7.8 & 6.9 & 7.0 \\
\hline 184 & Bear Creek near Oakes, N. Dak. & -- & 6.1 & 5.9 & 6.0 \\
\hline 186 & James River at N. Dak./S. Dak. State line & -- & 6.3 & 6.0 & 6.3 \\
\hline
\end{tabular}


Table 1-5. Median seasonal concentrations for selected constituents at selected sites in North Dakota from 1970 through 2008.Continued

[Winter, January-February, November-December; Spring, March-June; Summer, July-October; mg/L, milligrams per liter; --, not available; N, nitrogen; <. less than; P, phosphorus; C, carbon; $\mu \mathrm{g} / \mathrm{L}$, micrograms per liter]

\begin{tabular}{|c|c|c|c|c|c|}
\hline $\begin{array}{c}\text { Site } \\
\text { identification } \\
\text { number } \\
\text { (table 1) }\end{array}$ & Site name & Winter & Spring & Summer & $\begin{array}{c}\text { Period of } \\
\text { record }\end{array}$ \\
\hline \multicolumn{6}{|c|}{ Total suspended solids, in $\mathrm{mg} / \mathrm{L}$} \\
\hline 1 & Bois De Sioux River near Doran, Minn. & 11 & 36 & 46 & 36 \\
\hline 2 & Red River of the North at Wahpeton, N. Dak. & -- & 128 & -- & 128 \\
\hline 4 & Red River of the North at Brushville, Minn. & $<10$ & 38 & 46 & 36 \\
\hline 10 & Wild Rice River near Abercrombie, N. Dak. & 16 & 92 & 80 & 65 \\
\hline 11 & Red River of the North at Fargo, N. Dak. & $<10$ & 132 & 69 & 68 \\
\hline 12 & Red River of North below Fargo, N. Dak. & $<10$ & 70 & 41 & 41 \\
\hline 18 & Sheyenne River near Warwick, N. Dak. & $<10$ & 24 & 17 & 19 \\
\hline 20 & Mauvais Coulee near Cando, N. Dak. & -- & $<10$ & $<10$ & $<10$ \\
\hline 21 & Edmore Coulee near Edmore, N. Dak. & -- & $<10$ & -- & $<10$ \\
\hline 24 & Starkweather Coulee near Webster, N. Dak. & -- & $<10$ & 472 & $<10$ \\
\hline 28 & Big Coulee near Churchs Ferry, N. Dak. & -- & 49 & 99 & 53 \\
\hline 30 & Channel A near Penn, N. Dak. & -- & 24 & $<10$ & 14 \\
\hline 32 & Sheyenne River near Cooperstown, N. Dak. & $<10$ & 48 & 32 & 34 \\
\hline 33 & Baldhill Creek near Dazey, N. Dak. & & 19 & $<10$ & 17 \\
\hline 34 & Sheyenne River below Baldhill Dam, N. Dak. & $<10$ & 12 & $<10$ & $<10$ \\
\hline 51 & Elm River near Kelso, N. Dak. & -- & -- & 46 & 46 \\
\hline 52 & Red River of the North at Halstad, Minn. & 27 & 184 & 137 & 157 \\
\hline 53 & Beaver Creek near Finley, N. Dak. & 22 & 13 & 25 & 14 \\
\hline 55 & Goose River at Hillsboro, N. Dak. & $<10$ & 43 & 44 & 40 \\
\hline 56 & Red River of the North at Grand Forks, N. Dak. & $<10$ & 170 & 69 & 79 \\
\hline 58 & Turtle River at Manvel, N. Dak. & $<10$ & 55 & 33 & 34 \\
\hline 62 & Forest River near Minto, N. Dak. & $<10$ & 37 & 19 & 19 \\
\hline 67 & Park River at Grafton, N. Dak. & $<10$ & 36 & 22 & 23 \\
\hline 68 & Red River of the North at Drayton, N. Dak. & -- & 112 & 58 & 58 \\
\hline 75 & Pembina River at Neche, N. Dak. & $<10$ & 719 & 72 & 83 \\
\hline 77 & Red River of the North at Pembina, N. Dak., site 1 & 25 & 140 & 123 & 90 \\
\hline 78 & Red River of the North at Pembina, N. Dak., site 2 & 12 & 217 & 122 & 139 \\
\hline 80 & Long Creek near Noonan, N. Dak. & 11 & $<10$ & 19 & 14 \\
\hline
\end{tabular}


Table 1-5. Median seasonal concentrations for selected constituents at selected sites in North Dakota from 1970 through 2008 . Continued

[Winter, January-February, November-December; Spring, March-June; Summer, July-October; mg/L, milligrams per liter; --, not available; N, nitrogen; <. less than; $\mathrm{P}$, phosphorus; $\mathrm{C}$, carbon; $\mu \mathrm{g} / \mathrm{L}$, micrograms per liter]

\begin{tabular}{|c|c|c|c|c|c|}
\hline $\begin{array}{c}\text { Site } \\
\text { identification } \\
\text { number } \\
\text { (table 1) } \\
\end{array}$ & Site name & Winter & Spring & Summer & $\begin{array}{l}\text { Period of } \\
\text { record }\end{array}$ \\
\hline \multicolumn{6}{|c|}{ Total suspended solids, in mg/L-Continued } \\
\hline 82 & Souris River near Sherwood, N. Dak. & $<10$ & 19 & 18 & 13 \\
\hline 83 & Souris River near Foxholm, N. Dak. & $<10$ & $<10$ & $<10$ & $<10$ \\
\hline 85 & Souris River above Minot, N. Dak. & $<10$ & $<10$ & $<10$ & $<10$ \\
\hline 87 & Souris River near Verendrye, N. Dak. & $<10$ & 34 & 23 & 20 \\
\hline 88 & Wintering River near Karlsruhe, N. Dak. & $<10$ & $<10$ & $<10$ & $<10$ \\
\hline 89 & Souris River near Bantry, N. Dak. & $<10$ & 17 & 35 & 16 \\
\hline 96 & Deep River below Cut Bank Creek near Upham, N. Dak. & -- & -- & 15 & 15 \\
\hline 97 & Boundary Creek near Landa, N. Dak. & -- & 11 & $<10$ & 11 \\
\hline 98 & Souris River near Westhope, N. Dak. & $<10$ & $<10$ & 18 & 12 \\
\hline 100 & Missouri River near Williston, N. Dak. & 22 & -- & 77 & 58 \\
\hline 101 & Little Muddy River below Cow Creek near Williston, N. Dak. & -- & 26 & 19 & 24 \\
\hline 106 & Bear Den Creek near Mandaree, N. Dak. & 33 & 180 & 66 & 48 \\
\hline 110 & Little Missouri River at Marmarth, N. Dak. & -- & 2,212 & 81 & 257 \\
\hline 112 & Little Missouri River at Medora, N. Dak. & 74 & 643 & 96 & 198 \\
\hline 113 & Beaver Creek near Trotters, N. Dak. & -- & $<10$ & $<10$ & $<10$ \\
\hline 127 & Knife River at Hazen, N. Dak. & $<10$ & 47 & 18 & 19 \\
\hline 128 & Antelope Creek above Hazen, N. Dak. & 23 & $<10$ & 20 & 16 \\
\hline 129 & West Branch Antelope Creek No. 4 near Zap, N. Dak. & 28 & 13 & $<10$ & $<10$ \\
\hline 132 & Alderin Creek near Fort Clark, N. Dak. & 67 & 38 & 49 & 49 \\
\hline 133 & Coal Lake Coulee near Hensler, N. Dak. & $<10$ & 29 & $<10$ & 25 \\
\hline 142 & Missouri River at Bismarck, N. Dak. & 17 & -- & 56 & 37 \\
\hline 147 & Green River near New Hradec, N. Dak. & -- & 93 & -- & 93 \\
\hline 149 & Heart River near Richardton, N. Dak. & $<10$ & 32 & 25 & 25 \\
\hline 156 & Heart River near Mandan, N. Dak. & $<10$ & 22 & $<10$ & 12 \\
\hline 157 & Long Lake Creek above Long Lake near Moffit, N. Dak. & -- & 14 & 35 & 17 \\
\hline 158 & Apple Creek near Menoken, N. Dak. & -- & 30 & 36 & 32 \\
\hline 159 & Missouri River near Schmidt, N. Dak. & 11 & 31 & 23 & 23 \\
\hline 161 & Coal Bank Creek near Havelock, N. Dak. & -- & -- & $<10$ & $<10$ \\
\hline
\end{tabular}


Table 1-5. Median seasonal concentrations for selected constituents at selected sites in North Dakota from 1970 through 2008.Continued

[Winter, January-February, November-December; Spring, March-June; Summer, July-October; mg/L, milligrams per liter; --, not available; N, nitrogen; <. less than; P, phosphorus; $\mathrm{C}$, carbon; $\mu \mathrm{g} / \mathrm{L}$, micrograms per liter]

\begin{tabular}{|c|c|c|c|c|c|}
\hline $\begin{array}{c}\text { Site } \\
\text { identification } \\
\text { number } \\
\text { (table 1) } \\
\end{array}$ & Site name & Winter & Spring & Summer & $\begin{array}{c}\text { Period of } \\
\text { record }\end{array}$ \\
\hline \multicolumn{6}{|c|}{ Total suspended solids, in mg/L_Continued } \\
\hline 164 & Cannonball River near Raleigh, N. Dak. & 13 & 47 & 43 & 39 \\
\hline 169 & Cedar Creek near Raleigh, N. Dak. & 11 & 48 & 26 & 31 \\
\hline 170 & Cannonball River at Breien, N. Dak. & 19 & 78 & 24 & 35 \\
\hline 174 & Buffalo Creek Tributary near Gascoyne, N. Dak. & -- & -- & 125 & 125 \\
\hline 175 & James River near Manfred, N. Dak. & $<10$ & $<10$ & $<10$ & $<10$ \\
\hline 176 & James River near Grace City, N. Dak. & 11 & 14 & 17 & 13 \\
\hline 177 & James River above Arrowwood Lake near Kensal, N. Dak. & 11 & 16 & 29 & 16 \\
\hline 178 & Kelly Creek below Niccum Reservoir near Bordulac, N. Dak. & -- & 11 & 45 & 11 \\
\hline 179 & James River near Pingree, N. Dak. & $<10$ & 21 & 38 & 28 \\
\hline 180 & Pipestem Creek near Pingree, N. Dak. & -- & 11 & 39 & 26 \\
\hline 182 & James River at Jamestown, N. Dak. & $<10$ & 19 & 26 & 17 \\
\hline 183 & James River at Lamoure, N. Dak. & $<10$ & 56 & 54 & 42 \\
\hline 184 & Bear Creek near Oakes, N. Dak. & -- & 16 & 31 & 18 \\
\hline 185 & James River at Oakes, N. Dak. & 11 & 42 & 77 & 43 \\
\hline 186 & James River at N. Dak./S. Dak. State line & -- & 60 & 93 & 62 \\
\hline \multicolumn{6}{|c|}{ Suspended-sediment concentration, in $\mathrm{mg} / \mathrm{L}$} \\
\hline 5 & Red River of the North below Wahpeton, N. Dak. & 10 & 62 & 49 & 49 \\
\hline 6 & Red River of the North at Hickson, N. Dak. & 10 & 108 & 91 & 72 \\
\hline 10 & Wild Rice River near Abercrombie, N. Dak. & 24 & 44 & 51 & 42 \\
\hline 11 & Red River of the North at Fargo, N. Dak. & 10 & 177 & 103 & 115 \\
\hline 12 & Red River of North below Fargo, N. Dak. & 13 & 114 & 61 & 43 \\
\hline 14 & Red River of the North near Harwood, N. Dak. & 12 & 179 & 179 & 164 \\
\hline 18 & Sheyenne River near Warwick, N. Dak. & -- & 21 & 95 & 58 \\
\hline 24 & Starkweather Coulee near Webster, N. Dak. & -- & 394 & 6 & 200 \\
\hline 34 & Sheyenne River below Baldhill Dam, N. Dak. & -- & 17 & 17 & 17 \\
\hline 36 & Sheyenne River at Lisbon, N. Dak. & 14 & 169 & 48 & 102 \\
\hline 37 & Sheyenne River near Kindred, N. Dak. & 35 & 105 & 74 & 74 \\
\hline 44 & Maple River near Mapleton, N. Dak. & -- & -- & 120 & 120 \\
\hline 46 & Sheyenne River at Harwood, N. Dak. & 30 & 296 & 198 & 225 \\
\hline 52 & Red River of the North at Halstad, Minn. & 16 & 169 & 107 & 76 \\
\hline 53 & Beaver Creek near Finley, N. Dak. & 23 & 40 & 16 & 24 \\
\hline 55 & Goose River at Hillsboro, N. Dak. & 9 & 30 & 35 & 28 \\
\hline 56 & Red River of the North at Grand Forks, N. Dak. & 10 & 151 & 98 & 125 \\
\hline 57 & Turtle River at Turtle River State Park near Arvilla, N. Dak. & 16 & 17 & 12 & 15 \\
\hline 58 & Turtle River at Manvel, N. Dak. & 16 & 41 & 21 & 34 \\
\hline 59 & Red River of the North at Oslo, Minn. & 12 & 115 & 83 & 76 \\
\hline 62 & Forest River near Minto, N. Dak. & 38 & 42 & 18 & 38 \\
\hline 65 & Middle Branch Park River near Edinburg, N. Dak. & -- & 266 & 52 & 192 \\
\hline
\end{tabular}


Table 1-5. Median seasonal concentrations for selected constituents at selected sites in North Dakota from 1970 through 2008.Continued

[Winter, January-February, November-December; Spring, March-June; Summer, July-October; mg/L, milligrams per liter; --, not available; N, nitrogen; <. less than; P, phosphorus; $\mathrm{C}$, carbon; $\mu \mathrm{g} / \mathrm{L}$, micrograms per liter]

\begin{tabular}{|c|c|c|c|c|c|}
\hline $\begin{array}{c}\text { Site } \\
\text { identification } \\
\text { number } \\
\text { (table 1) }\end{array}$ & Site name & Winter & Spring & Summer & $\begin{array}{l}\text { Period of } \\
\text { record }\end{array}$ \\
\hline \multicolumn{6}{|c|}{ Suspended-sediment concentration, in $\mathrm{mg} / \mathrm{L}$-Continued } \\
\hline 66 & Cart Creek at Mountain, N. Dak. & -- & 574 & -- & 574 \\
\hline 67 & Park River at Grafton, N. Dak. & 4 & 22 & 12 & 14 \\
\hline 72 & Pembina River near Vang, N. Dak. & 44 & 668 & 38 & 210 \\
\hline 73 & Little South Pembina River near Walhalla, N. Dak. & 19 & 476 & 22 & 122 \\
\hline 74 & Pembina River at Walhalla, N. Dak. & 136 & 817 & 150 & 681 \\
\hline 75 & Pembina River at Neche, N. Dak. & 4 & 77 & 39 & 44 \\
\hline 76 & Tongue River at Akra, N. Dak. & -- & 136 & 14 & 42 \\
\hline 78 & Red River of the North at Pembina, N. Dak., site 2 & 86 & 189 & 230 & 190 \\
\hline 79 & Red River of the North at Emerson, Manitoba & 19 & 205 & 153 & 115 \\
\hline 81 & West Branch Short Creek near Columbus, N. Dak. & 36 & 19 & 15 & 22 \\
\hline 82 & Souris River near Sherwood, N. Dak. & 16 & 41 & 18 & 23 \\
\hline 83 & Souris River near Foxholm, N. Dak. & 10 & 17 & 12 & 13 \\
\hline 87 & Souris River near Verendrye, N. Dak. & 10 & 31 & 18 & 16 \\
\hline 88 & Wintering River near Karlsruhe, N. Dak. & 7 & 6 & 8 & 8 \\
\hline 89 & Souris River near Bantry, N. Dak. & 35 & 25 & 33 & 26 \\
\hline 90 & Willow Creek near Willow City, N. Dak. & 28 & 11 & 37 & 12 \\
\hline 91 & Stone Creek near Kramer, N. Dak. & -- & 14 & 7 & 14 \\
\hline 92 & Deep River near Upham, N. Dak. & -- & 11 & 16 & 12 \\
\hline 95 & Cut Bank Creek at Upham, N. Dak. & -- & 7 & 2 & 7 \\
\hline 96 & Deep River below Cut Bank Creek near Upham, N. Dak. & -- & 11 & 49 & 11 \\
\hline 97 & Boundary Creek near Landa, N. Dak. & -- & 16 & 24 & 16 \\
\hline 98 & Souris River near Westhope, N. Dak. & 28 & 20 & 17 & 21 \\
\hline 100 & Missouri River near Williston, N. Dak. & 46 & 444 & 182 & 178 \\
\hline 102 & Stony Creek near Williston, N. Dak. & 121 & 84 & 72 & 78 \\
\hline 104 & Beaver Creek near Ray, N. Dak. & 48 & 32 & 25 & 32 \\
\hline 106 & Bear Den Creek near Mandaree, N. Dak. & 94 & 103 & 108 & 104 \\
\hline 111 & Deep Creek near Amidon, N. Dak. & 88 & 95 & 41 & 71 \\
\hline 113 & Beaver Creek near Trotters, N. Dak. & 64 & 107 & 66 & 80 \\
\hline 114 & Little Missouri River near Watford City, N. Dak. & 192 & 2,122 & 1,060 & 1,028 \\
\hline 115 & Missouri River at Garrison Dam, N. Dak. & $<1$ & $<1$ & 3 & 2 \\
\hline 116 & Knife River at Manning, N. Dak. & 69 & 65 & 43 & 62 \\
\hline 117 & Stray Creek near Manning, N. Dak. & 50 & 27 & 30 & 31 \\
\hline 118 & Knife River at Marshall, N. Dak. & 54 & 106 & 107 & 89 \\
\hline 119 & Elm Creek near Golden Valley, N. Dak. & 78 & 69 & 88 & 78 \\
\hline 121 & Coyote Creek near Zap, N. Dak. & 57 & 43 & 45 & 48 \\
\hline 122 & Brush Creek near Beulah, N. Dak. & 25 & 30 & 17 & 25 \\
\hline 123 & Spring Creek below Lake Ilo near Dunn Center, N. Dak. & 18 & 41 & 54 & 41 \\
\hline 124 & Spring Creek near Halliday, N. Dak. & 52 & 70 & 45 & 53 \\
\hline
\end{tabular}


Table 1-5. Median seasonal concentrations for selected constituents at selected sites in North Dakota from 1970 through 2008.Continued

[Winter, January-February, November-December; Spring, March-June; Summer, July-October; mg/L, milligrams per liter; --, not available; N, nitrogen; <. less than; P, phosphorus; $\mathrm{C}$, carbon; $\mu \mathrm{g} / \mathrm{L}$, micrograms per liter]

\begin{tabular}{|c|c|c|c|c|c|}
\hline $\begin{array}{c}\text { Site } \\
\text { identification } \\
\text { number } \\
\text { (table 1) }\end{array}$ & Site name & Winter & Spring & Summer & $\begin{array}{c}\text { Period of } \\
\text { record }\end{array}$ \\
\hline \multicolumn{6}{|c|}{ Suspended-sediment concentration, in $\mathrm{mg} / \mathrm{L}$-Continued } \\
\hline 125 & Spring Creek at Zap, N. Dak. & 21 & 56 & 53 & 39 \\
\hline 127 & Knife River at Hazen, N. Dak. & 41 & 85 & 68 & 66 \\
\hline 129 & West Branch Antelope Creek No. 4 near Zap, N. Dak. & 39 & 28 & 114 & 29 \\
\hline 130 & West Branch Antelope Creek near Hazen, N. Dak. & 112 & 59 & -- & 60 \\
\hline 131 & Coal Creek near Stanton, N. Dak. & 81 & 44 & 25 & 47 \\
\hline 132 & Alderin Creek near Fort Clark, N. Dak. & 170 & 161 & 139 & 159 \\
\hline 138 & Square Butte Creek above Nelson Lake near Center, N. Dak. & 40 & 53 & 43 & 44 \\
\hline 139 & Hagel Creek near Center, N. Dak. & 34 & 28 & 41 & 29 \\
\hline 142 & Missouri River at Bismarck, N. Dak. & 84 & 136 & 91 & 97 \\
\hline 143 & South Branch Heart River near South Heart, N. Dak. & 1,901 & 157 & 200 & 179 \\
\hline 144 & North Creek near South Heart, N. Dak. & 57 & 68 & 191 & 68 \\
\hline 145 & Heart River near South Heart, N. Dak. & 135 & 103 & 112 & 125 \\
\hline 147 & Green River near New Hradec, N. Dak. & 43 & 33 & 38 & 34 \\
\hline 156 & Heart River near Mandan, N. Dak. & 26 & 120 & 34 & 42 \\
\hline 158 & Apple Creek near Menoken, N. Dak. & 13 & 24 & 20 & 16 \\
\hline 174 & Buffalo Creek Tributary near Gascoyne, N. Dak. & 98 & 54 & 37 & 63 \\
\hline 175 & James River near Manfred, N. Dak. & 71 & 12 & 8 & 11 \\
\hline 176 & James River near Grace City, N. Dak. & 75 & 10 & 10 & 11 \\
\hline 177 & James River above Arrowwood Lake near Kensal, N. Dak. & 51 & 21 & 38 & 30 \\
\hline 178 & Kelly Creek below Niccum Reservoir near Bordulac, N. Dak. & -- & 8 & 8 & 8 \\
\hline 179 & James River near Pingree, N. Dak. & 22 & 22 & 39 & 32 \\
\hline 182 & James River at Jamestown, N. Dak. & 79 & 28 & 42 & 39 \\
\hline 183 & James River at Lamoure, N. Dak. & 47 & 67 & 69 & 65 \\
\hline 185 & James River at Oakes, N. Dak. & 87 & 59 & 109 & 89 \\
\hline
\end{tabular}



Table 1-4. Summary statistics for field measurements, total suspended solids, and suspended sediment at selected sites in North Dakota from 1970 through 2008.

$[\mu \mathrm{S} / \mathrm{cm}$, microsiemens per centimeter; deg. C, degrees Celsius; --, not available; mg/L, milligrams per liter; <, less than]

\begin{tabular}{|c|c|c|c|c|c|c|}
\hline $\begin{array}{c}\text { Site } \\
\text { identification } \\
\text { number } \\
\text { (table 1) }\end{array}$ & Site name & $\begin{array}{l}\text { Number of } \\
\text { samples }\end{array}$ & $\begin{array}{l}\text { Period of } \\
\text { record }\end{array}$ & Median & Minimum & Maximum \\
\hline \multicolumn{7}{|c|}{ Specific conductance, field, in $\mu \mathrm{S} / \mathrm{cm}$ at $25 \mathrm{deg}$. C } \\
\hline 1 & Bois De Sioux River near Doran, Minn. & 27 & $2003-2007$ & 1,228 & 586 & 2,130 \\
\hline 2 & Red River of the North at Wahpeton, N. Dak. & 334 & $1971-2008$ & 526 & 200 & 1,050 \\
\hline 4 & Red River of the North at Brushville, Minn. & 32 & $2000-2007$ & 655 & 490 & 811 \\
\hline 5 & Red River of the North below Wahpeton, N. Dak. & 65 & 1970-1999 & 478 & 396 & 672 \\
\hline 6 & Red River of the North at Hickson, N. Dak. & 349 & $1975-2008$ & 550 & 238 & 1,590 \\
\hline 7 & Wild Rice River near Rutland, N. Dak. & 166 & $1971-2008$ & 1,245 & 100 & 3,810 \\
\hline 11 & Red River of the North at Fargo, N. Dak. & 535 & $1970-2008$ & 572 & 180 & 1,400 \\
\hline 12 & Red River of North below Fargo, N. Dak. & 176 & $1970-1986$ & 557 & 290 & 1,140 \\
\hline 13 & Red River of the North at Harwood, N. Dak. & 32 & 2003-2007 & 752 & 612 & 1,072 \\
\hline 14 & Red River of the North near Harwood, N. Dak. & 30 & $1997-2007$ & 759 & 352 & 964 \\
\hline 15 & Sheyenne River above Harvey, N. Dak. & 323 & $1971-2008$ & 1,298 & 210 & 2,300 \\
\hline 16 & Big Coulee near Fort Totten, N. Dak. & 43 & $1970-1975$ & 600 & 190 & 932 \\
\hline 17 & Sheyenne River at Warwick, N. Dak. & 17 & $2003-2006$ & 1,186 & 635 & 1,506 \\
\hline 18 & Sheyenne River near Warwick, N. Dak. & 420 & 1970-2008 & 852 & 210 & 1,900 \\
\hline 19 & Mauvais Coulee Tributary No. 3 near Cando, N. Dak. & 98 & $1987-2008$ & 764 & 268 & 2,130 \\
\hline 26 & Little Coulee near Leeds, N. Dak. & 36 & $1998-2008$ & 887 & 392 & 2,390 \\
\hline 27 & Little Coulee near Brinsmade, N. Dak. & 97 & $1976-1998$ & 785 & 225 & 2,920 \\
\hline 28 & Big Coulee near Churchs Ferry, N. Dak. & 247 & 1970-1997 & 799 & 300 & 2,800 \\
\hline 29 & Big Coulee at Graham Island inlet near Fort Totten, N. Dak. & 46 & $1970-1986$ & 2,745 & 608 & 8,400 \\
\hline 30 & Channel A near Penn, N. Dak. & 175 & 1983-2008 & 938 & 260 & 3,590 \\
\hline 31 & Devils Lake Outlet to Stump Lake near Lakota, N. Dak. & 41 & 1999-2007 & 3,976 & 418 & 9,360 \\
\hline 32 & Sheyenne River near Cooperstown, N. Dak. & 542 & $1970-2008$ & 945 & 220 & 2,000 \\
\hline 33 & Baldhill Creek near Dazey, N. Dak. & 371 & $1971-2008$ & 900 & 210 & 1,750 \\
\hline 34 & Sheyenne River below Baldhill Dam, N. Dak. & 406 & $1971-2008$ & 810 & 285 & 1,630 \\
\hline 35 & Sheyenne River at Valley City, N. Dak. & 99 & $1971-2005$ & 725 & 235 & 1,480 \\
\hline 36 & Sheyenne River at Lisbon, N. Dak. & 482 & $1970-2008$ & 890 & 110 & 1,865 \\
\hline 37 & Sheyenne River near Kindred, N. Dak. & 589 & $1971-2008$ & 800 & 180 & 1,770 \\
\hline 38 & $\begin{array}{l}\text { Sheyenne River above Sheyenne River Diversion near Horace, } \\
\text { N. Dak. }\end{array}$ & 175 & 1992-2008 & 955 & 418 & 2,000 \\
\hline
\end{tabular}


Table 1-4. Summary statistics for field measurements, total suspended solids, and suspended sediment at selected sites in North Dakota from 1970 through 2008. - Continued

$[\mu \mathrm{S} / \mathrm{cm}$, microsiemens per centimeter; deg. $\mathrm{C}$, degrees Celsius; --, not available; mg/L, milligrams per liter; <, less than]

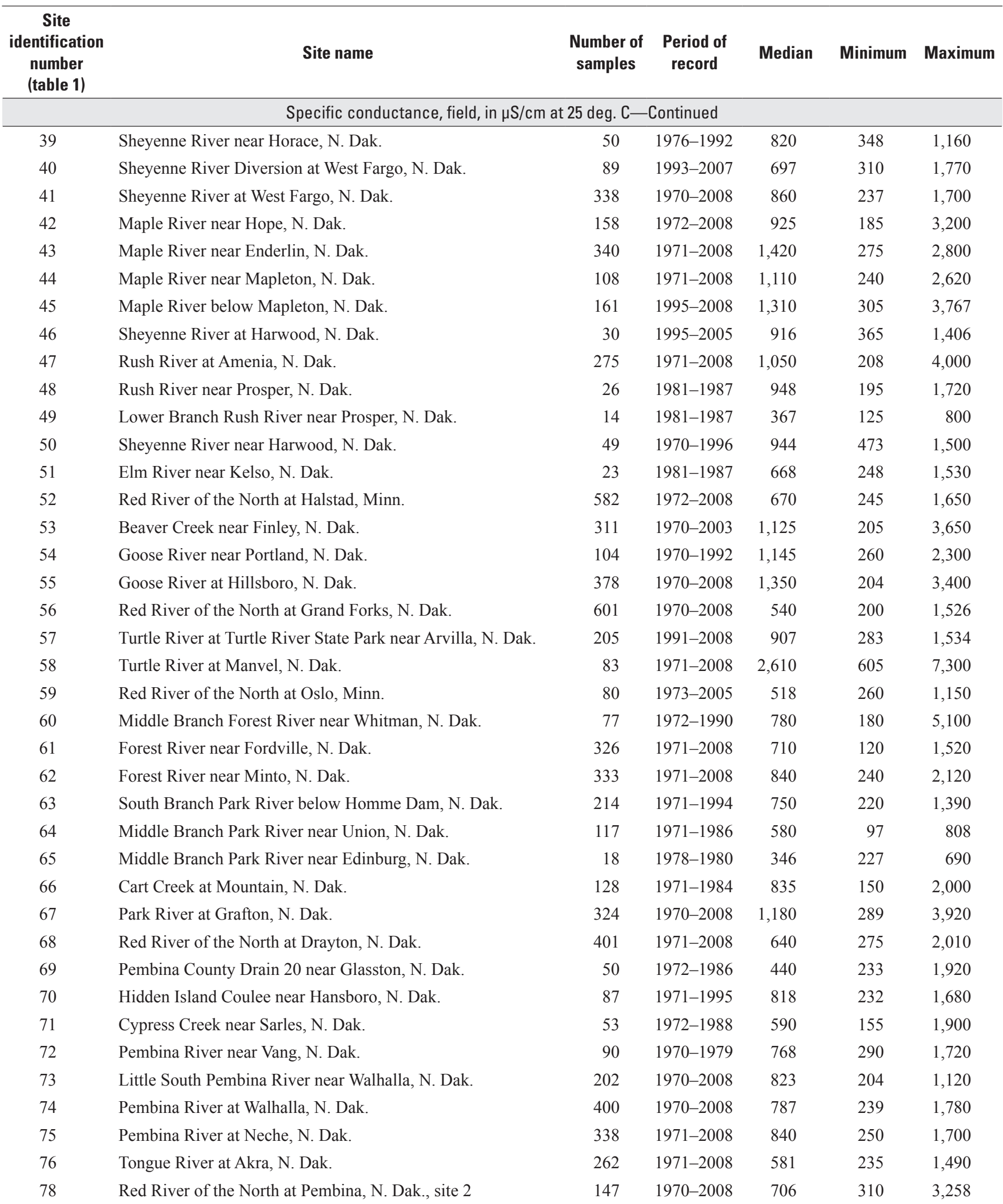


Table 1-4. Summary statistics for field measurements, total suspended solids, and suspended sediment at selected sites in North Dakota from 1970 through 2008. - Continued

$[\mu \mathrm{S} / \mathrm{cm}$, microsiemens per centimeter; deg. C, degrees Celsius; --, not available; mg/L, milligrams per liter; <, less than]

\begin{tabular}{|c|c|c|c|c|c|c|}
\hline $\begin{array}{c}\text { Site } \\
\text { identification } \\
\text { number } \\
\text { (table 1) }\end{array}$ & Site name & $\begin{array}{c}\text { Number of } \\
\text { samples }\end{array}$ & $\begin{array}{l}\text { Period of } \\
\text { record }\end{array}$ & Median & Minimum & Maximum \\
\hline \multicolumn{7}{|c|}{ Specific conductance, field, in $\mu \mathrm{S} / \mathrm{cm}$ at $25 \mathrm{deg}$. C-Continued } \\
\hline 79 & Red River of the North at Emerson, Manitoba & 403 & 1974-2004 & 600 & 130 & 1,810 \\
\hline 80 & Long Creek near Noonan, N. Dak. & 276 & $1971-2008$ & 1,210 & 200 & 11,920 \\
\hline 81 & West Branch Short Creek near Columbus, N. Dak. & 22 & $1978-1981$ & 1,590 & 565 & 3,850 \\
\hline 82 & Souris River near Sherwood, N. Dak. & 502 & 1970-2008 & 1,130 & 128 & 3,500 \\
\hline 83 & Souris River near Foxholm, N. Dak. & 380 & 1971-2008 & 883 & 260 & 2,900 \\
\hline 84 & Des Lacs River at Foxholm, N. Dak. & 362 & 1971-2008 & 1,471 & 150 & 3,330 \\
\hline 85 & Souris River above Minot, N. Dak. & 410 & 1970-2008 & 1,045 & 248 & 5,293 \\
\hline 86 & Bonnes Creek near Velva, N. Dak. & 18 & $1987-2005$ & 1,100 & 260 & 2,860 \\
\hline 87 & Souris River near Verendrye, N. Dak. & 477 & 1970-2008 & 1,140 & 315 & 3,482 \\
\hline 88 & Wintering River near Karlsruhe, N. Dak. & 342 & 1971-2008 & 713 & 225 & 2,630 \\
\hline 89 & Souris River near Bantry, N. Dak. & 413 & 1970-2008 & 990 & 355 & 3,100 \\
\hline 90 & Willow Creek near Willow City, N. Dak. & 267 & 1971-2008 & 875 & 140 & 3,210 \\
\hline 91 & Stone Creek near Kramer, N. Dak. & 85 & $1986-2000$ & 928 & 170 & 3,640 \\
\hline 92 & Deep River near Upham, N. Dak. & 149 & $1972-2007$ & 862 & 208 & 1,930 \\
\hline 93 & Egg Creek near Granville, N. Dak. & 69 & $1971-1981$ & 1,100 & 186 & 2,450 \\
\hline 94 & Cut Bank Creek at North Lake Outlet near Granville, N. Dak. & 59 & 1972-1980 & 1,100 & 322 & 2,080 \\
\hline 95 & Cut Bank Creek at Upham, N. Dak. & 74 & $1975-2000$ & 1,032 & 215 & 2,470 \\
\hline 96 & Deep River below Cut Bank Creek near Upham, N. Dak. & 99 & $1975-1989$ & 865 & 280 & 2,120 \\
\hline 97 & Boundary Creek near Landa, N. Dak. & 163 & $1972-2000$ & 1,000 & 200 & 3,200 \\
\hline 98 & Souris River near Westhope, N. Dak. & 663 & 1970-2008 & 980 & 58 & 4,220 \\
\hline 99 & Charbonneau Creek near Charbonneau, N. Dak. & 126 & 1971-2006 & 2,020 & 280 & 4,750 \\
\hline 100 & Missouri River near Williston, N. Dak. & 103 & 1970-1992 & 700 & 370 & 900 \\
\hline 101 & Little Muddy River below Cow Creek near Williston, N. Dak. & 283 & 1971-2008 & 2,050 & 220 & 3,450 \\
\hline 102 & Stony Creek near Williston, N. Dak. & 36 & $1977-1981$ & 1,960 & 220 & 2,800 \\
\hline 103 & Tobacco Garden Creek near Watford City, N. Dak. & 66 & $1976-1983$ & 2,425 & 220 & 4,500 \\
\hline 104 & Beaver Creek near Ray, N. Dak. & 63 & $1977-1982$ & 2,000 & 200 & 2,400 \\
\hline 105 & White Earth River at White Earth, N. Dak. & 141 & $1970-1982$ & 1,790 & 220 & 3,000 \\
\hline 106 & Bear Den Creek near Mandaree, N. Dak. & 491 & 1970-2008 & 2,500 & 230 & 4,080 \\
\hline 107 & Shell Creek near Parshall, N. Dak. & 106 & 1971-1981 & 2,400 & 235 & 3,500 \\
\hline 108 & East Fork Shell Creek near Parshall, N. Dak. & 97 & 1991-2008 & 3,090 & 230 & 4,220 \\
\hline 109 & Deepwater Creek near Mandaree, N. Dak. & 112 & 1990-2008 & 1,998 & 170 & 3,680 \\
\hline 110 & Little Missouri River at Marmarth, N. Dak. & 347 & 1971-2008 & 1,530 & 250 & 3,960 \\
\hline 111 & Deep Creek near Amidon, N. Dak. & 60 & $1977-1983$ & 3,775 & 795 & 5,250 \\
\hline 112 & Little Missouri River at Medora, N. Dak. & 138 & 1971-2008 & 1,746 & 376 & 5,316 \\
\hline 113 & Beaver Creek near Trotters, N. Dak. & 197 & $1977-2008$ & 2,260 & 310 & 6,200 \\
\hline 114 & Little Missouri River near Watford City, N. Dak. & 724 & $1971-2008$ & 1,630 & 400 & 5,000 \\
\hline 115 & Missouri River at Garrison Dam, N. Dak. & 488 & $1971-2007$ & 650 & 530 & 810 \\
\hline 116 & Knife River at Manning, N. Dak. & 385 & 1972-2008 & 1,750 & 129 & 4,200 \\
\hline 117 & Stray Creek near Manning, N. Dak. & 23 & 1975-1981 & 2,100 & 550 & 5,430 \\
\hline
\end{tabular}


Table 1-4. Summary statistics for field measurements, total suspended solids, and suspended sediment at selected sites in North Dakota from 1970 through 2008. - Continued

$[\mu \mathrm{S} / \mathrm{cm}$, microsiemens per centimeter; deg. $\mathrm{C}$, degrees Celsius; --, not available; mg/L, milligrams per liter; <, less than]

\begin{tabular}{|c|c|c|c|c|c|c|}
\hline $\begin{array}{c}\text { Site } \\
\text { identification } \\
\text { number } \\
\text { (table 1) }\end{array}$ & Site name & $\begin{array}{l}\text { Number of } \\
\text { samples }\end{array}$ & $\begin{array}{l}\text { Period of } \\
\text { record }\end{array}$ & Median & Minimum & Maximum \\
\hline \multicolumn{7}{|c|}{ Specific conductance, field, in $\mu \mathrm{S} / \mathrm{cm}$ at $25 \mathrm{deg}$. C-Continued } \\
\hline 118 & Knife River at Marshall, N. Dak. & 182 & $1972-1981$ & 1,865 & 285 & 3,680 \\
\hline 119 & Elm Creek near Golden Valley, N. Dak. & 98 & $1972-1995$ & 1,018 & 185 & 3,740 \\
\hline 121 & Coyote Creek near Zap, N. Dak. & 67 & $1977-1983$ & 1,760 & 120 & 3,300 \\
\hline 122 & Brush Creek near Beulah, N. Dak. & 170 & $1974-1990$ & 1,750 & 150 & 2,500 \\
\hline 123 & Spring Creek below Lake Ilo near Dunn Center, N. Dak. & 52 & $1977-1981$ & 2,028 & 150 & 4,400 \\
\hline 124 & Spring Creek near Halliday, N. Dak. & 52 & $1977-1981$ & 1,875 & 250 & 2,200 \\
\hline 128 & Antelope Creek above Hazen, N. Dak. & 60 & $1977-1985$ & 1,380 & 150 & 3,180 \\
\hline 129 & West Branch Antelope Creek No. 4 near Zap, N. Dak. & 82 & 1977-1986 & 395 & 65 & 1,400 \\
\hline 130 & West Branch Antelope Creek near Hazen, N. Dak. & 21 & $1978-1983$ & 760 & 110 & 2,740 \\
\hline 131 & Coal Creek near Stanton, N. Dak. & 32 & $1975-1981$ & 2,000 & 278 & 3,380 \\
\hline 132 & Alderin Creek near Fort Clark, N. Dak. & 56 & $1977-1983$ & 1,445 & 210 & 3,440 \\
\hline 133 & Coal Lake Coulee near Hensler, N. Dak. & 64 & 1978-1988 & 1,050 & 140 & 2,300 \\
\hline 134 & Buffalo Creek near Washburn, N. Dak. & 51 & 1978-1983 & 2,180 & 210 & 4,010 \\
\hline 135 & Turtle Creek above Washburn, N. Dak. & 118 & $1986-2003$ & 1,410 & 182 & 5,100 \\
\hline 136 & Painted Woods Creek near Wilton, N. Dak. & 324 & $1970-2003$ & 1,522 & 112 & 4,600 \\
\hline 143 & South Branch Heart River near South Heart, N. Dak. & 38 & 1979-1996 & 658 & 222 & 2,500 \\
\hline 144 & North Creek near South Heart, N. Dak. & 26 & 1978-1996 & 1,603 & 243 & 4,740 \\
\hline 145 & Heart River near South Heart, N. Dak. & 108 & $1975-2005$ & 2,130 & 226 & 4,450 \\
\hline 146 & Heart River at Dickinson, N. Dak. & 90 & 1984-1994 & 1,945 & 585 & 2,770 \\
\hline 147 & Green River near New Hradec, N. Dak. & 408 & $1971-2008$ & 1,062 & 150 & 6,930 \\
\hline 148 & Green River near Gladstone, N. Dak. & 64 & $1970-1975$ & 1,635 & 150 & 2,850 \\
\hline 149 & Heart River near Richardton, N. Dak. & 386 & $1971-2008$ & 1,670 & 220 & 4,800 \\
\hline 150 & Heart River above Lake Tschida near Glen Ullin, N. Dak. & 160 & $1988-2008$ & 1,700 & 398 & 3,500 \\
\hline 151 & Antelope Creek near Carson, N. Dak. & 103 & $1971-2008$ & 909 & 357 & 2,030 \\
\hline 152 & Big Muddy Creek near Almont, N. Dak. & 111 & 1991-2008 & 1,850 & 161 & 2,900 \\
\hline 153 & Heart River near Lark, N. Dak. & 229 & $1971-1995$ & 1,150 & 118 & 2,900 \\
\hline 154 & Heart River at Stark Bridge near Judson, N. Dak. & 152 & 1988-2008 & 1,380 & 260 & 2,660 \\
\hline 155 & Sweetbriar Creek near Judson, N. Dak. & 127 & $1971-2008$ & 1,050 & 200 & 2,140 \\
\hline 156 & Heart River near Mandan, N. Dak. & 492 & $1971-2008$ & 1,350 & 240 & 3,500 \\
\hline
\end{tabular}


Table 1-4. Summary statistics for field measurements, total suspended solids, and suspended sediment at selected sites in North Dakota from 1970 through 2008. - Continued

$[\mu \mathrm{S} / \mathrm{cm}$, microsiemens per centimeter; deg. $\mathrm{C}$, degrees Celsius; --, not available; mg/L, milligrams per liter; <, less than]

\begin{tabular}{|c|c|c|c|c|c|c|}
\hline $\begin{array}{c}\text { Site } \\
\text { identification } \\
\text { number } \\
\text { (table 1) }\end{array}$ & Site name & $\begin{array}{l}\text { Number of } \\
\text { samples }\end{array}$ & $\begin{array}{l}\text { Period of } \\
\text { record }\end{array}$ & Median & Minimum & Maximum \\
\hline \multicolumn{7}{|c|}{ Specific conductance, field, in $\mu \mathrm{S} / \mathrm{cm}$ at $25 \mathrm{deg}$. C-Continued } \\
\hline 157 & Long Lake Creek above Long Lake near Moffit, N. Dak. & 117 & 1988-2004 & 1,074 & 121 & 2,400 \\
\hline 158 & Apple Creek near Menoken, N. Dak. & 339 & $1971-2008$ & 1,260 & 100 & 2,570 \\
\hline 160 & Cannonball River at New England, N. Dak. & 39 & $1978-1981$ & 2,480 & 405 & 4,240 \\
\hline 161 & Coal Bank Creek near Havelock, N. Dak. & 109 & $1974-1983$ & 1,930 & 145 & 4,800 \\
\hline 162 & Cannonball River at Regent, N. Dak. & 407 & 1970-2008 & 1,830 & 172 & 3,310 \\
\hline 163 & Cannonball River below Bentley, N. Dak. & 129 & $1972-1982$ & 1,930 & 305 & 6,930 \\
\hline 167 & Timber Creek near Bentley, N. Dak. & 50 & $1977-1981$ & 2,600 & 810 & 4,250 \\
\hline 168 & Cedar Creek near Pretty Rock, N. Dak. & 63 & $1971-1976$ & 2,500 & 447 & 6,700 \\
\hline 169 & Cedar Creek near Raleigh, N. Dak. & 333 & $1972-2008$ & 1,600 & 200 & 4,400 \\
\hline 170 & Cannonball River at Breien, N. Dak. & 573 & 1970-2008 & 1,632 & 190 & 4,860 \\
\hline 171 & Beaver Creek near Linton, N. Dak. & 185 & $1972-1989$ & 880 & 130 & 2,400 \\
\hline 172 & Beaver Creek below Linton, N. Dak. & 141 & $1989-2008$ & 1,088 & 150 & 1,650 \\
\hline 173 & Porcupine Creek near Fort Yates, N. Dak. & 87 & 1991-1999 & 1,163 & 100 & 2,610 \\
\hline 174 & Buffalo Creek Tributary near Gascoyne, N. Dak. & 111 & $1974-1987$ & 4,900 & 555 & 9,490 \\
\hline 175 & James River near Manfred, N. Dak. & 126 & $1971-1998$ & 800 & 75 & 1,360 \\
\hline 182 & James River at Jamestown, N. Dak. & 349 & $1972-2008$ & 860 & 185 & 1,915 \\
\hline 183 & James River at Lamoure, N. Dak. & 386 & 1970-2008 & 820 & 160 & 2,585 \\
\hline 184 & Bear Creek near Oakes, N. Dak. & 167 & $1972-2008$ & 1,200 & 140 & 3,327 \\
\hline 185 & James River at Oakes, N. Dak. & 166 & $1970-2008$ & 920 & 250 & 4,650 \\
\hline 186 & James River at N. Dak./S. Dak. State line & 115 & 1974-2008 & 840 & 255 & 3,900 \\
\hline \multicolumn{7}{|c|}{ Specific conductance, laboratory, in $\mu S / \mathrm{cm}$ at $25 \mathrm{deg}$. C } \\
\hline 1 & Bois De Sioux River near Doran, Minn. & 40 & $2000-2007$ & 1,275 & 261 & 3,690 \\
\hline 2 & Red River of the North at Wahpeton, N. Dak. & 53 & $1991-2008$ & 604 & 414 & 1,320 \\
\hline 3 & Red River of the North near Wahpeton, N. Dak. & 4 & 1993-2006 & 720 & 583 & 1,640 \\
\hline 4 & Red River of the North at Brushville, Minn. & 61 & 1993-2007 & 608 & 338 & 879 \\
\hline 5 & Red River of the North below Wahpeton, N. Dak. & 20 & 1997-1999 & 528 & 423 & 1,160 \\
\hline 6 & Red River of the North at Hickson, N. Dak. & 78 & $1980-2008$ & 549 & 261 & 957 \\
\hline 7 & Wild Rice River near Rutland, N. Dak. & 35 & 1984-2008 & 1,540 & 261 & 3,820 \\
\hline 9 & Antelope Creek at Dwight, N. Dak. & 13 & 2001-2008 & 944 & 579 & 1,400 \\
\hline
\end{tabular}


Table 1-4. Summary statistics for field measurements, total suspended solids, and suspended sediment at selected sites in North Dakota from 1970 through 2008. - Continued

$[\mu \mathrm{S} / \mathrm{cm}$, microsiemens per centimeter; deg. $\mathrm{C}$, degrees Celsius; --, not available; mg/L, milligrams per liter; <, less than]

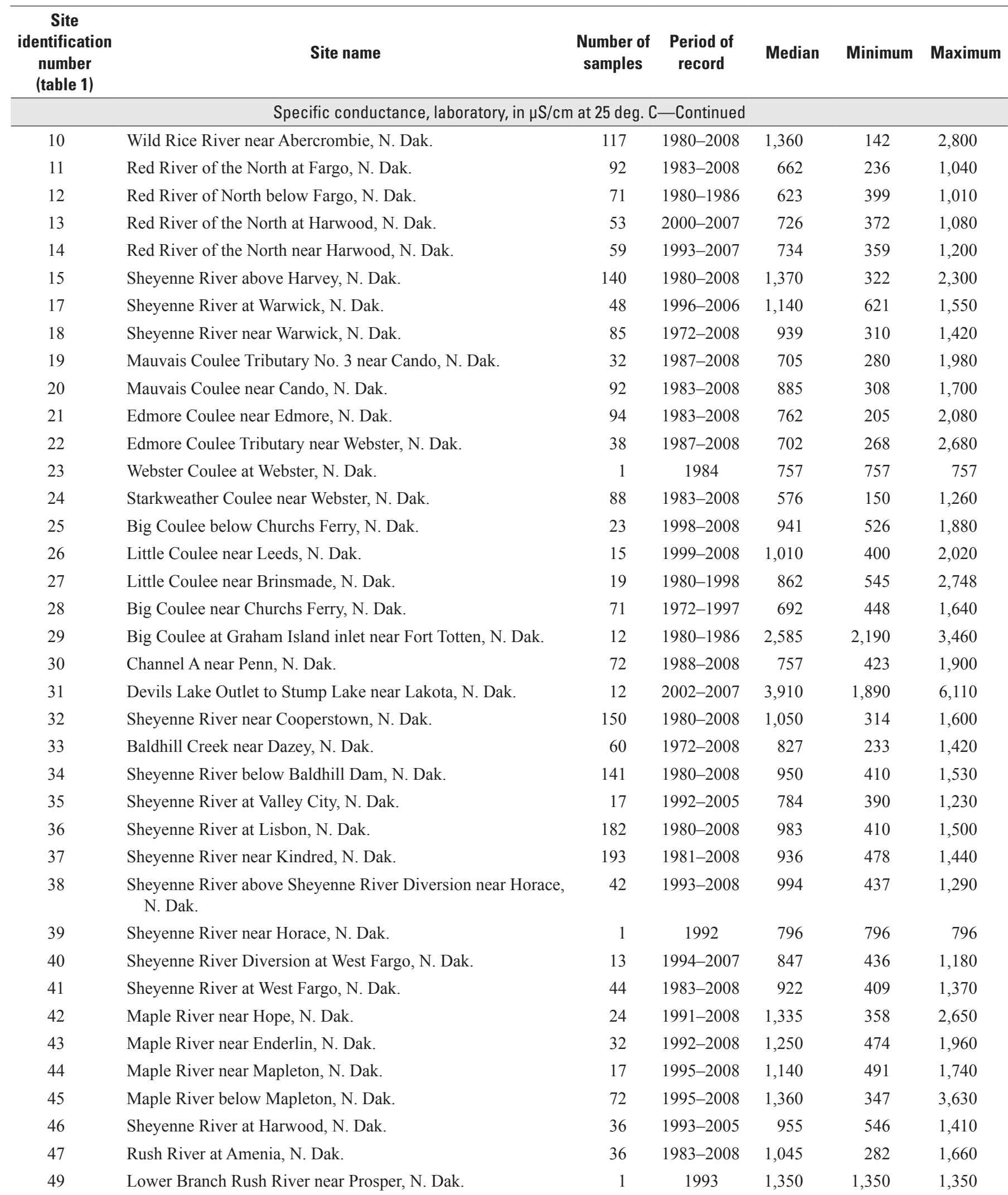


Table 1-4. Summary statistics for field measurements, total suspended solids, and suspended sediment at selected sites in North Dakota from 1970 through 2008. - Continued

$[\mu \mathrm{S} / \mathrm{cm}$, microsiemens per centimeter; deg. $\mathrm{C}$, degrees Celsius; --, not available; mg/L, milligrams per liter; <, less than]

\begin{tabular}{|c|c|c|c|c|c|c|}
\hline $\begin{array}{c}\text { Site } \\
\text { identification } \\
\text { number } \\
\text { (table 1) }\end{array}$ & Site name & $\begin{array}{l}\text { Number of } \\
\text { samples }\end{array}$ & $\begin{array}{l}\text { Period of } \\
\text { record }\end{array}$ & Median & Minimum & Maximum \\
\hline \multicolumn{7}{|c|}{ Specific conductance, laboratory, in $\mu \mathrm{S} / \mathrm{cm}$ at $25 \mathrm{deg}$. C-Continued } \\
\hline 50 & Sheyenne River near Harwood, N. Dak. & 1 & 1996 & 468 & 468 & 468 \\
\hline 51 & Elm River near Kelso, N. Dak. & 3 & 1993 & 1,160 & 1,080 & 1,290 \\
\hline 52 & Red River of the North at Halstad, Minn. & 136 & 1980-2008 & 703 & 299 & 1,090 \\
\hline 53 & Beaver Creek near Finley, N. Dak. & 58 & $1981-2003$ & 1,124 & 225 & 2,740 \\
\hline 55 & Goose River at Hillsboro, N. Dak. & 117 & 1988-2008 & 1,380 & 402 & 2,120 \\
\hline 56 & Red River of the North at Grand Forks, N. Dak. & 145 & 1983-2008 & 626 & 274 & 1,060 \\
\hline 57 & Turtle River at Turtle River State Park near Arvilla, N. Dak. & 99 & 1991-2008 & 905 & 282 & 1,540 \\
\hline 58 & Turtle River at Manvel, N. Dak. & 78 & 1981-2008 & 2,690 & 820 & 7,280 \\
\hline 59 & Red River of the North at Oslo, Minn. & 16 & 1991-2005 & 552 & 343 & 832 \\
\hline 60 & Middle Branch Forest River near Whitman, N. Dak. & 1 & 1987 & 504 & 504 & 504 \\
\hline 61 & Forest River near Fordville, N. Dak. & 33 & 1992-2008 & 824 & 448 & 1,470 \\
\hline 62 & Forest River near Minto, N. Dak. & 106 & 1987-2008 & 965 & 487 & 4,820 \\
\hline 63 & South Branch Park River below Homme Dam, N. Dak. & 7 & 1981-1994 & 618 & 294 & 866 \\
\hline 67 & Park River at Grafton, N. Dak. & 99 & 1988-2008 & 1,210 & 336 & 3,330 \\
\hline 68 & Red River of the North at Drayton, N. Dak. & 50 & 1991-2008 & 677 & 394 & 1,450 \\
\hline 70 & Hidden Island Coulee near Hansboro, N. Dak. & 5 & 1992-1995 & 676 & 328 & 1,070 \\
\hline 73 & Little South Pembina River near Walhalla, N. Dak. & 16 & 2001-2008 & 811 & 473 & 1,000 \\
\hline 74 & Pembina River at Walhalla, N. Dak. & 80 & 1980-2008 & 791 & 412 & 1,190 \\
\hline 75 & Pembina River at Neche, N. Dak. & 111 & 1990-2008 & 854 & 350 & 1,270 \\
\hline 76 & Tongue River at Akra, N. Dak. & 36 & 1980-2008 & 584 & 305 & 697 \\
\hline 77 & Red River of the North at Pembina, N. Dak., site 1 & 10 & 1993 & 709 & 440 & 786 \\
\hline 78 & Red River of the North at Pembina, N. Dak., site 2 & 150 & 1994-2008 & 693 & 316 & 1,580 \\
\hline 79 & Red River of the North at Emerson, Manitoba & 114 & 1980-2004 & 704 & 413 & 1,780 \\
\hline 80 & Long Creek near Noonan, N. Dak. & 41 & 1982-2008 & 1,350 & 338 & 2,850 \\
\hline 81 & West Branch Short Creek near Columbus, N. Dak. & 3 & 1981 & 1,330 & 628 & 2,120 \\
\hline 82 & Souris River near Sherwood, N. Dak. & 217 & 1980-2008 & 1,110 & 265 & 3,560 \\
\hline 83 & Souris River near Foxholm, N. Dak. & 113 & 1972-2008 & 996 & 470 & 2,370 \\
\hline 84 & Des Lacs River at Foxholm, N. Dak. & 168 & 1983-2008 & 1,625 & 196 & 3,210 \\
\hline 85 & Souris River above Minot, N. Dak. & 174 & 1981-2008 & 1,196 & 324 & 5,120 \\
\hline 86 & Bonnes Creek near Velva, N. Dak. & 18 & 1987-2005 & 1,115 & 269 & 2,880 \\
\hline 87 & Souris River near Verendrye, N. Dak. & 249 & $1981-2008$ & 1,390 & 312 & 3,400 \\
\hline 88 & Wintering River near Karlsruhe, N. Dak. & 89 & 1980-2008 & 762 & 315 & 1,990 \\
\hline 89 & Souris River near Bantry, N. Dak. & 158 & 1981-2008 & 919 & 398 & 2,080 \\
\hline 90 & Willow Creek near Willow City, N. Dak. & 121 & 1983-2008 & 959 & 290 & 2,290 \\
\hline 91 & Stone Creek near Kramer, N. Dak. & 84 & 1986-2000 & 760 & 220 & 3,650 \\
\hline 92 & Deep River near Upham, N. Dak. & 54 & 1987-2007 & 945 & 271 & 1,450 \\
\hline 93 & Egg Creek near Granville, N. Dak. & 1 & 1981 & 679 & 679 & 679 \\
\hline 95 & Cut Bank Creek at Upham, N. Dak. & 16 & 1999-2000 & 1,145 & 444 & 1,460 \\
\hline 96 & Deep River below Cut Bank Creek near Upham, N. Dak. & 49 & 1986-1989 & 660 & 285 & 1,520 \\
\hline
\end{tabular}


Table 1-4. Summary statistics for field measurements, total suspended solids, and suspended sediment at selected sites in North Dakota from 1970 through 2008. - Continued

$[\mu \mathrm{S} / \mathrm{cm}$, microsiemens per centimeter; deg. $\mathrm{C}$, degrees Celsius; --, not available; mg/L, milligrams per liter; <, less than]

\begin{tabular}{|c|c|c|c|c|c|c|}
\hline $\begin{array}{c}\text { Site } \\
\text { identification } \\
\text { number } \\
\text { (table 1) }\end{array}$ & Site name & $\begin{array}{c}\text { Number of } \\
\text { samples }\end{array}$ & $\begin{array}{l}\text { Period of } \\
\text { record }\end{array}$ & Median & Minimum & Maximum \\
\hline \multicolumn{7}{|c|}{ Specific conductance, laboratory, in $\mu \mathrm{S} / \mathrm{cm}$ at $25 \mathrm{deg}$. C-Continued } \\
\hline 97 & Boundary Creek near Landa, N. Dak. & 86 & $1986-2000$ & 920 & 268 & 2,830 \\
\hline 98 & Souris River near Westhope, N. Dak. & 143 & 1980-2008 & 960 & 415 & 3,110 \\
\hline 99 & Charbonneau Creek near Charbonneau, N. Dak. & 1 & 2006 & 4,060 & 4,060 & 4,060 \\
\hline 100 & Missouri River near Williston, N. Dak. & 19 & 1980-1992 & 720 & 417 & 913 \\
\hline 101 & Little Muddy River below Cow Creek near Williston, N. Dak. & 44 & $1983-2008$ & 1,975 & 260 & 2,530 \\
\hline 102 & Stony Creek near Williston, N. Dak. & 6 & $1980-1981$ & 1,670 & 217 & 2,750 \\
\hline 104 & Beaver Creek near Ray, N. Dak. & 18 & 1980-1982 & 2,020 & 221 & 2,320 \\
\hline 106 & Bear Den Creek near Mandaree, N. Dak. & 113 & 1980-2008 & 2,600 & 256 & 3,630 \\
\hline 108 & East Fork Shell Creek near Parshall, N. Dak. & 60 & 1991-2008 & 3,140 & 219 & 3,920 \\
\hline 109 & Deepwater Creek near Mandaree, N. Dak. & 57 & $1991-2008$ & 1,890 & 176 & 3,360 \\
\hline 110 & Little Missouri River at Marmarth, N. Dak. & 43 & 1990-2008 & 1,400 & 320 & 3,120 \\
\hline 111 & Deep Creek near Amidon, N. Dak. & 16 & $1980-1983$ & 3,705 & 839 & 5,320 \\
\hline 112 & Little Missouri River at Medora, N. Dak. & 104 & 1993-2008 & 1,700 & 405 & 5,300 \\
\hline 113 & Beaver Creek near Trotters, N. Dak. & 50 & 1980-2008 & 2,295 & 336 & 3,380 \\
\hline 114 & Little Missouri River near Watford City, N. Dak. & 164 & $1980-2008$ & 1,680 & 401 & 4,070 \\
\hline 115 & Missouri River at Garrison Dam, N. Dak. & 156 & 1980-2007 & 647 & 547 & 812 \\
\hline 116 & Knife River at Manning, N. Dak. & 50 & $1980-2008$ & 1,400 & 144 & 2,980 \\
\hline 117 & Stray Creek near Manning, N. Dak. & 4 & 1981 & 1,175 & 675 & 2,170 \\
\hline 118 & Knife River at Marshall, N. Dak. & 11 & 1980-1981 & 1,410 & 813 & 3,880 \\
\hline 119 & Elm Creek near Golden Valley, N. Dak. & 7 & 1981-1995 & 947 & 359 & 2,990 \\
\hline 120 & Knife River near Golden Valley, N. Dak. & 96 & $1987-2008$ & 1,980 & 290 & 4,550 \\
\hline 121 & Coyote Creek near Zap, N. Dak. & 21 & $1980-1983$ & 1,590 & 250 & 2,740 \\
\hline 122 & Brush Creek near Beulah, N. Dak. & 50 & $1980-1990$ & 1,850 & 260 & 2,250 \\
\hline 123 & Spring Creek below Lake Ilo near Dunn Center, N. Dak. & 17 & $1980-1993$ & 2,120 & 714 & 2,710 \\
\hline 124 & Spring Creek near Halliday, N. Dak. & 12 & 1980-1981 & 1,981 & 505 & 2,220 \\
\hline 125 & Spring Creek at Zap, N. Dak. & 129 & $1980-2008$ & 1,620 & 230 & 2,440 \\
\hline 126 & West Branch Otter Creek near Beulah, N. Dak. & 4 & $1992-1995$ & 644 & 388 & 948 \\
\hline 127 & Knife River at Hazen, N. Dak. & 181 & 1980-2008 & 1,660 & 295 & 2,570 \\
\hline 128 & Antelope Creek above Hazen, N. Dak. & 24 & $1980-1985$ & 1,195 & 145 & 3,120 \\
\hline 129 & West Branch Antelope Creek No. 4 near Zap, N. Dak. & 13 & $1981-1985$ & 769 & 93 & 1,370 \\
\hline 130 & West Branch Antelope Creek near Hazen, N. Dak. & 7 & $1981-1983$ & 721 & 192 & 2,700 \\
\hline 131 & Coal Creek near Stanton, N. Dak. & 6 & $1980-1981$ & 2,065 & 515 & 3,480 \\
\hline 132 & Alderin Creek near Fort Clark, N. Dak. & 19 & $1980-1983$ & 1,440 & 274 & 3,290 \\
\hline 133 & Coal Lake Coulee near Hensler, N. Dak. & 29 & $1980-1988$ & 1,590 & 150 & 2,280 \\
\hline 134 & Buffalo Creek near Washburn, N. Dak. & 17 & $1980-1983$ & 2,160 & 240 & 2,650 \\
\hline 135 & Turtle Creek above Washburn, N. Dak. & 100 & $1987-2003$ & 1,585 & 369 & 5,100 \\
\hline 136 & Painted Woods Creek near Wilton, N. Dak. & 135 & $1982-2003$ & 1,700 & 292 & 4,060 \\
\hline 137 & Square Butte Creek near Hannover, N. Dak. & 5 & $1980-1981$ & 807 & 169 & 1,240 \\
\hline 138 & Square Butte Creek above Nelson Lake near Center, N. Dak. & 18 & 1980-1982 & 1,205 & 174 & 1,400 \\
\hline
\end{tabular}


Table 1-4. Summary statistics for field measurements, total suspended solids, and suspended sediment at selected sites in North Dakota from 1970 through 2008. - Continued

$[\mu \mathrm{S} / \mathrm{cm}$, microsiemens per centimeter; deg. $\mathrm{C}$, degrees Celsius; --, not available; mg/L, milligrams per liter; <, less than]

\begin{tabular}{|c|c|c|c|c|c|c|}
\hline $\begin{array}{c}\text { Site } \\
\text { identification } \\
\text { number } \\
\text { (table 1) }\end{array}$ & Site name & $\begin{array}{l}\text { Number of } \\
\text { samples }\end{array}$ & $\begin{array}{l}\text { Period of } \\
\text { record }\end{array}$ & Median & Minimum & Maximum \\
\hline \multicolumn{7}{|c|}{ Specific conductance, laboratory, in $\mu \mathrm{S} / \mathrm{cm}$ at $25 \mathrm{deg}$. C-Continued } \\
\hline 139 & Hagel Creek near Center, N. Dak. & 14 & 1980-1982 & 1,265 & 173 & 2,030 \\
\hline 140 & Square Butte Creek below Center, N. Dak. & 38 & 1983-2008 & 1,585 & 560 & 1,870 \\
\hline 141 & Burnt Creek near Bismarck, N. Dak. & 31 & 1992-2008 & 1,070 & 196 & 1,680 \\
\hline 142 & Missouri River at Bismarck, N. Dak. & 52 & 1988-2008 & 650 & 441 & 734 \\
\hline 143 & South Branch Heart River near South Heart, N. Dak. & 22 & $1980-1996$ & 461 & 237 & 1,990 \\
\hline 144 & North Creek near South Heart, N. Dak. & 14 & 1980-1996 & 1,159 & 274 & 4,130 \\
\hline 145 & Heart River near South Heart, N. Dak. & 31 & 1980-2005 & 2,010 & 265 & 4,500 \\
\hline 146 & Heart River at Dickinson, N. Dak. & 14 & 1988-1994 & 1,855 & 791 & 2,990 \\
\hline 147 & Green River near New Hradec, N. Dak. & 50 & 1980-2008 & 889 & 154 & 2,880 \\
\hline 148 & Green River near Gladstone, N. Dak. & 4 & 1993 & 1,555 & 834 & 2,740 \\
\hline 149 & Heart River near Richardton, N. Dak. & 110 & $1987-2008$ & 1,560 & 402 & 3,000 \\
\hline 150 & Heart River above Lake Tschida near Glen Ullin, N. Dak. & 33 & 1992-2008 & 1,340 & 417 & 2,330 \\
\hline 151 & Antelope Creek near Carson, N. Dak. & 18 & 1999-2008 & 810 & 583 & 1,990 \\
\hline 152 & Big Muddy Creek near Almont, N. Dak. & 37 & 1991-2008 & 1,760 & 460 & 2,730 \\
\hline 153 & Heart River near Lark, N. Dak. & 9 & $1990-1995$ & 1,000 & 417 & 1,400 \\
\hline 154 & Heart River at Stark Bridge near Judson, N. Dak. & 33 & 1992-2008 & 1,330 & 281 & 1,750 \\
\hline 155 & Sweetbriar Creek near Judson, N. Dak. & 13 & $2002-2008$ & 1,470 & 591 & 2,010 \\
\hline 156 & Heart River near Mandan, N. Dak. & 183 & 1980-2008 & 1,460 & 360 & 2,760 \\
\hline 157 & Long Lake Creek above Long Lake near Moffit, N. Dak. & 27 & 1988-2004 & 922 & 212 & 1,520 \\
\hline 158 & Apple Creek near Menoken, N. Dak. & 43 & 1980-2008 & 1,180 & 252 & 2,010 \\
\hline 159 & Missouri River near Schmidt, N. Dak. & 10 & $1980-1981$ & 773 & 749 & 809 \\
\hline 160 & Cannonball River at New England, N. Dak. & 12 & $1980-1981$ & 1,980 & 443 & 4,060 \\
\hline 161 & Coal Bank Creek near Havelock, N. Dak. & 18 & $1980-1983$ & 2,000 & 466 & 4,590 \\
\hline 162 & Cannonball River at Regent, N. Dak. & 45 & 1980-2008 & 1,690 & 514 & 3,030 \\
\hline 164 & Cannonball River near Raleigh, N. Dak. & 89 & 1993-2008 & 1,750 & 434 & 5,360 \\
\hline 165 & White Butte Fork Cedar Creek near Scranton, N. Dak. & 6 & 1992-1995 & 1,185 & 562 & 3,140 \\
\hline 166 & Cedar Creek near Haynes, N. Dak. & 34 & 1983-2008 & 1,700 & 322 & 3,200 \\
\hline 167 & Timber Creek near Bentley, N. Dak. & 12 & $1980-1981$ & 2,450 & 1,520 & 3,950 \\
\hline 169 & Cedar Creek near Raleigh, N. Dak. & 95 & 1992-2008 & 1,620 & 364 & 3,910 \\
\hline 170 & Cannonball River at Breien, N. Dak. & 164 & 1980-2008 & 1,610 & 256 & 3,220 \\
\hline 171 & Beaver Creek near Linton, N. Dak. & 7 & 1984-1993 & 651 & 219 & 914 \\
\hline 172 & Beaver Creek below Linton, N. Dak. & 34 & 1990-2008 & 1,002 & 183 & 1,460 \\
\hline 173 & Porcupine Creek near Fort Yates, N. Dak. & 40 & 1992-1999 & 1,160 & 101 & 2,090 \\
\hline 174 & Buffalo Creek Tributary near Gascoyne, N. Dak. & 31 & $1980-1987$ & 5,480 & 1,127 & 10,500 \\
\hline 175 & James River near Manfred, N. Dak. & 41 & 1985-1998 & 970 & 197 & 1,360 \\
\hline 176 & James River near Grace City, N. Dak. & 141 & $1985-2008$ & 996 & 202 & 3,290 \\
\hline 177 & James River above Arrowwood Lake near Kensal, N. Dak. & 172 & $1985-2008$ & 970 & 275 & 2,339 \\
\hline 178 & Kelly Creek below Niccum Reservoir near Bordulac, N. Dak. & 9 & 1986-1989 & 572 & 360 & 772 \\
\hline 179 & James River near Pingree, N. Dak. & 134 & 1981-2008 & 816 & 321 & 1,601 \\
\hline
\end{tabular}


Table 1-4. Summary statistics for field measurements, total suspended solids, and suspended sediment at selected sites in North Dakota from 1970 through 2008. - Continued

$[\mu \mathrm{S} / \mathrm{cm}$, microsiemens per centimeter; deg. C, degrees Celsius; --, not available; mg/L, milligrams per liter; <, less than]

\begin{tabular}{|c|c|c|c|c|c|c|}
\hline $\begin{array}{c}\text { Site } \\
\text { identification } \\
\text { number } \\
\text { (table 1) }\end{array}$ & Site name & $\begin{array}{l}\text { Number of } \\
\text { samples }\end{array}$ & $\begin{array}{l}\text { Period of } \\
\text { record }\end{array}$ & Median & Minimum & Maximum \\
\hline \multicolumn{7}{|c|}{ Specific conductance, laboratory, in $\mu \mathrm{S} / \mathrm{cm}$ at $25 \mathrm{deg}$. C-Continued } \\
\hline 180 & Pipestem Creek near Pingree, N. Dak. & 49 & $1992-2008$ & 1,050 & 304 & 1,820 \\
\hline 181 & Pipestem Creek near Buchanan, N. Dak. & 5 & 1993 & 599 & 135 & 851 \\
\hline 183 & James River at Lamoure, N. Dak. & 205 & 1980-2008 & 938 & 293 & 2,600 \\
\hline 184 & Bear Creek near Oakes, N. Dak. & 42 & 1990-2008 & 1,330 & 422 & 1,830 \\
\hline 185 & James River at Oakes, N. Dak. & 97 & $1982-2008$ & 928 & 238 & 4,580 \\
\hline 186 & James River at N. Dak./S. Dak. State line & 30 & 1980-2008 & 928 & 527 & 1,520 \\
\hline 3 & Red River of the North near Wahpeton, N. Dak. & 5 & 1993-2006 & 8.0 & 7.9 & 8.5 \\
\hline 4 & Red River of the North at Brushville, Minn. & 60 & 1993-2007 & 8.2 & 7.5 & 9.0 \\
\hline 5 & Red River of the North below Wahpeton, N. Dak. & 65 & 1970-1999 & 8.1 & 7.4 & 9.1 \\
\hline 6 & Red River of the North at Hickson, N. Dak. & 171 & 1975-2008 & 8.1 & 7.2 & 9.4 \\
\hline 7 & Wild Rice River near Rutland, N. Dak. & 47 & $1971-2008$ & 8.1 & 6.8 & 8.6 \\
\hline 8 & Wild Rice River near Cayuga, N. Dak. & 15 & $1970-1977$ & 8.0 & 7.4 & 9.4 \\
\hline 9 & Antelope Creek at Dwight, N. Dak. & 13 & $2001-2008$ & 7.9 & 7.2 & 8.4 \\
\hline 10 & Wild Rice River near Abercrombie, N. Dak. & 246 & 1970-2008 & 8.0 & 6.8 & 8.9 \\
\hline 11 & Red River of the North at Fargo, N. Dak. & 274 & 1970-2008 & 8.0 & 7.2 & 8.8 \\
\hline 18 & Sheyenne River near Warwick, N. Dak. & 226 & 1970-2008 & 8.1 & 6.7 & 9.2 \\
\hline 19 & Mauvais Coulee Tributary No. 3 near Cando, N. Dak. & 38 & $1987-2008$ & 7.8 & 6.9 & 8.9 \\
\hline 20 & Mauvais Coulee near Cando, N. Dak. & 98 & 1972-2008 & 7.9 & 6.8 & 9.6 \\
\hline 21 & Edmore Coulee near Edmore, N. Dak. & 104 & $1972-2008$ & 7.9 & 6.7 & 9.8 \\
\hline 22 & Edmore Coulee Tributary near Webster, N. Dak. & 38 & $1987-2008$ & 7.8 & 6.8 & 8.8 \\
\hline 23 & Webster Coulee at Webster, N. Dak. & 2 & 1983-1984 & 8.2 & 8.1 & 8.2 \\
\hline 24 & Starkweather Coulee near Webster, N. Dak. & 93 & 1983-2008 & 8.0 & 6.8 & 9.4 \\
\hline 25 & Big Coulee below Churchs Ferry, N. Dak. & 23 & 1998-2008 & 8.4 & 7.3 & 9.0 \\
\hline 26 & Little Coulee near Leeds, N. Dak. & 14 & 1998-2008 & 8.4 & 6.8 & 9.0 \\
\hline 27 & Little Coulee near Brinsmade, N. Dak. & 31 & 1976-1998 & 7.9 & 7.2 & 8.9 \\
\hline 28 & Big Coulee near Churchs Ferry, N. Dak. & 151 & 1970-1997 & 8.0 & 6.8 & 9.5 \\
\hline 29 & Big Coulee at Graham Island inlet near Fort Totten, N. Dak. & 46 & $1970-1986$ & 8.5 & 7.3 & 9.2 \\
\hline 30 & Channel A near Penn, N. Dak. & 99 & 1984-2008 & 8.1 & 6.6 & 10.1 \\
\hline 31 & Devils Lake Outlet to Stump Lake near Lakota, N. Dak. & 12 & 1999-2007 & 8.7 & 8.1 & 9.2 \\
\hline
\end{tabular}


Table 1-4. Summary statistics for field measurements, total suspended solids, and suspended sediment at selected sites in North Dakota from 1970 through 2008. - Continued

$[\mu \mathrm{S} / \mathrm{cm}$, microsiemens per centimeter; deg. C, degrees Celsius; --, not available; mg/L, milligrams per liter; <, less than]

\begin{tabular}{|c|c|c|c|c|c|c|}
\hline $\begin{array}{c}\text { Site } \\
\text { identification } \\
\text { number } \\
\text { (table 1) }\end{array}$ & Site name & $\begin{array}{c}\text { Number of } \\
\text { samples }\end{array}$ & $\begin{array}{l}\text { Period of } \\
\text { record }\end{array}$ & Median & Minimum & Maximum \\
\hline \multicolumn{7}{|c|}{$\mathrm{pH}$, field, in standard units-Continued } \\
\hline 32 & Sheyenne River near Cooperstown, N. Dak. & 364 & $1970-2008$ & 8.1 & 6.8 & 8.9 \\
\hline 33 & Baldhill Creek near Dazey, N. Dak. & 102 & 1972-2008 & 8.1 & 6.7 & 8.7 \\
\hline 34 & Sheyenne River below Baldhill Dam, N. Dak. & 178 & 1972-2008 & 8.4 & 7.4 & 9.2 \\
\hline 35 & Sheyenne River at Valley City, N. Dak. & 38 & $1972-2005$ & 8.0 & 7.3 & 8.8 \\
\hline 36 & Sheyenne River at Lisbon, N. Dak. & 338 & 1970-2008 & 8.1 & 7.1 & 9.0 \\
\hline 37 & Sheyenne River near Kindred, N. Dak. & 378 & $1972-2008$ & 8.2 & 6.9 & 8.9 \\
\hline 38 & $\begin{array}{l}\text { Sheyenne River above Sheyenne River Diversion near Horace, } \\
\text { N. Dak. }\end{array}$ & 40 & 1993-2008 & 8.2 & 7.5 & 8.6 \\
\hline 39 & Sheyenne River near Horace, N. Dak. & 46 & 1976-1992 & 8.0 & 7.0 & 8.5 \\
\hline 40 & Sheyenne River Diversion at West Fargo, N. Dak. & 14 & 1994-2007 & 8.1 & 7.1 & 8.5 \\
\hline 41 & Sheyenne River at West Fargo, N. Dak. & 63 & 1970-2008 & 8.0 & 6.7 & 8.6 \\
\hline 42 & Maple River near Hope, N. Dak. & 39 & 1972-2008 & 7.8 & 7.1 & 8.5 \\
\hline 43 & Maple River near Enderlin, N. Dak. & 66 & $1972-2008$ & 7.8 & 6.9 & 8.4 \\
\hline 44 & Maple River near Mapleton, N. Dak. & 27 & $1972-2008$ & 8.0 & 6.5 & 8.6 \\
\hline 45 & Maple River below Mapleton, N. Dak. & 68 & 1995-2008 & 8.2 & 6.5 & 8.9 \\
\hline 46 & Sheyenne River at Harwood, N. Dak. & 35 & 1993-2005 & 8.0 & 7.1 & 8.5 \\
\hline 47 & Rush River at Amenia, N. Dak. & 50 & $1972-2008$ & 8.0 & 6.8 & 8.8 \\
\hline 48 & Rush River near Prosper, N. Dak. & 6 & 1983-1987 & 8.0 & 7.6 & 8.4 \\
\hline 49 & Lower Branch Rush River near Prosper, N. Dak. & 6 & $1983-1993$ & 7.8 & 6.6 & 8.4 \\
\hline 50 & Sheyenne River near Harwood, N. Dak. & 49 & 1970-1996 & 8.0 & 7.1 & 8.5 \\
\hline 51 & Elm River near Kelso, N. Dak. & 6 & $1983-1993$ & 8.1 & 6.5 & 8.5 \\
\hline 52 & Red River of the North at Halstad, Minn. & 328 & $1972-2008$ & 8.1 & 6.7 & 9.3 \\
\hline 53 & Beaver Creek near Finley, N. Dak. & 204 & $1970-2003$ & 8.2 & 7.1 & 9.4 \\
\hline 54 & Goose River near Portland, N. Dak. & 34 & 1970-1992 & 7.8 & 7.1 & 8.8 \\
\hline 55 & Goose River at Hillsboro, N. Dak. & 162 & $1970-2008$ & 8.1 & 7.3 & 9.0 \\
\hline 56 & Red River of the North at Grand Forks, N. Dak. & 233 & $1970-2008$ & 8.0 & 7.2 & 9.0 \\
\hline 57 & Turtle River at Turtle River State Park near Arvilla, N. Dak. & 121 & 1991-2008 & 8.1 & 7.0 & 8.6 \\
\hline 58 & Turtle River at Manvel, N. Dak. & 107 & $1971-2008$ & 8.1 & 6.7 & 8.9 \\
\hline 59 & Red River of the North at Oslo, Minn. & 75 & $1973-2005$ & 8.0 & 7.3 & 8.8 \\
\hline 60 & Middle Branch Forest River near Whitman, N. Dak. & 18 & $1972-1990$ & 7.9 & 7.0 & 8.2 \\
\hline 61 & Forest River near Fordville, N. Dak. & 67 & $1972-2008$ & 8.1 & 7.2 & 8.8 \\
\hline 62 & Forest River near Minto, N. Dak. & 156 & $1971-2008$ & 8.2 & 7.1 & 9.0 \\
\hline 63 & South Branch Park River below Homme Dam, N. Dak. & 26 & 1972-1994 & 8.0 & 7.1 & 8.3 \\
\hline 64 & Middle Branch Park River near Union, N. Dak. & 17 & 1972-1984 & 7.8 & 7.4 & 8.4 \\
\hline 65 & Middle Branch Park River near Edinburg, N. Dak. & 14 & $1978-1980$ & 8.2 & 7.7 & 8.5 \\
\hline 66 & Cart Creek at Mountain, N. Dak. & 20 & $1972-1984$ & 8.0 & 6.9 & 8.4 \\
\hline 67 & Park River at Grafton, N. Dak. & 144 & 1970-2008 & 8.1 & 7.1 & 9.0 \\
\hline 68 & Red River of the North at Drayton, N. Dak. & 74 & $1972-2008$ & 8.1 & 7.4 & 8.7 \\
\hline 69 & Pembina County Drain 20 near Glasston, N. Dak. & 9 & 1974-1984 & 7.7 & 7.6 & 8.1 \\
\hline
\end{tabular}


Table 1-4. Summary statistics for field measurements, total suspended solids, and suspended sediment at selected sites in North Dakota from 1970 through 2008. - Continued

$[\mu \mathrm{S} / \mathrm{cm}$, microsiemens per centimeter; deg. $\mathrm{C}$, degrees Celsius; --, not available; mg/L, milligrams per liter; <, less than]

\begin{tabular}{|c|c|c|c|c|c|c|}
\hline $\begin{array}{c}\text { Site } \\
\text { identification } \\
\text { number } \\
\text { (table 1) }\end{array}$ & Site name & $\begin{array}{c}\text { Number of } \\
\text { samples }\end{array}$ & $\begin{array}{l}\text { Period of } \\
\text { record }\end{array}$ & Median & Minimum & Maximum \\
\hline \multicolumn{7}{|c|}{$\mathrm{pH}$, field, in standard units-Continued } \\
\hline 70 & Hidden Island Coulee near Hansboro, N. Dak. & 20 & 1972-1995 & 7.7 & 7.1 & 8.2 \\
\hline 71 & Cypress Creek near Sarles, N. Dak. & 13 & $1972-1988$ & 7.7 & 6.7 & 8.0 \\
\hline 72 & Pembina River near Vang, N. Dak. & 87 & 1970-1979 & 8.0 & 7.2 & 8.6 \\
\hline 73 & Little South Pembina River near Walhalla, N. Dak. & 115 & 1970-2008 & 8.0 & 7.1 & 8.7 \\
\hline 74 & Pembina River at Walhalla, N. Dak. & 293 & 1970-2008 & 8.0 & 6.9 & 8.9 \\
\hline 75 & Pembina River at Neche, N. Dak. & 127 & $1972-2008$ & 8.2 & 6.7 & 8.8 \\
\hline 76 & Tongue River at Akra, N. Dak. & 88 & $1972-2008$ & 8.0 & 7.1 & 9.0 \\
\hline 77 & Red River of the North at Pembina, N. Dak., site 1 & 10 & 1993 & 8.0 & 7.3 & 8.4 \\
\hline 78 & Red River of the North at Pembina, N. Dak., site 2 & 181 & 1970-2008 & 8.2 & 6.6 & 9.0 \\
\hline 79 & Red River of the North at Emerson, Manitoba & 389 & 1978-2004 & 8.0 & 6.8 & 8.9 \\
\hline 80 & Long Creek near Noonan, N. Dak. & 68 & $1972-2008$ & 8.1 & 6.6 & 10.3 \\
\hline 81 & West Branch Short Creek near Columbus, N. Dak. & 21 & 1978-1981 & 8.4 & 7.4 & 8.9 \\
\hline 82 & Souris River near Sherwood, N. Dak. & 420 & $1972-2008$ & 8.1 & 6.9 & 9.2 \\
\hline 83 & Souris River near Foxholm, N. Dak. & 209 & $1972-2008$ & 8.4 & 6.8 & 9.8 \\
\hline 84 & Des Lacs River at Foxholm, N. Dak. & 187 & $1972-2008$ & 8.3 & 7.2 & 9.3 \\
\hline 85 & Souris River above Minot, N. Dak. & 216 & 1970-2008 & 8.2 & 7.2 & 9.5 \\
\hline 86 & Bonnes Creek near Velva, N. Dak. & 18 & $1987-2005$ & 8.1 & 7.4 & 8.7 \\
\hline 87 & Souris River near Verendrye, N. Dak. & 394 & $1970-2008$ & 8.1 & 7.0 & 9.1 \\
\hline 88 & Wintering River near Karlsruhe, N. Dak. & 137 & $1972-2008$ & 8.0 & 7.2 & 8.8 \\
\hline 89 & Souris River near Bantry, N. Dak. & 169 & 1970-2008 & 8.0 & 7.0 & 8.9 \\
\hline 90 & Willow Creek near Willow City, N. Dak. & 91 & $1972-2008$ & 8.2 & 7.2 & 9.8 \\
\hline 91 & Stone Creek near Kramer, N. Dak. & 30 & $1986-2000$ & 8.0 & 7.1 & 8.4 \\
\hline 92 & Deep River near Upham, N. Dak. & 65 & $1972-2007$ & 8.1 & 7.1 & 9.9 \\
\hline 93 & Egg Creek near Granville, N. Dak. & 14 & $1972-1981$ & 7.9 & 7.3 & 8.3 \\
\hline 94 & Cut Bank Creek at North Lake Outlet near Granville, N. Dak. & 15 & 1972-1976 & 8.0 & 7.4 & 9.3 \\
\hline 95 & Cut Bank Creek at Upham, N. Dak. & 31 & $1975-2000$ & 7.8 & 7.0 & 9.6 \\
\hline 96 & Deep River below Cut Bank Creek near Upham, N. Dak. & 43 & $1975-1989$ & 8.1 & 7.3 & 9.7 \\
\hline 97 & Boundary Creek near Landa, N. Dak. & 38 & $1972-2000$ & 8.0 & 7.3 & 8.9 \\
\hline 98 & Souris River near Westhope, N. Dak. & 559 & $1970-2008$ & 8.4 & 7.0 & 9.8 \\
\hline 99 & Charbonneau Creek near Charbonneau, N. Dak. & 23 & $1972-2006$ & 8.4 & 8.0 & 8.9 \\
\hline 100 & Missouri River near Williston, N. Dak. & 102 & 1970-1992 & 8.3 & 7.8 & 9.4 \\
\hline 101 & Little Muddy River below Cow Creek near Williston, N. Dak. & 74 & $1972-2008$ & 8.3 & 7.2 & 9.0 \\
\hline 102 & Stony Creek near Williston, N. Dak. & 36 & $1977-1981$ & 8.4 & 8.0 & 8.7 \\
\hline 103 & Tobacco Garden Creek near Watford City, N. Dak. & 14 & 1976-1977 & 8.4 & 7.4 & 8.8 \\
\hline 104 & Beaver Creek near Ray, N. Dak. & 57 & 1977-1982 & 8.3 & 7.8 & 9.2 \\
\hline 105 & White Earth River at White Earth, N. Dak. & 25 & 1970-1977 & 8.3 & 7.5 & 8.8 \\
\hline 106 & Bear Den Creek near Mandaree, N. Dak. & 399 & 1970-2008 & 8.4 & 7.1 & 9.6 \\
\hline 107 & Shell Creek near Parshall, N. Dak. & 21 & 1972-1977 & 8.3 & 7.8 & 8.9 \\
\hline 108 & East Fork Shell Creek near Parshall, N. Dak. & 54 & 1991-2008 & 8.5 & 7.1 & 9.2 \\
\hline
\end{tabular}


Table 1-4. Summary statistics for field measurements, total suspended solids, and suspended sediment at selected sites in North Dakota from 1970 through 2008. - Continued

$[\mu \mathrm{S} / \mathrm{cm}$, microsiemens per centimeter; deg. $\mathrm{C}$, degrees Celsius; --, not available; mg/L, milligrams per liter; <, less than]

\begin{tabular}{|c|c|c|c|c|c|c|}
\hline $\begin{array}{c}\text { Site } \\
\text { identification } \\
\text { number } \\
\text { (table 1) }\end{array}$ & Site name & $\begin{array}{c}\text { Number of } \\
\text { samples }\end{array}$ & $\begin{array}{l}\text { Period of } \\
\text { record }\end{array}$ & Median & Minimum & Maximum \\
\hline \multicolumn{7}{|c|}{$\mathrm{pH}$, field, in standard units-Continued } \\
\hline 109 & Deepwater Creek near Mandaree, N. Dak. & 62 & 1990-2008 & 8.5 & 7.6 & 9.3 \\
\hline 110 & Little Missouri River at Marmarth, N. Dak. & 88 & 1971-2008 & 8.4 & 6.9 & 10.5 \\
\hline 111 & Deep Creek near Amidon, N. Dak. & 59 & $1977-1983$ & 8.3 & 6.9 & 8.7 \\
\hline 112 & Little Missouri River at Medora, N. Dak. & 109 & $1972-2008$ & 8.3 & 7.2 & 10.7 \\
\hline 113 & Beaver Creek near Trotters, N. Dak. & 96 & 1977-2008 & 8.3 & 7.5 & 10.4 \\
\hline 114 & Little Missouri River near Watford City, N. Dak. & 681 & 1971-2008 & 8.4 & 7.3 & 9.2 \\
\hline 115 & Missouri River at Garrison Dam, N. Dak. & 474 & $1971-2007$ & 8.1 & 7.2 & 8.8 \\
\hline 116 & Knife River at Manning, N. Dak. & 120 & 1972-2008 & 8.2 & 6.7 & 9.2 \\
\hline 117 & Stray Creek near Manning, N. Dak. & 22 & $1975-1981$ & 8.4 & 7.4 & 9.6 \\
\hline 118 & Knife River at Marshall, N. Dak. & 70 & $1972-1981$ & 8.3 & 7.3 & 8.8 \\
\hline 119 & Elm Creek near Golden Valley, N. Dak. & 37 & $1973-1995$ & 8.1 & 7.1 & 8.7 \\
\hline 120 & Knife River near Golden Valley, N. Dak. & 127 & 1971-2008 & 8.4 & 6.6 & 9.4 \\
\hline 121 & Coyote Creek near Zap, N. Dak. & 62 & $1977-1983$ & 8.3 & 7.2 & 8.7 \\
\hline 122 & Brush Creek near Beulah, N. Dak. & 131 & $1974-1990$ & 8.0 & 6.9 & 8.8 \\
\hline 123 & Spring Creek below Lake Ilo near Dunn Center, N. Dak. & 58 & $1977-1993$ & 8.2 & 7.1 & 8.8 \\
\hline 124 & Spring Creek near Halliday, N. Dak. & 52 & 1977-1981 & 8.3 & 7.0 & 8.7 \\
\hline 125 & Spring Creek at Zap, N. Dak. & 233 & 1970-2008 & 8.2 & 6.6 & 9.2 \\
\hline 126 & West Branch Otter Creek near Beulah, N. Dak. & 15 & 1972-1994 & 8.0 & 6.9 & 9.3 \\
\hline 127 & Knife River at Hazen, N. Dak. & 480 & 1970-2008 & 8.3 & 7.0 & 9.2 \\
\hline 128 & Antelope Creek above Hazen, N. Dak. & 51 & $1977-1985$ & 8.0 & 6.6 & 8.7 \\
\hline 129 & West Branch Antelope Creek No. 4 near Zap, N. Dak. & 22 & 1977-1985 & 7.5 & 7.0 & 8.2 \\
\hline 130 & West Branch Antelope Creek near Hazen, N. Dak. & 18 & $1978-1983$ & 7.8 & 6.7 & 8.6 \\
\hline 131 & Coal Creek near Stanton, N. Dak. & 32 & $1975-1981$ & 8.3 & 7.9 & 9.0 \\
\hline 132 & Alderin Creek near Fort Clark, N. Dak. & 53 & $1977-1983$ & 8.3 & 7.3 & 8.7 \\
\hline 133 & Coal Lake Coulee near Hensler, N. Dak. & 45 & 1978-1988 & 8.3 & 6.6 & 8.7 \\
\hline 134 & Buffalo Creek near Washburn, N. Dak. & 48 & $1978-1983$ & 8.3 & 7.2 & 8.8 \\
\hline 135 & Turtle Creek above Washburn, N. Dak. & 99 & $1987-2003$ & 8.4 & 7.0 & 9.2 \\
\hline 136 & Painted Woods Creek near Wilton, N. Dak. & 193 & 1970-2003 & 8.3 & 6.7 & 9.7 \\
\hline 137 & Square Butte Creek near Hannover, N. Dak. & 26 & 1977-1981 & 8.2 & 7.6 & 8.6 \\
\hline 138 & Square Butte Creek above Nelson Lake near Center, N. Dak. & 64 & $1977-1982$ & 8.1 & 6.8 & 8.8 \\
\hline 139 & Hagel Creek near Center, N. Dak. & 44 & 1977-1982 & 8.2 & 6.7 & 9.8 \\
\hline 140 & Square Butte Creek below Center, N. Dak. & 86 & 1972-2008 & 8.0 & 7.1 & 9.3 \\
\hline 141 & Burnt Creek near Bismarck, N. Dak. & 49 & $1972-2008$ & 8.0 & 6.8 & 8.5 \\
\hline 142 & Missouri River at Bismarck, N. Dak. & 195 & 1970-2008 & 8.3 & 7.4 & 8.7 \\
\hline 143 & South Branch Heart River near South Heart, N. Dak. & 33 & 1979-1996 & 8.1 & 6.9 & 8.8 \\
\hline 144 & North Creek near South Heart, N. Dak. & 26 & 1978-1996 & 8.2 & 7.3 & 9.1 \\
\hline 145 & Heart River near South Heart, N. Dak. & 83 & $1975-2005$ & 8.3 & 6.8 & 8.8 \\
\hline 146 & Heart River at Dickinson, N. Dak. & 21 & 1986-1994 & 8.3 & 7.4 & 9.0 \\
\hline 147 & Green River near New Hradec, N. Dak. & 120 & 1972-2008 & 8.1 & 6.5 & 8.9 \\
\hline
\end{tabular}


Table 1-4. Summary statistics for field measurements, total suspended solids, and suspended sediment at selected sites in North Dakota from 1970 through 2008. - Continued

$[\mu \mathrm{S} / \mathrm{cm}$, microsiemens per centimeter; deg. $\mathrm{C}$, degrees Celsius; --, not available; mg/L, milligrams per liter; <, less than]

\begin{tabular}{|c|c|c|c|c|c|c|}
\hline $\begin{array}{c}\text { Site } \\
\text { identification } \\
\text { number } \\
\text { (table 1) }\end{array}$ & Site name & $\begin{array}{c}\text { Number of } \\
\text { samples }\end{array}$ & $\begin{array}{l}\text { Period of } \\
\text { record }\end{array}$ & Median & Minimum & Maximum \\
\hline \multicolumn{7}{|c|}{$\mathrm{pH}$, field, in standard units-Continued } \\
\hline 148 & Green River near Gladstone, N. Dak. & 33 & 1970-1993 & 7.9 & 6.8 & 8.3 \\
\hline 149 & Heart River near Richardton, N. Dak. & 149 & $1972-2008$ & 8.3 & 7.4 & 8.9 \\
\hline 151 & Antelope Creek near Carson, N. Dak. & 25 & $1972-2008$ & 8.2 & 7.4 & 9.1 \\
\hline 152 & Big Muddy Creek near Almont, N. Dak. & 39 & 1991-2008 & 8.3 & 7.3 & 9.3 \\
\hline 153 & Heart River near Lark, N. Dak. & 45 & 1971-1995 & 8.1 & 6.6 & 8.6 \\
\hline 154 & Heart River at Stark Bridge near Judson, N. Dak. & 40 & $1988-2008$ & 8.3 & 6.9 & 8.7 \\
\hline 158 & Apple Creek near Menoken, N. Dak. & 133 & $1972-2008$ & 8.1 & 6.9 & 9.5 \\
\hline 159 & Missouri River near Schmidt, N. Dak. & 143 & 1974-1981 & 8.3 & 7.7 & 8.8 \\
\hline 160 & Cannonball River at New England, N. Dak. & 39 & 1978-1981 & 8.2 & 7.3 & 8.6 \\
\hline 161 & Coal Bank Creek near Havelock, N. Dak. & 91 & 1974-1983 & 8.1 & 7.0 & 10.1 \\
\hline 162 & Cannonball River at Regent, N. Dak. & 118 & 1970-2008 & 8.2 & 6.8 & 8.9 \\
\hline 163 & Cannonball River below Bentley, N. Dak. & 31 & $1972-1977$ & 8.1 & 6.8 & 8.8 \\
\hline 164 & Cannonball River near Raleigh, N. Dak. & 86 & 1993-2008 & 8.4 & 7.0 & 9.1 \\
\hline 165 & White Butte Fork Cedar Creek near Scranton, N. Dak. & 26 & 1972-1995 & 8.0 & 7.2 & 8.8 \\
\hline 166 & Cedar Creek near Haynes, N. Dak. & 76 & $1971-2008$ & 8.2 & 7.1 & 9.5 \\
\hline 173 & Porcupine Creek near Fort Yates, N. Dak. & 42 & 1991-1999 & 8.0 & 7.4 & 8.5 \\
\hline 174 & Buffalo Creek Tributary near Gascoyne, N. Dak. & 96 & 1974-1987 & 8.3 & 7.2 & 9.6 \\
\hline 175 & James River near Manfred, N. Dak. & 43 & 1972-1998 & 8.0 & 7.1 & 9.1 \\
\hline 176 & James River near Grace City, N. Dak. & 145 & 1972-2008 & 8.3 & 7.0 & 9.9 \\
\hline 177 & James River above Arrowwood Lake near Kensal, N. Dak. & 163 & $1985-2008$ & 8.2 & 7.0 & 9.5 \\
\hline 178 & Kelly Creek below Niccum Reservoir near Bordulac, N. Dak. & 9 & 1986-1989 & 8.4 & 7.6 & 9.4 \\
\hline 179 & James River near Pingree, N. Dak. & 129 & 1979-2008 & 8.5 & 7.3 & 9.7 \\
\hline 180 & Pipestem Creek near Pingree, N. Dak. & 71 & 1974-2008 & 8.1 & 7.1 & 9.5 \\
\hline 181 & Pipestem Creek near Buchanan, N. Dak. & 9 & 1972-1993 & 8.0 & 7.5 & 8.5 \\
\hline 182 & James River at Jamestown, N. Dak. & 187 & $1972-2008$ & 8.1 & 6.7 & 9.1 \\
\hline 183 & James River at Lamoure, N. Dak. & 277 & 1970-2008 & 8.2 & 6.6 & 9.8 \\
\hline 184 & Bear Creek near Oakes, N. Dak. & 54 & $1972-2008$ & 8.1 & 6.5 & 9.0 \\
\hline 185 & James River at Oakes, N. Dak. & 143 & 1970-2008 & 8.1 & 6.9 & 9.4 \\
\hline 186 & James River at N. Dak./S. Dak. State line & 92 & $1974-2008$ & 8.4 & 7.5 & 9.6 \\
\hline
\end{tabular}


Table 1-4. Summary statistics for field measurements, total suspended solids, and suspended sediment at selected sites in North Dakota from 1970 through 2008. - Continued

$[\mu \mathrm{S} / \mathrm{cm}$, microsiemens per centimeter; deg. C, degrees Celsius; --, not available; mg/L, milligrams per liter; <, less than]

\begin{tabular}{|c|c|c|c|c|c|c|}
\hline $\begin{array}{c}\text { Site } \\
\text { identification } \\
\text { number } \\
\text { (table 1) } \\
\end{array}$ & Site name & $\begin{array}{l}\text { Number of } \\
\text { samples }\end{array}$ & $\begin{array}{l}\text { Period of } \\
\text { record }\end{array}$ & Median & Minimum & Maximum \\
\hline \multicolumn{7}{|c|}{$\mathrm{pH}$, laboratory, in standard units } \\
\hline 1 & Bois De Sioux River near Doran, Minn. & 40 & 2000-2007 & 8.3 & 6.8 & 8.8 \\
\hline 2 & Red River of the North at Wahpeton, N. Dak. & 56 & $1983-2008$ & 8.1 & 6.6 & 9.1 \\
\hline 3 & Red River of the North near Wahpeton, N. Dak. & 4 & 1993-2006 & 8.0 & 7.8 & 8.4 \\
\hline 4 & Red River of the North at Brushville, Minn. & 61 & 1993-2007 & 8.2 & 7.0 & 8.6 \\
\hline 5 & Red River of the North below Wahpeton, N. Dak. & 15 & 1997-1999 & 8.1 & 7.6 & 8.4 \\
\hline 6 & Red River of the North at Hickson, N. Dak. & 72 & 1980-2008 & 8.1 & 6.7 & 9.1 \\
\hline 7 & Wild Rice River near Rutland, N. Dak. & 31 & 1984-2008 & 8.0 & 6.7 & 8.4 \\
\hline 9 & Antelope Creek at Dwight, N. Dak. & 11 & 2001-2008 & 7.9 & 7.6 & 8.4 \\
\hline 10 & Wild Rice River near Abercrombie, N. Dak. & 107 & 1980-2008 & 8.1 & 6.5 & 8.5 \\
\hline 11 & Red River of the North at Fargo, N. Dak. & 81 & 1983-2008 & 8.1 & 6.7 & 8.6 \\
\hline 12 & Red River of North below Fargo, N. Dak. & 45 & $1980-1986$ & 8.0 & 7.4 & 8.9 \\
\hline 13 & Red River of the North at Harwood, N. Dak. & 53 & $2000-2007$ & 8.1 & 6.9 & 8.5 \\
\hline 14 & Red River of the North near Harwood, N. Dak. & 54 & 1993-2007 & 8.0 & 7.5 & 8.6 \\
\hline 15 & Sheyenne River above Harvey, N. Dak. & 135 & 1980-2008 & 8.2 & 7.0 & 9.0 \\
\hline 17 & Sheyenne River at Warwick, N. Dak. & 48 & 1996-2006 & 8.3 & 7.4 & 8.6 \\
\hline 18 & Sheyenne River near Warwick, N. Dak. & 62 & 1983-2008 & 8.2 & 6.9 & 8.9 \\
\hline 19 & Mauvais Coulee Tributary No. 3 near Cando, N. Dak. & 27 & $1987-2007$ & 7.6 & 6.7 & 9.1 \\
\hline 20 & Mauvais Coulee near Cando, N. Dak. & 68 & 1983-2008 & 7.9 & 6.8 & 9.1 \\
\hline 21 & Edmore Coulee near Edmore, N. Dak. & 74 & 1983-2008 & 7.8 & 6.5 & 9.6 \\
\hline 22 & Edmore Coulee Tributary near Webster, N. Dak. & 21 & $1987-2007$ & 7.8 & 6.7 & 8.9 \\
\hline 23 & Webster Coulee at Webster, N. Dak. & 2 & $1983-1984$ & 7.9 & 7.8 & 8.0 \\
\hline 24 & Starkweather Coulee near Webster, N. Dak. & 61 & 1983-2008 & 7.9 & 6.5 & 9.0 \\
\hline 25 & Big Coulee below Churchs Ferry, N. Dak. & 16 & 1998-2008 & 8.1 & 7.7 & 8.8 \\
\hline 26 & Little Coulee near Leeds, N. Dak. & 12 & 1999-2008 & 8.1 & 6.9 & 8.6 \\
\hline 27 & Little Coulee near Brinsmade, N. Dak. & 16 & $1980-1998$ & 7.9 & 7.5 & 8.5 \\
\hline 28 & Big Coulee near Churchs Ferry, N. Dak. & 37 & $1983-1997$ & 7.8 & 6.8 & 8.7 \\
\hline 29 & Big Coulee at Graham Island inlet near Fort Totten, N. Dak. & 12 & $1980-1986$ & 8.5 & 8.0 & 8.8 \\
\hline 30 & Channel A near Penn, N. Dak. & 49 & 1984-2008 & 7.8 & 6.8 & 8.7 \\
\hline 31 & Devils Lake Outlet to Stump Lake near Lakota, N. Dak. & 12 & $2002-2007$ & 8.5 & 8.0 & 9.1 \\
\hline 32 & Sheyenne River near Cooperstown, N. Dak. & 153 & $1980-2008$ & 8.3 & 6.7 & 8.6 \\
\hline 33 & Baldhill Creek near Dazey, N. Dak. & 49 & 1980-2008 & 8.0 & 6.7 & 8.5 \\
\hline 34 & Sheyenne River below Baldhill Dam, N. Dak. & 132 & $1980-2008$ & 8.3 & 6.9 & 9.0 \\
\hline 35 & Sheyenne River at Valley City, N. Dak. & 15 & $1987-2005$ & 8.0 & 6.7 & 8.3 \\
\hline 36 & Sheyenne River at Lisbon, N. Dak. & 175 & 1980-2008 & 8.1 & 6.6 & 8.8 \\
\hline 37 & Sheyenne River near Kindred, N. Dak. & 185 & 1980-2008 & 8.1 & 7.4 & 8.6 \\
\hline 38 & $\begin{array}{l}\text { Sheyenne River above Sheyenne River Diversion near Horace, } \\
\text { N. Dak. }\end{array}$ & 40 & 1993-2008 & 8.2 & 7.4 & 8.6 \\
\hline 39 & Sheyenne River near Horace, N. Dak. & 7 & 1982-1991 & 7.8 & 6.6 & 8.2 \\
\hline 40 & Sheyenne River Diversion at West Fargo, N. Dak. & 9 & 1995-2007 & 8.0 & 7.0 & 8.5 \\
\hline
\end{tabular}


Table 1-4. Summary statistics for field measurements, total suspended solids, and suspended sediment at selected sites in North Dakota from 1970 through 2008. - Continued

$[\mu \mathrm{S} / \mathrm{cm}$, microsiemens per centimeter; deg. C, degrees Celsius; --, not available; mg/L, milligrams per liter; <, less than]

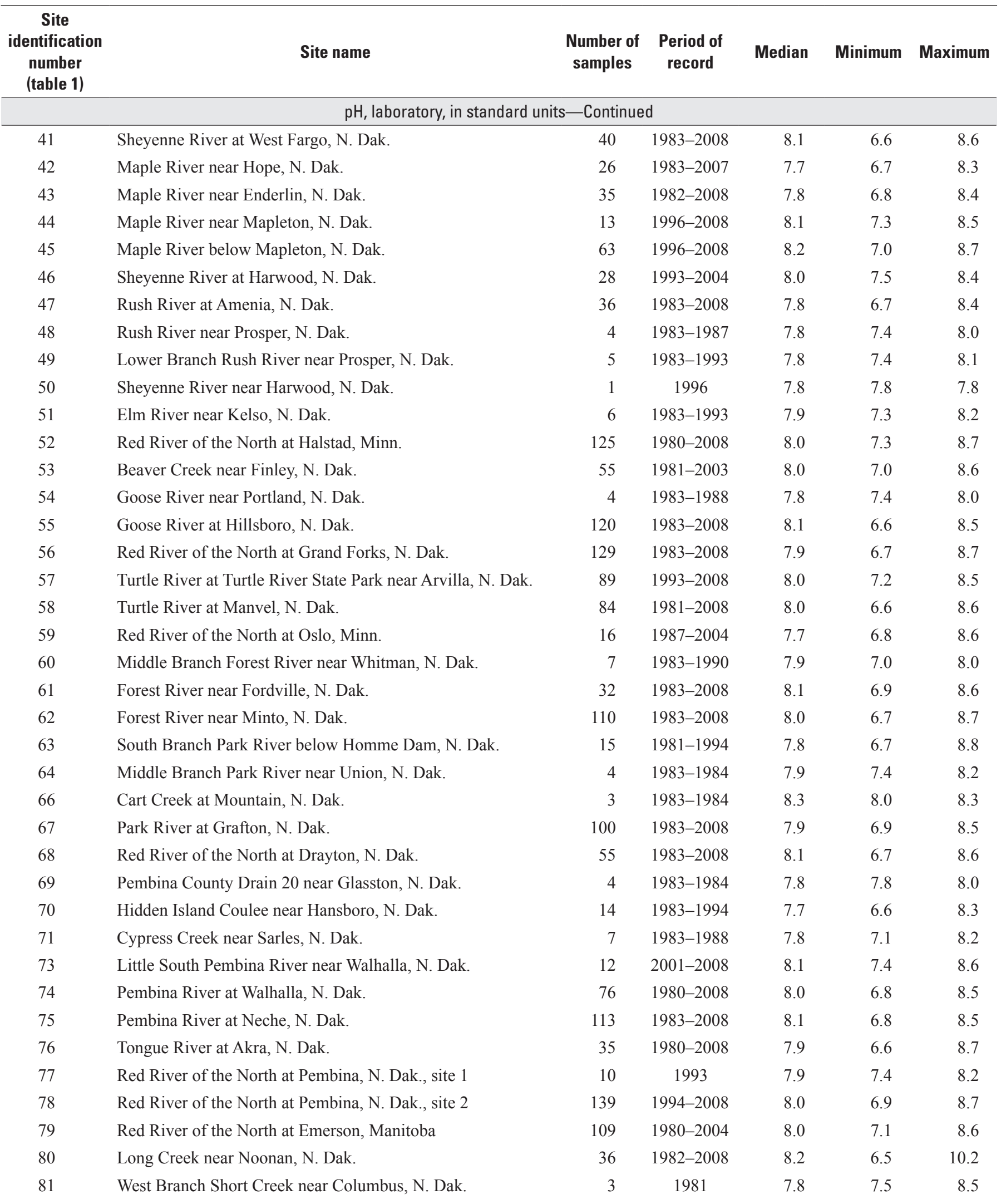


Table 1-4. Summary statistics for field measurements, total suspended solids, and suspended sediment at selected sites in North Dakota from 1970 through 2008. - Continued

$[\mu \mathrm{S} / \mathrm{cm}$, microsiemens per centimeter; deg. C, degrees Celsius; --, not available; mg/L, milligrams per liter; <, less than]

\begin{tabular}{|c|c|c|c|c|c|c|}
\hline $\begin{array}{c}\text { Site } \\
\text { identification } \\
\text { number } \\
\text { (table 1) }\end{array}$ & Site name & $\begin{array}{c}\text { Number of } \\
\text { samples }\end{array}$ & $\begin{array}{l}\text { Period of } \\
\text { record }\end{array}$ & Median & Minimum & Maximum \\
\hline \multicolumn{7}{|c|}{$\mathrm{pH}$, laboratory, in standard units-Continued } \\
\hline 82 & Souris River near Sherwood, N. Dak. & 212 & 1980-2008 & 8.1 & 7.0 & 9.2 \\
\hline 83 & Souris River near Foxholm, N. Dak. & 108 & 1972-2008 & 8.2 & 7.4 & 9.9 \\
\hline 84 & Des Lacs River at Foxholm, N. Dak. & 157 & 1983-2008 & 8.3 & 6.7 & 9.2 \\
\hline 85 & Souris River above Minot, N. Dak. & 171 & 1981-2008 & 8.2 & 6.7 & 9.5 \\
\hline 86 & Bonnes Creek near Velva, N. Dak. & 13 & $1987-2005$ & 7.9 & 7.2 & 8.3 \\
\hline 87 & Souris River near Verendrye, N. Dak. & 249 & 1981-2008 & 8.1 & 7.0 & 8.9 \\
\hline 88 & Wintering River near Karlsruhe, N. Dak. & 85 & 1980-2008 & 8.1 & 6.6 & 8.7 \\
\hline 89 & Souris River near Bantry, N. Dak. & 112 & 1981-2008 & 8.0 & 7.0 & 9.1 \\
\hline 90 & Willow Creek near Willow City, N. Dak. & 60 & 1983-2008 & 8.2 & 7.1 & 9.7 \\
\hline 91 & Stone Creek near Kramer, N. Dak. & 25 & $1986-2000$ & 8.1 & 6.9 & 8.8 \\
\hline 92 & Deep River near Upham, N. Dak. & 45 & $1987-2007$ & 8.2 & 6.6 & 9.8 \\
\hline 93 & Egg Creek near Granville, N. Dak. & 1 & 1981 & 7.7 & 7.7 & 7.7 \\
\hline 95 & Cut Bank Creek at Upham, N. Dak. & 16 & $1987-2000$ & 7.7 & 6.9 & 8.8 \\
\hline 96 & Deep River below Cut Bank Creek near Upham, N. Dak. & 13 & $1986-1989$ & 8.2 & 7.5 & 8.8 \\
\hline 97 & Boundary Creek near Landa, N. Dak. & 27 & $1986-2000$ & 7.9 & 7.0 & 8.7 \\
\hline 98 & Souris River near Westhope, N. Dak. & 84 & 1980-2008 & 8.2 & 7.3 & 10.0 \\
\hline 100 & Missouri River near Williston, N. Dak. & 20 & 1980-1992 & 8.2 & 8.1 & 8.7 \\
\hline 101 & Little Muddy River below Cow Creek near Williston, N. Dak. & 45 & $1983-2008$ & 8.3 & 6.7 & 8.8 \\
\hline 102 & Stony Creek near Williston, N. Dak. & 7 & $1980-1981$ & 8.4 & 7.3 & 8.5 \\
\hline 104 & Beaver Creek near Ray, N. Dak. & 17 & 1980-1982 & 8.2 & 7.7 & 8.5 \\
\hline 106 & Bear Den Creek near Mandaree, N. Dak. & 110 & 1980-2008 & 8.4 & 6.7 & 9.1 \\
\hline 108 & East Fork Shell Creek near Parshall, N. Dak. & 56 & 1991-2008 & 8.4 & 7.1 & 9.1 \\
\hline 109 & Deepwater Creek near Mandaree, N. Dak. & 54 & 1991-2008 & 8.4 & 7.0 & 9.3 \\
\hline 110 & Little Missouri River at Marmarth, N. Dak. & 44 & $1983-2008$ & 8.2 & 6.6 & 8.8 \\
\hline 111 & Deep Creek near Amidon, N. Dak. & 16 & $1980-1983$ & 8.2 & 7.8 & 8.5 \\
\hline 112 & Little Missouri River at Medora, N. Dak. & 98 & 1993-2007 & 8.3 & 6.7 & 8.7 \\
\hline 113 & Beaver Creek near Trotters, N. Dak. & 48 & 1980-2008 & 8.3 & 6.9 & 8.9 \\
\hline 114 & Little Missouri River near Watford City, N. Dak. & 153 & 1980-2007 & 8.3 & 6.9 & 8.7 \\
\hline 115 & Missouri River at Garrison Dam, N. Dak. & 147 & 1980-2007 & 8.3 & 7.5 & 8.5 \\
\hline 116 & Knife River at Manning, N. Dak. & 48 & 1980-2008 & 8.2 & 6.7 & 8.9 \\
\hline 117 & Stray Creek near Manning, N. Dak. & 5 & 1980-1981 & 8.2 & 7.2 & 8.4 \\
\hline 118 & Knife River at Marshall, N. Dak. & 11 & $1980-1981$ & 8.1 & 7.8 & 8.5 \\
\hline 119 & Elm Creek near Golden Valley, N. Dak. & 7 & $1981-1990$ & 7.4 & 6.8 & 7.6 \\
\hline 120 & Knife River near Golden Valley, N. Dak. & 91 & 1983-2008 & 8.3 & 6.8 & 8.8 \\
\hline 121 & Coyote Creek near Zap, N. Dak. & 21 & 1980-1983 & 8.3 & 7.4 & 8.6 \\
\hline 122 & Brush Creek near Beulah, N. Dak. & 50 & $1980-1990$ & 8.0 & 6.8 & 8.5 \\
\hline 123 & Spring Creek below Lake Ilo near Dunn Center, N. Dak. & 17 & $1980-1993$ & 8.3 & 7.5 & 8.6 \\
\hline 124 & Spring Creek near Halliday, N. Dak. & 11 & $1980-1981$ & 8.2 & 7.2 & 8.6 \\
\hline 125 & Spring Creek at Zap, N. Dak. & 132 & 1980-2008 & 8.2 & 6.9 & 8.9 \\
\hline
\end{tabular}


Table 1-4. Summary statistics for field measurements, total suspended solids, and suspended sediment at selected sites in North Dakota from 1970 through 2008. - Continued

$[\mu \mathrm{S} / \mathrm{cm}$, microsiemens per centimeter; deg. $\mathrm{C}$, degrees Celsius; --, not available; mg/L, milligrams per liter; <, less than]

\begin{tabular}{|c|c|c|c|c|c|c|}
\hline $\begin{array}{c}\text { Site } \\
\text { identification } \\
\text { number } \\
\text { (table 1) }\end{array}$ & Site name & $\begin{array}{l}\text { Number of } \\
\text { samples }\end{array}$ & $\begin{array}{l}\text { Period of } \\
\text { record }\end{array}$ & Median & Minimum & Maximum \\
\hline \multicolumn{7}{|c|}{$\mathrm{pH}$, laboratory, in standard units - Continued } \\
\hline 126 & West Branch Otter Creek near Beulah, N. Dak. & 7 & 1983-1995 & 7.7 & 6.9 & 8.7 \\
\hline 127 & Knife River at Hazen, N. Dak. & 170 & 1980-2008 & 8.2 & 6.9 & 8.6 \\
\hline 129 & West Branch Antelope Creek No. 4 near Zap, N. Dak. & 13 & $1981-1985$ & 7.8 & 7.2 & 8.2 \\
\hline 130 & West Branch Antelope Creek near Hazen, N. Dak. & 7 & $1981-1983$ & 8.0 & 7.3 & 8.3 \\
\hline 131 & Coal Creek near Stanton, N. Dak. & 6 & $1980-1981$ & 8.2 & 7.5 & 8.3 \\
\hline 132 & Alderin Creek near Fort Clark, N. Dak. & 20 & $1980-1983$ & 8.2 & 7.6 & 8.6 \\
\hline 136 & Painted Woods Creek near Wilton, N. Dak. & 132 & $1982-2003$ & 8.2 & 7.2 & 8.8 \\
\hline 137 & Square Butte Creek near Hannover, N. Dak. & 5 & 1980-1981 & 8.1 & 6.7 & 8.1 \\
\hline 138 & Square Butte Creek above Nelson Lake near Center, N. Dak. & 18 & 1980-1982 & 8.0 & 7.5 & 8.4 \\
\hline 139 & Hagel Creek near Center, N. Dak. & 14 & $1980-1982$ & 8.2 & 7.5 & 9.3 \\
\hline 140 & Square Butte Creek below Center, N. Dak. & 41 & $1983-2008$ & 8.2 & 7.1 & 9.0 \\
\hline 141 & Burnt Creek near Bismarck, N. Dak. & 20 & $1983-2008$ & 8.2 & 7.2 & 8.5 \\
\hline 142 & Missouri River at Bismarck, N. Dak. & 44 & 1986-2008 & 8.2 & 6.9 & 8.7 \\
\hline 143 & South Branch Heart River near South Heart, N. Dak. & 22 & 1980-1996 & 8.0 & 7.1 & 9.1 \\
\hline 144 & North Creek near South Heart, N. Dak. & 14 & 1980-1996 & 7.9 & 6.9 & 8.6 \\
\hline 151 & Antelope Creek near Carson, N. Dak. & 14 & 1999-2008 & 8.3 & 7.4 & 8.9 \\
\hline 152 & Big Muddy Creek near Almont, N. Dak. & 29 & 1991-2008 & 8.4 & 7.6 & 8.8 \\
\hline 153 & Heart River near Lark, N. Dak. & 19 & 1983-1995 & 8.2 & 7.6 & 8.7 \\
\hline 154 & Heart River at Stark Bridge near Judson, N. Dak. & 32 & 1988-2008 & 8.3 & 7.5 & 8.6 \\
\hline 155 & Sweetbriar Creek near Judson, N. Dak. & 11 & $2002-2008$ & 8.5 & 8.0 & 8.8 \\
\hline 156 & Heart River near Mandan, N. Dak. & 174 & $1980-2008$ & 8.3 & 6.9 & 8.7 \\
\hline 157 & Long Lake Creek above Long Lake near Moffit, N. Dak. & 22 & $1988-2004$ & 8.1 & 6.7 & 8.5 \\
\hline 158 & Apple Creek near Menoken, N. Dak. & 45 & 1980-2008 & 8.2 & 7.0 & 9.3 \\
\hline 159 & Missouri River near Schmidt, N. Dak. & 11 & 1980-1981 & 8.2 & 7.7 & 8.3 \\
\hline 160 & Cannonball River at New England, N. Dak. & 12 & $1980-1981$ & 8.1 & 6.8 & 8.3 \\
\hline 161 & Coal Bank Creek near Havelock, N. Dak. & 18 & $1980-1983$ & 8.1 & 7.0 & 8.6 \\
\hline 162 & Cannonball River at Regent, N. Dak. & 48 & 1980-2008 & 8.2 & 6.9 & 8.8 \\
\hline 164 & Cannonball River near Raleigh, N. Dak. & 87 & 1993-2008 & 8.4 & 6.9 & 9.1 \\
\hline 165 & White Butte Fork Cedar Creek near Scranton, N. Dak. & 15 & $1983-1995$ & 7.8 & 6.8 & 8.5 \\
\hline
\end{tabular}


Table 1-4. Summary statistics for field measurements, total suspended solids, and suspended sediment at selected sites in North Dakota from 1970 through 2008. - Continued

$[\mu \mathrm{S} / \mathrm{cm}$, microsiemens per centimeter; deg. C, degrees Celsius; --, not available; mg/L, milligrams per liter; <, less than]

\begin{tabular}{|c|c|c|c|c|c|c|}
\hline $\begin{array}{c}\text { Site } \\
\text { identification } \\
\text { number } \\
\text { (table 1) }\end{array}$ & Site name & $\begin{array}{c}\text { Number of } \\
\text { samples }\end{array}$ & $\begin{array}{l}\text { Period of } \\
\text { record }\end{array}$ & Median & Minimum & Maximum \\
\hline \multicolumn{7}{|c|}{$\mathrm{pH}$, laboratory, in standard units-Continued } \\
\hline 166 & Cedar Creek near Haynes, N. Dak. & 32 & $1982-2008$ & 8.4 & 6.8 & 9.5 \\
\hline 167 & Timber Creek near Bentley, N. Dak. & 12 & $1980-1981$ & 8.1 & 7.9 & 8.4 \\
\hline 169 & Cedar Creek near Raleigh, N. Dak. & 99 & 1983-2008 & 8.3 & 6.7 & 9.0 \\
\hline 170 & Cannonball River at Breien, N. Dak. & 158 & 1980-2008 & 8.3 & 6.5 & 9.0 \\
\hline 171 & Beaver Creek near Linton, N. Dak. & 11 & $1983-1993$ & 7.9 & 6.6 & 8.2 \\
\hline 172 & Beaver Creek below Linton, N. Dak. & 23 & 1990-2008 & 8.1 & 6.4 & 8.6 \\
\hline 173 & Porcupine Creek near Fort Yates, N. Dak. & 38 & 1991-1999 & 8.1 & 7.1 & 8.4 \\
\hline 174 & Buffalo Creek Tributary near Gascoyne, N. Dak. & 31 & $1980-1987$ & 8.2 & 7.3 & 8.9 \\
\hline 175 & James River near Manfred, N. Dak. & 42 & 1983-1998 & 8.1 & 6.9 & 8.6 \\
\hline 176 & James River near Grace City, N. Dak. & 136 & 1983-2008 & 8.3 & 6.7 & 9.8 \\
\hline 177 & James River above Arrowwood Lake near Kensal, N. Dak. & 168 & $1985-2008$ & 8.1 & 7.2 & 9.0 \\
\hline 178 & Kelly Creek below Niccum Reservoir near Bordulac, N. Dak. & 9 & 1986-1989 & 8.2 & 7.5 & 9.1 \\
\hline 179 & James River near Pingree, N. Dak. & 132 & $1981-2008$ & 8.3 & 7.3 & 9.8 \\
\hline 180 & Pipestem Creek near Pingree, N. Dak. & 52 & $1982-2008$ & 8.1 & 6.8 & 8.9 \\
\hline 181 & Pipestem Creek near Buchanan, N. Dak. & 5 & 1993 & 7.8 & 7.0 & 8.2 \\
\hline 182 & James River at Jamestown, N. Dak. & 185 & 1983-2008 & 8.0 & 7.3 & 8.9 \\
\hline 183 & James River at Lamoure, N. Dak. & 200 & 1980-2008 & 8.1 & 7.1 & 9.3 \\
\hline 184 & Bear Creek near Oakes, N. Dak. & 40 & 1984-2008 & 8.0 & 6.5 & 8.8 \\
\hline 185 & James River at Oakes, N. Dak. & 98 & $1982-2008$ & 8.0 & 7.0 & 8.8 \\
\hline 186 & James River at N. Dak./S. Dak. State line & 31 & 1980-2008 & 8.4 & 7.7 & 8.8 \\
\hline \multicolumn{7}{|c|}{ Water temperature, in deg. C } \\
\hline 1 & Bois De Sioux River near Doran, Minn. & 40 & 2000-2007 & 16.9 & -2.2 & 26.7 \\
\hline 2 & Red River of the North at Wahpeton, N. Dak. & 350 & 1971-2008 & 9.0 & 0.0 & 30.0 \\
\hline 3 & Red River of the North near Wahpeton, N. Dak. & 5 & 1993-2006 & 8.2 & 0.0 & 15.0 \\
\hline 4 & Red River of the North at Brushville, Minn. & 60 & 1993-2007 & 15.0 & -0.1 & 27.2 \\
\hline 5 & Red River of the North below Wahpeton, N. Dak. & 66 & 1970-1999 & 11.9 & -0.2 & 26.0 \\
\hline 6 & Red River of the North at Hickson, N. Dak. & 355 & 1975-2008 & 12.0 & -0.2 & 32.0 \\
\hline 7 & Wild Rice River near Rutland, N. Dak. & 172 & $1971-2008$ & 12.0 & 0.0 & 29.5 \\
\hline 8 & Wild Rice River near Cayuga, N. Dak. & 54 & 1970-1979 & 10.5 & 0.0 & 29.0 \\
\hline 9 & Antelope Creek at Dwight, N. Dak. & 23 & 2001-2008 & 14.1 & 0.0 & 33.0 \\
\hline 10 & Wild Rice River near Abercrombie, N. Dak. & 408 & 1970-2008 & 10.5 & -0.1 & 29.5 \\
\hline 11 & Red River of the North at Fargo, N. Dak. & 507 & 1970-2008 & 13.7 & -0.5 & 32.0 \\
\hline 12 & Red River of North below Fargo, N. Dak. & 176 & $1970-1986$ & 9.0 & 0.0 & 28.0 \\
\hline 13 & Red River of the North at Harwood, N. Dak. & 53 & $2000-2007$ & 15.2 & -1.9 & 27.4 \\
\hline 14 & Red River of the North near Harwood, N. Dak. & 58 & 1993-2007 & 15.4 & -0.2 & 26.7 \\
\hline 15 & Sheyenne River above Harvey, N. Dak. & 329 & 1971-2008 & 7.2 & -0.5 & 28.5 \\
\hline 16 & Big Coulee near Fort Totten, N. Dak. & 43 & $1970-1975$ & 6.0 & 0.0 & 24.5 \\
\hline 17 & Sheyenne River at Warwick, N. Dak. & 44 & 1996-2006 & 13.1 & -0.8 & 26.0 \\
\hline 18 & Sheyenne River near Warwick, N. Dak. & 377 & 1970-2008 & 8.0 & -1.2 & 30.0 \\
\hline
\end{tabular}


Table 1-4. Summary statistics for field measurements, total suspended solids, and suspended sediment at selected sites in North Dakota from 1970 through 2008. - Continued

$[\mu \mathrm{S} / \mathrm{cm}$, microsiemens per centimeter; deg. C, degrees Celsius; --, not available; mg/L, milligrams per liter; <, less than]

\begin{tabular}{|c|c|c|c|c|c|c|}
\hline $\begin{array}{c}\text { Site } \\
\text { identification } \\
\text { number } \\
\text { (table 1) }\end{array}$ & Site name & $\begin{array}{l}\text { Number of } \\
\text { samples }\end{array}$ & $\begin{array}{l}\text { Period of } \\
\text { record }\end{array}$ & Median & Minimum & Maximum \\
\hline \multicolumn{7}{|c|}{ Water temperature, in deg. $\mathrm{C}$-Continued } \\
\hline 19 & Mauvais Coulee Tributary No. 3 near Cando, N. Dak. & 100 & $1987-2008$ & 11.8 & 0.0 & 26.5 \\
\hline 20 & Mauvais Coulee near Cando, N. Dak. & 268 & $1971-2008$ & 10.3 & 0.0 & 27.5 \\
\hline 22 & Edmore Coulee Tributary near Webster, N. Dak. & 131 & $1986-2008$ & 10.7 & 0.0 & 27.5 \\
\hline 23 & Webster Coulee at Webster, N. Dak. & 11 & $1980-1985$ & 10.0 & 1.0 & 13.0 \\
\hline 24 & Starkweather Coulee near Webster, N. Dak. & 168 & 1980-2008 & 11.5 & 0.0 & 26.0 \\
\hline 25 & Big Coulee below Churchs Ferry, N. Dak. & 35 & $1998-2008$ & 12.0 & 1.0 & 26.0 \\
\hline 29 & Big Coulee at Graham Island inlet near Fort Totten, N. Dak. & 46 & 1970-1986 & 10.0 & 0.0 & 24.5 \\
\hline 30 & Channel A near Penn, N. Dak. & 177 & 1983-2008 & 7.5 & 0.0 & 29.5 \\
\hline 31 & Devils Lake Outlet to Stump Lake near Lakota, N. Dak. & 42 & 1999-2007 & 12.5 & 0.0 & 26.5 \\
\hline 32 & Sheyenne River near Cooperstown, N. Dak. & 541 & $1970-2008$ & 9.0 & -1.5 & 28.0 \\
\hline 33 & Baldhill Creek near Dazey, N. Dak. & 382 & $1971-2008$ & 6.8 & 0.0 & 29.0 \\
\hline 34 & Sheyenne River below Baldhill Dam, N. Dak. & 444 & $1971-2008$ & 8.5 & -1.0 & 26.5 \\
\hline 35 & Sheyenne River at Valley City, N. Dak. & 108 & $1971-2005$ & 5.5 & 0.0 & 26.0 \\
\hline 36 & Sheyenne River at Lisbon, N. Dak. & 463 & $1970-2008$ & 9.0 & -2.2 & 30.0 \\
\hline 37 & Sheyenne River near Kindred, N. Dak. & 632 & $1971-2008$ & 9.5 & -0.5 & 28.0 \\
\hline 43 & Maple River near Enderlin, N. Dak. & 338 & $1971-2008$ & 6.0 & 0.0 & 30.0 \\
\hline 44 & Maple River near Mapleton, N. Dak. & 104 & $1971-2008$ & 10.4 & 0.0 & 31.0 \\
\hline 45 & Maple River below Mapleton, N. Dak. & 169 & $1995-2008$ & 12.5 & -2.2 & 31.5 \\
\hline 46 & Sheyenne River at Harwood, N. Dak. & 42 & 1993-2005 & 9.5 & -0.2 & 26.5 \\
\hline 47 & Rush River at Amenia, N. Dak. & 281 & $1971-2008$ & 9.0 & 0.0 & 28.5 \\
\hline 48 & Rush River near Prosper, N. Dak. & 28 & $1981-1987$ & 13.8 & 0.0 & 27.0 \\
\hline 49 & Lower Branch Rush River near Prosper, N. Dak. & 19 & 1981-1993 & 8.0 & 0.0 & 27.0 \\
\hline 50 & Sheyenne River near Harwood, N. Dak. & 49 & 1970-1996 & 8.0 & 0.0 & 25.5 \\
\hline 51 & Elm River near Kelso, N. Dak. & 28 & 1981-1993 & 8.3 & 0.0 & 27.5 \\
\hline 52 & Red River of the North at Halstad, Minn. & 586 & $1972-2008$ & 9.5 & 0.0 & 28.5 \\
\hline 53 & Beaver Creek near Finley, N. Dak. & 336 & 1970-2003 & 10.0 & -0.5 & 30.5 \\
\hline 54 & Goose River near Portland, N. Dak. & 100 & 1970-1988 & 7.0 & 0.0 & 27.0 \\
\hline 55 & Goose River at Hillsboro, N. Dak. & 419 & 1970-2008 & 9.0 & -2.2 & 27.8 \\
\hline 56 & Red River of the North at Grand Forks, N. Dak. & 590 & 1970-2008 & 9.5 & -0.1 & 29.0 \\
\hline
\end{tabular}


Table 1-4. Summary statistics for field measurements, total suspended solids, and suspended sediment at selected sites in North Dakota from 1970 through 2008. - Continued

$[\mu \mathrm{S} / \mathrm{cm}$, microsiemens per centimeter; deg. C, degrees Celsius; --, not available; mg/L, milligrams per liter; <, less than]

\begin{tabular}{|c|c|c|c|c|c|c|}
\hline $\begin{array}{c}\text { Site } \\
\text { identification } \\
\text { number } \\
\text { (table 1) }\end{array}$ & Site name & $\begin{array}{c}\text { Number of } \\
\text { samples }\end{array}$ & $\begin{array}{l}\text { Period of } \\
\text { record }\end{array}$ & Median & Minimum & Maximum \\
\hline \multicolumn{7}{|c|}{ Water temperature, in deg. C-Continued } \\
\hline 57 & Turtle River at Turtle River State Park near Arvilla, N. Dak. & 202 & $1991-2008$ & 9.3 & 0.0 & 28.2 \\
\hline 58 & Turtle River at Manvel, N. Dak. & 104 & 1971-2008 & 11.8 & -0.1 & 28.6 \\
\hline 59 & Red River of the North at Oslo, Minn. & 81 & $1973-2005$ & 7.0 & 0.0 & 26.5 \\
\hline 60 & Middle Branch Forest River near Whitman, N. Dak. & 76 & $1972-1990$ & 6.0 & 0.0 & 28.0 \\
\hline 61 & Forest River near Fordville, N. Dak. & 327 & 1971-2008 & 6.0 & 0.0 & 27.5 \\
\hline 62 & Forest River near Minto, N. Dak. & 363 & $1971-2008$ & 8.8 & -2.2 & 27.0 \\
\hline 63 & South Branch Park River below Homme Dam, N. Dak. & 210 & 1971-1994 & 6.3 & 0.0 & 27.0 \\
\hline 64 & Middle Branch Park River near Union, N. Dak. & 114 & 1971-1986 & 8.0 & 0.0 & 30.0 \\
\hline 65 & Middle Branch Park River near Edinburg, N. Dak. & 18 & 1978-1980 & 4.3 & 0.0 & 27.0 \\
\hline 66 & Cart Creek at Mountain, N. Dak. & 125 & 1971-1984 & 3.5 & 0.0 & 29.5 \\
\hline 67 & Park River at Grafton, N. Dak. & 351 & 1970-2008 & 9.0 & -2.1 & 28.0 \\
\hline 68 & Red River of the North at Drayton, N. Dak. & 424 & 1971-2008 & 9.5 & 0.0 & 28.5 \\
\hline 69 & Pembina County Drain 20 near Glasston, N. Dak. & 48 & $1972-1986$ & 6.8 & 0.0 & 31.5 \\
\hline 70 & Hidden Island Coulee near Hansboro, N. Dak. & 99 & 1971-1995 & 8.0 & 0.0 & 28.0 \\
\hline 71 & Cypress Creek near Sarles, N. Dak. & 54 & 1972-1988 & 8.5 & 0.5 & 25.0 \\
\hline 72 & Pembina River near Vang, N. Dak. & 107 & 1970-1979 & 6.5 & 0.0 & 26.5 \\
\hline 73 & Little South Pembina River near Walhalla, N. Dak. & 229 & 1970-2008 & 4.5 & 0.0 & 28.5 \\
\hline 74 & Pembina River at Walhalla, N. Dak. & 392 & 1970-2008 & 6.0 & 0.0 & 28.5 \\
\hline 75 & Pembina River at Neche, N. Dak. & 405 & $1971-2008$ & 9.0 & -2.2 & 28.0 \\
\hline 76 & Tongue River at Akra, N. Dak. & 272 & $1971-2008$ & 10.0 & -0.5 & 28.5 \\
\hline 77 & Red River of the North at Pembina, N. Dak., site 1 & 10 & 1993 & 10.0 & 0.0 & 21.0 \\
\hline 78 & Red River of the North at Pembina, N. Dak., site 2 & 178 & 1970-2008 & 14.0 & -2.2 & 27.4 \\
\hline 79 & Red River of the North at Emerson, Manitoba & 404 & 1974-2004 & 9.2 & 0.0 & 29.0 \\
\hline 80 & Long Creek near Noonan, N. Dak. & 285 & $1971-2008$ & 7.0 & 0.0 & 28.9 \\
\hline 81 & West Branch Short Creek near Columbus, N. Dak. & 23 & $1978-1981$ & 6.5 & 0.0 & 22.5 \\
\hline 82 & Souris River near Sherwood, N. Dak. & 500 & $1970-2008$ & 8.0 & 0.0 & 29.0 \\
\hline 83 & Souris River near Foxholm, N. Dak. & 380 & $1971-2008$ & 8.0 & 0.0 & 27.0 \\
\hline 84 & Des Lacs River at Foxholm, N. Dak. & 403 & $1971-2008$ & 8.0 & -1.9 & 28.0 \\
\hline 85 & Souris River above Minot, N. Dak. & 428 & 1970-2008 & 8.0 & -1.8 & 31.5 \\
\hline 86 & Bonnes Creek near Velva, N. Dak. & 18 & $1987-2005$ & 2.8 & 0.0 & 24.0 \\
\hline 87 & Souris River near Verendrye, N. Dak. & 513 & 1970-2008 & 8.8 & -2.1 & 30.2 \\
\hline 88 & Wintering River near Karlsruhe, N. Dak. & 344 & $1971-2008$ & 8.3 & 0.0 & 30.0 \\
\hline 89 & Souris River near Bantry, N. Dak. & 457 & $1970-2008$ & 9.5 & 0.0 & 27.5 \\
\hline 90 & Willow Creek near Willow City, N. Dak. & 307 & $1971-2008$ & 10.0 & 0.0 & 29.3 \\
\hline 91 & Stone Creek near Kramer, N. Dak. & 137 & $1986-2000$ & 7.3 & 0.0 & 27.0 \\
\hline 92 & Deep River near Upham, N. Dak. & 148 & 1972-2007 & 13.5 & 0.0 & 29.0 \\
\hline 93 & Egg Creek near Granville, N. Dak. & 68 & 1971-1981 & 9.0 & 0.0 & 26.5 \\
\hline 94 & Cut Bank Creek at North Lake Outlet near Granville, N. Dak. & 58 & 1972-1980 & 11.0 & 0.0 & 28.5 \\
\hline 95 & Cut Bank Creek at Upham, N. Dak. & 74 & $1975-2000$ & 12.0 & 0.0 & 26.0 \\
\hline
\end{tabular}


Table 1-4. Summary statistics for field measurements, total suspended solids, and suspended sediment at selected sites in North Dakota from 1970 through 2008. - Continued

$[\mu \mathrm{S} / \mathrm{cm}$, microsiemens per centimeter; deg. C, degrees Celsius; --, not available; mg/L, milligrams per liter; <, less than]

\begin{tabular}{|c|c|c|c|c|c|c|}
\hline $\begin{array}{c}\text { Site } \\
\text { identification } \\
\text { number } \\
\text { (table 1) }\end{array}$ & Site name & $\begin{array}{c}\text { Number of } \\
\text { samples }\end{array}$ & $\begin{array}{l}\text { Period of } \\
\text { record }\end{array}$ & Median & Minimum & Maximum \\
\hline \multicolumn{7}{|c|}{ Water temperature, in deg. C-Continued } \\
\hline 96 & Deep River below Cut Bank Creek near Upham, N. Dak. & 131 & 1975-1989 & 10.5 & 0.0 & 24.0 \\
\hline 97 & Boundary Creek near Landa, N. Dak. & 214 & $1972-2000$ & 7.0 & 0.0 & 28.0 \\
\hline 98 & Souris River near Westhope, N. Dak. & 734 & 1970-2008 & 7.5 & 0.0 & 27.0 \\
\hline 99 & Charbonneau Creek near Charbonneau, N. Dak. & 126 & 1971-2006 & 5.0 & -0.2 & 28.0 \\
\hline 100 & Missouri River near Williston, N. Dak. & 103 & 1970-1992 & 10.0 & 0.0 & 25.0 \\
\hline 101 & Little Muddy River below Cow Creek near Williston, N. Dak. & 285 & $1971-2008$ & 8.5 & 0.0 & 26.0 \\
\hline 102 & Stony Creek near Williston, N. Dak. & 40 & $1977-1981$ & 4.0 & 0.0 & 26.5 \\
\hline 103 & Tobacco Garden Creek near Watford City, N. Dak. & 66 & $1976-1983$ & 4.0 & 0.0 & 23.0 \\
\hline 104 & Beaver Creek near Ray, N. Dak. & 65 & 1977-1982 & 7.5 & 0.0 & 28.0 \\
\hline 105 & White Earth River at White Earth, N. Dak. & 136 & 1970-1982 & 3.0 & 0.0 & 25.0 \\
\hline 106 & Bear Den Creek near Mandaree, N. Dak. & 505 & $1970-2008$ & 6.5 & 0.0 & 31.1 \\
\hline 107 & Shell Creek near Parshall, N. Dak. & 107 & 1971-1981 & 6.0 & 0.0 & 28.0 \\
\hline 108 & East Fork Shell Creek near Parshall, N. Dak. & 105 & 1991-2008 & 7.3 & 0.0 & 25.5 \\
\hline 109 & Deepwater Creek near Mandaree, N. Dak. & 117 & 1990-2008 & 9.0 & 0.0 & 26.0 \\
\hline 110 & Little Missouri River at Marmarth, N. Dak. & 354 & $1971-2008$ & 9.0 & 0.0 & 30.1 \\
\hline 111 & Deep Creek near Amidon, N. Dak. & 66 & $1977-1983$ & 7.5 & 0.0 & 29.0 \\
\hline 112 & Little Missouri River at Medora, N. Dak. & 178 & $1971-2008$ & 12.0 & -2.1 & 30.5 \\
\hline 113 & Beaver Creek near Trotters, N. Dak. & 213 & $1977-2008$ & 9.0 & 0.0 & 26.2 \\
\hline 114 & Little Missouri River near Watford City, N. Dak. & 830 & $1971-2008$ & 10.0 & -2.1 & 33.5 \\
\hline 115 & Missouri River at Garrison Dam, N. Dak. & 463 & $1971-2007$ & 7.7 & 0.0 & 17.1 \\
\hline 116 & Knife River at Manning, N. Dak. & 382 & $1972-2008$ & 5.0 & 0.0 & 28.5 \\
\hline 117 & Stray Creek near Manning, N. Dak. & 24 & $1975-1981$ & 7.3 & 0.0 & 23.5 \\
\hline 118 & Knife River at Marshall, N. Dak. & 180 & $1972-1981$ & 6.8 & 0.0 & 28.0 \\
\hline 119 & Elm Creek near Golden Valley, N. Dak. & 97 & 1972-1994 & 2.0 & 0.0 & 22.0 \\
\hline 120 & Knife River near Golden Valley, N. Dak. & 401 & $1971-2008$ & 8.0 & -2.1 & 27.5 \\
\hline 121 & Coyote Creek near Zap, N. Dak. & 70 & $1977-1983$ & 8.3 & 0.0 & 30.0 \\
\hline 122 & Brush Creek near Beulah, N. Dak. & 174 & 1974-1990 & 7.3 & 0.0 & 27.5 \\
\hline 123 & Spring Creek below Lake Ilo near Dunn Center, N. Dak. & 62 & $1977-1993$ & 9.8 & 0.0 & 26.0 \\
\hline 124 & Spring Creek near Halliday, N. Dak. & 51 & $1977-1981$ & 12.0 & 0.0 & 26.0 \\
\hline 125 & Spring Creek at Zap, N. Dak. & 427 & $1970-2008$ & 6.0 & -2.0 & 33.0 \\
\hline 126 & West Branch Otter Creek near Beulah, N. Dak. & 97 & 1972-1994 & 2.0 & 0.0 & 26.0 \\
\hline 127 & Knife River at Hazen, N. Dak. & 646 & 1970-2008 & 9.9 & -2.0 & 30.5 \\
\hline 128 & Antelope Creek above Hazen, N. Dak. & 66 & $1977-1985$ & 3.5 & 0.0 & 29.0 \\
\hline 129 & West Branch Antelope Creek No. 4 near Zap, N. Dak. & 98 & $1977-1986$ & 2.0 & 0.0 & 22.0 \\
\hline 130 & West Branch Antelope Creek near Hazen, N. Dak. & 27 & $1978-1983$ & 6.6 & 0.0 & 26.0 \\
\hline 131 & Coal Creek near Stanton, N. Dak. & 34 & $1975-1981$ & 5.3 & 0.0 & 23.0 \\
\hline 132 & Alderin Creek near Fort Clark, N. Dak. & 61 & $1977-1983$ & 9.0 & 0.0 & 25.0 \\
\hline 133 & Coal Lake Coulee near Hensler, N. Dak. & 68 & $1978-1988$ & 4.0 & -0.5 & 26.5 \\
\hline 134 & Buffalo Creek near Washburn, N. Dak. & 55 & $1978-1983$ & 10.5 & 0.0 & 25.5 \\
\hline
\end{tabular}


Table 1-4. Summary statistics for field measurements, total suspended solids, and suspended sediment at selected sites in North Dakota from 1970 through 2008. - Continued

$[\mu \mathrm{S} / \mathrm{cm}$, microsiemens per centimeter; deg. C, degrees Celsius; --, not available; mg/L, milligrams per liter; <, less than]

\begin{tabular}{|c|c|c|c|c|c|c|}
\hline $\begin{array}{c}\text { Site } \\
\text { identification } \\
\text { number } \\
\text { (table 1) }\end{array}$ & Site name & $\begin{array}{c}\text { Number of } \\
\text { samples }\end{array}$ & $\begin{array}{l}\text { Period of } \\
\text { record }\end{array}$ & Median & Minimum & Maximum \\
\hline \multicolumn{7}{|c|}{ Water temperature, in deg. C-Continued } \\
\hline 135 & Turtle Creek above Washburn, N. Dak. & 139 & 1986-2003 & 6.5 & -0.5 & 28.0 \\
\hline 136 & Painted Woods Creek near Wilton, N. Dak. & 325 & 1970-2003 & 7.5 & 0.0 & 28.5 \\
\hline 137 & Square Butte Creek near Hannover, N. Dak. & 30 & $1977-1981$ & 7.3 & 0.0 & 27.0 \\
\hline 138 & Square Butte Creek above Nelson Lake near Center, N. Dak. & 75 & $1977-1982$ & 6.5 & 0.0 & 26.5 \\
\hline 139 & Hagel Creek near Center, N. Dak. & 51 & $1977-1982$ & 4.0 & 0.0 & 31.5 \\
\hline 140 & Square Butte Creek below Center, N. Dak. & 333 & 1971-2008 & 9.5 & 0.0 & 28.0 \\
\hline 141 & Burnt Creek near Bismarck, N. Dak. & 205 & $1972-2008$ & 4.8 & 0.0 & 27.5 \\
\hline 142 & Missouri River at Bismarck, N. Dak. & 335 & 1970-2008 & 10.0 & 0.0 & 22.7 \\
\hline 143 & South Branch Heart River near South Heart, N. Dak. & 41 & 1979-1996 & 12.0 & 0.0 & 27.0 \\
\hline 144 & North Creek near South Heart, N. Dak. & 27 & $1978-1996$ & 5.5 & 0.0 & 25.5 \\
\hline 145 & Heart River near South Heart, N. Dak. & 114 & $1975-2005$ & 6.0 & 0.0 & 28.5 \\
\hline 146 & Heart River at Dickinson, N. Dak. & 96 & 1984-1994 & 8.5 & -0.5 & 28.0 \\
\hline 147 & Green River near New Hradec, N. Dak. & 408 & $1971-2008$ & 6.0 & 0.0 & 31.0 \\
\hline 148 & Green River near Gladstone, N. Dak. & 68 & $1970-1993$ & 5.8 & 0.0 & 27.0 \\
\hline 149 & Heart River near Richardton, N. Dak. & 421 & $1971-2008$ & 8.5 & -2.0 & 29.0 \\
\hline 150 & Heart River above Lake Tschida near Glen Ullin, N. Dak. & 163 & 1988-2008 & 9.5 & 0.0 & 32.5 \\
\hline 151 & Antelope Creek near Carson, N. Dak. & 104 & 1971-2008 & 7.8 & 0.0 & 29.5 \\
\hline 152 & Big Muddy Creek near Almont, N. Dak. & 112 & 1991-2008 & 11.3 & 0.0 & 27.0 \\
\hline 153 & Heart River near Lark, N. Dak. & 222 & $1971-1995$ & 6.5 & 0.0 & 28.5 \\
\hline 154 & Heart River at Stark Bridge near Judson, N. Dak. & 148 & 1988-2008 & 10.8 & 0.0 & 28.9 \\
\hline 155 & Sweetbriar Creek near Judson, N. Dak. & 126 & 1971-2008 & 8.0 & 0.0 & 26.5 \\
\hline 156 & Heart River near Mandan, N. Dak. & 543 & 1971-2008 & 10.2 & -2.0 & 31.0 \\
\hline 157 & Long Lake Creek above Long Lake near Moffit, N. Dak. & 127 & 1988-2004 & 6.0 & 0.0 & 27.5 \\
\hline 158 & Apple Creek near Menoken, N. Dak. & 345 & 1971-2008 & 5.5 & -0.5 & 29.0 \\
\hline 159 & Missouri River near Schmidt, N. Dak. & 143 & $1974-1981$ & 8.0 & 0.0 & 18.0 \\
\hline 160 & Cannonball River at New England, N. Dak. & 43 & $1978-1981$ & 9.5 & 0.0 & 29.0 \\
\hline 161 & Coal Bank Creek near Havelock, N. Dak. & 110 & $1974-1983$ & 8.0 & 0.0 & 27.5 \\
\hline 162 & Cannonball River at Regent, N. Dak. & 414 & 1970-2008 & 8.5 & 0.0 & 28.5 \\
\hline 163 & Cannonball River below Bentley, N. Dak. & 129 & 1972-1982 & 6.0 & 0.0 & 29.0 \\
\hline 164 & Cannonball River near Raleigh, N. Dak. & 115 & 1993-2008 & 12.0 & -2.1 & 30.0 \\
\hline 165 & White Butte Fork Cedar Creek near Scranton, N. Dak. & 141 & 1972-1995 & 7.0 & 0.0 & 27.0 \\
\hline 166 & Cedar Creek near Haynes, N. Dak. & 347 & 1971-2008 & 8.0 & 0.0 & 28.6 \\
\hline 167 & Timber Creek near Bentley, N. Dak. & 55 & $1977-1981$ & 8.5 & -0.5 & 28.0 \\
\hline 168 & Cedar Creek near Pretty Rock, N. Dak. & 63 & $1971-1976$ & 6.5 & 0.0 & 28.5 \\
\hline 169 & Cedar Creek near Raleigh, N. Dak. & 376 & $1972-2008$ & 7.5 & -2.1 & 31.1 \\
\hline 170 & Cannonball River at Breien, N. Dak. & 650 & 1970-2008 & 11.0 & -2.1 & 30.5 \\
\hline 171 & Beaver Creek near Linton, N. Dak. & 191 & $1972-1993$ & 5.0 & 0.0 & 32.0 \\
\hline 172 & Beaver Creek below Linton, N. Dak. & 143 & 1989-2008 & 7.9 & 0.0 & 29.5 \\
\hline 173 & Porcupine Creek near Fort Yates, N. Dak. & 84 & 1991-1999 & 13.8 & 0.0 & 25.5 \\
\hline
\end{tabular}


Table 1-4. Summary statistics for field measurements, total suspended solids, and suspended sediment at selected sites in North Dakota from 1970 through 2008. - Continued

$[\mu \mathrm{S} / \mathrm{cm}$, microsiemens per centimeter; deg. $\mathrm{C}$, degrees Celsius; --, not available; mg/L, milligrams per liter; <, less than]

\begin{tabular}{|c|c|c|c|c|c|c|}
\hline $\begin{array}{c}\text { Site } \\
\text { identification } \\
\text { number } \\
\text { (table 1) }\end{array}$ & Site name & $\begin{array}{l}\text { Number of } \\
\text { samples }\end{array}$ & $\begin{array}{l}\text { Period of } \\
\text { record }\end{array}$ & Median & Minimum & Maximum \\
\hline 174 & Buffalo Creek Tributary near Gascoyne, N. Dak. & 113 & $1974-1987$ & 9.0 & 0.0 & 30.0 \\
\hline 175 & James River near Manfred, N. Dak. & 126 & 1971-1998 & 6.5 & 0.0 & 28.0 \\
\hline 177 & James River above Arrowwood Lake near Kensal, N. Dak. & 221 & $1985-2008$ & 9.0 & 0.0 & 27.0 \\
\hline 178 & Kelly Creek below Niccum Reservoir near Bordulac, N. Dak. & 17 & $1986-1989$ & 4.5 & 0.5 & 21.5 \\
\hline 179 & James River near Pingree, N. Dak. & 146 & $1978-2008$ & 14.6 & 0.0 & 27.5 \\
\hline 180 & Pipestem Creek near Pingree, N. Dak. & 228 & 1974-2008 & 8.0 & 0.0 & 28.5 \\
\hline 181 & Pipestem Creek near Buchanan, N. Dak. & 29 & $1971-1993$ & 6.5 & 0.0 & 25.5 \\
\hline 185 & James River at Oakes, N. Dak. & 172 & $1970-2008$ & 9.0 & 0.0 & 28.0 \\
\hline 186 & James River at N. Dak./S. Dak. State line & 113 & $1974-2008$ & 12.0 & 0.0 & 28.1 \\
\hline \multicolumn{7}{|c|}{ Dissolved oxygen, in mg/L } \\
\hline 1 & Bois De Sioux River near Doran, Minn. & 39 & $2000-2007$ & 9.1 & 4.5 & 16.0 \\
\hline 2 & Red River of the North at Wahpeton, N. Dak. & 23 & $1991-2004$ & 9.5 & 4.2 & 13.6 \\
\hline 3 & Red River of the North near Wahpeton, N. Dak. & 5 & $1993-2006$ & 11.9 & 8.3 & 13.4 \\
\hline 4 & Red River of the North at Brushville, Minn. & 60 & $1993-2007$ & 9.8 & 5.4 & 15.6 \\
\hline 5 & Red River of the North below Wahpeton, N. Dak. & 66 & $1970-1999$ & 10.1 & 6.0 & 15.2 \\
\hline 14 & Red River of the North near Harwood, N. Dak. & 52 & $1993-2006$ & 9.4 & 6.0 & 15.1 \\
\hline 15 & Sheyenne River above Harvey, N. Dak. & 72 & $1982-2004$ & 8.8 & 0.0 & 16.6 \\
\hline 17 & Sheyenne River at Warwick, N. Dak. & 46 & $1996-2006$ & 9.1 & 4.2 & 14.6 \\
\hline 18 & Sheyenne River near Warwick, N. Dak. & 28 & $1986-2007$ & 7.9 & 2.1 & 14.2 \\
\hline 19 & Mauvais Coulee Tributary No. 3 near Cando, N. Dak. & 3 & $1989-1995$ & 10.1 & 2.4 & 12.8 \\
\hline 20 & Mauvais Coulee near Cando, N. Dak. & 61 & $1987-2001$ & 9.1 & 0.9 & 13.8 \\
\hline 21 & Edmore Coulee near Edmore, N. Dak. & 59 & $1983-2001$ & 9.4 & 2.6 & 13.5 \\
\hline 22 & Edmore Coulee Tributary near Webster, N. Dak. & 2 & 1993 & 9.2 & 9.0 & 9.4 \\
\hline 24 & Starkweather Coulee near Webster, N. Dak. & 62 & $1987-2001$ & 10.0 & 3.2 & 15.1 \\
\hline 25 & Big Coulee below Churchs Ferry, N. Dak. & 11 & $1998-2001$ & 8.6 & 2.1 & 17.5 \\
\hline 27 & Little Coulee near Brinsmade, N. Dak. & 2 & $1993-1995$ & 6.6 & 6.2 & 7.0 \\
\hline 28 & Big Coulee near Churchs Ferry, N. Dak. & 61 & $1985-1997$ & 8.1 & 1.3 & 12.3 \\
\hline 29 & Big Coulee at Graham Island inlet near Fort Totten, N. Dak. & 9 & $1982-1986$ & 10.8 & 9.3 & 13.8 \\
\hline 30 & Channel A near Penn, N. Dak. & 70 & 1985-1999 & 9.4 & 2.1 & 17.8 \\
\hline
\end{tabular}


Table 1-4. Summary statistics for field measurements, total suspended solids, and suspended sediment at selected sites in North Dakota from 1970 through 2008. - Continued

$[\mu \mathrm{S} / \mathrm{cm}$, microsiemens per centimeter; deg. C, degrees Celsius; --, not available; mg/L, milligrams per liter; <, less than]

\begin{tabular}{|c|c|c|c|c|c|c|}
\hline $\begin{array}{c}\text { Site } \\
\text { identification } \\
\text { number } \\
\text { (table 1) }\end{array}$ & Site name & $\begin{array}{l}\text { Number of } \\
\text { samples }\end{array}$ & $\begin{array}{l}\text { Period of } \\
\text { record }\end{array}$ & Median & Minimum & Maximum \\
\hline \multicolumn{7}{|c|}{ Dissolved oxygen, in $\mathrm{mg} / \mathrm{L}$-Continued } \\
\hline 31 & Devils Lake Outlet to Stump Lake near Lakota, N. Dak. & 1 & 1999 & 10.5 & 10.5 & 10.5 \\
\hline 32 & Sheyenne River near Cooperstown, N. Dak. & 165 & 1979-2007 & 8.7 & 1.9 & 15.8 \\
\hline 33 & Baldhill Creek near Dazey, N. Dak. & 49 & 1979-2005 & 10.4 & 2.8 & 15.4 \\
\hline 34 & Sheyenne River below Baldhill Dam, N. Dak. & 121 & 1979-2007 & 10.7 & 5.1 & 15.0 \\
\hline 35 & Sheyenne River at Valley City, N. Dak. & 12 & $1992-1993$ & 10.7 & 7.2 & 15.0 \\
\hline 36 & Sheyenne River at Lisbon, N. Dak. & 127 & $1982-2007$ & 10.0 & 6.0 & 14.7 \\
\hline 37 & Sheyenne River near Kindred, N. Dak. & 380 & 1976-2007 & 9.1 & 4.0 & 17.3 \\
\hline 38 & $\begin{array}{l}\text { Sheyenne River above Sheyenne River Diversion near Horace, } \\
\text { N. Dak. }\end{array}$ & 10 & 2003-2006 & 10.0 & 7.5 & 13.9 \\
\hline 39 & Sheyenne River near Horace, N. Dak. & 38 & $1976-1979$ & 7.4 & 2.2 & 13.3 \\
\hline 41 & Sheyenne River at West Fargo, N. Dak. & 13 & $1991-2006$ & 8.7 & 5.7 & 12.4 \\
\hline 43 & Maple River near Enderlin, N. Dak. & 1 & 1992 & 12.4 & 12.4 & 12.4 \\
\hline 45 & Maple River below Mapleton, N. Dak. & 45 & 1998-2007 & 9.8 & 3.4 & 21.2 \\
\hline 46 & Sheyenne River at Harwood, N. Dak. & 29 & 1993-1999 & 9.9 & 5.2 & 15.9 \\
\hline 49 & Lower Branch Rush River near Prosper, N. Dak. & 2 & 1993 & 10.4 & 9.2 & 11.5 \\
\hline 50 & Sheyenne River near Harwood, N. Dak. & 48 & 1970-1974 & 8.0 & 3.8 & 14.4 \\
\hline 51 & Elm River near Kelso, N. Dak. & 3 & 1993 & 10.4 & 9.0 & 10.6 \\
\hline 52 & Red River of the North at Halstad, Minn. & 313 & 1978-2006 & 8.2 & 1.9 & 16.2 \\
\hline 53 & Beaver Creek near Finley, N. Dak. & 185 & 1970-1996 & 9.7 & 4.8 & 13.8 \\
\hline 54 & Goose River near Portland, N. Dak. & 1 & 1992 & 5.4 & 5.4 & 5.4 \\
\hline 55 & Goose River at Hillsboro, N. Dak. & 90 & $1991-2008$ & 9.4 & 4.1 & 18.1 \\
\hline 56 & Red River of the North at Grand Forks, N. Dak. & 107 & $1991-2008$ & 9.7 & 3.9 & 14.8 \\
\hline 57 & Turtle River at Turtle River State Park near Arvilla, N. Dak. & 96 & $1991-2000$ & 9.9 & 5.8 & 19.3 \\
\hline 58 & Turtle River at Manvel, N. Dak. & 67 & 1993-2008 & 9.6 & 0.9 & 18.1 \\
\hline 59 & Red River of the North at Oslo, Minn. & 55 & 1973-2004 & 9.4 & 5.0 & 14.1 \\
\hline 61 & Forest River near Fordville, N. Dak. & 1 & 1992 & 6.8 & 6.8 & 6.8 \\
\hline 62 & Forest River near Minto, N. Dak. & 88 & 1974-2008 & 9.3 & 1.8 & 14.1 \\
\hline 67 & Park River at Grafton, N. Dak. & 71 & 1991-2008 & 9.3 & 0.8 & 14.2 \\
\hline 68 & Red River of the North at Drayton, N. Dak. & 19 & 1991-2004 & 9.3 & 5.8 & 13.4 \\
\hline 72 & Pembina River near Vang, N. Dak. & 55 & 1976-1979 & 9.7 & 0.5 & 13.2 \\
\hline 73 & Little South Pembina River near Walhalla, N. Dak. & 63 & $1976-1995$ & 10.8 & 3.6 & 13.8 \\
\hline 74 & Pembina River at Walhalla, N. Dak. & 134 & 1976-1995 & 10.0 & 6.2 & 13.2 \\
\hline 75 & Pembina River at Neche, N. Dak. & 85 & 1994-2008 & 9.7 & 3.3 & 14.8 \\
\hline 76 & Tongue River at Akra, N. Dak. & 12 & 1979-2005 & 10.1 & 6.5 & 13.2 \\
\hline 77 & Red River of the North at Pembina, N. Dak., site 1 & 10 & 1993 & 10.6 & 2.8 & 15.0 \\
\hline 78 & Red River of the North at Pembina, N. Dak., site 2 & 166 & $1970-2008$ & 9.3 & 4.6 & 16.7 \\
\hline 79 & Red River of the North at Emerson, Manitoba & 328 & 1978-2004 & 8.2 & 1.3 & 18.2 \\
\hline 80 & Long Creek near Noonan, N. Dak. & 8 & 1997 & 10.1 & 7.8 & 12.8 \\
\hline 81 & West Branch Short Creek near Columbus, N. Dak. & 21 & $1978-1981$ & 9.6 & 3.9 & 13.1 \\
\hline
\end{tabular}


Table 1-4. Summary statistics for field measurements, total suspended solids, and suspended sediment at selected sites in North Dakota from 1970 through 2008. - Continued

$[\mu \mathrm{S} / \mathrm{cm}$, microsiemens per centimeter; deg. C, degrees Celsius; --, not available; mg/L, milligrams per liter; <, less than]

\begin{tabular}{|c|c|c|c|c|c|c|}
\hline $\begin{array}{c}\text { Site } \\
\text { identification } \\
\text { number } \\
\text { (table 1) }\end{array}$ & Site name & $\begin{array}{c}\text { Number of } \\
\text { samples }\end{array}$ & $\begin{array}{l}\text { Period of } \\
\text { record }\end{array}$ & Median & Minimum & Maximum \\
\hline \multicolumn{7}{|c|}{ Dissolved oxygen, in $\mathrm{mg} / \mathrm{L}$-Continued } \\
\hline 82 & Souris River near Sherwood, N. Dak. & 407 & 1974-2008 & 8.0 & 0.0 & 19.4 \\
\hline 83 & Souris River near Foxholm, N. Dak. & 180 & 1972-1999 & 9.1 & 0.0 & 21.0 \\
\hline 84 & Des Lacs River at Foxholm, N. Dak. & 137 & $1981-2007$ & 9.8 & 0.0 & 16.7 \\
\hline 85 & Souris River above Minot, N. Dak. & 164 & $1981-2008$ & 9.5 & 0.1 & 20.0 \\
\hline 86 & Bonnes Creek near Velva, N. Dak. & 3 & $1994-2000$ & 11.1 & 10.5 & 14.5 \\
\hline 87 & Souris River near Verendrye, N. Dak. & 309 & $1970-2008$ & 8.2 & 0.3 & 16.7 \\
\hline 88 & Wintering River near Karlsruhe, N. Dak. & 57 & $1981-1998$ & 8.3 & 1.3 & 13.6 \\
\hline 89 & Souris River near Bantry, N. Dak. & 107 & $1981-2001$ & 8.3 & 0.0 & 25.8 \\
\hline 90 & Willow Creek near Willow City, N. Dak. & 46 & $1982-2000$ & 9.1 & 3.7 & 15.0 \\
\hline 91 & Stone Creek near Kramer, N. Dak. & 30 & $1986-2000$ & 8.8 & 5.3 & 21.6 \\
\hline 92 & Deep River near Upham, N. Dak. & 27 & $1989-2000$ & 8.9 & 3.4 & 19.0 \\
\hline 95 & Cut Bank Creek at Upham, N. Dak. & 16 & 1999-2000 & 8.4 & 1.6 & 10.7 \\
\hline 96 & Deep River below Cut Bank Creek near Upham, N. Dak. & 16 & 1982-1989 & 7.8 & 0.4 & 11.4 \\
\hline 97 & Boundary Creek near Landa, N. Dak. & 30 & $1986-2000$ & 9.7 & 1.0 & 21.0 \\
\hline 98 & Souris River near Westhope, N. Dak. & 513 & $1970-2008$ & 9.0 & 0.0 & 22.5 \\
\hline 99 & Charbonneau Creek near Charbonneau, N. Dak. & 1 & 2006 & 15.6 & 15.6 & 15.6 \\
\hline 100 & Missouri River near Williston, N. Dak. & 97 & 1974-1992 & 9.8 & 7.0 & 13.0 \\
\hline 101 & Little Muddy River below Cow Creek near Williston, N. Dak. & 9 & 1999-2005 & 8.9 & 6.5 & 11.5 \\
\hline 102 & Stony Creek near Williston, N. Dak. & 36 & $1977-1981$ & 11.5 & 5.3 & 13.5 \\
\hline 104 & Beaver Creek near Ray, N. Dak. & 56 & 1977-1982 & 10.7 & 7.6 & 13.8 \\
\hline 106 & Bear Den Creek near Mandaree, N. Dak. & 372 & 1970-1996 & 10.5 & 5.8 & 16.4 \\
\hline 108 & East Fork Shell Creek near Parshall, N. Dak. & 46 & $1991-2001$ & 8.9 & 4.6 & 12.5 \\
\hline 109 & Deepwater Creek near Mandaree, N. Dak. & 50 & 1990-2001 & 8.9 & 4.9 & 13.3 \\
\hline 110 & Little Missouri River at Marmarth, N. Dak. & 9 & 1995-1999 & 8.8 & 7.2 & 10.9 \\
\hline 111 & Deep Creek near Amidon, N. Dak. & 58 & $1977-1983$ & 11.2 & 5.5 & 17.9 \\
\hline 112 & Little Missouri River at Medora, N. Dak. & 86 & 1979-2007 & 9.5 & 0.6 & 15.4 \\
\hline 113 & Beaver Creek near Trotters, N. Dak. & 57 & 1977-1999 & 10.9 & 6.2 & 14.0 \\
\hline 114 & Little Missouri River near Watford City, N. Dak. & 666 & $1974-2007$ & 10.0 & 3.8 & 19.4 \\
\hline 115 & Missouri River at Garrison Dam, N. Dak. & 423 & $1974-2007$ & 11.0 & 5.8 & 15.4 \\
\hline 116 & Knife River at Manning, N. Dak. & 55 & $1977-1982$ & 9.5 & 2.6 & 12.7 \\
\hline 117 & Stray Creek near Manning, N. Dak. & 22 & 1975-1981 & 10.4 & 2.6 & 14.8 \\
\hline 118 & Knife River at Marshall, N. Dak. & 46 & $1977-1981$ & 9.4 & 6.0 & 16.2 \\
\hline 119 & Elm Creek near Golden Valley, N. Dak. & 16 & $1977-1981$ & 10.1 & 3.6 & 12.7 \\
\hline 120 & Knife River near Golden Valley, N. Dak. & 58 & $1997-2007$ & 9.5 & 5.5 & 15.0 \\
\hline 121 & Coyote Creek near Zap, N. Dak. & 61 & $1977-1983$ & 11.4 & 6.6 & 14.4 \\
\hline 122 & Brush Creek near Beulah, N. Dak. & 130 & 1974-1990 & 9.4 & 2.7 & 13.4 \\
\hline 123 & Spring Creek below Lake Ilo near Dunn Center, N. Dak. & 55 & $1977-1993$ & 9.1 & 2.5 & 15.4 \\
\hline 124 & Spring Creek near Halliday, N. Dak. & 50 & $1977-1981$ & 9.9 & 6.2 & 14.0 \\
\hline 125 & Spring Creek at Zap, N. Dak. & 167 & $1974-2007$ & 9.5 & 4.8 & 16.6 \\
\hline
\end{tabular}


Table 1-4. Summary statistics for field measurements, total suspended solids, and suspended sediment at selected sites in North Dakota from 1970 through 2008. - Continued

$[\mu \mathrm{S} / \mathrm{cm}$, microsiemens per centimeter; deg. $\mathrm{C}$, degrees Celsius; --, not available; mg/L, milligrams per liter; <, less than]

\begin{tabular}{|c|c|c|c|c|c|c|}
\hline $\begin{array}{c}\text { Site } \\
\text { identification } \\
\text { number } \\
\text { (table 1) }\end{array}$ & Site name & $\begin{array}{c}\text { Number of } \\
\text { samples }\end{array}$ & $\begin{array}{l}\text { Period of } \\
\text { record }\end{array}$ & Median & Minimum & Maximum \\
\hline \multicolumn{7}{|c|}{ Dissolved oxygen, in $\mathrm{mg} / \mathrm{L}$-Continued } \\
\hline 127 & Knife River at Hazen, N. Dak. & 424 & 1974-2007 & 9.3 & 5.6 & 19.6 \\
\hline 128 & Antelope Creek above Hazen, N. Dak. & 52 & $1977-1985$ & 11.1 & 2.0 & 16.2 \\
\hline 129 & West Branch Antelope Creek No. 4 near Zap, N. Dak. & 11 & $1982-1985$ & 12.2 & 4.8 & 21.2 \\
\hline 130 & West Branch Antelope Creek near Hazen, N. Dak. & 20 & $1978-1983$ & 9.7 & 3.9 & 13.4 \\
\hline 131 & Coal Creek near Stanton, N. Dak. & 31 & $1977-1981$ & 11.0 & 5.2 & 17.0 \\
\hline 132 & Alderin Creek near Fort Clark, N. Dak. & 53 & $1977-1983$ & 10.8 & 7.0 & 14.6 \\
\hline 133 & Coal Lake Coulee near Hensler, N. Dak. & 45 & $1978-1988$ & 10.9 & 7.0 & 16.0 \\
\hline 134 & Buffalo Creek near Washburn, N. Dak. & 47 & $1978-1983$ & 10.8 & 5.6 & 15.8 \\
\hline 135 & Turtle Creek above Washburn, N. Dak. & 87 & 1988-2003 & 8.9 & 0.8 & 15.2 \\
\hline 136 & Painted Woods Creek near Wilton, N. Dak. & 147 & 1970-2003 & 9.6 & 2.8 & 15.8 \\
\hline 137 & Square Butte Creek near Hannover, N. Dak. & 26 & 1977-1981 & 11.1 & 4.6 & 12.8 \\
\hline 138 & Square Butte Creek above Nelson Lake near Center, N. Dak. & 64 & 1977-1982 & 9.5 & 3.2 & 12.8 \\
\hline 139 & Hagel Creek near Center, N. Dak. & 45 & 1977-1982 & 10.0 & 4.7 & 14.6 \\
\hline 140 & Square Butte Creek below Center, N. Dak. & 9 & $1983-1993$ & 9.0 & 6.9 & 13.4 \\
\hline 142 & Missouri River at Bismarck, N. Dak. & 151 & 1974-2001 & 10.6 & 7.8 & 13.4 \\
\hline 143 & South Branch Heart River near South Heart, N. Dak. & 33 & 1979-1996 & 9.3 & 4.3 & 13.4 \\
\hline 144 & North Creek near South Heart, N. Dak. & 25 & 1978-1996 & 10.1 & 2.3 & 14.6 \\
\hline 145 & Heart River near South Heart, N. Dak. & 63 & 1975-1994 & 9.1 & 4.7 & 14.2 \\
\hline 146 & Heart River at Dickinson, N. Dak. & 5 & 1993 & 9.0 & 6.8 & 14.0 \\
\hline 147 & Green River near New Hradec, N. Dak. & 55 & 1977-2006 & 9.1 & 4.2 & 14.1 \\
\hline 148 & Green River near Gladstone, N. Dak. & 4 & 1993 & 9.5 & 7.1 & 14.2 \\
\hline 149 & Heart River near Richardton, N. Dak. & 73 & 1994-2007 & 9.6 & 5.2 & 23.3 \\
\hline 152 & Big Muddy Creek near Almont, N. Dak. & 3 & 1993 & 9.0 & 8.8 & 11.0 \\
\hline 156 & Heart River near Mandan, N. Dak. & 283 & $1977-2007$ & 10.1 & 0.5 & 14.0 \\
\hline 157 & Long Lake Creek above Long Lake near Moffit, N. Dak. & 3 & 1988-1989 & 10.7 & 8.2 & 10.8 \\
\hline 158 & Apple Creek near Menoken, N. Dak. & 40 & 1977-1993 & 7.5 & 3.8 & 12.1 \\
\hline 159 & Missouri River near Schmidt, N. Dak. & 140 & 1974-1981 & 10.5 & 8.4 & 13.6 \\
\hline 160 & Cannonball River at New England, N. Dak. & 36 & $1978-1981$ & 9.8 & 6.4 & 14.8 \\
\hline 161 & Coal Bank Creek near Havelock, N. Dak. & 86 & 1974-1983 & 10.3 & 1.4 & 19.2 \\
\hline 162 & Cannonball River at Regent, N. Dak. & 47 & $1977-1981$ & 9.1 & 5.9 & 15.9 \\
\hline 164 & Cannonball River near Raleigh, N. Dak. & 72 & 1993-2007 & 9.7 & 6.2 & 14.8 \\
\hline 166 & Cedar Creek near Haynes, N. Dak. & 1 & 1996 & 11.3 & 11.3 & 11.3 \\
\hline 167 & Timber Creek near Bentley, N. Dak. & 47 & $1977-1981$ & 10.3 & 3.7 & 14.5 \\
\hline 169 & Cedar Creek near Raleigh, N. Dak. & 65 & $1993-2007$ & 9.8 & 5.3 & 14.3 \\
\hline 170 & Cannonball River at Breien, N. Dak. & 334 & 1974-2007 & 10.0 & 3.4 & 17.0 \\
\hline 171 & Beaver Creek near Linton, N. Dak. & 4 & 1993 & 9.1 & 7.7 & 12.8 \\
\hline 173 & Porcupine Creek near Fort Yates, N. Dak. & 39 & 1991-1999 & 7.8 & 4.2 & 13.3 \\
\hline 174 & Buffalo Creek Tributary near Gascoyne, N. Dak. & 87 & 1974-1987 & 9.7 & 3.8 & 21.0 \\
\hline 175 & James River near Manfred, N. Dak. & 41 & 1985-1998 & 10.3 & 3.8 & 17.0 \\
\hline
\end{tabular}


Table 1-4. Summary statistics for field measurements, total suspended solids, and suspended sediment at selected sites in North Dakota from 1970 through 2008. - Continued

$[\mu \mathrm{S} / \mathrm{cm}$, microsiemens per centimeter; deg. C, degrees Celsius; --, not available; mg/L, milligrams per liter; <, less than]

\begin{tabular}{|c|c|c|c|c|c|c|}
\hline $\begin{array}{c}\text { Site } \\
\text { identification } \\
\text { number } \\
\text { (table 1) }\end{array}$ & Site name & $\begin{array}{l}\text { Number of } \\
\text { samples }\end{array}$ & $\begin{array}{l}\text { Period of } \\
\text { record }\end{array}$ & Median & Minimum & Maximum \\
\hline \multicolumn{7}{|c|}{ Dissolved oxygen, in $\mathrm{mg} / \mathrm{L}$-Continued } \\
\hline 176 & James River near Grace City, N. Dak. & 113 & $1985-2007$ & 9.4 & 0.5 & 18.3 \\
\hline 177 & James River above Arrowwood Lake near Kensal, N. Dak. & 166 & $1985-2008$ & 9.2 & 0.8 & 17.8 \\
\hline 179 & James River near Pingree, N. Dak. & 127 & $1980-2008$ & 9.9 & 1.9 & 22.4 \\
\hline 180 & Pipestem Creek near Pingree, N. Dak. & 21 & 1987-1999 & 10.7 & 6.2 & 16.4 \\
\hline 181 & Pipestem Creek near Buchanan, N. Dak. & 5 & 1993 & 9.7 & 4.6 & 13.5 \\
\hline 182 & James River at Jamestown, N. Dak. & 154 & 1984-2007 & 9.2 & 1.0 & 17.5 \\
\hline 186 & James River at N. Dak./S. Dak. State line & 50 & 1976-1999 & 9.5 & 0.6 & 30.4 \\
\hline \multicolumn{7}{|c|}{ Total suspended solids, in mg/L } \\
\hline 1 & Bois De Sioux River near Doran, Minn. & 40 & $2000-2007$ & 36 & $<10$ & 174 \\
\hline 2 & Red River of the North at Wahpeton, N. Dak. & 2 & 1996 & 128 & 72 & 183 \\
\hline 3 & Red River of the North near Wahpeton, N. Dak. & 1 & 2006 & $<10$ & $<10$ & $<10$ \\
\hline 4 & Red River of the North at Brushville, Minn. & 56 & $1993-2007$ & 36 & $<10$ & 261 \\
\hline 10 & Wild Rice River near Abercrombie, N. Dak. & 49 & 1993-2007 & 65 & $<10$ & 269 \\
\hline 11 & Red River of the North at Fargo, N. Dak. & 67 & $1971-2008$ & 68 & $<10$ & 709 \\
\hline 12 & Red River of North below Fargo, N. Dak. & 19 & 1973-1977 & 38 & $<10$ & 130 \\
\hline 24 & Starkweather Coulee near Webster, N. Dak. & 5 & 1988 & $<10$ & $<10$ & 472 \\
\hline 28 & Big Coulee near Churchs Ferry, N. Dak. & 5 & $1987-1988$ & 53 & 17 & 99 \\
\hline 30 & Channel A near Penn, N. Dak. & 7 & 1987-1992 & 14 & $<10$ & 191 \\
\hline 32 & Sheyenne River near Cooperstown, N. Dak. & 92 & 1995-2007 & 34 & $<10$ & 159 \\
\hline 33 & Baldhill Creek near Dazey, N. Dak. & 11 & 1995-1996 & 17 & $<10$ & 74 \\
\hline 34 & Sheyenne River below Baldhill Dam, N. Dak. & 87 & $1995-2007$ & $<10$ & $<10$ & 94 \\
\hline 36 & Sheyenne River at Lisbon, N. Dak. & 71 & 1997-2007 & 40 & $<10$ & 415 \\
\hline 37 & Sheyenne River near Kindred, N. Dak. & 72 & 1996-2007 & 65 & $<10$ & 991 \\
\hline 38 & $\begin{array}{l}\text { Sheyenne River above Sheyenne River Diversion near Horace, } \\
\text { N. Dak. }\end{array}$ & 13 & $2005-2007$ & 102 & $<10$ & 767 \\
\hline 41 & Sheyenne River at West Fargo, N. Dak. & 6 & 1996-2006 & 159 & 19 & 245 \\
\hline 45 & Maple River below Mapleton, N. Dak. & 49 & $1997-2007$ & 62 & $<10$ & 489 \\
\hline 46 & Sheyenne River at Harwood, N. Dak. & 10 & 1993 & 102 & 19 & 250 \\
\hline 51 & Elm River near Kelso, N. Dak. & 2 & 1993 & 46 & $<10$ & 81 \\
\hline
\end{tabular}


Table 1-4. Summary statistics for field measurements, total suspended solids, and suspended sediment at selected sites in North Dakota from 1970 through 2008. - Continued

$[\mu \mathrm{S} / \mathrm{cm}$, microsiemens per centimeter; deg. C, degrees Celsius; --, not available; mg/L, milligrams per liter; <, less than]

\begin{tabular}{|c|c|c|c|c|c|c|}
\hline $\begin{array}{c}\text { Site } \\
\text { identification } \\
\text { number } \\
\text { (table 1) }\end{array}$ & Site name & $\begin{array}{c}\text { Number of } \\
\text { samples }\end{array}$ & $\begin{array}{l}\text { Period of } \\
\text { record }\end{array}$ & Median & Minimum & Maximum \\
\hline \multicolumn{7}{|c|}{ Total suspended solids, in $\mathrm{mg} / \mathrm{L}-$ Continued } \\
\hline 52 & Red River of the North at Halstad, Minn. & 12 & 1978-2007 & 157 & 19 & 621 \\
\hline 53 & Beaver Creek near Finley, N. Dak. & 7 & 1970-1976 & 14 & $<10$ & 40 \\
\hline 55 & Goose River at Hillsboro, N. Dak. & 74 & $1996-2008$ & 40 & $<10$ & 503 \\
\hline 56 & Red River of the North at Grand Forks, N. Dak. & 78 & $1997-2008$ & 79 & $<10$ & 708 \\
\hline 58 & Turtle River at Manvel, N. Dak. & 67 & $1997-2008$ & 34 & $<10$ & 799 \\
\hline 62 & Forest River near Minto, N. Dak. & 73 & $1996-2008$ & 19 & $<10$ & 430 \\
\hline 67 & Park River at Grafton, N. Dak. & 67 & 1996-2008 & 23 & $<10$ & 878 \\
\hline 68 & Red River of the North at Drayton, N. Dak. & 5 & 1996 & 58 & 31 & 174 \\
\hline 75 & Pembina River at Neche, N. Dak. & 77 & 1996-2008 & 83 & $<10$ & 3,740 \\
\hline 77 & Red River of the North at Pembina, N. Dak., site 1 & 10 & 1993 & 90 & $<10$ & 320 \\
\hline 78 & Red River of the North at Pembina, N. Dak., site 2 & 90 & 1997-2008 & 139 & $<10$ & 1,900 \\
\hline 80 & Long Creek near Noonan, N. Dak. & 10 & 1997 & 14 & $<10$ & 34 \\
\hline 82 & Souris River near Sherwood, N. Dak. & 178 & $1978-2008$ & 13 & $<10$ & 96 \\
\hline 83 & Souris River near Foxholm, N. Dak. & 47 & $1982-1998$ & $<10$ & $<10$ & 62 \\
\hline 84 & Des Lacs River at Foxholm, N. Dak. & 83 & $1996-2007$ & 31 & $<10$ & 105 \\
\hline 85 & Souris River above Minot, N. Dak. & 134 & $1982-2008$ & $<10$ & $<10$ & 99 \\
\hline 87 & Souris River near Verendrye, N. Dak. & 189 & $1982-2008$ & 20 & $<10$ & 170 \\
\hline 88 & Wintering River near Karlsruhe, N. Dak. & 14 & 1982-1998 & $<10$ & $<10$ & 35 \\
\hline 89 & Souris River near Bantry, N. Dak. & 59 & $1982-1998$ & 16 & $<10$ & 63 \\
\hline 90 & Willow Creek near Willow City, N. Dak. & 15 & 1982-1997 & 14 & $<10$ & 24 \\
\hline 91 & Stone Creek near Kramer, N. Dak. & 5 & 1992-1993 & $<10$ & $<10$ & 54 \\
\hline 92 & Deep River near Upham, N. Dak. & 7 & 1997 & $<10$ & $<10$ & 14 \\
\hline 96 & Deep River below Cut Bank Creek near Upham, N. Dak. & 3 & 1982 & 15 & $<10$ & 33 \\
\hline 97 & Boundary Creek near Landa, N. Dak. & 6 & $1992-1993$ & 11 & $<10$ & 26 \\
\hline 98 & Souris River near Westhope, N. Dak. & 116 & $1971-2008$ & 12 & $<10$ & 116 \\
\hline 100 & Missouri River near Williston, N. Dak. & 3 & 1975-1977 & 58 & 22 & 96 \\
\hline 101 & Little Muddy River below Cow Creek near Williston, N. Dak. & 9 & 1999 & 24 & 18 & 88 \\
\hline 106 & Bear Den Creek near Mandaree, N. Dak. & 9 & $1970-1977$ & 53 & 30 & 180 \\
\hline 110 & Little Missouri River at Marmarth, N. Dak. & 8 & 1999 & 257 & 28 & 3,750 \\
\hline 112 & Little Missouri River at Medora, N. Dak. & 73 & 1996-2007 & 198 & $<10$ & 20,000 \\
\hline 113 & Beaver Creek near Trotters, N. Dak. & 9 & 1999 & $<10$ & $<10$ & 98 \\
\hline 114 & Little Missouri River near Watford City, N. Dak. & 68 & $1996-2007$ & 314 & $<10$ & 56,000 \\
\hline 115 & Missouri River at Garrison Dam, N. Dak. & 4 & $1975-1977$ & $<10$ & $<10$ & $<10$ \\
\hline 120 & Knife River near Golden Valley, N. Dak. & 60 & 1997-2007 & 41 & $<10$ & 467 \\
\hline 122 & Brush Creek near Beulah, N. Dak. & 14 & 1974-1990 & $<10$ & $<10$ & 26 \\
\hline 123 & Spring Creek below Lake Ilo near Dunn Center, N. Dak. & 4 & 2008 & 15 & $<10$ & 36 \\
\hline 125 & Spring Creek at Zap, N. Dak. & 80 & $1975-2008$ & 11 & $<10$ & 289 \\
\hline 127 & Knife River at Hazen, N. Dak. & 76 & 1975-2007 & 19 & $<10$ & 735 \\
\hline 128 & Antelope Creek above Hazen, N. Dak. & 16 & $1982-1985$ & 16 & $<10$ & 233 \\
\hline
\end{tabular}


Table 1-4. Summary statistics for field measurements, total suspended solids, and suspended sediment at selected sites in North Dakota from 1970 through 2008. - Continued

$[\mu \mathrm{S} / \mathrm{cm}$, microsiemens per centimeter; deg. C, degrees Celsius; --, not available; mg/L, milligrams per liter; <, less than]

\begin{tabular}{|c|c|c|c|c|c|c|}
\hline $\begin{array}{c}\text { Site } \\
\text { identification } \\
\text { number } \\
\text { (table 1) }\end{array}$ & Site name & $\begin{array}{l}\text { Number of } \\
\text { samples }\end{array}$ & $\begin{array}{l}\text { Period of } \\
\text { record }\end{array}$ & Median & Minimum & Maximum \\
\hline \multicolumn{7}{|c|}{ Total suspended solids, in mg/L_-Continued } \\
\hline 129 & West Branch Antelope Creek No. 4 near Zap, N. Dak. & 11 & $1982-1985$ & $<10$ & $<10$ & 222 \\
\hline 132 & Alderin Creek near Fort Clark, N. Dak. & 6 & $1982-1983$ & 49 & 18 & 170 \\
\hline 142 & Missouri River at Bismarck, N. Dak. & 2 & $1975-1978$ & 37 & 17 & 56 \\
\hline 147 & Green River near New Hradec, N. Dak. & 1 & 1982 & 93 & 93 & 93 \\
\hline 149 & Heart River near Richardton, N. Dak. & 67 & 1996-2007 & 25 & $<10$ & 206 \\
\hline 156 & Heart River near Mandan, N. Dak. & 68 & $1996-2007$ & 12 & $<10$ & 475 \\
\hline 161 & Coal Bank Creek near Havelock, N. Dak. & 2 & 1974-1977 & $<10$ & $<10$ & $<10$ \\
\hline 164 & Cannonball River near Raleigh, N. Dak. & 61 & 1996-2007 & 39 & $<10$ & 885 \\
\hline 169 & Cedar Creek near Raleigh, N. Dak. & 53 & 1996-2007 & 31 & $<10$ & 701 \\
\hline 170 & Cannonball River at Breien, N. Dak. & 63 & $1996-2007$ & 35 & $<10$ & 4,500 \\
\hline 174 & Buffalo Creek Tributary near Gascoyne, N. Dak. & 4 & $1974-1977$ & 125 & 60 & 260 \\
\hline 175 & James River near Manfred, N. Dak. & 34 & 1985-1998 & $<10$ & $<10$ & 44 \\
\hline 176 & James River near Grace City, N. Dak. & 99 & $1985-2007$ & 13 & $<10$ & 96 \\
\hline 177 & James River above Arrowwood Lake near Kensal, N. Dak. & 169 & $1985-2008$ & 16 & $<10$ & 206 \\
\hline 178 & Kelly Creek below Niccum Reservoir near Bordulac, N. Dak. & 8 & 1986-1989 & 11 & $<10$ & 81 \\
\hline 186 & James River at N. Dak./S. Dak. State line & 9 & 1998-1999 & 62 & 12 & 207 \\
\hline \multicolumn{7}{|c|}{ Suspended-sediment concentration, in $\mathrm{mg} / \mathrm{L}$} \\
\hline 5 & Red River of the North below Wahpeton, N. Dak. & 21 & 1997-1999 & 49 & 4 & 125 \\
\hline 6 & Red River of the North at Hickson, N. Dak. & 102 & $1975-2003$ & 72 & 3 & 379 \\
\hline 10 & Wild Rice River near Abercrombie, N. Dak. & 62 & 1975-1994 & 42 & 4 & 540 \\
\hline 11 & Red River of the North at Fargo, N. Dak. & 62 & $1975-2008$ & 115 & 4 & 21,400 \\
\hline 12 & Red River of North below Fargo, N. Dak. & 56 & 1973-1977 & 43 & 4 & 436 \\
\hline 14 & Red River of the North near Harwood, N. Dak. & 21 & 1997-1999 & 164 & 7 & 310 \\
\hline 18 & Sheyenne River near Warwick, N. Dak. & 2 & 2001 & 58 & 21 & 95 \\
\hline 24 & Starkweather Coulee near Webster, N. Dak. & 2 & 2001 & 200 & 6 & 394 \\
\hline 34 & Sheyenne River below Baldhill Dam, N. Dak. & 2 & 2001 & 17 & 17 & 17 \\
\hline 36 & Sheyenne River at Lisbon, N. Dak. & 42 & 1977-1995 & 102 & 8 & 730 \\
\hline 37 & Sheyenne River near Kindred, N. Dak. & 113 & $1975-2001$ & 74 & 10 & 2,850 \\
\hline 44 & Maple River near Mapleton, N. Dak. & 1 & 1975 & 120 & 120 & 120 \\
\hline
\end{tabular}


Table 1-4. Summary statistics for field measurements, total suspended solids, and suspended sediment at selected sites in North Dakota from 1970 through 2008. - Continued

$[\mu \mathrm{S} / \mathrm{cm}$, microsiemens per centimeter; deg. C, degrees Celsius; --, not available; mg/L, milligrams per liter; <, less than]

\begin{tabular}{|c|c|c|c|c|c|c|}
\hline $\begin{array}{c}\text { Site } \\
\text { identification } \\
\text { number } \\
\text { (table 1) }\end{array}$ & Site name & $\begin{array}{c}\text { Number of } \\
\text { samples }\end{array}$ & $\begin{array}{l}\text { Period of } \\
\text { record }\end{array}$ & Median & Minimum & Maximum \\
\hline \multicolumn{7}{|c|}{ Suspended-sediment concentration, in $\mathrm{mg} / \mathrm{L}$-Continued } \\
\hline 46 & Sheyenne River at Harwood, N. Dak. & 21 & 1997-1999 & 225 & 10 & 810 \\
\hline 52 & Red River of the North at Halstad, Minn. & 135 & $1976-2001$ & 76 & 6 & 730 \\
\hline 53 & Beaver Creek near Finley, N. Dak. & 101 & $1971-1996$ & 24 & $<1$ & 633 \\
\hline 55 & Goose River at Hillsboro, N. Dak. & 10 & 1994-2008 & 28 & 9 & 610 \\
\hline 56 & Red River of the North at Grand Forks, N. Dak. & 29 & $1993-2008$ & 125 & 6 & 1,110 \\
\hline 57 & Turtle River at Turtle River State Park near Arvilla, N. Dak. & 68 & $1993-2000$ & 15 & $<1$ & 981 \\
\hline 58 & Turtle River at Manvel, N. Dak. & 24 & $1980-2008$ & 34 & 6 & 106 \\
\hline 59 & Red River of the North at Oslo, Minn. & 54 & $1973-1977$ & 76 & 5 & 1,060 \\
\hline 62 & Forest River near Minto, N. Dak. & 21 & $1974-2008$ & 38 & 4 & 158 \\
\hline 65 & Middle Branch Park River near Edinburg, N. Dak. & 14 & $1978-1980$ & 192 & 6 & 1,340 \\
\hline 66 & Cart Creek at Mountain, N. Dak. & 9 & 1978-1979 & 574 & 138 & 2,920 \\
\hline 67 & Park River at Grafton, N. Dak. & 8 & $2007-2008$ & 14 & 4 & 31 \\
\hline 72 & Pembina River near Vang, N. Dak. & 91 & $1970-1979$ & 210 & 4 & 6,760 \\
\hline 73 & Little South Pembina River near Walhalla, N. Dak. & 115 & $1970-1995$ & 122 & 3 & 8,460 \\
\hline 74 & Pembina River at Walhalla, N. Dak. & 249 & $1970-1995$ & 681 & 4 & 19,900 \\
\hline 75 & Pembina River at Neche, N. Dak. & 8 & $2007-2008$ & 44 & 4 & 1,210 \\
\hline 76 & Tongue River at Akra, N. Dak. & 16 & 2003-2004 & 42 & 9 & 813 \\
\hline 78 & Red River of the North at Pembina, N. Dak., site 2 & 54 & 1994-2008 & 190 & 8 & 1,243 \\
\hline 79 & Red River of the North at Emerson, Manitoba & 123 & $1978-2001$ & 115 & 6 & 911 \\
\hline 81 & West Branch Short Creek near Columbus, N. Dak. & 22 & 1978-1981 & 22 & 3 & 70 \\
\hline 82 & Souris River near Sherwood, N. Dak. & 64 & $1974-1981$ & 23 & 4 & 1,400 \\
\hline 83 & Souris River near Foxholm, N. Dak. & 90 & 1972-1980 & 13 & 2 & 80 \\
\hline 87 & Souris River near Verendrye, N. Dak. & 93 & 1976-1986 & 16 & 4 & 1,150 \\
\hline 88 & Wintering River near Karlsruhe, N. Dak. & 28 & $1977-1980$ & 8 & 3 & 20 \\
\hline 89 & Souris River near Bantry, N. Dak. & 139 & $1985-2000$ & 26 & 2 & 746 \\
\hline 90 & Willow Creek near Willow City, N. Dak. & 134 & $1986-2000$ & 12 & 2 & 399 \\
\hline 91 & Stone Creek near Kramer, N. Dak. & 132 & $1986-2000$ & 14 & 2 & 310 \\
\hline 92 & Deep River near Upham, N. Dak. & 19 & 1989-2000 & 12 & 4 & 92 \\
\hline 95 & Cut Bank Creek at Upham, N. Dak. & 16 & 1999-2000 & 7 & 2 & 167 \\
\hline 96 & Deep River below Cut Bank Creek near Upham, N. Dak. & 102 & 1986-1989 & 11 & $<1$ & 100 \\
\hline 97 & Boundary Creek near Landa, N. Dak. & 134 & $1986-2000$ & 16 & $<1$ & 76 \\
\hline 98 & Souris River near Westhope, N. Dak. & 250 & 1974-1994 & 21 & 3 & 1,045 \\
\hline 100 & Missouri River near Williston, N. Dak. & 68 & 1975-1982 & 178 & 16 & 6,740 \\
\hline 102 & Stony Creek near Williston, N. Dak. & 39 & $1977-1981$ & 78 & 16 & 1,370 \\
\hline 104 & Beaver Creek near Ray, N. Dak. & 51 & 1977-1982 & 32 & 4 & 180 \\
\hline 106 & Bear Den Creek near Mandaree, N. Dak. & 177 & $1971-1995$ & 104 & 8 & 11,300 \\
\hline 111 & Deep Creek near Amidon, N. Dak. & 53 & 1977-1982 & 71 & 9 & 978 \\
\hline 113 & Beaver Creek near Trotters, N. Dak. & 56 & $1977-1981$ & 80 & 14 & 2,560 \\
\hline 114 & Little Missouri River near Watford City, N. Dak. & 126 & 1971-1994 & 1,028 & 27 & 40,400 \\
\hline
\end{tabular}


Table 1-4. Summary statistics for field measurements, total suspended solids, and suspended sediment at selected sites in North Dakota from 1970 through 2008. - Continued

$[\mu \mathrm{S} / \mathrm{cm}$, microsiemens per centimeter; deg. $\mathrm{C}$, degrees Celsius; --, not available; mg/L, milligrams per liter; <, less than]

\begin{tabular}{|c|c|c|c|c|c|c|}
\hline $\begin{array}{c}\text { Site } \\
\text { identification } \\
\text { number } \\
\text { (table 1) }\end{array}$ & Site name & $\begin{array}{l}\text { Number of } \\
\text { samples }\end{array}$ & $\begin{array}{l}\text { Period of } \\
\text { record }\end{array}$ & Median & Minimum & Maximum \\
\hline \multicolumn{7}{|c|}{ Suspended-sediment concentration, in $\mathrm{mg} / \mathrm{L}$-Continued } \\
\hline 115 & Missouri River at Garrison Dam, N. Dak. & 67 & 1974-2007 & 2 & $<1$ & 11 \\
\hline 116 & Knife River at Manning, N. Dak. & 50 & $1977-1982$ & 62 & 11 & 173 \\
\hline 118 & Knife River at Marshall, N. Dak. & 46 & $1977-1981$ & 89 & 23 & 1,510 \\
\hline 119 & Elm Creek near Golden Valley, N. Dak. & 16 & 1977-1981 & 78 & 15 & 864 \\
\hline 121 & Coyote Creek near Zap, N. Dak. & 55 & $1977-1983$ & 48 & 6 & 638 \\
\hline 122 & Brush Creek near Beulah, N. Dak. & 90 & 1974-1988 & 25 & 2 & 388 \\
\hline 127 & Knife River at Hazen, N. Dak. & 132 & 1974-1993 & 66 & 8 & 3,780 \\
\hline 128 & Antelope Creek above Hazen, N. Dak. & 35 & $1977-1982$ & 28 & 3 & 406 \\
\hline 129 & West Branch Antelope Creek No. 4 near Zap, N. Dak. & 196 & 1977-1982 & 29 & 3 & 325 \\
\hline 130 & West Branch Antelope Creek near Hazen, N. Dak. & 17 & $1978-1983$ & 60 & 10 & 1,120 \\
\hline 131 & Coal Creek near Stanton, N. Dak. & 34 & $1977-1981$ & 47 & 6 & 894 \\
\hline 132 & Alderin Creek near Fort Clark, N. Dak. & 40 & $1977-1982$ & 159 & 9 & 6,100 \\
\hline 133 & Coal Lake Coulee near Hensler, N. Dak. & 18 & 1978-1982 & 35 & 5 & 887 \\
\hline 134 & Buffalo Creek near Washburn, N. Dak. & 42 & $1978-1983$ & 68 & $<1$ & 824 \\
\hline 137 & Square Butte Creek near Hannover, N. Dak. & 29 & 1977-1981 & 17 & 3 & 425 \\
\hline 147 & Green River near New Hradec, N. Dak. & 49 & $1977-1982$ & 34 & 9 & 222 \\
\hline 156 & Heart River near Mandan, N. Dak. & 120 & $1972-1993$ & 42 & 4 & 2,690 \\
\hline 158 & Apple Creek near Menoken, N. Dak. & 20 & 1979-1981 & 16 & 3 & 53 \\
\hline 159 & Missouri River near Schmidt, N. Dak. & 6 & 1975-1976 & 146 & 26 & 352 \\
\hline 160 & Cannonball River at New England, N. Dak. & 42 & 1978-1981 & 58 & 19 & 1,560 \\
\hline 161 & Coal Bank Creek near Havelock, N. Dak. & 74 & $1975-1982$ & 22 & 3 & 481 \\
\hline 162 & Cannonball River at Regent, N. Dak. & 51 & $1977-1981$ & 67 & 6 & 770 \\
\hline 167 & Timber Creek near Bentley, N. Dak. & 54 & 1977-1981 & 44 & 10 & 572 \\
\hline 170 & Cannonball River at Breien, N. Dak. & 139 & 1972-1992 & 90 & 6 & 9,100 \\
\hline 174 & Buffalo Creek Tributary near Gascoyne, N. Dak. & 75 & $1974-1987$ & 63 & 2 & 1,200 \\
\hline 175 & James River near Manfred, N. Dak. & 32 & 1985-1995 & 11 & $<1$ & 92 \\
\hline 176 & James River near Grace City, N. Dak. & 54 & 1985-1995 & 11 & 2 & 243 \\
\hline 177 & James River above Arrowwood Lake near Kensal, N. Dak. & 155 & $1985-2008$ & 30 & 2 & 288 \\
\hline 178 & Kelly Creek below Niccum Reservoir near Bordulac, N. Dak. & 10 & 1986-1989 & 8 & 3 & 24 \\
\hline
\end{tabular}




\begin{tabular}{|c|c|c|c|c|c|c|}
\hline $\begin{array}{c}\text { Site } \\
\text { identification } \\
\text { number } \\
\text { (table 1) }\end{array}$ & Site name & $\begin{array}{c}\text { Number of } \\
\text { samples }\end{array}$ & $\begin{array}{l}\text { Period of } \\
\text { record }\end{array}$ & Median & Minimum & Maximum \\
\hline \multicolumn{7}{|c|}{ Suspended-sediment concentration, in $\mathrm{mg} / \mathrm{L}$-Continued } \\
\hline 179 & James River near Pingree, N. Dak. & 107 & 1984-2008 & 32 & 2 & 784 \\
\hline 182 & James River at Jamestown, N. Dak. & 79 & $1985-1995$ & 39 & 8 & 154 \\
\hline 185 & James River at Oakes, N. Dak. & 61 & 1985-1995 & 89 & 2 & 544 \\
\hline
\end{tabular}




\section{Appendix 2. Weighted regression methods for relating flow and concentration differences}

Figure 2-1. Weighted linear regression model for mean relative differences in streamflow and the logarithm of mean relative differences in sulfate concentration.

Figure 2-2. Partially weighted residuals from the weighted regression model for sulfate and sample size used to compute mean relative streamflow and concentration differences. SD is the standard deviation of the partially weighted residuals as a function of sample size

Figure 2-3. Partially weighted residuals from the weighted regression model for sulfate and mean relative streamflow differences. SD is the standard deviation of the partially weighted residuals as a function of the mean absolute difference between downstream and upstream flow

Figure 2-4. Weighted linear regression model for mean relative differences in streamflow and the logarithm of mean relative differences in sulfate concentration, standardized to a sample size of $n=40$. 
In this appendix, the methods used to determine the percentile lines in figures 31 and 32 are described. Consider sulfate first (fig. 31). Let $X$ be the mean absolute difference between the downstream and upstream flow, as a percent of downstream flow, and let $Y$ be the mean absolute difference between the downstream and upstream sulfate concentration, as a percent of downstream concentration. It was first noted by examining graphs of $X$ and $\log (Y)$ that $X$ was linearly related to $\log (Y)$ (base-10 logarithm). This is shown in figure 2-1, where the vertical axis is in log units. The regression line was obtained from a weighted simple linear regression model,

$$
\log (Y)=\alpha+\beta X+\omega(X, n) e
$$

where

$$
\begin{aligned}
\alpha & \text { is the intercept; } \\
\beta & \text { is the slope; } \\
\omega(X, n) & \text { is a weight that depends on } X \text { and } n ; \\
n & \text { is the sample size (the number of pairs of } \\
& \text { values used to compute the mean); and } \\
e & \text { is the error, assumed to be normally } \\
& \text { distributed with mean zero and constant } \\
& \text { variance. }
\end{aligned}
$$

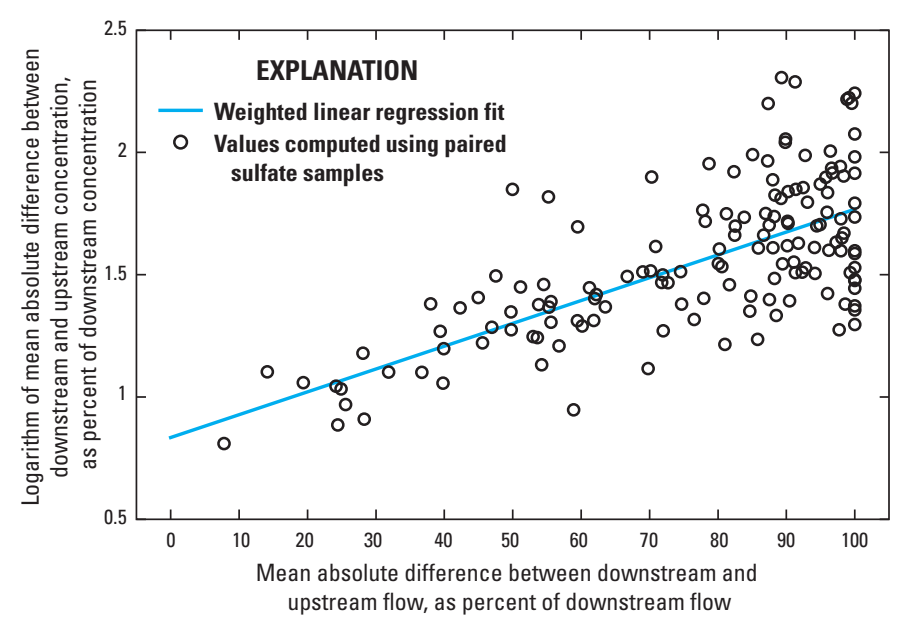

Figure 2-1. Weighted linear regression model for mean relative differences in streamflow and the logarithm of mean relative differences in sulfate concentration.

The weights were determined through trial and error, to make the residual variance as close to a constant (with respect to $X$ and $n$ ) as possible. The weight function is given by:

$$
\omega(X, n)=[0.32+8 / n]^{1 / 2}[1+(X-50) / 100]
$$

The weights decrease as the sample size increases (smaller error variance for larger sample size) and increase as $X$ increases. The relation between the partially weighted residuals (PWR) and sample size:

$$
\operatorname{PWR}(n)=\omega(X, n) e /[1+(X-50) / 100]=[0.32+8 / n]^{1 / 2} e
$$

is shown in figure 2-2. The points in this figure show a scatterplot of the square root of $\mathrm{n}$ and $\operatorname{PWR}(n)$ and the lines are plus and minus 2 standard deviations (2SD), where $\mathrm{SD}=[0.32+8 / n]^{1 / 2}$ sterr, which are approximate 95 -percent limits on the partial residuals assuming e is normally distributed and sterr is the standard error of the regression (the estimate of the standard deviation of the errors from the regression model, in this case sterr $=0.196$ ). Although the errors are somewhat positively skewed, the weights provide a good representation of the decrease in variance as the sample size increases. The relation between the partially weighted residuals and $X$,

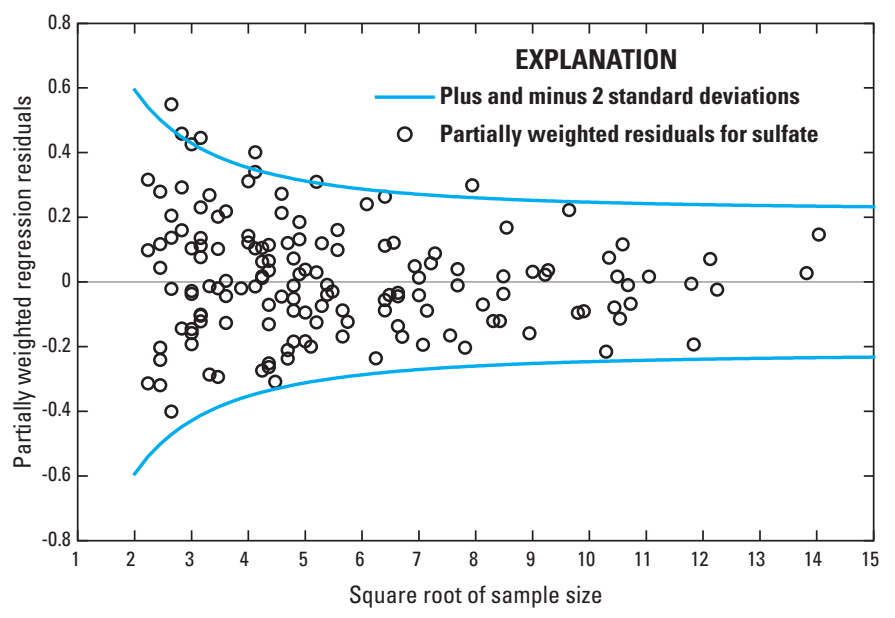

Figure 2-2. Partially weighted residuals from the weighted regression model for sulfate and sample size used to compute mean relative streamflow and concentration differences. SD is the standard deviation of the partially weighted residuals as a function of sample size

$$
\operatorname{PWR}(X)=\omega(X, n) \mathrm{e} /[0.32+8 / n]^{1 / 2}=[1+(X-50) / 100] e
$$

is shown in figure $2-3$. The points in this figure show a scatterplot of $X$ and $\operatorname{PWR}(X)$ and the lines are plus and minus 2SD, where $\mathrm{SD}=[1+(X-50) / 100]$ sterr, which are approximate 95-percent limits on the partial residuals assuming e is normally distributed. Again, although the errors are somewhat positively skewed, the weights provide a good representation of the increase in variance as $X$ increases.

Next, the errors were adjusted to remove the effects of variable sample sizes. A reference sample size of $n=40$ was chosen (for example, 8 samples per year over 5 years or 4 samples per year over 10 years). The adjusted errors are:

$$
\left[e^{*} \mid n=40\right]=[0.32+8 / 40]^{1 / 2} e /[0.32+8 / n]^{1 / 2}
$$

and the final fitted adjusted regression model is: 


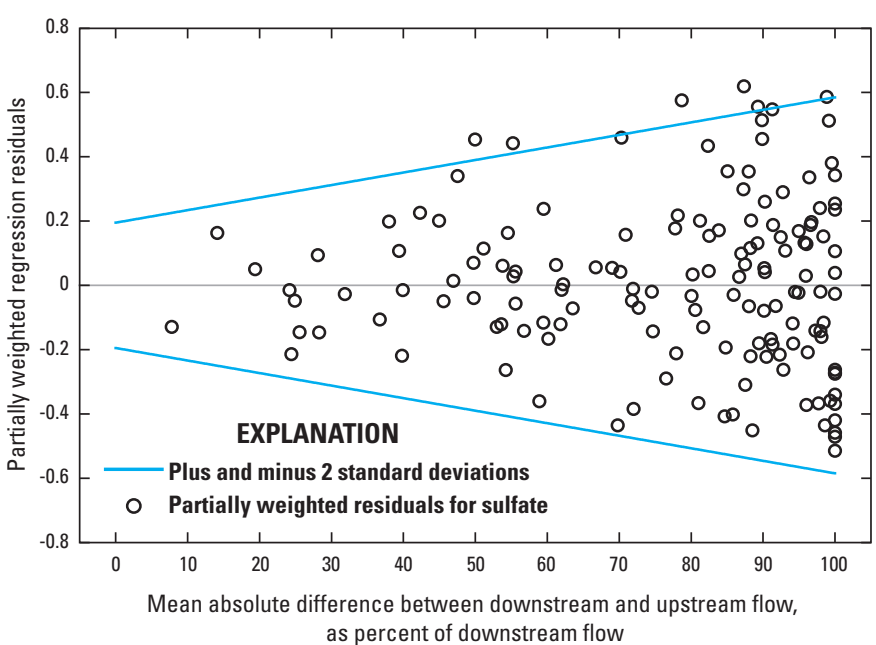

Figure 2-3. Partially weighted residuals from the weighted regression model for sulfate and mean relative streamflow differences. SD is the standard deviation of the partially weighted residuals as a function of the mean absolute difference between downstream and upstream flow

$$
\log (Y)=0.83+0.0093 X+[1+(X-50) / 100]\left[e^{*} \mid n=40\right]
$$

where the estimated standard deviation of $\left[e^{*} \mid n=40\right]$ is 0.196 . The adjusted regression model is illustrated in figure $2-4$. The 50th percentile is just the fitted regression line and the 90th percentile is:

$$
\begin{gathered}
\text { 90th pct }[\log (Y)]=0.83+0.0093 X+1.28 \\
{[1+(X-50) / 100] 0.196}
\end{gathered}
$$

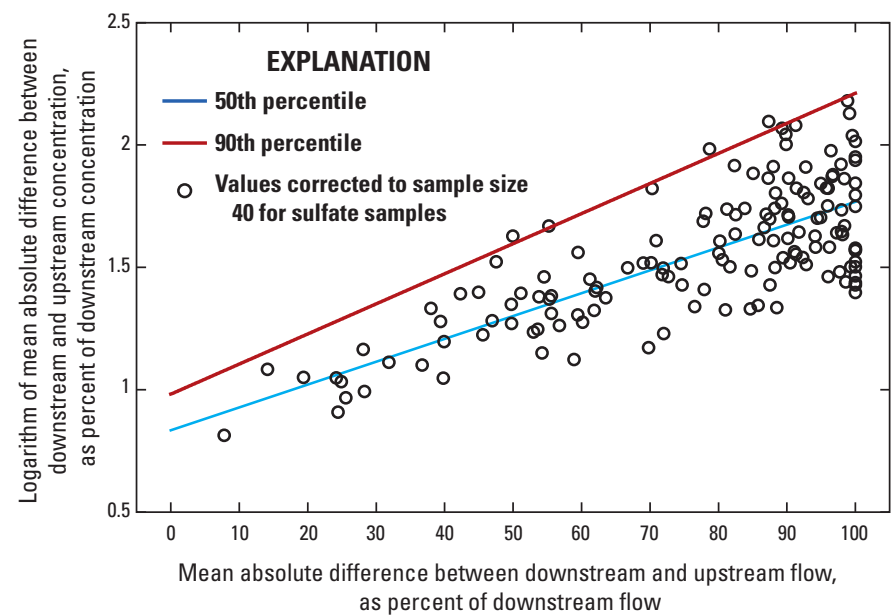

Figure 2-4. Weighted linear regression model for mean relative differences in streamflow and the logarithm of mean relative differences in sulfate concentration, standardized to a sample size of $n=40$. where 1.28 is the 90th percentile of a standard normal distribution. Figure 31 is simply figure $2-4$ with the values expressed in arithmetic rather than log-transformed scale.

The same procedure used for sulfate was used to perform a weighted regression analysis for total phosphorus, but with different weights. The weight function for total phosphorus was:

$$
\omega(X, n)=[0.016+8 / n]^{1 / 2}[1+(X-50) / 100]
$$

the adjusted errors for phosphorus were:

$$
\left[e^{*} \mid n=40\right]=[0.016+8 / 40]^{1 / 2} e /[0.016+8 / n]^{1 / 2}
$$

and the final fitted adjusted regression model was:

$\log (Y)=1.43+0.0034 X+[1+(X-50) / 100]\left[e^{*} \mid n=40\right]$

and the estimated standard deviation of $\left[e^{*} \mid n=40\right]$ was 0.161 . Figure 32 was obtained using this regression model and reversing the transformed resulting percentiles in a similar manner to sulfate. 



\section{Appendix 3. Water quality data used in this report}

Appendix3.xlsx contains the four data tables listed below and is available for download at: http://pubs.usgs.gov/sir/2012/5216/downloads/appendix3.xlsx

Table 3-1. Major ion constituent data at selected sites in North Dakota from 1970 through 2008.

Table 3-2. Nutrient constituent data at selected sites in North Dakota from 1970 through 2008.

Table 3-3 Trace metal constituent data at selected sites in North Dakota from 1970 through 2008.

Table 3-4. Field measurement, total suspended solids, and suspended-sediment data at selected sites in North Dakota from 1970 through 2008. 
Publishing support provided by:

Rolla Publishing Service Center

For more information concerning this publication, contact:

Director, USGS North Dakota Water Science Center

821 East Interstate Avenue

Bismarck, North Dakota 58503

(701) 250-7400

Or visit the North Dakota Water Science Center Web site at: http://nd.water.usgs.gov/ 



$$
\begin{aligned}
& \frac{\mathbb{2}}{3} \\
& \frac{3}{3}
\end{aligned}
$$

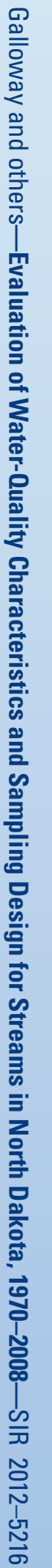

